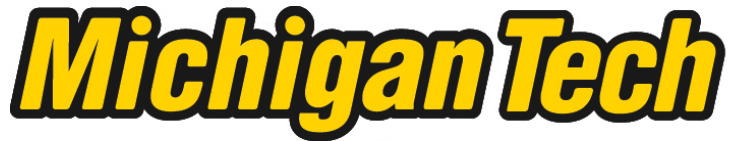 \\ Michigan Technological University Create the Future Digital Commons @ Michigan Tech
}

2015

DESIGN AND DEVELOPMENT OF BODIPY-BASED FLUORESCENT PROBES FOR SENSING AND IMAGING OF CYANIDE, Zn (II) IONS, LYSOSOMAL pH AND CANCER CELLS

Jingtuo Zhang

Michigan Technological University

Follow this and additional works at: https://digitalcommons.mtu.edu/etds

Part of the Analytical Chemistry Commons, and the Organic Chemistry Commons

Copyright 2015 Jingtuo Zhang

Recommended Citation

Zhang, Jingtuo, "DESIGN AND DEVELOPMENT OF BODIPY-BASED FLUORESCENT PROBES FOR SENSING AND IMAGING OF CYANIDE, Zn (II) IONS, LYSOSOMAL pH AND CANCER CELLS", Master's Thesis, Michigan Technological University, 2015.

https://doi.org/10.37099/mtu.dc.etds/913

Follow this and additional works at: https://digitalcommons.mtu.edu/etds

Part of the Analytical Chemistry Commons, and the Organic Chemistry Commons 


\title{
DESIGN AND DEVELOPMENT OF BODIPY-BASED FLUORESCENT PROBES FOR SENSING AND IMAGING OF CYANIDE, Zn (II) IONS, LYSOSOMAL pH AND CANCER CELLS
}

\author{
By \\ Jingtuo Zhang
}

\begin{abstract}
A DISSERTATION
Submitted in partial fulfillment of the requirements for degree of DOCTOR OF PHILOSOPHY In Chemistry
\end{abstract} MICHIGAN TECHNOLOGICAL UNIVERSITY 2015

(C) 2015 Jingtuo Zhang 
This dissertation has been approved in partial fulfillment of the requirements for the Degree of DOCTOR OF PHILOSOPHY in Chemistry.

Department of Chemistry

Dissertation Advisor: $\quad$ Dr. Haiying Liu

Committee member: $\quad$ Dr. Ashutosh Tiwari

Committee member: $\quad$ Dr. Patricia A. Heiden

Committee member: $\quad$ Dr. Xiaoqing Tang

Department Chair: $\quad$ Dr. Cary F. Chabalowski 


\section{Table of Contents}

Table of Contents ................................................................................................ ii

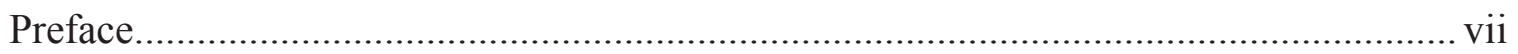

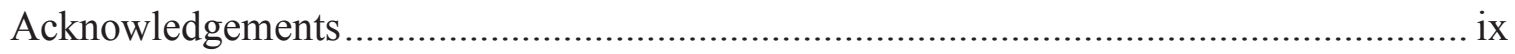

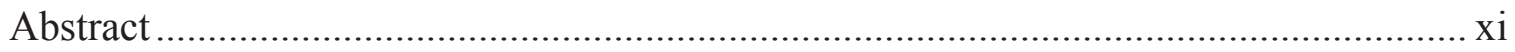

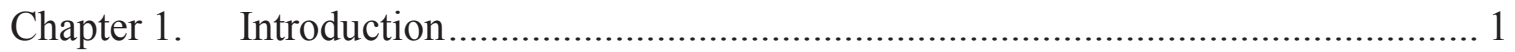

1.1 Fluorescence sensing and fluorescent probes............................................. 1

1.2 Design criteria of fluorescent probes ............................................................ 3

1.3 BODIPY-based fluorescent probes .............................................................. 5

1.3.1 Merits of BODIPY-based fluorescent probes ........................................... 5

1.3.2 Drawbacks and challenges of current BODIPY-based fluorescent probes .. 6

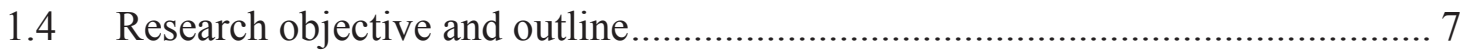

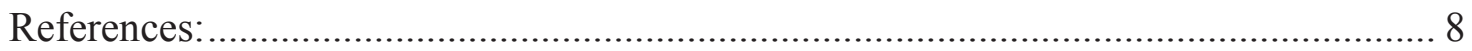

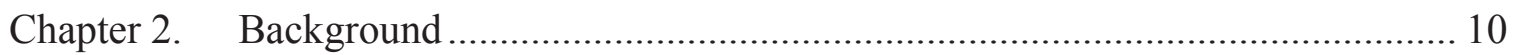

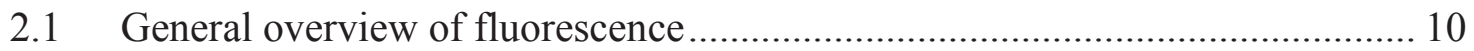

2.2 Common organic fluorophores for fluorescent sensing and labeling ............... 12

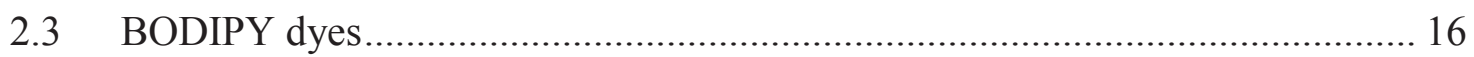

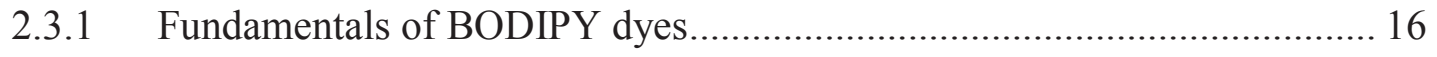

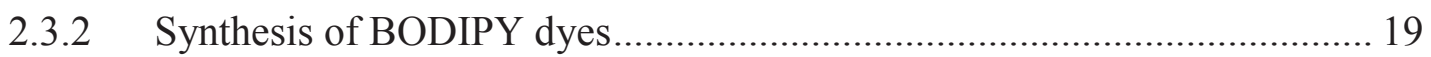

2.4 Modification strategies of BODIPY core: functional sites and reactions ......... 22

2.4.1 Modifications to meso-aromatic substituents on the BODIPY core ........... 22

2.4.2 Functionalization at the 2,6-positions of BODIPY core .......................... 24

2.4.3 Functionalization at the 3,5-positions of BODIPY core .......................... 28

2.4.4 Substitution of fluorine atoms at 4-position of BODIPY core .................. 31

2.5 Tuning the absorption and emission wavelengths......................................... 33

2.5.1 Tuning through introducing electron donating and halogen atoms ........... 33

2.5.2 Tuning through extension of $\pi$-conjugation ...................................... 34

2.6 Modulation of fluorescence of BODIPY-based fluorescent probes.................. 38

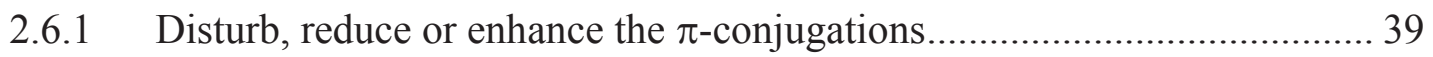

2.6.2 Photoinduced electron transfer (PET) .................................................... 40

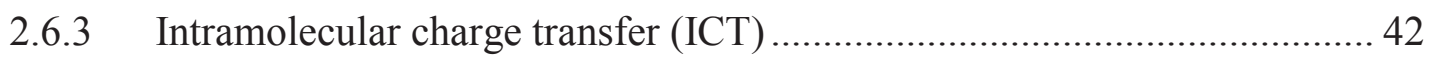

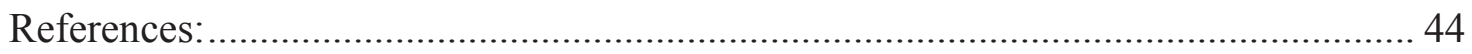


Chapter 3. Highly Water-soluble Neutral BODIPY Dyes with Controllable Fluorescence Quantum Yields ................................................................................ 54

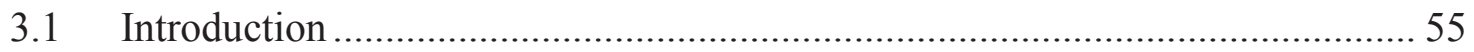

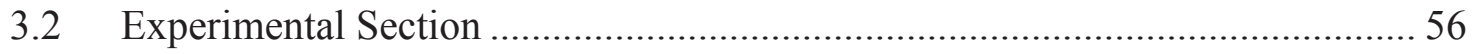

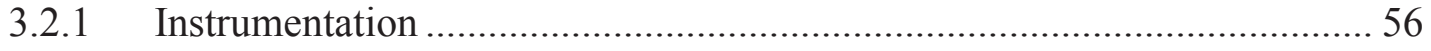

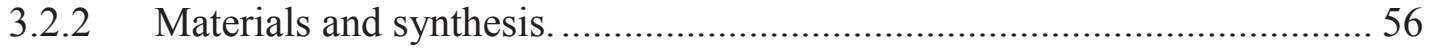

3.2.3 Optical Measurements ............................................................................ 71

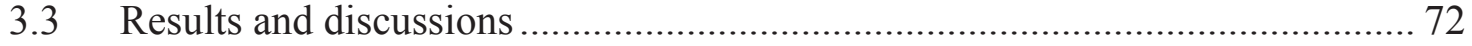

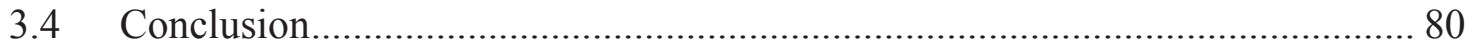

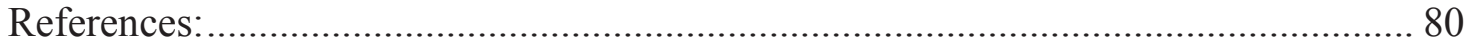

Chapter 4. Controlled Knoevenagel Reactions of Methyl Groups of 1,3,5,7Tetramethyl BODIPY Dyes for Unique BODIPY Dyes ................................................ 83

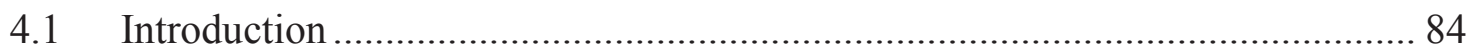

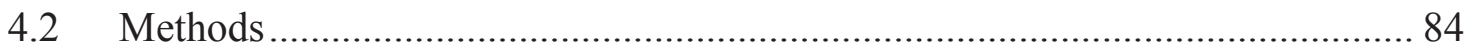

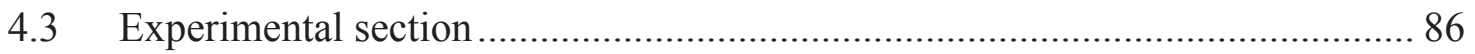

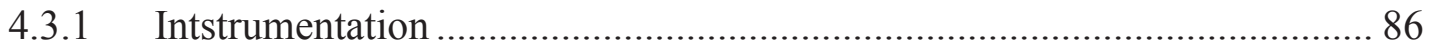

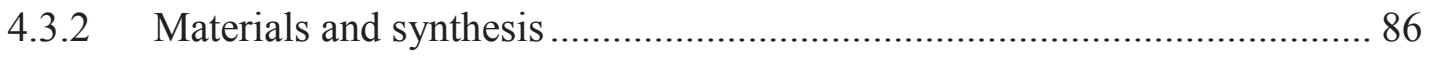

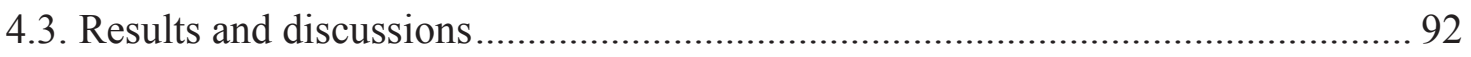

4.3.1 Synthesis approach and optical properties .................................................. 92

4.3.2 Spectroscopic responses of 2,6-diformal BODIPY dyes to Cysteine......... 97

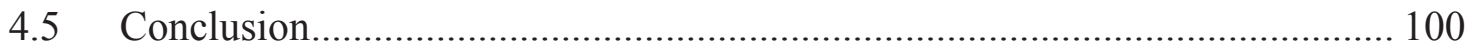

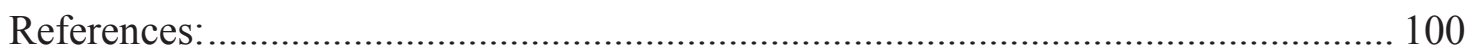

Chapter 5. BODIPY-based Ratiometric Fluorescent Probes for Sensitive and Selective

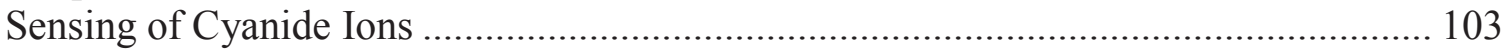

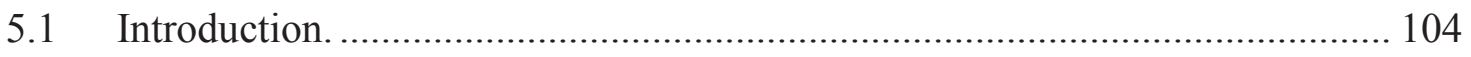

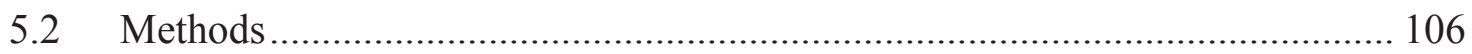

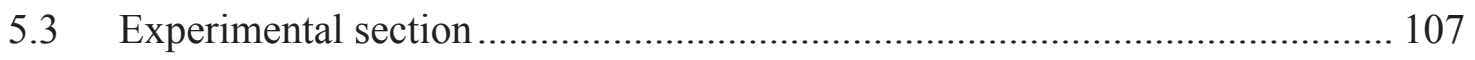

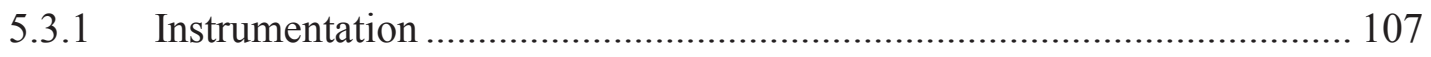

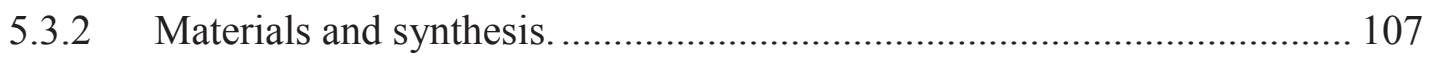

5.3.3 Optical Measurements ....................................................................... 110

5.3.4 Non-linear fitting of absorption titration data of probe 5-A ..................... 111

5.3.5 Computational Methods...................................................................... 112

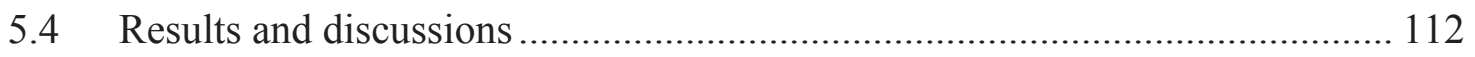

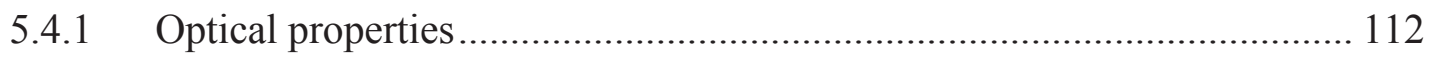


5.4.2 Spectroscopic responses to cyanide ion and concentration dependency .. 113

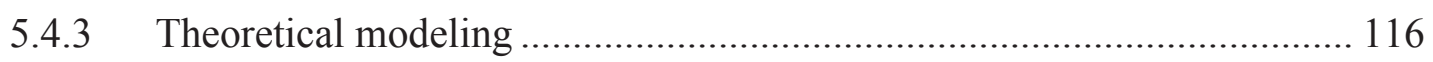

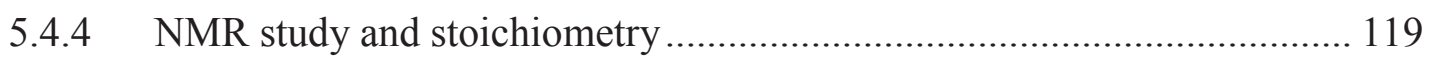

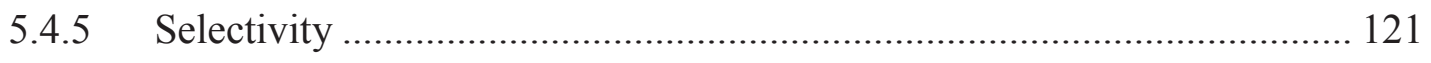

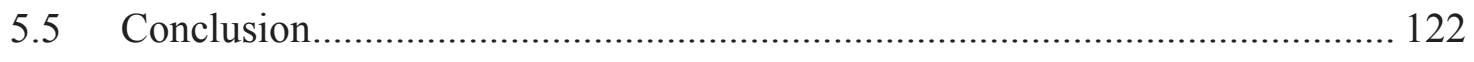

References:

Chapter 6. Highly Water-soluble BODIPY-based Fluorescent Probes for Sensitive Fluorescent Sensing of Zinc (II) ........................................................................ 125

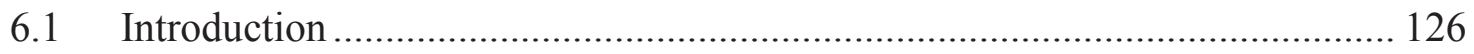

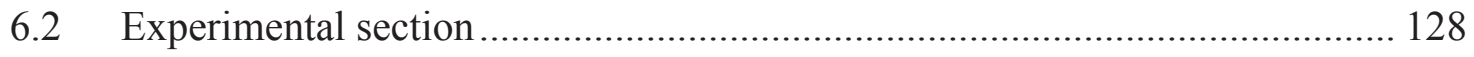

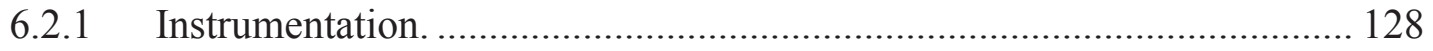

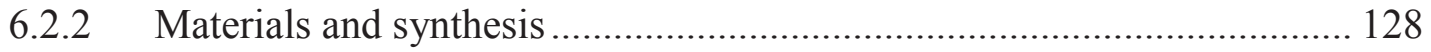

6.2.3 Optical measurements and buffer conditions:............................................ 131

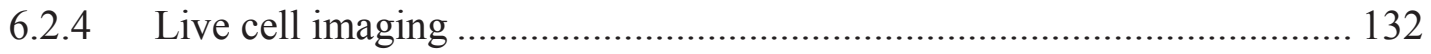

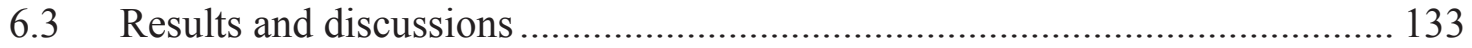

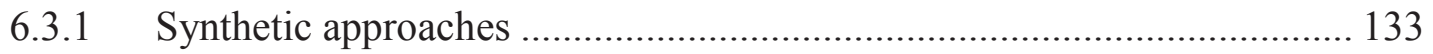

6.3.2 Spectroscopic responses to zinc (II) and concentration dependency........ 135

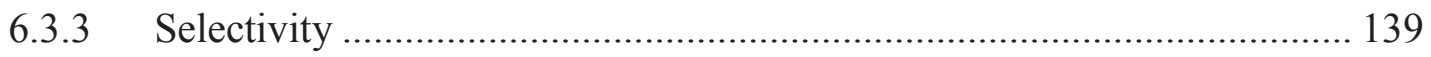

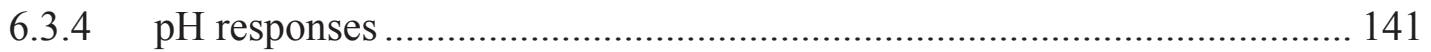

6.3.5 Fluorescence responses of probe 6-C to zinc (II) in living cells............... 142

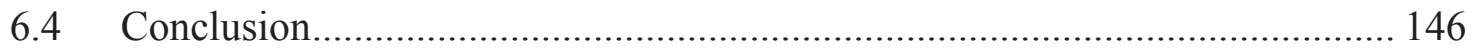

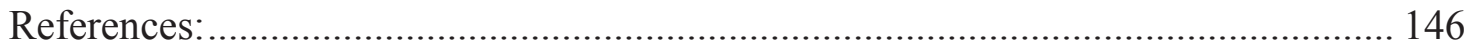

Chapter 7. Near-infrared Fluorescent Probes Based on Piperazine-Functionalized BODIPY Dyes for Sensitive Detection of Lysosomal pH.............................................. 151

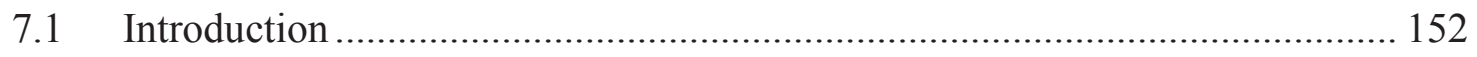

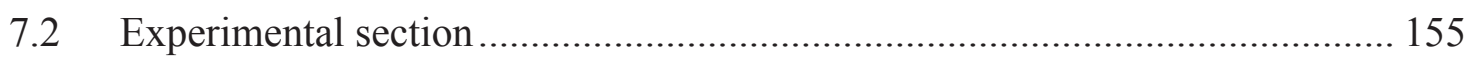

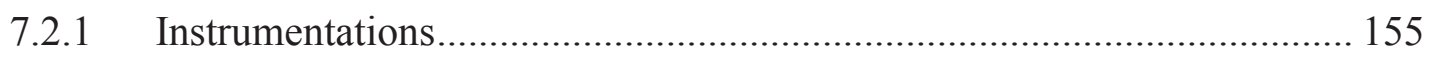

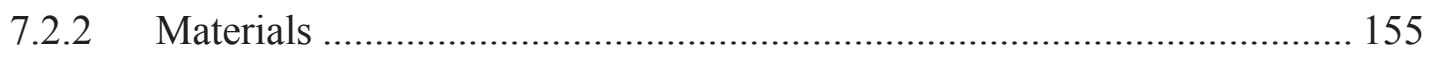

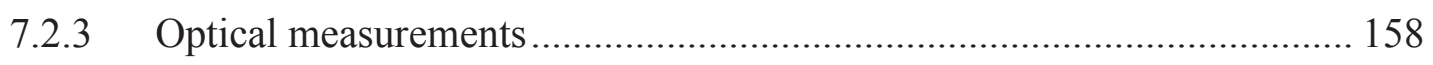

7.2.4 Determination of $\mathrm{p} K_{\mathrm{a}}$ by fluorometric titration....................................... 159

7.2.5 Cell culture and fluorescence imaging................................................... 159

7.2.6 Determination of cellular uptake efficiency ............................................ 160

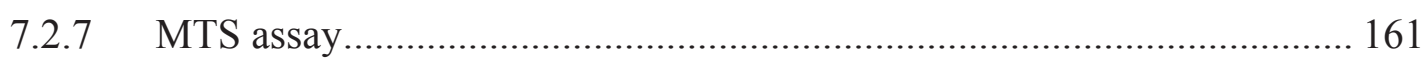

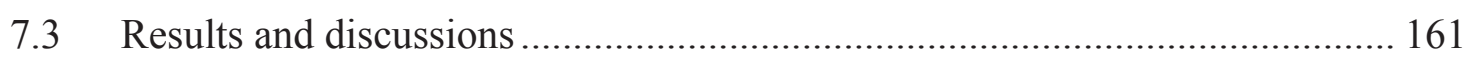




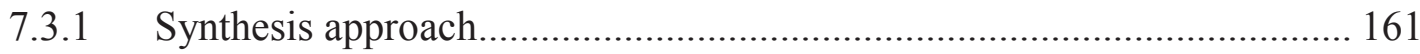

7.3.2 Optical properties of fluorescent probes ................................................. 163

7.3.3 Optical responses of fluorescent probes to $\mathrm{pH}$......................................... 164

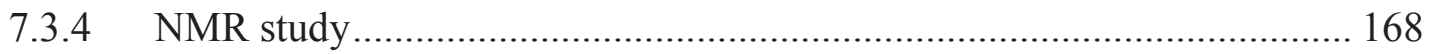

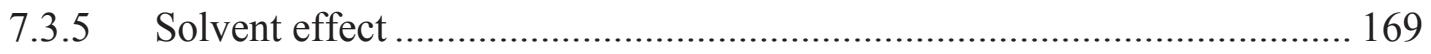

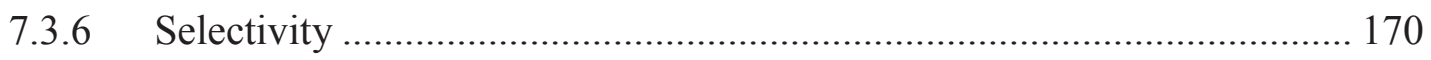

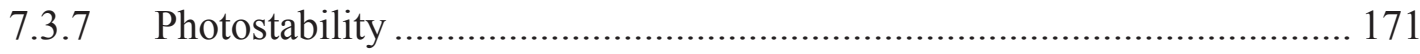

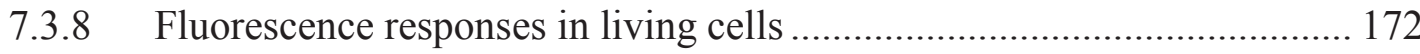

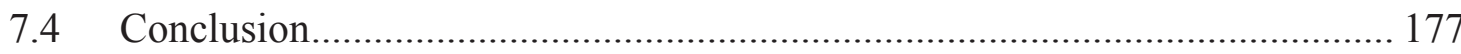

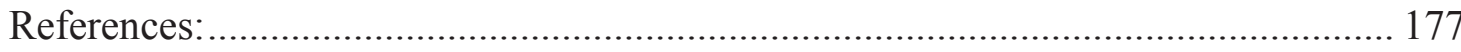

Chapter 8. Highly Water-soluble, Near-infrared Emissive Copolymeric BODIPY Dye Bearing RGD Peptide Residues for Cancer Imaging.................................................. 182

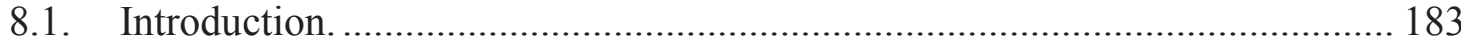

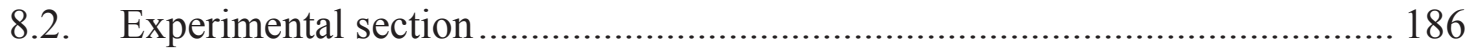

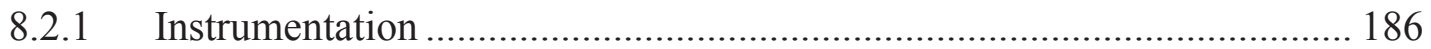

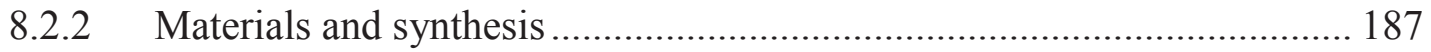

8.2.3 Cell Culture and Fluorescence Imaging ................................................... 190

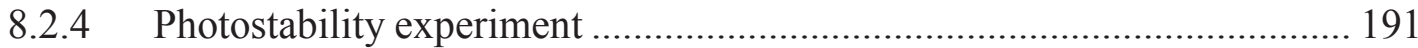

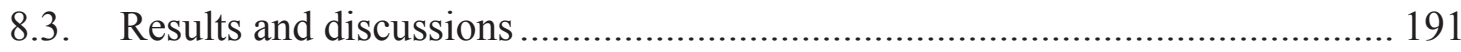

8.3.1 Synthesis of BODIPY Polymeric Dyes ...................................................... 191

8.3.2 Optical properties of BODIPY-based polymeric dyes............................... 194

8.3.3 Application of BODIPY polymeric dye bearing cancer-homing RGD peptides in near-infrared imaging of cancer cells ................................................... 197

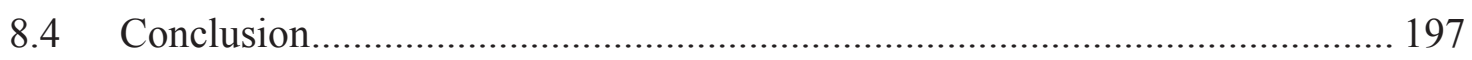

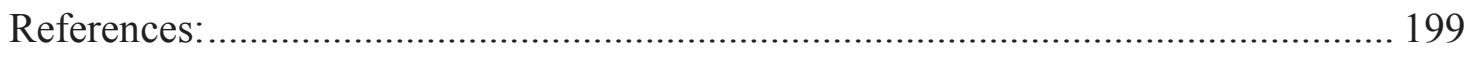

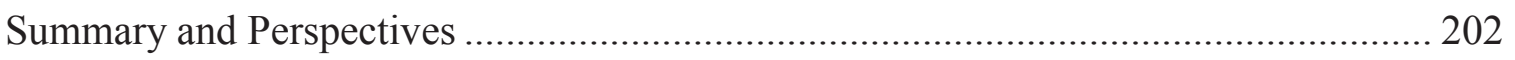

Appendix A Supporting Information for Chapter 3 …………………………........ 205

Appendix B Supporting Information for Chapter 4 ............................................... 227

Appendix C Supporting Information for Chapter 5 ............................................... 250

Appendix D Supporting Information for Chapter 6............................................... 273

Appendix E Supporting Information for Chapter 7 ............................................... 285

Appendix F Supporting Information for Chapter 8 ................................................. 305

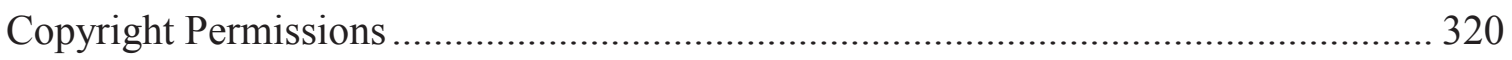




\section{Preface}

All the contents in chapter 1, 2 and summary section were written by the author of this dissertation. The contents in other six chapters were edited and organized by the author of this dissertation based on previously published papers, which are described in detail as below.

Chapter 3 is based on the multi-author article that was previously published in "Organic Letters 2011, 13 (3), 438-441". The author of this dissertation was responsible for collection and analysis of all spectroscopic data and a portion of synthesis work of compounds. The major synthetic work was done by Dr. Shilei Zhu. Dr. Giri Vegesna and Dr. Sarah Green also participated in the data analysis. Dr. Haiying Liu is the corresponding author and he revised the manuscript.

Chapter 4 is based on the multi-author article that was previously published in " $R S C$ Advances 2012, 2 (2), 404-407'. The author of this dissertation was responsible for collection and analysis of all spectroscopic data of all compounds and conducting experiments for cysteine sensing. All the syntheses of compounds were conducted by Dr. Shilei Zhu. Dr. Matthias Zeller and Dr. Rudy Luck conducted of X-ray single crystal structure measurements. Dr. Sarah Green, Dr. Ashutosh Tiwari, Dr. Giri Vegesna and Mrs. Haihua Li also participated in the data analysis. Dr. Haiying Liu is the corresponding author and he revised the manuscript.

Chapter 5 is based on the multi-author article that was previously published in " $R S C$ Advances 2013, 3 (1), 68-72." The author of this dissertation was responsible for the synthetic work and all spectroscopic properties collection, fluorescence sensing measurements and data analysis. Dr. Shilei Zhu helped to synthesize some starting materials. Computational modeling work was done by Dr. Loredana Valenzano. Dr. Haiying Liu is the corresponding author and he revised the manuscript.

Chapter 6 is based on the multi-author article that was previously published in "Journal of Materials Chemistry B 2013, 1 (12), 1722-1728". The author of this dissertation was responsible for all spectroscopic properties collection, $\mathrm{Zn}$ (II) ions 
fluorescence sensing measurements and data analysis. Dr. Shilei Zhu did major synthetic work. Dr. Giri Vegesna provided some starting material for synthesis. Dr. Jagadeesh Janjanam, who was the postdoctoral associate in Dr. Ashutosh Tiwari's group, did all cell imaging work. Dr. Haiying Liu and Dr. Ashutosh Tiwari are the corresponding authors and they revised the manuscript.

Chapter 7 is based on the multi-author article that was previously published in "Journal of Materials Chemistry B 2015, 3 (10), 2173-2184". The author of this dissertation is responsible for all synthetic work, NMR study, spectroscopic analysis and majority of manuscript writing. Mr. Cong Li helped to conducted spectroscopic data collection and Mr. Fei Xie helped to synthesize starting materials. Mrs. Mu Yang and Mr. Nethaniah Dorh did all cell imaging work. Dr. Haiying Liu, Dr. Ashutosh Tiwari and Dr. Fen-Tair Luo are the corresponding authors and they revised the manuscript.

Chapter 8 is based on the multi-author article that was previously published in "Analytica Chimica Acta 2013, 758, 138-144". The author of this dissertation was responsible for all spectroscopic properties collection and partial of synthesis. Dr. Shilei Zhu did majority of synthetic work. Cancer cell imaging was done by Dr. Jianjun Wei, Dr. Jagadeesh Janjanam and Dr. Ashutosh Tiwari. Mr. Jianheng Bi and Dr. Giri Vegesna participated in the data analysis and manuscript revision. Dr. Haiying Liu and Dr. Jianjun Wei are the corresponding authors and they revised the manuscript.

In addition, all high-resolution mass spectrometry (HRMS) of compounds were conducted by Dr. Fen-Tair Luo (Academia Sinica). 


\section{Acknowledgements}

There are numbers of great people to whom I would like to express my gratitude during my Ph.D. journey. Without their help and support, it is even not possible to complete this brain-wretch task.

First, I would like to express my heartfelt thanks to my advisor, Dr. Haiying Liu for his guidance and support towards my graduate study. From course-work study to research projects, Dr. Liu was always there giving me patient and concise advice when I encountered problems. His enthusiasm, confidence, earnest endeavors, diligent work and keen insight in research have always influenced and guided me to be a successful researcher.

I would also like to thank my committee members Dr. Ashutosh Tiwari, Dr. Patricia A. Heiden and Dr. Xiaoqing Tang for their precious time, efforts and excellent suggestions on my Ph.D. dissertation and defense. I also appreciate former department chair Dr. Sarah A. Green and current department chair Dr. Cary F. Chabalowski for their help during my Ph.D. study.

My Ph.D research projects have involved lots of collaborative work. I am very much grateful to my research collaborators Dr. Ashutosh Tiwari (Chemistry Department, MTU) and his group members, Dr. Loredana Valenzano (Chemistry Department, MTU), Dr. Rudy Luck and Dr. Fen-Tair Luo (Academia Sinica). Their efforts made indispensable contributions to the projects in this dissertation. Here I also would like to show my special acknowledgement to Dr. Ashutosh Tiwari because he gave me many advices on not only my research projects but also my long-term career plan.

I could not complete my Ph.D. without the help from my labmates: Dr. Shilei Zhu, Dr. Giri K. Vegesna, Dr. Venkat R. Donuru, Jianheng Bi, Fei Xie, Cong Li, Mingxi Fang, Wafa Mazi and Bhaskar Halami. I sincerely show my appreciation to their great efforts for helping me. 
Teaching is a precious experience during my graduate study. I would like to show my deepest thank to my teaching supervisors Lorri A. Reilly, Aparna Pandey and Dr. Haiying Liu for their great help and efforts to train me to be a better teacher that I believe will be a lifelong benefit to me.

I would like to thank Department of Chemistry for five years financial support. I truly appreciate all the help from staffs of Department of Chemistry including Celine Grace, Denise Laux, Margaret Dunsten, Charlene Page, Kelly Smith, Jerry Lutz, Andrew Galerneau, Dean Seppala and Joel Smith. I also would like to thank my colleagues and friends Ning Chen, Zezhou Wang, Xiang Zhang, Xu Xiang, Nethaniah Dorh, Mimi Yang, Ni Fan, Durga Pokharel, Suntara (Boat) Fueangfung, Ashok Knanal, Rashmi Adhikari, Kapil Adhikari, Zichen Qian and Jie Zhou for their great support and help.

Finally, I would like to thank my family for their always support, encouragements and understandings for this five and half year Ph.D. study. Particularly, a million words would be too short to say how grateful I am to my beloved wife, Mu (Molly) Yang. She has always been helping me on research, sharing my happiness and sadness, gaving me confidence and motivations. Without her support, I could imagine how pale and struggle my study life would be. So I really want to say: Thank you, my dear!

$\mathrm{Ph} . \mathrm{D}$. study is an incredible journey and I am so grateful to all of the amazing people who have helped me along the way. 


\begin{abstract}
BODIPY (4,4-Difluoro-3a,4a-diaza-s-indacene) dyes have gained lots of attention in application of fluorescence sensing and imaging in recent years because they possess many distinctive and desirable properties such as high extinction coefficient, narrow absorption and emission bands, high quantum yield and low photobleaching effect. However, most of BODIPY-based fluorescent probes have very poor solubilities in aqueous solution, emit less than $650 \mathrm{~nm}$ fluorescence that can cause cell and tissue photodamages compared with bio-desirable near infrared (650-900 nm) light. These undesirable properties extremely limit the applications of BODIPY-based fluorescent probes in sensing and imaging applications. In order to overcome these drawbacks, we have developed a very effective strategy to prepare a series of neutral highly watersoluble BODIPY dyes by enhancing the water solubilities of BODIPY dyes via incorporation of tri(ethylene glycol)methyl ether (TEG) and branched oligo(ethylene glycol)methyl ether (BEG) residues onto BODIPY dyes at 1,7-, 2,6-, 3,5-, 4- and mesopositions. We also have effectively tuned absorptions and emissions of BOIDPY dyes to red, deep red and near infrared regions via significant extension of $\pi$-conjugation of BODIPY dyes by condensation reactions of aromatic aldehydes with 2,6-diformyl BODIPY dyes at 1,3,5,7-positions. Based on the foundation that we built for enhancing water solubility and tuning wavelength, we have designed and developed a series of water-soluble, BODIPY-based fluorescent probes for sensitive and selective sensing and imaging of cyanide, $\mathrm{Zn}$ (II) ions, lysosomal $\mathrm{pH}$ and cancer cells.
\end{abstract}

We have developed three BODIPY-based fluorescent probes for sensing of cyanide ions by incorporating indolium moieties onto the 6-position of TEG- or BEG-modified BOIDPY dyes. Two of them are highly water-soluble. These fluorescent probes showed selective and fast ratiometric fluorescent responses to cyanide ions with a dramatic fluorescence color change from red to green accompanying a significant increase in fluorescent intensity. The detection limit was measured as $0.5 \mu \mathrm{M}$ of cyanide ions. We also have prepared three highly water-soluble fluorescent probes for sensing of $\mathrm{Zn}$ (II) ions by introducing dipicoylamine (DPA, Zn ion chelator) onto 2- and/or 6-positions of 
BEG-modified BODIPY dyes. These probes showed selective and sensitive responses to $\mathrm{Zn}$ (II) ion in the range from $0.5 \mu \mathrm{M}$ to $24 \mu \mathrm{M}$ in aqueous solution at $\mathrm{pH}$ 7.0. Particularly, one of the probes displayed ratiometric responses to $\mathrm{Zn}$ (II) ions with fluorescence quenching at $661 \mathrm{~nm}$ and fluorescence enhancement at $521 \mathrm{~nm}$. This probe has been successfully applied to the detection of intracellular Zn (II) ions inside the living cells. Then, we have further developed three acidotropic, near infrared emissive BODIPYbased fluorescent probes for detection of lysosomal $\mathrm{pH}$ by incorporating piperazine moiety at 3,5-positions of TEG- or BEG-modified BODIPY dyes as parts of conjugation. The probes have low auto-fluorescence at physiological neutral condition while their fluorescence intensities will significant increase at $715 \mathrm{~nm}$ when $\mathrm{pH}$ shift to acidic condition. These three probes have been successfully applied to the in vitro imaging of lysosomes inside two types of living cells. At the end, we have synthesized one watersoluble, near infrared emissive cancer cell targetable BODIPY-based fluorescent polymer bearing cancer homing peptide (cRGD) residues for cancer cell imaging applications. This polymer exhibited excellent water-solubility, near infrared emission $(712 \mathrm{~nm})$, good biocompatibility. It also showed low nonspecific interactions to normal endothelial cells and can effectively detect breast tumor cells. 


\section{Chapter 1. Introduction}

\subsection{Fluorescence sensing and fluorescent probes}

In the past 20 years, there has been a remarkable growth in the use of fluorescence probes for fluorescence sensing and imaging in various fields of modern sciences. So far, fluorescent sensing technology has become a very powerful and indispensable tool to be extensively used in the areas of clinical diagnostics, biotechnology, molecular biology and biochemistry, materials and environmental sciences. ${ }^{1-2}$ Compared with traditional methods like chemo-absorptive detections, electrochemical detections, NMR and radioactive tracings, many features of fluorescence sensing distinguish itself to be one of most powerful transduction mechanisms to report the chemical recognition events, ${ }^{3}$ especially for sensing and visualization of analytes inside of living cells. For instance, fluorescence signal does not consume or destruct analytes and surrounding objectives. This non-invasive measurement is ideal for bio-specimens. The fluorescence sensing has ultra-high sensitivity and specificity which even allows the detection of the signal from single molecule. ${ }^{4}$ With rapid response time in the scale of $10^{-8} \mathrm{~s}$ and diversity of emission wavelengths, fluorescence sensing is possible to provide the real-time monitoring of various analytes simultaneously. ${ }^{2}$ In addition, fluorescence travels without specific physical guide and no reference is required, which enormously simplifies the technical and instrumental requirements and reduces the measurement costs. Thus, the fluorescence sensing technology is considered as one of the most important methods for future miniaturized detections and screenings. ${ }^{5}$

There is no doubt that the fluorescent probe is the key component of fluorescence sensing technology because it is the molecule capable of converting of chemical events (binding, reactions, conformation changes etc.) to detectable fluorescence signals, which is in direct relation to the sensitivity, specificity, response range and many other aspects of the sensing. Although some natural products with intrinsic fluorescence have been widely used, their numbers still are very limited. Therefore, development of synthetic fluorescent probes are very important because they extensively enlarge the diversity of fluorescent probes and extend the areas of fluorescent technology. 


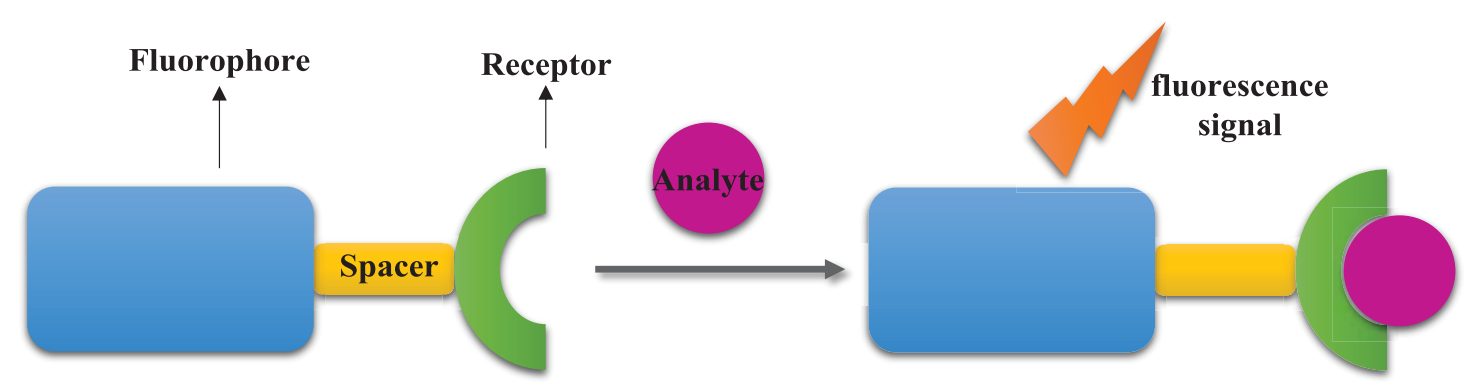

Figure 1.1. Illustration of fluorescent probe.

The typical structure of synthetic fluorescent probe consists of three essential parts fluorophore, spacer and receptor (Figure 1.1). The receptor is an analyte recognition site that is also called chelator, ligand or binding site. The fluorophore is a fluorescent reporter moiety which translates the recognition events between the analyte and the receptor into changed or amplified fluorescent output signals. The spacer (also called linker) is the moiety which links fluorophore and receptor (Figure 1.1). The length of spacer is variable and highly dependent on the mechanism of the fluorescence modulation. In some cases of fluorescent probes, the spacers are shortened to be a single bond. The sensing mechanisms of fluorescent probes are experimentally versatile so the output fluorescent signals can be monitored in the form of absorption and emission spectra, fluorescence intensities, fluorescent lifetimes, quantum yields and even as anisotropies. ${ }^{6}$ With the rapid developments of advanced instrumentations including various types of fluorescence microscopes, the design and development of novel fluorescent probes with high sensitivities, specificities and spatial sampling capability is particularly important for further investigating the cellular and subcellular functions. This area attracts lots of attention of researchers and it continues to be the one of most vibrant and multidisciplinary topics. However, there are still many molecules, especially in living cells, which are either hard to recognize or have too low concentrations to be detected by current fluorescent probes. Therefore new artificial fluorescent probes are highly desired. ${ }^{7}$ 


\subsection{Design criteria of fluorescent probes}

Since the fluorescence sensing and imaging are critically dependent on the fluorescent probes, design of appropriate probes with desired properties is particularly important. There are some criteria need to be considered in the design of fluorescent probe, which will be discussed in below.

1) Selective response: The first and foremost need to consider for design of fluorescent probe is the ability of the probe to selectively and specifically fluorescence respond to the analyte of interest without interference by interferents. For example, specific chelation pocket design of the receptor moiety on the fluorescent probe will only allow specific metal cation to bind when other cations are present at same time.

2) The brightness of fluorescence: The fluorescence should be as bright as possible. It means the high quantum yield, large extinction coefficient at excitation wavelength are very important and should be desired properties of the fluorophore or the entire fluorescent probe.

3) Robustness of fluorescent probes: Stability against chemicals and light is an obvious criterion for the fluorescent probe. Many categories of fluorescent probes still suffer from the high photobleaching effect (the irreversible destruction of the excited fluorophore under high-intensity illumination conditions), which significantly limit their fluorescence detectabilities. Therefore, chemostability and photostability of fluorescent probes are highly desired properties and must be considered.

4) Turn-on fluorescence: the turn-on output fluorescence signal (fluorescence enhancement) of fluorescent probe is much more favored than the turn-off response (fluorescence quenching) because it is able to significantly elevate the signal-tonoise $\operatorname{ratio}(\mathrm{S} / \mathrm{N})$ and make the fluorescence visualization easier to be realized.

Living organisms are much more complicated and easier to be damaged than the regular analytical specimens. Thus, beside of the basic criteria discussed above there are 
more issues and requirements need to be concerned for those fluorescent probes which function inside of living cells or bio-tissues.

a) Good aqueous solubility and membrane permeability: Considering the character of aqueous environment in cytosol and plasma of cells and tissues, good aqueous solubility of the fluorescent probe is highly desired for biological applications, especially for in vivo measurements. The Probe with too much hydrophobicity/lipophilicity will easily aggregate together and accumulate within membrane structures of the cells, which extremely limits the performance of the probe such as targeting localization, quantum yield and sensitivity. However, sufficient lipophilicity is also necessary for the fluorescent probe to achieve good permeability to pass through the membranes so that it can go into the cells or subcellular organelles as specific targets. ${ }^{8}$ So, appropriate hydrophilic-lipophilic balance is a crucial factor to control the performance of the fluorescent probe. ${ }^{9}$

b) Long-wavelength absorption and emission: Compared with the fluorescence in the region of ultraviolet (UV) and short wavelength of visible light $(<500 \mathrm{~nm})$, longwavelength absorption/emission is highly desired for fluorescent probe in biological applications since it generates less photodamage to cells and tissues and there is less interference caused by absorptions and auto-fluorescence of biomolecules. Particularly, near infrared region (NIR, defined as $650 \mathrm{~nm}-900 \mathrm{~nm}$ ) is a golden range for bio-applicable fluorescent probes. This is because near infrared light not only minimizes the photodamage but also dramatically reduces the light scattering (Rayleigh and Raman) and absorbing interferences. It also generates deep penetrations for biological specimens (up to 1-2 cm in depth), which extremely facilitates in vivo imaging of molecular processes. ${ }^{10-12}$ Thus this region is also called "biological window". 13-14

c) Ratiometric fluorescence responses: Ratiometric response means the fluorescence changes at two different wavelengths simultaneously. This two-channel fluorescence response usually offers high signal-to-noise ratio $(\mathrm{S} / \mathrm{N})$ and it is very helpful for intracellular measurements because it could eliminate most fluorescence change caused by the effects which are not related to the analytes of interest, such as 
unequal dye loading, different cell thickness, photobleaching and dye leakage, instrumental efficiency etc.

Although no fluorescent indicator has been reported that perfectly satisfies all these criteria at once, partial fulfillment for particular usage will be helpful.

\subsection{BODIPY-based fluorescent probes}

Among numerous synthetic fluorescent probes, those sets based on 4,4-Difluoro-4bora-3a,4a-diaza-s-indacene (Difluoroboron dipyrromethene, abbreviated as BODIPY) have shown almost highest potential and attracted tremendous attentions in recent years. BODIPY is actually a structural analogue of porphyrins. Figure 1.2 shows the structure of the BODIPY core.

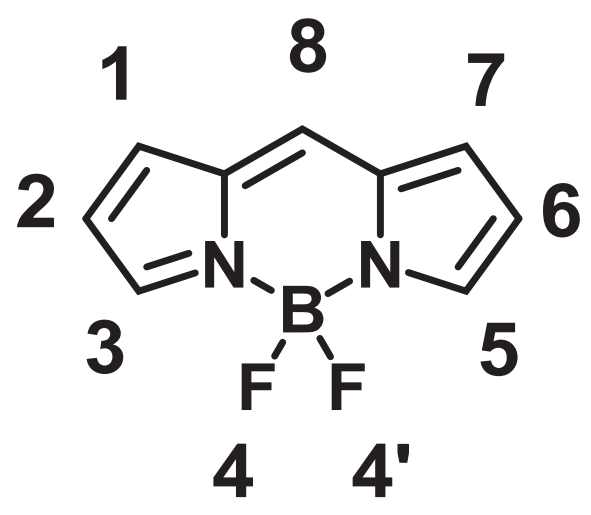

Figure 1.2. Structure of BODIPY dye.

\subsubsection{Merits of BODIPY-based fluorescent probes}

The reason of BODIPY dyes being the focus of the research in fluorescent sensing nowadays is that this highly fluorescent dye possesses many remarkable chemical and spectroscopic properties that are ideal for the design of next generation fluorescent probes. For instance, the BODIPY core absorbs and emits sharp bands of light around $500 \mathrm{~nm}$ with high extinction coefficient (usually $>80000 \mathrm{M}^{-1} \mathrm{~cm}^{-1}$ ) and high quantum yields. BODIPY dye is chemically and physicochemically robust with moderate redox potential, excellent photostability and negligible sensitivity to solvents and temperatures. ${ }^{15}$ More importantly, the unique structure of BODIPY dye endows itself 
with eight functionalizable positions surrounding the core. Many different types of reactions can be applied through these positions, such as electrophilic substitutions at 2,6positions, nucleophilic subsititutions at 3,5- or 4- or 8-positions, condensation reactions at 3,5- or 2,6-positions, palladium catalyzed cross coupling reactions like Suzuki and Sonogashira at all positions except 4-position etc. ${ }^{15}$ These synthetic pathways extremely extend the diversity of BODIPY derivatives so that they could incorporate various functionalities with different receptors for many sensing applications. In addition, BODIPY dye is a highly tunable molecule. The original BODIPY core does not emit long wavelength or NIR fluorescence, but facile modifications of BODIPY core can tune their absorption and emission wavelengths to red, deep red and even NIR regions with extended the conjugations. These excellent characteristics of BODIPY dye have inspired researchers for developing BODIPY-based fluorescent probes for environmental, biological sensing and imaging applications and many of them have been reported by in recent years. The detailed the properties and current applications of BODIPY dyes and BODIPY-based fluorescent probes are described in Chapter 2.

\subsubsection{Drawbacks and challenges of current BODIPY-based fluorescent probes}

However, the discovery about BODIPY dyes and their application for fluorescence sensing is far away from mature, especially in the area of biosensing and imaging, and still facing many challenges because of many undesirable characteristics of BODIPY dyes. For example, many analytes such as transition metal ions, toxic species, intracellular $\mathrm{pH}$ etc. are still lack of efficient sensing by fluorescent probes with high sensitivities (low detection limits) in biological conditions although some good probes (including BODIPY-based probes) have been reported. This means the sensitivities and specificities of BODIPY-based fluorescent probes to these analytes eagerly need further improvement. Besides, BODIPY core has highly hydrophobic nature. This character makes the most of current BODIPY base fluorescent probes too hydrophobic (with very low aqueous solubility) to be applied into aqueous conditions such as biological system. Moreover, most BODIPY fluorescent probes absorb and emit in short wavelength regions which causes potential photodamage to cells and tissues. Only a few NIR emissive BODIPY dyes have been reported. All of these challenges and drawbacks extremely limit 
the applications of BODIPY-based fluorescent probes in in vitro and in vivo biosensing and labeling applications.

\subsection{Research objective and outline}

In order to overcome the current challenges which have been mentioned above and better apply BODIPY dyes to fluorescence sensing, the research in this dissertation focuses on the modification of BODIPY dyes and development of novel, highly sensitive and selective BODIPY-based fluorescent probes for various sensing applications, especially for biosensing and imaging in living cells. The aim of this research concentrates on three directions: enhancing water solubility of BODIPY dyes, tuning BODIPY dyes to be long-wavelength (especially in NIR region) emissive, and developing BODIPY-based fluorescent probes for fluorescent sensing and in vitro bioimaging applications (Figure 1.3).

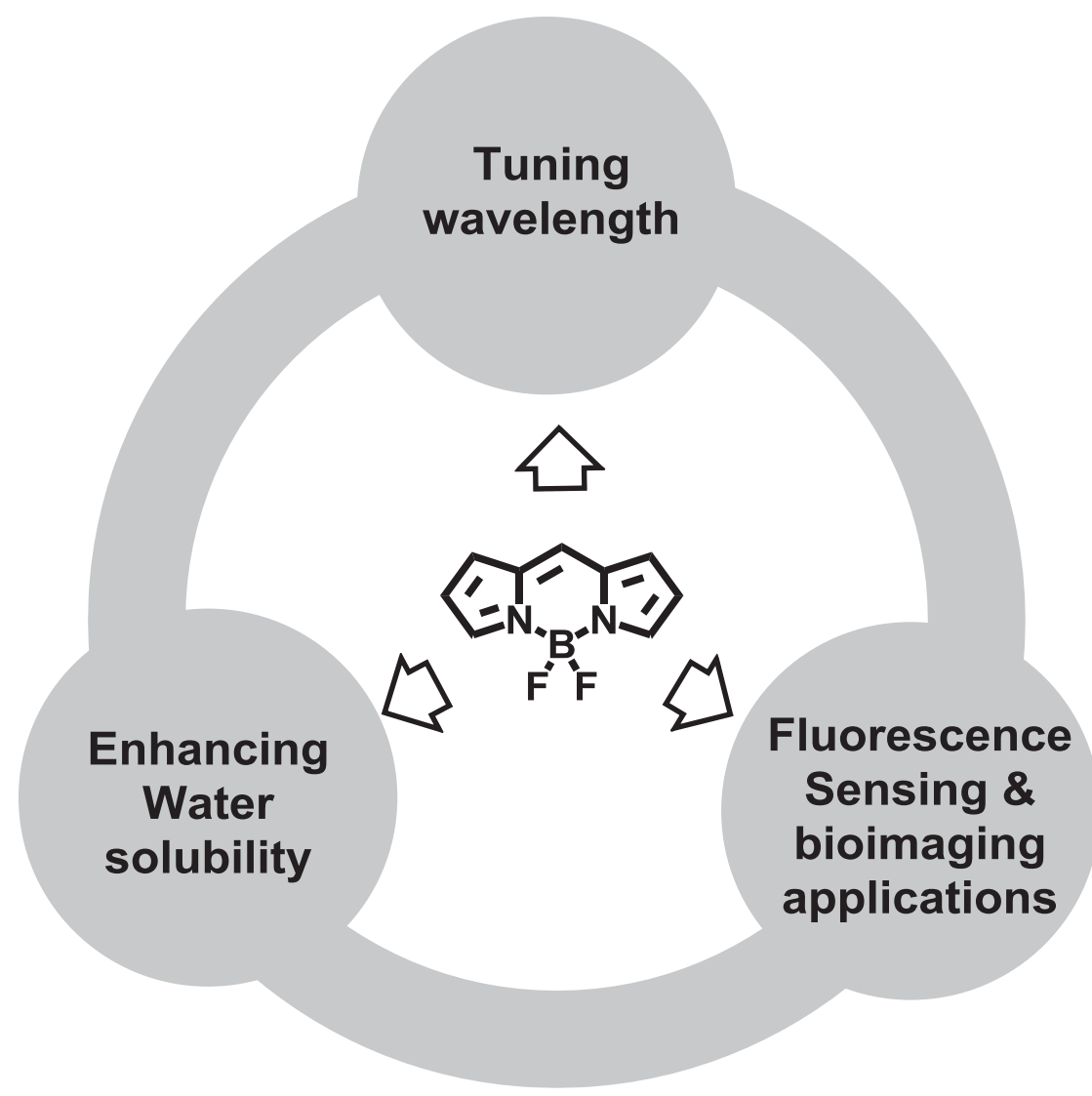

Figure 1.3. Research outline based on BODIPY dye. 
In detail, to overcome the drawback of the high hydrophobicity of regular BODIPY dye we explored novel methods and modifications strategies to enhance the hydrophilicity of BODIPY core. Based on this, a series of neutral, highly water-soluble BODIPY dyes were synthesized. This was discussed in Chapter 3. In Chapter 4, we investigated the strategies of further tuning the absorption and emission wavelength of BODIPY dyes to the deep red, and NIR regions through activated condensation reactions at 1,3,5,7-positions of BODIPY dyes. With the foundations built in Chapter 3 and 4, we designed and synthesized various novel, highly sensitive BODIPY fluorescent probes for detection of cyanide ion, $\mathrm{Zn}$ (II) ion, lysosomal $\mathrm{pH}$ and cancer cells. The detections of these analytes are extremely important and eagerly desired to improve from current sensing methods, especially in biological conditions. All the probes were fully characterized in chemical structures, spectroscopic properties and fluorescent responses to analytes. We applied our BODIPY fluorescent probes for $\mathrm{Zn}$ (II) ion, lysosomal $\mathrm{pH}$ and cancer cells to in vitro fluorescence sensing and imaging in living cells. All design, investigations and applications of these probes were discussed in Chapter 5 to 8 .

\section{References:}

1. Mason, W. T., Fluorescent and Luminescent Probes for Biological Activity (Second Edition) Academic Press: 1999.

2. Lakowicz, J. R., Principles of Fluorescence Spectroscopy. 3 ed.; Springer Science and Business Media: 2006.

3. Lakowicz, J. R., Principles. In Topics in Fluorescence Spectroscopy, Plenum Press: New York, 1991; Vol. 2.

4. Demchenko, A. P., Introduction to Fluorescence Sensing. Springer: 2009.

5. Lakowicz, J. R., Applications. In Topics in Fluorescence Spectroscopy, Plenum Press: New York, 1991; Vol. 3.

6. Lakowicz, J. R., Techniques. In Topics in Fluorescence Spectroscopy, Plenum Press: New York, 1991; Vol. 4.

7. Czarnik, A. W., Desperately Seeking Sensors. Chemistry \& Biology 1995, 2 (7), 423-428. 
8. Thompson, K., Dockery, P., Horobin, R. W., Predicting and Avoiding Subcellular Compartmentalization Artifacts Arising from Acetoxymethyl Ester Calcium Imaging Probes. The Case of Fluo-3 AM and A General Account of The Phenomenon Including A Problem Avoidance Chart. Biotechnic \& Histochemistry 2012, 87 (7), 468-483.

9. Carter, K. P., Young, A. M., Palmer, A. E., Fluorescent Sensors for Measuring Metal Ions in Living Systems. Chemical Reviews 2014, 114 (8), 4564-4601.

10. Gao, X. H., Cui, Y. Y., Levenson, R. M., Chung, L. W. K., Nie, S. M., In Vivo Cancer Targeting and Imaging with Semiconductor Quantum Dots. Nature Biotechnology 2004, 22 (8), 969-976.

11. Escobedo, J. O., Rusin, O., Lim, S., Strongin, R. M., NIR Dyes for Bioimaging Applications. Current Opinion in Chemical Biology 2010, 14 (1), 64-70.

12. Hilderbrand, S. A., Weissleder, R., Near-infrared Fluorescence: Application to In Vivo Molecular Imaging. Current Opinion in Chemical Biology 2010, 14 (1), 7179.

13. Weissleder, R., A Clearer Vision for In Vivo Imaging. Nature Biotechnology 2001, 19 (4), 316-317.

14. Frangioni, J. V., In Vivo Near-infrared Fluorescence Imaging. Current Opinion in Chemical Biology 2003, 7 (5), 626-634.

15. Loudet, A., Burgess, K., BODIPY Dyes and Their Derivatives: Syntheses and Spectroscopic Properties. Chemical Reviews 2007, 107 (11), 4891-4932. 


\section{Chapter 2. Background}

\subsection{General overview of fluorescence}

In 1845, the fluorescence phenomenon was first reported by Sir John Frederick William Herschel by observation of "superficial color" light from quinine aqueous solution under sunlight ${ }^{1}$. But until 1950s, people started to scientifically reveal, understand and develop fluorescence and fluorescence technology with the rapid development of modern physics and innovation of spectrofluorometer ${ }^{2}$. Generally speaking, fluorescence is belong to one type of luminescence which is the emission of light from any substances from their electronically excited states after absorbing of light or other electromagnetic radiations. Molecules with fluorescence properties are called fluorophores and typically they are aromatic compounds. Their $\pi$-electrons on the ground orbital can be excited to higher energy leveled excited orbitals under the radiations of light and these unstable electrons rapidly return to their ground state orbital by energy lease with emission of photons.

The classic way for illustration of this light absorption and emission process of fluorescence is by using Jablonski diagram. This diagram is named after Professor Alexander Jablonski, who is regarded as the father of fluorescence spectroscopy because of his many accomplishments on fluorescence. Figure 2.1 shows a typical Jablonski diagram. The ground, first and second electronic states are depicted by $S_{0}, S_{1}$ and $S_{2}$, respectively. At each of these energy levels, the fluorophores can exist in many vibrational energy levels. Under the light irradiation, the fluorophore is excited to numbers of different vibrational levels on excited state of either $S_{1}$ or $S_{2}$ which depends on the irradiation energy. Then the molecule very rapidly relaxes to its lowest vibrational level of excited state $S_{1}$ through vibrational relaxation and internal conversion processes from $S_{2}$ to $S_{1}$. This process occurs within $10^{-12}$ second that is several orders of magnitude smaller than the fluorescence emission rate $\left(\sim 10^{-8} \mathrm{~s}\right)$. So this transition usually completes prior to the fluorescence and it will not give any emission. From a thermally equilibrated excited state, which is the lowest vibrational state of $\mathrm{S}_{1}$, fluorophore returns to different vibrational levels of ground states $\mathrm{S}_{0}$, releasing the energy through emission of photons. 
This process is called fluorescence. The fluorophore in $\mathrm{S}_{1}$ state also can undergo an electron spin conversion via intersystem crossing process to be converted to its triplet state $T_{1}$. The transition from $T_{1}$ state to ground state $S_{0}$ is termed phosphorescence, another type of luminescence, giving a longer wavelength emission relative to fluorescence due to the lower energy levels of $T_{1}$ relative to $S_{1}$ states. Because of the same spin orientation of electrons at $\mathrm{T}_{1}$ and $\mathrm{S}_{0}$ states, the transition from $\mathrm{T}_{1}$ to singlet ground state is forbidden by the principle of quantum mechanics. As a result, the emission rate of phosphorescence is several orders of magnitude smaller than that of fluorescence.

\section{Singlet Excited States}

Vibrational

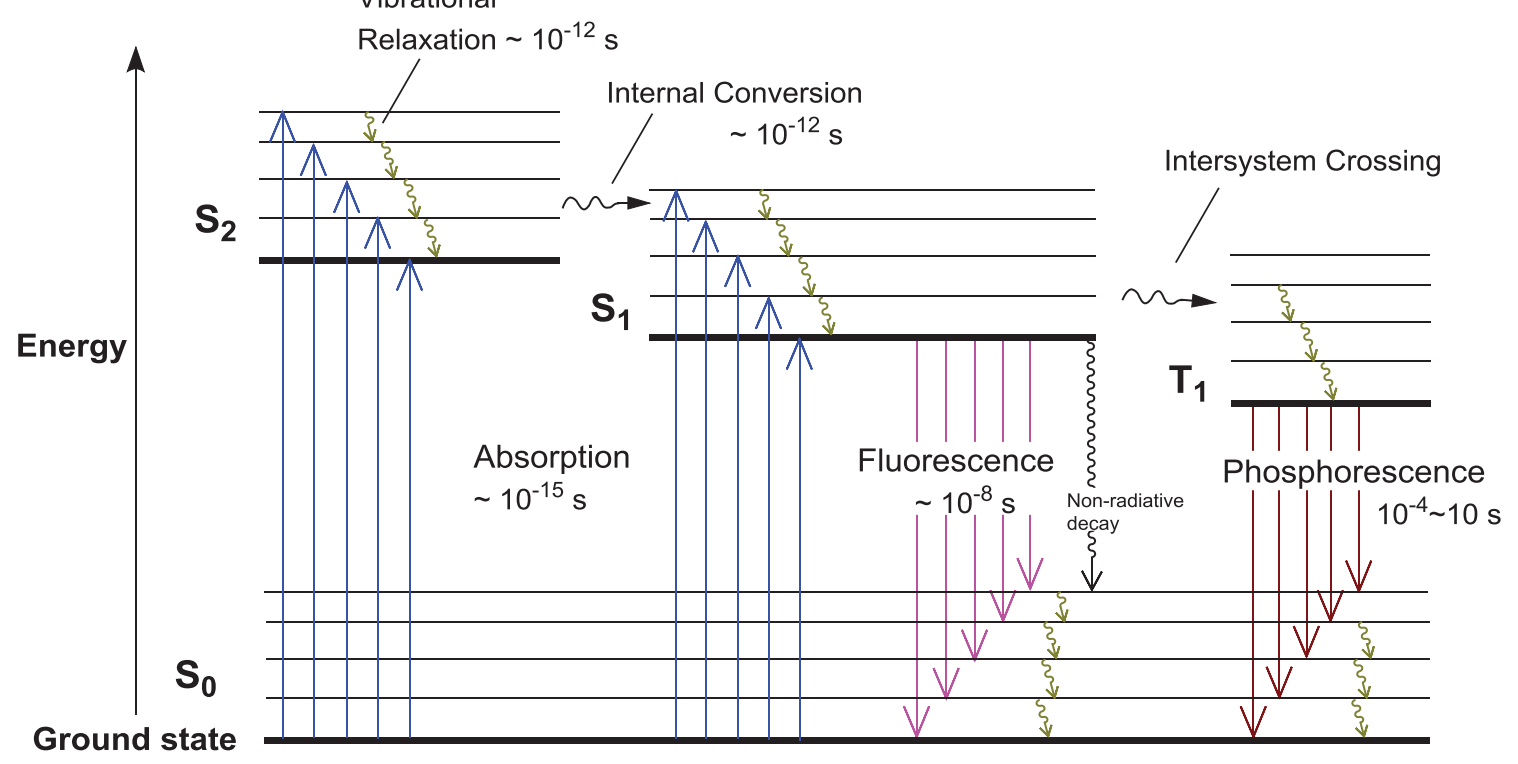

Figure 2.1. Jablonski diagram.

Jablonski diagram also reveals that the energy of fluorescence emission is always less than that of absorption. Because of this, fluorescence typically occurs at longer wavelengths than absorption. This wavelength shift was first discovered by Sir. G. G. Stokes in 1852 and named bear his name "Stokes shift"3. A typical fluorescence spectrum contains absorption and emission curves in one plot. Emission spectra are relative broad and usually a mirror image of absorption spectrum of $\mathrm{S}_{0}-\mathrm{S}_{1}$ transitions. The shape and 
intensity of fluorescence emission spectra are highly depend on the chemical structure of fluorophore, solvents and some other factors.

The characteristics of fluorescent molecules usually are described by several important parameters including: maximum absorption and emission wavelength $\left(\lambda_{\text {abs }}\right.$, $\lambda_{\mathrm{em}}$ ), molar extinction coefficient $(\varepsilon)$, fluorescence quantum yield $\left(\Phi_{\mathrm{f}}\right)$ and fluorescent life time $(\tau)$. Maximum absorption and emission wavelength is the wavelength of highest peak of absorption and emission spectra, respectively. Molar extinction coefficient $(\varepsilon)$ is the constant of Lambert-Beers law, describing the light absorption ability of fluorescent molecules at certain wavelength. Fluorescence quantum yield is the ratio of photons absorbed by fluorophore to photons emitted through fluorescence. In other words, the fluorescence quantum yield gives the probability of deactivation of excited states through fluorescence emission rather than by another, non-radiative transitions. Fluorescent quantum yield governs the brightness of the fluorescent molecules and it is one of the most important characteristics fluorophores. High quantum yield is always highly desired for fluorescent probes design and other fluorescence technologies. Fluorescence life time $(\tau)$ is average time fluorophore spend for staying at excited states before it returns to the ground states through fluorescence emission. The typical fluorescent lifetime is around several nanoseconds.

\subsection{Common organic fluorophores for fluorescent sensing and labeling}

The unique absorption and emission properties of fluorescent molecules make them ideal for environmental, biological sensing and labeling applications. Relative to the absorbance based dye labeling, radioactive labeling and electrochemical sensing, fluorescent sensing and labeling technique is much more informative, sensitive and noninvasive, which make it feasible to do the things that were not previously possible. Although some of the natural biomolecules like NADH, tryptophan, green fluorescent proteins (GFP) are intrinsic fluorescent, using synthetic organic fluorophores extensively extends the diversity of fluorescent sensing, the area of fluorescent technologies with more informative and sensitive visualizations. Obviously, the key factor of fluorescence sensing and labeling application rely on the fluorophores. Nowadays, thousands of 
organic fluorophores has been developed and most of them absorb and emit light in visible to near infrared region. Among them, four fluorophore families (Figure 2.2) including fluoresceins, rhodamines, cyanines and BODIPYs are most well-known and studied.

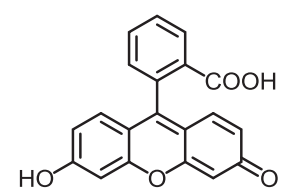

Fluorescein

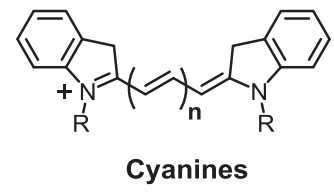

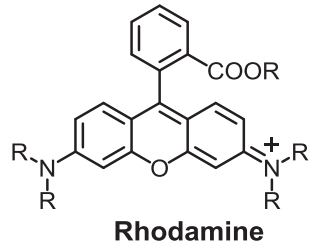

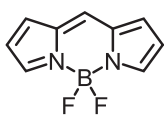

BODIPY

Figure 2.2. Commonly used organic fluorophore families.

Fluorescein belongs to the xanthene class of dyes. It absorb and emit light in visible region with maximum peaks at $490 \mathrm{~nm}$ and $512 \mathrm{~nm}$ in basic water for absorption and emission, respectively ${ }^{4}$. The remarkable properties of fluorescein like high extinction coefficient, high quantum yield and good water solubility make fluorescein become one of the most used fluorephore for bio-labeling applications ${ }^{5}$ and clinic diagnoses in the field of ophthalmology and optometry ${ }^{6}$. However, fluorescein has many disadvantages including high photo-bleaching rate, absorption and emission $\mathrm{pH}$-dependency, and relative short emission wavelength. These drawbacks limit its further applications.

Rhodamine is also one type of xanthene class of dyes. Most Rhodamine dyes have very strong absorptions and emissions at around $500 \mathrm{~nm}$ in visible region with high quantum yields. Compared with fluorescein, Rhodamine dyes have higher photostability and their core optical properties are less sensitive to $\mathrm{pH}^{7}$ Rhodamine dyes like Rhodamine 6G and Rhodamine B, Rhodamine 101 etc. (Figure 2.3) have been widely used in the area of fluorescence standards, fluorescent lifetime measurements, labeling agents and environmental examinations. More importantly, Rhodamine provides a very good skeleton for fluorescence modulations because of its unique spirolactam ring openclose mechanism (Figure 2.4). The amidation of Rhodamine usually results in spiro- 
cyclization that forms a five-membered spirolactam ring showing no fluorescence because the conjugation is disrupted. Changing of $\mathrm{pH}$ or adding of metal ions can open the spirolactam ring and rebuild the conjugation system so the fluorescent is recovered. Based on this strategy, thousands of functionalized Rhodamine derivatives with different optical and sensing properties have been developed for bio-labeling, subcellular imaging and ion sensing applications in last 30 years. ${ }^{8-11}$ To some extent, Rhodamine based fluorescent probes have been one of the most popular research area in chemistry, biochemistry and biology. However, Rhodamine based fluorophores still have many weak points. Low water solubility, limited modification sites on the core and short wavelength emissions (compared with near-infrared emission) of Rhodamine dyes limit their applications although many success has been accomplished in this area.
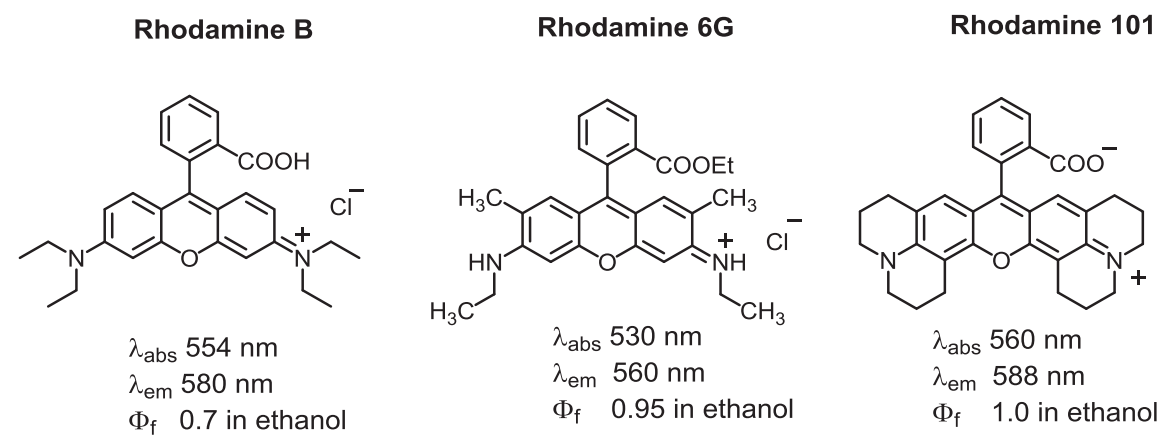

Figure 2.3. Examples of commercial Rhodamine dyes.
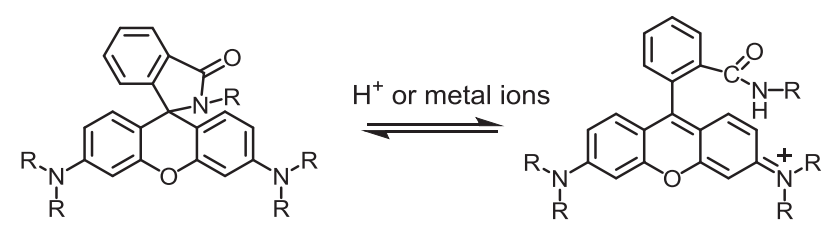

Figure 2.4. Fluorescent modulation of Rhodamine based fluorescent probes through spriolactam ring open/off process. 

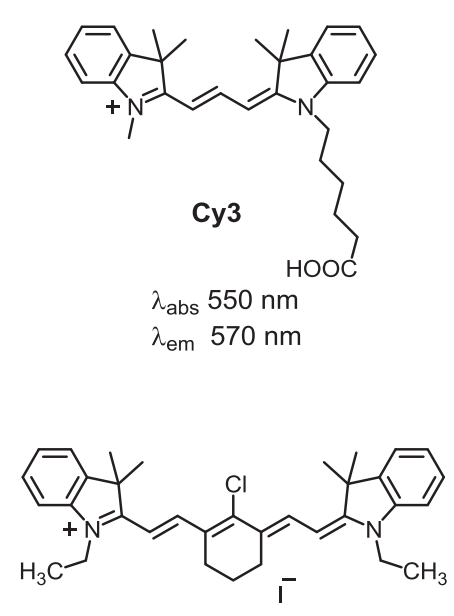

Cy7 (IR-780)

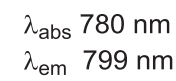

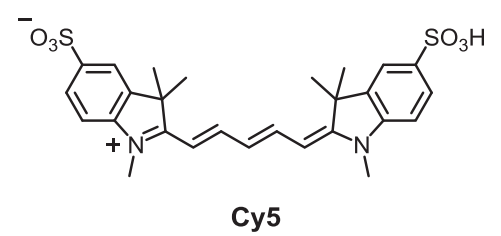

$\lambda_{\text {abs }} 650 \mathrm{~nm}$

$\lambda_{\text {em }} 670 \mathrm{~nm}$

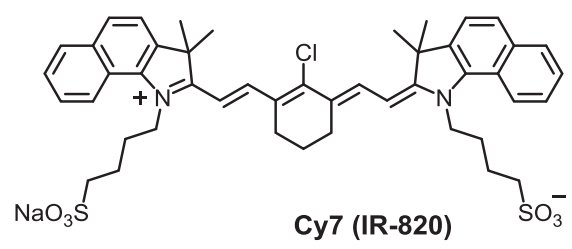

$\lambda_{\text {abs }} 820 \mathrm{~nm}$

$\lambda_{\text {em }} 820 \sim 830 \mathrm{~nm}$

Figure 2.5. Examples of Cyanine dyes.

Cyanine is a synthetic dye family belonging to polymethine group. Cyanine dye is one of the most commonly used long-wavelength fluorophore. It contains two heterocyclic rings linked by polymethine chain with conjugated double bonds as conjugation. The number of double bonds (typically 2-5) decides the wavelength it absorbs and emits, usually in the range from $600 \mathrm{~nm}$ to $900 \mathrm{~nm}$. Figure 2.5 shows several examples of Cyanine dyes with different absorption and emission wavelengths. The names of $\mathrm{Cy} 3,5$ or 7 come from the combination of abbreviation of cyanine and the number of carbons of methines that separate two heterocyclic rings. Cyanine dyes have been widely used in industry area and more recently in biological area by taking benefit of its near infrared (NIR) absorptions and emissions which ideally fits the ideal "biological window" (700-900 nm). Nevertheless, the main issue related to this fluorophore for biological sensing applications is that strong aggregation effect and concentration dependency in aqueous solution are often encountered, which significantly reduce the aqueous solubility, fluorescence brightness and the sensitivities of cyanine dyes. One way to solve this aggregation problem of cyanine dyes is to introduce anions such as sulfonate or carboxylate groups on the cyanine fluorophore that can significantly enhance the solubility and reduce the aggregations because of strong electrostatic 
repulsions. However, this ionic profile bring the problems of electrostatic non-specific bindings or other electrostatic interferences between fluorophores and analytes, especially during cellular or subcellular level biological sensing. In addition, cyanine dyes also suffer from their poor photostability, scanty functionalization methods and rotation/photoisomerization due to non-rigid structures that result in nonradiative decays. ${ }^{12-14}$

BODIPY is a structural analogue of the porphyrins (Figure 2.6). Compared with the other three type fluorophores discussed above, BODIPY dyes possess many advantages such as their facile synthesis and structural versatility, high extinction coefficient ( usually $>80000 \mathrm{M}^{-1} \mathrm{~cm}^{-1}$ ), sharp absorption and emission bands with high quantum yields, moderate redox potential, negligible sensitivity to solvent and temperature and excellent photostability. In this research, we chose BODIPY fluorophore as the base skeleton for further modification and probe design. Therefore, in the following section of this chapter, the detailed structure information, optical properties, reactions and functionalization of BODIPY fluorophore will be discussed.

\subsection{BODIPY dyes}

\subsubsection{Fundamentals of BODIPY dyes}

The IUPAC name of BODIPY is 4,4-Difluoro-4-bora-3a,4a-diaza-s-indacene. More concise and popular name "BODIPY" is come from the abbreviation of BOronDIPYrromethene. It is composed of dipyrromethene complexed with a disubstituted boron atom, typically a $\mathrm{BF}_{2}$ unit. Because of $\mathrm{BF}_{2}$ complexation, the dipyrromethene ligand is linked by a N-B-N bridge, forming fixed planarity of $\pi$-electrons system where the positive charge can be delocalized between two nitrogen atoms via resonance structures equilibrium (Figure 2.6). The single crystal X-ray structure of BODIPY indicates a fused planar system with strong chromophoric $\pi$-electrons delocalization within twelve-membered framework of BODIPY core (Figure 2.7). But two fluorine atoms, instead of co-planed with dipyrrole ring, is perpendicular to it with a distorted tetrahedron $\mathrm{BF}_{2} \mathrm{~N}_{2}$ configuration. ${ }^{15-16}$ Although BODIPY dyes do not obey Hückel's aromaticity rule, their properties are more similar to those of aromatic $\pi$-system because 
of the rigidity introduced by coordination of boron atom. The IUPAC numbering system of BODIPY core shows eight functionable positions, and in addition to it, the conventional numbering by $\alpha-, \beta$ - and meso-positions are also used, especially for the "meso-position" which is more popular than the statement of 8-postion in many papers.

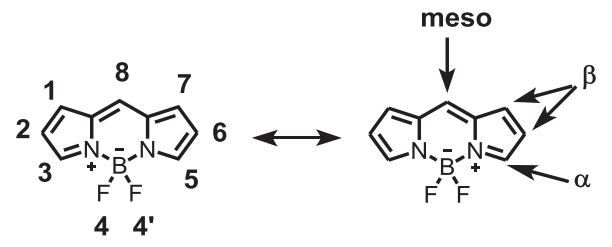

Figure 2.6. Structure of BODIPY core and its numbering system.

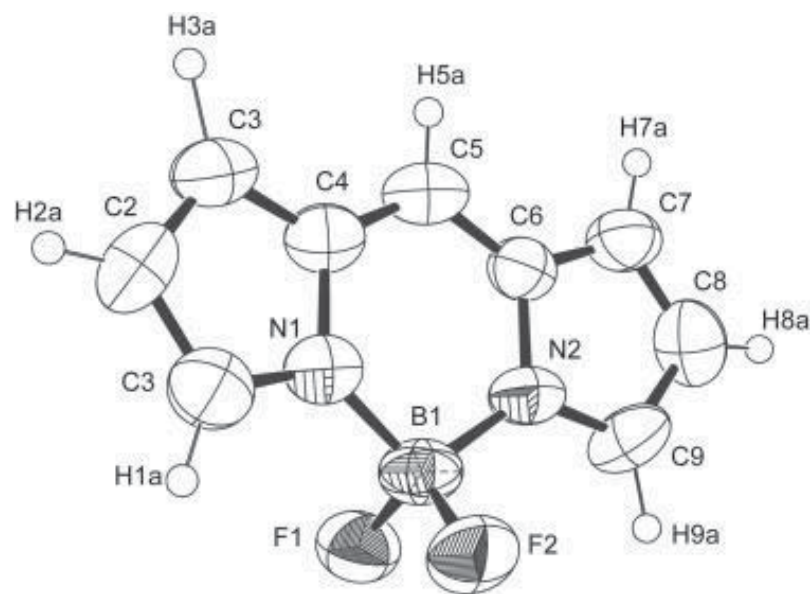

Figure 2.7. X-ray structure of unsubstituted BODIPY dye. ${ }^{16}$ (Graph reuse was permitted by Elsevier B.V, see detail at end of this dissertation)

Compared with classic fluorophores like fluorescein and rhodamine, BODIPY dyes have outstanding photophysical properties such as high extinction coefficients, narrow absorption and emission bands, $\mathrm{pH}$ insensitive, good stability, high fluorescence quantum yields etc. BODIPY and its derivatives are neutral, thermostable and highly colored small molecules with intensive and sharp-band absorptions and fluorescent emissions because of the rigid framework on the core. Typical absorption and emission bands of BODIPY dyes lie in the range of 480 - $550 \mathrm{~nm}$ with very high extinction coefficients and quantum 
yields in organic solvents (normally $\varepsilon>8000 \mathrm{M}^{-1} \mathrm{~cm}^{-1}$; $\Phi_{\mathrm{f}}>0.6$ ). Figure 2.8 shows the absorption and emission spectra of an unsubstituted BODIPY dye. The intense absorption band around $500 \mathrm{~nm}$ is attributed to $\mathrm{S}_{0}-\mathrm{S}_{1}\left(\pi-\pi^{*}\right)$ transition and the shoulder peak centered at $480 \mathrm{~nm}$ is due to the $0-1$ vibrational transition. Substituted BODIPY dyes containing electron donating groups like alkyl and amino, heavy atoms like iodine and bromine usually shows about 10 - $50 \mathrm{~nm}$ red-shifted absorptions and emissions due to the reduced the HOMO-LUMO gaps of the dyes. Further conjugation of BODIPY core with aromatic rings, double bonds and triple bonds through the electrophilic positions of pyrrole rings allow the absorption and emission of BODIPY dyes be tuned to longer wavelengths with 50-300 $\mathrm{nm}$ red shifts depending on the level of $\pi$-system extension. In summary, many different types of modifications can be applied on BODIPY dyes to tune their absorption and emission properties in a very broad range, which extensively expand the applications of BODIPY dyes in many fields. The detailed modifications, optical properties tuning of BODIPY dyes will be discussed in section of 2.4 in this dissertation.

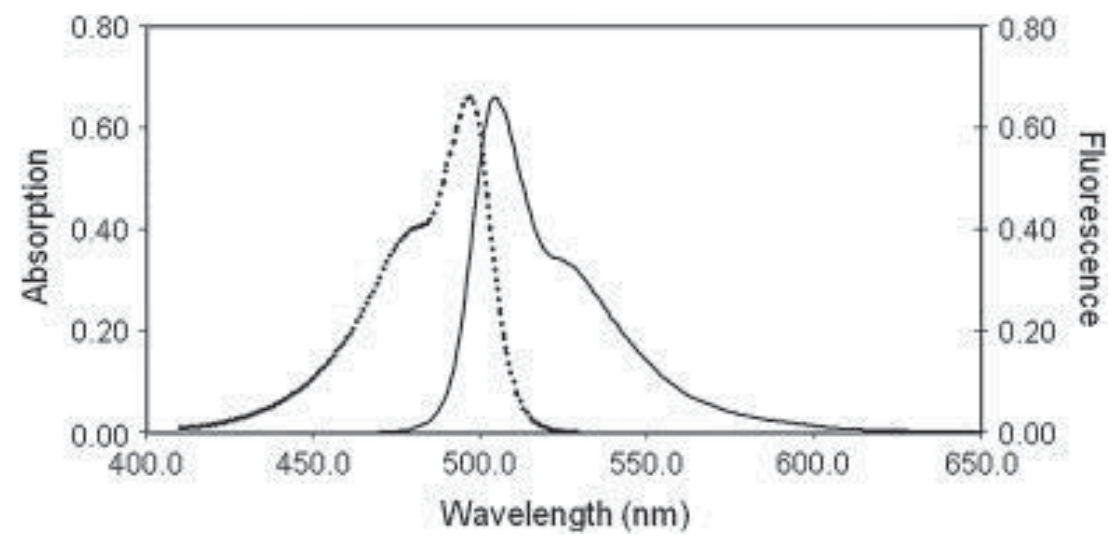

Figure 2.8. Absorption and fluorescence emission spectra of unsubstituted BODIPY dye in $\mathrm{MeOH}$ solution. ${ }^{16}$ (Graph reuse was permitted by Elsevier B.V, see detail at end of this dissertation)

The synthesis of BODIPY dyes were first reported by Treibs and Kreuzer in $1968 .{ }^{17}$ But the unsubstituted BODIPY dye (core only, Figure 2.9 BODIPY dye 2.1) had not been reported in literature until 2009 because of synthetic difficulty resulted from unblocked pyrrole carbons which can easily undergo electrophilic attack ${ }^{16,18-19}$. In another word, 
substituted BODIPY dyes are more stable and easier to be synthesized. Especially for $\alpha-$ positions of pyrrole rings on BODIPY core, in most cases, they are blocked by using 2methyl substituted pyrrole as starting material to prevent further electrophilic polymerizations. The most common substituted BODIPY dyes are those with alkylations on pyrrole rings through 1-8 positions. Particularly, the symmetrical di-, tetra-, hexa-, and hepta-alkylated BODIPY dyes (Figure 2.9 2.2-2.5) are widely used because they can be easily synthesized by alkylated pyrroles with alkylated aldehydes as the starting materials. Although some unsymmetrical alkylated BODIPY dyes (like 2.8 and 2.9 in Figure 2.9) were also reported, they are harder to synthesize and have been less used. ${ }^{20}$ In fact, phenyl or other aromatic groups are usually incorporated to the substitutions at 8- or meso-position of BODIPY core not only because of the synthetic convenience by just using corresponding aromatic aldehydes as starting materials but also because aromatic groups at meso-position provide diverse functionalities for BODIPY dyes with no special effect on the absorption and emission wavelengths due to their poor conjugations with BODIPY core. However, the quantum yields of 1,7-dimethyl-8-aryl-substituted BODIPY dyes (like 2.7 in Figure 2.9) are much higher than their counterparts without 1,7substituents (like 2.6 in Figure 2.9). This difference is attributed to that 1,7-substituents spatially prevent the free rotations of aryl groups so the loss of energy from non-radiative decay via molecular motions is reduced. Take this into consideration, 1,3,5,7-tetramethyl8-aryl-substituted BODIPY dyes are popular to be used as skeleton for further modifications.

\subsubsection{Synthesis of BODIPY dyes}

8- or meso-subsitituted BODIPY dyes can be synthesized either from reacting of pyrroles with aldehydes or pyrroles with acid chloride, which depends on the aryl or aliphatic substituents on meso-position. For meso-aryl-substituted BODIPY dyes, a typical one-pot synthetic route is shown in scheme 2.1. An acid catalyzed (typically trifluoroacetic acid) condensation of aromatic aldehyde 2.10 with pyrrole 2.11 affords dipyrrolemethane $\mathbf{2 . 1 2}$. This compound is relative unstable and sensitive to light, air and acid. So the oxidation step by using 2,3-dichloro-5,6-dicyano-1,4-benzoquinone (DDQ) is immediately conducted after preparation of dipyrrolemthane, yielding 
dipyrrolemethene or dipyrrin 2.13. Without further purification, dipyrrin 2.13 undergo complexation with boron by using boron trifluoride diethyl etherate $\left(\mathrm{BF}_{3} \cdot \mathrm{Et}_{2} \mathrm{O}\right)$ under base condition, giving the final highly fluorescent, brown color BODIPY product $\mathbf{2 . 1 4}$ with about $10-30 \%$ as final yield. For unsubstituted pyrrole $\left(\mathrm{R}_{1}-\mathrm{R}_{3}=\mathrm{H}\right)$, this reaction is usually carried in pyrrole as solvent to prevent potential polymerizations while the reaction with $\alpha$-substituted pyrrole does not require excess pyrrole and can react with aromatic aldehyde in normal organic solvent like $\mathrm{CH}_{2} \mathrm{Cl}_{2}$. This type of BODIPY dyes can carry many functional groups like hydroxyl, ester, carbocyclic acid, halogen etc. on the meso-aryl substituents, which typically are robust enough to allow many postmodifications such as oxidation, reduction, nucleophilic substitution and noble metalcatalyzed coupling reactions.

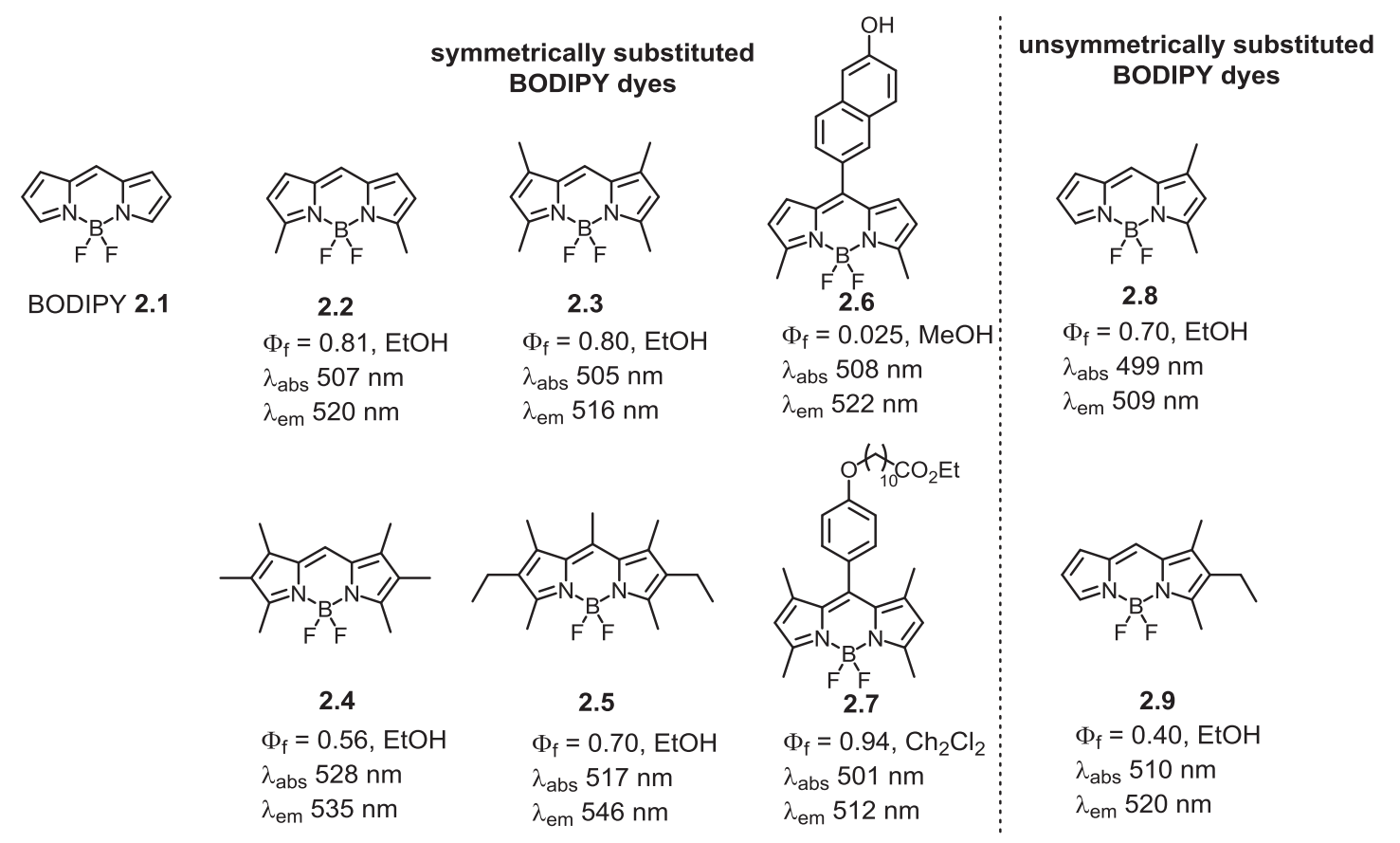

Figure 2.9. Chemical structures of BODIPY core and alkyl-, aryl-substituted BODIPY dyes. (The optical data in the figure are from the previous publications ${ }^{20-23}$ ) 


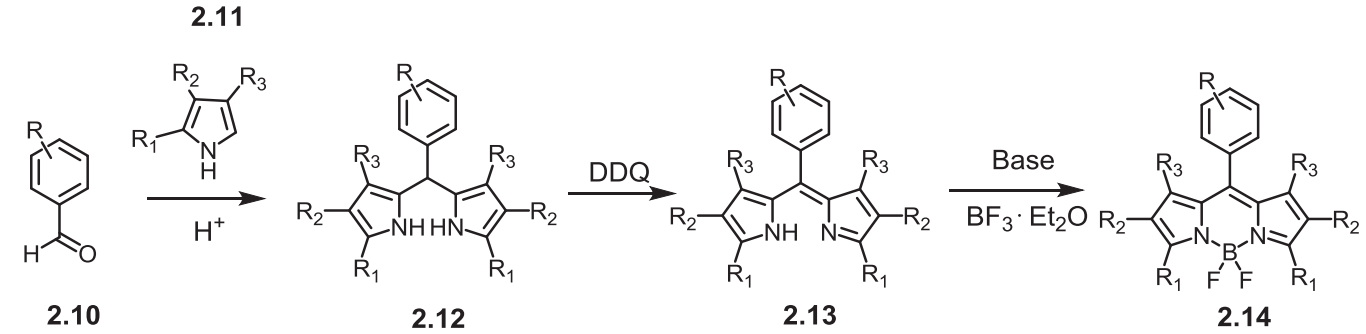

Scheme 2.1. Synthetic route to meso-aryl-substituted BODIPY dyes using aromatic aldehydes.

Another approach of BODIPY synthesis is by using pyrrole and acyl chloride as starting materials (Scheme 2.2). This approach is mainly for the preparations of mesoalkyl-substituted BODIPY dyes that cannot be synthesized by aldehyde route. The condensation of pyrrole $\mathbf{2 . 1 0}$ with acyl chloride $\mathbf{2 . 1 5}$ generates the intermediate acylpyrrole that is usually not isolated and can further react with pyrrole to afford dipyrrolemethene $\mathbf{2 . 1 6}$ without any oxidation step. Similarly, the final step is the complexation with boron under excess base condition, yielding the final BODIPY product 2.17. Other active carboxylic derivatives such as acid anhydride ${ }^{24}$ or orthoester $^{25}$ can also be used replacing acyl chloride in scheme 2.2.

Because of the functionalization diversity provided by meso-aryl-substituents on BODIPY core, in this dissertation, all the BODIPY dyes in chapter 3 - 9 were synthesized following the first route (Scheme 2.1) by using aromatic aldehydes.

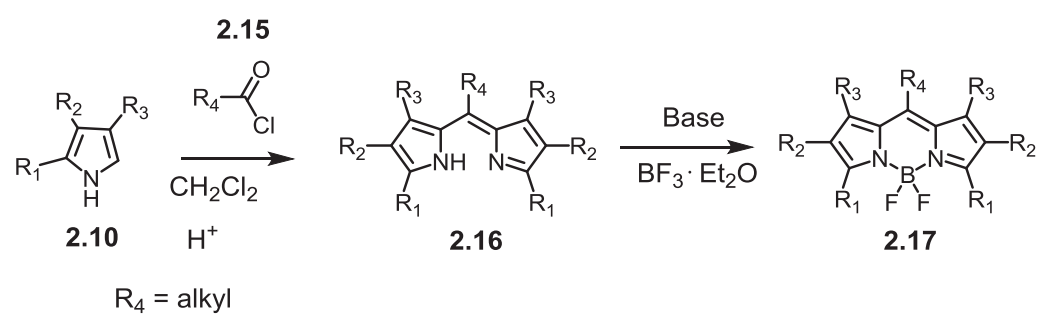

Scheme 2.2. Synthetic route to meso-alkyl-substituted BODIPY dyes using acyl chloride. 


\subsection{Modification strategies of BODIPY core: functional sites and reactions}

One of the biggest merits of using BODIPY skeleton for fluorescent probe design is the synthetic facileness for post-modifications and functionalization. The chemical robustness of BODIPY dyes and their many modification sites enable them to be functionalized with linkers, quenchers, receptors and many other organic or bioconjugates through different types of reactions.

\subsubsection{Modifications to meso-aromatic substituents on the BODIPY core}

Functionalization through meso-position is one of the most effective way to incorporate functional groups and to build larger architectures on to BODIPY core because it can be easily achieved by acid catalyzed condensation of pyrrole with appropriately substituted aromatic aldehyde. This strategy has been widely used to produce many different BODIPY dyes for many applications. For instance, functional phenyl groups at meso-postion containing triggers or sensitive groups to specific analytes have been introduced to generate many chemosensors and fluorescent sensors (Figure 2.10) such as $\mathrm{pH}$ probes $(\mathbf{2 . 1 8}, \mathbf{2 . 1 9}),{ }^{26-27}$ redox species probes $(\mathbf{2 . 2 0}, \mathbf{2 . 2 1}, \mathbf{2 . 2 4}),{ }^{28-30}$ biomolecule probes $(\mathbf{2 . 2 2}, \mathbf{2 . 2 3})^{31-32}$ and probes for different metal cations $(2.25$ 2.31) ${ }^{33-38}$ It has been noticed that aromatic substituent at meso-positions are poorly conjugated with a BODIPY core because of its orthogonal geometry. Therefore, in most cases, the fluorescence of probes in this category is manipulated through the modulations of the photo-induced electron transfer (PET) process from aromatic substituents to BODIPY cores. In addition, different functional groups can also be introduced to the BODIPY through this meso-aryl substituent strategy, allowing the post functionalization of BODIPY dyes. For examples, carboxylic acid group (2.32), ${ }^{39}$ halogen atoms (Br, I) $(\mathbf{2 . 3 3})^{40}$ and azide group $(\mathbf{2 . 3 4})^{41}$ are usually used as reacting sites at meso-position for further modifications through amidation, palladium-catalyzed cross coupling reactions and click reaction (azide-alkyne cycloaddition), respectively. 

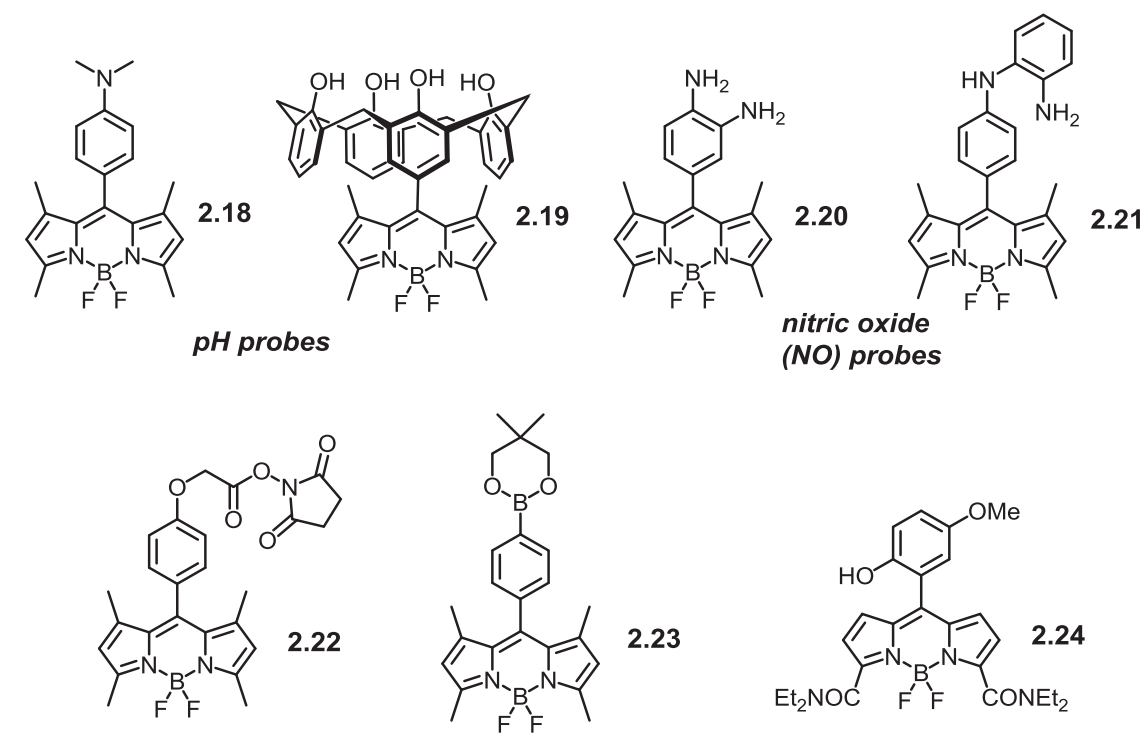

amine probe

monosacharide probe

hypochlorous acid probe
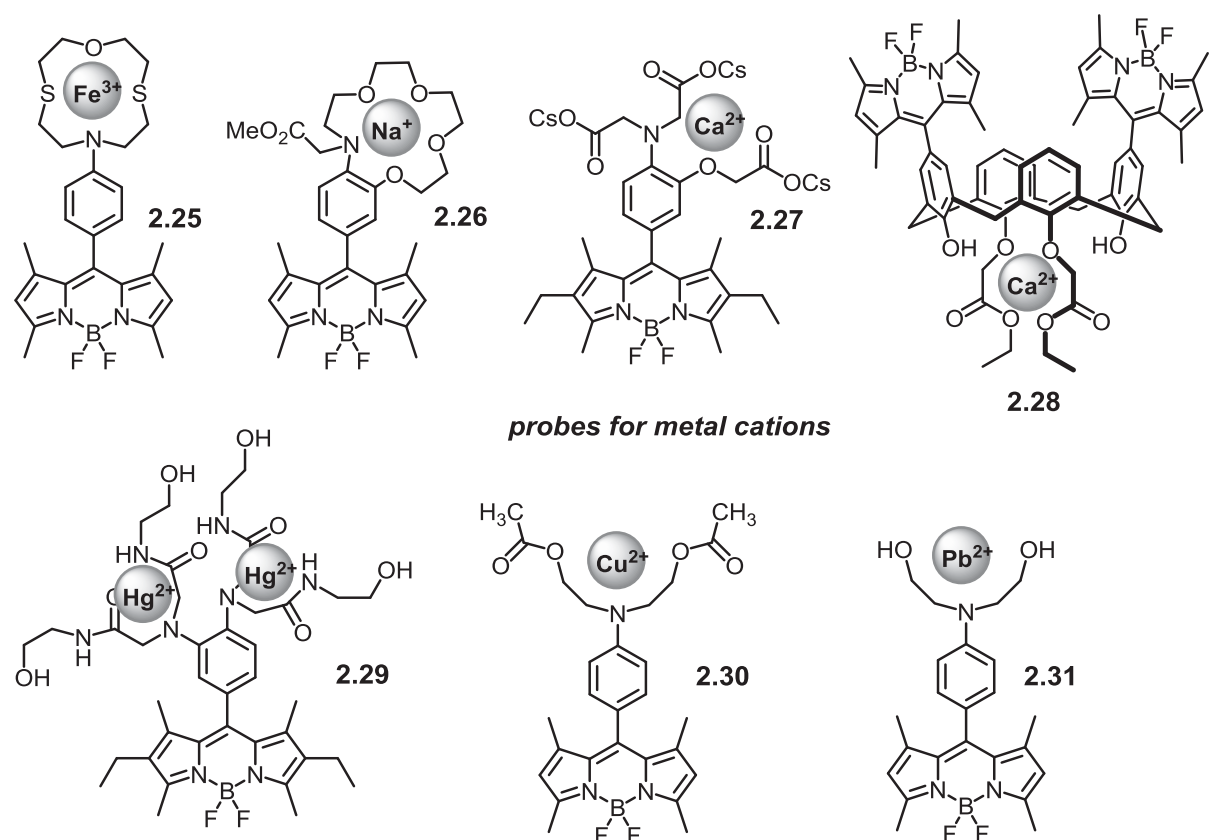

probes for metal cations
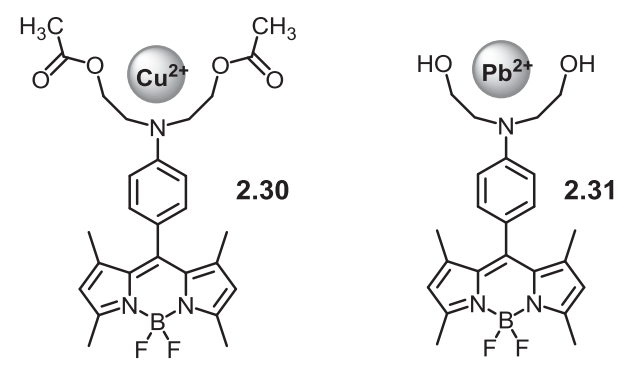

Figure 2.10. Selected BODIPY probes with different meso-modifications. 


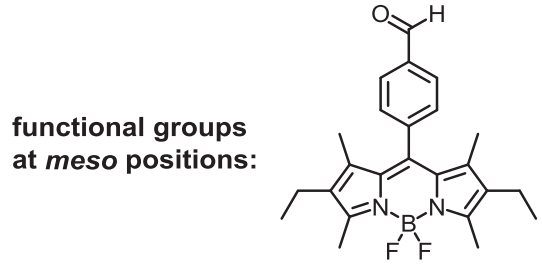

2.32

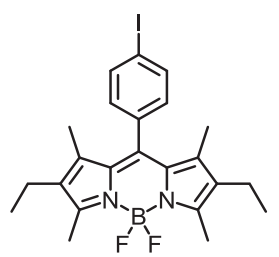

2.33

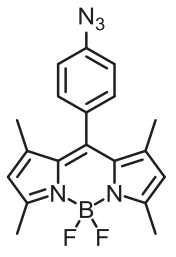

2.34

Figure 2.11. BODIPY dyes bearing functional groups at meso-positions.

\subsubsection{Functionalization at the 2,6-positions of BODIPY core}

Simple examination of resonance structures of BODIPY dyes reveals that the 2- and 6-positions of the BODIPY core bear the least positive charges (Figure 2.12). This electron rich character makes the 2- and 6-positions susceptible to electrophilic substitution reactions. Several kinds of this type reaction including halogenation, formylation, sulfonation and nitrations etc. have been reported.

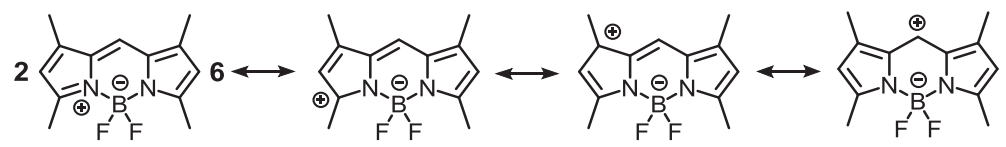

Figure 2.12. Illustration of electrophilic positions of 1,3,5,7-tetramethyl BODIPY dye by resonance structures.

Bromination and iodination are commonly used for 2,6-halogenation of BODIPY core. Slowly adding of bromine to the solution of 1,3,5,7,8-pentamethyl BODIPY dyes at room temperature can easily introduce bromo-substituents at 2,6-positions, giving the product 2.35. ${ }^{20}$ Iodination of BODIPY core can also be achieved by the route described in scheme 2.3. ${ }^{42}$ Predictably, the strong $p$ - $\pi$ conjugation of bromine and iodine atoms at 2,6positions with BODIPY core cause a significant red shifts of the maximum absorption and emission wavelengths, usually $20-50 \mathrm{~nm}$. Bromination and iodination of BOIDPY dye at 2,6-positions also significantly quench the quantum yields of the dyes due to the increased rate of intersystem crossing resulted from heavy atom effect. This effect make 
brominated or iodized BODIPY dyes more probability on their triplet state under excitation, and therefore, many of them have been used as photosensitizers. ${ }^{42-45}$

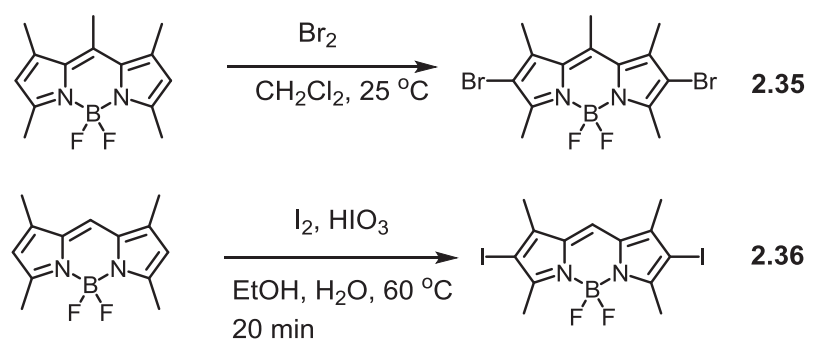

Scheme 2.3. Bromination and iodination of BODIPY dyes.

2,6-Brominated or 2,6-iodized BODIPY dyes are also one type of important intermediates and building blocks for further functionalization because they can further undergo palladium(0) mediated cross coupling reaction such as Sonogashira coupling, Suzuki coupling and Heck coupling to link the BODIPY core with other unsaturated architectures via newly generated carbon-carbon bonds (Figure 2.13).

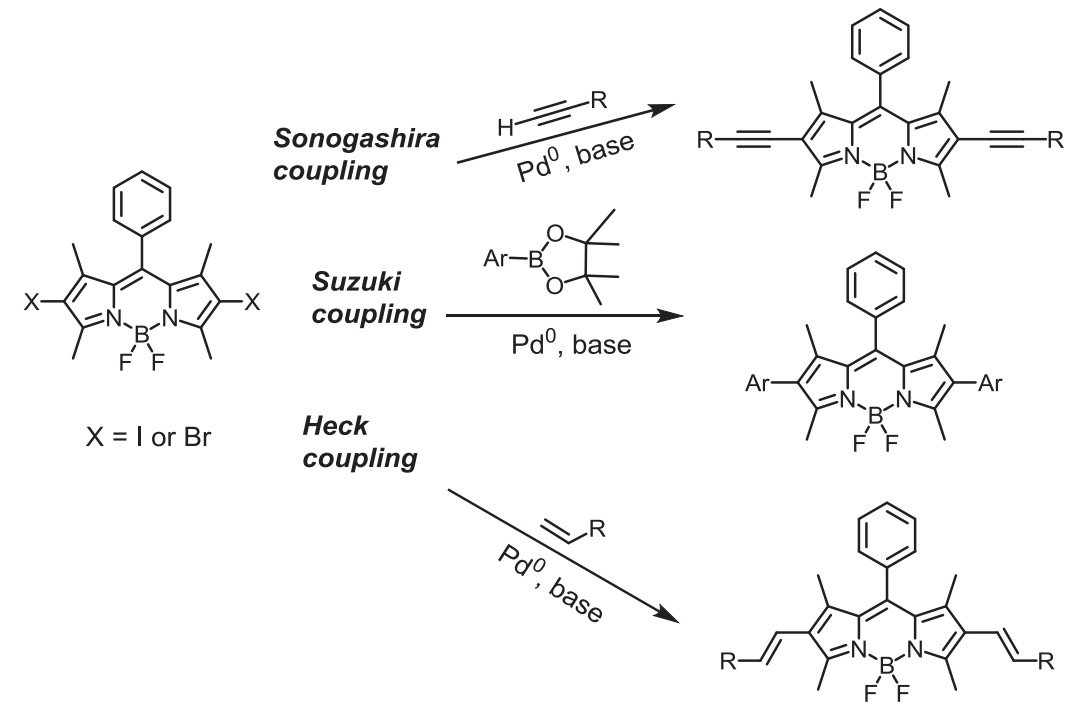

Figure 2.13. Palladium mediated cross coupling reactions of 2,6-halogenated ( $\mathrm{Br}$ and I) BODIPY dyes. 
Another important electrophilic substitution on 2,6-position of BODIPY core is formylation through Vilsmeier-Haack reaction. One of the popular conditions is: in the solvent of 1,2-dichloroethane, which has higher boiling point than dichloromethane, 2,6unblocked BODIPY dye react with the excess amount of Vilsmeier-Haack reagent which is freshly generated by reacting of DMF and phosphoryl chloride at $50{ }^{\circ} \mathrm{C}$ for 2 hours. After that, the solution is added into excess amount of weak base such as $\mathrm{NaHCO}_{3}$ aqueous solutions to hydrolyze the iminium intermediate, giving the $\beta$-formal-BODIPY dye with high yield (usually $>80 \%$ ). ${ }^{46}$ Since 2,6-position are electron rich, formylation at $\beta$-position is favored even on the $\alpha, \beta$-unsubstituted BODIPY core. ${ }^{46}$ The excess amount of Vilsmeier-Haack reagent does not generate the 2,6-diformyl-BODIPY dye because the formation of 2-mono-iminium intermediate during the reaction significantly deactivate the further electrophilic attack at its opposite, 6-position (Scheme 2.5). Introduction of $\beta$ formal groups usually does not case significantly change of absorption and emission maximum wavelength and normally small blue shifts $(3-10 \mathrm{~nm})$ will be observed. ${ }^{46-47}$ One of the benefits of $\beta$-formyl-BODIPY dyes is that they can participate in Knoevenagel condensation reaction with active hydrogen compound like cyanoacetic acid, propanedinitrile, methyl acetoacetate for further extension of $\pi$-conjugation with double bond formation at 2- or 6- position. Several of this type compounds have been reported for various applications. ${ }^{46,48}$

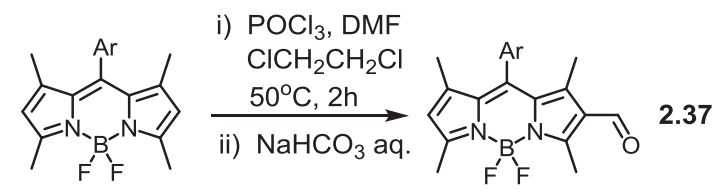

Scheme 2.4. Synthetic route to $\beta$-formyl-BODIPY dye via Vilsmeier-Haack reaction. 


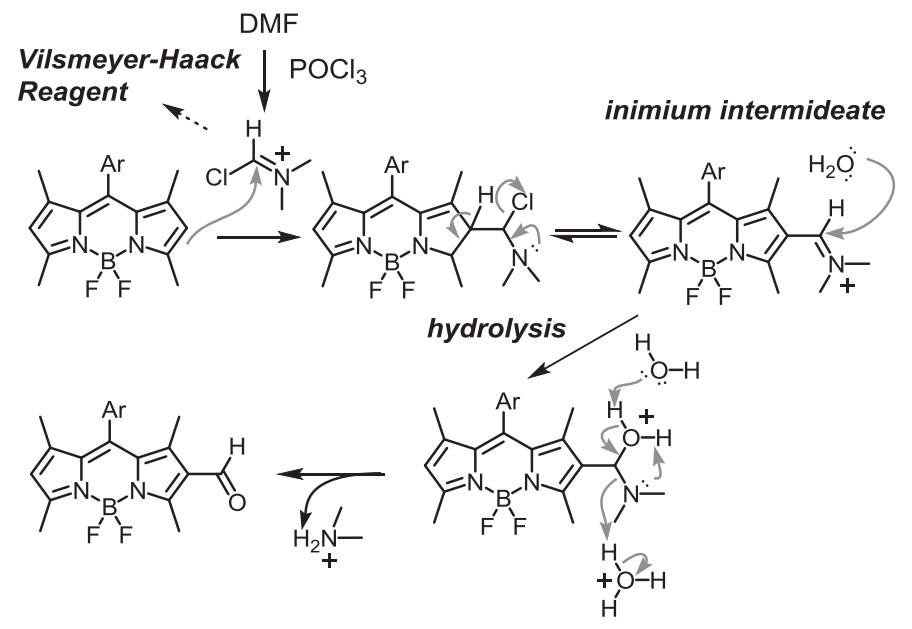

Scheme 2.5. Mechanism of $\beta$-formylation of BODIPY dye through Vilsmeier-Haack reactions.

Other electrophilic substitution such as sulfonation, nitration of BODIPY core at 2,6position also have been reported (Scheme 2.6). Since introduction of sulfonate groups do not change absorption and fluorescence emission significantly compared with their parent unsubstituted BODIPY dye, they are mainly for enhancing the dye solubility in water. ${ }^{49-52}$ Introduction of nitro groups on 2,6-positions can be achieved by the treatment of the dye with nitric acid at $0{ }^{\circ} \mathrm{C}$. The nitro groups significantly quench the fluorescent quantum yield due to their strong electron withdrawing property. Since the sulfonate and nitro groups at 2,6-positions of BODIPY dyes are hard to be further functionalized (to the best of our knowledge, nitro groups at 2,6-positions of BODIPY core are very hard to be reduced to amine groups), they are less used for modifications of BODIPY dyes.

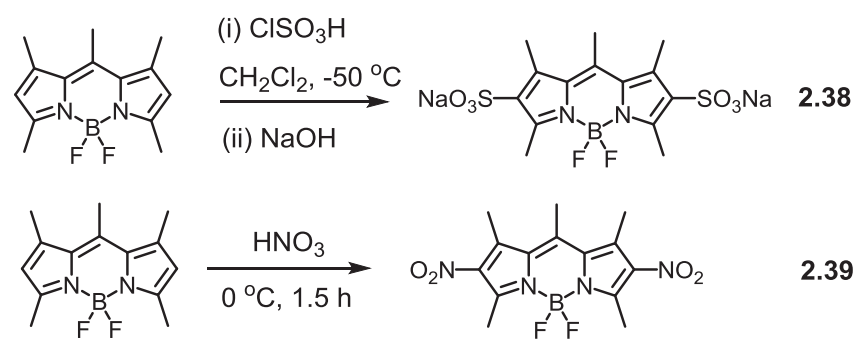

Scheme 2.6. Sulfonation and nitration of BODIPY dyes at 2,6-positions. 


\subsubsection{Functionalization at the 3,5-positions of BODIPY core}

\subsubsection{3,5-halogenated BODIPY dyes and their reactions}

Chlorine atoms can be preferentially introduced to the 3- and/or 5-positions of BODIPY core through the chlorination of dipyrrolmethane followed by oxidation with $p$ chloranil and complexation with boron trifluoride etherate (Scheme 2.7). The presence of good leaving group like chlorine enable BODIPY dyes to undergo nucleophilic substitution reactions. Many nucleophiles including alkoxides, amines, thioalkoxides and diethyl malonate anions have been applied to this type of reaction. ${ }^{53-54}$ Both mono-and disubstituted products can be obtained by controlling the stage of the reactions including reactant ratio and reaction time. Several example compounds are shown in Figure 2.14.

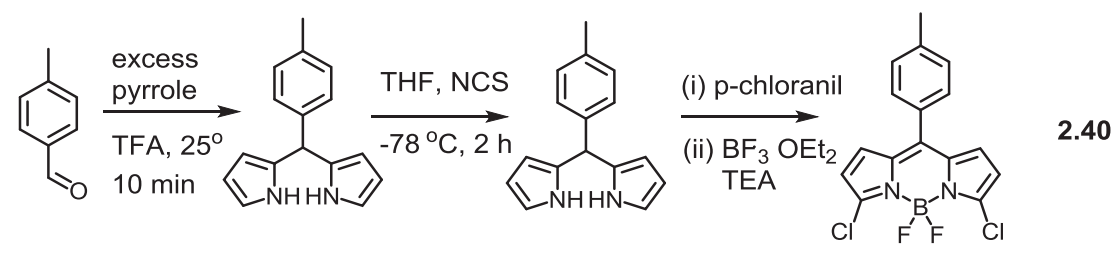

Scheme 2.7. Chlorination of BODIPY dye at 3,5-positions.

Regarding the spectroscopic properties, the electron donating substituents especially like amino, thioether at 3,5-positions significantly change both absorption and emission spectra by red shifting compared to their parent unsubstituted BODIPY core. The quantum yields of these compounds varied widely but in general will be reduced due to the intramolecular charge transfer (ICT) effect from the electron donating atoms (nitrogen, sulfur) of the 3,5-substituents to the BODIPY core. However, this effect does provide an inspiration for designing the fluorescent probes. Couple of BODIPY fluorescent probes for sensitive detection of metal cations have been developed based on the modulation of ICT effect by incorporating different cation chelators as 3- and/or 5substituents onto BODIPY core (Figure 2.14 2.47-2.49). ${ }^{54-56}$ In addition, 3- and/or 5chlorine or other halogen-substituted BODIPY dyes are also reactive for the palladium mediated cross coupling reactions such as Sonogashira coupling, Suzuki coupling and 
Heck coupling. Some 3- and/or 5-diaryl, styryl or alkynated BODIPY dyes have been reported based on these reactions. ${ }^{57-58}$

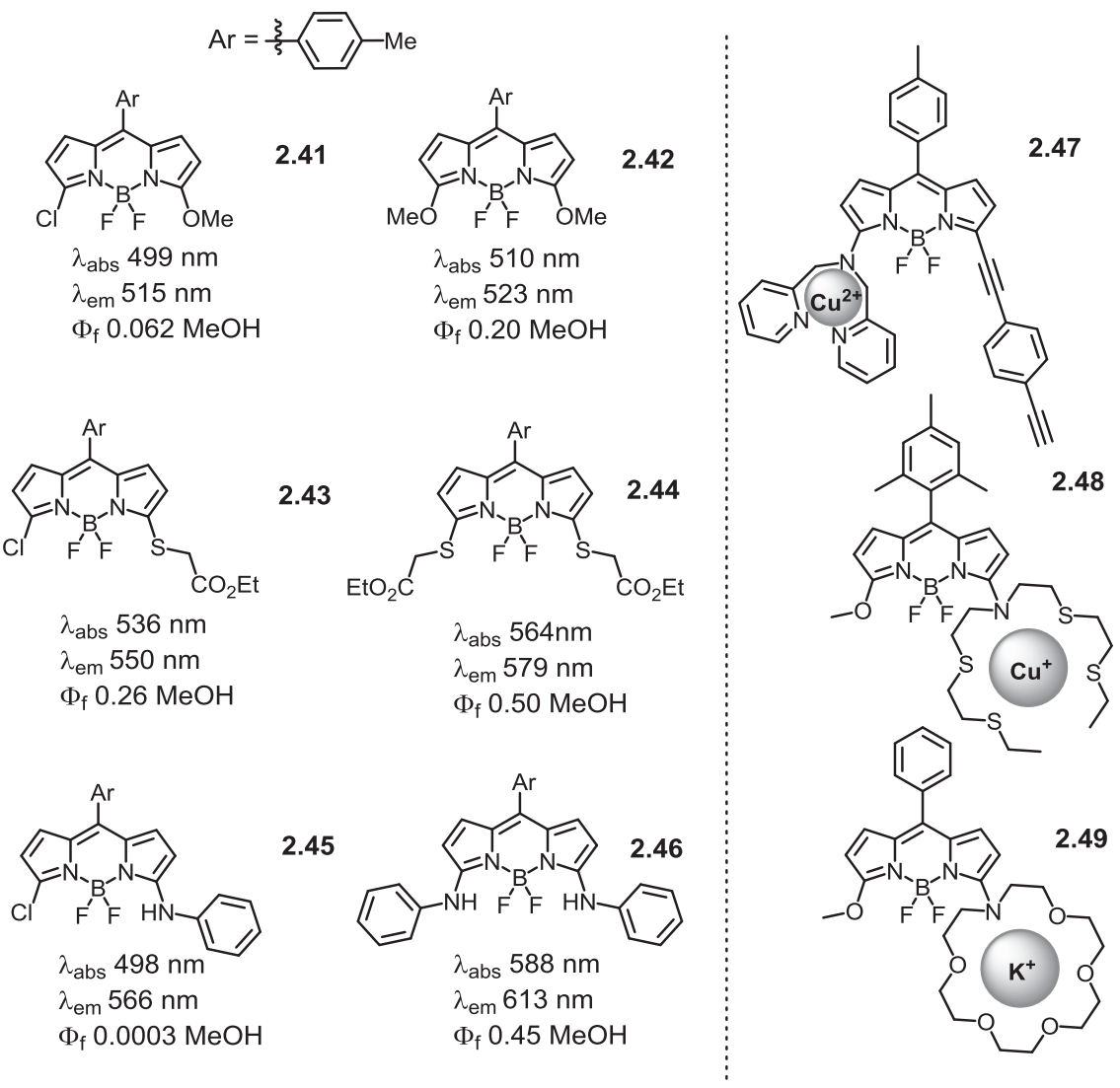

Figure 2.14. 3,5-Substituted BODIPY dyes through $\mathrm{S}_{N} \mathrm{Ar}$ reactions. (The optical data in the figure are from the previous publications ${ }^{54-56}$ )

\subsubsection{Condensation reactions of the 3,5-dimethyl derivatives with benzaldehyde derivatives}

Dimethyl substituents at 3,5-positions of BODIPY core are considered acidic enough to participate in Knoevenagel condensation reactions. Thus, various types of 3,5-distyrylBODIPY derivatives (abbreviated as styryl-BODIPYs) can be synthesized by Knoevenagel condensation of 3,5-dimethyl-BODIPY with different aromatic aldehydes (Figure 2.15). Piperidine and acetic acid are the typical catalysts used in this type of condensation. Water is formed as a byproduct during the condensation process and it can be removed azeotropically by using a Dean-Stark apparatus with benzene or toluene as solvent (Figure 2.15). This reaction usually gives mixture products of mono- and distyryl- 
BODIPY dyes. The yield of mono- and distyryl BODIPY dyes can be controlled by adjusting the equivalent of aromatic aldehyde added, reaction time, temperature and other reaction conditions.
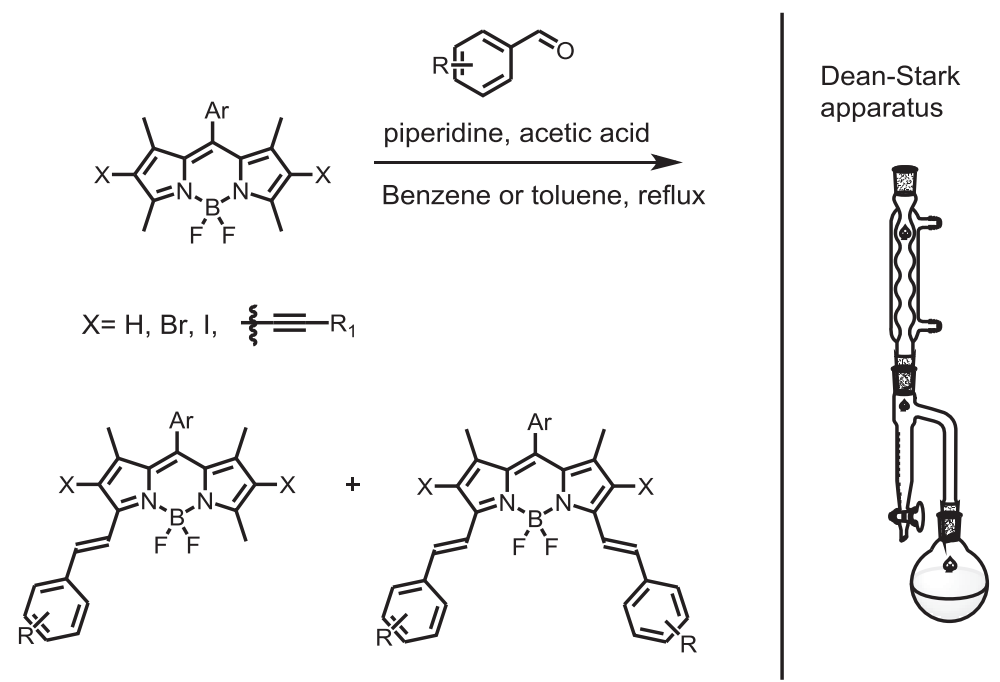

Figure 2.15. Knoevenagel condensation of 1,3,5,7-tetramethyl-BODIPY dyes with aromatic aldehyde derivatives.

Compared with the meso-position modification by aryl substitutions, condensation reaction at 3,5-positions not only offers numbers of functionalities to BODIPY dyes by using appropriate modified aromatic aldehyde, but also widely extend the $\pi$-conjugation of BODIPY core so that their absorption/emission wavelengths shift to red, deep red and even near infrared regions. So far, Knoevenagel condensation of 3,5-dimethyl BODIPY dyes with aromatic aldehyde has been considered one of the best strategies for obtaining long absorption and emission wavelength (especially in near infrared (NIR) region) BODIPY dyes because it is more convenient and less time consuming than other analogous synthetic routes. Reacting with aromatic aldehydes which have electron donating substituent like dimethylamino group at para-position causes further red-shifts of both absorption and emissions compared with those styryl-BODIPY dyes without para-substituents. Many fluorescent probes with long wavelength emission $\left(\lambda_{\mathrm{em}}>600\right.$ $\mathrm{nm}$ ) based on styryl-BODIPY dyes have been developed and extensive different types of analytes are involved (Figure 2.16). ${ }^{59-63}$ 

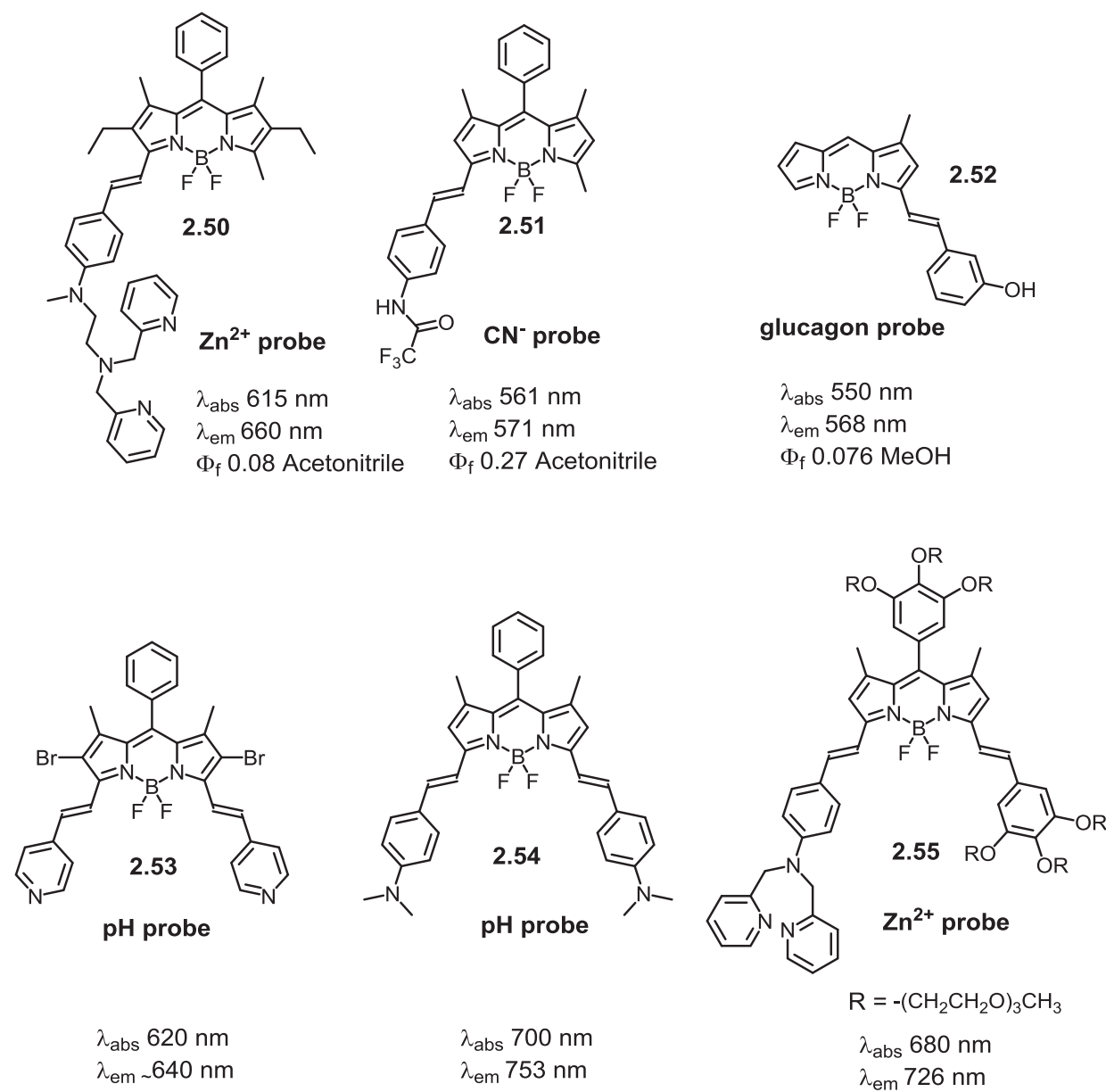

Figure 2.16. Selected fluorescent probes based on styryl-BODIPY scaffold. (The optical data in the figure are from the previous publications ${ }^{59-63}$ )

Methyl substituents at 1,7-positions of BODIPY also are also considered as acidic but not as strong as 3,5-methyl substituents so Knoevenagel condensation primarily occur at 3,5-position rather than 1,7-positions and 3,5-mono- and/or distyryl compounds usually dominate the reaction products. However, extending the reaction time and rising the temperature can activate 1,7-positions and stepwise afford 1,3,5-tristyryl- and 1,3,5,7tetrastyryl-BODIPY dyes through same condensation mechanism after formation of mono- and distyryl compounds. ${ }^{64}$

\subsubsection{Substitution of fluorine atoms at 4-position of BODIPY core}

Fluorine atoms on boron center of BODIPY core can be replaced by many different substituents including aryl, alkynyl and alkoxy groups via nucleophilic substitution 
reactions to generate a new family of photostable dyes called $C$-BODIPYs, $E$-BODIPYs and $O$-BODIPYs (Scheme 2.8). ${ }^{65-67}$ These reactions typically require very strong nucleophiles such as aryl lithium or Grignard, alkynyl lithium or Grignard, alkoxide reagents in a very basic condition. Among them, 4-diethynyl-BODIPY dyes (EBODIPYs) are more popular than other 4-subsitutied BODIPY dyes because the weak acidity of ethynyl group allows the fresh preparation of lithium acetylide or ethynyl Grignard reagents by simply reacting ethynyl compounds with lithium alkylide or alkylmagnesium bromide.
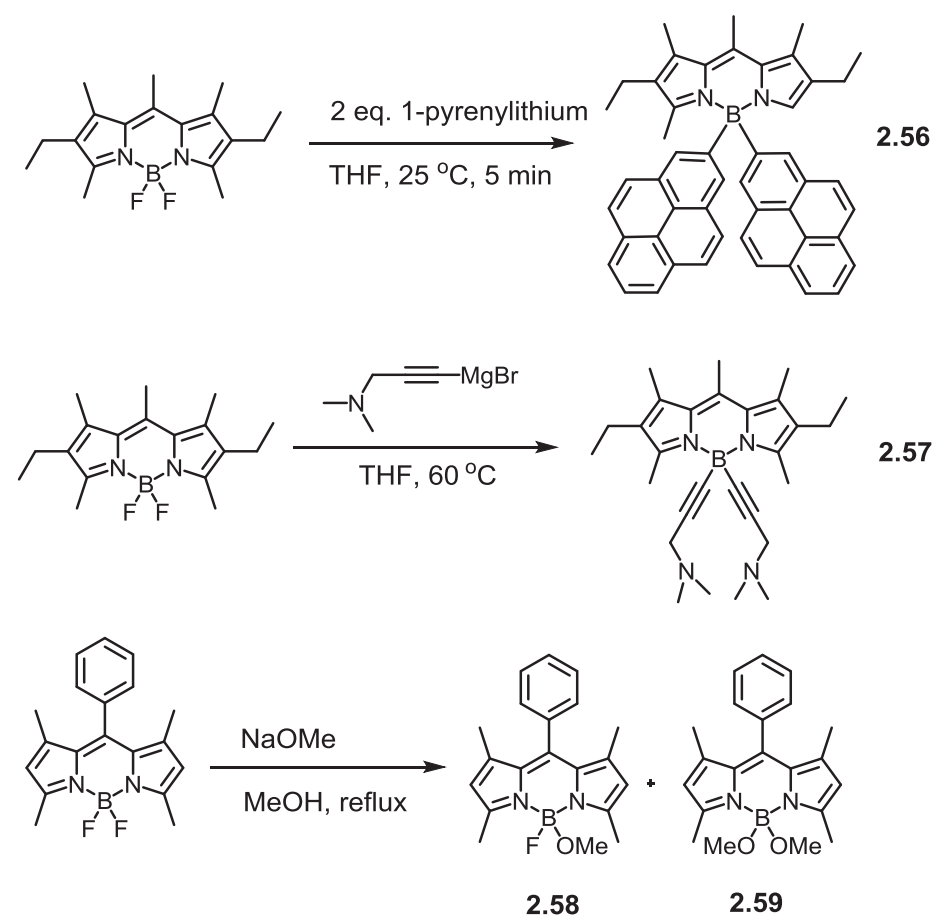

Scheme 2.8. Example substitution reactions on boron centers of BODIPY dyes.

The substitution on boron center of BODIPY core will not cause the many shifts on both absorption and emission wavelengths of the dye because those substituents are not engaged in the $\pi$-system of BODIPY frame due to the tetrahedron confirmation of boron center bonds. However, the substitutions on boron atom do affect the quantum yields and reactivity of the BODIPY dyes. 


\subsection{Tuning the absorption and emission wavelengths}

One of the major goals of modification of BODIPY core is to tune its absorption and emission to long wavelength region (> $500 \mathrm{~nm}$ ). BODIPY dyes with different absorption and emission wavelengths in visible region extensively extend the importance and diversity of their applications, for instance, different colored BODIPY probes, colorimetric and fluorescent dual-channel BODIPY probes and fluorescent probes based on förster resonance energy transfer (FRET). Particularly, tuning their absorption and emission wavelengths to deep red and near infrared region is more important for in vivo measurements in biological applications of BOIDPY fluorescent probes because there is less light scattering and absorption interference caused by cells and tissues in this region of wavelength, so called "biological window".

Based on the reactions and modification strategies discussed in section 2.4.3, there are several typical approaches to tune the absorption and emission wavelengths of BOIDPY dyes by extension of the $\pi$-conjugation and adjust the energy gap between its HOMO and LUMO orbitals.

\subsubsection{Tuning through introducing electron donating and halogen atoms}

Heteroatom substituents like nitrogen, sulfur and oxygen atoms can be introduced at 3- and/or 5-positions of BODIPY core via aromatic nucleophilic substitution reactions $\left(\mathrm{S}_{N} \mathrm{Ar}\right)$ with 3,5-halogenated BODIPY dyes. The strong electron donating character of these atom substituents significantly reduce the HOMO-LUMO energy gaps by increase the $\pi$-electron density of the dyes, leading to obvious red-shifts on both absorption and emission wavelengths, usually 30-50 $\mathrm{nm}$ shift. Compounds 2.41-2.46 are example BODIPY dyes in this type (Figure 2.14). The strong electron pushing effect also cause the high dipole moments on excited states of the dyes, which can be stabilized by polar solvent rather than non-polar ones. That means, the fluorescence intensities and emission wavelengths of this type of BODIPY dyes are usually solvent dependent.

Besides, heavy halogen substituents like bromo and iodo groups at 2,6-positions on BODIPY dyes also lead to red shifts of absorption and emissions because increased $p-\pi$ conjugations. The typical red shift of this kind of modification varies from $20-50 \mathrm{~nm}$. Usually iodization at 2,6-positions cause a little more red shift than corresponding 
bromination. However, both of bromo and iodo groups introduced on BODIPY framework will significantly quench the fluorescence quantum yield of the dye due to heave atom effect, and iodo group quench more than bromo group (Figure 2.17).
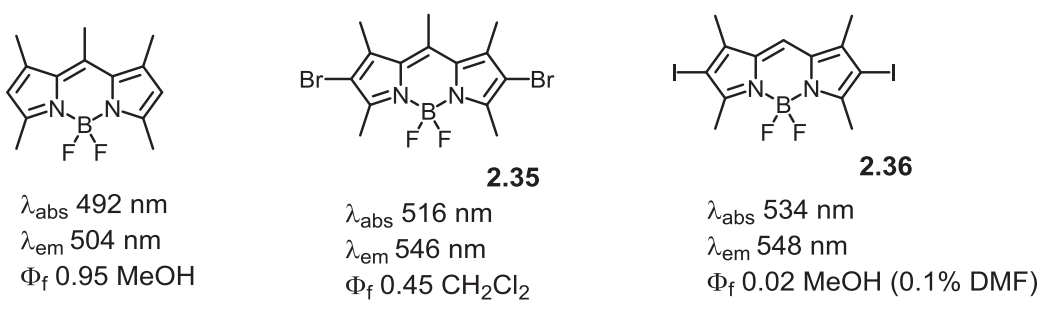

Figure 2.17. Photophysical properties of parent BODIPY core, 2,6-brominated and iodinated BODIPY dyes. (The optical data in the figure are from the previous publications $^{20,42}$ )

\subsubsection{Tuning through extension of $\pi$-conjugation}

Extension of $\pi$-conjugation of BODIPY dyes will significantly reduce their HOMOLUMO energy gaps and adjust their absorption and emission wavelengths to longer wavelength regions. Based on the structure of BODIPY core, several positions including 1-, 2-, 3-, 5-, 6-, and 7-positions are available for further modification to extend the $\pi$ conjugation via forming $\mathrm{C}-\mathrm{C}$ bonds with $\mathrm{sp}^{2}$ or $\mathrm{sp}$ hybridized carbons. However, mesoposition and 4-position are usually not appropriate for this type of modification because the orthogonal substituents at these two positions are poorly conjugated with BODIPY core. Compared with electron donating substituents on BODIPY core, the extension of $\pi$-conjugation will cause much more significant and larger red shift on major spectra wavelengths, which usually more than $100 \mathrm{~nm}$.

Introduction of aryl or alkynyl substituents at 2,6- or 3,5-positions has proven to be a very efficient way to extend $\pi$-conjugation of BODIPY core. These BODIPY dyes can be obtained either via direct synthesis of BODIPY dyes by using corresponding $\alpha$-arylsubstituted pyrroles or via palladium catalyzed cross coupling reactions (Suzuki, Sonogashira) with 2,6- and/or 3,5-halogenated BODIPY dyes and corresponding 
aromatic coupling reagents. Many different unsaturated architectures and polymers have been introduced via these positions to form larger $\pi$-conjugation that significantly tune the absorption and emission wavelength of the dye to red, deep red and even near infrared regions. For instance, 3,5-diphenyl-substituted BODIPY dye $\mathbf{2 . 6 1}$ has about $50 \mathrm{~nm}$ red shift on its main spectra bands relative to unsubstituted BODIPY dye 2.60 (Figure $2.18){ }^{68-69}$ The para-electron donating groups of $\mathbf{2 . 6 2}$ push more electrons to conjugation and make larger red shifts. However, both $\mathbf{2 . 6 1}$ and $\mathbf{2 . 6 2}$ do not have high quantum yields. One of the reasons is the energy loss caused by non-radiative decay due to the rotation of C-Ar bonds at 3,5-positions. ${ }^{69-70}$ The steric hindrance between arylsubstituents at 3,5-positions and $\mathrm{BF}_{2}$ unit also affects the conjugation degree between two aromatic systems. More steric hindrance results in larger constrains which make two $\pi$ system cannot remain coplanar. So, thiophene substituted BODIPY dye $\mathbf{2 . 6 3}$ absorbs and emits longer wavelength than $\mathbf{2 . 6 2}$ just because 5-membered rings have less space hindrance than 6-membered rings, although thiophene has similar electron properties with para-methoxy-phenyl ring. ${ }^{71}$ Significant red shifts of absorption and emission wavelengths also can be achieved by peripheral alkynyl substitution at 3,5- and/or 2,6positions, such as 2.64, 2.65 and 2.67. The 2,6-dialkynyl substitution of $\mathbf{2 . 6 5}$ give a spectra red shift about $70 \mathrm{~nm}$ relative to $\mathbf{2 . 6 0}$ but it is not as large as the red shift caused by its analog 2.64 with 3,5-dialkynyl substitutions. ${ }^{72-73}$ Further extending the $\pi$ conjugation by co-polymerization with other alkynyl-aryl unites through 2,6-positions of BODIPY dye $(\mathbf{2 . 6 7})^{74}$ leads to larger red shifts of main spectra compared with 2,6dialkynyl-substituted BODIPY dyes (2.65). Conjugated polymeric BODIPY dyes also can be formed through Ar-Ar single bonds connections at 2,6-positions of BODIPY dyes via Suzuki coupling reaction, for example 2.66. However, this type of polymeric BODIPY dyes does not offer significant spectra red shift. The main reason is that the steric repulsions between the hydrogens of 1,3- or 5,7-methyl groups and orthohydrogens of phenyl rings lower the degree of co-planarity between two aromatic units. ${ }^{75}$ Both polymers $\mathbf{2 . 6 6}$ and $\mathbf{2 . 6 7}$ have been synthesized and reported by our group. 


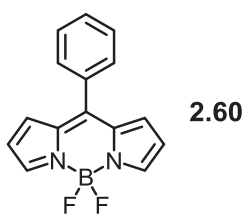

$\lambda_{\text {abs }} 503 \mathrm{~nm}$

$\lambda_{\text {em }} 521 \mathrm{~nm}$

$\Phi_{f} 0.053$ toluene
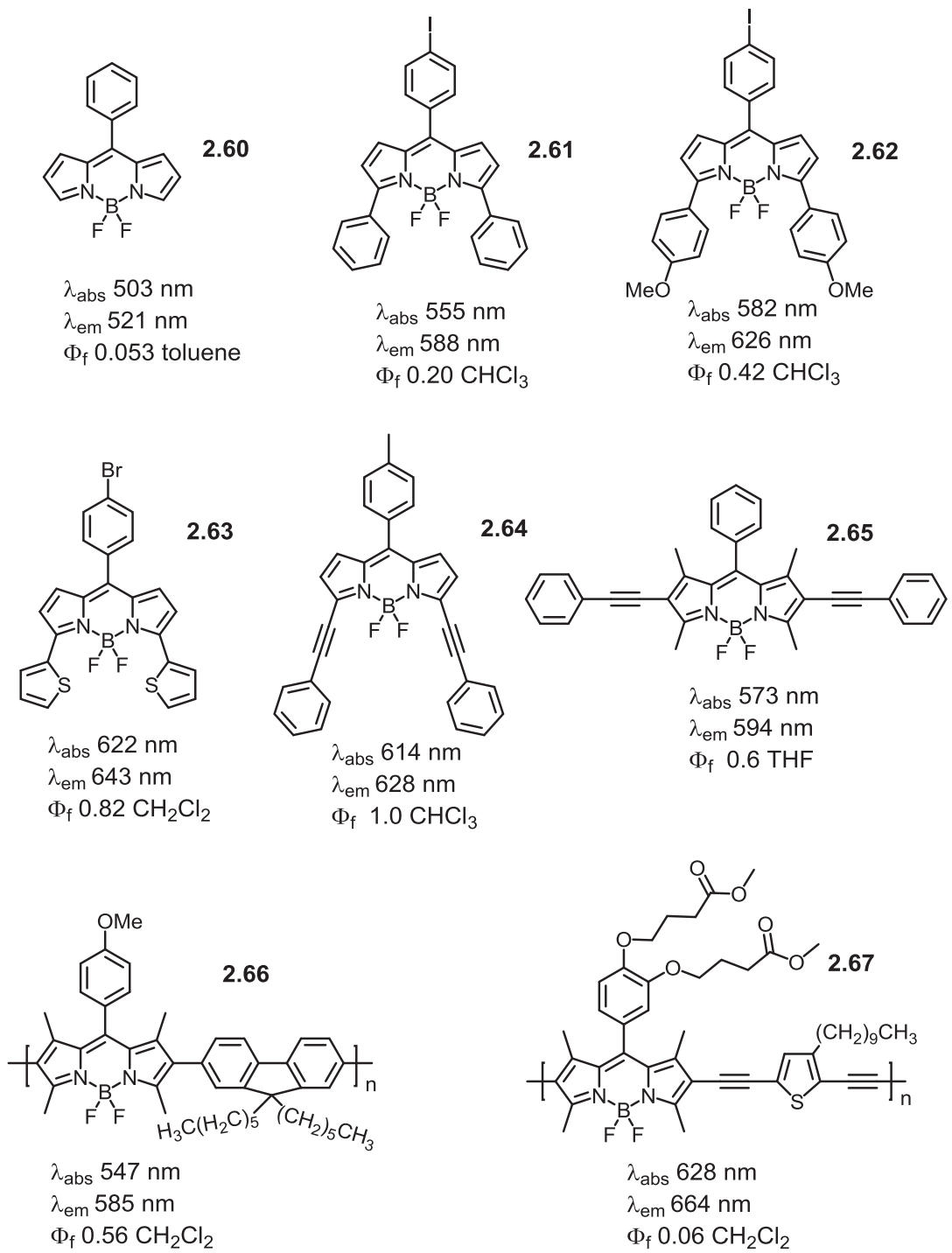

Figure 2.18. Extension of $\pi$-conjugation of BODIPY dyes thought 3,5-and 2,6positions.(The optical data in the figure are from the previous publications ${ }^{68-75}$ )

Styryl-substituted BODIPY dyes formed by Knoevenagel condensations with aromatic aldehydes is another extremely useful category of BODIPY dyes which afford deep red or near infrared (NIR) emissions. From mono- to tetrastyryl substitution, more styryl units are incorporated, larger red shifts will be obtained. When a monostyryl substituent is introduced to 3-position of BODIPY dye (2.69), its absorption and emission significantly shift to longer wavelength by $60 \mathrm{~nm}$ relative to its parent dye 2.68 (Figure 2.19). ${ }^{76-77}$ Strong electron donating groups like dimethylamino groups on the para- 
position of styryl substituents make further red shifts by $30 \mathrm{~nm}$ for absorption and $69 \mathrm{~nm}$ for emission respectively. Clearly, the stocks shift is significantly enlarged by introduce of dimethylamino groups. This is mainly attributed to the formation of low-lying charge transfer (CT) excited state caused by the intramolecular charge transfer (ICT) effect. ${ }^{78}$ However, introduce of weak electron donating groups like alkoxy groups on the same position (2.71) only give a very small red shifts on spectra wavelengths relative to $\mathbf{2 . 6 9} .^{79}$
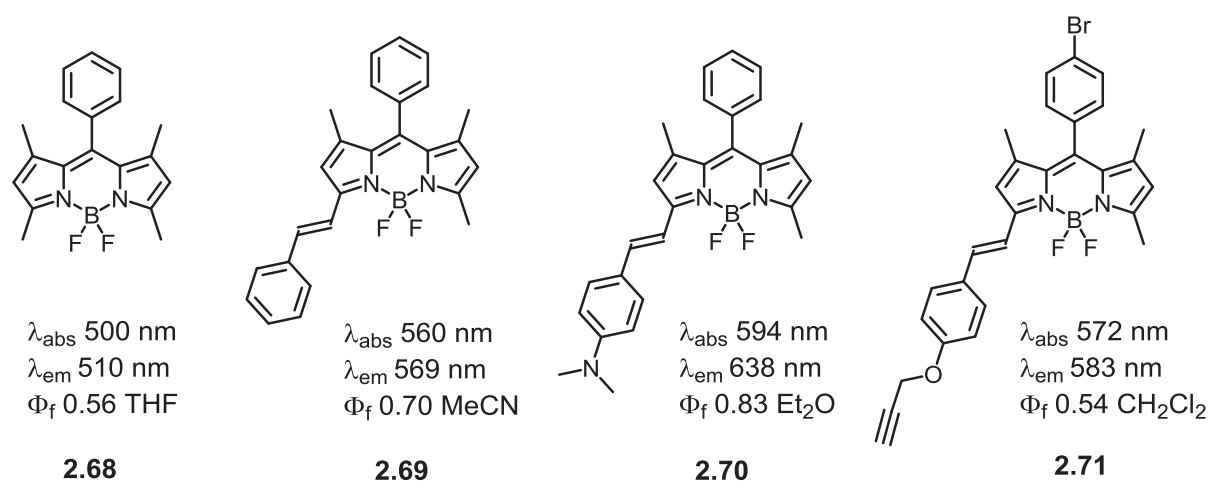

Figure 2.19. Selected 3-monostyryl-substituted BODIPY dyes. (The optical data in the figure are from the previous publications ${ }^{76-79}$ )

3,5-Distyryl substitution of BODIPY dyes can tune the absorption and emission wavelengths to far red and NIR regions with further significant red shifts of about $70 \mathrm{~nm}$ than mono-substituted one when comparing compounds $\mathbf{2 . 7 2}$ with $\mathbf{2 . 6 9}$ (Figure 2.20). ${ }^{80}$ These remarkable red shifts indicate the extreme importance of this type of BODIPY dyes as the scaffolds for design of NIR emissive fluorescent probes. Similar with compound 2.70, the dimethylamino substituents at para-position make further significant red shifts and drive the molecule to emit over $700 \mathrm{~nm}$ fluorescence (2.73). On the contrary, styryl substituents with electron withdrawing property like pyridine (2.74) will reduce the electron density on BODIPY core and cause blue shifts of spectra wavelengths relative to regular distyryl-substituted BODIPY like $2.72 .{ }^{81}$ As discussed in section 2.4.3.2, it is possible to obtain not only mono- and distyryl BODIPY dyes but also triand tetrastyryl BODIPY dyes through the extension of Knoevenagel condensations to 1and 7-positions. The spectroscopic properties of these dyes are also changed when 
different numbers of styryl substituents are attached. In general, more styryl substituents give longer absorption and emission wavelengths. For instance, tristyryl dye $\mathbf{2 . 7 6}$ absorbs and emits $20 \mathrm{~nm}$ longer than distyryl dye $\mathbf{2 . 7 5}$. And tetrastyryl dye $\mathbf{2 . 7 6}$ make another 20 $\mathrm{nm}$ red-shifts of both aborption and emissions relative to $\mathbf{2 . 7 6} .{ }^{64}$ However, the red shifts caused by 1,7-distyryl substituents are much less than the one caused by 3,5-distyryl substituents.
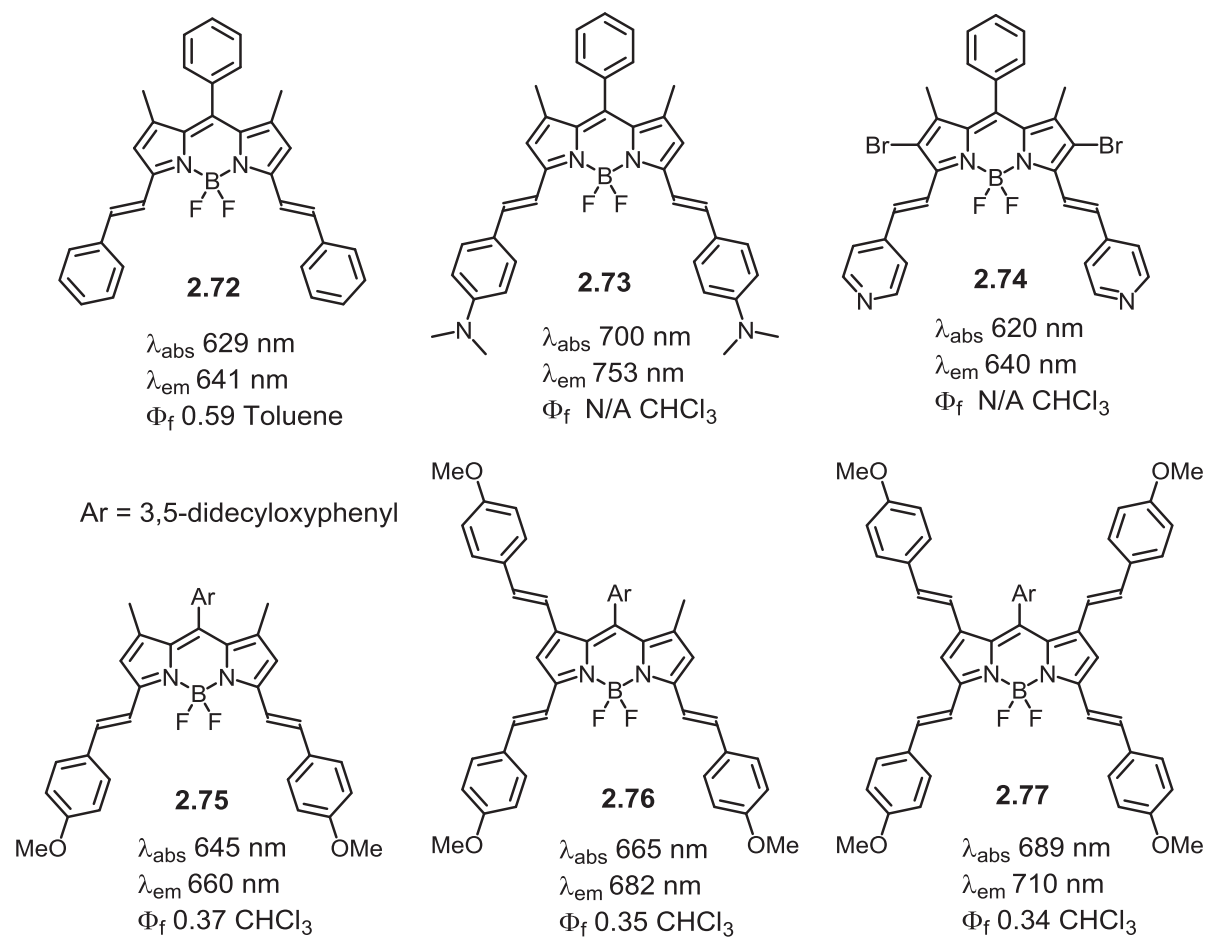

Figure 2.20. Selected 3,5-distyryl and 1,3,5,7-tetrastyryl-substituted BODIPY dyes. (The optical data in the figure are from the previous publications ${ }^{64,80-81}$ )

\subsection{Modulation of fluorescence of BODIPY-based fluorescent probes}

One of the key for designing a fluorescent probe is to modulate its fluorescence to form a "fluorescent switch" which can respond to the concentration or presence of the analyte. Many photophysical processes (such as quenching by collisions, exciplex formations, energy transfers, conjugation disturbance, intramolecular charge transfers, 
photoinduced electron transfers) can be involved in modulating the fluorescence of indicator upon recognizing analytes. The most popular and widely used mechanism for design of fluorescent probes will be briefly discussed in the following sections.

\subsubsection{Disturb, reduce or enhance the $\pi$-conjugations}

Changing the properties of their $\pi$-conjugations of fluorophores is probably the most direct way to alter the spectroscopic properties of fluorescent molecules. With this strategy in mind, many fluorescent indicators have been developed based on this mechanism. In general, the $\pi$-conjugation of fluorophore can be disturbed, reduced or enhanced depending on the recognition events of analytes (Figure 2.21). In many cases, disturbing and reducing of the $\pi$-conjugation require the chemical reactions occurring between fluorescent indicators with the analytes. For instance, the oxidation, reduction or nucleophilic attack reactions can change the key groups on conjugation or disrupt the conjugation with formation of $\mathrm{sp}^{3}$ hybridized carbons. In terms of spectroscopic properties, the direct reflection of altering conjugation system will be the significant shifts of absorption and emission spectra together with fluorescence intensity change. Thus, many dual-channel, ratiometric responsive fluorescent probes have been developed based on this mechanism. ${ }^{82-85}$ Fluorescent probes based on the spirocyclization of xanthene derivatives such as Rhodamines are a classic type of probes using the mechanism of enhancing $\pi$-conjugation. The spirocyclized form is non-fluorescent but the spiro-ring opening and re-aromatization caused by the reaction with analytes (for example: heavy metal ions) ${ }^{86-88}$ will rebuild the $\pi$-conjugation which recovers fluorescence. 


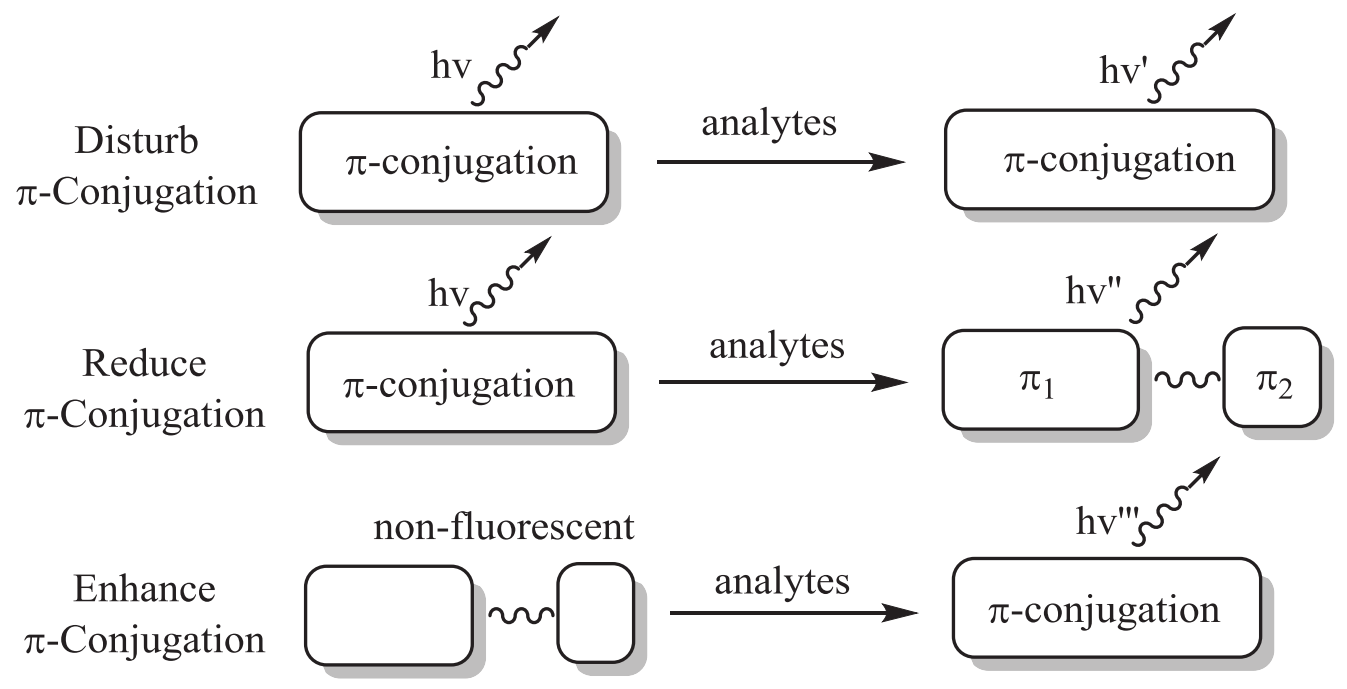

Figure 2.21. Illustration of fluorescence modulation mechanism by disturbing, reducing and enhancing $\pi$-conjugation of the fluorophore.

\subsubsection{Photoinduced electron transfer (PET)}

Fluorescent probes based on photoinduced electron transfer are important family of fluoro- and chemosensors. The probes in this category are often composed of fluorophore and receptor. The receptor can be chelating group, coordination site, receptor, host or ligand. Fluorophore and receptor are linked orthogonally or through a spacer. In other word, fluorophores and receptors are isolated or very poorly conjugated for PET based probes (Figure 2.22). The photoinduced electron transfer process, that is the electron transfer between fluorophore and receptor under excitation, usually cause fluorescence quenching. The binding event between receptor and analyte prohibits the PET process and recover the fluorescence of the probe. Thus, PET mechanism is quite useful for design of "off-on" responses of fluorescent probes.

The receptor will either function as electron donor or electron acceptor in PET. In the case of donor, the fluorophore is reduced whereas electron rich receptor is oxidized when PET process occur, so it is called reductive-PET. On the contrary, oxidized-PET is defined when the electron deficient receptor functions as an acceptor and fluorophore is oxidized whereas electron withdrawing receptor is reduced. However, only a few 
oxidized-PET based fluorescent probes have been reported in literature and the most of PET based BODIPY probes are dominated by reductive-PET process because the receptor as an electron donor is much easier to design and synthesize. Thus, reductivePET process will be discussed in detail below.
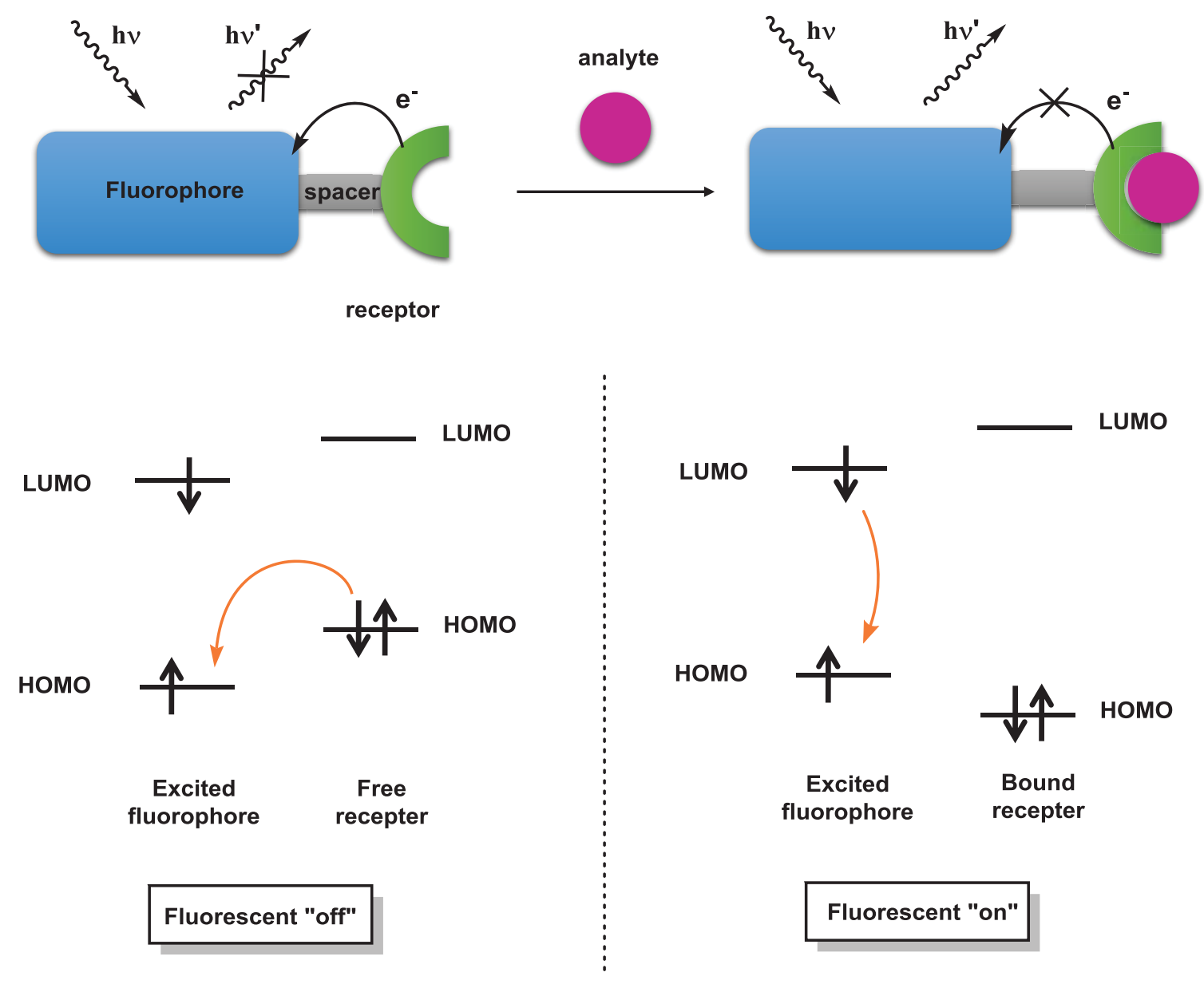

Figure 2.22. Illustration of mechanism of reductive-PET based fluorescent probes.

The reductive-PET process can be illustrated by simply using molecular frontier orbitals theory, which is a pictorial tool to show the mechanism of the fluorescence "offon" switching of the probe (Figure 2.22). The analyte-free receptors on these probes are electron rich groups (e.g. tertiary amines groups, para-amino-phenyl groups) relative to 
the flurophores and their highest occupied molecular orbital (HOMO) is just in between of the HOMO and lowest unoccupied molecular orbital (LUMO) of the fluorophore. Under the light excitation, an electron of fluorophore is promoted from its HOMO to LUMO. Subsequently, one of the receptor HOMO electron rapidly transfers to the HOMO orbital of fluorophore to fill the vacancy, which prohibits the radiative decay of the excited electron of the fluorophore and causes fluorescence quenching (or very weak fluorescence). The chelation or coordination of the receptor to analyte significantly reduce the electron density of receptor and lower its HOMO so that the electron cannot transfer to fluorophore anymore. In this situation, the intrinsic fluorescence of the fluorophore is turned on.

\subsubsection{Intramolecular charge transfer (ICT)}

When strong electron donating receptors are integrated with the electron deficient fluorophore with $\pi$-conjugation, photon excitation can cause another process occur intramolecular charge transfer (ICT). This process can be modulated by change the electron donating ability of the receptor. Thus, it is an appropriate mechanism for design of fluorescent probes.

ICT based fluorescent probe usually possesses receptor that serves as electron donor and this receptor is conjugated with an electron-withdrawing moiety that serves as electron acceptor. They undergo an intramolecular charge transfer from the electron donating part (receptor) to the electron withdrawing part (acceptor) under light excitation, significantly increasing the dipole moments. The proton-promoted locally excited state (or Franck-Condon state) is not thermodynamically stable in the surrounding polar solvent environment and will rapidly relax to the intramolecular charge transfer state (CT) where minimum free energy is reached by a thermodynamic equilibrium between solvent cage and fluorophore (Figure 2.23). Thus, the fluorescence emission (including fluorescence intensity, fluorescence wavelength, lifetime and quantum yield) from ICT state to ground state highly depends on the polarity of environment. For example, increasing solvent polarity causes red shifts of main spectra and low quantum yield $(\Phi)$ of the probe can be observed in polar solvent but not in nonpolar ones. 
Figure 2.23 shows the mechanism of ICT based fluorescent probes. It is expected that analyte such as a metal cation or a proton can chelate or bind with electron rich receptor moiety (donor), which significantly reduces the electron donating ability of receptors so that the efficiency of ICT is changed. This process alter properties of the fluorophore like maximum absorption wavelength $\left(\lambda_{\mathrm{abs}}\right)$, maximum emission wavelength $\left(\lambda_{\mathrm{em}}\right)$, fluorescent intensity, fluorescence quantum yield $(\Phi)$ and fluorescence lifetime $(\tau)$ with this binding event. Compared with PET-based probes, ICT-based probes not only generate fluorescence intensity changes, but more importantly lead to shifts for both absorption and emission spectra due to the change of energy gap before and after binding events. This character makes it possible to measure the change simultaneously at two different wavelengths, which is a ratiometric measurement. Many BODIPY-based probes for metal cations and $\mathrm{pH}$ are in this category. ${ }^{54-56,59-63}$
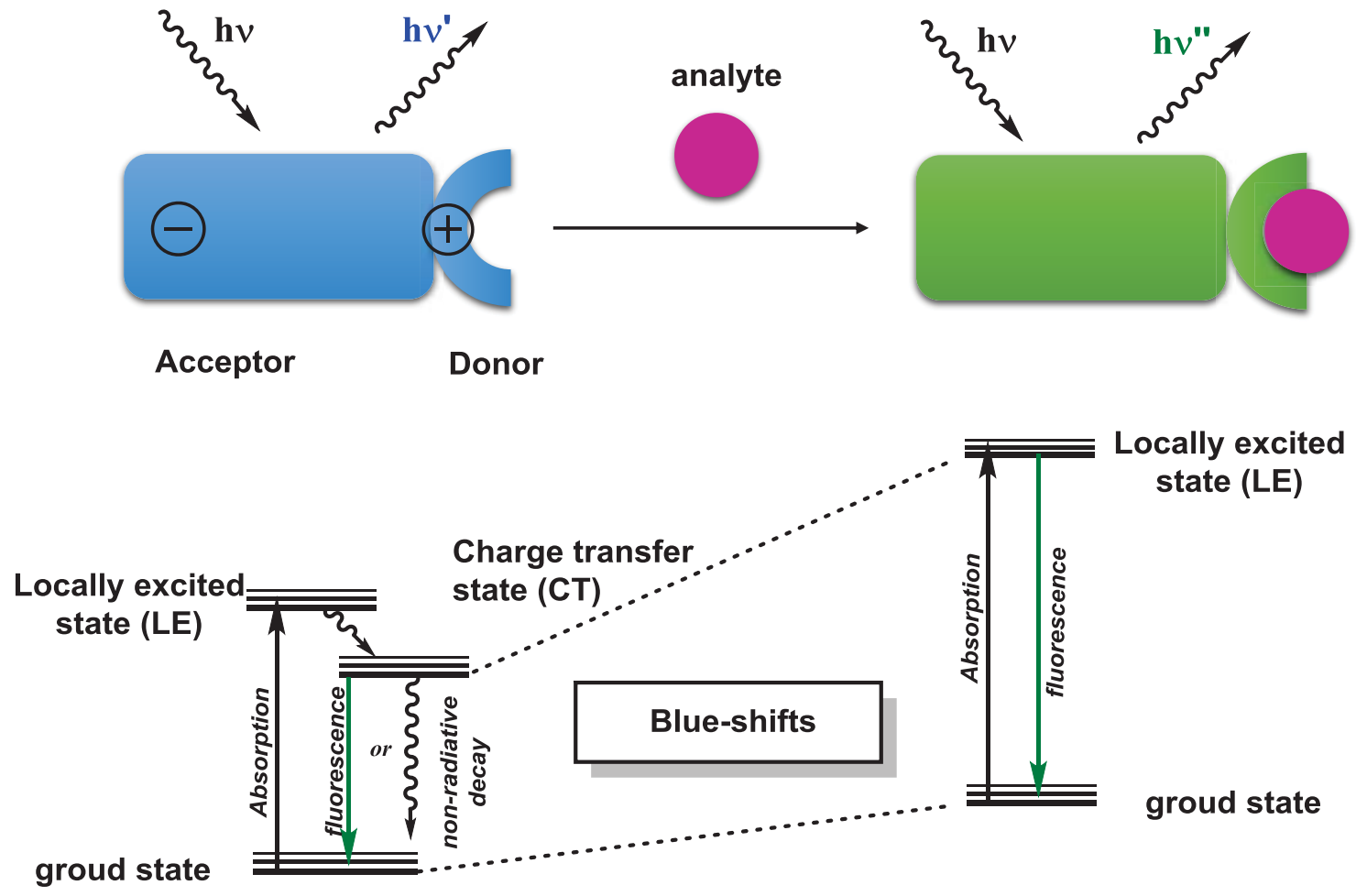

Excited fluorophore with ICT
Excited

fluorophore without ICT

Figure 2.23. Illustration of mechanism of ICT based fluorescent probes. 


\section{References:}

1. Herschel, J. F. W., On a Case of Superficial Colour Presented by a Homogeneous Liquid Internally Colourless. Phil. Trans. Roy. Soc. 1845, 135, 143-145.

2. Undenfriend, S., Development of The Spectrophotofluorometer and Its Commercialization. Protein. Sci. 1995, 4, 452-551.

3. Stokes, G. G., On the Change of Refrangibility of Light. Phil. Trans. R Soc. (London) 1852, 142, 463-562.

4. Lakowicz, J. R., Principles of Fluorescence Spectroscopy. 3 ed.; Springer Science and Business Media: 2006.

5. Noga, E. J., Udomkusonsri, P., Fluorescein: A Rapid, Sensitive, Nonlethal Method for Detecting Skin Ulceration in Fish. Veterinary Pathology 2002, 39 (6), 726-731.

6. Martonyi, C. L., Bahn, C. F., Meyer, R. F., Clinical Slit Lamp Biomicroscopy and Photo Slit Lamp Biomicrography. Time One Ink, Ltd: 1985.

7. Zondervan, R., Kulzer, F., Kol'chenko, M. A., Orrit, M., Photobleaching of Rhodamine 6G in Poly(Vinyl Alcohol) at The Ensemble and Single-Molecule Levels. Journal of Physical Chemistry A 2004, 108 (10), 1657-1665.

8. Dickinson, B. C., Srikun, D., Chang, C. J., Mitochondrial-targeted Fluorescent Probes for Reactive Oxygen Species. Current Opinion in Chemical Biology 2010, $14(1), 50-56$.

9. Du, P., Lippard, S. J., A Highly Selective Turn-On Colorimetric, Red Fluorescent Sensor for Detecting Mobile Zinc in Living Cells. Inorganic Chemistry 2010, 49 (23), 10753-10755.

10. Pires, M. M., Chmielewski, J., Fluorescence Imaging of Cellular Glutathione using A Latent Rhodamine. Organic Letters 2008, 10 (5), 837-840.

11. Yang, Y. K., Yook, K. J., Tae, J., A Rhodamine-Based Fluorescent and Colorimetric Chemodosimeter for The Rapid Detection of $\mathrm{Hg} 2+$ Ions in Aqueous Media. Journal of the American Chemical Society 2005, 127 (48), 16760-16761. 
12. Buston, J. E. H., Young, J. R., Anderson, H. L., Rotaxane-Encapsulated Cyanine Dyes: Enhanced Fluorescence Efficiency and Photostability. Chemical Communications 2000, (11), 905-906.

13. Tyutyulkov, N. J. F., Mehlhorn, A., Dietz, F., Tadjer, A., Polymethine Dyes. St. Kliment Ohridski University Press: 1991.

14. Soper, S. A., Mattingly, Q. L., Steady-State and Picosecond Laser Fluorescence Studies of Nonradiative Pathways in Tricarbocyanine Dyes - Implications to the Design of Near-IR Fluorochromes with High Fluorescence Efficiencies. Journal of the American Chemical Society 1994, 116 (9), 3744-3752.

15. Kubo, Y., Minowa, Y., Shoda, T., Takeshita, K., Synthesis of A New Type of Dibenzopyrromethene-boron Complex with Near-infrared Absorption Property. Tetrahedron Letters 2010, 51 (12), 1600-1602.

16. Tram, K., Yan, H., Jenkins, H. A., Vassiliev, S., Brucec, D., The Synthesis and Crystal Structure of Unsubstituted 4,4-Difluoro-4-Bora-3a, 4a-Diaza-S-Indacene (BODIPY). Dyes and Pigments 2009, 82 (3), 392-395.

17. Alfred Treibs, F.-H. K., Difluorboryl-Komplexe von Di- und Tripyrrylmethenen. Justus Liebigs Annalen der Chemie 1968, 718 (1), 208-223.

18. Arroyo, I. J., Hu, R., Merino, G., Tang, B. Z., Pena-Cabrera, E., The Smallest and One of The Brightest. Efficient Preparation and Optical Description of The Parent Borondipyrromethene System. Journal of Organic Chemistry 2009, 74 (15), 5719-5722.

19. Schmitt, A., Hinkeldey, B., Wild, M., Jung, G., Synthesis of The Core Compound of the BODIPY Dye Class: 4,4'-Difluoro-4-bora-(3a,4a)-diaza-s-indacene. Journal of Fluorescence 2009, 19 (4), 755-758.

20. Vos de Wael, E., Pardoen, J. A., Van Koeveringe, J. A., Lugtenburg, J., Pyrromethene-BF2 Complexes (4,4'-difluoro-4-bora-3a,4a-diaza-s-indacenes) Synthesis and Luminescence Properties. Recueil Des Travaux Chimiques Des Pays-Bas-Journal of the Royal Netherlands Chemical Society 1977, 96 (12), 306309. 
21. Bandichhor, R., Thivierge, C., Bhuvanesh, N. S. P., Burgess, K., 4,4-Difluoro1,3,5,7-tetramethyl-4-bora-3a,4a-diaza-s-indacene. Acta Crystallographica Section E-Structure Reports Online 2006, 62, O4310-O4311.

22. Baruah, M., Qin, W. W., Basaric, N., De Borggraeve, W. M., Boens, N., BODIPY-based Hydroxyaryl Derivatives as Fluorescent $\mathrm{pH}$ Probes. Journal of Organic Chemistry 2005, 70 (10), 4152-4157.

23. Yamada, K., Toyota, T., Takakura, K., Ishimaru, M., Sugawara, T., Preparation of BODIPY Probes for Multicolor Fluorescence Imaging Studies of Membrane Dynamics. New Journal of Chemistry 2001, 25 (5), 667-669.

24. Li, Z. G., Mintzer, E., Bittman, R., First Synthesis of Free Cholesterol - BODIPY Conjugates. Journal of Organic Chemistry 2006, 71 (4), 1718-1721.

25. Yakubovskyi, V. P., Shandura, M. P., Kovtun, Y. P., Boradipyrromethenecyanines. European Journal of Organic Chemistry 2009, (19), 3237-3243.

26. Werner, T., Huber, C., Heinl, S., Kollmannsberger, M., Daub, J., Wolfbeis, O. S., Novel Optical pH-Sensor Based on A Boradiaza-Indacene Derivative. Fresenius Journal of Analytical Chemistry 1997, 359 (2), 150-154.

27. Baki, C. N., Akkaya, E. U., Boradiazaindacene-appended Calix 4 Arene: Fluorescence Sensing of pH Near Neutrality. Journal of Organic Chemistry 2001, $66(4), 1512-1513$.

28. Zhang, X. A., Wang, H., Li, J. S., Zhang, H. S., Development of A Fluorescent Probe for Nitric Oxide Detection Based on Difluoroboradiaza-s-indacene Fluorophore. Anal. Chim. Acta 2003, 481 (1), 101-108.

29. Zhang, X., Zhang, H. S., Design, Synthesis and Characterization of A Novel Fluorescent Probe for Nitric Oxide Based on Difluoroboradiaza-S-Indacene Fluorophore. Spectrochimica Acta Part a-Molecular and Biomolecular Spectroscopy 2005, 61 (6), 1045-1049.

30. Sun, Z.-N., Liu, F.-Q., Chen, Y., Tam, P. K. H., Yang, D., A Highly Specific BODIPY-based Fluorescent Probe for The Detection of Hypochlorous Acid. Organic Letters 2008, 10 (11), 2171-2174. 
31. DiCesare, N., Lakowicz, J. R., Fluorescent Probe for Monosaccharides Based on A Functionalized Boron-Dipyrromethene with A Boronic Acid Group. Tetrahedron Letters 2001, 42 (52), 9105-9108.

32. Li, J.-S., Wang, H., Huang, K.-J., Zhang, H.-S., Determination of Biogenic Amines in Apples and Wine with 8-Phenyl-(4-oxy-acetic acid Nhydroxysuccinimide ester)-4,4-difluoro-1,3,5,7-tetramethyl-4-bora-3a,4a-diaza-sindacene by High Performance Liquid Chromatography. Anal. Chim. Acta 2006, 575 (2), 255-261.

33. Qi, X., Jun, E. J., Xu, L., Kim, S. J., Hong, J. S. J., Yoon, Y. J., Yoon, J. Y., New BODIPY Derivatives as OFF-ON Fluorescent Chemosensor and Fluorescent Chemodosimeter for $\mathrm{Cu} 2+$ : Cooperative Selectivity Enhancement toward $\mathrm{Cu} 2+$. Journal of Organic Chemistry 2006, 71 (7), 2881-2884.

34. Bricks, J. L., Kovalchuk, A., Trieflinger, C., Nofz, M., Buschel, M., Tolmachev, A. I., Daub, J., Rurack, K., On The Development of Sensor Molecules That Display Fe-III-Amplified Fluorescence. Journal of the American Chemical Society 2005, 127 (39), 13522-13529.

35. Martin, V. V., Rothe, A., Gee, K. R., Fluorescent Metal Ion Indicators Based On Benzoannelated Crown Systems: A Green Fluorescent Indicator for Intracellular Sodium Ions. Bioorganic \& Medicinal Chemistry Letters 2005, 15 (7), 18511855 .

36. Wang, J., Qian, X., A Series of Polyamide Receptor Based PET Fluorescent Sensor Molecules: Positively Cooperative Hg2+ Ion Binding with High Sensitivity. Organic Letters 2006, 8 (17), 3721-3724.

37. Kim, H. J., Kim, J. S., BODIPY Appended Cone-calix 4 Arene: Selective Fluorescence Changes upon Ca2+ Binding. Tetrahedron Letters 2006, 47 (39), 7051-7055.

38. Basaric, N., Baruah, M., Qin, W. W., Metten, B., Smet, M., Dehaen, W., Boens, N., Synthesis and Spectroscopic Characterisation of BODIPY (R) Based Fluorescent Off-On Indicators with Low Affinity for Calcium. Organic \& Biomolecular Chemistry 2005, 3 (15), 2755-2761. 
39. Niu, S. L., Ulrich, G., Ziessel, R., Kiss, A., Renard, P.-Y., Romieu, A., WaterSoluble BODIPY Derivatives. Organic Letters 2009, 11 (10), 2049-2052.

40. Ulrich, G., Ziessel, R., Convenient and Efficient Synthesis of Functionalized Oligopyridine Ligands Bearing Accessory Pyrromethene-BF2 Fluorophores. Journal of Organic Chemistry 2004, 69 (6), 2070-2083.

41. Leonardi, M. J., Topka, M. R., Dinolfo, P. H., Efficient Forster Resonance Energy Transfer in 1,2,3-Triazole Linked BODIPY-Zn(II) Meso-Tetraphenylporphyrin Donor-Acceptor Arrays. Inorganic Chemistry 2012, 51 (24), 13114-13122.

42. Yogo, T., Urano, Y., Ishitsuka, Y., Maniwa, F., Nagano, T., Highly Efficient and Photostable Photosensitizer Based on BODIPY Chromophore. Journal of the American Chemical Society 2005, 127 (35), 12162-12163.

43. Ozlem, S., Akkaya, E. U., Thinking Outside the Silicon Box: Molecular and Logic As an Additional Layer of Selectivity in Singlet Oxygen Generation for Photodynamic Therapy. Journal of the American Chemical Society 2009, 131 (1), $48-+$.

44. Lim, S. H., Thivierge, C., Nowak-Sliwinska, P., Han, J., van den Bergh, H., Wagnieres, G., Burgess, K., Lee, H. B., In Vitro and In Vivo Photocytotoxicity of Boron Dipyrromethene Derivatives for Photodynamic Therapy. Journal of Medicinal Chemistry 2010, 53 (7), 2865-2874.

45. Adarsh, N., Avirah, R. R., Ramaiah, D., Tuning Photosensitized Singlet Oxygen Generation Efficiency of Novel Aza-BODIPY Dyes. Organic Letters 2010, 12 (24), 5720-5723.

46. Jiao, L., Yu, C., Li, J., Wang, Z., Wu, M., Hao, E., Beta-Formyl-BODIPYs from The Vilsmeier-Haack Reaction. Journal of Organic Chemistry 2009, 74 (19), 7525-7528.

47. Yu, C., Jiao, L., Yin, H., Zhou, J., Pang, W., Wu, Y., Wang, Z., Yang, G., Hao, E., Alpha-/Beta-Formylated Boron-Dipyrrin (BODIPY) Dyes: Regioselective Syntheses and Photophysical Properties. European Journal of Organic Chemistry 2011, (28), 5460-5468. 
48. Kolemen, S., Cakmak, Y., Erten-Ela, S., Altay, Y., Brendel, J., Thelakkat, M., Akkaya, E. U., Solid-state Dye-sensitized Solar Cells using Red and Near-IR Absorbing Bodipy Sensitizers. Organic Letters 2010, 12 (17), 3812-3815.

49. Wories, H. J., Koek, J. H., Lodder, G., Lugtenburg, J., Fokkens, R., Driessen, O., Mohn, G. R., A Novel Water-Soluble Fluorescent Probe - Synthesis, Luminescence and Biological Properties of the Sodium-Salt of the 4-sulfonato3,3',5,5'-tetramethyl-2,2'-pyrromethen-1,1'-BF2 Complex. Recueil Des Travaux Chimiques Des Pays-Bas-Journal of the Royal Netherlands Chemical Society 1985, 104 (11), 288-291.

50. Shah, M., Thangaraj, K., Soong, M.-L., Wolford, L. T., Boyer, J. H., Politzer, I. R., Pavlopoulos, T. G., Pyrromethene-BF2 complexes as Laser Dyes: 1. Heteroatom Chemistry 1990, 1 (5), 389-399.

51. Urano, T., Nagasaka, H., Tsuchiyama, M., Ide, H. Polymerizable Composition Containing Pyrromethene Type Coloring Matter and Titanocene Compound. 1996.

52. Morgan, L. R., Boyer, J. H. Boron Difluoride Compounds Useful in Photodynamic Therapy and Production of Laser Light. 1995.

53. Rohand, T., Baruah, M., Qin, W. W., Boens, N., Dehaen, W., Functionalisation of Fluorescent BODIPY Dyes by Nucleophilic Substitution. Chemical Communications 2006, (3), 266-268.

54. Baruah, M., Qin, W. W., Vallee, R. A. L., Beljonne, D., Rohand, T., Dehaen, W., Boens, N., A Highly Potassium-selective Ratiometric Fluorescent Indicator Based on BODIPY Azacrown Ether Excitable with Visible Light. Organic Letters 2005, 7 (20), 4377-4380.

55. Zeng, L., Miller, E. W., Pralle, A., Isacoff, E. Y., Chang, C. J., A Selective TurnOn Fluorescent Sensor for Imaging Copper in Living Cells. Journal of the American Chemical Society 2006, 128 (1), 10-11.

56. Yin, S., Leen, V., Van Snick, S., Boens, N., Dehaen, W., A highly Sensitive, Selective, Colorimetric and Near-infrared Fluorescent Turn-On Chemosensor for Cu2+ Based on BODIPY. Chemical Communications 2010, 46 (34), 6329-6331. 
57. Leen, V., Braeken, E., Luckermans, K., Jackers, C., Van der Auweraer, M., Boens, N., Dehaen, W., A Versatile, Modular Synthesis of Monofunctionalized BODIPY Dyes. Chemical Communications 2009, (30), 4515-4517.

58. Han, J., Gonzalez, O., Aguilar-Aguilar, A., Pena-Cabrera, E., Burgess, K., 3- and 5-Functionalized BODIPYs via The Liebeskind-Srogl Reaction. Organic \& Biomolecular Chemistry 2009, 7 (1), 34-36.

59. Lee, J. S., Kang, N. Y., Kim, Y. K., Samanta, A., Feng, S. H., Kim, H. K., Vendrell, M., Park, J. H., Chang, Y. T., Synthesis of A BODIPY Library and Its Application to The Development of Live Cell Glucagon Imaging Probe. Journal of the American Chemical Society 2009, 131 (29), 10077-10082.

60. Coskun, A., Deniz, E., Akkaya, E. U., A Sensitive Fluorescent Chemosensor for Anions Based on A Styryl-boradiazaindacene Framework. Tetrahedron Letters 2007, 48 (31), 5359-5361.

61. Ekmekci, Z., Yilmaz, M. D., Akkaya, E. U., A Monostyryl-boradiazaindacene (BODIPY) Derivative as Colorimetric and Fluorescent Probe for Cyanide Ions. Organic Letters 2008, 10 (3), 461-464.

62. Dost, Z., Atilgan, S., Akkaya, E. U., Distyryl-Boradiazaindacenes: Facile Synthesis of Novel Near IR Emitting Fluorophores. Tetrahedron 2006, 62 (36), 8484-8488.

63. Atilgan, S., Ozdemir, T., Akkaya, E. U., A Sensitive and Selective Ratiometric Near IR Fluorescent Probe for Zinc Ions Based on The Distyryl-bodipy Fluorophore. Organic Letters 2008, 10 (18), 4065-4067.

64. Buyukcakir, O., Bozdemir, O. A., Kolemen, S., Erbas, S., Akkaya, E. U., Tetrastyryl-Bodipy Dyes: Convenient Synthesis and Characterization of Elusive Near IR Fluorophores. Organic Letters 2009, 11 (20), 4644-4647.

65. Goze, C., Ulrich, G., Mallon, L. J., Allen, B. D., Harriman, A., Ziessel, R., Synthesis and Photophysical Properties of Borondipyrromethene Dyes Bearing Aryl Substituents at The Boron Center. Journal of the American Chemical Society 2006, 128 (31), 10231-10239. 
66. Niu, S.-L., Ulrich, G., Retailleau, P., Harrowfield, J., Ziessel, R., New Insights into The Solubilization of Bodipy Dyes. Tetrahedron Letters 2009, 50 (27), 38403844.

67. Gabe, Y., Ueno, T., Urano, Y., Kojima, H., Nagano, T., Tunable Design Strategy for Fluorescence Probes Based on 4-Substituted BODIPY Chromophore: Improvement of Highly Sensitive Fluorescence Probe for Nitric Oxide. Analytical and Bioanalytical Chemistry 2006, 386 (3), 621-626.

68. Wagner, R. W., Lindsey, J. S., Boron-Dipyrromethene Dyes for Incorporation in Synthetic Multi-pigment Light-harvesting Arrays. Pure and Applied Chemistry 1998, 70 (8), AR1-AR1.

69. Thoresen, L. H., Kim, H. J., Welch, M. B., Burghart, A., Burgess, K., Synthesis of 3,5-Diaryl-4,4-difluoro-4-bora-3a,4a-diaza-s-indacene (BODIPY $\quad(R)$ ) Dyes. Synlett 1998, (11), 1276-+.

70. Burghart, A., Kim, H. J., Welch, M. B., Thoresen, L. H., Reibenspies, J., Burgess, K., Bergstrom, F., Johansson, L. B. A., 3,5-Diaryl-4,4-difluoro-4-bora-3a,4adiaza-s-Indacene (BODIPY) Dyes: Synthesis, Spectroscopic, Electrochemical, and Structural Properties. Journal of Organic Chemistry 1999, 64 (21), 78137819.

71. Rihn, S., Retailleau, P., Bugsaliewicz, N., De Nicola, A., Ziessel, R., Versatile Synthetic Methods for The Engineering of Thiophene-substituted Bodipy Dyes. Tetrahedron Letters 2009, 50 (50), 7008-7013.

72. Zhang, D., Wang, Y., Xiao, Y., Qian, S., Qian, X., Long-wavelength Boradiazaindacene Derivatives with Two-photon Absorption Activity and Strong Emission: Versatile Candidates for Biological Imaging Applications. Tetrahedron 2009, 65 (39), 8099-8103.

73. Qin, W., Rohand, T., Dehaen, W., Clifford, J. N., Driesen, K., Beljonne, D., Van Averbeke, B., Van der Auweraer, M., Boens, N., Boron Dipyrromethene Analogs with Phenyl, Styryl, and Ethynylphenyl Substituents: Synthesis, Photophysics, Electrochemistry, and Quantum-Chemical Calculations. Journal of Physical Chemistry A 2007, 111 (35), 8588-8597. 
74. Donuru, V. R., Vegesna, G. K., Velayudham, S., Green, S., Liu, H., Synthesis and Optical Properties of Red and Deep-Red Emissive Polymeric and Copolymeric BODIPY Dyes. Chemistry of Materials 2009, 21 (10), 2130-2138.

75. Meng, G., Velayudham, S., Smith, A., Luck, R., Liu, H., Color Tuning of Polyfluorene Emission with BODIPY Monomers. Macromolecules 2009, 42 (6), 1995-2001.

76. Kollmannsberger, M., Gareis, T., Heinl, S., Breu, J., Daub, J., Electrogenerated Chemiluminescence and Proton-Dependent Switching of Fluorescence: Functionalized Difluoroboradiaza-s-Indacenes. Angewandte Chemie-International Edition in English 1997, 36 (12), 1333-1335.

77. Rurack, K., Kollmannsberger, M., Daub, J., Molecular Switching inThe Near Infrared (NIR) with A Functionalized Boron - Dipyrromethene Dye. Angewandte Chemie-International Edition 2001, 40 (2), 385-387.

78. Zhang, D., Martin, V., Garcia-Moreno, I., Costela, A., Eugenia Perez-Ojeda, M., Xiao, Y., Development of Excellent Long-wavelength BODIPY Laser Dyes with A Strategy that Combines Extending Pi-Conjugation and Tuning ICT Effect. Physical Chemistry Chemical Physics 2011, 13 (28), 13026-13033.

79. Zhang, X., Xiao, Y., Qian, X., Highly efficient energy transfer in the light harvesting system composed of three kinds of boron-dipyrromethene derivatives. Organic Letters 2008, 10 (1), 29-32.

80. Rurack, K., Kollmannsberger, M., Daub, J., A Highly Efficient Sensor Molecule Emitting in The Near Infrared (NIR): 3,5-Distyryl-8-(pdimethylaminophenyl)difluoroboradiaza-s-indacene. New Journal of Chemistry 2001, 25 (2), 289-292.

81. Deniz, E., Isbasar, G. C., Bozdemir, O. A., Yildirim, L. T., Siemiarczuk, A., Akkaya, E. U., Bidirectional Switching of Near IR Emitting Boradiazaindacene Fluorophores. Organic Letters 2008, 10 (16), 3401-3403.

82. Huo, F.-J., Sun, Y.-Q., Su, J., Chao, J.-B., Zhi, H.-J., Yin, C.-X., Colorimetric Detection of Thiols using A Chromene Molecule. Organic Letters 2009, 11 (21), 4918-4921. 
83. Huang, S.-T., Ting, K.-N., Wang, K.-L., Development of A Long-wavelength Fluorescent Probe Based On Quinone-methide-type Reaction to Detect Physiologically Significant Thiols. Anal. Chim. Acta 2008, 620 (1-2), 120-126.

84. Zhao, H. T., Kalivendi, S., Zhang, H., Joseph, J., Nithipatikom, K., VasquezVivar, J., Kalyanaraman, B., Superoxide Reacts with Hydroethidine but Forms A Fluorescent Product That Is Distinctly Different From Ethidium: Potential Implications in Intracellular Fluorescence Detection of Superoxide. Free Radical Biology and Medicine 2003, 34 (11), 1359-1368.

85. Kim, H. J., Ko, K. C., Lee, J. H., Lee, J. Y., Kim, J. S., KCN Sensor: Unique Chromogenic and 'Turn-On' Fluorescent Chemodosimeter: Rapid Response and High Selectivity. Chemical Communications 2011, 47 (10), 2886-2888.

86. Ko, S.-K., Yang, Y.-K., Tae, J., Shin, I., In Vivo Monitoring Of Mercury Ions Using A Rhodamine-based Molecular Probe. Journal of the American Chemical Society 2006, 128 (43), 14150-14155.

87. Kim, H., Lee, S., Lee, J., Tae, J., Rhodamine Triazole-based Fluorescent Probe for The Detection of Pt2+. Organic Letters 2010, 12 (22), 5342-5345.

88. Wu, D., Huang, W., Duan, C., Lin, Z., Meng, Q., Highly Sensitive Fluorescent Probe for Selective Detection of $\mathrm{Hg} 2+$ in DMF Aqueous Media. Inorganic Chemistry 2007, 46 (5), 1538-1540. 


\section{Chapter 3. Highly Water-soluble Neutral BODIPY Dyes with Controllable Fluorescence Quantum Yields ${ }^{1}$}

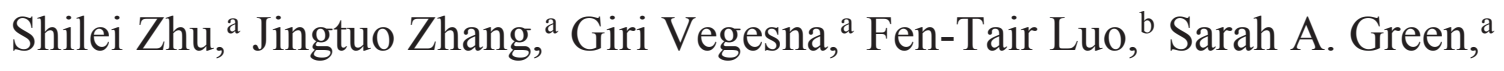
and Haiying Liu*a

${ }^{a}$ Department of Chemistry, Michigan Technological University, 1400 Townsend Drive, Houghton, MI 49931, USA

${ }^{b}$ Institute of Chemistry, Academia Sinica, Taipei, Taiwan 11529, Republic of China

${ }^{1}$ The material included in this chapter was previously published in the Organic Letters 2011, 13 (3), 438-441. DOI: 10.1021/o1102758z

Publication Date (Web): Dec. 22, 2010 Copyright (C) 2010 American Chemical Society http://pubs.acs.org/doi/abs/10.1021/ol102758z 


\subsection{Introduction}

BODIPY (4,4'-difluoro-4-bora-3a,4a-diaza-s-indacene) dyes have gained a great deal of attention recently because of their many distinctive and desirable properties such as high extinction coefficients, narrow absorption and emission bands, high quantum efficiencies of fluorescence, and relative insensitivity to environmental perturbations, and resistance to photobleaching. ${ }^{1-2}$ Biological and medical applications of the BODIPY dyes require good water solubility and resistance to the formation of nonfluorescent dimer and higher aggregates. Reported strategies to make these dyes water-soluble typically involve introducing oligo(ethylene glycol), ${ }^{3}$ N,N-bis(2-hydroxyethyl) amine, ${ }^{4}$ carbohydrates, ${ }^{5}$ nucleotides, ${ }^{6}$ or ionic hydrophilic groups such as carboxylic acid, ${ }^{7-8}$ sulfonate, ${ }^{9-11}$ and ammonium groups ${ }^{9-10}$ to BODIPY dyes. There are desirable advantages to prepare neutral water-soluble BODIPY dyes to prevent potential nonspecific interactions through electrostatic interactions between BODIPY dyes and other proteins in biological and medical applications. It is very important to develop general approaches to significantly enhance water solubility of neutral BODIPY dyes with controlled fluorescence quantum yields.

We hypothesize that incorporation of branched oligo(ethylene glycol)methyl ether into BODIPY dyes could effectively enhance enthalpic interactions of BODIPY dyes with water and significantly increase water solubility of BODIPY dyes, and that introduction of steric hindrance at meso- or 4-position of BODIPY dyes could significantly reduce their aggregation caused by potential $\pi-\pi$ stacking interactions between BOIDIPY cores in aqueous solution, and considerably enhance their fluorescence quantum yields. In this letter, we have introduced branched oligo(ethylene glycol)methyl ether to the meso, 2- and 6-, and 4-positions of BODIPY dyes and significantly enhance fluorescence quantum yields of BODIPY dyes (Scheme 3.1). These neutral BODIPY dyes are highly water-soluble because of the strong hydrophilic nature of oligo(ethylene glycol)methyl ether residues. 


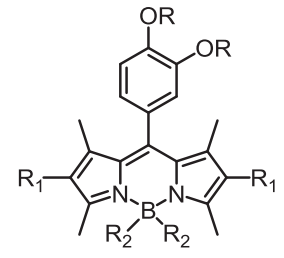

3-A: $R_{1}=H, R_{2}=F$

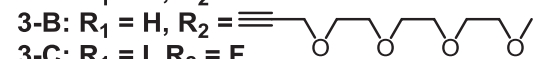

3-C: $R_{1}=I, R_{2}=F$

3-D: $\mathbf{R}_{\mathbf{1}}=\equiv \mathrm{H}, \mathbf{R}_{\mathbf{2}}=\mathbf{F}$

3-E: $\mathbf{R}_{1}=\equiv-\mathrm{H}, \mathbf{R}_{\mathbf{2}}=\mathrm{OCH}_{3}$

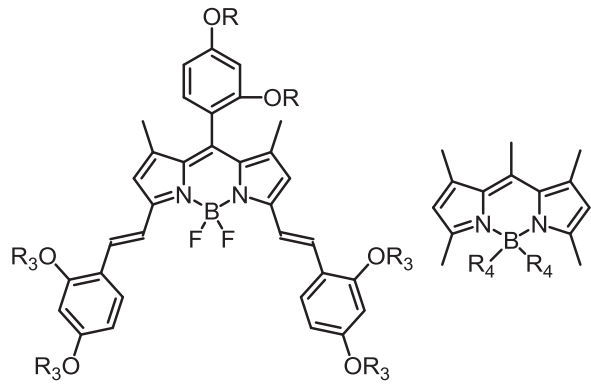

3-H

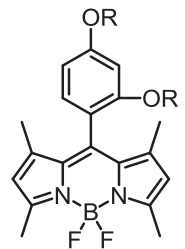

3-F

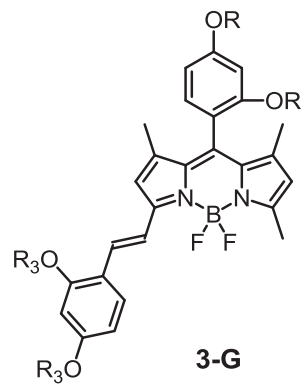

Where
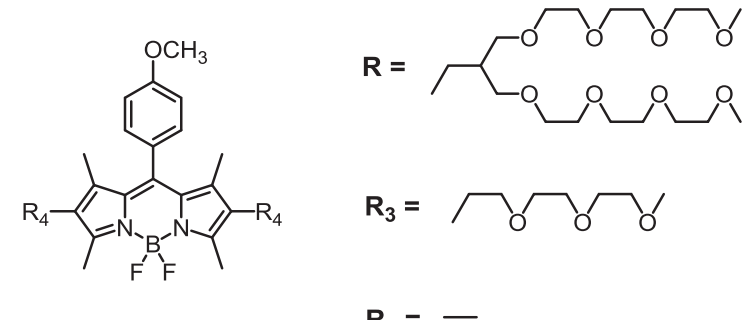

$\mathbf{R}_{4}=\equiv \overbrace{0} \mathrm{O} / \overbrace{\mathrm{OR}}$

Scheme 3.1. Chemical structures of highly water-soluble BODIPY dyes.

\subsection{Experimental Section}

\subsubsection{Instrumentation}

${ }^{1} \mathrm{H}$ NMR and ${ }^{13} \mathrm{C}$ NMR spectra were taken on a $400 \mathrm{MHz}$ Varian Unity Inova spectrophotometer instrument. ${ }^{1} \mathrm{H}$ and ${ }^{13} \mathrm{C}$ NMR spectra were recorded in $\mathrm{CDCl}_{3}$, chemical shifts $(\delta)$ are given in ppm relative to solvent peaks $\left({ }^{1} \mathrm{H}: \delta 7.26 ;{ }^{13} \mathrm{C}: \delta 77.3\right)$ as internal standard. Absorption spectra were taken on a Hewlett Packard 8452A Diode Array UV-visible spectrophotometer. Fluorescence spectra were recorded on a Spex Fluorolog $16810.22 \mathrm{~m}$ steady-state fluorometer.

\subsubsection{Materials and synthesis.}

Unless otherwise indicated, all reagents and solvents were obtained from commercial suppliers (Aldrich, Sigma, Fluka, Acros Organics, Fisher Scientific, Lancaster) and used without further purification. Air- and moisture-sensitive reactions were conducted in oven-dried glassware using a standard Schlenk line or drybox techniques under an inert atmosphere of dry nitrogen. Compound $\mathbf{3 . 8}$ was prepared and characterized according to 
the literature. ${ }^{12-13}$ Compound 3.11 was prepared and characterized according to the literature. ${ }^{14}$ Compound 3.15 was prepared and characterized according to the literature. ${ }^{15}$ Compound 3.16 was prepared and characterized according to the literature. ${ }^{16}$

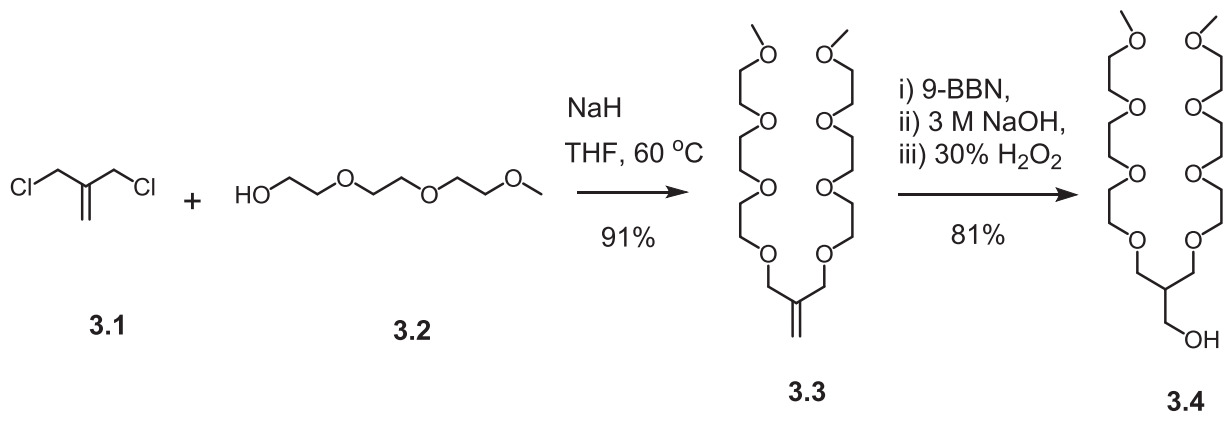

Scheme 3.2. Synthetic route to compound 3.4.

Compound 3.3: $60 \% \mathrm{NaH}$ (dispersion in mineral oil) (18.0 g, $0.45 \mathrm{~mol}$ ), ethylene glycol monomethyl ether (2) (50 g, $0.3 \mathrm{~mol})$, and freshly distilled dry THF (350 mL) were placed in a dry round-bottom flask in an ice bath and stirred for $0.5 \mathrm{~h}$ at $60{ }^{\circ} \mathrm{C}$ under $\mathrm{N}_{2}$ atmosphere. After the reaction was cooled down to room temperature, methallyl dichloride (1) (18.8 g, $0.15 \mathrm{~mol})$ was added dropwise to this mixture and the resulting mixture was stirred at $65{ }^{\circ} \mathrm{C}$ for $12 \mathrm{~h}$. When the reaction was cooled down to room temperature, the reaction mixture was quenched with water and extracted with $\mathrm{CH}_{2} \mathrm{Cl}_{2}$. After the organic layer was dried over anhydrous $\mathrm{MgSO}_{4}$, the solvent was removed by using a rotary evaporator. The product was quickly and simply purified by column chromatography using hexane/acetone/ $\mathrm{CH}_{2} \mathrm{Cl}_{2} / \mathrm{EtOH}(5 / 2 / 3 / 0.5, \mathrm{v} / \mathrm{v})$ to give compound 3.3 as a crude liquid. The compound $\mathbf{3 . 3}$ can be used in the next step without further purification.

Compound 3.4: Compound 3.3 (51.3 g, $135 \mathrm{mmol}$ ) was dissolved in $400 \mathrm{~mL}$ of dry THF under $\mathrm{N}_{2}$ atmosphere, and $360 \mathrm{~mL}$ of $0.5 \mathrm{M}$ 9-BBN solution in THF was added dropwise at room temperature. The reaction mixture was stirred for $45 \mathrm{~min}$, cooled to 0 ${ }^{\circ} \mathrm{C}$ in an ice bath, carefully quenched with $95 \mathrm{~mL}$ of $3 \mathrm{M} \mathrm{NaOH}$ aqueous solution, and then stirred for $30 \mathrm{~min}$ at room temperature. When the reaction mixture was cooled again to $0{ }^{\circ} \mathrm{C}, 95 \mathrm{~mL}$ of $30 \% \mathrm{H}_{2} \mathrm{O}_{2}$ aqueous solution was carefully added. After the mixture was stirred at room temperature $3 \mathrm{~h}$, THF solvent was removed in a rotary evaporator. 
The mixture was saturated with $\mathrm{K}_{2} \mathrm{CO}_{3}$ and extracted with $\mathrm{CH}_{2} \mathrm{Cl}_{2}$. When the organic layer was collected, the solvent was removed in a rotary evaporator. The resulting product was purified by column chromatography using hexane/acetone $/ \mathrm{CH}_{2} \mathrm{Cl}_{2} / \mathrm{EtOH}$ (5/2/3/0.5, v/v) to yield compound 3.4 as a colorless liquid (42.65 g, 81\%). ${ }^{1} \mathrm{H}$ NMR (400 $\left.\mathrm{MHz}, \mathrm{CDCl}_{3}\right): \delta 3.68$ (t, $\left.J=5.6 \mathrm{~Hz}, 1 \mathrm{H},-\mathrm{OH}\right), 3.60-3.47$ (m, 30H), 3.32 (s, $\left.6 \mathrm{H}\right), 2.10-$ $2.04(\mathrm{~m}, 1 \mathrm{H}) .{ }^{13} \mathrm{C} \mathrm{NMR}\left(100 \mathrm{MHz}, \mathrm{CDCl}_{3}\right): \delta 72.1,71.0,70.8,70.75,70.7,70.68,70.6$, 63.6, 59.2, 41.4. HRMS (FAB) calcd for $\mathrm{C}_{18} \mathrm{H}_{39} \mathrm{O}_{9}[\mathrm{M}+\mathrm{H}]^{+}$, 399.2594; found, 399.2601.

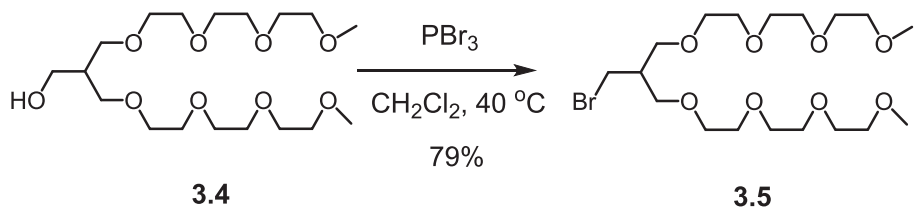

Scheme 3.3. Synthetic route to compound 3.5.

Compound 3.5: To the solution of compound 3.4 (20.0 g, $50 \mathrm{mmol})$ in $\mathrm{CH}_{2} \mathrm{Cl}_{2}$ (150 $\mathrm{mL})$ added $\mathrm{PBr}_{3}(7.1 \mathrm{~mL}, 75 \mathrm{mmol})$ dropwise at $0{ }^{\circ} \mathrm{C}$. After the mixture was stirred at 40 ${ }^{\circ} \mathrm{C}$ for $12 \mathrm{~h}$, it was cooled down to room temperature and dissolved in $\mathrm{CH}_{2} \mathrm{Cl}_{2}$. After water was added slowly to the mixture at $0{ }^{\circ} \mathrm{C}$, the mixture was transferred into a separation funnel. The organic layer was collected, washed with water, $\mathrm{NaHCO}_{3}$ solution, dried over anhydrous $\mathrm{Na}_{2} \mathrm{SO}_{4}$ and filtered. The filtrate was concentrated under reduced pressure and the crude product was purified by column chromatography using hexane/EtOAc/EtOH $(7 / 3 / 0.3, \mathrm{v} / \mathrm{v})$ as a eluent to yield compound $\mathbf{3 . 5}$ as colorless liquid (18.16 g, 79\%). ${ }^{1} \mathrm{H}$ NMR (400 MHz, $\mathrm{CDCl}_{3}$ ): $\delta 3.63-3.35$ (m, 30H), 3.35 (s, 6H), 2.30$2.21(\mathrm{~m}, 1 \mathrm{H}) .{ }^{13} \mathrm{C}$ NMR $\left(100 \mathrm{MHz}, \mathrm{CDCl}_{3}\right): \delta$ 72.1, 70.8, 70.7, 70.6, 70.2, 59.2, 41.5, 33.6. HRMS (FAB) calcd for $\mathrm{C}_{18} \mathrm{H}_{38} \mathrm{O} 8 \mathrm{Br}[\mathrm{M}+\mathrm{H}]^{+}, 461.1750$; found, 461.1744 .

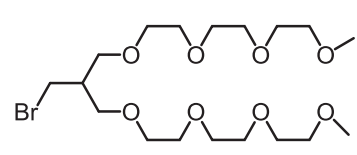

3.5

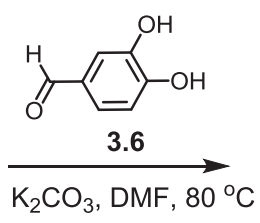

$75 \%$

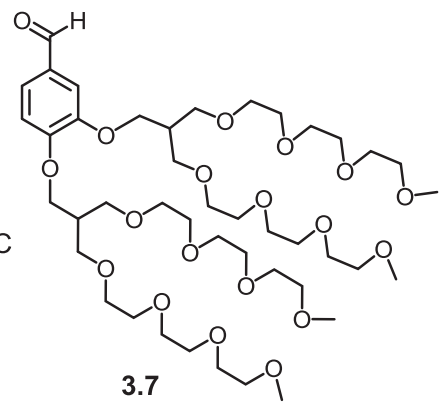

Scheme 3.3. Synthetic route to compound 3.7. 
Compound 3.7: When $15 \mathrm{~mL}$ of DMF solution was added to a $100-\mathrm{mL}$ roundbottom flask containing 3,4-dihydroxybezaldehyde (3.6) (228 mg, $1.65 \mathrm{mmol})$, compound 3.5 (1.9 g, $3.48 \mathrm{mmol})$, and $\mathrm{K}_{2} \mathrm{CO}_{3}(1.14 \mathrm{~g}, 8.25 \mathrm{mmol})$, the mixture was stirred for $12 \mathrm{~h}$ at $80{ }^{\circ} \mathrm{C}$ under a nitrogen atmosphere. After completion of the reaction (which was monitored by TLC), DMF was removed from the mixture under reduced pressure. The resulting crude product was purified by silica gel column chromatography using hexane/EtOAc/ $\mathrm{CH}_{2} \mathrm{Cl}_{2} / \mathrm{EtOH}(5 / 2 / 3 / 0.5$ to $5 / 1 / 3 / 1, \mathrm{v} / \mathrm{v})$ to yield compound 3.7 as a colorless liquid (1.10 g, 75\%). ${ }^{1} \mathrm{H}$ NMR (400 MHz, $\left.\mathrm{CDCl}_{3}\right): \delta 9.80(\mathrm{~s}, 1 \mathrm{H}), 7.41-7.38$ $(\mathrm{m}, 2 \mathrm{H}), 6.97(\mathrm{~d}, J=8.0 \mathrm{~Hz}, 1 \mathrm{H}), 4.12(\mathrm{~d}, J=5.6 \mathrm{~Hz}, 2 \mathrm{H}), 4.08$ (d, $J=5.6 \mathrm{~Hz}, 2 \mathrm{H}), 3.63-$ $3.50(\mathrm{~m}, 56 \mathrm{H}), 3.35$ (4 x CH, $12 \mathrm{H}), 2.48-2.39(\mathrm{~m}, 2 \mathrm{H}) .{ }^{13} \mathrm{C} \mathrm{NMR}\left(100 \mathrm{MHz}, \mathrm{CDCl}_{3}\right): \delta$ $191.1,154.7,149.5,130.1,126.6,111.9,111.0,72.1,70.9,70.8,70.7,70.6,69.5,69.3$, 67.0, 66.9, 59.2, 40.1, 40.0. HRMS (FAB) calcd for $\mathrm{C}_{43} \mathrm{H}_{79} \mathrm{O}_{19}[\mathrm{M}+\mathrm{H}]^{+}$, 899.5216; found, 899.5226.

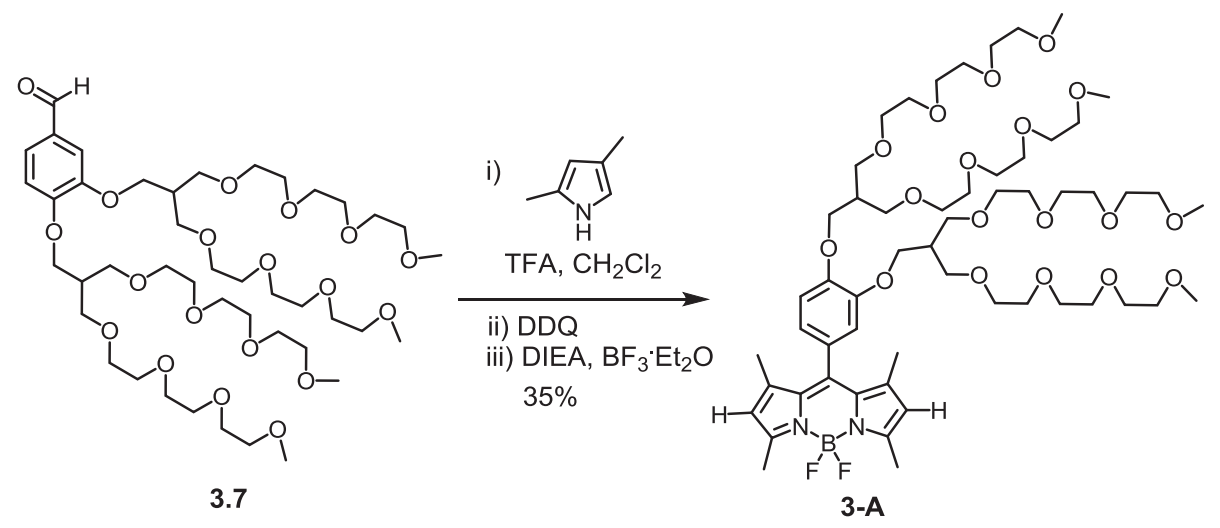

Scheme 3.4. Synthetic route to compound 3-A.

Compound 3-A: Compound 3.7 (2.7 g, $3.0 \mathrm{mmol})$ and 2,4-dimethylpyrrole (712 $\mathrm{mg}, 7.5 \mathrm{mmol}$ ) were dissolved in $500 \mathrm{~mL}$ of dry $\mathrm{CH}_{2} \mathrm{Cl}_{2}$ in a $2000-\mathrm{mL}$ flask. Two drops of trifluoroacetic acid (TFA) were added to the reaction mixture, and the resulting mixture was stirred in the dark for $12 \mathrm{~h}$ under a nitrogen atmosphere at room temperature. After the complete consumption of aldehyde 3.7 (which was monitored by TLC), DDQ (2,3-dichloro-5,6-dicyano-1,4-benzoquinone) (820 $\mathrm{mg}, 3.6 \mathrm{mmol})$ was added to the reaction mixture. After the mixture had stirred for $40 \mathrm{~min}, 10 \mathrm{~mL}$ of diisopropylethylamine (DIPEA) and $10 \mathrm{~mL}$ of $\mathrm{BF}_{3} \cdot \mathrm{OEt}_{2}$ were added to the mixture. After 
the mixture was further stirred for $40 \mathrm{~min}$, it was concentrated to $200 \mathrm{~mL}$ and filtered. When the filtrate was washed twice with water and brine solution, the organic layer was collected, dried over anhydrous $\mathrm{MgSO}_{4}$, and concentrated under reduced pressure. The crude product was purified by column chromatography using hexane/EtOAc/ $\mathrm{CH}_{2} \mathrm{Cl}_{2} / \mathrm{EtOH}(10 / 2 / 6 / 1, \mathrm{v} / \mathrm{v})$ to obtain BODIPY dye 3-A as yellow-red oil (1.16 g, 35\%). ${ }^{1} \mathrm{H}$ NMR (400 MHz, $\left.\mathrm{CDCl}_{3}\right): \delta 6.97$ (d, $\left.J=8.0 \mathrm{~Hz}, 1 \mathrm{H}\right), 6.79-6.75$ (m, 2H), 5.97 (s, 2H), 4.08 (d, $J=5.2 \mathrm{~Hz}, 2 \mathrm{H}), 3.98$ (d, $J=6.0 \mathrm{~Hz}, 2 \mathrm{H}), 3.64-3.50(\mathrm{~m}, 56 \mathrm{H})$, 3.36-3.34 (4 x CH $3,12 \mathrm{H}), 2.63$ (s, 6H), 2.48-2.38 (m, 2H), 1.47 (s, 6H). ${ }^{13} \mathrm{C}$ NMR (100 $\left.\mathrm{MHz}, \mathrm{CDCl}_{3}\right): \delta 155.5,149.9,149.8,143.3,142.0,131.9,127.3,121.2,120.7,113.9$, $113.5,72.1,72.1,70.8,70.8,70.7,70.7,70.6,69.6,69.5,67.7,67.3,59.2,59.2,40.2$, 14.7. IR $\left(\mathrm{cm}^{-1}\right): 2868,1543,1509,1467,1411,1363,1351,1305,1262,1249,1193$, 1094, 1026, 997, 847, 810, 761. ${ }^{11} \mathrm{~B}$ NMR $\left(128 \mathrm{~Hz}, \mathrm{CDCl}_{3}\right): \delta 5.75(\mathrm{t}, J=34 \mathrm{~Hz})$. HRMS (FAB) calcd for $\mathrm{C}_{55} \mathrm{H}_{91} \mathrm{~N}_{2} \mathrm{~F}_{2} \mathrm{~B} \mathrm{O} \mathrm{O}_{18}[\mathrm{M}+\mathrm{H}]^{+}, 1116.6328$; found, 1116.6332. It shows an absorption maximum at $501 \mathrm{~nm}, 500 \mathrm{~nm}$ and $499 \mathrm{~nm}$ in $\mathrm{CH}_{2} \mathrm{Cl}_{2}$, ethanol and PBS solution, respectively. It also shows a weak absorption peak at $370 \mathrm{~nm}$ in PBS solution. It shows an emission maximum at $511 \mathrm{~nm}, 509 \mathrm{~nm}$ and $510 \mathrm{~nm}$ in $\mathrm{CH}_{2} \mathrm{Cl}_{2}$, ethanol and PBS solution, respectively.

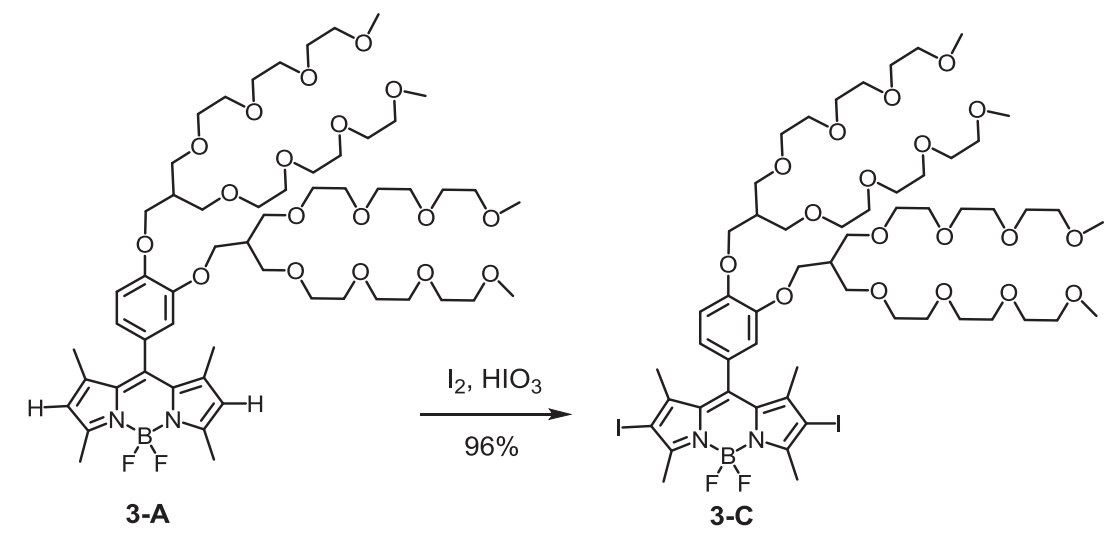

Scheme 3.5. Synthetic route to compound 3-C.

Compound 3-C: Iodic acid $(0.30 \mathrm{~g}, 1.70 \mathrm{mmol})$ in $3 \mathrm{~mL}$ of water was added dropwise to the ethanol solution $(15 \mathrm{~mL})$ containing compound 3-A $(0.84 \mathrm{~g}, 0.752 \mathrm{mmol})$ and iodine $(0.25 \mathrm{~g}, 1.95 \mathrm{mmol})$ over 30 minutes. Then the mixture was stirred for $4 \mathrm{~h}$. The mixture was concentrated under reduced pressure, dissolved in $\mathrm{CH}_{2} \mathrm{Cl}_{2}$, and washed twice with water and brine solution. The organic layer was collected, dried over 
anhydrous $\mathrm{Na}_{2} \mathrm{SO}_{4}$, and concentrated under reduced pressure. The crude product was purified by column chromatography to yield BODIPY dye 3-C as red oil (0.99 g, 96\%). ${ }^{1} \mathrm{H}$ NMR $\left(400 \mathrm{MHz}, \mathrm{CDCl}_{3}\right): \delta 6.98(\mathrm{~d}, J=8.0 \mathrm{~Hz}, 1 \mathrm{H}), 6.73-6.70(\mathrm{~m}, 2 \mathrm{H}), 4.09$ (d, $J=$ $6.0 \mathrm{~Hz}, 2 \mathrm{H}), 3.96$ (d, $J=5.2 \mathrm{~Hz}, 2 \mathrm{H}), 3.64-3.49$ (m, 56H), 3.35-3.34 (4 x CH, $12 \mathrm{H}), 2.61$ $(\mathrm{s}, 6 \mathrm{H}), 2.48-2.37(\mathrm{~m}, 2 \mathrm{H}), 1.47(\mathrm{~s}, 6 \mathrm{H}) .{ }^{13} \mathrm{C} \mathrm{NMR}\left(100 \mathrm{MHz}, \mathrm{CDCl}_{3}\right): \delta 156.7,150.2$, $145.6,141.8,131.8,126.9,120.6,113.9,113.1,72.1,70.8,70.8,70.7,70.7,70.6,69.6$, 69.5, 67.7, 67.3, 59.2, 59.2, 40.2, 17.4, 16.2. IR ( $\left.\mathrm{cm}^{-1}\right): 2868,1528,1457,1397,1345$, 1305, 1264, 1249, 1178, 1097, 995, 850, 759. HRMS (MAIDL) calcd for $\mathrm{C}_{55} \mathrm{H}_{89} \mathrm{BF}_{2} \mathrm{I}_{2} \mathrm{~N}_{2} \mathrm{O}_{18} \mathrm{Na}[\mathrm{M}+\mathrm{Na}]^{+}$, 1391.4159; found, 1391.4192. It shows an absorption maximum at $534 \mathrm{~nm}, 533 \mathrm{~nm}$ and $539 \mathrm{~nm}$ in $\mathrm{CH}_{2} \mathrm{Cl}_{2}$, ethanol and PBS solution, respectively. It also shows a weak absorption peak at $390 \mathrm{~nm}$ in $\mathrm{CH}_{2} \mathrm{Cl}_{2}$ and $\mathrm{PBS}$ solution, respectively. It shows an emission maximum at $548 \mathrm{~nm}, 550 \mathrm{~nm}$ and $552 \mathrm{~nm}$ in $\mathrm{CH}_{2} \mathrm{Cl}_{2}$, ethanol and PBS solution, respectively.
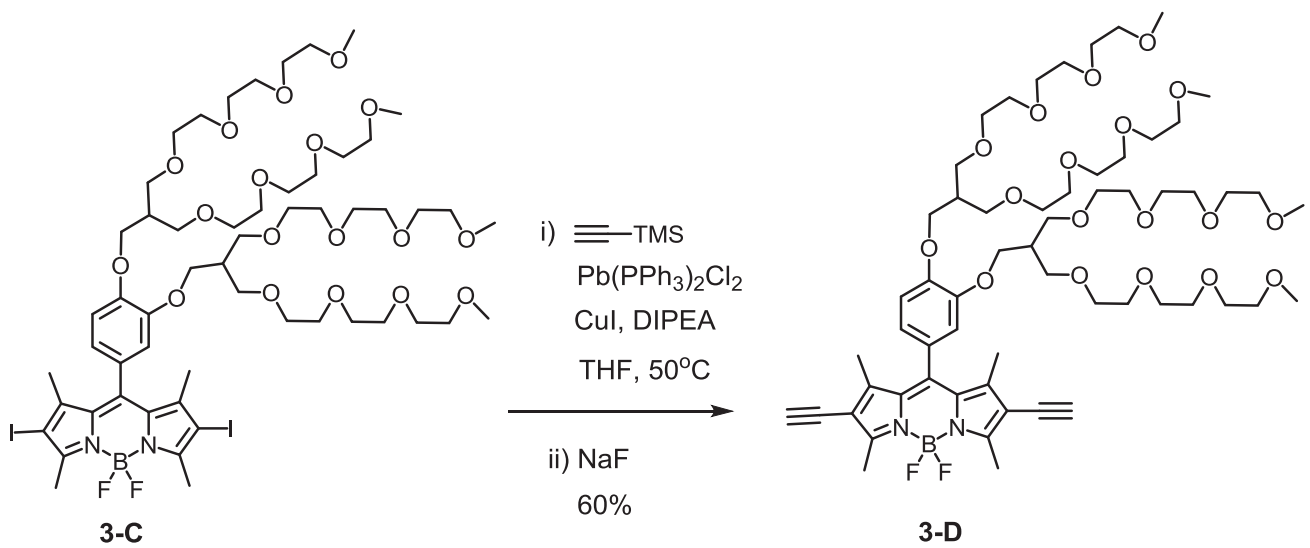

Scheme 3.6. Synthetic route to compound 3-D.

Compound 3-D: Compound 3-C (820 mg, $0.6 \mathrm{mmol}), \mathrm{CuI}(5 \mathrm{mg})$ and $\mathrm{Pd}\left(\mathrm{PPh}_{3}\right)_{2} \mathrm{Cl}_{2}$ $(8 \mathrm{mg})$ were added to a $50-\mathrm{mL}$ round-bottom flask under nitrogen atmosphere. When degassed anhydrous THF $(10 \mathrm{~mL})$, anhydrous diisopropylamine $(5 \mathrm{~mL})$, and trimethylsilylacetylene ( $425 \mathrm{uL}, 3 \mathrm{mmol})$ were added to the flask, the mixture was stirred at $50{ }^{\circ} \mathrm{C}$ overnight. When the reaction mixture was concentrated under reduced pressure, dissolved in the mixture of $\mathrm{MeOH}(15 \mathrm{~mL})$ and $\mathrm{H}_{2} \mathrm{O}(0.5 \mathrm{~mL}), \mathrm{NaF}(125 \mathrm{mg}, 3 \mathrm{mmol})$ was added to the mixed solution. After the mixture was stirred at $50{ }^{\circ} \mathrm{C}$ overnight, it was concentrated and purified by silica gel column chromatography using 
hexane/EtOAc/ $\mathrm{CH}_{2} \mathrm{Cl}_{2} / \mathrm{EtOH}(5 / 1 / 3 / 0.5, \mathrm{v} / \mathrm{v})$ to yield BODIPY dye 3-D as red oil (420 $\mathrm{mg}, 60 \%) .{ }^{1} \mathrm{H}$ NMR (400 MHz, $\left.\mathrm{CDCl}_{3}\right): \delta 6.98(\mathrm{~d}, J=8.0 \mathrm{~Hz}, 1 \mathrm{H}), 6.74-6.70(\mathrm{~m}, 2 \mathrm{H})$, $4.08(\mathrm{~d}, J=6.0 \mathrm{~Hz}, 2 \mathrm{H}), 3.96(\mathrm{~d}, J=5.6 \mathrm{~Hz}, 2 \mathrm{H}), 3.63-3.48(\mathrm{~m}, 56 \mathrm{H}), 3.34-3.32(4 \mathrm{x}$ $\left.\mathrm{CH}_{3}, 12 \mathrm{H}\right), 3.30$ (s, 2H), 2.61 (s, 6H), 2.48-2.37 (m, 2H), 1.55 (s, 6H). ${ }^{13} \mathrm{C}$ NMR (100 $\left.\mathrm{MHz}, \mathrm{CDCl}_{3}\right): \delta 158.8,150.2,150.1,145.8,143.5,131.4,126.4,120.5,115.2,113.9$, 113.1, 84.3, 76.2, 72.1, 72.1, 70.8, 70.8, 70.7, 70.7, 70.6, 69.6, 69.5, 67.7, 67.3, 59.2, 59.240.2, 13.7. IR $\left(\mathrm{cm}^{-1}\right): 2921,2856,1531,1468,1401,1365,1313,1249,1193,1092$, 1012, 942, 850, 762. ${ }^{11} \mathrm{~B}$ NMR $\left(128 \mathrm{~Hz}, \mathrm{CDCl}_{3}\right): \delta 5.14(\mathrm{t}, J=32 \mathrm{~Hz})$. HRMS (FAB) calcd for $\mathrm{C}_{59} \mathrm{H}_{91} \mathrm{~N}_{2} \mathrm{~F}_{2} \mathrm{BO}_{18}[\mathrm{M}+\mathrm{H}]^{+}, 1164.6328$; found, 1164.6322. It shows an absorption maximum at $539 \mathrm{~nm}$ and $536 \mathrm{~nm}$ in $\mathrm{CH}_{2} \mathrm{Cl}_{2}$ and $\mathrm{PBS}$ solution, respectively. It also exhibits a weak absorption peak at $390 \mathrm{~nm}$ and $389 \mathrm{~nm}$ in $\mathrm{CH}_{2} \mathrm{Cl}_{2}$ and $\mathrm{PBS}$ solution, respectively. It displays an emission maximum at $554 \mathrm{~nm}$ and $555 \mathrm{~nm}$ in $\mathrm{CH}_{2} \mathrm{Cl}_{2}$ and $\mathrm{PBS}$ solution, respectively.

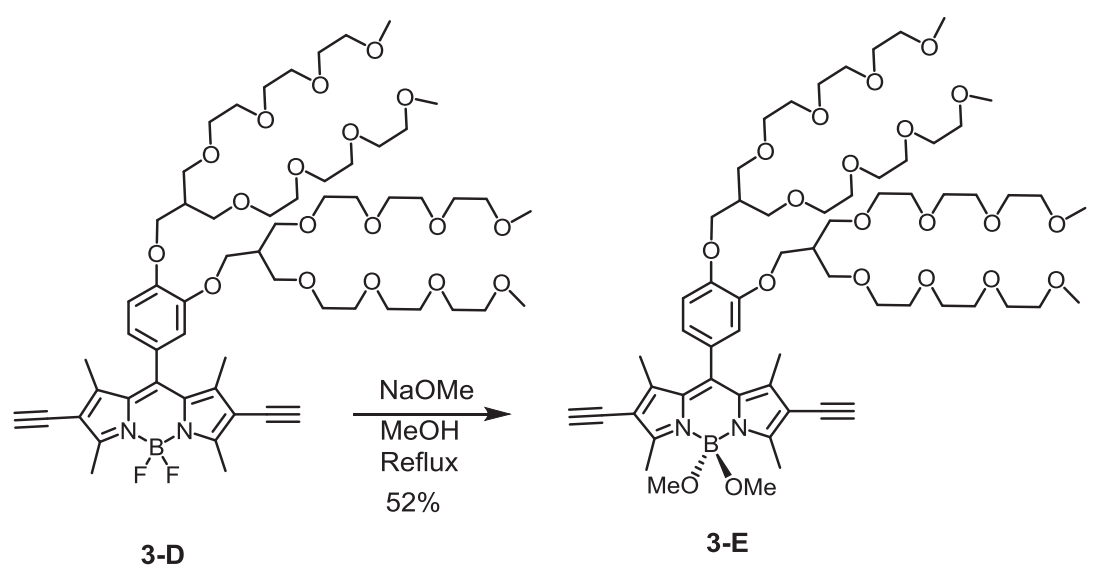

Scheme 3.7. Synthetic route to compound 3-E.

Compound 3-E: To the solution of compound 3-D (90.0 $\mathrm{mg}, 0.077 \mathrm{mmol})$ in dry $\mathrm{MeOH}(10 \mathrm{~mL})$ added $\mathrm{NaOMe}(21 \mathrm{mg}, 0.232 \mathrm{mmol})$. Then the mixture was stirred reflux under a nitrogen atmosphere overnight. The reaction mixtures were then cooled down to ambient temperature and were concentrated. The crude product was purified by column chromatography using hexane/ $\mathrm{CH}_{2} \mathrm{Cl}_{2} / \mathrm{EtOAc} / \mathrm{EtOH}$ (5/3/1/0.5, v/v) to yield BODIPY dye 3-E as a red oil $(48 \mathrm{mg}, 52 \%) .{ }^{1} \mathrm{H}$ NMR $\left(400 \mathrm{MHz}, \mathrm{CDCl}_{3}\right): \delta 6.98(\mathrm{~d}, J=8.8 \mathrm{~Hz}$, 1H), 6.73-6.70 (m, 2H), 4.08 (d, $J=6.0 \mathrm{~Hz}, 2 \mathrm{H}), 3.97$ (d, $J=6.0 \mathrm{~Hz}, 2 \mathrm{H}), 3.64-3.49$ (m, 56H), 3.34-3.33 (4 x CH $3,12 \mathrm{H}), 3.31(\mathrm{~s}, 2 \mathrm{H}), 2.92(\mathrm{~s}, 3 \mathrm{H}), 2.88(\mathrm{~s}, 3 \mathrm{H}), 2.61(\mathrm{~s}, 6 \mathrm{H})$, 
2.47-2.38 (m, 2H), 1.55 (s, 6H). ${ }^{13} \mathrm{C}$ NMR (100 MHz, $\left.\mathrm{CDCl}_{3}\right): \delta 158.9,150.1,150.0$, 144.3, 143.3, 132.9, 127.1, 120.8, 114.7, 113.9, 113.3, 84.1, 76.8, 72.1, 70.8, 70.7, 70.7, 70.6, 69.6, 69.5, 67.8, 67.3, 59.2, 49.5, 49.4, 40.2, 13.8, 13.6. IR $\left(\mathrm{cm}^{-1}\right): 2869,2815$, 1531, 1467, 1426, 1392, 1364, 1350, 1302, 1262, 1246, 1199, 1179, 1049, 1011, 960, 849, 752. ${ }^{11} \mathrm{~B}$ NMR $\left(128 \mathrm{~Hz}, \mathrm{CDCl}_{3}\right): \delta 5.41(\mathrm{~S})$. HRMS (FAB) calcd for $\mathrm{C}_{61} \mathrm{H}_{97} \mathrm{~N}_{2} \mathrm{BO}_{20}$ $[\mathrm{M}]^{+}, 1188.6728$; found, 1188.6725. It shows an absorption maximum at $538 \mathrm{~nm}$ and 534 $\mathrm{nm}$ in $\mathrm{CH}_{2} \mathrm{Cl}_{2}$ and $\mathrm{PBS}$ solution, respectively. It also exhibits a weak absorption peak at $390 \mathrm{~nm}$ and $387 \mathrm{~nm}$ in $\mathrm{CH}_{2} \mathrm{Cl}_{2}$ and PBS solution, respectively. It displays an emission maximum at $552 \mathrm{~nm}$ and $550 \mathrm{~nm}$ in $\mathrm{CH}_{2} \mathrm{Cl}_{2}$ and PBS solution, respectively.

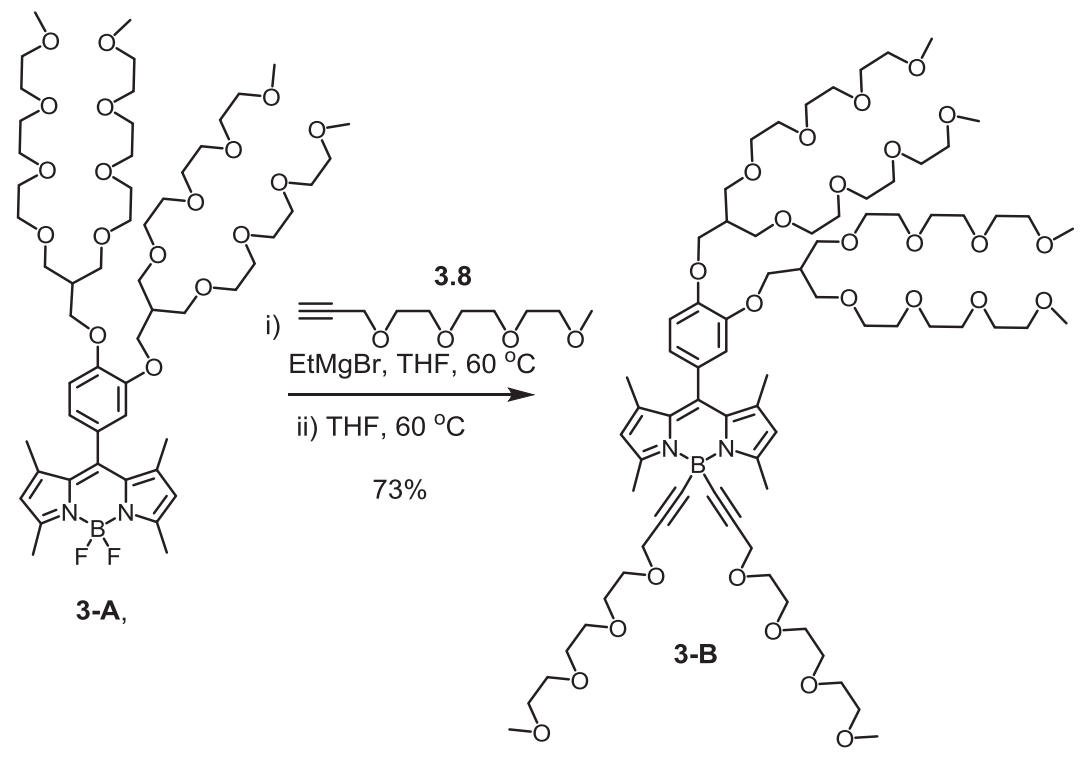

Scheme 3.8. Synthetic route to compound 3-B.

Compound 3-B: To a solution of compound $\mathbf{3 . 8}(1.60 \mathrm{mmol}, 323 \mathrm{mg})$ in anhydrous THF ( $2 \mathrm{~mL}$ ) was added ethylmagnesium bromide (1.90 mmol, $1.9 \mathrm{~mL}$ of $1.0 \mathrm{M}$ solution). When the mixture was heated at $60^{\circ} \mathrm{C}$ for 2 hours and cooled down to room temperature, a solution of compound 3-A (180 mg, $0.16 \mathrm{mmol})$ in anhydrous THF (3 mL) was added to the mixture. The resulting mixture was stirred at $60^{\circ} \mathrm{C}$ overnight until complete consumption of the starting material was observed by TLC. When water $(10 \mathrm{~mL})$ was added to the mixture, the resulting mixture was extracted with $\mathrm{CH}_{2} \mathrm{Cl}_{2}$. The organic layer was dried over anhydrous $\mathrm{Na}_{2} \mathrm{SO}_{4}$, and concentrated under reduced pressure. The crude product was purified by column chromatography using hexanes $/ \mathrm{CH}_{2} \mathrm{Cl}_{2} / \mathrm{EtOH}$ (3:6:0.5, 
$\mathrm{v} / \mathrm{v}$ ) to yield BODIPY dye 3-B as purple oil (172 mg, 73\%). ${ }^{1} \mathrm{H}$ NMR (400 $\mathrm{MHz}$, $\left.\mathrm{CDCl}_{3}\right): \delta 6.84(\mathrm{~d}, J=8.0 \mathrm{~Hz}, 1 \mathrm{H}), 6.73-6.69(\mathrm{~m}, 2 \mathrm{H}), 5.92(\mathrm{~s}, 2 \mathrm{H}), 4.09$ (s, 2H), 3.99 (d, $J=6.0 \mathrm{~Hz}, 2 \mathrm{H}), 3.88(\mathrm{~d}, J=5.6 \mathrm{~Hz}, 2 \mathrm{H}), 3.56-3.42(\mathrm{~m}, 80 \mathrm{H}), 3.27-3.26\left(6 \mathrm{x} \mathrm{CH}_{3}, 18 \mathrm{H}\right)$, $2.62(\mathrm{~s}, 6 \mathrm{H}), 2.40-2.29(\mathrm{~m}, 2 \mathrm{H}), 1.37$ (s, 6H). ${ }^{13} \mathrm{C} \mathrm{NMR}\left(100 \mathrm{MHz}, \mathrm{CDCl}_{3}\right): \delta$ 155.0, 149.8, 149.6, 141.8, 141.2, 130.0, 127.8, 121.4, 120.8, 113.8, 113.7, 90.8, 72.0, 70.8, $70.7,70.7,70.6,70.5,70.4,69.5,69.4,68.8,67.7,67.3,59.7,59.6,59.1,40.2,16.2,14.9$. IR ( $\left.\mathrm{cm}^{-1}\right): 2869,1548,1511,1465,1408,1350,1307,1260,1199,1180,1097,1027,980$, 849. ${ }^{11} \mathrm{~B}$ NMR $\left(128 \mathrm{~Hz}, \mathrm{CDCl}_{3}\right.$ ): $\delta-7.71$ (s). HRMS (FAB) calcd for $\mathrm{C}_{75} \mathrm{H}_{125} \mathrm{~N}_{2} \mathrm{BO}_{26}$ $[\mathrm{M}+\mathrm{H}]^{+}, 1480.8614$; found, 1480.8615. It shows an absorption maximum at $499 \mathrm{~nm}$ and $496 \mathrm{~nm}$ in $\mathrm{CH}_{2} \mathrm{Cl}_{2}$ and PBS solution, respectively. It also exhibits a weak absorption peak at $366 \mathrm{~nm}$ and $363 \mathrm{~nm}$ in $\mathrm{CH}_{2} \mathrm{Cl}_{2}$ and $\mathrm{PBS}$ solution, respectively. It displays an emission maximum at $508 \mathrm{~nm}$ and $507 \mathrm{~nm}$ in $\mathrm{CH}_{2} \mathrm{Cl}_{2}$ and PBS solution, respectively.

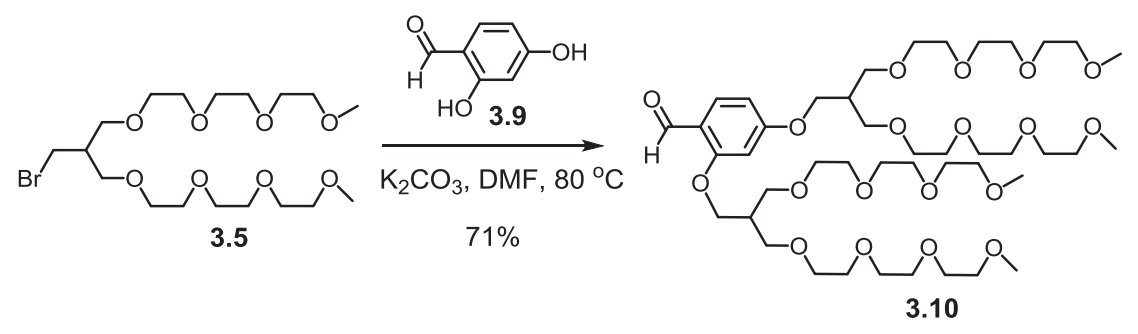

Scheme 3.9. Synthetic route to compound 3.10.

Compound 3.10: When $15 \mathrm{~mL}$ of DMF was added to a $100-\mathrm{mL}$ round-bottom flask containing 3,4-dihydroxybezaldehyde $\mathbf{3 . 9}$ (490 mg, $3.58 \mathrm{mmol}$ ), compound 3.5 (4.12 g, $8.94 \mathrm{mmol})$, and $\mathrm{K}_{2} \mathrm{CO}_{3}(1.47 \mathrm{~g}, 10.74 \mathrm{mmol})$ under a nitrogen atmosphere, the mixture was stirred at $80{ }^{\circ} \mathrm{C}$ for $12 \mathrm{~h}$ under a nitrogen atmosphere. After completion of the reaction (which was monitored by TLC), DMF was removed from the mixture under reduced pressure. The resulting crude product was purified by silica gel column chromatography using hexane/EtOAc/ $\mathrm{CH}_{2} \mathrm{Cl}_{2} / \mathrm{EtOH} \quad(5 / 2 / 3 / 0.5$ to $5 / 1 / 3 / 1, \mathrm{v} / \mathrm{v})$ to yield compound 3.10 as a colorless oil (2.3 g, 72\%). ${ }^{1} \mathrm{H}$ NMR (400 MHz, $\mathrm{CDCl}_{3}$ ): $\delta 10.23$ (s, $1 \mathrm{H}), 7.70(\mathrm{~d}, J=8.8 \mathrm{~Hz}, 1 \mathrm{H}), 6.48$ (dd, $J=8.8,2.0 \mathrm{~Hz}, 1 \mathrm{H}), 6.41(\mathrm{~d}, J=3.0 \mathrm{~Hz}, 1 \mathrm{H})$, 4.07-4.02 (m, 4H), 3.58-3.45 (m, 56H), 3.31-3.29 (4 x CH $3,12 \mathrm{H}), 2.42-2.33(\mathrm{~m}, 2 \mathrm{H}) .{ }^{13} \mathrm{C}$ NMR (100 MHz, $\left.\mathrm{CDCl}_{3}\right): \delta 188.2,165.9,163.4,130.4,119.1,106.8,99.3,72.1,71.1$, 
70.8, 70.7, 70.6, 69.4, 69.3, 66.8, 66.7, 59.2, 40.1. HRMS (FAB) calcd for $\mathrm{C}_{43} \mathrm{H}_{79} \mathrm{O}_{19}$ $[\mathrm{M}+\mathrm{H}]^{+}, 899.5216$; found, 899.5226.

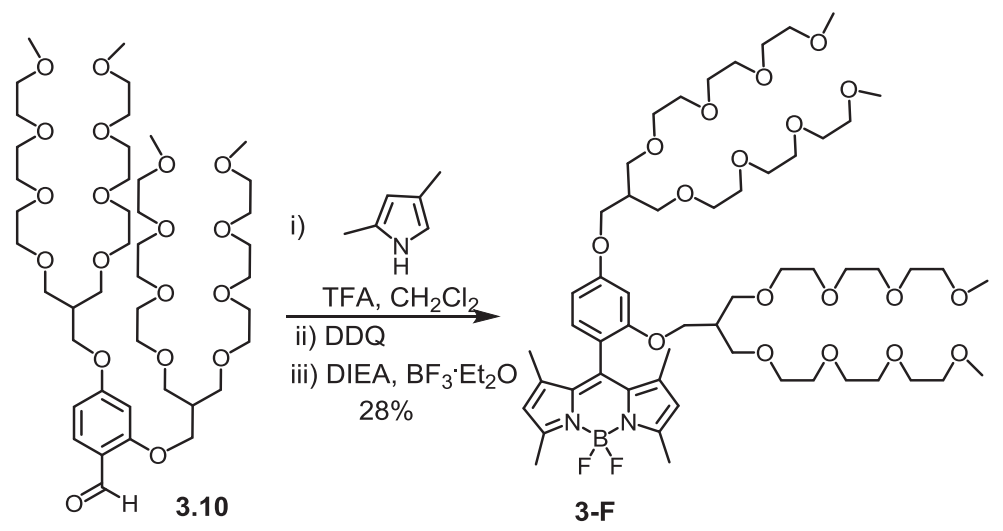

Scheme 3.10. Synthetic route to compound 3-F.

Compound 3-F: Compound 3.10 ( $2.0 \mathrm{~g}, 2.23 \mathrm{mmol}$ ) and 2,4-dimethylpyrrole (530 $\mathrm{mg}, 5.58 \mathrm{mmol}$ ) were dissolved in $400 \mathrm{~mL}$ of dry $\mathrm{CH}_{2} \mathrm{Cl}_{2}$ in a $1000 \mathrm{~mL}$ flask. Two drops of trifluoroacetic acid (TFA) were added to the reaction mixture, and the resulting mixture was stirred in the dark for $12 \mathrm{~h}$ under nitrogen atmosphere at room temperature. After the complete consumption of compound $\mathbf{3 . 1 0}$ (which was monitored by TLC), DDQ (2,3-dichloro-5,6-dicyano-1,4-benzoquinone) $(610 \mathrm{mg}, 2.68 \mathrm{mmol})$ was added to the reaction mixture. When the mixture was stirred for $40 \mathrm{~min}, 10 \mathrm{~mL}$ of diisopropylethylamine (DIEA) and $10 \mathrm{~mL}$ of $\mathrm{BF}_{3} \cdot \mathrm{OEt}_{2}$ were added to the mixture. After the mixture was further stirred for $40 \mathrm{~min}$, it was concentrated to $200 \mathrm{~mL}$ and filtered. When the filtrate was washed twice with water and brine solution, the organic layer was collected, dried over anhydrous $\mathrm{Na}_{2} \mathrm{SO}_{4}$, and concentrated under reduced pressure. The crude product was purified by column chromatography using hexane/EtOAc/ $\mathrm{CH}_{2} \mathrm{Cl}_{2} / \mathrm{EtOH}(10 / 2 / 6 / 1, \mathrm{v} / \mathrm{v})$ to obtain BODIPY dye 3-F as yellow-red oil (697 mg, 28\%). ${ }^{1} \mathrm{H}$ NMR (400 MHz, $\mathrm{CDCl}_{3}$ ): $\delta 6.86$ (d, $\left.J=8.4 \mathrm{~Hz}, 1 \mathrm{H}\right), 6.51-6.45$ (m, $2 \mathrm{H}), 5.84(\mathrm{~s}, 2 \mathrm{H}), 3.95(\mathrm{~d}, J=5.6 \mathrm{~Hz}, 2 \mathrm{H}), 3.86(\mathrm{~d}, J=5.2 \mathrm{~Hz}, 2 \mathrm{H}), 3.54-3.40(\mathrm{~m}, 56 \mathrm{H})$, 3.28-3.26 (4 x CH $3,12 \mathrm{H}), 2.41$ (s, 6H), 2.33-2.30 (m, 1H), 2.15-2.13 (m, 1H), 1.39 (s, $6 \mathrm{H}) .{ }^{13} \mathrm{C}$ NMR (100 MHz, $\left.\mathrm{CDCl}_{3}\right): \delta 161.5,156.7,154.6,142.8,139.5,132.2,129.7$, $120.7,116.4,106.3,100.4,72.0,70.8,70.7,70.6,70.5,70.4,69.5,69.1,66.5,66.2,59.1$, 40.1, 39.6, 14.6, 14.2. IR ( $\left.\mathrm{cm}^{-1}\right): 2868,1608,1543,1509,1467,1410,1363,1351,1303$, 
1275, 1261, 1192, 1026, 979, 836, 764, 750, 705. HRMS (FAB) calcd for $\mathrm{C}_{55} \mathrm{H}_{91} \mathrm{~N}_{2} \mathrm{~F}_{2} \mathrm{BO}_{18}[\mathrm{M}+\mathrm{H}]^{+}, 1116.6328$; found, 1116.6332. It shows an absorption maximum at $504 \mathrm{~nm}$ and $501 \mathrm{~nm}$ in $\mathrm{CH}_{2} \mathrm{Cl}_{2}$ and PBS solution, respectively. It also displays a weak absorption peak at $370 \mathrm{~nm}$ and $368 \mathrm{~nm}$ in $\mathrm{CH}_{2} \mathrm{Cl}_{2}$ and PBS solution, respectively. It exhibits an emission maximum at $514 \mathrm{~nm}$ and $511 \mathrm{~nm}$ in $\mathrm{CH}_{2} \mathrm{Cl}_{2}$ and $\mathrm{PBS}$ solution, respectively.

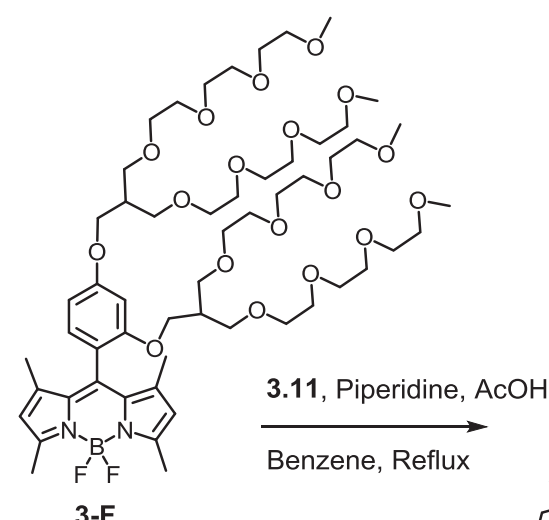

3-F

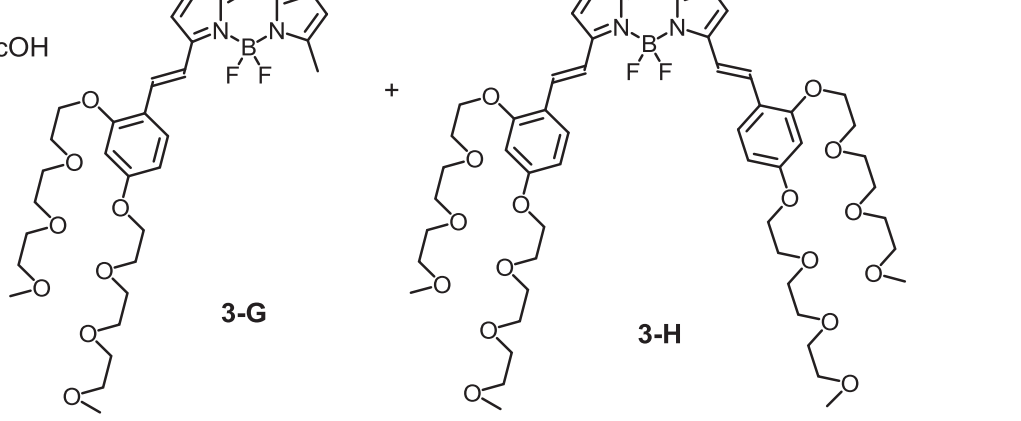

Scheme 3.11. Synthetic route to compounds $3-\mathbf{G}$ and $\mathbf{3}-\mathbf{H}$.

Compound 3-G and 3-H: When BODIPY dye 3-F (550 mg, $0.49 \mathrm{mmol}$ ), and compound $3.11(847 \mathrm{mg}, 1.97 \mathrm{mmol})$ were dissovled in a mixture of benzene $(30 \mathrm{~mL})$, toluene $(10 \mathrm{~mL})$, piperidine $(1.0 \mathrm{~mL})$ and $\mathrm{AcOH}(0.8 \mathrm{~mL})$, the mixture was heated under reflux for $8 \mathrm{~h}$. Any water formed during the reaction was removed azeotropically by using a Dean-Stark apparatus. The mixture was concentrated in vacuo, diluted with EtOAc and washed with water and brine, respectively. The organic layer was collected, dried over $\mathrm{Na}_{2} \mathrm{SO}_{4}$ and concentrated in vacuo. The crude product was purified by silica gel column chromatography by using mobile phase (hexane/EtOAc/ $\mathrm{CH}_{2} \mathrm{Cl}_{2} / \mathrm{EtOH}$, 4/1/3/0.5 to hexane/acetone/ $\mathrm{CH}_{2} \mathrm{Cl}_{2} / \mathrm{EtOH}, 4 / 1 / 3 / 1$ ) to obtain BODIPY dye 3-G (92 mg, $12 \%)$ as purple oil and BODIPY dye $\mathbf{3}-\mathbf{H}(20 \mathrm{mg}, 2 \%)$ as green oil. Monostyryl BODIPY dye 3-G, ${ }^{1} \mathrm{H}$ NMR (400 MHz, $\left.\mathrm{CDCl}_{3}\right): \delta 7.60(\mathrm{~d}, J=16.4 \mathrm{~Hz}, 1 \mathrm{H}), 7.55(\mathrm{~d}, J=8.8 \mathrm{~Hz}$, 
1H), 7.47 (d, $J=16.4 \mathrm{~Hz}, 1 \mathrm{H}), 6.97$ (d, $J=8.4 \mathrm{~Hz}, 1 \mathrm{H}), 6.57-6.43(\mathrm{~m}, 5 \mathrm{H}), 5.91(\mathrm{~s}, 1 \mathrm{H})$, 4.16-4.11 (m, 4H), 4.03 (d, $J=6.0 \mathrm{~Hz}, 2 \mathrm{H}), 3.94-3.92(\mathrm{~m}, 4 \mathrm{H}), 3.83$ (t, $J=5.2 \mathrm{~Hz}, 2 \mathrm{H})$, 3.75-3.47 (m, 72H), 3.35-3.32 (6 x CH $3,18 \mathrm{H}), 2.52$ (s, 3H), 2.44-2.37 (m, 1H), 2.23-2.19 $(\mathrm{m}, 1 \mathrm{H}), 1.52(\mathrm{~s}, 3 \mathrm{H}), 1.46(\mathrm{~s}, 3 \mathrm{H}) .{ }^{13} \mathrm{C} \mathrm{NMR}\left(100 \mathrm{MHz}, \mathrm{CDCl}_{3}\right): \delta 161.5,161.0,158.2$, 157.0, 154.0, 153.3, 142.4, 141.4, 137.3, 133.7, 132.2, 131.4, 130.1, 129.2, 120.4, 119.5, $117.7,116.8,106.7,106.2,100.4,100.3,77.4,72.2,72.1,71.0,70.9,70.8,70.7,70.7$, $70.6,70.5,69.8,69.7,69.6,69.2,69.2,68.3,67.7,66.7,66.3,59.2,59.2,40.2,39.7,14.8$, 14.6, 14.2. IR (cm $\left.{ }^{-1}\right): 2869,1606,1576,1543,1507,1466,1411,1364,1351,1302,1275$, 1192, 1027, 980, 836, 765, 750, 706. HRMS (MAIDL) calcd for $\mathrm{C}_{76} \mathrm{H}_{123} \mathrm{BF}_{2} \mathrm{~N}_{2} \mathrm{O}_{26}[\mathrm{M}]^{+}$, 1528.8425; found, 1528.8413. It shows an absorption maximum at $580 \mathrm{~nm}$ and $577 \mathrm{~nm}$ in $\mathrm{CH}_{2} \mathrm{Cl}_{2}$ solution and PBS solution, respectively. It also shows a weak absorption peak at $350 \mathrm{~nm}$, and absorption shoulder peaks at $505 \mathrm{~nm}$ and $540 \mathrm{~nm}$ in $\mathrm{CH}_{2} \mathrm{Cl}_{2}$ solution. It displays a weak absorption peak at $347 \mathrm{~nm}$, and absorption shoulder peaks at $505 \mathrm{~nm}$ and $539 \mathrm{~nm}$ in PBS solution. It shows an emission maximum at $596 \mathrm{~nm}$ and $598 \mathrm{~nm}$ in $\mathrm{CH}_{2} \mathrm{Cl}_{2}$ solution and PBS solution, respectively.

Distyryl BODIPY dye 3-H, ${ }^{1} \mathrm{H}$ NMR (400 MHz, $\left.\mathrm{CDCl}_{3}\right): \delta$ 7.64-7.60 (m, 4H), 7.50 $(\mathrm{d}, J=16.4 \mathrm{~Hz}, 2 \mathrm{H}), 7.00(\mathrm{~d}, J=8.4 \mathrm{~Hz}, 1 \mathrm{H}), 6.56-6.47(\mathrm{~m}, 8 \mathrm{H}), 5.27-5.14(\mathrm{~m}, 8 \mathrm{H}), 4.08$ $(\mathrm{d}, J=6.0 \mathrm{~Hz}, 2 \mathrm{H}), 4.04-3.92(\mathrm{~m}, 6 \mathrm{H}), 3.86-3.84(\mathrm{~m}, 4 \mathrm{H}), 3.75-3.45$ (m, 88H), 3.36-3.31 (8 x CH $3,24 \mathrm{H}), 2.52(\mathrm{~s}, 3 \mathrm{H}), 2.49-2.40(\mathrm{~m}, 1 \mathrm{H}), 2.28-2.22\left(\mathrm{~m}, 1.53(\mathrm{~s}, 3 \mathrm{H}) .{ }^{13} \mathrm{C} \mathrm{NMR}\right.$ $\left(100 \mathrm{MHz} \mathrm{CDCl}_{3}\right): \delta 161.5,160.9,158.0,157.2,152.9,141.3,135.1,133.9,130.4$, 130.3, 128.6, 119.8, 117.8, 117.2, 117.0, 106.8, 106.1, 100.4, 77.4, 72.2, 72.1, 71.0, 70.9, 70.8, 70.7, 70.7, 70.6, 70.5, 69.9, 69.7, 69.6, 69.2, 68.4, 67.8, 66.6, 66.4, 59.2, 59.1, 53.6, 40.2, 39.7, 14.5. IR $\left(\mathrm{cm}^{-1}\right): 2869,1607,1577,1543,1508,1463,1412,1364,1351,1303$, 1275, 1261, 1192, 1099, 1027, 980, 835, 764, 706. HRMS (MAIDL) calcd for $\mathrm{C}_{97} \mathrm{H}_{155} \mathrm{BF}_{2} \mathrm{~N}_{2} \mathrm{O}_{34}[\mathrm{M}]^{+}, 1941.0522$; found, 1941.0518. It shows an absorption maximum at $659 \mathrm{~nm}$ and $660 \mathrm{~nm}$ in $\mathrm{CH}_{2} \mathrm{Cl}_{2}$ solution and PBS solution, respectively. It also shows two absorption peaks of phenyl groups at meso and 3,5-positions at $325 \mathrm{~nm}$ and $383 \mathrm{~nm}$ respectively, a weak absorption peak of BODIPY core at $508 \mathrm{~nm}$, and an absorption shoulder peak at $602 \mathrm{~nm}$ in $\mathrm{CH}_{2} \mathrm{Cl}_{2}$ solution. It also displays two strong absorption peaks of phenyl groups at meso and 3,5-positions at $326 \mathrm{~nm}$ and $383 \mathrm{~nm}$, respectively, a weak absorption peak of BODIPY core at $507 \mathrm{~nm}$, and an absorption shoulder peak $609 \mathrm{~nm}$ in 
PBS solution. It shows an emission maximum at $676 \mathrm{~nm}$ and $676 \mathrm{~nm}$ in $\mathrm{CH}_{2} \mathrm{Cl}_{2}$ solution and PBS solution, respectively.

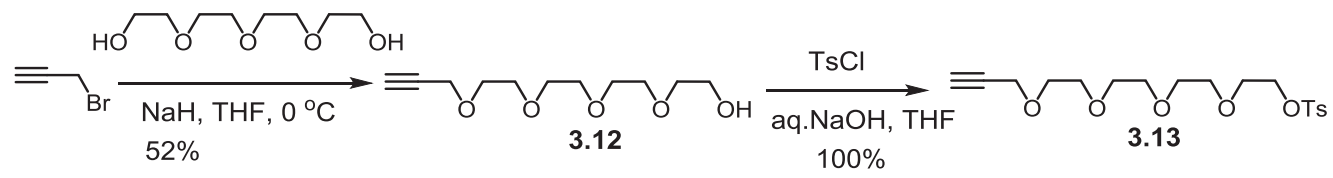

Scheme 3.12. Synthetic route to compound 3.13.

Compound 3.12: $\quad$ Tetra(ethylene glycol) $(6.98 \mathrm{~g}, 36.0 \mathrm{mmol})$ was dissolved in anhydrous THF $(80 \mathrm{~mL})$. When sodium hydride $(1.76 \mathrm{~g}, 60 \%)$ was added into the mixture at $0{ }^{\circ} \mathrm{C}$, the solution was stirred until no hydrogen gas was released. When propargyl bromide (5.95 g, 80\% in toluene) was added dropwise into the mixture at $0{ }^{\circ} \mathrm{C}$, the reaction mixture was at room temperature overnight. After the mixture was filtered, the filtrate was concentrated under reduced pressure and purified by silica gel column chromatography using hexanes/ $\mathrm{CH}_{2} \mathrm{Cl}_{2} / \mathrm{EtOH}(3: 6: 0.5, \mathrm{v} / \mathrm{v})$ to give compound $\mathbf{3 . 1 2}$ as pale yellow oil (4.32 g, 52\%). ${ }^{1} \mathrm{H}$ NMR (400 MHz, $\left.\mathrm{CDCl}_{3}\right): \delta 4.14$ (d, $\left.J=2.8 \mathrm{~Hz}, 2 \mathrm{H}\right)$, 3.67-3.60 (m, 14H), 3.56-3.53 (m, 2H), $1.06(\mathrm{~s}, 1 \mathrm{H},-\mathrm{OH}), 2.39(\mathrm{t}, J=2.8 \mathrm{~Hz}, 1 \mathrm{H}) .{ }^{13} \mathrm{C}$ NMR (100 MHz, $\left.\mathrm{CDCl}_{3}\right): \delta 94.6,79.8,74.7,72.7,70.8,70.7,70.7,70.6,70.5,69.3,61.9$, 58.6 .

Compound 3.13: $\quad$ To a solution of compound $3.12(4.32 \mathrm{~g}, 18.7 \mathrm{mmol})$ in THF (20 mL) and aqueous $\mathrm{NaOH}(2.2 \mathrm{~g} / 22 \mathrm{~mL})$ was added the solution of $\mathrm{TsCl}(6.0 \mathrm{~g})$ in THF $(30 \mathrm{~mL})$ at $0{ }^{\circ} \mathrm{C}$. After the mixture was stirred for $2 \mathrm{~h}, 200 \mathrm{~mL}$ of $\mathrm{CH}_{2} \mathrm{Cl}_{2}$ was added to the mixture. When the mixture was washed with water, the organic layer was collected, dried over $\mathrm{Na}_{2} \mathrm{SO}_{4}$ and concentrated. The crude product was purified by column chromatography using hexane/EtOAc $(1 / 2, \mathrm{v} / \mathrm{v})$ to yield compound $\mathbf{3 . 1 3}$ as oil. ${ }^{1} \mathrm{H}$ NMR (400 MHz, $\mathrm{CDCl}_{3}$ ): $\delta 7.75$ (d, $\left.J=6.8 \mathrm{~Hz}, 2 \mathrm{H}\right), 7.30$ (d, $\left.J=6.8 \mathrm{~Hz}, 2 \mathrm{H}\right), 4.15$ (d, $J=2.4 \mathrm{~Hz}, 2 \mathrm{H}), 4.12$ (t, $J=4.8 \mathrm{~Hz}, 2 \mathrm{H}), 3.66-3.57$ (m, 10H), 3.56-3.54 (m, 4H), 2.40 (s, $3 \mathrm{H}), 2.39(\mathrm{t}, J=2.4 \mathrm{~Hz}, 1 \mathrm{H}) .{ }^{13} \mathrm{C} \mathrm{NMR}\left(100 \mathrm{MHz}, \mathrm{CDCl}_{3}\right): \delta 150.0,133.2,130.0,128.2$, 79.9, 74.7, 70.9, 70.8, 70.7, 70.6, 69.5, 69.3, 68.9, 58.6, 21.8. HRMS (FAB) calcd for $\mathrm{C}_{18} \mathrm{H}_{27} \mathrm{O} 7 \mathrm{~S}[\mathrm{M}+\mathrm{H}]^{+}, 387.1478$; found, 387.1473. 


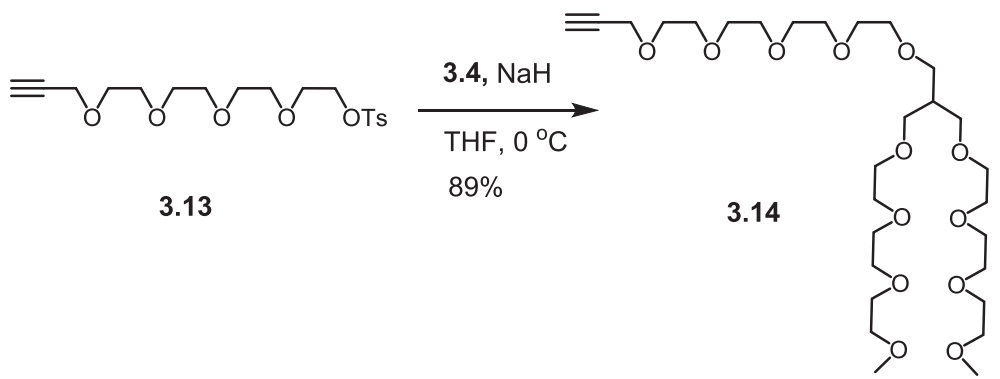

Scheme 3.13. Synthetic route to compound 3.14.

Compound 3.14: $\quad$ Compound 3.4 (3.4 g, $8.55 \mathrm{mmol})$ was dissolved in anhydrous tetrahydrofuran $(60 \mathrm{~mL})$. When sodium hydride $(372 \mathrm{mg}, 60 \%$ dispersion in mineral oil) was added into the mixture in an ice-water bath, the mixture was stirred until no hydrogen gas was released. After compound 3.13 (3.0 g, $7.77 \mathrm{mmol})$ was added to the mixture in an ice-water bath, the resulting mixture was stirred at $0{ }^{\circ} \mathrm{C}$ for $2 \mathrm{~h}$, and then at room temperature overnight, and filtered. The filtrate was concentrated under reduced pressure and purified by silica gel column chromatography using hexanes $/ \mathrm{CH}_{2} \mathrm{Cl}_{2} / \mathrm{EtOH}$ $(6: 3: 0.5, \mathrm{v} / \mathrm{v})$ to give compound $\mathbf{3 . 1 4}$ as light yellow oil $(4.23 \mathrm{~g}, 89 \%)$. ${ }^{1} \mathrm{H}$ NMR (400 $\left.\mathrm{MHz}, \mathrm{CDCl}_{3}\right): \delta 4.16(\mathrm{~d}, J=2.4 \mathrm{~Hz}, 2 \mathrm{H}), 3.68-3.50(\mathrm{~m}, 40 \mathrm{H}), 3.45(\mathrm{~d}, J=6.0 \mathrm{~Hz}, 6 \mathrm{H})$, $3.34(\mathrm{~s}, 6 \mathrm{H}), 2.41(\mathrm{t}, J=2.4 \mathrm{~Hz}, 1 \mathrm{H}), 2.18-2.12(\mathrm{~m}, 1 \mathrm{H}) .{ }^{13} \mathrm{C} \mathrm{NMR}\left(100 \mathrm{MHz}, \mathrm{CDCl}_{3}\right): \delta$ $79.9,74.7,72.1,70.8,70.8,70.7,70.7,70.6,69.8,69.3,59.2,58.6,40.3$. HRMS (FAB) calcd for $\mathrm{C}_{29} \mathrm{H}_{57} \mathrm{O}_{13}[\mathrm{M}+\mathrm{H}]^{+}, 613.3799$; found, 613.3802 .

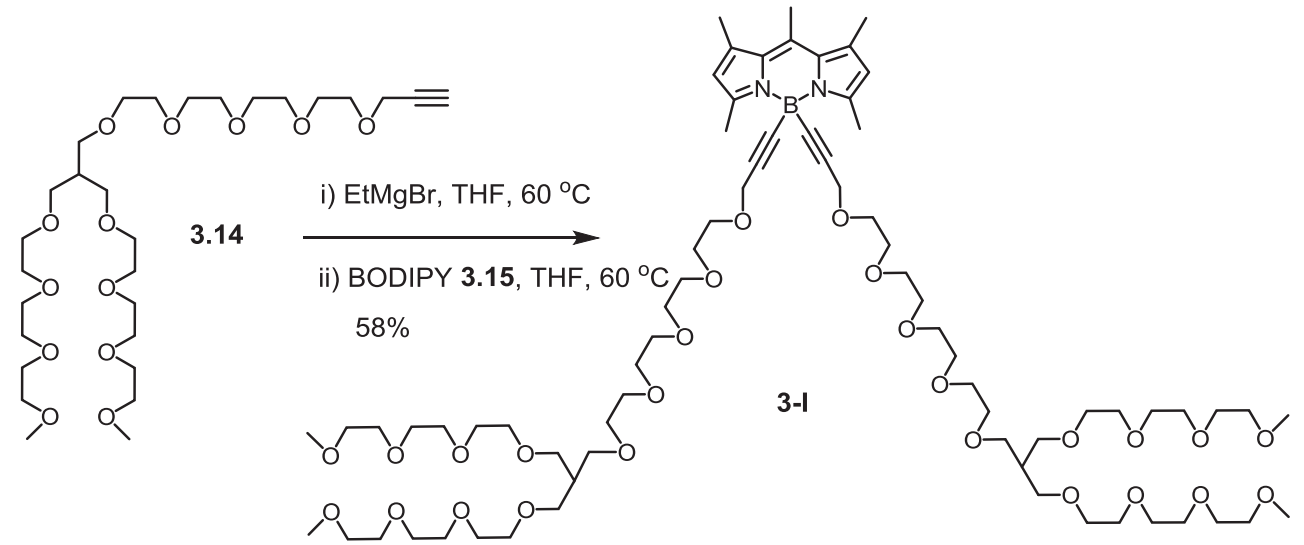

Scheme 3.14. Synthetic route to compound 3-I.

Compound 3-I: To a solution of compound $3.14(1.20 \mathrm{mmol}, 734 \mathrm{mg})$ in anhydrous THF $(2 \mathrm{~mL})$ was added ethylmagnesium bromide $(1.50 \mathrm{mmol}, 1.5 \mathrm{~mL}$ of 1.0 
$\mathrm{M}$ solution). After the mixture was heated at $60^{\circ} \mathrm{C}$ for 2 hours, and cooled down to room temperature, a solution of compound 3.15 (180 $\mathrm{mg}, 0.16 \mathrm{mmol})$ in anhydrous THF (3 $\mathrm{mL}$ ) was added to the mixture. The resulting mixture was stirred at $60^{\circ} \mathrm{C}$ overnight until complete consumption of the starting material was observed by TLC. When water (10 $\mathrm{mL}$ ) was added to the mixture, the resulting mixture was extracted with $\mathrm{CH}_{2} \mathrm{Cl}_{2}$. The organic layer was collected, dried over anhydrous $\mathrm{Na}_{2} \mathrm{SO}_{4}$, and concentrated under reduced pressure. The crude product was purified by column chromatography using hexanes/ $\mathrm{CH}_{2} \mathrm{Cl}_{2} / \mathrm{EtOH}(3: 6: 0.5, \mathrm{v} / \mathrm{v})$ to yield BODIPY dye 3-I as purple oil (168 mg, 58\%). ${ }^{1} \mathrm{H}$ NMR (400 MHz, $\left.\mathrm{CDCl}_{3}\right): \delta 6.05$ (s, 2H), 4.10 (s, 4H), 3.72-3.49 (m, 80H), 3.44 $(\mathrm{d}, J=6.0 \mathrm{~Hz}, 12 \mathrm{H}), 3.34\left(4 \mathrm{x} \mathrm{CH}_{3}, 12 \mathrm{H}\right), 2.64(\mathrm{~s}, 6 \mathrm{H}), 2.53(\mathrm{~s}, 3 \mathrm{H}), 2.38(\mathrm{~s}, 6 \mathrm{H}), 2.17-$ $2.11(\mathrm{~m}, 2 \mathrm{H}) .{ }^{13} \mathrm{C}$ NMR (100 MHz, $\left.\mathrm{CDCl}_{3}\right): \delta 153.5,141.4,139.2,130.5,121.8,90.7$, $72.1,70.8,70.8,70.7,69.8,68.8,68.1,59.7,59.2,40.3,25.8,17.8,16.7,16.1 . \mathrm{IR}\left(\mathrm{cm}^{-1}\right)$ : 2865, 1558, 1538, 1513, 1465, 1408, 1350, 1308, 1247, 1188, 1097, 1040, 983, 850, 715. ${ }^{11} \mathrm{~B}$ NMR $\left(128 \mathrm{~Hz}, \mathrm{CDCl}_{3}\right): \delta-9.41$ (s). HRMS (FAB) calcd for $\mathrm{C}_{72} \mathrm{H}_{127} \mathrm{~N}_{2} \mathrm{BO}_{26}[\mathrm{M}+\mathrm{H}]^{+}$, 1446.8770; found, 1446.8745. It shows an absorption maximum at $495 \mathrm{~nm}$ and $491 \mathrm{~nm}$ in $\mathrm{CH}_{2} \mathrm{Cl}_{2}$ and PBS solution, respectively. It also shows a weak absorption peak at $355 \mathrm{~nm}$ and $353 \mathrm{~nm}$ in $\mathrm{CH}_{2} \mathrm{Cl}_{2}$ and $\mathrm{PBS}$ solution, respectively. It shows an emission maximum at $507 \mathrm{~nm}$ and $501 \mathrm{~nm}$ in $\mathrm{CH}_{2} \mathrm{Cl}_{2}$ and PBS solution, respectively.

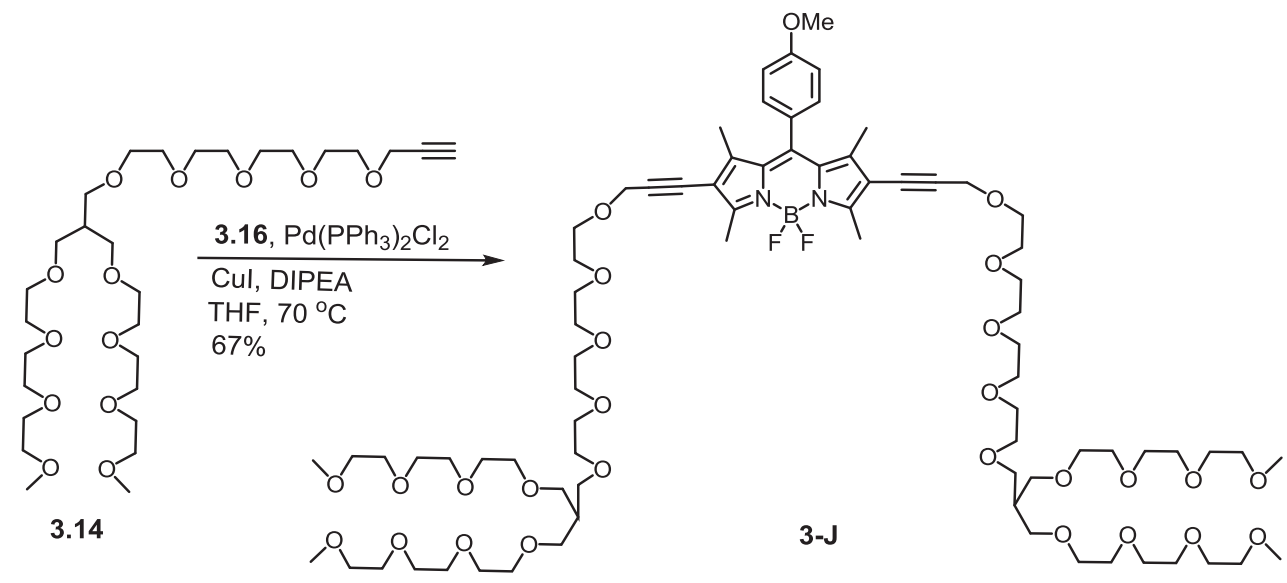

Scheme 3.15. Synthetic route to compound 3-J.

Compound 3-J: Compound 3.14 (171 mg, $0.28 \mathrm{mmol}$ ), compound 3.16 (68 mg, $0.112 \mathrm{mmol})$, CuI $(2.0 \mathrm{mg})$ and $\mathrm{Pd}\left(\mathrm{PPh}_{3}\right)_{2} \mathrm{Cl}_{2}(3.0 \mathrm{mg})$ were added to a $50-\mathrm{mL}$ roundbottom flask under a nitrogen atmosphere. When degassed anhydrous THF (5 mL) and 
diisopropylamine $(3 \mathrm{~mL})$ were added to the flask, the mixture was stirred at $70{ }^{\circ} \mathrm{C}$ overnight. After the completion of the reaction (which was monitored by TLC), the reaction mixture was concentrated under reduced pressure and diluted with $\mathrm{CH}_{2} \mathrm{Cl}_{2}$. The organic phase was washed with aqueous $1 \mathrm{~N} \mathrm{HCl}$, saturated aqueous $\mathrm{NaHCO}_{3}$, and brine respectively. The organic layer was collected, dried over $\mathrm{Na}_{2} \mathrm{SO}_{4}$ and concentrated in vacuo. The crude product was purified by silica gel column chromatography (hexane/ $\left.\mathrm{CH}_{2} \mathrm{Cl}_{2} / \mathrm{EtOH}, 3 / 6 / 0.5\right)$ to obtain BODIPY dye 3-J as red oil (118 mg, 67\%). ${ }^{1} \mathrm{H}$ NMR (400 MHz, $\left.\mathrm{CDCl}_{3}\right): \delta 7.10(\mathrm{~d}, J=8.8 \mathrm{~Hz}, 2 \mathrm{H}), 7.00(\mathrm{~d}, J=8.8 \mathrm{~Hz}, 2 \mathrm{H}), 4.39(\mathrm{~s}, 4 \mathrm{H})$, $3.86(\mathrm{~s}, 3 \mathrm{H}), 3.73-3.50(\mathrm{~m}, 80 \mathrm{H}), 3.45$ (d, $J=6.0 \mathrm{~Hz}, 12 \mathrm{H}), 3.34\left(4 \mathrm{x} \mathrm{CH}_{3}, 12 \mathrm{H}\right), 2.59$ (s, $6 \mathrm{H}), 2.17-2.12(\mathrm{~m}, 2 \mathrm{H}), 1.46(\mathrm{~s}, 6 \mathrm{H}) .{ }^{13} \mathrm{C} \mathrm{NMR}\left(100 \mathrm{MHz}, \mathrm{CDCl}_{3}\right): \delta 160.7,158.5$, $145.0,143.2,131.6,129.2,126.5,115.6,115.0,92.2,78.7,72.1,70.8,70.8,70.7,70.6$, 69.8, 69.2, 68.1, 59.5, 59.2, 55.6, 40.3, 25.8, 13.7. IR $\left(\mathrm{cm}^{-1}\right): 2958,2921,2854,1724$, 1533, 1462, 1377, 1317, 1248, 1217, 1103, 1029, 887, 721. HRMS (MAIDL) calcd for $\mathrm{C}_{78} \mathrm{H}_{129} \mathrm{BF}_{2} \mathrm{~N}_{2} \mathrm{O}_{27} \mathrm{Na}[\mathrm{M}+\mathrm{Na}]^{+}, 1597.8740$; found, 1597.8788. It shows an absorption maximum at $547 \mathrm{~nm}$ and $543 \mathrm{~nm}$ in $\mathrm{CH}_{2} \mathrm{Cl}_{2}$ and $\mathrm{PBS}$ solution, respectively. It also shows a weak absorption peak at $396 \mathrm{~nm}$ and $393 \mathrm{~nm}$ in $\mathrm{CH}_{2} \mathrm{Cl}_{2}$ and $\mathrm{PBS}$ solution, respectively.

It displays an emission maximum at $563 \mathrm{~nm}$ and $563 \mathrm{~nm}$ in $\mathrm{CH}_{2} \mathrm{Cl}_{2}$ and $\mathrm{PBS}$ solution, respectively.

\subsubsection{Optical Measurements}

The absorption and emission spectra of all compounds were scanned at room temperature with standard $1 \mathrm{~cm}$ path length quartz cuvette. The excitation and emission slit width was set to $1 \mathrm{~nm}$ and all the samples were scanned with an increment of $1 \mathrm{~nm}$. Fluorescence quantum yields of BODIPY dyes were measured in dichloromethane and $0.5 \mathrm{M}$ phosphate buffer solution (PBS) $(\mathrm{pH}=7.4)$, which were calculated by using fluorescein excited at $490 \mathrm{~nm}$ in $0.1 \mathrm{~N} \mathrm{NaOH}$ as the reference quantum efficiency $\left(\phi_{\mathrm{n}}=\right.$ $85 \%) .{ }^{17-20}$ Concentrations of BODIPY dyes ranging from $1.0 \times 10^{-7} \mathrm{~mol} / \mathrm{L}$ to $5.0 \times 10^{-8}$ $\mathrm{mol} / \mathrm{L}$ (in which the quantum yields do not change with varying concentration) were used to measure fluorescence quantum yields, absorption and fluorescent spectra. Excitation wavelength at $470 \mathrm{~nm}$ was used for measurement of fluorescence spectra of BODIPY dyes 3-A, 3-B, 3-F and 3-I. Excitation wavelength at $510 \mathrm{~nm}$ was used for measurement 
of fluorescence spectra of BODIPY dyes 3-C, 3-D and 3-E. Excitation wavelength at 540 $\mathrm{nm}$ was used for measurement of fluorescence spectrum of BODIPY dye 3-G. Excitation wavelengths at $610 \mathrm{~nm}$ and $620 \mathrm{~nm}$ were used for measurement of fluorescence spectra of BODIPY dye 3-H in dichloromethane solution and 0.5 M PBS solution, respectively. Excitation wavelengths at $500 \mathrm{~nm}$ and $520 \mathrm{~nm}$ were used for measurement of fluorescence spectra of BODIPY dye 3-J in dichloromethane solution and 0.5 M PBS solution, respectively.

\subsection{Results and discussions}

In order to demonstrate the feasibility of using branched oligo(ethylene glycol)methyl ether to enhance the water solubility of BODIPY dyes, we first introduced branched oligo(ethylene glycol)methyl ether to BODIPY dyes at the meso position (Scheme 3.16). Compound 3.4 was prepared according to a reported procedure ${ }^{21}$ and further brominated with $\mathrm{PBr}_{3}$ in methylene chloride at $40{ }^{\circ} \mathrm{C}$, affording brominated branched oligo(ethylene glycol)methyl ether (3.5). The benzaldehyde deriviative bearing branched oligo(ethylene glycol)methyl ether residues (3.7) was prepared by reacting 3,4dihydroxybenzaldehyde with compound $\mathbf{3 . 5}$ in a basic condition. BODIPY dye substituted with branched oligo(ethylene glycol)methyl ether at meso-position (3-A) was prepared through the condensation of the benzaldehyde derivative (3.7) with 2,4dimethylpyrrole in the presence of a catalytic amount of trifluoroacetic acid (TFA), and followed by oxidation with 2,3-dichloro-5,6-dicyano-1,4-benzoquinone (DDQ) and chelation with $\mathrm{BF}_{3}$-etherate in the presence of $N, N$-diisopropylethylamine (DIEA). ${ }^{22}$ BODIPY dye (3-A) is highly water-soluble. Highly fluorescent E-BODIPY dye (3-B) in aqueous solution was also prepared by replacing the usual fluorine atoms of BODIPY dye (3-A) with ethynyl tri(ethylene glycol)methyl ether (3.8) in Grignard reaction. In order to evaluate the effect of functionalization of BODIPY dye (3-A) on water solubility, we iodized BODIPY dye 3-A at 2- and 6-positions, affording 2,6-diiodo BODIPY dye (3-C), palladium-catalyzed Sonogashira coupled 2,6-diiodo BODIPY dye (3-C) with trimethylsilylacetylene and followed by deprotection of trimethylsilyl groups

in the presence of NaF, affording 2,6-diethynyl BODIPY dye (3-D). ${ }^{23-24}$ Replacement of the usual fluorine atoms of BODIPY dye 3-A with methyloxy subunits introduced some 
steric hindrance to the BODIPY core and resulted in BODIPY dye with methyloxy subunits at 4-position (3-E). BODIPY dyes (3-B, 3-C, 3-D and 3-E) are highly watersoluble and further functionalization of BODIPY dye (3-A) does not affect water solubility of new BODIPY dyes, indicating that strong hydrophilic feature of branched oligo(ethylene glycol)methyl ether residues considerably enhance interactions of BODIPY dyes with water and significantly increase water solubility of BODIPY dyes.

BODIPY dye 3-A displays weak fluorescence in $0.5 \mathrm{M}$ phosphate buffer solution (PBS) (pH 7.40) with fluorescence quantum yield of $4.2 \%$ while it becomes highly fluorescent with fluorescence quantum yield of $68 \%$ and $61 \%$ in dichlormethane and ethanol, respectively. The low fluorescence quantum yield of BODIPY dye 3-A may be attributed to self-quenching because of likely aggregation of the dye in aqueous solution through $\pi-\pi$ stacking or hydrophobic interactions between BODIPY cores. In order to prove this hypothesis, we replaced the usual fluorine atoms of BODIPY dye 3-A with ethynyl subunits to introduce steric hindrance to the BODIPY core, resulting in BODIPY dye (3-B) with significantly enhanced fluorescence quantum yield of $35.7 \%$ in PBS solution. The side chains of ethynyl tri(ethylene glycol)methyl ether in BODIPY dye (3B) provide steric hindrance and reduce aggregation of BODIPY dye in aqueous solution. Iodization of BODIPY dye (3-A) at positions 2 and 6 results in 2,6-diiodo BODIPY dye (3-C), which has an extremely low fluorescence quantum yield of $1.2 \%$ in PBS solution because of efficient intersystem crossing induced by the heavy atom effect of iodine. ${ }^{24}$ Replacement of iodo groups of BODIPY dye (3-C) at positions 2 and 6 with ethynyl groups yielded 2,6-diethynyl BODIPY dye (3-D), which shows low fluorescence quantum yield of $1.9 \%$, attributed to potential aggregation of BODIPY dye (3-D) with enhanced $\pi$-conjugation in aqueous solution. Replacement of the fluorine atoms of BODIPY dye (3-D) with methyloxy subunits gave BODIPY dye (3-E), which displays enhanced fluorescence quantum yield of $8.9 \%$ due to the slightly enhanced steric hindrance of the methyloxy subunits on the BODIPY core. 
(ii)

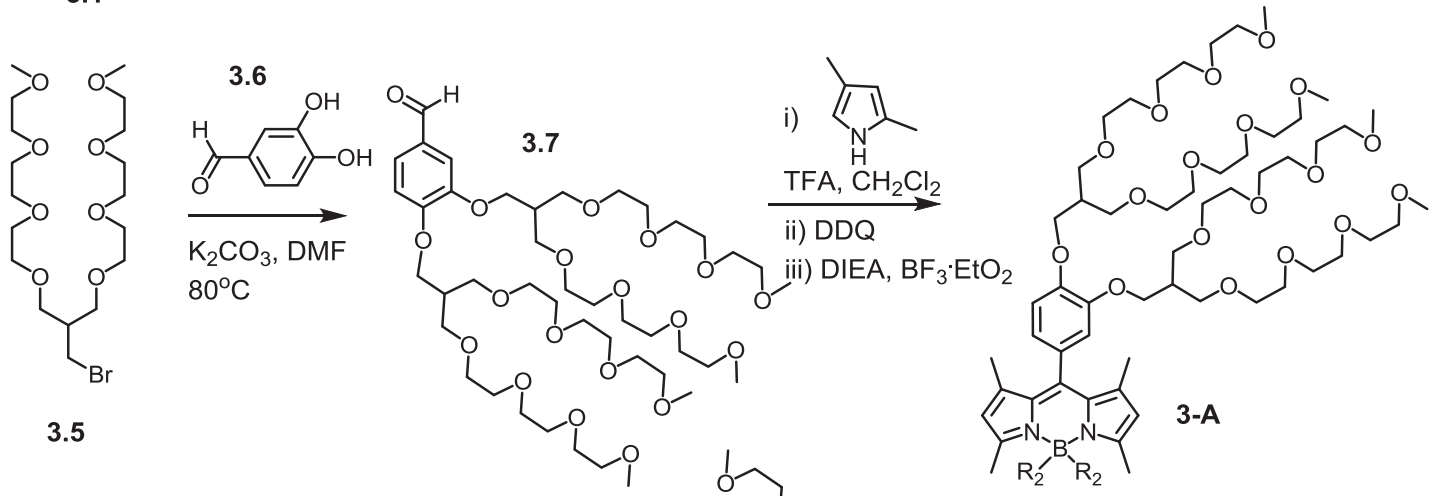

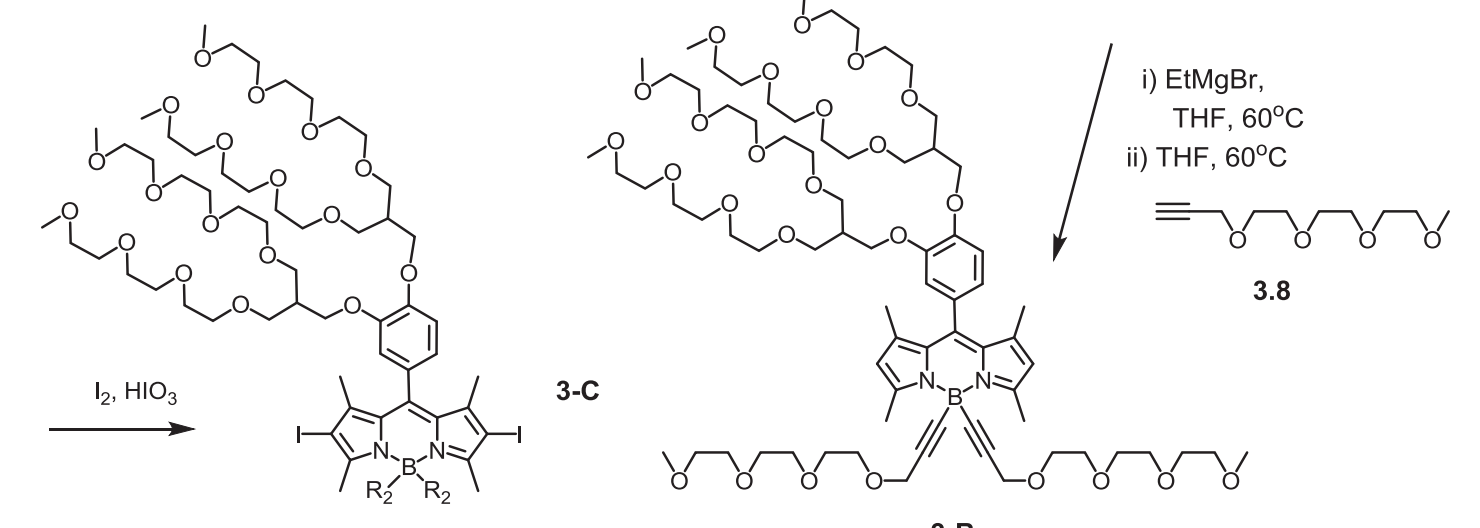

i) $\mathrm{H}=\mathrm{Si}\left(\mathrm{CH}_{3}\right)_{3}$
$\mathrm{Pd}\left(\mathrm{PPh}_{3}\right)_{2} \mathrm{Cl}_{2}, \mathrm{Cul}$
$\mathrm{DIPEA}, \mathrm{THF}, 50^{\circ} \mathrm{C}$
ii) $\mathrm{NaF}$<smiles>CCOCCOCCCOCCOC</smiles>

$\sum_{R_{2}^{B} B_{R_{2}}}{ }^{-N}$

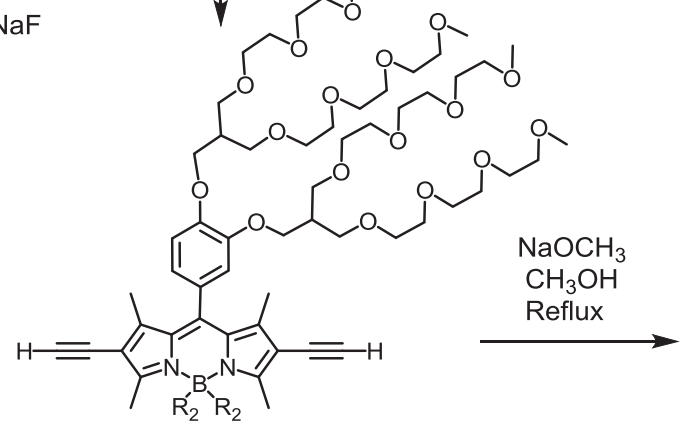

3-D

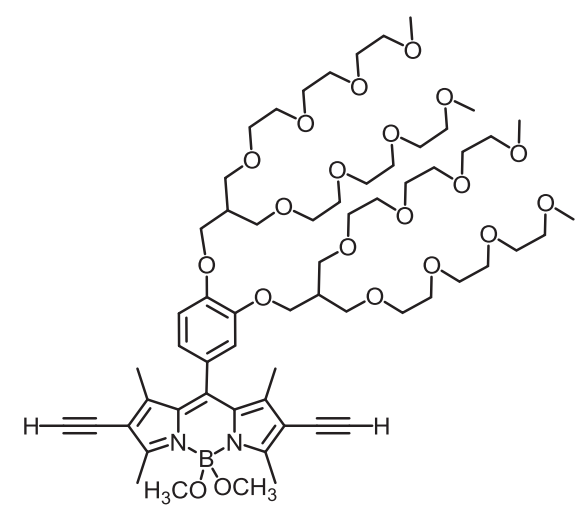

3-E

Scheme 3.16. Synthetic routes to highly water-soluble BODIPY dyes (3-A, 3-B, 3-C, 3D and 3-E) with branched oligo(ethylene glycol)methyl ether residues at meso-position. 

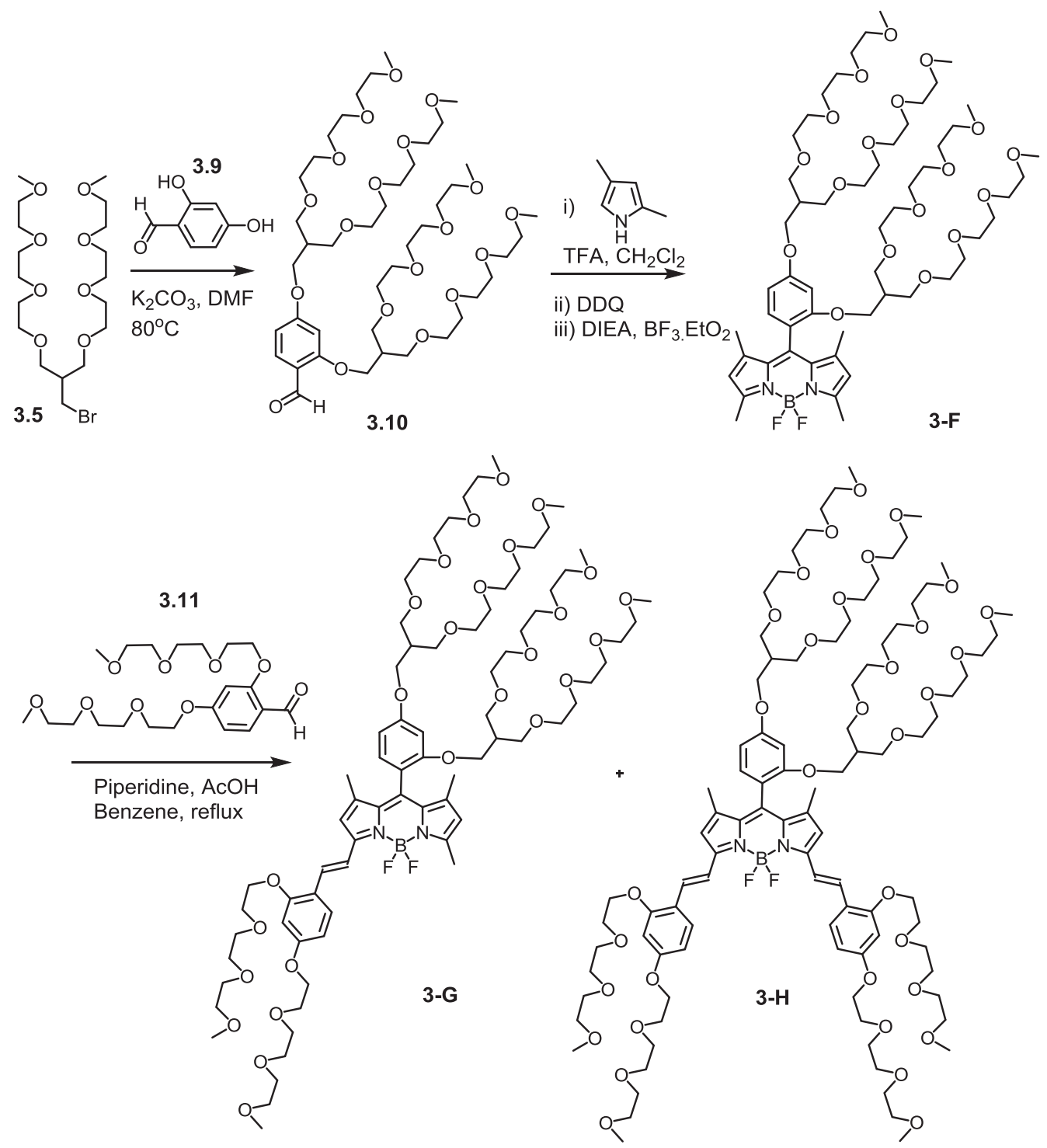

Scheme 3.17. Synthetic routes to water-soluble BODIPY dyes (3-F, 3-G and 3-H) bearing monostryl and distyryl groups at 3- and 5-positions.

We hypothesized that introduction of oligo(ethylene glycol)methyl ether group to an ortho-position on the meso-phenyl ring of BODIPY dyes would additionally enhance fluorescence quantum yields of BODIPY dyes through significantly enhanced steric hindrance to prevent potential $\pi-\pi$ stacking interactions between the BODIPY cores. In order to prove our hypothesis, we prepared BODIPY dye (3-F) with one ortho-substituent group of branched oligo(ethylene glycol)methyl ether from the aldehyde (3.10) via a similar approach to that used to prepare BODIPY dye (3-A) (Scheme 3.17). As we 
expected, BODIPY dye (3-F) is highly fluorescent with fluorescence quantum yield of $31.2 \%$ in PBS solution which we attribute its enhanced steric hindrance of the bulky ortho-substituent group on the meso-phenyl ring of BODIPY dye (3-F). BODIPY dyes with extended $\pi$-conjugation systems have strong hydrophobic features and are typically insoluble in water. In order to further demonstrate feasibility of using branched oligo(ethylene glycol)methyl ether to promote water-solubility of BODIPY dyes with extended $\pi$-conjugation system, we prepared BODIPY dyes bearing monostyryl and distyryl groups at 3-, 5-positions (3-G and 3-H) by condensation of methyl substituents of BODIPY dye (3-F) at 3- and 5-positions with aldehyde derivative (3.11) (Scheme 3.17). ${ }^{25}$ Both BODIPY dyes $\mathbf{3 - G}$ and $\mathbf{3}-\mathbf{H}$ are readily soluble in aqueous solution and have fluorescence quantum yields of $21.2 \%$ and $1.3 \%$, respectively.

The unexpectedly low fluorescence quantum yield of BODIPY dye 3-H may arise from rotation of vinyl bonds caused by interactions between flexible oligo(ethylene glycol)-methyl ether at the meso-position with those at 3-and 5-positions, suggested by a strong absorption peak at $383 \mathrm{~nm}$, attributed to phenyl units at 3,5-positions, the relatively weak absorption of the BODIPY core at $507 \mathrm{~nm}$, and a shoulder peak at 605 nm.

In order to further show the versatility of these approaches, we introduced branched oligo(ethylene glycol)methy ether to BODIPY dyes at 4,4'-, 2- and 6-positions. We introduced a tethered spacer of tetra(ethylene glycol) to the branched oligo(ethylene glycol)methyl ether in order to reduce space hindrance around the BODIPY dye by reacting compound 3.4 with ethynyl-functionalized tetra(ethylene glycol) tosylate (3.13) in dry THF in the presence of sodium hydride. BODIPY dye (3-I) bearing branched oligo(ethylene glycol)methyl ether residues at 4-, 4'-positions was prepared by replacing the usual fluorine atoms of F-BODIPY dye (3.15) with ethynyl-functionalized oligo(ethylene glycol)methyl ether (3.14) via a Grignard reaction (Scheme 3.18). BODIPY dye (3-J) bearing branched oligo(ethylene glycol)methyl ether residues at 2and 6-positions was prepared by palladium-catalyzed Sonogashira coupling of 2,6-diiodo BODIPY dye (3.16) with ethynyl-functionalized branched oligo(ethylene glycol)methyl ether (3.14) (Scheme 3.18). Both BODIPY dyes (3-I and 3-J) are extremely soluble in water and both are highly fluorescent in aqueous solution. Thus, introduction of side 
chain serves the dual purposes of enhancing aqueous solubility and enhancing fluorescence quantum yields by reducing aggregation. BODIPY dye (3-I) with the size chains at the 4,4'-position is considerably more fluorescent in PBS solution (fluorescence quantum yield 68\%), than BODIPY dye (3-J) substituted at the 2,6-positions (fluorescence quantum yield 34\%). So, substitution at the 4,4'-positions is more effective at preventing aggregation than 2,6-subsitution (Table 3.1).

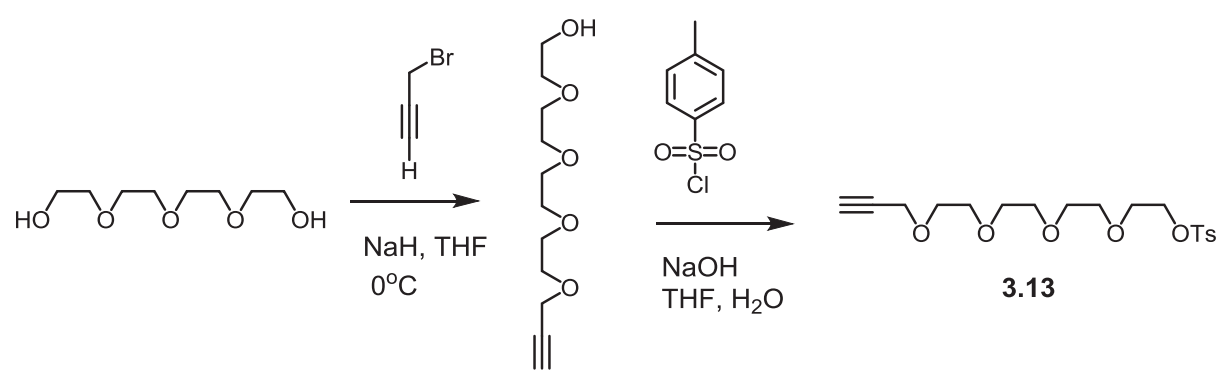

3.12
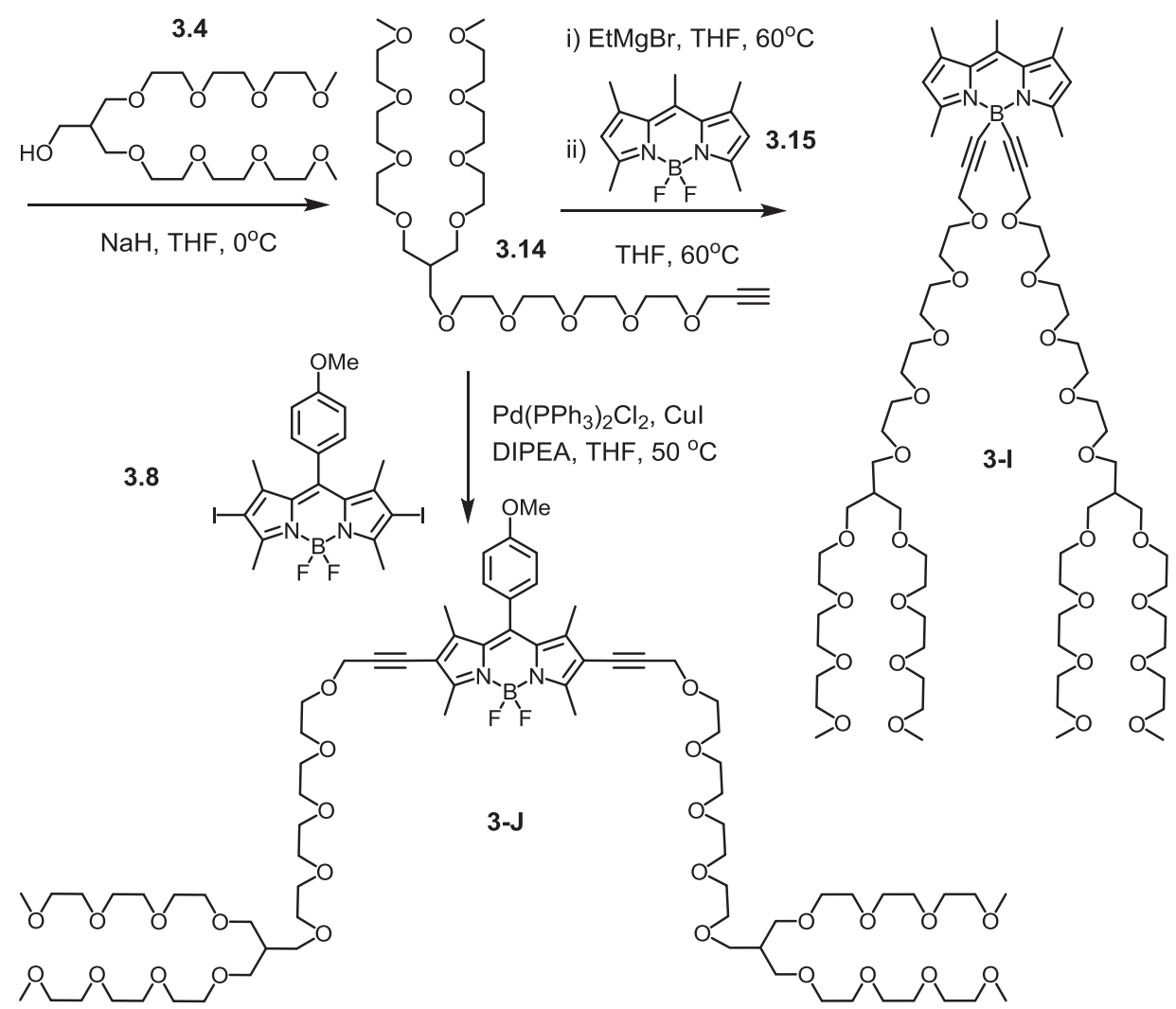

Scheme 3.18. Synthetic route to BODIPY dyes (3-I and 3-J) bearing branched oligo(ethylene glycol)ether at 4-, 2- and 6-positions. 
Table 3.1. Absorption and emission maxima, and fluorescence quantum yields of BODIPY dyes in organic solvents and 0.5 M phosphate buffer solution (PBS) (pH 7.4). Quantum yields of BODIPY dyes were determined by use of fluorescein (quantum yield of 0.85 in $0.1 \mathrm{~N} \mathrm{NaOH}$ ) as a standard. ${ }^{20}$

\begin{tabular}{|c|c|c|c|c|}
\hline $\begin{array}{c}\text { BODIPY } \\
\text { dye }\end{array}$ & Solvent & $\begin{array}{l}\text { Absorption } \\
\text { maximum } \\
(\mathrm{nm})\end{array}$ & $\begin{array}{l}\text { Emission } \\
\text { maximum } \\
(\mathrm{nm})\end{array}$ & $\begin{array}{c}\text { Fluorescence } \\
\text { quantum yield } \\
(\%)\end{array}$ \\
\hline \multirow{4}{*}{ 3-A } & Dichloromethane & 501 & 511 & 68.0 \\
\hline & Ethanol & 500 & 509 & 61.0 \\
\hline & $\mathrm{H}_{2} \mathrm{O}+10 \%$ ethanol & 500 & 509 & 8.2 \\
\hline & PBS & 499 & 510 & 4.2 \\
\hline \multirow{2}{*}{ 3-B } & Dichloromethane & 499 & 508 & 81.0 \\
\hline & PBS & 496 & 507 & 35.7 \\
\hline \multirow{2}{*}{$3-\mathrm{C}$} & Dichloromethane & 534 & 548 & 1.9 \\
\hline & PBS & 533 & 550 & 1.2 \\
\hline \multirow{4}{*}{ 3-D } & Dichloromethane & 539 & 552 & 47.9 \\
\hline & Ethanol & 539 & 554 & 43.0 \\
\hline & $\mathrm{H}_{2} \mathrm{O}+10 \%$ ethanol & 538 & 556 & 4.2 \\
\hline & PBS & 536 & 555 & 1.9 \\
\hline \multirow{2}{*}{$3-\mathrm{E}$} & Dichloromethane & 538 & 552 & 51.4 \\
\hline & PBS & 534 & 550 & 8.9 \\
\hline \multirow{2}{*}{$3-F$} & Dichloromethane & 504 & 514 & 91.5 \\
\hline & PBS & 501 & 511 & 31.2 \\
\hline \multirow{2}{*}{ 3-G } & Dichloromethane & 580 & 596 & 30.7 \\
\hline & PBS & 577 & 598 & 21.2 \\
\hline \multirow{2}{*}{ 3-H } & Dichloromethane & 659 & 676 & 6.1 \\
\hline & PBS & 660 & 676 & 1.3 \\
\hline \multirow{2}{*}{ 3-I } & Dichloromethane & 495 & 507 & 79.0 \\
\hline & PBS & 491 & 501 & 68.0 \\
\hline \multirow{2}{*}{ 3-J } & Dichloromethane & 547 & 563 & 39 \\
\hline & PBS & 543 & 563 & 33.6 \\
\hline
\end{tabular}

All BODIPY dyes (3-A-3-J) are easily soluble not only in water but also in common solvents such as acetone, ethanol, methanol, dimethylformamide, dimethyl sulfoxide, ethyl acetate, tetrahydrofuran, methylene chloride and chloroform. The absorption 
properties of BODIPY dye (3-A) in PBS solution are characterized by a strong $\mathrm{S}_{0} \rightarrow \mathrm{S}_{1}(\pi$ $\pi^{*}$ ) transition at $499 \mathrm{~nm}$ and a weaker broad band around $350 \mathrm{~nm}$ attributed to the $\mathrm{S}_{0} \rightarrow \mathrm{S}_{2}$ $\left(\pi-\pi^{*}\right)$ transition (Table 3.1). ${ }^{1}$ Replacement of the usual fluorine atoms of BODIPY dye (3-A) with ethynyl subunits at 4,4'-positions cause slight blue shifts of BODIPY dye (3B) in both absorption and emission compared with its precursor BODIPY dye 3-A. These shifts may arise from less aggregation of BODIPY dye (3-B) in PBS solution due to bulking ethynyl substituents, resulting in significantly enhanced fluorescence quantum yields of BODIPY dye (3-B). Introduction of 2,6-diiodo substituents to the BODIPY dye (3-A) leads to a large red shifts (33 nm and $38 \mathrm{~nm}$ ) of BODIPY dye (3-C) in both the UV-absorption and fluorescence maxima, respectively, as 2,6-diiodo substituents function as auxochromes (Table 3.1). 2,6-Diethynylation of 2,6-diiodo-tetramethyl BODIPY dye (3-C) results in a little red shift for BODIPY dye (3-D) due to the enhanced conjugation compared with BODIPY dye (3-A). Replacement of the usual fluorine atoms of BODIPY dye (3-D) with methyloxy subunits at positions 4,4' causes a little blue shifts of BODIPY dye (3-E) in both absorption and emission of compared with its precursor BODIPY dye 3-D (Table 3.1), which may arise from less aggregation of BODIPY dye (3-E) due to slightly enhanced steric hindrance from the methyloxy subunits.

BODIPY dye (3-F) in PBS solution displays absorption and emission peak at $501 \mathrm{~nm}$ and $511 \mathrm{~nm}$, respectively. BODIPY dyes with monostyryl and distyryl substituents at 3, 3 and 5 positions (3-G and 3-H) in PBS solution show significant red shifts in both absorption and emission compared with their precursor BODIPY dye (3-F). Monostyryland distyryl-substituted BODIPY dyes ( $\mathrm{G}$ and $\mathrm{H}$ ) in aqueous solution show absorption at $577 \mathrm{~nm}$ and $660 \mathrm{~nm}$, and fluorescence maxima at $598 \mathrm{~nm}$ and $676 \mathrm{~nm}$, respectively (Table 3.1 and Appendix A). BODIPY dye (3-I) in aqueous solution shows absorption and fluorescence maxima at $491 \mathrm{~nm}$ and $501 \mathrm{~nm}$, respectively. Absorption and fluorescence spectra of BODIPY dye (3-J) in PBS solution are red-shifted with absorption and fluorescence maxima at $543 \mathrm{~nm}$ and $563 \mathrm{~nm}$, respectively, compared with those of its precursor BODIPY (3.15) in methylene chloride because of its extended $\pi$ conjugation. 


\subsection{Conclusion}

In conclusion, we have developed effective ways to prepare highly water-soluble BODIPY dyes with emissions from green to deep red regions through functionalization of BODIPY dyes at positions 8,2 and 6 , or 4 and 4' with branched oligo(ethylene glycol)methyl ether residues. Fluorescence quantum yields of BODIPY dyes can be manipulated by introducing branched oligo(ethylene glycol)methyl ether residues to an ortho position on the meso-phenyl ring of BODIPY dyes, replacing the fluorine atoms of BODIPY dyes at positions 4 and 4' with methyloxy or ethynyl subunits or attaching branched oligo(ethylene glycol)methyl ether residues to positions 2 and 6 of BODIPY dyes.

\section{References:}

1. Loudet, A., Burgess, K., BODIPY Dyes and Their Derivatives: Syntheses and Spectroscopic Properties. Chemical Reviews 2007, 107, 4891-4932.

2. Ulrich, G., Ziessel, R., Harriman, A., The Chemistry of Fluorescent Bodipy Dyes: Versatility Unsurpassed. Angewandte Chemie-International Edition 2008, 47, 1184-1201.

3. Atilgan, S., Ozdemir, T., Akkaya, E. U., A sensitive and selective ratiometric near IR fluorescent probe for zinc ions based on the distyryl-bodipy fluorophore. Org. Lett. 2008, 10 (18), 4065-4067.

4. Jiao, L. J., Li, J. L., Zhang, S. Z., Wei, C., Hao, E. H., Vicente, M. G. H., A Selective Fluorescent Sensor for Imaging Cu2+ in Living Cells. New Journal of Chemistry 2009, 33 (9), 1888-1893.

5. Yen, V. H., Micouin, L., Ronet, C., Gachelin, G., Bonin, M., Total Enantioselective Synthesis and In Vivo Biological Evaluation of A Novel Fluorescent BODIPY Alpha-Galactosylceramide. Chembiochem 2003, 4 (1), 27 33.

6. Giessler, K., Griesser, H., Gohringer, D., Sabirov, T., Richert, C., Synthesis of 3 'BODIPY-Labeled Active Esters of Nucleotides and A Chemical Primer Extension Assay on Beads. European Journal of Organic Chemistry 2010, (19), 3611-3620. 
7. Dilek, O., Bane, S. L., Synthesis, Spectroscopic Properties and Protein Labeling of Water Soluble 3,5-Disubstituted Boron Dipyrromethenes. Bioorganic and Medicinal Chemistry Letters 2009, 19 (24).

8. Dodani, S. C., He, Q., Chang, C. J., A Turn-On Fluorescent Sensor for Detecting Nickel in Living Cells. Journal of the American Chemical Society 2009, 131 (50), 18020-18021.

9. Niu, S. L., Ulrich, G., Retailleau, P., Harrowfield, J., Ziessel, R., New Insights into The Solubilization of Bodipy Dyes. Tetrahedron Letters 2009, 50 (27), 38403844.

10. Niu, S. L., Ulrich, G., Ziessel, R., Kiss, A., Renard, P. Y., Romieu, A., WaterSoluble BODIPY Derivatives. Organic Letters 2009, 11 (10), 2049-2052.

11. Li, L. L., Han, J. Y., Nguyen, B., Burgess, K., Syntheses and Spectral Properties of Functionalized, Water-soluble BODIPY Derivatives. Journal of Organic Chemistry 2008, 73 (5), 1963-1970.

12. Jahnke, E., Weiss, J., Neuhaus, S., Hoheisel, T. N., Frauenrath, H., Synthesis of Diacetylene-Containing Peptide Building Blocks and Amphiphiles, Their SelfAssembly and Topochemical Polymerization in Organic Solvents. Chemistry- $a$ European Journal 2009, 15 (2), 388-404.

13. Scates, B. A., Lashbrook, B. L., Chastain, B. C., Tominaga, K., Elliott, B. T., Theising, N. J., Baker, T. A., Fitch, R. W., Polyethylene Glycol-based Homologated Ligands for Nicotinic Acetylcholine Receptors. Bioorganic \& Medicinal Chemistry 2008, 16 (24), 10295-10300.

14. Nielsen, C. B., Johnsen, M., Arnbjerg, J., Pittelkow, M., McIlroy, S. P., Ogilby, P. R., Jorgensen, M., Synthesis and Characterization of Water-soluble Phenylenevinylene-based Singlet Oxygen Sensitizers For Two-photon Excitation. Journal of Organic Chemistry 2005, 70 (18), 7065-7079.

15. Shah, M., Thangaraj, K., Soong, M.-L., Wolford, L. T., Boyer, J. H., Politzer, I. R., Pavlopoulos, T. G., Pyrromethene-BF2 Complexes as Laser Dyes:1. Heteroatom Chemistry 1990, 1 (5), 389-399. 
16. Meng, G., Velayudham, S., Smith, A., Luck, R., Liu, H., Color Tuning of Polyfluorene Emission with BODIPY Monomers. Macromolecules 2009, 42 (6), 1995-2001.

17. Parker, C. A., Rees, W. T., Correction of Fluorescence Spectra and Measurement of Fluorescence Quantum Efficiency. Analyst 1960, 85, 587 - 600.

18. Yogo, T., Urano, Y., Ishitsuka, Y., Maniwa, F., Nagano, T., Highly Efficient and Photostable Photosensitizer Based on BODIPY Chromophore. Journal of the American Chemical Society 2005, 127 (35), 12162-12163.

19. Maruyama, S., Kikuchi, K., Hirano, T., Urano, Y., Nagano, T., A Novel, Cellpermeable, Fluorescent Probe for Ratiometric Imaging of Zinc Ion. Journal of the American Chemical Society 2002, 124 (36), 10650-10651.

20. Gabe, Y., Urano, Y., Kikuchi, K., Kojima, H., Nagano, T., Highly Sensitive Fluorescence Probes for Nitric Oxide Based on Boron Dipyrromethene Chromophore-rational Design of Potentially Useful Bioimaging Fluorescence Probe. Journal of the American Chemical Society 2004, 126 (10), 3357-3367.

21. Lee, M., Jeong, Y. S., Cho, B. K., Oh, N. K., Zin, W. C., Self-assembly of Molecular Dumbbells into Organized Bundles with Tunable Size. Chemistry-a European Journal 2002, 8 (4), 876-883.

22. Meng, G., Velayudham, S., Smith, A., Luck, R., Liu, H. Y., Color Tuning of Polyfluorene Emission with BODIPY Monomers. Macromolecules 2009, 42 (6), 1995-2001.

23. Donuru, V. R., Vegesna, G. K., Velayudham, S., Meng, G., Liu, H. Y., Deep-Red Emissive Conjugated Poly(2,6-BODIPY-ethynylene)s Bearing Alkyl Side Chains. J. Polym. Sci., Part A: Polym. Chem. 2009, 47 (20), 5354-5366.

24. Donuru, V. R., Vegesna, G. K., Velayudham, S., Green, S., Liu, H. Y., Synthesis and Optical Properties of Red and Deep-Red Emissive Polymeric and Copolymeric BODIPY Dyes. Chemistry of Materials 2009, 21 (10), 2130-2138.

25. Donuru, V. R., Zhu, S., Green, S., Liu, H., Near-Infrared Emissive BODIPY Polymeric and Co-polymeric Dyes. Polymer 2010, 51 (23), 5359-5368. 


\section{Chapter 4. Controlled Knoevenagel Reactions of Methyl Groups of 1,3,5,7-Tetramethyl BODIPY Dyes for Unique BODIPY Dyes ${ }^{1}$}

Shilei Zhu, ${ }^{a}$ Jingtuo Zhang, ${ }^{a}$ Giri Vegesna, ${ }^{a}$ Ashutosh Tiwari, ${ }^{a}$ Fen-Tair Luo, ${ }^{\mathrm{b}}$ Matthias Zeller, ${ }^{\mathrm{c}}$ Rudy Luck, ${ }^{\mathrm{a}}$ Haihua Li, ${ }^{\mathrm{d}}$ Sarah Green ${ }^{\mathrm{a}}$ and Haiying Liu*a $^{* a}$

${ }^{a}$ Department of Chemistry, Michigan Technological University, 1400 Townsend Drive, Houghton, MI 49931, USA

${ }^{\mathrm{b}}$ Institute of Chemistry, Academia Sinica, Taipei, Taiwan 11529, Republic of China

${ }^{\mathrm{c}}$ Department of Chemistry, Youngstown State University, Youngstown, OH 44555, USA

${ }^{\mathrm{d}}$ University Libraries, University of Alabama, Tuscaloosa, AL, 35487, USA

1 The material included in this chapter was previously published in [RSC Advances 2012, 2 (2), 404-407. DOI: 10.1039/C1RA00678A Publication Date (Web): Nov 04, 2011]Reproduced by permission of The Royal Society of Chemistry http://pubs.rsc.org/en/Content/ArticleLanding/2012/RA/C1RA00678A\#!divAbstract 


\subsection{Introduction}

BODIPY (4,4-difluoro-4-bora-3a,4a-diaza-s-indacene) dyes have received considerable attention recently because they possess many distinctive and desirable properties such as high extinction coefficients, narrow absorption and emission bands, high quantum efficiencies of fluorescence, relative insensitivity to environmental perturbations, and high resistance to photobleaching. ${ }^{1}$ BODIPY dyes typically absorb light in the visible region and emit fluorescence between 470 and $550 \mathrm{~nm}$. Tuning of their optical properties for the absorption and emission maxima in the far-red and near-infrared region have been achieved by functionalizing BODIPY cores at the meso-, 2,6- and 3,5positions, fusing some aromatic rings to the BODIPY core, and replacing pyrrole by isoindole. ${ }^{1-3}$ Among these approaches, monostyryl- and distyryl modifications via the Knoevenagel reaction are facile and versatile routes to effectively tune BODIPY dyes for near-infrared emission. Methyl groups at the 3,5-positions of BODIPY dyes are active in the Knoevenagel reaction, and often results in 3-monostyryl- and 3,5-distyryl-BODIPY dyes. ${ }^{1-6}$ Recently, the Akkaya group has creatively prepared 3,5,7-tristyryl- and 1,3,5,7tetrastyryl-BODIPY dyes by activating methyl groups at the 1,7-positions via the introduction of phenylethynyl groups at the 2,6-positions. ${ }^{7}$ Ziessel's group successfully prepared 1,3,5,7-tetrastyryl-BODIPY dyes by using high temperature of $140{ }^{\circ} \mathrm{C} .{ }^{8}$ However, methyl groups at the 3,5-positions in these reported approaches are more reactive and undergo Knoevenagel reactions faster than those at 1,7-positions. ${ }^{7,8}$

\subsection{Methods}

In this chapter, we reported a simple and efficient approach to control the Knoevenagel reaction sequence of methyl groups at 1,3,5,7-positions of BODIPY dyes. The method is utilized to prepare novel building blocks of monostyryl, distyryl-, tristyryland tetrastyryl-BODIPY dyes with unique chemical structures (Scheme 4.1). To achieve this goal we employed electron withdrawing formyl groups at 6-, and 2,6-positions of BODIPY dyes to initiate Knoevenagel reaction of methyl groups at the 7-, and the 1,7positions with aromatic aldehyde derivatives. 

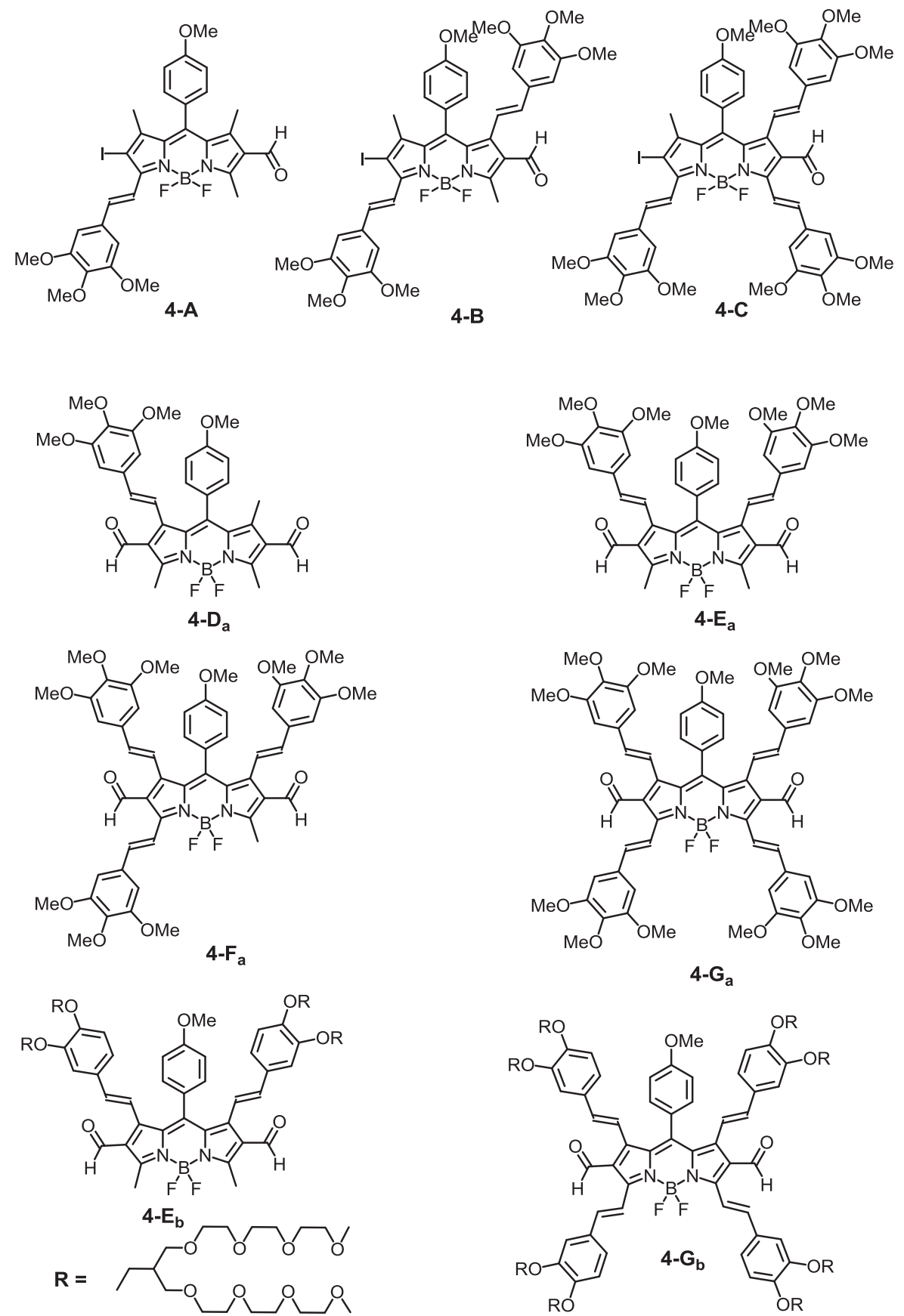

Scheme 4.1. Monostyryl, distyryl-, tristyryl- and tetrastyryl-BODIPY dyes with unique chemical structures.

These formyl groups may provide steric hindrance and prevent initial Knoevenagel reaction of methyl groups at the 5, and the 3,5-positions with aromatic aldehydes, resulting in 2-iodo-6-formyl-3,7-distyryl-BODIPY dye, 2,6-diformyl-1-monostyryl- 
BODIPY dye and, 2,6-diformyl-1,7-distyryl-BODIPY dye. Formation of vinyl bonds at 7-, and 1,7-positions facilitates further Knoevenagel reaction of methyl groups at the 3, and the 3,5-positions with the aromatic aldehydes, producing 2-iodo-6-formyl-3,5,7tristyryl-BODIPY dye, 2,6-diformyl-1,3,7-tristyryl-BODIPY dye, and 2,6-diformyl1,3,5,7-tetrastyryl-BODIPY dye. Branched oligo(ethylene glycol)methyl ether residues were used to facilitate water solubility of 1,7-distyryl and 1,3,5,7-tetrastyryl-BODIPY dyes. These BODIPY dyes can serve as novel potential building blocks for BODIPYbased conjugated oligomers, dendrimers, and highly water-soluble near-infrared emissive sensing materials.

\subsection{Experimental section}

\subsubsection{Intstrumentation}

${ }^{1} \mathrm{H}$ NMR and ${ }^{13} \mathrm{C}$ NMR spectra were taken on a $400 \mathrm{MHz}$ Varian Unity Inova spectrophotometer instrument. ${ }^{1} \mathrm{H}$ and ${ }^{13} \mathrm{C}$ NMR spectra were recorded in $\mathrm{CDCl}_{3}$, chemical shifts $(\delta)$ are given in ppm relative to solvent peaks $\left({ }^{1} \mathrm{H}: \delta 7.26 ;{ }^{13} \mathrm{C}: \delta 77.3\right)$ as internal standard. Absorption spectra were taken on a Perkin Elmer Lambda 35 UV/VIS spectrometer. Fluorescence spectra were recorded on a Jobin Yvon Fluoromax-4 spectrofluorometer. The excitation and emission slit width were set to $1 \mathrm{~nm}$ for quantum yield calculations and all samples were scanned with increments of $1 \mathrm{~nm}$. Fluorescence quantum yields of BODIPY dyes were measured in dichloromethane and aqueous solution by using standard $1 \mathrm{~cm}$ path-length quartz fluorescence cell. And they were calculated by using fluorescein excited at $490 \mathrm{~nm}$ in $0.1 \mathrm{~N} \mathrm{NaOH}$ aqueous solution as the reference quantum efficiency $\left(\phi_{\mathrm{n}}=85 \%\right) \cdot{ }^{9-11}$ Concentrations of BODIPY dyes ranging from $1.0 \times 10^{-6} \mathrm{~mol} / \mathrm{L}$ to $5.0 \times 10^{-6} \mathrm{~mol} / \mathrm{L}$ (in which the quantum yields do not change with varying concentration) were used to measure fluorescence quantum yields, absorption and fluorescent spectra.

\subsubsection{Materials and synthesis}

Unless otherwise indicated, all reagents and solvents were obtained from commercial suppliers (Aldrich, Sigma, Fluka, Acros Organics, Fisher Scientific, Lancaster) and used without further purification. Air- and moisture-sensitive reactions were conducted in 
oven-dried glassware using a standard Schlenk line or drybox techniques under an inert atmosphere of dry nitrogen. Compound 4.3 was prepared and characterized according to the literature. ${ }^{12}$

Compound 4.4: When iodic acid (597 mg, $3.39 \mathrm{mmol})$ in $5 \mathrm{~mL}$ of water was added dropwise to the ethanol $(40 \mathrm{~mL})$ and THF $(10 \mathrm{~mL})$ solution containing compound $\mathbf{4 . 3}$ (1.08 $\mathrm{g}, 2.83 \mathrm{mmol})$ and iodine $(1.08 \mathrm{~g}, 4.24 \mathrm{mmol})$ in 30 minutes, the mixture was stirred for $5 \mathrm{~h}$. The mixture was concentrated under reduced pressure, dissolved in $\mathrm{CH}_{2} \mathrm{Cl}_{2}$, and washed twice with water and saturated saline solution. The organic layer was collected, dried over anhydrous $\mathrm{Na}_{2} \mathrm{SO}_{4}$, and concentrated under reduced pressure. The crude product was purified by column chromatography to yield BODIPY dye 4.4 as red crystal solid (1.28 g, 89\%). ${ }^{1} \mathrm{H}$ NMR (400 MHz, $\left.\mathrm{CDCl}_{3}\right): \delta 10.01(\mathrm{~s}, 1 \mathrm{H}), 7.14(\mathrm{~d}, J=$ $6.4 \mathrm{~Hz}, 2 \mathrm{H}), 7.05$ (d, $J=6.8 \mathrm{~Hz}, 2 \mathrm{H}), 3.89(\mathrm{~s}, 3 \mathrm{H}), 2.81(\mathrm{~s}, 3 \mathrm{H}), 2.68$ (s, 3H), 1.70 (s, $3 \mathrm{H}), 1.48(\mathrm{~s}, 3 \mathrm{H}) .{ }^{13} \mathrm{C} \mathrm{NMR}\left(100 \mathrm{MHz}, \mathrm{CDCl}_{3}\right): \delta 185.9,161.0,160.6,158.0,148.0$, $145.5,144.2$, 134.1, 130.6, 129.2, 127.1, 126.2, 115.3, 115.1, 55.6, 17.8, 16.6, 13.5, 12.2. IR $\left(\mathrm{cm}^{-1}\right): 2834,1667,1608,1545,1514,1476,1425,1399,1349,1310,1288,1265$, 1247, 1209, 1176, 1107, 1084, 1023, 994, 960, 890, 835, 815, 783, 766, 735, 707, 673. HRMS (FAB) calcd for $\mathrm{C}_{21} \mathrm{H}_{20} \mathrm{BF}_{2} \mathrm{I}_{2} \mathrm{~N}_{2} \mathrm{O}_{2} \mathrm{I}[\mathrm{M}]^{+}, 508.0631$; found, 508.0627.

Compound 4-A, 4-B, 4-C: When BODIPY dye 4.4 (270 mg, $0.53 \mathrm{mmol})$, and compound 4.5a (2.0 g, $10.2 \mathrm{mmol})$ were dissovled in a mixture of toluene $(250 \mathrm{~mL})$, piperidine $(0.8 \mathrm{~mL})$ and $\mathrm{AcOH}(0.6 \mathrm{~mL})$, the mixture was stirred at $108{ }^{\circ} \mathrm{C}$ under reflux for $4 \mathrm{~h}$. Any water formed during the reaction was removed azeotropically by using a Dean-Stark apparatus. After the reaction was quenched with water at room temperature, the mixture was diluted with EtOAc and washed with water and brine, respectively. The organic layer was collected, dried over $\mathrm{Na}_{2} \mathrm{SO}_{4}$ and concentrated in vacuo. The crude product was purified by silica gel column chromatography by using mobile phase (hexane/EtOAc/ $\mathrm{CH}_{2} \mathrm{Cl}_{2}, 4 / 0.5 / 2$ to $3 / 1 / 2, \mathrm{v} / \mathrm{v}$ ) to obtain monostyryl BODIPY dye 4-A (36 mg, 10\%) as purple crystal solid, distyryl BODIPY dye 4-B (168 mg, 37\%) as blue powder and tristyryl BODIPY dye 4-C (23 mg, 5\%) as green powder and recovered compound 4.5a (1.5 g). Monostyryl BODIPY dye 4-A, ${ }^{1} \mathrm{H}$ NMR (400 $\left.\mathrm{MHz}, \mathrm{CDCl}_{3}\right): \delta$ $10.02(\mathrm{~s}, 1 \mathrm{H}), 8.10(\mathrm{~d}, J=16.8 \mathrm{~Hz}, 1 \mathrm{H}), 7.54(\mathrm{~d}, J=16.8 \mathrm{~Hz}, 1 \mathrm{H}), 7.18(\mathrm{~d}, J=6.8 \mathrm{~Hz}$, 
2H), $7.06(\mathrm{~d}, J=6.8 \mathrm{~Hz}, 2 \mathrm{H}), 6.85(\mathrm{~s}, 2 \mathrm{H}), 3.94(\mathrm{~s}, 6 \mathrm{H}), 3.90(\mathrm{~s}, 3 \mathrm{H}), 3.89(\mathrm{~s}, 3 \mathrm{H}), 2.85$ (s, 3H), 1.72 (s, 3H), 1.54 (s, 3H). ${ }^{13} \mathrm{C}$ NMR (100 MHz, $\left.\mathrm{CDCl}_{3}\right): \delta$ 186.0, 161.0, 158.4, 153.7, 153.0, 148.5, 145.6, 143.2, 141.3, 140.2, 134.9, 132.2, 131.3, 129.4, 127.4, 126.4, 118.0, 115.3, 105.2, 61.2, 56.5, 55.6, 18.2, 13.7, 12.3. IR ( $\left.\mathrm{cm}^{-1}\right): 2923,2844,1649,1613$, 1580, 1504, 1470, 1413, 1354, 1312, 1269, 1248, 1210, 1182, 1157, 1100, 1047, 1004, 993, 952, 900, 844, 817, 797, 771, 747, 712, 675, 662. HRMS (FAB) calcd for $\mathrm{C}_{31} \mathrm{H}_{30} \mathrm{BF}_{2} \mathrm{~N}_{2} \mathrm{O}_{5} \mathrm{I}[\mathrm{M}]^{+}, 686.1260$; found, 686.1259. Distyryl BODIPY dye 4-B, ${ }^{1} \mathrm{H}$ NMR (400 MHz, $\left.\mathrm{CDCl}_{3}\right): \delta 9.85$ (s, 1H), $8.12(\mathrm{~d}, J=16.8 \mathrm{~Hz}, 1 \mathrm{H}), 7.55(\mathrm{~d}, J=16.4 \mathrm{~Hz}, 1 \mathrm{H})$, $7.21(\mathrm{~d}, J=7.6 \mathrm{~Hz}, 2 \mathrm{H}), 6.99$ (d, $J=7.6 \mathrm{~Hz}, 2 \mathrm{H}), 6.84(\mathrm{~s}, 2 \mathrm{H}), 6.40(\mathrm{~d}, J=16.0 \mathrm{~Hz}, 1 \mathrm{H})$, $6.24(\mathrm{~s}, 2 \mathrm{H}), 5.75(\mathrm{~d}, J=16.0 \mathrm{~Hz}, 1 \mathrm{H}), 3.92-3.81\left(\mathrm{~s} \times 6 \mathrm{OCH}_{3}, 18 \mathrm{H}\right), 3.71(\mathrm{~s}, 3 \mathrm{H}), 2.90$ (s, 3H), 1.53 (s, 3H). ${ }^{13} \mathrm{C}$ NMR (100 MHz, $\left.\mathrm{CDCl}_{3}\right): \delta 187.3,160.8,158.0,153.7,153.5$, 153.1, 148.3, 145.3, 141.6, 140.2, 139.2, 138.2, 135.1, 132.1, 132.0, 131.0, 130.5, 126.4, $126.3,118.9,118.0,115.1,105.3,104.1,94.6,85.0,61.2,61.1,56.5,56.2,55.4,18.3$, 14.7. IR ( $\left.\mathrm{cm}^{-1}\right): 2938,2839,1731,1661,1618,1579,1505,1468,1418,1382,1360$, $1321,1270,1249,1162,1151,1128,1103,1073,1005,956,856,839,819,798,763$, 753, 714, 699, 685. HRMS (FAB) calcd for $\mathrm{C}_{41} \mathrm{H}_{40} \mathrm{BF}_{2} \mathrm{~N}_{2} \mathrm{O}_{8} \mathrm{I}[\mathrm{M}]^{+}, 864.1890$; found, 864.1883. Tristyryl BODIPY dye 4-C, ${ }^{1} \mathrm{H}$ NMR (400 MHz, $\left.\mathrm{CDCl}_{3}\right): \delta 9.85$ (s, $\left.1 \mathrm{H}\right), 8.15$ (d, J = 16.8 Hz, 1H), $8.11(\mathrm{~d}, J=16.4 \mathrm{~Hz}, 1 \mathrm{H}), 7.73(\mathrm{~d}, \mathrm{~J}=16.0 \mathrm{~Hz}, 1 \mathrm{H}), 7.62(\mathrm{~d}, J=$ $16.4 \mathrm{~Hz}, 1 \mathrm{H}), 7.25$ (d, $J=7.2 \mathrm{~Hz}, 1 \mathrm{H}), 7.01$ (d, $J=7.6 \mathrm{~Hz}, 1 \mathrm{H}), 6.90$ (s, 2H), 6.86 (s, 2H), $6.49(\mathrm{~d}, J=16.0 \mathrm{~Hz}, 1 \mathrm{H}), 6.28(\mathrm{~s}, 2 \mathrm{H}), 5.83(\mathrm{~d}, J=16.0 \mathrm{~Hz}, 1 \mathrm{H}), 3.96-3.84(\mathrm{~s} \times 9$ $\left.\mathrm{OCH}_{3}, 27 \mathrm{H}\right), 3.71(\mathrm{~s}, 3 \mathrm{H}), 1.57$ (s, 3H). ${ }^{13} \mathrm{C} \mathrm{NMR}\left(100 \mathrm{MHz}, \mathrm{CDCl}_{3}\right): \delta 190.9,186.8$, $160.8,153.8,153.7,153.5,153.1,148.1,147.5,147.0,142.8,141.6,140.4,140.0,139.2$, 139.1, 135.6, 132.7, 132.2, 132.0, 130.7, 128.6, 126.9, 126.5, 119.0, 118.1, 117.1, 115.2, $106.9,105.5,105.4,104.2,61.3,61.2,61.1,56.7,56.5,56.4,56.2,55.4,18.3 . \quad$ IR $\left(\mathrm{cm}^{-1}\right)$ : 2937, 2837, 1671, 1618, 1579, 1505, 1463, 1418, 1360, 1327, 1246, 1175, 1151, 1126, 1103, 1006, 959, 821, 762, 704. HRMS (FAB) calcd for $\mathrm{C}_{51} \mathrm{H}_{50} \mathrm{BF}_{2} \mathrm{~N}_{2} \mathrm{O}_{11} \mathrm{I}[\mathrm{M}]^{+}$, 1042.2520; found, 1042.2523.

Compound 4.6: A mixture of DMF $(6 \mathrm{~mL})$ and $\mathrm{POCl}_{3}(6 \mathrm{~mL})$ was stirred in an ice bath for 5 min under argon. After the solution was warmed to room temperature, it was stirred for additional $30 \mathrm{~min}$. To this reaction mixture was added compound 4.3 (160 mg, $0.42 \mathrm{mmol}$ ) in dichloroethane $(50 \mathrm{~mL})$, the temperature was raised to $60{ }^{\circ} \mathrm{C}$, and the 
mixture was stirred for an additional $5 \mathrm{~h}$. The reaction mixture was cooled to room temperature and slowly poured into saturated aqueous $\mathrm{NaHCO}_{3}(300 \mathrm{~mL})$ under ice-cold conditions. After being warmed to room temperature, the reaction mixture was further stirred for $60 \mathrm{~min}$ and washed with water. The organic layers were combined, dried over anhydrous $\mathrm{Na}_{2} \mathrm{SO}_{4}$, and evaporated in vacuo. The crude product was further purified using column chromatography (silica gel, hexane/EtOAc/ $\mathrm{CH}_{2} \mathrm{Cl}_{2}, 4 / 0.5 / 2$, v/v) to give BODIPY 4.6 (141 mg, 82\%) as red crystal solid. ${ }^{1} \mathrm{H}$ NMR (400 MHz, $\left.\mathrm{CDCl}_{3}\right): \delta 10.01(\mathrm{~s}$, 2H), 7.15 (d, $J=8.4 \mathrm{~Hz}, 2 \mathrm{H}), 7.06$ (d, $J=8.4 \mathrm{~Hz}, 2 \mathrm{H}), 3.87$ (s, 3H), 2.82 (s, 6H), 1.73 (s, $6 \mathrm{H}) .{ }^{13} \mathrm{C}$ NMR $\left(100 \mathrm{MHz}, \mathrm{CDCl}_{3}\right): \delta 185.9,161.2,160.7,148.6,147.8,132.5,190.0$, 128.2, 125.6, 115.5, 55.7, 13.9, 12.6. IR ( $\left.\mathrm{cm}^{-1}\right): 2925,2840,1670,1608,1569,1514$, $1471,1443,1406,1380,1307,1292,1250,1175,1082,1026,1009,964,949,900,851$, $838,820,803,787,768,733,720,705$. HRMS (FAB) calcd for $\mathrm{C}_{22} \mathrm{H}_{22} \mathrm{~N}_{2} \mathrm{~F}_{2} \mathrm{BO}_{3}$ $[\mathrm{M}+\mathrm{H}]^{+}, 411.1692$; found, 411.1692 .

Compound 4-Da, 4-Ea, 4-Fa and 4-Ga: When BODIPY dye 4.6 (200 mg, 0.5 $\mathrm{mmol})$, and compound $4.5 \mathrm{a}(2.0 \mathrm{~g}, 10.2 \mathrm{mmol})$ were dissovled in a mixture of toluene $(350 \mathrm{~mL})$, piperidine $(1.0 \mathrm{~mL})$ and $\mathrm{AcOH}(0.8 \mathrm{~mL})$, the mixture was stirred at $112{ }^{\circ} \mathrm{C}$ for $3 \mathrm{~h}$. Any water formed during the reaction was removed azeotropically by using a DeanStark apparatus. After the reaction was quenched with water at room temperature, the mixture was diluted with EtOAc and washed with water and brine, respectively. The organic layer was collected, dried over $\mathrm{Na}_{2} \mathrm{SO}_{4}$ and concentrated in vacuo. The crude product was purified by silica gel column chromatography by using mobile phase (hexane/EtOAc/ $\mathrm{CH}_{2} \mathrm{Cl}_{2}, 4 / 0.5 / 2$ to $3 / 1 / 2$, v/v) to obtain monostyryl BODIPY dye 4-Da (40 mg, 14\%) as red-purple powder, distyryl BODIPY dye 4-E a (105 mg, 29\%) as bluepurple powder, tristyryl BODIPY dye $\mathbf{4 - F}$ a (48 $\mathrm{mg}, 11 \%$ ) as blue powder and tetrastyryl BODIPY dye 4-Ga (16 mg, 3\%) as green powder and recovered compound 4.5a (1.6 g). Monostyryl BODIPY dye 4-Da, ${ }^{1} \mathrm{H}$ NMR (400 MHz, $\mathrm{CDCl}_{3}$ ): $\delta 10.03$ (s, $\left.1 \mathrm{H}\right), 9.85(\mathrm{~s}, 1 \mathrm{H})$, 7.22 (d, $J=8.4 \mathrm{~Hz}, 2 \mathrm{H}), 7.01$ (d, $J=8.4 \mathrm{~Hz}, 2 \mathrm{H}), 6.44$ (d, $J=16.0 \mathrm{~Hz}, 1 \mathrm{H}), 6.25$ (s, 2H), $5.75(\mathrm{~d}, J=16.0 \mathrm{~Hz}, 1 \mathrm{H}), 3.83$ (s, 3H), 3.81 (s, 6H), 3.72 (s, 3H), 2.90 (s, 3H), 2.85 (s, 3H), 1.77 (s, 3H). ${ }^{13} \mathrm{C}$ NMR (100 MHz, $\left.\mathrm{CDCl}_{3}\right): \delta$ 187.1, 185.8, 161.0, 160.9, 160.6, $153.5,148.1,146.3,139.5,139.4,132.7,132.1,131.6,130.1,128.3,127.1,125.5,118.4$, 115.3, 104.3, 61.2, 56.2, 55.5, 14.8, 14.0, 12.6. IR $\left(\mathrm{cm}^{-1}\right)$ : 2936, 2839, 1670, 1609, 1579, 
$1507,1419,1380,1317,1292,1249,1165,1150,1127,1085,1019,915,837,798,728$, 712, 692. HRMS (FAB) calcd for $\mathrm{C}_{32} \mathrm{H}_{32} \mathrm{BF}_{2} \mathrm{~N}_{2} \mathrm{O}_{6}[\mathrm{M}+\mathrm{H}]^{+}$, 589.2321; found, 589.2325. Distyryl BODIPY dye 4-Ea, ${ }^{1} \mathrm{H}$ NMR (400 MHz, $\mathrm{CDCl}_{3}$ ): $\delta 9.86$ (s, 2H), 7.28 (d, $J=8.8$ Hz, 2H), 6.97 (d, $J=8.4 \mathrm{~Hz}, 2 \mathrm{H}), 6.50$ (d, $J=16.0 \mathrm{~Hz}, 2 \mathrm{H}), 6.24$ (s, 4H), 5.80 (d, $J=$ $16.0 \mathrm{~Hz}, 2 \mathrm{H}), 3.81$ (s, 6H), 3.79 (s, 12H), 3.72 (s, 3H), 2.90 (s, 6H). ${ }^{13} \mathrm{C}$ NMR (100 MHz, $\left.\mathrm{CDCl}_{3}\right): \delta 187.0,160.8,153.5,147.6,144.7,139.6,139.5,132.3,131.6,131.2,127.0$, 125.6, 118.4, 115.2, 104.3, 61.2, 56.2, 55.2, 14.8. IR ( $\left.\mathrm{cm}^{-1}\right):$ 2939, 2838, 1669, 1610, 1578, 1502, 1465, 1427, 1378, 1321, 1272, 1246, 1142, 1128, 1025, 1005, 963, 925, 866, 837, 729, 706, 693. HRMS (FAB) calcd for $\mathrm{C}_{42} \mathrm{H}_{42} \mathrm{BF}_{2} \mathrm{~N}_{2} \mathrm{O}_{9}[\mathrm{M}+\mathrm{H}]^{+}, 767.2951$; found, 767.2946. Tristyryl BODIPY dye 4-Fa, ${ }^{1} \mathrm{H}$ NMR $\left(400 \mathrm{MHz}, \mathrm{CDCl}_{3}\right): \delta 9.91(\mathrm{~s}, 1 \mathrm{H}), 9.87$ (s, 1H), 8.20 (d, $J=16.0 \mathrm{~Hz}, 1 \mathrm{H}), 7.76(\mathrm{~d}, J=16.0 \mathrm{~Hz}, 1 \mathrm{H}), 7.31$ (d, $J=8.4 \mathrm{~Hz}, 2 \mathrm{H})$, $6.97(\mathrm{~d}, J=8.4 \mathrm{~Hz}, 2 \mathrm{H}), 6.93(\mathrm{~s}, 2 \mathrm{H}), 6.54(\mathrm{~d}, J=16.0 \mathrm{~Hz}, 1 \mathrm{H}), 6.50$ (d, $J=15.6 \mathrm{~Hz}$, $1 \mathrm{H}), 6.28(\mathrm{~s}, 2 \mathrm{H}), 6.25(\mathrm{~s}, 2 \mathrm{H}), 5.87(\mathrm{~d}, J=15.6 \mathrm{~Hz}, 1 \mathrm{H}), 5.84(\mathrm{~d}, J=16.0 \mathrm{~Hz}, 1 \mathrm{H}), 3.95-$ $3.81\left(\mathrm{~s} \times 9 \mathrm{OCH}_{3}, 27 \mathrm{H}\right), 3.56(\mathrm{~s}, 3 \mathrm{H}), 2.98(\mathrm{~s}, 3 \mathrm{H}) \cdot{ }^{13} \mathrm{C} \mathrm{NMR}\left(100 \mathrm{MHz}, \mathrm{CDCl}_{3}\right): \delta$ 187.1, 186.6, 160.8, 155.9, 153.7, 153.6, 153.5, 149.4, 147.2, 144.6, 143.2, 140.4, 140.2, $139.6,139.5,133.4,132.3,131.6,131.4,127.8,125.8,118.5,118.4,116.7,115.2,105.7$, 104.4, 104.3, 61.3, 61.2, 56.5, 56.2, 55.2, 15.0. IR $\left(\mathrm{cm}^{-1}\right): 2928,2840,1672,1608,1579$, 1504, 1463, 1417, 1377, 1328, 1245, 1176, 1128, 1006, 836, 764, 709. HRMS (FAB) calcd for $\mathrm{C}_{52} \mathrm{H}_{51} \mathrm{BF}_{2} \mathrm{~N}_{2} \mathrm{O}_{12}[\mathrm{M}]^{+}, 944.3503$; found, 944.3499. Tetrastyryl BODIPY dye 4Ga,${ }^{1} \mathrm{H}$ NMR $\left(400 \mathrm{MHz}, \mathrm{CDCl}_{3}\right): \delta 9.88(\mathrm{~s}, 2 \mathrm{H}), 8.20(\mathrm{~d}, J=16.0 \mathrm{~Hz}, 2 \mathrm{H}), 7.79(\mathrm{~d}, J=$ $16.4 \mathrm{~Hz}, 2 \mathrm{H}), 7.33$ (d, $J=8.4 \mathrm{~Hz}, 2 \mathrm{H}), 6.96$ (d, $J=8.8 \mathrm{~Hz}, 2 \mathrm{H}), 6.93$ (s, 4H), 6.57 (d, $J=$ $16.0 \mathrm{~Hz}, 2 \mathrm{H}), 6.28(\mathrm{~s}, 4 \mathrm{H}), 5.90(\mathrm{~d}, J=16.0 \mathrm{~Hz}, 2 \mathrm{H}), 3.93-3.73\left(\mathrm{~s} \times 12 \mathrm{OCH}_{3}, 36 \mathrm{H}\right), 3.55$ (s, 3H). ${ }^{13} \mathrm{C}$ NMR (100 MHz, $\left.\mathrm{CDCl}_{3}\right): \delta 186.6,160.8,155.7,153.7,153.5,149.0,144.5$, $141.8,140.5,140.3,139.6,133.6,132.4,131.6,131.5,127.8,126.0,118.7,116.8,115.2$, 105.8, 104.4, 61.3, 61.2, 56.5, 56.2, 55.2. IR ( $\left.\mathrm{cm}^{-1}\right): 2918,2848,1730,1670,1619,1579$, 1501, 1463, 1419, 1381, 1325, 1267, 1244, 1127, 1028, 1003, 964, 822, 735, 701. HRMS (FAB) calcd for $\mathrm{C}_{62} \mathrm{H}_{61} \mathrm{BF}_{2} \mathrm{~N}_{2} \mathrm{O}_{15}[\mathrm{M}]^{+}, 1122.4133$; found, 1122.4128 .

Compound 4-E: $\quad$ When BODIPY dye 4.6 (100 $\mathrm{mg}, 0.244 \mathrm{mmol})$, and compound 4.5b (876 mg, $0.976 \mathrm{mmol})$ were dissovled in a mixture of toluene $(100 \mathrm{~mL})$, piperidine $(0.6 \mathrm{~mL})$ and $\mathrm{AcOH}(0.5 \mathrm{~mL})$, the mixture was stirred at $110^{\circ} \mathrm{C}$ for $2 \mathrm{~h}$. Any water formed during the reaction was removed azeotropically by using a Dean-Stark 
apparatus. After the reaction was quenched with water at room temperature, the mixture was diluted with EtOAc and then washed with water and brine, respectively. The organic layer was collected, dried over $\mathrm{Na}_{2} \mathrm{SO}_{4}$ and concentrated in vacuo. The crude product was purified by silica gel column chromatography by using mobile phase $\left(\mathrm{MeOH} / \mathrm{h}\right.$ exane/EtOAc/ $\left.\mathrm{CH}_{2} \mathrm{Cl}_{2}, 0.5 / 5 / 1 / 3, \mathrm{v} / \mathrm{v}\right)$ to obtain distyryl BODIPY dye 4-E $\mathbf{b}$ (190 mg, 36\%) as purple oil. ${ }^{1} \mathrm{H}$ NMR (400 MHz, $\left.\mathrm{CDCl}_{3}\right): \delta 9.83$ (s, 2H), 7.23 (d, $J=7.6 \mathrm{~Hz}$, $7.00(\mathrm{~d}, J=8.4 \mathrm{~Hz}, 2 \mathrm{H}), 6.69$ (d, $J=8.0 \mathrm{~Hz}, 2 \mathrm{H}), 6.50-6.45$ (m, 6H) 5.75 (d, $J=16.0 \mathrm{~Hz}$, 2H), 3.98 (d, $J=5.6 \mathrm{~Hz}, 4 \mathrm{H}), 3.90$ (d, $J=5.6 \mathrm{~Hz}, 4 \mathrm{H}), 3.68(\mathrm{~s}, 3 \mathrm{H}), 3.58-3.44(\mathrm{~m}, 112 \mathrm{H})$, 3.31-3.28 (s× $\left.8 \mathrm{OCH}_{3}, 24 \mathrm{H}\right), 2.88(\mathrm{~s}, 6 \mathrm{H}), 2.38-2.35(\mathrm{~m}, 4 \mathrm{H}) .{ }^{13} \mathrm{C} \mathrm{NMR}(100 \mathrm{MHz}$, $\left.\mathrm{CDCl}_{3}\right): \delta 187.1,161.1,160.5,155.1,150.5,149.2,148.0,144.6,139.6,132.2,130.9$, 130.1, 129.2, 126.8, 125.6, 120.3, 117.1, 115.5, 113.1, 112.1, 77.5, 72.1, 70.8, 70.7, 70.7, 70.6, 69.5, 69.4, 69.2, 67.1, 59.2, 55.8, 55.7, 40.2, 40.1, 14.8. HRMS (MAIDL) calcd for $\mathrm{C}_{108} \mathrm{H}_{173} \mathrm{BF}_{2} \mathrm{~N}_{2} \mathrm{O}_{39} \mathrm{Na}[\mathrm{M}+\mathrm{Na}]^{+}$, 2194.1572; found, 2194.1599 .

Compound 4-Gb: When BODIPY dye 4.6 (100 $\mathrm{mg}, 0.244 \mathrm{mmol}$ ), and compound 5 $\mathbf{b}(2.2 \mathrm{~g}, 2.44 \mathrm{mmol})$ were dissovled in a mixture of toluene $(100 \mathrm{~mL})$, piperidine $(0.6 \mathrm{~mL})$ and $\mathrm{AcOH}(0.5 \mathrm{~mL})$, the mixture was stirred at $116^{\circ} \mathrm{C}$ for $4 \mathrm{~h}$. Any water formed during the reaction was removed azeotropically by using a Dean-Stark apparatus. The mixture was directly concentrated in vacuo, diluted with EtOAc and then washed with water and brine, respectively. The organic layer was collected, dried over $\mathrm{Na}_{2} \mathrm{SO}_{4}$ and concentrated in vacuo. The crude product was purified by silica gel column chromatography by using mobile phase $\left(\mathrm{MeOH} /\right.$ hexane/EtOAc/ $\mathrm{CH}_{2} \mathrm{Cl}_{2}, 1 / 5 / 1 / 3$, v/v) to obtain tetrastyryl BODIPY dye 4-Gb (403 mg, 42\%) as dark green oil. ${ }^{1} \mathrm{H}$ NMR (400 MHz, $\left.\mathrm{CDCl}_{3}\right): \delta 9.79$ (s, 2H), $8.10(\mathrm{~d}, J=16.0 \mathrm{~Hz}, 2 \mathrm{H}), 7.68(\mathrm{~d}, J=16.0 \mathrm{~Hz}, 2 \mathrm{H}), 7.33$ (d, $J=8.0 \mathrm{~Hz}, 2 \mathrm{H}), 7.23$ (d, $J=5.6 \mathrm{~Hz}, 2 \mathrm{H}), 7.07$ (s, 2H), 6.96-6.90 (m, 4H), 6.68 (d, $J=$ $8.4 \mathrm{~Hz}, 2 \mathrm{H}), 6.57-6.50(\mathrm{~m}, 6 \mathrm{H}), 5.82$ (d, $J=16.0 \mathrm{~Hz}, 2 \mathrm{H}) .4 .05$ (br, 8H), 3.98 (d, $J=5.2$ $\mathrm{Hz}, 4 \mathrm{H}), 3.90(\mathrm{~d}, J=5.2 \mathrm{~Hz}, 4 \mathrm{H}), 3.65-3.43(\mathrm{~m}, 224 \mathrm{H}), 3.29-3.27\left(\mathrm{~s} \times 16 \mathrm{OCH}_{3}, 48 \mathrm{H}\right)$, 2.41-2.33 (m, 8H). ${ }^{13} \mathrm{C}$ NMR (100 MHz, $\left.\mathrm{CDCl}_{3}\right): \delta$ 186.5, 161.0, 155.9, 151.1, 150.4, 149.2, 149.1, 148.9, 144.4, 140.1, 133.7, 131.4, 130.0, 129.4, 127.4, 126.3, 121.2, 120.6, 117.6, 115.5, 114.5, 113.5, 113.0, 112.1, 77.5, 72.1, 72.0, 70.8, 70.8, 70.7, 70.6, 70.6, 69.5, 69.4, 67.3, 67.1, 59.1, 55.7, 40.3, 40.2. HRMS (MAIDL) calcd for $\mathrm{C}_{194} \mathrm{H}_{325} \mathrm{BF}_{2} \mathrm{~N}_{2} \mathrm{O}_{75} \mathrm{Na}[\mathrm{M}+\mathrm{Na}]^{+}$, 3955.1633; found, 3955.1687. 


\subsection{Results and discussions}

\subsubsection{Synthesis approach and optical properties}

We chose reported BODIPY dye (4.2) to illustrate the feasibility of our unique approach. A formyl group was introduced to BODIPY dye 4.2 at the 6-position via the Vilsmeier-Haack reaction, affording 6-formyl-BODIPY dye (4.3). Iodination of BODIPY dye 4.3 at the 2-position yields the 2-iodo-6-formyl-BODIPY dye (4). A three-hour Knoevenagel condensation of BODIPY dye 4 with excess 3,4,5-trimethoxybenzaldehyde (5) afforded 2-iodo-6-formyl-3-monostyryl-BODIPY dye (4-A), 2-iodo-6-formyl-3,7distyryl-BODIPY dye (4-B) and 2-iodo-6-formyl-3,5,7-tristyryl-BODIPY dye (4-C) in diluted solution (Scheme 4.2). Introduction of an iodo group at 2-position helps activate methyl groups at 3,7-position of BODIPY dye 4. These heterobifunctional BODIPY dyes are expected to be potential and useful building blocks for BODIPY-based donor-piacceptor organic dyes, oligomers, and dendrimers through palladium-catalyzed Suzuki or Sonogashira reactions. The aldehyde groups can be further functionalized with a variety of functional groups such as electron acceptors.

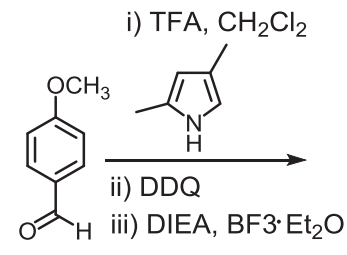

4.1

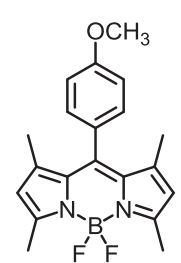

4.2

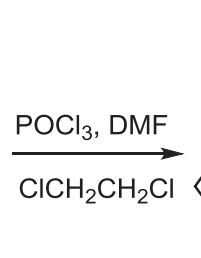

4.3

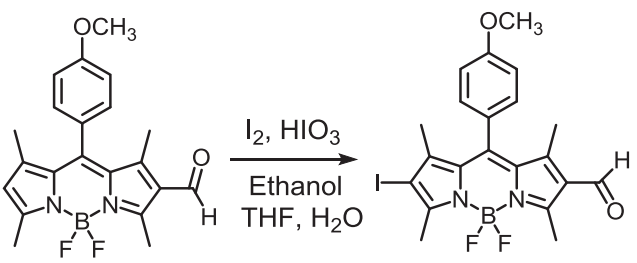

4.4

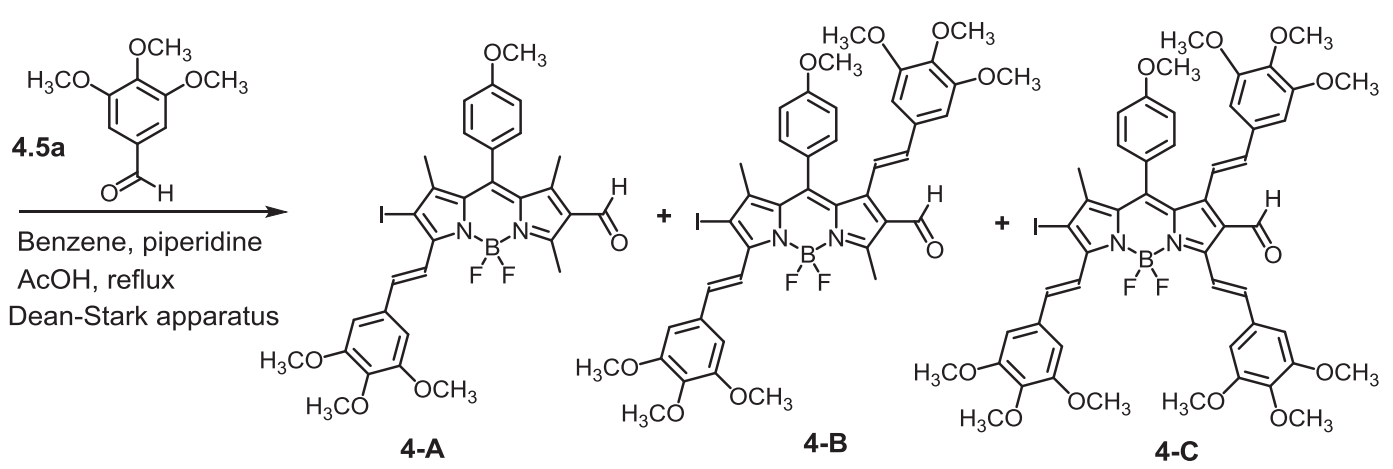

Scheme 4.2. Synthetic route to heterobifunctional BODIPY dyes. 


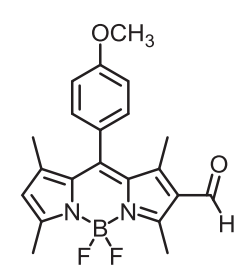

4.3

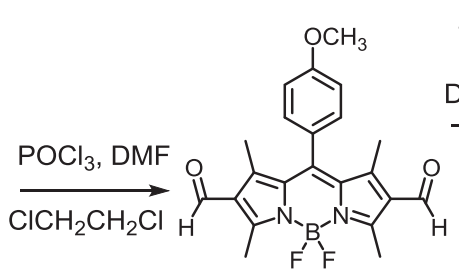

4.6

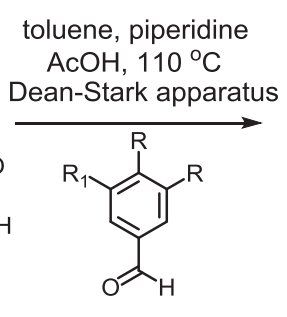

$4.5 \mathrm{a}, 4.5 \mathrm{~b}$

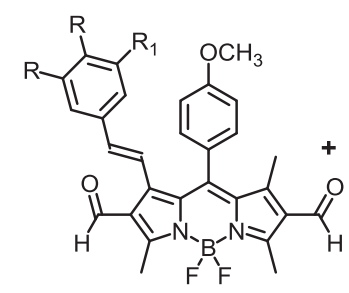

4- $D_{a}$

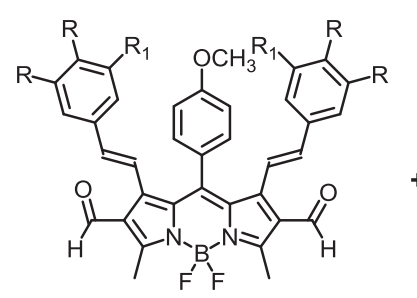

$4-E_{a}, 4-E_{b}$
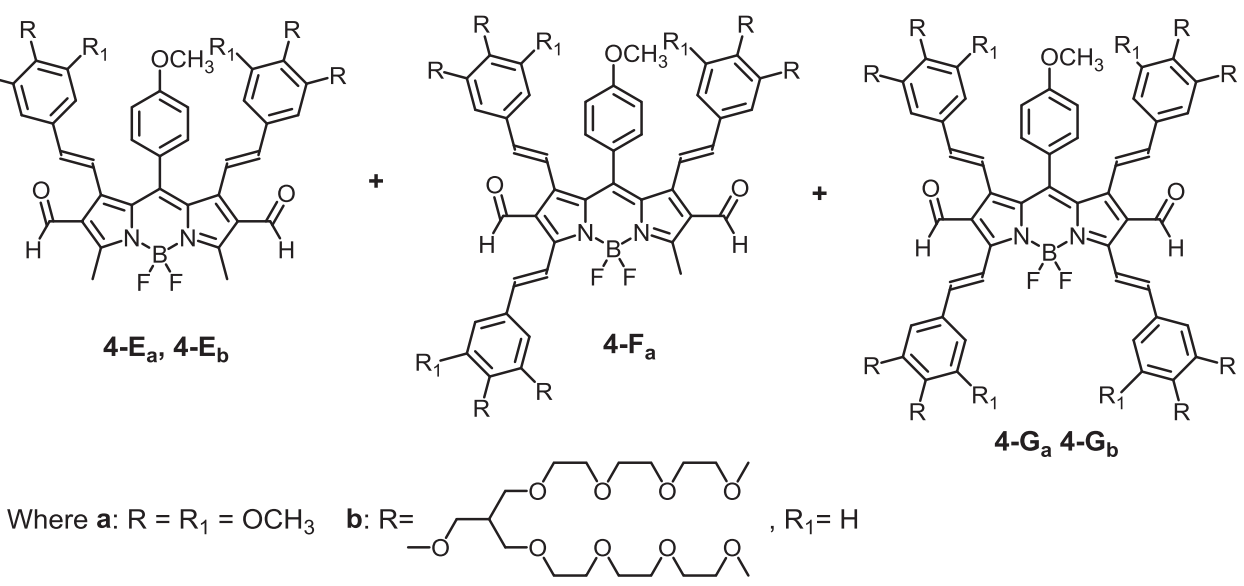

Scheme 4.3. Synthetic route to 2,6-diformyl BODIPY dyes bearing different numbers of styryl groups.

Existing studies in this area suggest that it may not be possible to prepare 2,6diformyl- BODIPY dyes by one-pot reaction of BODIPY dyes with a large excess amount of $\mathrm{POCl}_{3}$ in 1,2-dichloroethane solution. ${ }^{13}$ However, we have successfully obtained the 2,6-diformyl-BODIPY dye (4.6) by a Vilsmeier-Haack reaction using 6formyl-BODIPY dye (4.3). One- or three-hour Knoevenagel condensation of 2,6diformyl-BODIPY dye (4.6) with different equivalents of benzaldehyde derivatives (4.5a, 4.5b) in diluted toluene solution afforded the 2,6-formyl-1-monostyryl-BODIPY dye (4Da), 2,6-diformyl-1,7-distyryl-BODIPY dyes (4-Ea , 4-E $\mathbf{b})$, 2,6-formyl-1,3,7-tristyrylBODIPY dye (4-Fa), and 2,6-diformyl-1,3,5,7-tetrastyryl-BODIPY dyes (4-Ga, 4-G $\mathbf{G})$, respectively (Scheme 4.3). The presence of formyl groups at the 2,6-positions may provide steric hindrance and prevent initial Knoevenagel reactions of methyl groups at 3,5-positions with aldehyde compounds $(4.5 \mathrm{a}, 4.5 \mathrm{~b})$. Formation of distyryl groups at the 1,7-positions activates methyl groups at the 3,5-positions for further Knoevenagel reaction with aldehyde derivatives $(\mathbf{4 . 5 a}, \mathbf{4 . 5 b})$, resulting in 2,6-diformyl-1,3,7-tristyryl-, 
and 2,6-diformyl-1,3,5,7-tetrastyryl-BODIPY dyes. The water solubility of BODIPY dyes can be easily enhanced by using branched oligo(ethylene glycol)methyl ether residues. Reaction times (less than one and an half hour), and the ratio of BODIPY dye 6 to aldehyde derivatives $(\mathbf{4 . 5 a}, \mathbf{4 . 5 b})$ have to be carefully controlled in order to obtain 2,6 formyl-1-monostyryl- and 2,6-diformyl-1,7-distyryl-BODIPY dyes because of the high reactivity of methyl groups at the 1,7-positions. Further reaction beyond 3 hours resulted in the complete disappearance of 2,6-formyl-1-monostyryl- and 2,6-diformyl-1,7distyryl-BODIPY dyes, and formation of 2,6- formyl-1,3,7-tristyryl-, and 2,6-diformyl1,3,5,7-tetrastyryl-BODIPY dyes (Scheme 4.3). The formyl groups at the 2,6-positions of water-soluble BODIPY dyes can be further functionalized with a variety of functional groups such as crown ethers and $\mathrm{Zn}^{2+}$ ion binding moiety for sensing and imaging applications. ${ }^{14-15}$ Our preliminary results show that the formyl groups of the BODIPY dyes are active and easy to further be functionalized with different functional groups.

Introduction of formyl groups in BODIPY dye 4.2 at the 6- and 2,6-positions causes proton NMR peaks corresponding to methyl groups at the 7- and 1,7-positions in BODIPY dye 4.2 to move to lower fields by $0.27 \mathrm{ppm}$ and $0.30 \mathrm{ppm}$ compared with those of BODIPY dyes 4.3 and 4.6. This indicates that the formyl groups at the 6-, and 2,6-positions of BODIPY dyes may activate the methyl groups at the 7-, and 1,7positions for initial Knoevenagel reaction with aromatic aldehyde derivatives. Crystal structures of BODIPY dyes $\mathbf{4 . 4}$ and $\mathbf{4 . 6}$ that the carbonyl group in BODIPY dye $\mathbf{4}$ is oriented trans to the methyl group at the 1-position while the two carbonyl groups in BODIPY dye 4.6 are oriented cis to the methyl groups at the 1,7-positions (please see Figure B.24 in Appendix B). Formation of two vinyl bonds in BODIPY dye 4-Ea at the 1,7-positions cause the resonances for the methyl protons at the 3,5-positions to shift to lower field by $0.08 \mathrm{ppm}$ compared with those of 2,6-diformyl-BODIPY dye $\mathbf{4 . 6}$ (please see in Figure B.10 and B.14 in Appendix B). Formation of vinyl bonds in the 7-, and the 1,7-positions facilitates further Knoevenagel reaction of methyl groups at 3, and 3,5positions with the aromatic aldehydes. These reactions have to be conducted in dilution solution to prevent the Knoevenagel self-condensation of BODIPY dye 4.2 or 4.6. As evident in the NMR spectra of BODIPY dyes, the formyl groups of BODIPY dyes (4.4, 
4.6) at the 2-, and the 2,6-positions remain intact during Knoevenagel reactions (please see Appendix B).

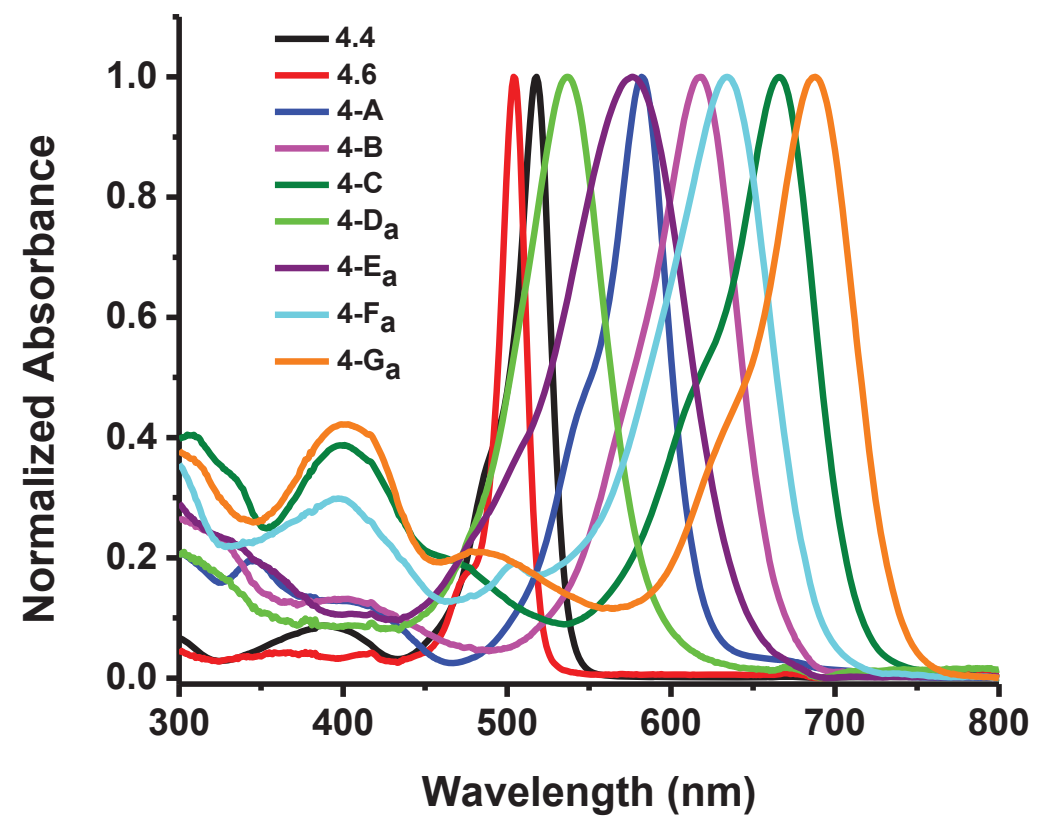

Figure 4.1. Normalized absorption spectra of BODIPY dyes (4.4, 4.6, 4-A - 4-C, 4-Da 4-Ga) in methylene chloride.

The 6-Formyl-BODIPY dye (4.3) displays a lower fluorescence quantum yield and exhibits a slight blue shifts by $3 \mathrm{~nm}$ in absorption compared to BODIPY dye (4.2). 2,6Diformyl-BODIPY dye (4.6) shows a slightly lower fluorescence quantum yield than the 6-formyl-BODIPY dye (4.3), indicating that the presence of formyl groups quenches fluorescence of the BODIPY dyes. 2-Iodo-6-formyl-BODIPY dye (4) shows a lower fluorescence quantum yield of 1.5\% compared to 6-formyl-BODIPY dye (4.3) because its fluorescence is significantly quenched by the heavy element iodine (Table 1). Extension of the $\pi$-conjugation systems in BODIPY dyes from monostyryl-, distyryl-, tristyryl- to tetrastyryl-BODIPY dyes causes significantly red shifts in their absorption and emission. The molar absorptivities of new BODIPY dyes are a little lower than their precursor BODIPPY dyes (Figures 2 and 3 and Table 1). These new BODIPY dyes display much broader absorption peaks than their precursor BODIPY dyes as the formyl 
groups at 2, and 2,6-positions affect the $\pi$-conjugation of BODIPY dyes (Figure 4.1). 2,6Heterbifunctional BODIPY dyes (4-A - 4-C) show slightly narrower absorption and emission peaks than those with diformyl groups at the 2,6-positions (4-E - 4-G). BODIPY dyes 4-E $\mathbf{E}_{\mathbf{b}}$ and $\mathbf{4}-\mathbf{G}_{\mathbf{b}}$ show broad absorption peaks (please see Figures B.34 and B.37 in Appendix B), which may result from dye aggregation in aqueous solutions, and bulky branched oligo(ethylene glycol)methyl ether residues that affect $\pi$-conjugation of BODIPY dyes. 2,6-Diformyl-1,3,5,7-tetrastyryl-BODIPY dye (4-Gb) is the first reported water-soluble neutral near-infrared emissive BODIPY dye to the best of our knowledge. All new BODIPY dyes (4-A - 4-G) show low fluorescence quantum yields. Fluorescence of BODIPY dyes 4-A - 4-C are expected to be quenched both by the heavy element iodine and an aldehyde group, while BODIPY dyes 4-D - 4-G are expected to be quenched by two aldehyde groups

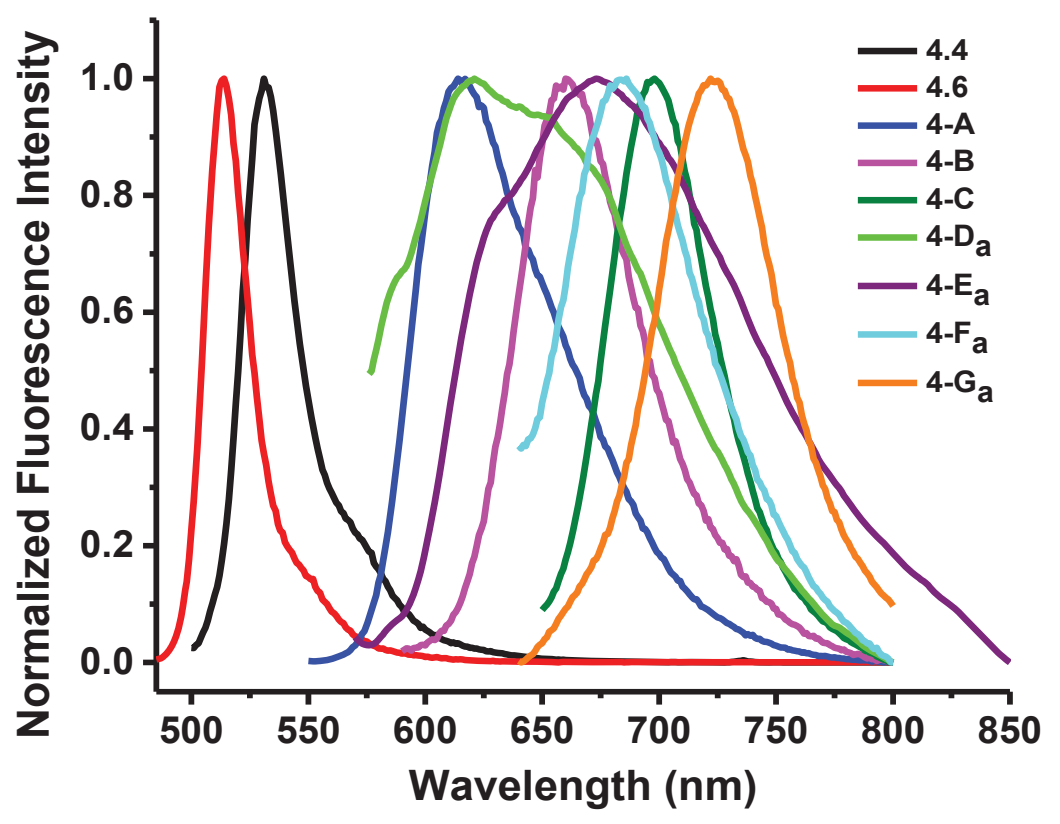

Figure 4.2. Normalized fluorescence spectra of BODIPY dyes $\left(\mathbf{4}, \mathbf{4 . 6}, \mathbf{4 - A}-\mathbf{4}-\mathrm{C}, \mathbf{4 - D _ { \mathbf { a } } -}\right.$ 4-Ga) in methylene chloride. 
Table 4.1. Absorption and emission peaks, fluorescence quantum yield $\left(\Phi_{\mathrm{f}}\right)$, and absorption coefficients $\left(\varepsilon_{\max }\right)$ of BODIPY dyes $\left(\mathbf{4 . 2}-\mathbf{4 . 4}, \mathbf{4 . 6}, \mathbf{4 - A}-\mathbf{4}-\mathbf{D}_{\mathbf{a}}, \mathbf{4}-\mathbf{E}_{\mathbf{a}}, \mathbf{4}-\mathbf{F}_{\mathbf{a}}\right.$ and 4-Ga) in methylene chloride solution and BODIPY dyes (4-E $\mathbf{b}, \mathbf{4 - G})$ aqueous solution. Fluorescence quantum yields of BODIPY dyes were calculated by using fluorescein excited at $490 \mathrm{~nm}$ in $0.1 \mathrm{~N} \mathrm{NaOH}$ as the reference quantum efficiency $\left(\Phi_{\mathrm{f}}=85 \%\right) .{ }^{9-11,16}$

\begin{tabular}{ccccc}
\hline BODIPY dye & $\lambda_{\text {abs }}(\mathrm{nm})$ & $\lambda_{\mathrm{em}}(\mathrm{nm})$ & $\Phi_{\mathrm{f}}(\%)$ & $\varepsilon_{\max }\left(10^{4} \mathrm{M}^{-1} \mathrm{~cm}^{-1}\right)$ \\
\hline 4.2 & 500 & 510 & 80 & 8.86 \\
4.3 & 497 & 508 & 65.5 & 8.24 \\
4.4 & 518 & 530 & 1.5 & 8.26 \\
$\mathbf{4 . 6}$ & 504 & 514 & 48.7 & 12.67 \\
4-A & 582 & 615 & 3.5 & 7.99 \\
4-B & 617 & 660 & 2.1 & 3.97 \\
4-C & 665 & 698 & 0.9 & 5.86 \\
4-Da & 537 & 621 & 0.5 & 2.53 \\
4-Ea & 576 & 673 & 0.2 & 3.69 \\
4-E & 557 & 695 & 0.1 & 1.29 \\
4-Fa & 634 & 686 & 0.1 & 6.06 \\
4-Ga & 688 & 722 & 0.4 & 7.27 \\
4-Gb & 661 & 710 & 0.1 & 4.58 \\
\hline
\end{tabular}

\subsubsection{Spectroscopic responses of 2,6-diformal BODIPY dyes to Cysteine}

It seems that the formyl groups quench fluorescence of BODIPY dyes with extended $\pi$-conjugation systems more efficiently than typical BODIPY dyes (Table 4.1). In order to test our hypothesis that formyl groups quench fluorescence of BODIPY dyes, we measured the optical properties of BODIPY dyes $\left(\mathbf{4}-\mathbf{E}_{\mathbf{b}}\right.$ and $\left.\mathbf{4}-\mathbf{G}_{\mathbf{b}}\right)$ in the presence of cysteine as the reaction of aldehyde groups with cysteine resulted in thiazolidine formation ${ }^{17}$. The presence of a 500-fold equivalent of cysteine in ten hours significantly enhances fluorescence intensity (up to 210 times) of the BODIPY dye $\mathbf{4}-\mathbf{E}_{\mathbf{b}}$ and causes blue shifts in both absorption and emission by $64 \mathrm{~nm}$ and $96 \mathrm{~nm}$ (Figures 3 and 4), respectively as the reaction of aldehyde groups with cysteine resulted in thiazolidine formation $^{17}$ and reduced $\pi$-conjugation of the BODIPY dyes. The presence of cysteine 
also causes color changes of the aqueous solutions of BODIPY dye $\mathbf{4}-\mathbf{E}_{\mathbf{b}}$ from blue to purple, and colorless to red in the absence (Figure 4.5a) and presence of a transilluminator, respectively (please see Figure B.38 in Appendix B). The presence of a 500 -fold equivalent of cysteine resulted in a 60-fold increase in fluorescence intensity of BODIPY dye 4-G $\mathbf{G}$, caused the disappearance of an absorption peak at $400 \mathrm{~nm}$, and led to blue shifts in both absorption and emission by $37 \mathrm{~nm}$ and $33 \mathrm{~nm}$, respectively (see Figures B.40 and B.41 in Appendix B). The presence of cysteine also results in color changes of the aqueous solution of BODIPY dye $\mathbf{4 - G} \mathbf{b}$ from green to blue (Figure $4.5 b$ ), and colorless to red in the absence and presence of a transilluminator, respectively (please see Figure B.39 in Appendix B). These experiments demonstrate that the presence of the formyl groups quenches the dye fluorescence.

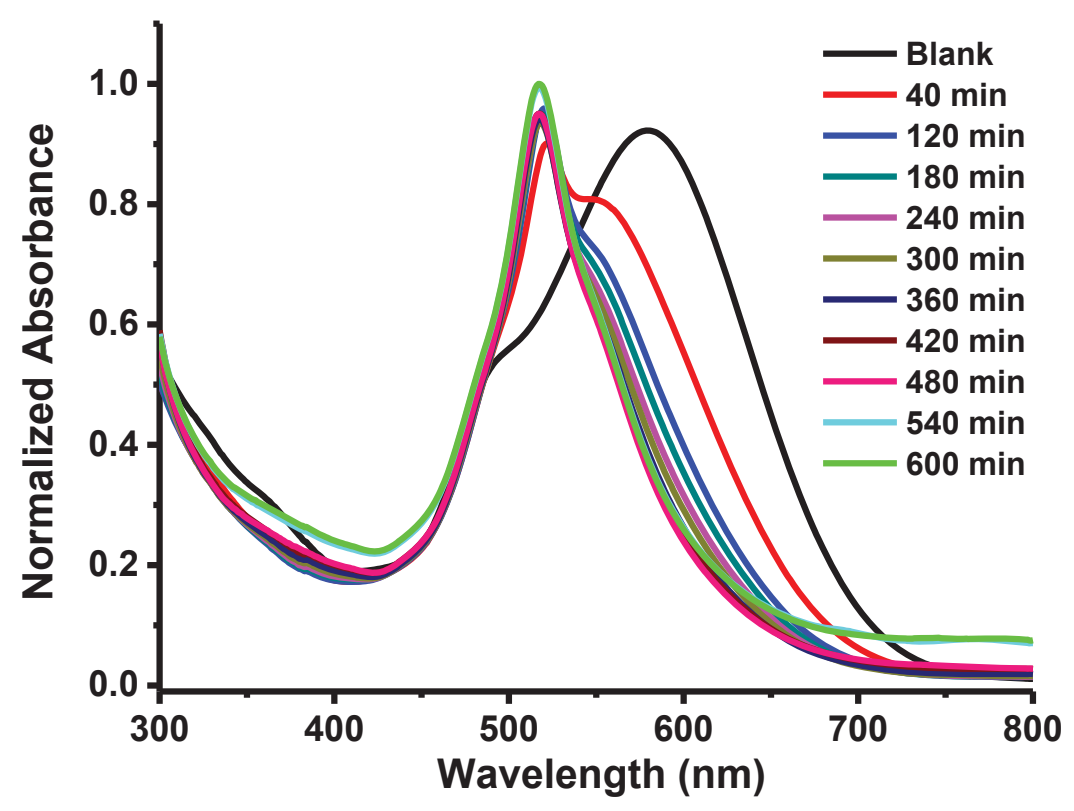

Figure 4.3. Normalized absorption spectra of BODIPY dye 4- $\mathbf{E}_{\mathbf{b}}(20 \mu \mathrm{M})$ in $0.01 \mathrm{M}$ PBS buffer $\mathrm{pH} 7.4$ in the absence and presence of L-cysteine $(10 \mathrm{mM})$ at different times. 


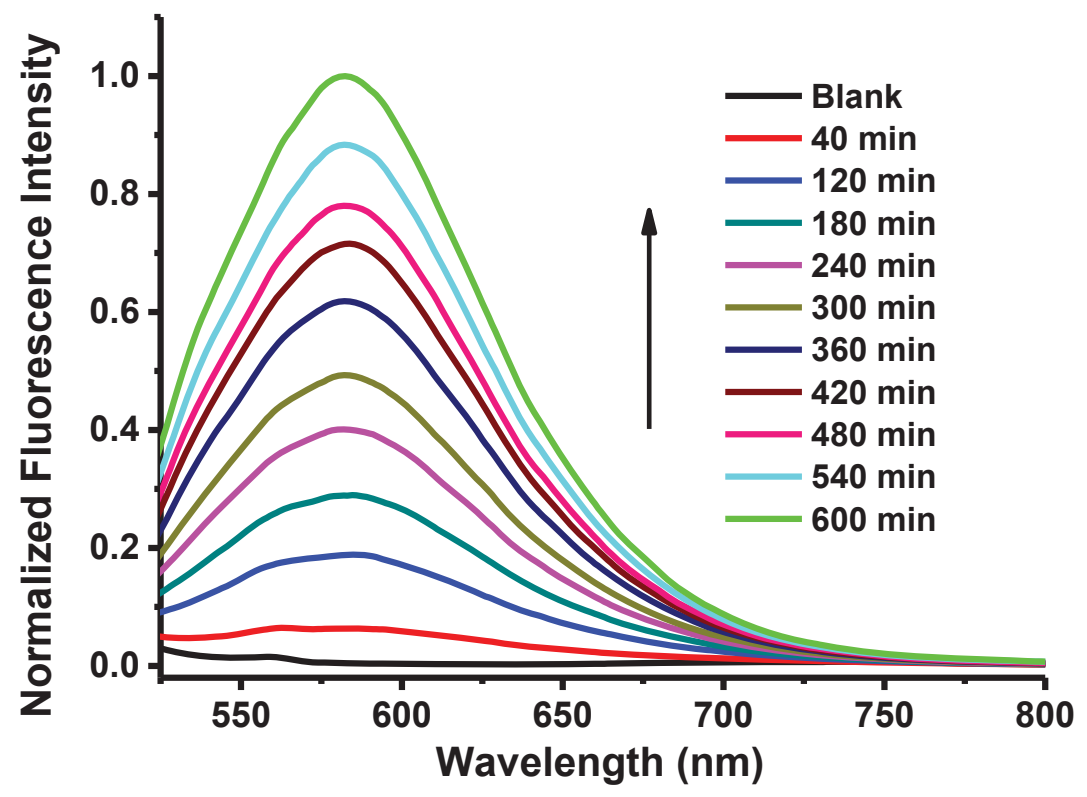

Figure 4.4. Normalized fluorescence spectra of BODIPY dye 4-E $\mathbf{E}_{\mathbf{b}}(20 \mu \mathrm{M})$ in $0.01 \mathrm{M}$ PBS buffer pH 7.4 in the absence and presence of L-cysteine (10 mM) at different times. Excitation wavelength was at $470 \mathrm{~nm}$.

a
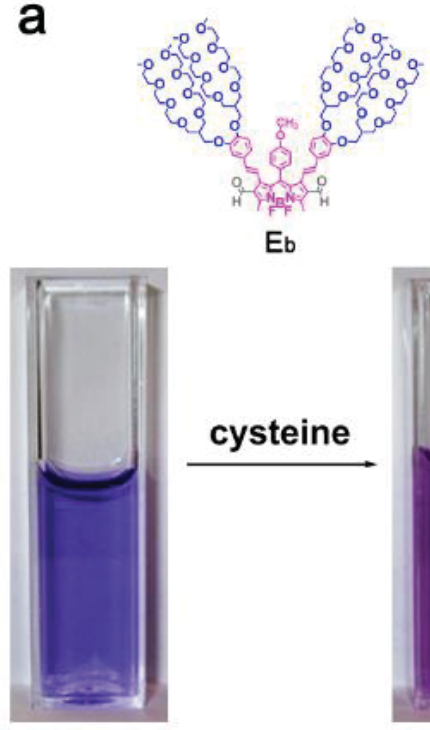

Eb

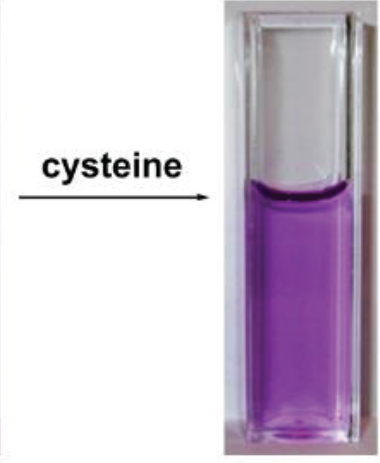

b
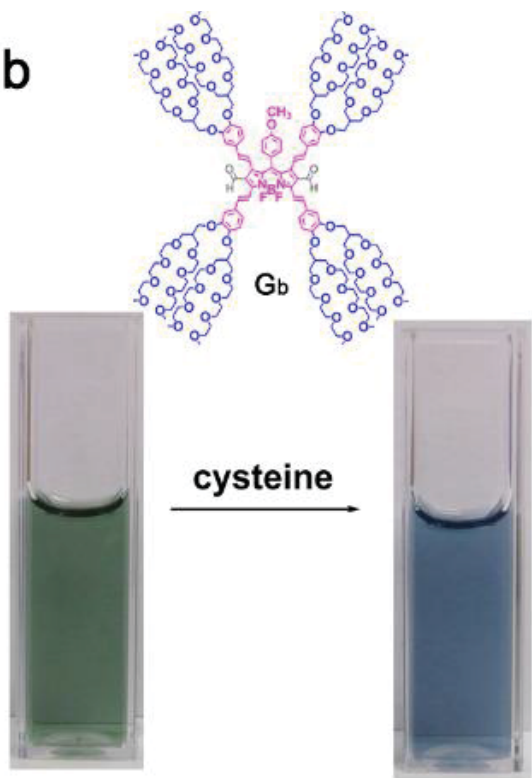

Figure 4.5. (a) Visualized images of BODIPY dye $4-\mathbf{E}_{\mathbf{b}}(20 \mu \mathrm{M})$ in $0.01 \mathrm{M}$ PBS buffer pH 7.4 in the absence and presence of L-cysteine $(10 \mathrm{mM})$. (b) Visualized images of 
BODIPY dye 4-G $\mathbf{G}(20 \mu \mathrm{M})$ in $0.01 \mathrm{M}$ PBS buffer $\mathrm{pH} 7.4$ in the absence and presence of L-cysteine (10 mM).

\subsection{Conclusion}

In conclusion, we have developed an effective approach to control the Knoevenagel reaction sequence of methyl groups of 1,3,5,7-tetramethyl BODIPY dyes by placing formyl groups at the 2- and the 2,6-positions. This allows for the preparation of novel near-infrared emissive BODIPY dyes, which can serve as potential useful building blocks for oligomers, dendrimers and sensing and imaging materials.

\section{References:}

1. Loudet, A., Burgess, K., BODIPY Dyes and Their Derivatives: Syntheses and Spectroscopic Properties. Chemical Reviews 2007, 107, 4891-4932.

2. Ziessel, R., Ulrich, G., Harriman, A., The Chemistry of Bodipy: A New El Dorado for Fluorescence Tools. New Journal of Chemistry 2007, 31 (4), 496-501.

3. Ulrich, G., Ziessel, R., Harriman, A., The Chemistry of Fluorescent Bodipy Dyes: Versatility Unsurpassed. Angewandte Chemie-International Edition 2008, 47, 1184-1201.

4. Zhu, S., Zhang, J., Vegesna, G. K., Pandey, R., Luo, F.-T., Green, S. A., Liu, H., One-pot Efficient Synthesis of Dimeric, Trimeric, and Tetrameric BODIPY Dyes for Panchromatic Absorption. Chemical Communications 2011, 47 (12), 35083510 .

5. Zhu, S. L., Zhang, J. T., Vegesna, G., Luo, F. T., Green, S. A., Liu, H. Y., Highly Water-soluble Neutral BODIPY Dyes with Controllable Fluorescence Quantum Yields. Organic Letters 2011, 13 (3), 438-441.

6. Ekmekci, Z., Yilmaz, M. D., Akkaya, E. U., A Monostyryl-boradiazaindacene (BODIPY) Derivative as Colorimetric and Fluorescent Probe for Cyanide Ions. Organic Letters 2008, 10 (3), 461-464. 
7. Buyukcakir, O., Bozdemir, O. A., Kolemen, S., Erbas, S., Akkaya, E. U., Tetrastyryl-Bodipy Dyes: Convenient Synthesis and Characterization of Elusive Near IR Fluorophores. Organic Letters 2009, 11 (20), 4644-4647.

8. Bura, T., Retailleau, P., Ulrich, G., Ziessel, R., Highly Substituted Bodipy Dyes with Spectroscopic Features Sensitive to The Environment. Journal of Organic Chemistry 2011, 76 (4), 1109-1117.

9. Maruyama, S., Kikuchi, K., Hirano, T., Urano, Y., Nagano, T., A Novel, Cellpermeable, Fluorescent Probe for Ratiometric Imaging of Zinc Ion. Journal of the American Chemical Society 2002, 124 (36), 10650-10651.

10. Gabe, Y., Urano, Y., Kikuchi, K., Kojima, H., Nagano, T., Highly Sensitive Fluorescence Probes for Nitric Oxide Based on Boron Dipyrromethene Chromophore-rational Design of Potentially Useful Bioimaging Fluorescence Probe. Journal of the American Chemical Society 2004, 126 (10), 3357-3367.

11. Yogo, T., Urano, Y., Ishitsuka, Y., Maniwa, F., Nagano, T., Highly Efficient and Photostable Photosensitizer Based on BODIPY Chromophore. Journal of the American Chemical Society 2005, 127 (35), 12162-12163.

12. Jiao, L., Yu, C., Li, J., Wang, Z., Wu, M., Hao, E., Beta-Formyl-BODIPYs from The Vilsmeier-Haack Reaction. Journal of Organic Chemistry 2009, 74 (19), 7525-7528.

13. Jiao, L. J., Yu, C. J., Li, J. L., Wang, Z. Y., Wu, M., Hao, E. H., Beta-FormylBODIPYs from The Vilsmeier-Haack Reaction. Journal of Organic Chemistry 2009, 74 (19), 7525-7528.

14. Bozdemir, O. A., Guliyev, R., Buyukcakir, O., Selcuk, S., Kolemen, S., Gulseren, G., Nalbantoglu, T., Boyaci, H., Akkaya, E. U., Selective Manipulation of ICT and PET Processes in Styryl-Bodipy Derivatives: Applications in Molecular Logic and Fluorescence Sensing of Metal Ions. Journal of the American Chemical Society 2010, 132 (23), 8029-8036.

15. Buccella, D., Horowitz, J. A., Lippard, S. J., Understanding Zinc Quantification with Existing and Advanced Ditopic Fluorescent Zinpyr Sensors. Journal of the American Chemical Society 2011, 133 (11), 4101-4114. 
16. Parker, C. A., Rees, W. T., Correction of Fluorescence Spectra and Measurement of Fluorescence Quantum Efficiency. Analyst 1960, 85, 587 - 600.

17. Rusin, O., St Luce, N. N., Agbaria, R. A., Escobedo, J. O., Jiang, S., Warner, I. M., Dawan, F. B., Lian, K., Strongin, R. M., Visual Detection of Cysteine and Homocysteine. Journal of the American Chemical Society 2004, 126 (2), 438-439. 


\section{Chapter 5. BODIPY-based Ratiometric Fluorescent Probes for Sensitive and Selective Sensing of Cyanide Ions ${ }^{1}$}

Jingtuo Zhang, ${ }^{\mathrm{a}}$ Shilei Zhu, ${ }^{\mathrm{a}}$ Loredana Valenzano, ${ }^{\mathrm{a}}$ Fen-Tair Luo $^{\mathrm{b}}$ and Haiying Liu*a

${ }^{a}$ Department of Chemistry, Michigan Technological University, 1400 Townsend Drive, Houghton, MI 49931, USA

${ }^{\mathrm{b}}$ Institute of Chemistry, Academia Sinica, Taipei, Taiwan 11529, Republic of China

\footnotetext{
${ }^{1}$ The material included in this chapter was previously published in [RSC Advances 2013, 3 (1), 68-72. DOI: 10.1039/C2RA22205A Publication Date (Web): Oct 24, 2012]Reproduced by permission of The Royal Society of Chemistry http://pubs.rsc.org/en/content/articlelanding/2013/ra/c2ra22205a\#!divAbstract
} 


\subsection{Introduction.}

Cyanide is a well-known highly toxic substance and very harmful to human health. ${ }^{1}$ Its small amount can result in extremely lethal poisoning by inhibiting the cellular respiration in living creatures via its binding to a heme unit in active site of cytochrome $\mathrm{c}$ when it is absorbed through the gastrointestinal track, skin or lungs. ${ }^{2-3}$ As a result, any accidental release of cyanide salts from industries involved in gold mining, electroplating, metallurgy and the synthesis of nylon and other synthetic fibers and resins can cause serious environmental disaster, ${ }^{4}$ especially under potential terrorist threats. Therefore, it is highly desirable to develop sensitive, selective and quick detection methods for toxic cyanide anion. Fluorescent probes have shown many promising advantages including high sensitivity, low cost, fast response and easy operation for potential in vitro assay and in vivo imaging applications among the different chemical sensing approaches for cyanide ion. ${ }^{5-6}$ These fluorescent probes are mainly based on the Benzil rearrangement reaction, ${ }^{5}$ nucleophilic addition, ${ }^{7-8}$ hydrogen-bonding interactions, ${ }^{9-}$

${ }^{10}$ cyanide complexes/addition, ${ }^{11}$ and coordination of copper ion with cyanide ${ }^{12-13}$ for sensing applications. However, most of these organic dyes are not soluble in aqueous solution, and there are still needs to address some limitations concerning the sensitivity, selectivity, longer wavelength emission, and compatibility within an aqueous environment.

We chose BODIPY dyes as the fluorescent transducers to develop three ratiometric fluorescent probes (5-A - 5-C) (Scheme 5.1) for highly sensitive and selective sensing of cyanide ion because BODIPY dyes possess many advantages such as high quantum yields and large extinction coefficients and easy tuning for longer wavelength emission. ${ }^{14-17}$ Fluorescent probe 5-A was used to systematically study its response mechanism to cyanide ion by using ${ }^{1} \mathrm{H}$ NMR, mass spectrometry, absorption and fluorescence spectrometry because its structural simplicity with tri(ethylene glycol)methyl ether residues at the meso-position. For practical application, highly watersoluble fluorescent probe 5-C was prepared to sensitively detect cyanide ion by introducing branched oligo(ethylene glycol)methyl ether residues and propanesulfonate to BODIPY dye. Fluorescent probe 5-B was used as a control to demonstrate the 
importance of propanesulfonate residue in probe 5-C to enhance water solubility of the reaction product of the fluorescent probe with cyanide ion in order to achieve sensitive detection of cyanide ion without any precipitation of the probe 5-C. The fluorescent probes show fast ratiometric fluorescent responses to cyanide ion with a dramatic fluorescence color change from red to green accompanying a significant increase in fluorescent intensity because addition reaction of cyanide ion with the iminium ion of the probes disrupts $\pi$-conjugation between BODIPY core and indole moiety, which leads to significant dual color and fluorescence changes.

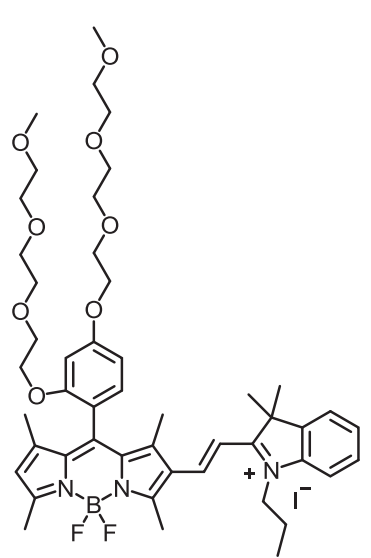

Probe 5-A

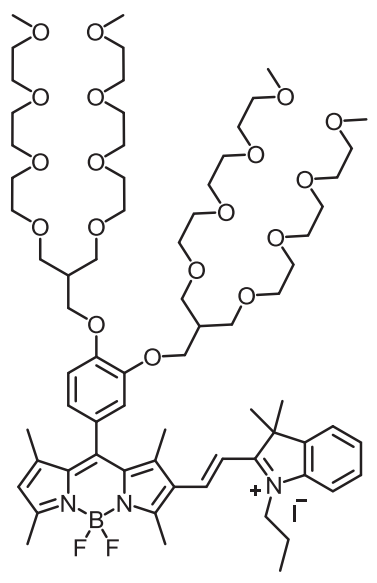

Probe 5-B

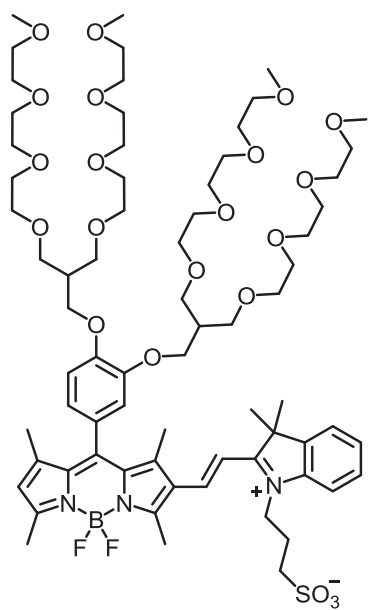

Probe 5-C

Scheme 5.1. BODIPY-based fluorescent probes for cyanide ion.

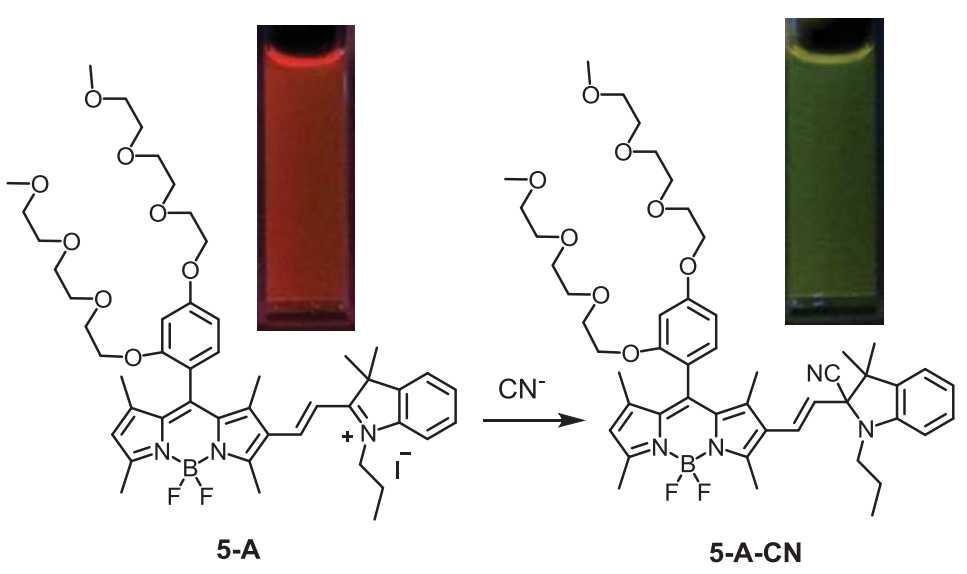

Scheme 5.2. BODIPY-based Fluorescent probe for cyanide ion sensing. 


\subsection{Methods}

The strategy to prepare the fluorescent probes was outlined in scheme 5.3. Use of an ortho-substituent group of tri(ethylene glycol)methyl ether on the meso-phenyl ring of BODIPY dyes was used to increase fluorescence quantum yield and hydrophilic properties of the BODIPY dye. ${ }^{18}$ BODIPY dyes (5.2a, 5.2b) were prepared via the condensation of the benzaldehyde derivatives $(\mathbf{5 . 1 a}, \mathbf{5 . 1 b})$ with 2,4-dimethylpyrrole according to the reported procedure. ${ }^{18-20}$ 2-Formyl-BODIPY dyes $(\mathbf{5 . 3 a}, \mathbf{5 . 3 b})$ was prepared by the Vilsmeier-Haack reaction of BODIPY dyes 5.2a and 5.2b, respectively. ${ }^{19}$ Fluorescent probes 5-A, 5-B and 5-C was readily prepared by a condensation of 2-formyl-BODIPY dyes (5.3a, 5.3b) with 2,3,3-trimethyl-1-propyl-3Hindolium iodide (5.4a) or 2,3,3-trimethyl-1-(3-sulfonatepropyl)-3H-indolium (5.4b) in ethanol solution under reflux condition (Scheme 5.3). All compounds have been characterized by ${ }^{1} \mathrm{H}$ NMR, ${ }^{13} \mathrm{C}$ NMR, and high-resolution mass spectroscopy (see the Appendix C).

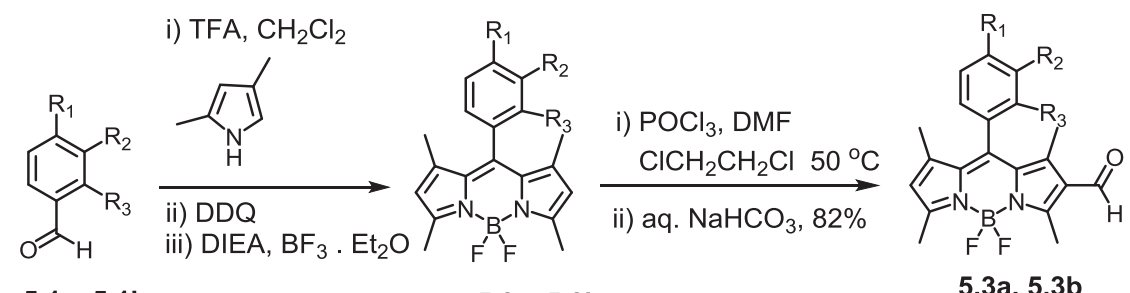

$$
5.1 \mathrm{a}, 5.1 \mathrm{~b}
$$

$5.2 \mathrm{a}, 5.2 \mathrm{~b}$

5.3a, 5.3b
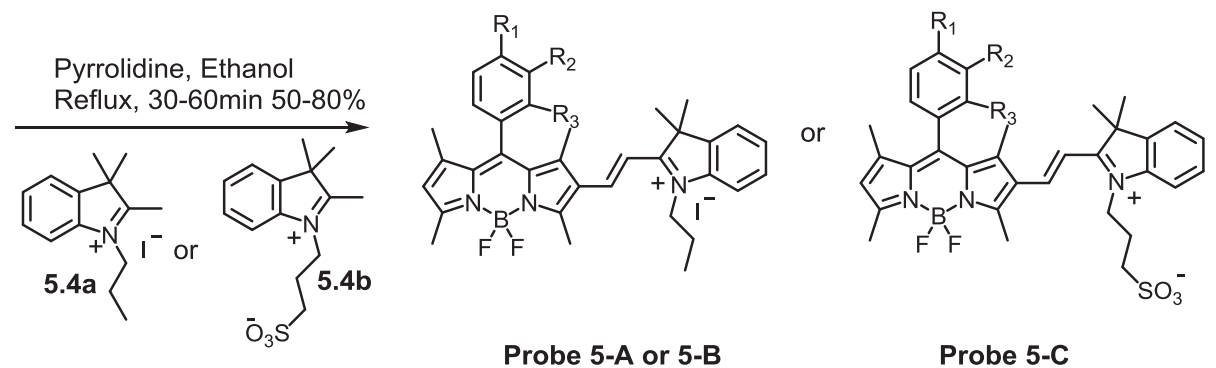

Probe 5-C

$$
\begin{array}{ll}
\text { a, probe 5-A: } & \\
\mathrm{R}_{1}=\mathrm{R}_{3}= & -\mathrm{O} O \mathrm{O} \quad \mathrm{OCH}_{3} \\
\mathrm{R}_{2}=-\mathrm{H} &
\end{array}
$$

b, probe 5-B, 5-C:

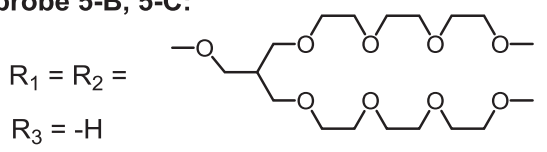

Scheme 5.3. Synthetic route to BODIPY-based fluorescent probe 5-A, 5-B and 5-C for cyanide ion. 


\subsection{Experimental section}

\subsubsection{Instrumentation}

${ }^{1} \mathrm{H}$ NMR and ${ }^{13} \mathrm{C}$ NMR spectra were taken on a $400 \mathrm{MHz}$ Varian Unity Inova spectrophotometer instrument. ${ }^{1} \mathrm{H}$ and ${ }^{13} \mathrm{C}$ NMR spectra were recorded in $\mathrm{CDCl}_{3}$, chemical shifts $(\delta)$ are given in ppm relative to solvent peaks $\left({ }^{1} \mathrm{H}: \delta 7.26 ;{ }^{13} \mathrm{C}: \delta 77.3\right)$ as internal standard. Absorption spectra were taken on a Perkin Elmer Lambda 35 UV-Vis spectrometer. Fluorescence spectra were recorded on a Jobin Yvon Fluoromax-4 spectrofluorometer.

\subsubsection{Materials and synthesis.}

Materials. Unless otherwise indicated, all reagents and solvents were obtained from commercial suppliers (Aldrich, Sigma, Fluka, Acros Organics, Fisher Scientific, Lancaster) and used without further purification. Air- and moisture-sensitive reactions were conducted in oven-dried glassware using a standard Schlenk line or drybox techniques under an inert atmosphere of dry nitrogen. Compound 5.1a and 5.2a was prepared according to our reported procedure. ${ }^{18}$

Compound 5.2a: $\quad$ Compound 5.1a was prepared according general procedure. The aldehyde compound 5.1a (5.0 g, $11.6 \mathrm{mmol})$ and 2,4-dimethylpyrrole (2.75 g, 29 mmol) were dissolved in dry $\mathrm{CH}_{2} \mathrm{Cl}_{2}(800 \mathrm{~mL})$. Five drops of trifluoroacetic acid (TFA) were added to the reaction mixture, and the resulting mixture was stirred in the dark overnight under nitrogen atmosphere at room temperature. Then DDQ (2,3-dichloro-5,6dicyano-1,4-benzoquinone) ( $3.2 \mathrm{~g}$ ) was added to the reaction mixture. When the mixture was stirred for $40 \mathrm{~min}, 16 \mathrm{~mL}$ of diisopropylethylamine (DIPEA) and $16 \mathrm{~mL}$ of $\mathrm{BF}_{3} \cdot \mathrm{OEt}_{2}$ were added to the mixture. After the mixture was further stirred for $40 \mathrm{~min}$, it was concentrated, and then was washed twice with water, dried over anhydrous $\mathrm{Na}_{2} \mathrm{SO}_{4}$, and concentrated under reduced pressure. The crude product was purified by column chromatography to obtain orange red oil 5.2a (2.9 g, 38\%). ${ }^{1} \mathrm{H}$ NMR (400 $\left.\mathrm{MHz}, \mathrm{CDCl}_{3}\right)$ : $\delta 6.97(\mathrm{~d}, J=7.6 \mathrm{~Hz}, 1 \mathrm{H}), 6.59-6.57(\mathrm{~m}, 2 \mathrm{H}), 5.92(\mathrm{~s}, 2 \mathrm{H}), 4.14(\mathrm{t}, J=4.0 \mathrm{~Hz}, 2 \mathrm{H}), 4.03$ (t, $J=4.8 \mathrm{~Hz}, 2 \mathrm{H}), 3.87$ (t, $J=5.2 \mathrm{~Hz}, 2 \mathrm{H}), 3.76-3.64(\mathrm{~m}, 8 \mathrm{H}), 3.55-3.39$ (m, $8 \mathrm{H}), 3.38-$ $3.36(\mathrm{~m}, 5 \mathrm{H}), 3.33$ (s, 3H), 2.51 (s, 6H), 1.47 (s, 6H). $\left.{ }^{13} \mathrm{C} \mathrm{NMR} \mathrm{(100} \mathrm{MHz,} \mathrm{CDCl}_{3}\right): \delta$ $161.2,156.9,154.9,142.9,139.2,132.2,130.1,120.9,116.8,106.4,100.6,72.1,72.0$, 
71.2, 71.1, 70.9, 70.8, 70.7, 70.4, 69.9, 69.2, 67.8, 59.3, 59.1, 14.7, 14.2. IR ( $\left.\mathrm{cm}^{-1}\right): 2872$, $1609,1577,1542,1507,1468,1409,1363,1303,1269,1189,1155,1105,1083,1043$, 971, 835, 766, 705. HRMS (FAB) calcd for $\mathrm{C}_{33} \mathrm{H}_{47} \mathrm{BF}_{2} \mathrm{~N}_{2} \mathrm{O}_{8}[\mathrm{M}]^{+}$, 648.3394; found, 648.3396 .

Compound 5.3a: A mixture of DMF $(7.5 \mathrm{~mL})$ and $\mathrm{POCl}_{3}(7.5 \mathrm{~mL})$ was stirred in an ice bath for 5 min under argon. After being warmed to room temperature, it was stirred for additional $30 \mathrm{~min}$. To this reaction mixture was added compound 5.2a (400 mg, $0.617 \mathrm{mmol})$ in dichloroethane $(70 \mathrm{~mL})$, the temperature was raised to $50{ }^{\circ} \mathrm{C}$, and the mixture was stirred for an additional $2 \mathrm{~h}$. The reaction mixture was cooled to room temperature and slowly poured into saturated aqueous $\mathrm{NaHCO}_{3}$ under ice-cold conditions till no gas formed. After being warmed to room temperature, the reaction mixture was further stirred for $30 \mathrm{~min}$ and washed with water. The organic layers were combined, dried over anhydrous $\mathrm{Na}_{2} \mathrm{SO}_{4}$, and evaporated in vacuum. The crude product was further purified using column chromatography to give BODIPY 5.3a (340 mg, 82\%). ${ }^{1} \mathrm{H}$ NMR (400 MHz, $\left.\mathrm{CDCl}_{3}\right): \delta 9.97$ (s, 1H), 6.94 (d, $\left.J=7.6 \mathrm{~Hz}, 1 \mathrm{H}\right), 6.61-6.58(\mathrm{~m}, 2 \mathrm{H})$, $6.08(\mathrm{~s}, 1 \mathrm{H}), 4.14(\mathrm{t}, J=4.4 \mathrm{~Hz}, 2 \mathrm{H}), 4.03(\mathrm{t}, J=4.8 \mathrm{~Hz}, 2 \mathrm{H}), 3.87(\mathrm{t}, J=4.8 \mathrm{~Hz}, 2 \mathrm{H})$, 3.74-3.62 (m, 8H), 3.54-3.35 (m, $13 \mathrm{H}), 3.30(\mathrm{~s}, 3 \mathrm{H}), 2.76(\mathrm{~s}, 3 \mathrm{H}), 2.56(\mathrm{~s}, 3 \mathrm{H}), 1.75(\mathrm{~s}$, 3H), 1.50 (s, 3H). ${ }^{13} \mathrm{C}$ NMR (100 $\left.\mathrm{MHz}, \mathrm{CDCl}_{3}\right)$ : 185.9, 161.4, 160.8, 156.5, 155.7, 147.1, $142.2,141.0,134.7,130.2,129.6,125.9,123.4,115.6,106.4,100.6,71.9,71.7,70.9$, 70.8, 70.6, 70.5, 70.4, 70.2, 69.6, 69.0, 68.7, 67.6, 59.0, 58.9, 14.9, 14.5, 12.9, 11.0. HRMS (ESI) calcd for $\mathrm{C}_{34} \mathrm{H}_{47} \mathrm{BF}_{2} \mathrm{~N}_{2} \mathrm{O}_{9} \mathrm{Na}[\mathrm{M}+\mathrm{Na}]^{+}$, 699.3240; found, 699.3252.

Compound 5.3b: $\quad$ Compound 5.3b was prepared by the same procedure of synthesis of compound 5.3a. DMF $(8 \mathrm{~mL})$ and $\mathrm{POCl}_{3}(8 \mathrm{~mL})$ compound 5.2b $(750 \mathrm{mg}$, $0.67 \mathrm{mmol})$ in dichloroethane $(40 \mathrm{~mL})$, give BODIPY 5.3b $(620 \mathrm{mg}, 83 \%) .{ }^{1} \mathrm{H}$ NMR (400 MHz, $\left.\mathrm{CDCl}_{3}\right): \delta 9.89(\mathrm{~s}, 1 \mathrm{H}), 6.92(\mathrm{~d}, J=8 \mathrm{~Hz}, 1 \mathrm{H}), 6.65-6.70(\mathrm{~m}, 2 \mathrm{H}), 6.05$ (s, $1 \mathrm{H}), 4.00(\mathrm{~d}, J=5.6 \mathrm{~Hz}, 2 \mathrm{H}), 4.89$ (d, $J=5.6 \mathrm{~Hz}, 2 \mathrm{H}), 3.55-3.39(\mathrm{~m}, 56 \mathrm{H}), 3.39-3.23$ (m, 12H), 2.69 (s, 3H), 2.48 (s, 3H), 1.65 (s, 3H), 1.43 (s, 3H). $\left.{ }^{13} \mathrm{C} \mathrm{NMR} \mathrm{(100} \mathrm{MHz,} \mathrm{CDCl}_{3}\right)$ : $185.4,161.0,155.9,149.7,147.0,143.4,142.5,134.0,129.7,125.9,125.8,123.5,120.0$, $113.5,112.6,71.6,71.5,70.3,70.3,70.2,70.2,70.1,70.1,69.5,69.0,67.2,66.8,58.7$, 58.6, 39.7, 14.8, 12.7, 11.4. HRMS (ESI) calcd for $\mathrm{C}_{56} \mathrm{H}_{91} \mathrm{BF}_{2} \mathrm{~N}_{2} \mathrm{O}_{19} \mathrm{Na}[\mathrm{M}+\mathrm{Na}]^{+}$, 1167.6175; found, 1167.6182 . 
Probe 5-A: $\quad$ BODIPY dye 5.3a $(800 \mathrm{mg}, 1.18 \mathrm{mmol})$ and indolium derivative 5.4a (0.96 g, 4 eq.) were dissolved in $30 \mathrm{~mL}$ of $\mathrm{EtOH}$, then pyrrolidine $(0.1 \mathrm{~mL})$ was added to the solution. The reaction mixture was stirring at $86{ }^{\circ} \mathrm{C}$ for $40-60$ min till starting materials was consumed by TLC monitoring and then evaporated in vacuum. The resulting solid was dissolved in $\mathrm{CH}_{2} \mathrm{Cl}_{2}$, and the organic layer was washed three times with water, dried over anhydrous $\mathrm{Na}_{2} \mathrm{SO}_{4}$, and evaporated in vacuum. The residue was purified by column chromatography on silica (DCM / MeOH, 90:0.5 v/v) and then was further purified by TLC plate (Hexanes/DCM/EtoAc/EtOH, 5/3/1/0.5) to attain compound 5-A (882 mg, $78 \%$ ) as dark purple oil. ${ }^{1} \mathrm{H}$ NMR (400 MHz, $\left.\mathrm{CDCl}_{3}\right): \delta 8.05$ $(\mathrm{d}, J=16.0 \mathrm{~Hz}, 1 \mathrm{H}), 7.63$ (d, $J=7.6 \mathrm{~Hz}, 1 \mathrm{H}), 7.53$ (d, $J=7.2 \mathrm{~Hz}, 1 \mathrm{H}), 7.49-7.40$ (m, 2H), $6.94(\mathrm{~d}, J=4.4 \mathrm{~Hz}, 1 \mathrm{H}), 6.90(\mathrm{~d}, J=16.0 \mathrm{~Hz}, 1 \mathrm{H}), 6.18(\mathrm{~s}, 1 \mathrm{H}), 4.55$ (t, $J=6.8 \mathrm{~Hz}$, 2H), $4.16(\mathrm{t}, J=4.4 \mathrm{~Hz}, 2 \mathrm{H}), 4.06(\mathrm{q}, J=4.4 \mathrm{~Hz}, 2 \mathrm{H}), 3.86(\mathrm{t}, J=4.4 \mathrm{~Hz}, 2 \mathrm{H}), 3.73-3.59$ $(\mathrm{m}, 8 \mathrm{H}), 3.52-3.31(\mathrm{~m}, 13 \mathrm{H}), 3.25(\mathrm{~s}, 3 \mathrm{H}), 2.78(\mathrm{~s}, 3 \mathrm{H}), 2.56(\mathrm{~s}, 3 \mathrm{H}), 1.95(\mathrm{~m}, 2 \mathrm{H}), 1.76$ $(\mathrm{s}, 3 \mathrm{H}), 1.75(\mathrm{~s}, 3 \mathrm{H}), 1.74(\mathrm{~s}, 3 \mathrm{H}), 1.53(\mathrm{~s}, 3 \mathrm{H}), 1.00(\mathrm{t}, J=7.2 \mathrm{~Hz}, 3 \mathrm{H}) .{ }^{13} \mathrm{C}$ NMR $(100$ $\left.\mathrm{MHz}_{,} \mathrm{CDCl}_{3}\right): \delta 180.7,163.8,162.0,156.8,155.6,149.2,146.1,142.4,141.1,140.5$, 139.2, 136.2, 132.1, 130.0, 129.8, 129.1, 125.0, 122.9, 115.1, 114.8, 108.4, 107.2, 101.0, 72.1, 72.0, 71.0, 70.9, 70.8, 70.7, 70.6, 70.5, 69.8, 69.3, 69.0, 68.0, 59.2, 59.1, 51.8, 49.5, 28.1, 21.8, 15.5, 15.0, 14.5, 13.4, 11.9. HRMS (FAB) calcd for $\mathrm{C}_{48} \mathrm{H}_{65} \mathrm{O}_{8} \mathrm{~N}_{3} \mathrm{~F}_{2} \mathrm{~B}[\mathrm{M}-\mathrm{I}]^{+}$, 860.4833; found, 860.4830.

Probe 5-B: $\quad$ Probe 5-B was prepared by BODIPY dye 5.3b and indolium derivative 5.4a with the same synthesis procedure of probe 5-A. BODIPY dye 5.3b (240 $\mathrm{mg}, 0.21 \mathrm{mmol})$, indolium derivative 5.4a (170 mg, 4 eq.), $6 \mathrm{~mL}$ of EtOH, pyrrolidine $(18 \mu \mathrm{L})$, give compound 5-B (230 mg, $75 \%)$ as dark purple oil. ${ }^{1} \mathrm{H}$ NMR (400 MHz, DMSO-d $)): \delta 8.10(\mathrm{~d}, J=15.6 \mathrm{~Hz}, 1 \mathrm{H}), 7.90(\mathrm{~d}, J=7.6 \mathrm{~Hz}, 1 \mathrm{H}), 7.85(\mathrm{~d}, J=7.6 \mathrm{~Hz}$, 1H), 7.62-7.55 (m, 2H), $7.20(\mathrm{~d}, J=8.4 \mathrm{~Hz}), 7.19(\mathrm{~s}, 1 \mathrm{H}), 7.00-6.92(\mathrm{~m}, 2 \mathrm{H}), 6.50$ (s, $1 \mathrm{H}), 4.50(\mathrm{t}, J=6.4 \mathrm{~Hz}, 2 \mathrm{H}), 4.07(\mathrm{~d}, J=5.2 \mathrm{~Hz}, 2 \mathrm{H}), 4.00(\mathrm{~s}, 2 \mathrm{H}), 3.57-3.33(\mathrm{~m}, 56 \mathrm{H})$, 3.21-3.18 (m, 12H), $2.82(\mathrm{~s}, 3 \mathrm{H}), 2.57$ (s, 3H), $1.86(\mathrm{sex}, 2 \mathrm{H}), 1.75(\mathrm{~s}, 6 \mathrm{H}), 1.72(\mathrm{~s}, 3 \mathrm{H})$, $1.54(\mathrm{~s}, 3 \mathrm{H}), 0.94(\mathrm{t}, J=7.2 \mathrm{~Hz}, 3 \mathrm{H}) .{ }^{13} \mathrm{C}$ NMR (100 MHz, DMSO-d6): $\delta$ 181.0, 173.3, 162.2 , 155.2, 153.0, 149.6, 149.5, 147.7, 145.2, 143.2, 142.8, 140.9, 139.9, 134.1, 130.7, $129.1,128.9,125.5,125.2,124.7,123.0,120.6,114.8,114.1,113.3,110.6,71.3,71.2$, $70.0,69.8,69.8,69.7,69.6,68.4,67.1,66.7,58.0,51.6,47.4,26.4,21.3,14.8,14.6,13.8$, 109 
12.9, 10.9. HRMS (ESI) calcd for $\mathrm{C}_{70} \mathrm{H}_{109} \mathrm{O}_{18} \mathrm{~N}_{3} \mathrm{~F}_{2} \mathrm{~B}$ [M-I] $]^{+}$, 1328.7767; found, 1328.7756.

Probe 5-C: Probe 5-C was prepared by BODIPY dye 5.3b and indolium derivative 5.4b with the same synthesis procedure of probe 5-A. BODIPY dye 5.3b (160 $\mathrm{mg}, 0.14 \mathrm{mmol}$ ), indolium derivative 5.4b (120 mg, 4 eq.), $5 \mathrm{~mL}$ of EtOH, pyrrolidine $(12 \mu \mathrm{L})$, give compound $3(100 \mathrm{mg}, 50 \%)$ as dark purple oil. ${ }^{1} \mathrm{H}$ NMR (400 MHz, DMSO- $\left.d_{6}\right): \delta 8.08(\mathrm{~d}, J=16 \mathrm{~Hz}, 1 \mathrm{H}), 7.96(\mathrm{~d}, J=7.6 \mathrm{~Hz}, 1 \mathrm{H}) .7 .81$ (d, $\left.J=7.6 \mathrm{~Hz}, 1 \mathrm{H}\right)$, 7.57 (m, 2H), 7.18 (d, $J=8.4 \mathrm{~Hz}, 1 \mathrm{H}) 7.11-7.05(\mathrm{~m}, 2 \mathrm{H}), 6.93(\mathrm{~d}, J=8 \mathrm{~Hz}, 1 \mathrm{H}), 6.47$ (s, 1H), $4.71(\mathrm{t}, 2 \mathrm{H}), 4.07$ (d, $J=5.6 \mathrm{~Hz}, 1 \mathrm{H}), 3.99$ (d, $J=4.8 \mathrm{~Hz}, 1 \mathrm{H}), 3.57-3.27(\mathrm{~m}, 56 \mathrm{H})$, 3.23-3.15 (m, 12H), 2.86 (s, 3H), 2.60-2.56 (m, 5H), 2.11 (quin, 2H), $1.72(\mathrm{~s}, 6 \mathrm{H}), 1.70$ (s, 3H), 1.53 (s, 3H). ${ }^{13} \mathrm{C}$ NMR (100 MHz, DMSO-d $)$ : $\delta 180.9,161.8,155.5,149.6$, $149.5,142.8,140.7,133.9,130.6,129.1,125.5,124.5,122.9,120.5,114.8,113.3,110.4$, $99.8,71.3,71.2,70.0,69.9,69.8,69.7,69.6,68.4,67.1,66.7,58.0,57.9,51.6,47.4,26.4$, 15.2, 14.6, 12.6. HRMS (ESI) calcd for $\mathrm{C}_{70} \mathrm{H}_{108} \mathrm{BF}_{2} \mathrm{~N}_{3} \mathrm{O}_{21} \mathrm{SNa}[\mathrm{M}+\mathrm{Na}]^{+}, 1430.7155$; found, 1430.7163 .

\subsubsection{Optical Measurements}

All the absorption and emission spectra were recorded by using standard $1 \mathrm{~cm}$ path length quartz fluorescence cuvette at room temperature. The slit width of excitation and emission were set to $3 \mathrm{~nm}$ and excitation wavelength is set at $470 \mathrm{~nm}$ for fluorescence spectroscopy. $100 \mathrm{mM}$ and $10 \mathrm{mM}$ stock solution of sodium cyanide in water and 2.0 $\mathrm{mM}$ stock solution of probe 5-A, 5-B and 5-C in acetonitrile were prepared for spectra titration studies. Quantitative amount of stock solution of probe (5-A, 5-B and 5-C) was transferred into small vials by pipettor. After drying of acetonitrile in vials, probe 5-A, 5B and 5-C was then diluted to the corresponding concentration $(20 \mu \mathrm{M})$ with mix solution of $\mathrm{CH}_{3} \mathrm{CN}$-Tris $(10 \mathrm{mM}, \mathrm{pH}=9.3,9: 1, \mathrm{v} / \mathrm{v})$ or Tris buffer $(10 \mathrm{mM}, \mathrm{pH}=9.3)$. Various sodium salts were used for anions selectivity measurements except for $\mathrm{HSO}_{4}^{-}$for which its potassium salt was used. Each absorption and fluorescence spectrum was recorded after stirring the solution for $30 \mathrm{~min}$ at room temperature. 


\subsubsection{Non-linear fitting of absorption titration data of probe 5-A}

This reaction is almost irreversible and has thermodynamic equilibrium. We assume 1:1 reaction of probe 5-A and $\mathrm{CN}^{-}$which can be expressed by the equation:

$$
5-\mathrm{A}+\mathrm{CN}^{-} \stackrel{K_{\text {ass }}}{\longrightarrow} 5-\mathrm{A}-\mathrm{CN}
$$

This reaction will give:

$$
K_{a s s}=\frac{[5-\mathrm{A}-\mathrm{CN}]}{[5-\mathrm{A}]\left[\mathrm{CN}^{-}\right]} \ldots \ldots \ldots . . .(\text { eq. } 5.1)
$$

Where $[5-\mathrm{A}-\mathrm{CN}],[5-\mathrm{A}]$ and $\left[\mathrm{CN}^{-}\right]$is the equilibrium concentration of addition product 5-A $-\mathrm{CN}$, probe 5-A and cyanide ion in buffer solution, respectively. Considering that $[5-\mathrm{A}]=[5-\mathrm{A}]_{0}-[5-\mathrm{A}-\mathrm{CN}]$ and $\left[\mathrm{CN}^{-}\right]=\left[\mathrm{CN}^{-}\right]_{0}-[5-\mathrm{A}-\mathrm{CN}]\left([5-\mathrm{A}]_{0}\right.$ and $\left[\mathrm{CN}^{-}\right]_{0}$ is the initial concentration of probe 5-A and cyanide ion respectively), the following equation can be obtained:

$$
[5-\mathrm{A}-\mathrm{CN}]=K_{\text {ass }} \times\left([5-\mathrm{A}]_{0}-[5-\mathrm{A}-\mathrm{CN}]\right)\left(\left[\mathrm{CN}^{-}\right]_{0}-[5-\mathrm{A}-\mathrm{CN}]\right)
$$

Solve equation 2 will give:

$$
\begin{aligned}
& {[5-\mathrm{A}-\mathrm{CN}]=\frac{1}{2}\left[\frac{1}{K_{\text {ass }}}+[5-\mathrm{A}]_{0}+\left[\mathrm{CN}^{-}\right]_{0}-\right.} \\
& \left.\sqrt{\left(\frac{1}{K_{\text {ass }}}+[5-\mathrm{A}]_{0}+\left[\mathrm{CN}^{-}\right]_{0}\right)^{2}-4[5-\mathrm{A}]_{0}\left[\mathrm{CN}^{-}\right]_{0}}\right]=\mathcal{F}\left([5-\mathrm{A}]_{0},\left[\mathrm{CN}^{-}\right]_{0}\right)
\end{aligned}
$$

From Beer's law, the absorbance at $546 \mathrm{~nm}$ is given by follow:

$$
\begin{aligned}
& A=\varepsilon_{1} l[5-\mathrm{A}]+\varepsilon_{2} l[5-\mathrm{A}-\mathrm{CN}] \\
& A_{0}=\varepsilon_{1} l[5-\mathrm{A}]_{0} \\
& A_{\text {min }}=\varepsilon_{2} l[5-\mathrm{A}-\mathrm{CN}]_{0}
\end{aligned}
$$

$A_{0}$ is the absorbance of probe 5-A without addition of cyanide ion. $A_{\min }$ is the absorbance of probe 5-A with addition of excess amount of cyanide ion. $\varepsilon_{1}$ and $\varepsilon_{2}$ is the 
extinction coefficient of 5-A and 5-A-CN, respectively. The equation 5.2 can be replaced as follows:

$$
\frac{A-A_{0}}{\left(A-A_{\min }\right) K_{\text {ass }}}+\left[\mathrm{CN}^{-}\right]_{0}=[5-\mathrm{A}-\mathrm{CN}]
$$

From equation (3) and (4), the following equation can be obtained:

$$
A=\frac{A_{\min } K_{\text {ass }}\left\{\mathcal{F}\left([5-\mathrm{A}]_{0},\left[\mathrm{CN}^{-}\right]_{0}\right)-\left[\mathrm{CN}^{-}\right]_{0}\right\}-A_{0}}{K_{\text {ass }}\left\{\mathcal{F}\left([5-\mathrm{A}]_{0},\left[\mathrm{CN}^{-}\right]_{0}\right)-\left[\mathrm{CN}^{-}\right]_{0}\right\}-1} \ldots \ldots . . .(\text { eq. 5.5) }
$$

The equation 5.5 was used for non-linear fitting of absorption titration data of probe 5-A with $\left[\mathrm{CN}^{-}\right]_{0}$. The parameter $K_{\text {ass }}$ was determined to be $K_{\text {ass }}=(3.3 \pm 0.6) \times 10^{5} \mathrm{M}^{-1}$

\subsubsection{Computational Methods}

Results for the optimized structures and the relative electronic properties were obtained at B3LYP and 6-311G(d,p) level of theory as implemented in Gaussian09. The Continuum Polarizable Conductor Model (CPCM) was employed to consider the effect of acetonitrile as the solvent used to experimentally determine the optical properties of probe 5-A and 5-A-CN. For both the structures, TD-DFT calculations were performed to investigate excitations to the first singlet excited state on the geometries as previously optimized at DFT level.

\subsection{Results and discussions}

\subsubsection{Optical properties}

BODIPY dye 5.2a shows absorption and emission peaks at $504 \mathrm{~nm}$ and $513 \mathrm{~nm}$, respectively, and possesses high fluorescence quantum yield of $92 \%$ in methylene chloride solution. 2-Formyl-BODIPY dye (5.3a) displays a lower fluorescence quantum yield and shows a slight shift by $9 \mathrm{~nm}$ in absorption compared to BODIPY dye (5.2a) (please see Figure C.21 in the Appendix C). Fluorescent probe 5-A exhibits two emission peaks at $515 \mathrm{~nm}$ and $605 \mathrm{~nm}$ (see Table 5.1 and Figure C.22 in the Appendix C). The formation of the emission peak at $515 \mathrm{~nm}$ likely arises from the steric hindrance between methyl group of BODIPY core at 1,3-positions and vinylic protons, which makes them be 
slightly nonplanar and deconjugated, and thus causes appearance of emission peak of BODIPY core at $515 \mathrm{~nm}$. This steric hindrance is further confirmed by the broad absorption peak of the probe (please see Figure C.22 in the Appendix C). Fluorescent probes 5-B and 5-C display similar optical properties in Tris buffer (10 mM, pH 9.3) as fluorescent probe 5-A (Table 5.1).

Table 5.1. Maximum absorption peaks, emission peaks and extinction coefficients of probe 5-A - 5-C and their $\mathrm{CN}$ adducts

\begin{tabular}{|c|c|c|c|c|}
\hline & $\lambda_{\text {abs }}(\mathrm{nm})$ & $\lambda_{\text {em }}(\mathrm{nm})$ & $\varepsilon_{\max }\left(\times 10^{4} \mathrm{M}^{-1} \mathrm{~cm}^{-1}\right)$ & Solvent \\
\hline 5-A & 564 & 605 & 6.8 & ACN-Tris buffer mix \\
\hline 5-A-CN & 521 & 555 & 5.8 & ACN-Tris buffer mix \\
\hline 5-B & 558 & 596 & 3.7 & Tris buffer \\
\hline 5-B-CN & 522 & 559 & 2.3 & Tris buffer \\
\hline 5-C & 560 & 600 & 5.2 & Tris buffer \\
\hline 5-C-CN & 520 & 550 & 3.7 & Tris buffer \\
\hline
\end{tabular}

\subsubsection{Spectroscopic responses to cyanide ion and concentration dependency}

We studied the concentration-dependent changes of fluorescence and absorption spectra of probe 5-A in the absence and presence of different equivalents of cyanide ion (Figures 5.1-5.3). Addition of sodium cyanide results in appearance of a new peak at 555 $\mathrm{nm}$, and a drastic decrease in the peak at $605 \mathrm{~nm}$. The new peak increases considerably with gradual addition of cyanide ion and a well-defined isoemissive point shows up at $598 \mathrm{~nm}$ (Figure 5.1). 4.5 Equivalents of cyanide ion is sufficient to make the reaction completed, showing that the probe is highly reactive to cyanide ions. There is an up to 17 -fold increase in the fluorescence ratio $\left(I_{555} / I_{605}\right)$ in the presence of 4.5 equivalents of cyanide ion (Figure 5.1). The detection limit $(\mathrm{S} / \mathrm{N}=3)$ was calculated to be $0.5 \mu \mathrm{M}$ with a linear ratio response $\left(I_{555} / I_{605}\right)$ of the fluorescence intensity at $555 \mathrm{~nm}$ and $605 \mathrm{~nm}$ to cyanide concentration ranging from $5.0 \mu \mathrm{M}$ to $50.0 \mu \mathrm{M}$ (Figure 5.1, inset). The nucleophilic addition of the cyanide anion toward the iminium ion causes breakage of the indole conjugation to BODIPY core in the probe 5-A, resulting in significant dual color and fluorescence changes. Fluorescent probe 5-C shows highly sensitive response to 
cyanide ion with lower detection limit of $0.1 \mu \mathrm{M}$, significant decrease of emission peak at $600 \mathrm{~nm}$, and appearance of a new emission peak at $550 \mathrm{~nm}$ in $10 \mathrm{mM}$ Tris buffer (pH 9.3) (please see Figure C.34 in Appendix C). A very good linear ratio response $\left(I_{550} / I_{600}\right)$ of the fluorescence intensity at $550 \mathrm{~nm}$ and $600 \mathrm{~nm}$ to cyanide concentration was observed ranging from $0.5 \mu \mathrm{M}$ to $50.0 \mu \mathrm{M}$ in $10 \mathrm{mM}$ Tris buffer ( $\mathrm{pH}$ 9.3) (Figure 5.2) which indicates that the probe 5-C has potential useful application in the quantitative detection of cyanide ion in aqueous solution. Addition of cyanide ion to the probe 5-B in Tris buffer ( $\mathrm{pH}$ 9.3) resulted in similar fluorescence ratio responses to cyanide ion with poor linearship of the fluorescence ratio to cyanide concentration as the precipitation of the reaction product was observed due to the lack of charge repulsion of the neutral reaction product. These results indicate that propanesulfonate residue in probe 5-C plays a very important role in enhancing water solubility of the reaction product of the fluorescent probe with cyanide ion and preventing the dye aggregation via charge repulsion between propansulfonate groups in order to achieve sensitive detection of cyanide ion.

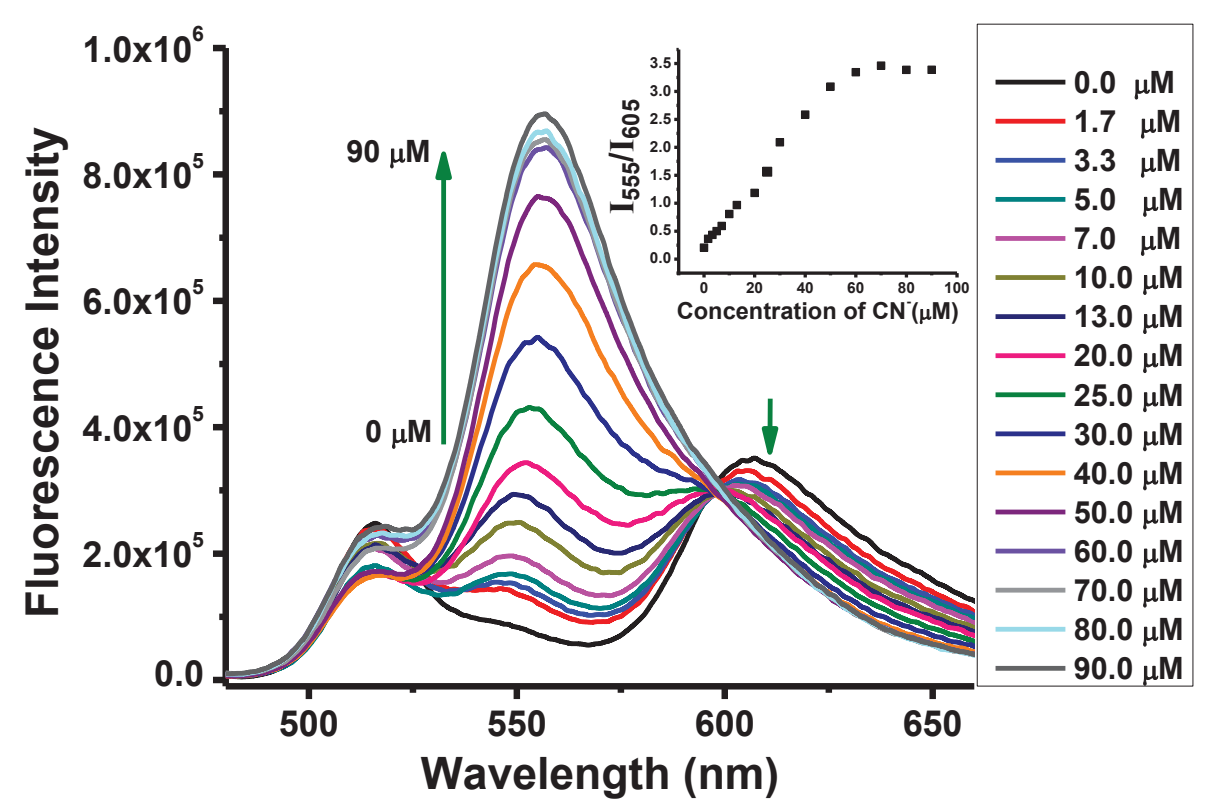

Figure 5.1. Fluorescence spectra of the fluorescent probe 5-A $(20 \mu \mathrm{M})$ in the absence and presence of different amounts of cyanide ion in a mixed solution of $\mathrm{CH}_{3} \mathrm{CN}$ and Tris buffer $(10 \mathrm{mM}, \mathrm{pH}=9.3)(9: 1, \mathrm{v} / \mathrm{v})$. Inset curve is plot of fluorescent intensity ratio 
change at $555 \mathrm{~nm}$ and $605 \mathrm{~nm}\left(\mathrm{I}_{555} / \mathrm{I}_{605}\right)$ of the fluorescent probe 5-A upon titration of cyanide ion.

Figure 5.3 shows the absorption spectral changes of fluorescent probe 5-A on addition of cyanide ion in a mixed solution of $\mathrm{CH}_{3} \mathrm{CN}$-Tris buffer $(10 \mathrm{mM} \mathrm{pH}=9.3,9$ : $1, \mathrm{v} / \mathrm{v})$. Gradual addition of cyanide ion to probe 5-A also causes emergence of a new absorption band at $521 \mathrm{~nm}$, and significant decreases of the absorption band at $564 \mathrm{~nm}$, which completely disappears with addition of 4.5 equivalents of cyanide ion (Figure 5.3). A clean isosbestic point at $528 \mathrm{~nm}$ showed an interconversion into single distinct chemical species during the titration. This 1:1 stoichiometry was further confirmed by a good non-linear fitting of the titration data at $564 \mathrm{~nm}$ by assuming a 1:1 association between probe 5-A and cyanide (Figure 5.3 inset). The apparent association constant was determined to be $3.3 \times 10^{5} \mathrm{M}^{-1}$. Probes 5-B and 5-C display similar absorption responses to cyanide ion in $10 \mathrm{mM}$ Tris buffer (pH 9.3) (Figures C.29 and C.35 in Appendix C).

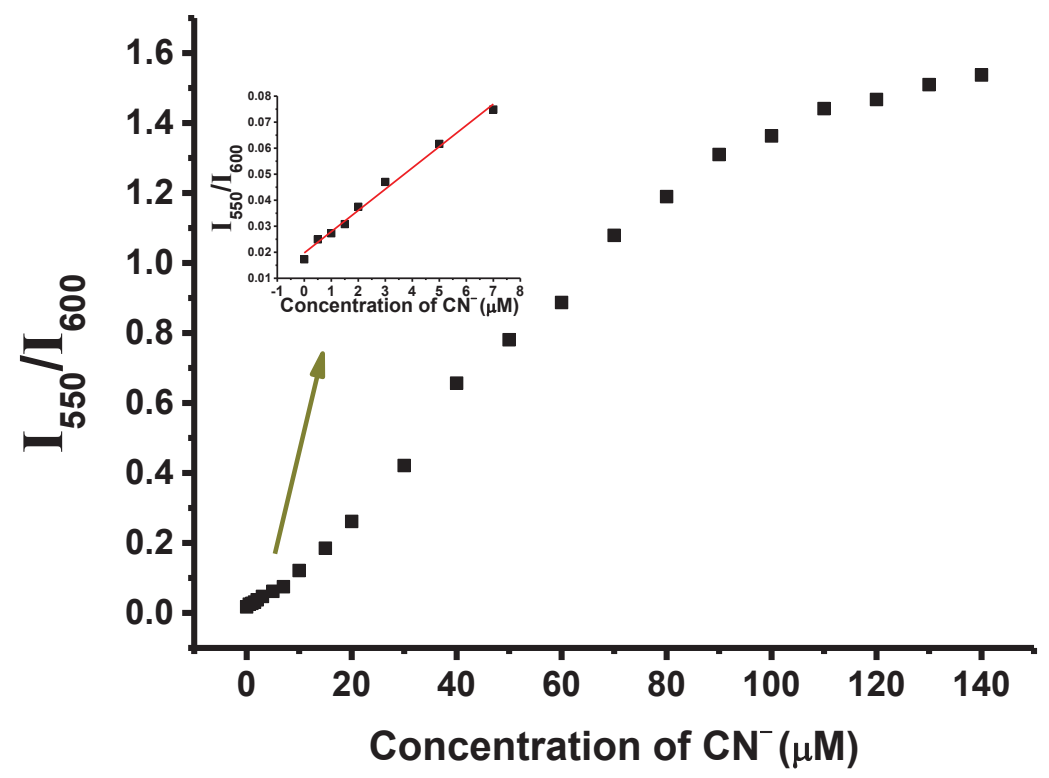

Figure 5.2. Fluorescent intensity ratio change at $550 \mathrm{~nm}$ and $600 \mathrm{~nm}\left(\mathrm{I}_{550} / \mathrm{I}_{600}\right)$ of the fluorescent probe 5-C $(20 \mu \mathrm{M})$ upon titration of cyanide ion in Tris buffer $(10 \mathrm{mM}, \mathrm{pH}$ 9.3) solution. 


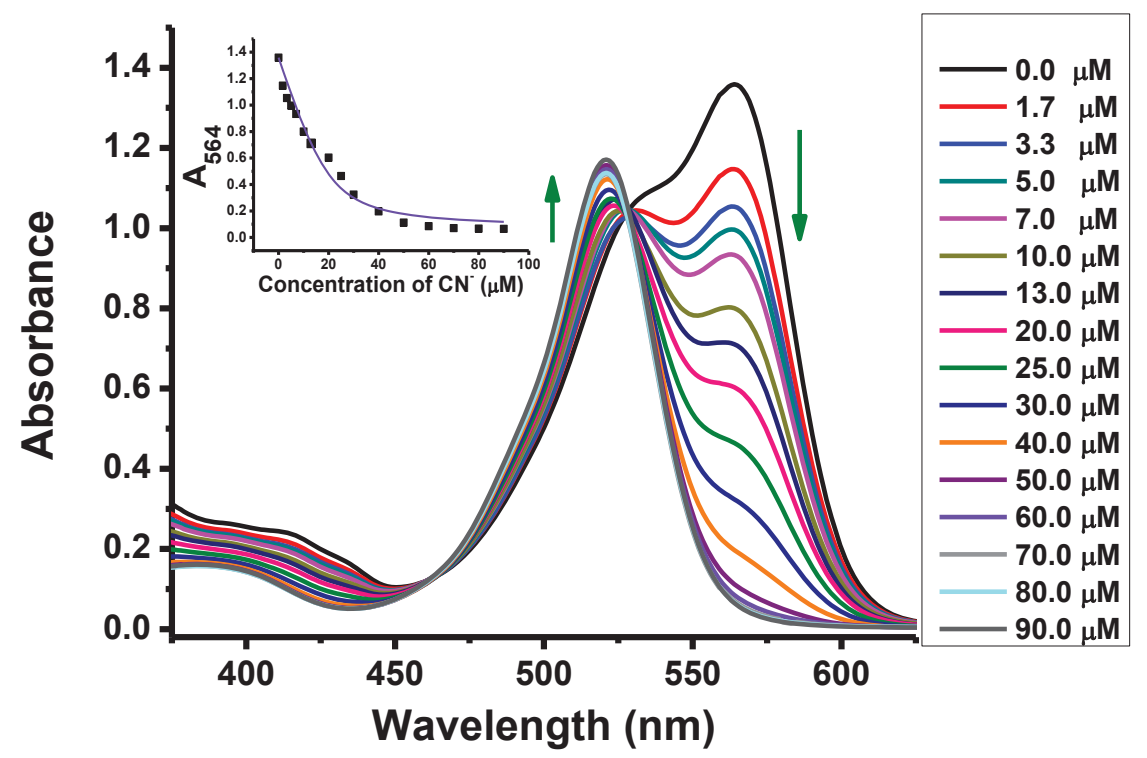

Figure 5.3. Absorption spectra of the probe 5-A $(20 \mu \mathrm{M})$ in the absence and presence of different amount of cyanide ion in a mixed solution of $\mathrm{CH}_{3} \mathrm{CN}$-Tris buffer $(10 \mathrm{mM} \mathrm{pH}=$ 9.3, $9: 1, \mathrm{v} / \mathrm{v})$. Inset: plot of absorbance change $\left(A_{564}\right)$ of probe 5-A upon addition of cyanide ion, where the line is the non-linear fitting curve obtained assuming a $1: 1$ reaction of probe $\mathbf{5 - A}$ with cyanide ion.

\subsubsection{Theoretical modeling}

In order to shed light on the interesting absorption and emission feature of the fluorescent probe 5-A and the reaction product $\mathbf{5 - A - C N}$, we investigated their structures by using density functional theory (DFT) and time-dependent DFT (TD-DFT) computational approaches with the Gaussian 09 programs. ${ }^{21}$ Figure 5.4 shows the optimized geometries for both structures. In the probe $\mathbf{5 - A}$, the expected steric hindrance is confirmed and it translates into a slightly non-planar arrangement of the structure (Figure 5.4). The DFT and TD-DFT optimized structures of probe 5-A and the reaction production 5-A-CN show that the probe 5-A possesses a conjugated $\mathrm{sp}^{2}$ hydridized carbon bond between BODIPY core and indole group while a $\mathrm{sp}^{3}$ hybridized carbon in the reaction product $\mathbf{1 - C N}$ is perpendicular to the BODIPY core (Figure 5.4). The significant structure difference between the probe 5-A and the reaction product 5-A-CN results in considerable difference in their $\pi$-conjugations. As a result, the electron 
densities of LUMO of 5-A-CN are only located on the BODIPY core while those of the probe 5-A are spread over BODIPY core through the indole moiety. The fluorescence enhancement of the reaction product $\mathbf{5}-\mathbf{A}-\mathbf{C N}$ arises from the prevention of intramolecular charge transfer (ICT) between BODIPY core and indole moiety because of the conjugation disruption between the BODIPY core and indole group via the $\mathrm{sp}^{3}$ hybridized carbon. Detailed understanding regarding the absorption and emission blue shift upon the formation of 5-A-CN can be obtained via TD-DFT calculation as well. The lowest singlet electronic transitions of both probe 5-A and reaction product $\mathbf{5}-\mathbf{A}-\mathbf{C N}$ are mainly contributed by HOMO-LUMO transitions (Figure 5.4). The calculated energy gap of probe $\mathbf{5 - A}$ is $2.25 \mathrm{eV}(551 \mathrm{~nm})$ whereas the gap of $\mathbf{5 - A}-\mathrm{CN}$ is $2.45 \mathrm{eV}(505 \mathrm{~nm})$. This energy gap difference gives a $44 \mathrm{~nm}$ blue shift in absorption bands which is in excellent agreement with experimental data $(43 \mathrm{~nm})$. 


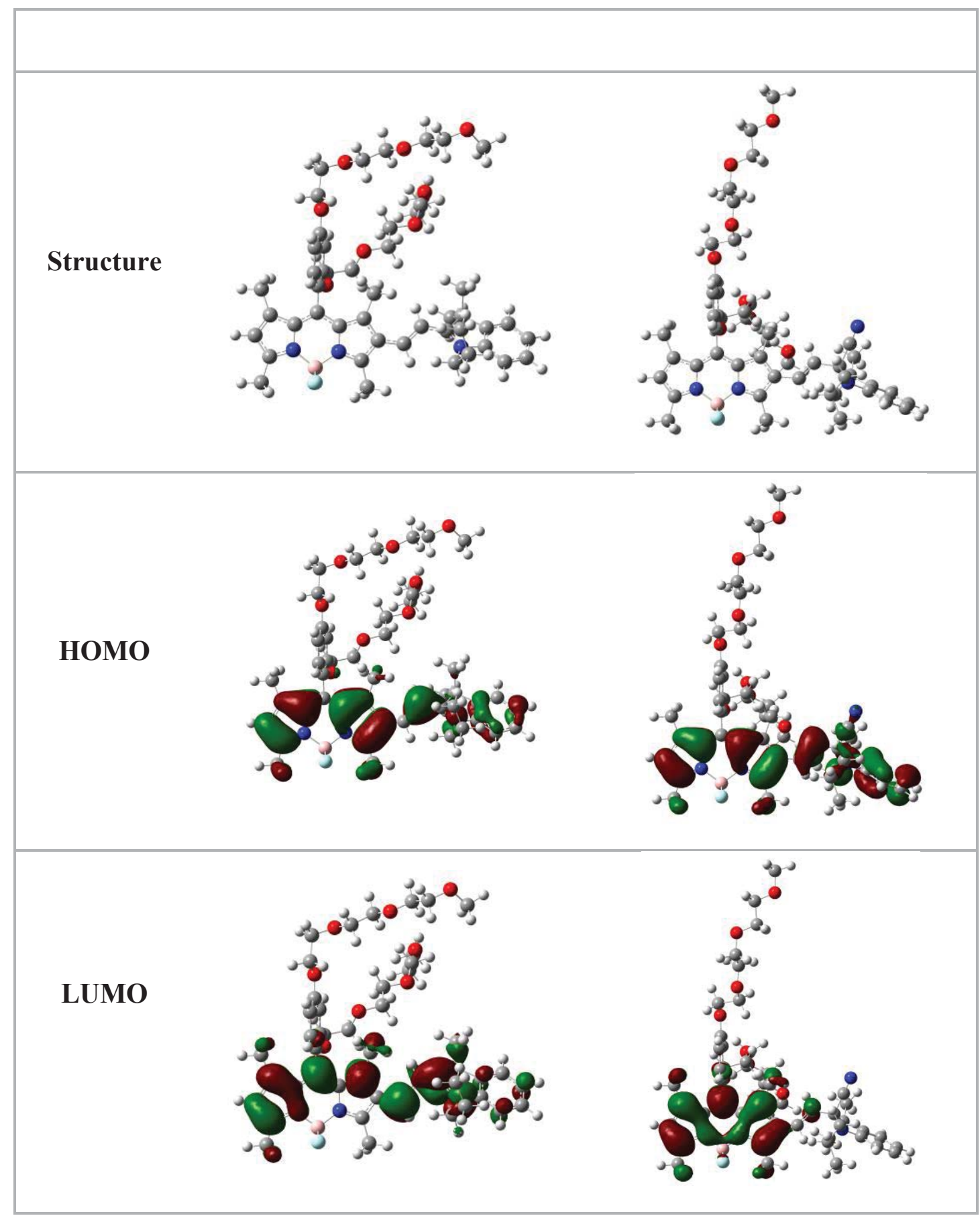

Figure 5.4. Optimized structures Optimized structures of probe 5-A (left) and reaction product 5-A-CN (right) and their electron distributions for the HOMO and LUMO levels as obtained from TD-DFT calculations. 


\subsubsection{NMR study and stoichiometry}

We used ${ }^{1} \mathrm{H}$ NMR and mass spectroscopy to investigate the potential interaction mechanism of probe 5-A with cyanide ion. A FAB-MS spectrometric analysis of the fluorescent probe treated with $\mathrm{CN}^{-}$in aqueous solution showed signals for the formation of 1-CN adduct $(m / z, 886.49)$. We also studied and compared the ${ }^{1} \mathrm{H}$ NMR spectra of the fluorescent probe 5-A in the absence and presence of cyanide anions. Addition of one equivalent of cyanide ion to the fluorescent probe at room temperature causes significant upfield shifts of several different proton peaks, which include changes of doublet peaks corresponding to vinylic proton at a-position from $\delta 8.10 \mathrm{ppm}$ to $\delta 6.75 \mathrm{ppm}$, doublet peaks corresponding to vinylic proton at b-position from $\delta 6.96 \mathrm{ppm}$ to $\delta 5.84 \mathrm{ppm}$, singlet peak corresponding to proton at g-position from $\delta 6.47 \mathrm{ppm}$ to $\delta 6.22 \mathrm{ppm}$, doublet peaks corresponding to proton of indolium moiety at d-position from $\delta 7.84 \mathrm{ppm}$ to $\delta 7.07 \mathrm{ppm}$, and doublet peaks related to proton of indolium moiety at c-position from $\delta 7.89 \mathrm{ppm}$ to $\delta 6.67 \mathrm{ppm}$ (Figure 5.5), indicating the formation of the 5-A-CN adduct. These upfield chemical shifts arise from disappearance of a positive charge in the fluorescent probe after the formation of the 5-A-CN adduct, and are consistent with the formation of the cyanoindolium moiety by the nucleophilic attack of cyanide anion toward iminium carbon. ${ }^{1} \mathrm{H}$ NMR analysis implied that the cyanide anion functions as a nucleophile in DMSO solution. Addition of more than one equivalent of cyanide doesn't cause further chemical shift, implying that that a 1:1 adduct between probe $\mathbf{1}$ and $\mathrm{CN}^{-}$is formed. Job's analysis for the addition reaction of the fluorescent probe with cyanide ion also corroborated the 1:1 reaction stoichiometry (Figure 5.6). 

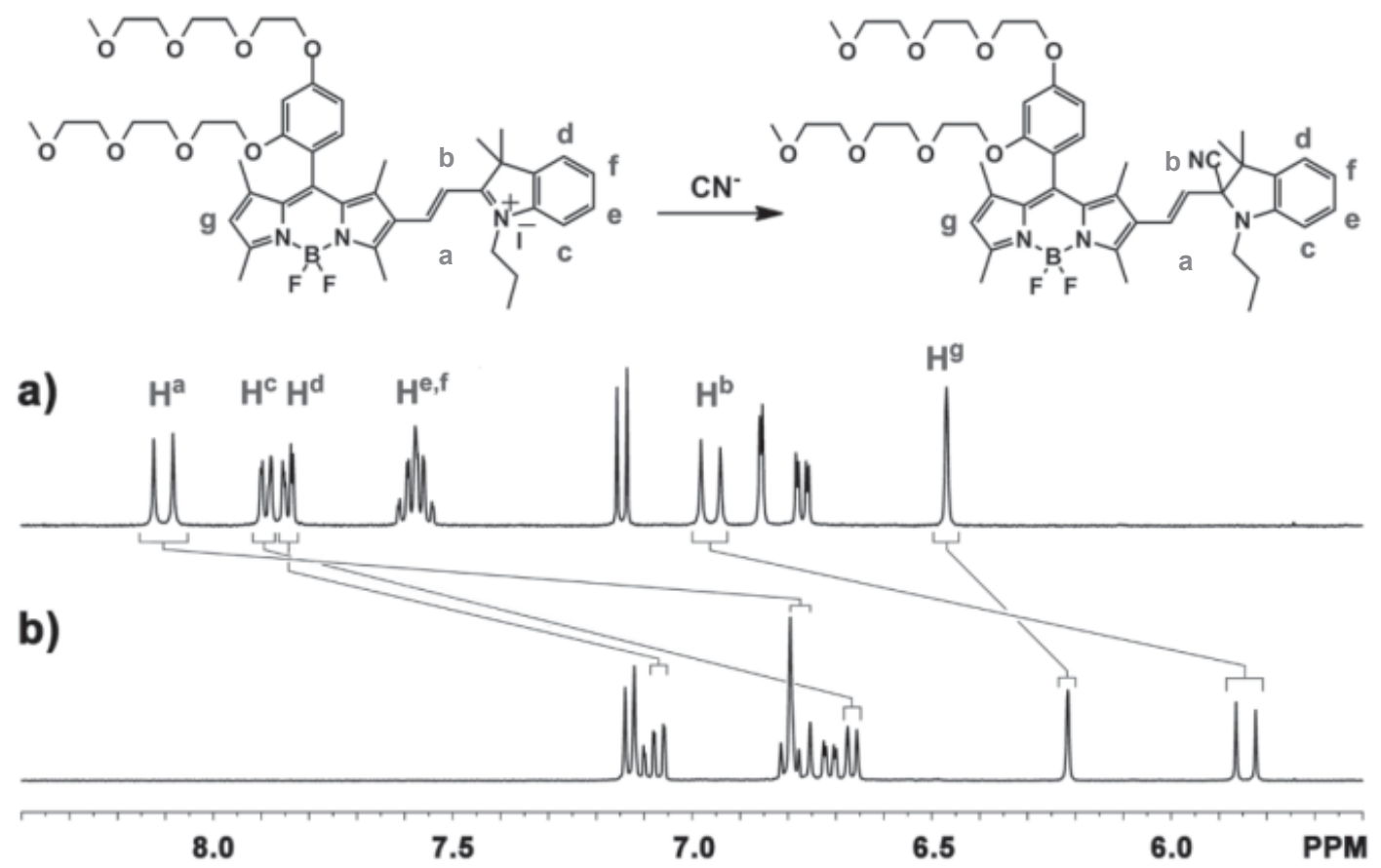

Figure 5.5. ${ }^{1} \mathrm{H}$ NMR spectra of probe 5-A $(10 \mathrm{mM})$ in the absence (a) and presence (b) of cyanide ion (one equivalent) in DMSO- $d_{6}$ solution at room temperature.
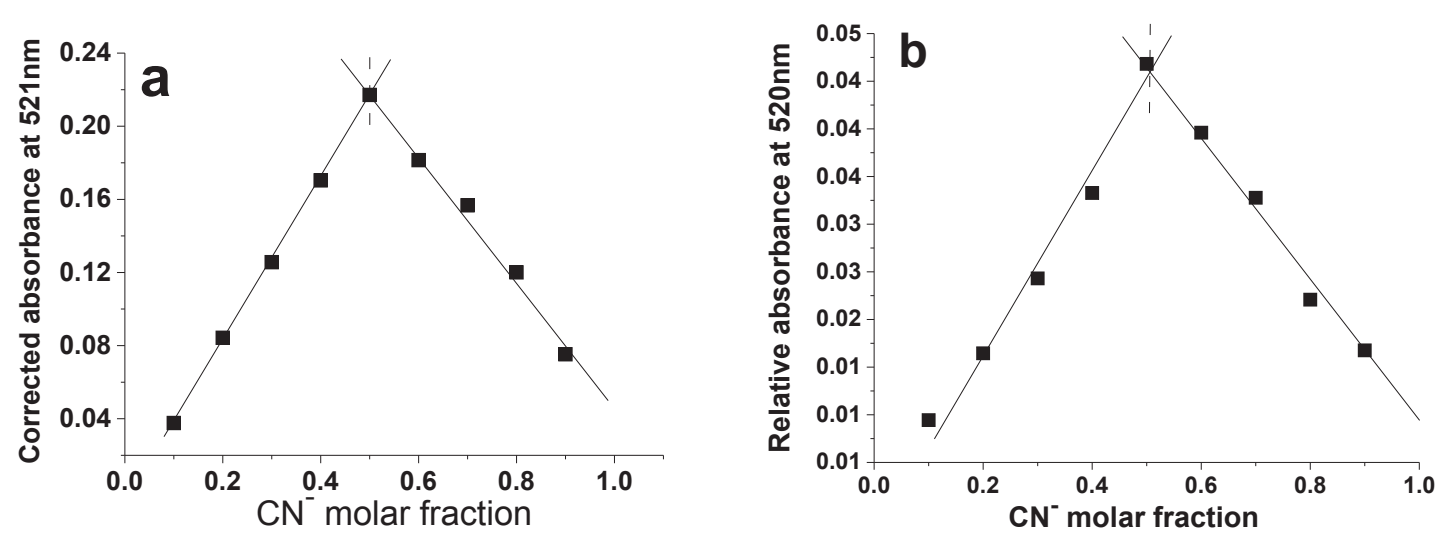

Figure 5.6. Job's plot of probe 5-A (a) with cyanide ion in mixed solution of $\mathrm{CH}_{3} \mathrm{CN}$ and Tris buffer $(10 \mathrm{mM} \mathrm{pH}=9.3,9: 1, \mathrm{v} / \mathrm{v})$ and 5-C (b) with cyanide ion Tris buffer $(10 \mathrm{mM}$ $\mathrm{pH}=9.3$ ). Total concentration of probe $\mathbf{5 - A}$ or probe $\mathbf{5 - C}$ and cyanide ion is $50 \mu \mathrm{M}$. 


\subsubsection{Selectivity}
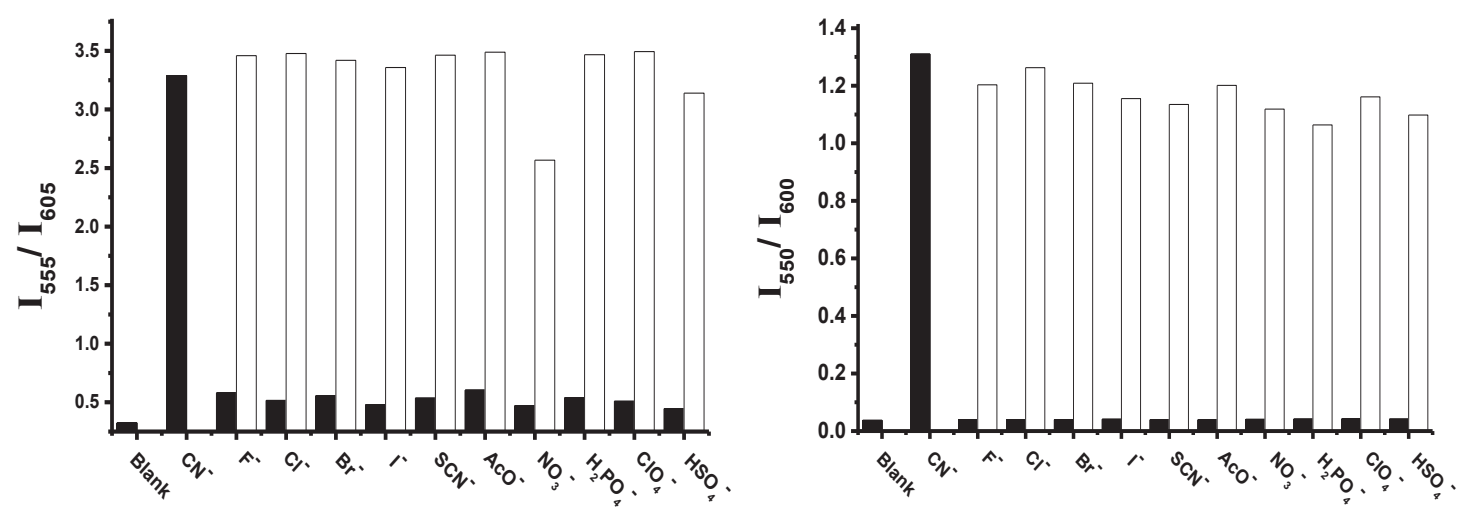

Figure. 5.7. The fluorescent ratiometric responses of probe 5-A (left) $(20 \mu \mathrm{M})$ and probe 5-C (right) $(20 \mu \mathrm{M})$ to different anions $(100 \mu \mathrm{M})$ in the presence and presence of $60 \mu \mathrm{M}$ and $90 \mu \mathrm{M}$ cyanide ion in mixed solution of $\mathrm{CH}_{3} \mathrm{CN}$ and Tris buffer $(10 \mathrm{mM}, \mathrm{pH}=9.3)$ $(9: 1, \mathrm{v} / \mathrm{v})$ and $100 \%$ Tris buffer $(10 \mathrm{mM}, \mathrm{pH}=9.3)$ respectively. Black bars show the fluorescent responses of the probes to different anions while white bars display the fluorescent responses of the probes to different anions in the presence of cyanide ion.

We used different anionic species such as $\mathrm{F}^{-}, \mathrm{Cl}^{-}, \mathrm{Br}^{-}, \mathrm{I}^{-}, \mathrm{SCN}^{-}, \mathrm{NO}_{3}{ }^{-}, \mathrm{CHCOO}^{-}$, $\mathrm{SO}_{4}{ }^{2-}, \mathrm{HSO}_{4}^{-}$, and $\mathrm{H}_{2} \mathrm{PO}_{4}^{-}$to study the selectivity of the probe 5-A in the absence and presence of cyanide ions (Figures 5.7, left). Among these various anion species tested, only cyanide ions responds to fluorescent probe 5-A with large blue shifts by $50 \mathrm{~nm}$ and $43 \mathrm{~nm}$ in emission and absorption spectra, respectively (Figures 6, left and Figure C.23C.24 in Appendix C), and causes a remarkable visible color change from purple to pink (see Figure C.38 in Appendix C), and red to green in the absence and presence of a transilluminator, respectively. The presence of other anions such as $\mathrm{F}^{-}, \mathrm{Cl}^{-}, \mathrm{Br}^{-}, \mathrm{I}^{-}, \mathrm{SCN}^{-}$ , $\mathrm{NO}_{3}{ }^{-}, \mathrm{CHCOO}^{-}, \mathrm{SO}_{4}{ }^{2-}, \mathrm{HSO}_{4}^{-}$or $\mathrm{H}_{2} \mathrm{PO}_{4}{ }^{-}$did not cause any significant change in emission peak at $605 \mathrm{~nm}$, but result in slight fluorescence enhancement at $515 \mathrm{~nm}$, and slight decrease in absorption peak at $564 \mathrm{~nm}$. The fluorescence ratio data $\left(I_{555} / I_{605}\right)$ reveal that probe 5-A is highly selective toward cyanide over other common inorganic anions, which is due to the fact that cyanide is more nucleophilic than other anions in aqueous solution. These results indicate that fluorescent probe 5-A may have potential and promising application in detection of cyanide ion in the presence of other different 
anions. The probes 5-B and 5-C display the same good selectivity to cyanide ion over other anionic species as the probe 5-A (please see Appendix C and the Figure 5.7, right).

\subsection{Conclusion}

In summary, we have successfully prepared selective and sensitive BODIPY-based probes for ratiometric fluorescent detection of cyanide ion. The highly water-soluble fluorescent probe 5-C shows great potential application in detection of cyanide ion in aqueous solution.

\section{References:}

1. Baskin, S. I., Brewer, T. G., In Medical Aspects of Chemical and Biological Warfare. TMM Publications: Washington, DC, 1997; Vol. Chapter 10.

2. Vennesland, B., Comm, E. E., Knownles, C. J., Westly, J., Wissing, F., Cyanide in Biology. Academic Press: London, 1981.

3. Bhattacharya, P. K., Metal Ions in Biochemistry. Alpha Science International Ltd.: Harrow, U.K., 2005.

4. Young, C., Tidwell, L., Anderson, C., Cyanide: Social, Industrial, and Economic Aspects. Minerals, Metals, and Materials Society: Warrendale, 2001.

5. Sessler, J. L., Cho, D.-G., The Benzil Rearrangement Reaction: Trapping of A Hitherto Minor Product and Its Application to The Development of A Selective Cyanide Anion Indicator. Organic Letters 2008, 10 (1), 73-75.

6. Lv, X., Liu, J., Liu, Y., Zhao, Y., Sun, Y.-Q., Wang, P., Guo, W., Ratiometric Fluorescence Detection of Cyanide Based on A Hybrid Coumarin-Hemicyanine Dye: The Large Emission Shift and The High Selectivity. Chemical Communications 2011, 47 (48), 12843-12845.

7. Yuan, L., Lin, W., Yang, Y., Song, J., Wang, J., Rational Design of A Highly Reactive Ratiometric Fluorescent Probe for Cyanide. Organic Letters 2011, 13 (14), 3730-3733. 
8. Lee, K.-S., Kim, H. J., Kim, G.-H., Shin, I., Hong, J.-I., Fluorescent Chemodosimeter for Selective Detection of Cyanide in Water. Organic Letters 2008, 10 (1), 49-51.

9. Chen, C.-L., Lin, T.-P., Chen, Y.-S., Sun, S.-S., Probing Receptor-anion Interactions by Ratiometric Chemosensors Containing Pyrrolecarboxamide Interacting Sites. European Journal of Organic Chemistry 2007, (24), 39994010 .

10. Chung, Y. M., Raman, B., Kim, D. S., Ahn, K. H., Fluorescence Modulation in Anion Sensing By Introducing Intramolecular H-Bonding Interactions in HostGuest Adducts. Chemical Communications 2006, (2), 186-188.

11. Jamkratoke, M., Ruangpornvisuti, V., Tumcharern, G., Tuntulani, T., Tomapatanaget, B., A-D-A Sensors Based on Naphthoimidazoledione and Boronic Acid as Turn-On Cyanide Probes in Water. Journal of Organic Chemistry 2009, 74 (10), 3919-3922.

12. Jung, H. S., Han, J. H., Kim, Z. H., Kang, C., Kim, J. S., Coumarin-Cu(II) Ensemble-Based Cyanide Sensing Chemodosimeter. Organic Letters 2011, 13 (19), 5056-5059.

13. Lou, X., Qiang, L., Qin, J., Li, Z., A New Rhodamine-Based Colorimetric Cyanide Chemosensor: Convenient Detecting Procedure and High Sensitivity and Selectivity. Acs Applied Materials \& Interfaces 2009, 1 (11), 2529-2535.

14. Loudet, A., Burgess, K., BODIPY Dyes and Their Derivatives: Syntheses and Spectroscopic Properties. Chemical Reviews 2007, 107, 4891-4932.

15. Ekmekci, Z., Yilmaz, M. D., Akkaya, E. U., A Monostyryl-boradiazaindacene (BODIPY) Derivative as Colorimetric and Fluorescent Probe for Cyanide Ions. Organic Letters 2008, 10 (3), 461-464.

16. Ziessel, R., Insights into A Largely Unexplored Chemistry: That of Boradipyrromethenes. Comptes Rendus Chimie 2007, 10 (7), 622-629.

17. Ziessel, R., Ulrich, G., Harriman, A., The Chemistry of Bodipy: A New El Dorado for Fluorescence Tools. New Journal of Chemistry 2007, 31 (4), 496-501. 
18. Zhu, S., Zhang, J., Vegesna, G., Luo, F.-T., Green, S. A., Liu, H., Highly Watersoluble Neutral BODIPY Dyes with Controllable Fluorescence Quantum Yields. Organic Letters 2011, 13 (3), 438-441.

19. Zhu, S., Zhang, J., Vegesna, G., Tiwari, A., Luo, F.-T., Zeller, M., Luck, R., Li, H., Green, S., Liu, H., Controlled Knoevenagel Reactions of Methyl Groups of 1,3,5,7-Tetramethyl BODIPY Dyes for Unique BODIPY Dyes. RSC Adv., 2011, 2, 404-407.

20. Zhu, S., Zhang, J., Vegesna, G. K., Pandey, R., Luo, F.-T., Green, S. A., Liu, H., One-pot Efficient Synthesis of Dimeric, Trimeric, and Tetrameric BODIPY Dyes for Panchromatic Absorption. Chemical Communications 2011, 47 (12), 35083510.

21. Frisch, M. J., Trucks, G. W., Schlegel, H. B., Scuseria, G. E., Robb, M. A., Cheeseman, J. R., Scalmani, G., Barone, V., Mennucci, B., Petersson, G. A., Nakatsuji, H., Caricato, M., Li, X., Hratchian, H. P., Izmaylov, A. F., Bloino, J., Zheng, G., Sonnenberg, J. L., Hada, M., Ehara, M., Toyota, K., Fukuda, R., Hasegawa, J., Ishida, M., Nakajima, T., Honda, Y., Kitao, O., Nakai, H., Vreven, T., Montgomery, J. A., Peralta, J., J. E. , Ogliaro, F., Bearpark, M., Heyd, J. J., Brothers, E., Kudin, K. N., Staroverov, V. N., Kobayashi, R., Normand, J., Raghavachari, K., Rendell, A., Burant, J. C., Iyengar, S. S., Tomasi, J., Cossi, M., Rega, N., Millam, J. M., Klene, M., Knox, J. E., Cross, J. B., Bakken, V., Adamo, C., Jaramillo, J., Gomperts, R., Stratmann, R. E., Yazyev, O., Austin, A. J., Cammi, R., Pomelli, C., Ochterski, J. W., Martin, R. L., Morokuma, K., Zakrzewski, V. G., Voth, G. A., Salvador, P., Dannenberg, J. J., Dapprich, S., Daniels, A. D., Farkas, Ö., Foresman, J. B., Ortiz, J. V., Cioslowski, J., Fox, D. J., Gaussian 09. Gaussian, Inc.: Wallingford CT, 2009. , 2009. 


\section{Chapter 6. Highly Water-soluble BODIPY-based Fluorescent Probes for Sensitive Fluorescent Sensing of Zinc (II) ${ }^{1}$}

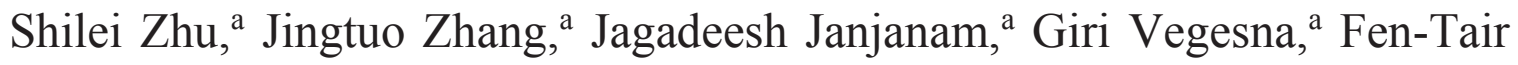
Luo, ${ }^{\mathrm{b}}$ Ashutosh Tiwari*a and Haiying Liu*a

${ }^{a}$ Department of Chemistry, Michigan Technological University, 1400 Townsend Drive, Houghton, MI 49931, USA

${ }^{\mathrm{b}}$ Institute of Chemistry, Academia Sinica, Taipei, Taiwan 11529, Republic of China

\footnotetext{
${ }^{1}$ The material included in this chapter was previously published in [Journal of Materials Chemistry B 2013, $l$ (12), 1722-1728. DOI: 10.1039/C3TB00249G Publication Date (Web): Jan 21, 2013]-Reproduced by permission of The Royal Society of Chemistry http://pubs.rsc.org/en/content/articlelanding/2013/tb/c3tb00249g\#!divAbstract
} 


\subsection{Introduction}

There are wide variety of essential metal ions such as copper, manganese, iron, magnesium and zinc required for normal functioning of the living organisms. Zinc is an essential trace element, and is necessary for the normal functioning of all living systems including plants, animals, and even microorganisms. ${ }^{1}$ In the human body, zinc is the second most abundant transition-metal ion and plays very important roles as structural and catalytic cofactor of more than 300 metaloproteins associated with gene expression, apoptosis, enzyme regulation, and neuronal transmission. ${ }^{1}$ There are pools of loosely bound or chelatable zinc in some organs such as brain, pancreas and spermatozoa. Zinc (II) plays numerous critical roles in cellular metabolism, gene expression, apoptosis, and co-factors in metalloenzyme catalysis, and neurotransmission in biological systems. ${ }^{1-3}$ The pathology of neurological disorders such as Alzheimer's disease, cerebral ischemia and epilepsy may also arise from the disruption of $\mathrm{Zn}$ (II) homeostasis. Zinc (II) is reported to be in the micro- to millimolar range in synaptic vesicles of many excitatory forebrain neurons. ${ }^{4}$ This vesicular zinc (II) is also released into synaptic space with estimated concentration ranging from $10 \mu \mathrm{M}$ to $30 \mu \mathrm{M} .^{5-6}$ Therefore, effective in vivo detection of $\mathrm{Zn}$ (II) with its concentration range from $10^{-10} \mathrm{M}$ in the cytoplasm ${ }^{7-8}$ to $10^{-4}$ $\mathrm{M}$ in some vesicles ${ }^{4,9}$ is very important in monitoring biological processes. ${ }^{10-13}$ As a result, development of fluorescent sensors for zinc (II) ions has attracted significant attention because of their high sensitivity and operational simplicity for real-time analysis. Several studies focusing on improving and increasing the sensitivity and selectivity of the fluorescent probes for zinc ions have been reported in literature. ${ }^{3-12}$ Among these incorporation of 2,2'-dipicolylamine residue into different fluorophores such as 2-aryl benzimidazoles, benzoxazoles, ${ }^{14}$ indoles, benzofurans, quinoline,${ }^{15}$ dansyl, anthracene,${ }^{16}$ coumarin, ${ }^{17-18}$ naphthalimide, ${ }^{19}$ fluorescein, ${ }^{20-25}$ Rhodamine, ${ }^{25-27}$ cyanine dye, ${ }^{9}$ BODIPY dyes, ${ }^{28}$ and porphyrins ${ }^{29}$ for fluorescent detection of zinc (II) ions has been widely reported due to its ease of introduction, high sensitivity, and selectivity. These fluorescent probes respond to zinc (II) ions through photoinduced electron transfer, internal charge transfer, excimer/exciplex formation and extinction, chelation-enhanced fluorescence, or fluorescence resonance energy transfer. ${ }^{3-12}$ However, many fluorescent 
probes have poor solubility in water, especially those with longer wavelength emissions at greater than $600 \mathrm{~nm}$. The search for readily accessible fluorescent probes with excellent water solubility, $\mathrm{pH}$-independent response under physiological conditions, large dynamic range, high specificity, ratiometric responses with significant ratio changes of the two emission peaks, and long-wavelength absorption and emission region is still a challenging task for intact in vivo detection of zinc (II) in living cells.

BODIPY (4,4'-difluoro-4-bora-3a,4a-diaza-s-indacene) dyes with unique properties such as relatively high absorption coefficients, fluorescence quantum yields, relatively narrow absorption and emission bands, have been successfully applied as ion sensors including zinc sensors. $\mathrm{Zn}^{2+}$ binding moieties were introduced to BODIPY dyes at the meso or 3,5-positions, and most of these fluorescent probes are not completely soluble in aqueous solution. As a result, development of new BODIPY-based fluorescent probes with controlled number of binding sites for zinc (II) ions at 2,6-positions, and excellent water solubility will enable them more suitable for biological and medical applications. With these in mind, we introduced branched oligo(ethylene glycol)methyl ether residues to the meso- or 1,7-positions to achieve excellent water solubility of BODIPY dyes with different emission wavelengths, and incorporated zinc (II) chelator 2,2'-dipicolylamine residues into BOIDPY dyes at 2- or 2,6-positions, obtaining three highly water-soluble fluorescent probes (6-A, 6-B and 6-C) for zinc (II) ions with high solubility (minimum solubility of $50 \mathrm{mg} / \mathrm{mL}$ in aqueous solution). Fluorescent probes 6-A, 6-B and 6-C display weak fluorescence in the absence of zinc ions because of their photo-induced electron transfer mechanism. However, the presence of zinc (II) ions significantly enhances fluorescence of probes 6-A and 6-B with slight blue shifts in the emission spectra because the binding of bis(pyridin-2-ylmethyl)amine moieties with zinc (II) ions prevents the photo-induced electron transfer. The presence of different amounts of zinc ions shows significant ratiometric responses of fluorescent probe 6-C to zinc (II) ions with fluorescence increase at $521 \mathrm{~nm}$ and fluorescence quenching at $661 \mathrm{~nm}$. This is because binding of zinc (II) ions may prevents the photo-induced electron transfer and may interrupt $\pi$-conjugation between two styryl groups at 1,7-positions and BODIPY core. These fluorescent probes show excellent sensitivity and selectivity to zinc (II) ion in HEPES buffer $\mathrm{pH} 7.0$ while fluorescent probe 6-C may offer most promising application 
because of its ratiometric responses to zinc (II) ions. We also tested fluorescent probe 6C with cancer cell line (SW-620) to see its incorporation and also labeling of the cellular zinc (II). These fluorescent probes may have potential applications in measuring zinc (II) concentration in the micromolar ranges in synaptic vesicles and synaptic space. ${ }^{4-6}$
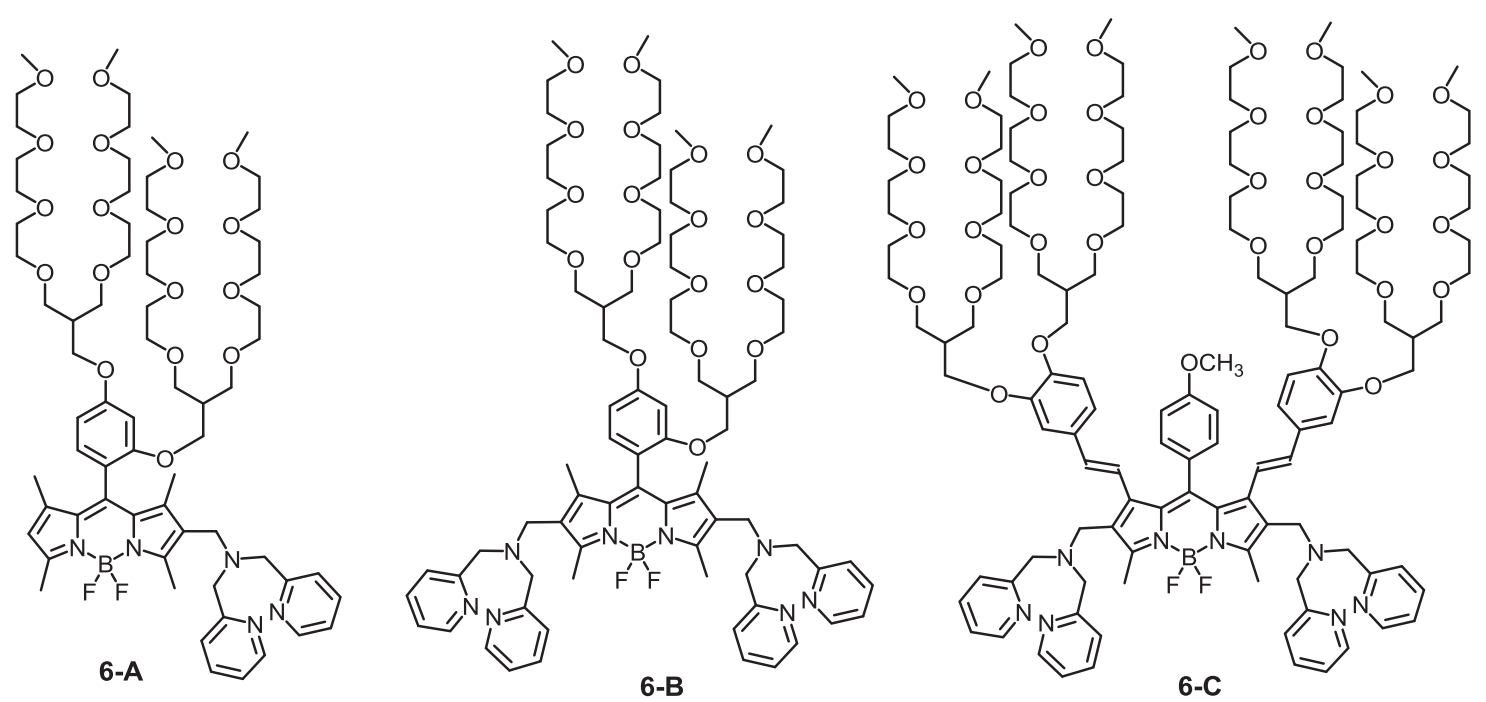

Scheme 6.1. Chemical structures of BODIPY-based fluorescent probes for zinc (II) ions.

\subsection{Experimental section}

\subsubsection{Instrumentation.}

${ }^{1} \mathrm{H}$ NMR and ${ }^{13} \mathrm{C}$ NMR spectra were taken on a $400 \mathrm{MHz}$ Varian Unity Inova spectrophotometer instrument. Absorption spectra were taken on a Perkin Elmer Lambda 35 UV/VIS spectrometer. Fluorescence spectra were recorded on a Jobin Yvon Fluoromax-4 spectrofluorometer with excitation wavelength of $470 \mathrm{~nm}$. All the absorption and emission spectra were recorded at room temperature.

\subsubsection{Materials and synthesis}

Unless otherwise indicated, all reagents and solvents were obtained from commercial suppliers and used without further purification. Air- and moisture-sensitive reactions were conducted in oven-dried glassware using a standard Schlenk line or drybox techniques under an inert atmosphere of dry nitrogen. Aldehyde derivative (6.1) and 
highly water-soluble BODIPY dye (6.2) were prepared according to our reported procedure. $^{30}$

Compound 6.3: A mixture of DMF $(2.0 \mathrm{~mL})$ and $\mathrm{POCl}_{3}(1.0 \mathrm{~mL})$ was stirred on ice for five minutes under argon atmosphere. The mixture was warmed to room temperature and was stirred further for 30 minutes. After adding compound 6.2 (300 mg, $0.27 \mathrm{mmol}$ ) in dichloroethane $(50 \mathrm{~mL})$ to the reaction mixture, the resulting mixture was stirred at 50 ${ }^{\circ} \mathrm{C}$ for two hours. The reaction mixture was cooled down to room temperature and then was slowly poured into saturated $\mathrm{NaHCO}_{3}$ aqueous solution at $0{ }^{\circ} \mathrm{C}$ on ice. The mixture was warmed to room temperatureand was further stirred for 30 minutes and then washed with water. The organic layers were combined, dried over anhydrous $\mathrm{Na}_{2} \mathrm{SO}_{4}$, and concentrated under reduced pressure. The crude product was purified by using a mixed mobile phase (hexane: $\mathrm{CH}_{2} \mathrm{Cl}_{2}$ : EtOAc: $\mathrm{MeOH}=5: 3: 1: 0.5, \mathrm{~V} / \mathrm{V}$ ) to attain BODIPY 6.3 (275 mg, $89 \%$ ) as red oil. ${ }^{1} \mathrm{H}$ NMR (400 MHz, $\left.\mathrm{CDCl}_{3}\right): \delta 9.97(\mathrm{~s}, 1 \mathrm{H}), 6.92(\mathrm{~d}, J=8.0$ $\mathrm{Hz}, 1 \mathrm{H}), 6.60$ (d, $J=8.4 \mathrm{~Hz}, 1 \mathrm{H}), 6.54(\mathrm{~s}, 1 \mathrm{H}), 6.09(\mathrm{~s}, 1 \mathrm{H}), 4.03$ (d, $J=5.6 \mathrm{~Hz}, 2 \mathrm{H})$, $3.94(\mathrm{~d}, J=5.2 \mathrm{~Hz}, 2 \mathrm{H}), 3.61-3.15(\mathrm{~m}, 68 \mathrm{H}), 2.84(\mathrm{~s}, 3 \mathrm{H}), 2.56(\mathrm{~s}, 3 \mathrm{H}), 2.43-2.37$ (m, $1 \mathrm{H}), 2.23-2.17(\mathrm{~m}, 1 \mathrm{H}), 1.75(\mathrm{~s}, 3 \mathrm{H}), 1.51(\mathrm{~s}, 3 \mathrm{H}) .{ }^{13} \mathrm{C} \mathrm{NMR}\left(100 \mathrm{For} \mathrm{MHz}, \mathrm{CDCl}_{3}\right): \delta$ $186.1,162.0,161.0,156.6,156.0,147.2,142.3,141.6,135.0,130.5,129.6,126.1,123.6$, $115.5,106.6,100.6,72.1,70.8,70.8,70.8,70.7,70.7,70.6,70.5,69.5,69.2,69.1,66.7$, 66.4, 59.2, 40.2, 39.7, 15.2, 14.8, 13.1, 11.3. HRMS (FAB) calcd for $\mathrm{C}_{56} \mathrm{H}_{92} \mathrm{BF}_{2} \mathrm{~N}_{2} \mathrm{O}_{19}$ $[\mathrm{M}+\mathrm{H}]^{+}, 1145.6355$; found, 1145.6335 .

Compound 6.5: BODIPY 6.5 was prepared using BODIPY dye 6.3 by a procedure similar to that used for preparing BODIPY dye $\mathbf{3}$ but the mixture was stirred at a higher temperature of $60{ }^{\circ} \mathrm{C}$ instead of $50{ }^{\circ} \mathrm{C}$ for five hours ( $82 \%$ of reaction yield). ${ }^{1} \mathrm{H}$ NMR (400 MHz, $\left.\mathrm{CDCl}_{3}\right): \delta 10.04(\mathrm{~s}, 2 \mathrm{H}), 6.93(\mathrm{~d}, J=8.4 \mathrm{~Hz}, 1 \mathrm{H}), 6.64(\mathrm{dd}, \mathrm{J}=8.8,2.0 \mathrm{~Hz}$, 1H), $6.59(\mathrm{~d}, J=2.0 \mathrm{~Hz}, 1 \mathrm{H}), 4.07$ (d, $J=6.0 \mathrm{~Hz}, 2 \mathrm{H}), 3.97$ (d, $J=5.2 \mathrm{~Hz}, 2 \mathrm{H}), 3.63-$ $3.15(\mathrm{~m}, 68 \mathrm{H}), 2.84(\mathrm{~s}, 6 \mathrm{H}), 2.44-2.40(\mathrm{~m}, 1 \mathrm{H}), 2.23-2.20(\mathrm{~m}, 1 \mathrm{H}), 1.83(\mathrm{~s}, 6 \mathrm{H}) .{ }^{13} \mathrm{C}$ NMR (100 MHz, $\left.\mathrm{CDCl}_{3}\right): \delta 186.0,162.5,160.2,156.5,148.1,145.6,132.7,129.3,127.9$, $114.8,107.0,100.9,72.1,70.9,70.8,70.8,70.7,70.5,69.5,69.1,66.8,66.5,59.3,59.2$, 40.2, 39.6, 13.9, 11.8. HRMS (FAB) calcd for $\mathrm{C}_{57} \mathrm{H}_{92} \mathrm{BF}_{2} \mathrm{~N}_{2} \mathrm{O}_{20}[\mathrm{M}+\mathrm{H}]^{+}, 1173.6305$; found, 1173.6310 . 
Probe 6-A: $\quad$ After $10 \mathrm{~mL}$ of dry $\mathrm{ClCH}_{2} \mathrm{CH}_{2} \mathrm{Cl}(10 \mathrm{~mL})$ was added to a $50-\mathrm{mL}$ flask containing BODIPY dye $\mathbf{6 . 3}$ (110 mg, $0.1 \mathrm{mmol})$ and 2-pyridinyl-N-(2pyridinylmethyl)methanamine $6.4(47 \mathrm{mg}, 0.2 \mathrm{mmol})$, the mixture was stirred at $40{ }^{\circ} \mathrm{C}$ for four hours. When $\mathrm{NaBH}(\mathrm{OAc})_{3}(40 \mathrm{mg}, 0.18 \mathrm{mmol})$ and acetic acid (1 drop) were added to the flask at room temperature, the mixture was stirred overnight at room temperature, diluted with $\mathrm{CH}_{2} \mathrm{Cl}_{2}$ and washed with water and brine, respectively. The organic layer was collected, dried over $\mathrm{Na}_{2} \mathrm{SO}_{4}$ and concentrated in vacuo. The crude product was purified by TLC plate (hexane/EtOAc/ $\mathrm{CH}_{2} \mathrm{Cl}_{2} / \mathrm{MeOH}, 5 / 1 / 3 / 0.5$, v/v) to obtain BODIPY dye 6-A (97 mg, 74\%) as red oil. ${ }^{1} \mathrm{H}$ NMR (400 MHz, $\left.\mathrm{CDCl}_{3}\right): \delta 8.43(\mathrm{~d}$, $J=4.8 \mathrm{~Hz}, 2 \mathrm{H}), 7.59(\mathrm{td}, J=7.6,1.6 \mathrm{~Hz}, 2 \mathrm{H}), 7.36$ (d, $J=7.6 \mathrm{~Hz}, 2 \mathrm{H}), 7.10-7.07$ (m, 2H), $6.87(\mathrm{~d}, J=8.4 \mathrm{~Hz}, 1 \mathrm{H}), 6.54(\mathrm{dd}, J=8.4,2.0 \mathrm{~Hz}, 1 \mathrm{H}), 6.49$ (d, $J=2.0 \mathrm{~Hz}, 1 \mathrm{H})$, $5.87(\mathrm{~s}, 1 \mathrm{H}), 4.01(\mathrm{~d}, J=5.6 \mathrm{~Hz}, 2 \mathrm{H}), 3.94-3.86(\mathrm{~m}, 2 \mathrm{H}), 3.64-3.08(\mathrm{~m}, 70 \mathrm{H}), 2.46$ (s, $3 \mathrm{H}), 2.45(\mathrm{~s}, 3 \mathrm{H}), 2.43-2.37(\mathrm{~m}, 1 \mathrm{H}), 2.17-2.11(\mathrm{~m}, 1 \mathrm{H}), 1.41(\mathrm{~s}, 3 \mathrm{H}), 1.40(\mathrm{~s}, 3 \mathrm{H}) .{ }^{13} \mathrm{C}$ NMR (100 MHz, $\left.\mathrm{CDCl}_{3}\right): \delta 161.5,159.9,156.7,155.7,154.2,149.0,142.4,141.4,139.2$, $136.5,132.1,131.7,129.9,127.1,123.3,122.2,120.6,116.7,106.3,100.5,72.1,70.8$, 70.7, 70.7, 70.5, 70.5, 69.6, 69.2, 69.1, 66.6, 66.4, 60.7, 59.2, 48.4, 40.2, 39.6, 14.7, 14.2, 13.0, 11.9. HRMS (FAB) calcd for $\mathrm{C}_{68} \mathrm{H}_{105} \mathrm{BF}_{2} \mathrm{~N}_{5} \mathrm{O}_{18}[\mathrm{M}+\mathrm{H}]^{+}, 1328.7516$; found, 1328.7502.

Probe 6-B: When $15 \mathrm{~mL}$ of dry $\mathrm{ClCH}_{2} \mathrm{CH}_{2} \mathrm{Cl}(15 \mathrm{~mL})$ was added to a $50-\mathrm{mL}$ flask containing BODIPY dye 6.5 (100 mg, $0.085 \mathrm{mmol})$ and 2-pyridinyl-N-(2pyridinylmethyl)methanamine $6.4(90 \mathrm{mg}, 0.45 \mathrm{mmol})$, The mixture was stirred at $40{ }^{\circ} \mathrm{C}$ for four hours. After $\mathrm{NaBH}(\mathrm{OAc})_{3}(80 \mathrm{mg}, 0.36 \mathrm{mmol})$ and acetic acid (2 drop) were added to the flask at room temperature, the mixture was stirred overnight at room temperature, diluted with $\mathrm{CH}_{2} \mathrm{Cl}_{2}$ and washed with water and brine, respectively. The organic layer was collected, dried over $\mathrm{Na}_{2} \mathrm{SO}_{4}$ and concentrated in vacuo. The crude product was purified by TLC plate using a mixed mobile phase (hexane/acetone $/ \mathrm{CH}_{2} \mathrm{Cl}_{2} / \mathrm{MeOH}, 5 / 1 / 3 / 0.6, \mathrm{v} / \mathrm{v}$ ) to obtain amino BODIPY dye 6-B (68 mg, 52\%) as red oil. ${ }^{1} \mathrm{H}$ NMR (400 MHz, $\left.\mathrm{CDCl}_{3}\right): \delta 8.40(\mathrm{~d}, J=4.8 \mathrm{~Hz}, 4 \mathrm{H}), 7.55$ (t, $J=$ $7.6 \mathrm{~Hz}, 4 \mathrm{H}), 7.32$ (d, $J=7.6 \mathrm{~Hz}, 4 \mathrm{H}), 7.06-7.03(\mathrm{~m}, 4 \mathrm{H}), 6.81(\mathrm{~d}, J=8.0 \mathrm{~Hz}, 1 \mathrm{H}), 6.52$ $(\mathrm{d}, J=8.4 \mathrm{~Hz}, 1 \mathrm{H}), 6.46(\mathrm{~s}, 1 \mathrm{H}), 4.00(\mathrm{~d}, J=5.2 \mathrm{~Hz}, 2 \mathrm{H}), 3.82$ (d, $J=5.6 \mathrm{~Hz}, 2 \mathrm{H}), 3.61$ $3.01(\mathrm{~m}, 72 \mathrm{H}), 2.43-2.35(\mathrm{~m}, 7 \mathrm{H}), 2.06-2.03(\mathrm{~m}, 1 \mathrm{H}), 1.35(\mathrm{~s}, 6 \mathrm{H}) .{ }^{13} \mathrm{C}$ NMR $(100 \mathrm{MHz}$, 130 
$\left.\mathrm{CDCl}_{3}\right): \delta 161.5,159.9,156.7,155.1,149.0,141.1,138.9,136.5,131.6,129.9,126.9$, 123.3, 122.2, 116.9, 106.4, 100.5, 72.1, 72.0, 70.8, 70.8, 70.7, 70.6, 70.6, 70.4, 70.4, 69.6, 69.1, 66.6, 66.5, 60.5, 59.2, 59.1, 48.3, 40.2, 39.6, 12.9, 11.8. HRMS (FAB) calcd for $\mathrm{C}_{81} \mathrm{H}_{118} \mathrm{BF}_{2} \mathrm{~N}_{8} \mathrm{O}_{18}[\mathrm{M}+\mathrm{H}]^{+}, 1539.8625$; found, 1539.8622 .

Probe 6-C: $\quad$ A mixed solution of $\mathbf{6 . 8}(60 \mathrm{mg}, 0.028 \mathrm{mmol})$ and 2-pyridinyl-N(2-pyridinylmethyl)methanamine $6.4(33 \mathrm{mg}, 0.168 \mathrm{mmol})$ in $10 \mathrm{~mL}$ of dry $\mathrm{ClCH}_{2} \mathrm{CH}_{2} \mathrm{Cl}$ was stirred at $50{ }^{\circ} \mathrm{C}$ for four hours. When $\mathrm{NaBH}(\mathrm{OAc})_{3}(30 \mathrm{mg}, 0.112 \mathrm{mmol})$ and acetic acid ( 1 drop) were added to the flask at room temperature, the mixture was stirred overnight at room temperature, diluted with $\mathrm{CH}_{2} \mathrm{Cl}_{2}$ and washed with water and brine, respectively. The organic layer was collected, dried over $\mathrm{Na}_{2} \mathrm{SO}_{4}$ and concentrated in vacuo. The crude product was purified by TLC plate (hexane/acetone $/ \mathrm{CH}_{2} \mathrm{Cl}_{2} / \mathrm{MeOH}$, 5/1/3/1, v/v) to obtain amino BODIPY dye 6-C (46 mg, 65\%) as dark purple oil. ${ }^{1} \mathrm{H}$ NMR (400 MHz, $\mathrm{CDCl}_{3}$ ): $\delta 8.43$ (d, $\left.J=5.2 \mathrm{~Hz}, 2 \mathrm{H}\right), 7.52$ (t-like, $\left.J=8.0 \mathrm{~Hz}, 2 \mathrm{H}\right), 7.35$ (d, $J=8.0 \mathrm{~Hz}, 2 \mathrm{H}), 7.15$ (d, $J=8.8 \mathrm{~Hz}, 2 \mathrm{H}), 7.05$ (t-like, $J=6.0 \mathrm{~Hz}, 2 \mathrm{H}), 6.88$ (d, $J=8.8$ $\mathrm{Hz}, 2 \mathrm{H}), 6.77$ (d, $J=16.0 \mathrm{~Hz}, 2 \mathrm{H}), 6.67$ (d, $J=8.4 \mathrm{~Hz}, 2 \mathrm{H}), 6.50$ (s, 2H), 6.44 (d, $J=8.4$ $\mathrm{Hz}, 2 \mathrm{H}), 5.67$ (d, $J=16.4 \mathrm{~Hz}, 2 \mathrm{H}), 3.98$ (d, $J=5.6 \mathrm{~Hz}, 4 \mathrm{H}), 3.90$ (d, $J=5.6 \mathrm{~Hz}, 4 \mathrm{H})$, $3.73(\mathrm{~s}, 3 \mathrm{H}), 3.62-3.44(\mathrm{~m}, 108 \mathrm{H}), 3.34-3.31(\mathrm{~m}, 28 \mathrm{H}), 2.51(\mathrm{~s}, 6 \mathrm{H}), 2.43-2.36(\mathrm{~m}, 4 \mathrm{H})$. ${ }^{13} \mathrm{C}$ NMR $\left(100 \mathrm{MHz}, \mathrm{CDCl}_{3}\right): \delta 165.6,164.6,161.5,153.6,153.4,146.5,144.2,140.1$, $139.9,137.9,135.2,134.7,134.0,128.4,126.1,125.7,124.6,122.4,121.8,116.9,115.2$, $114.0,71.2,71.1,69.8,69.7,69.6,69.6,68.4,58.6,57.4,57.3,37.2,37.1$. HRMS (MAIDL) calcd for $\mathrm{C}_{132} \mathrm{H}_{200} \mathrm{BF}_{2} \mathrm{~N}_{8} \mathrm{O}_{37}[\mathrm{M}+\mathrm{H}]^{+}, 2538.4076$; found, 2538.7638 .

\subsubsection{Optical measurements and buffer conditions:}

All buffer solutions were prepared by using deionized distilled water. Three different buffers at concentration of $10 \mathrm{mM}$ were used to evaluate effect of $\mathrm{pH}$ on the fluorescent properties of fluorescent probes 6-A, 6-B and 6-C. These buffers are citric acid buffer (acidic range), HEPES buffer (neutral range), tris buffer (basic range). No meaurable changes of optical property of fluorescent probe 6-A, 6-B or 6-C were found when differnent buffers (citric, HEPES or tris) were used at same $\mathrm{pH}$ values. 
Rhodamine $6 \mathrm{G}\left(\Phi_{\mathrm{f}}=0.95 \text { in ethanol }\right)^{31-32}$ was used as a reference standard to determine the fluorescence quantum yields of BODIPY dye 6.3, 6.5 and probe 6-A, 6-B, 6-C. The corresponding fluorescence spectra of fluorescent probes 6-A, 6-B and 6-C were collected at the excitation wavelength of $470 \mathrm{~nm}$. Both the samples and references were prepared fresh under identical conditions. The quantum yields were calculated using the following equation:

$$
\Phi_{\mathrm{X}}=\Phi_{\mathrm{st}}\left(\operatorname{Grad}_{\mathrm{X}} / \operatorname{Grad}_{\mathrm{st}}\right)\left(\eta_{\mathrm{X}}^{2} / \eta_{\mathrm{st}}^{2}\right) \ldots \ldots(\text { eq. 6.1) }
$$

Where the subscripts 'st' and ' $\mathrm{x}$ ' stand for standard and test, respectively, $\Phi$ is the fluorescence quantum yield, Grad represents the gradient from the plot of integrated fluorescence intensity versus absorbance and $\eta$ is the refractive index of the solvent.

\subsubsection{Live cell imaging}

SW 620 cells $\left(\right.$ ATCC $^{\circledR}$ Catalog No. CCL-2277) were cultured in Dulbecco's Modified Eagle's Medium/Nutrient Mixture F12 Ham's Liquid Media (DMEM/F-12) supplemented with 10\% fetal bovine serum (FBS, GIBCO), penicillin (100 U/mL) and streptomycin $(100 \mu \mathrm{g} / \mathrm{mL})\left(1 \mathrm{X}\right.$ PenStrep, GIBCO) at $37^{\circ} \mathrm{C}$ in humidified incubator with $5 \% \mathrm{CO}_{2}$. The cells were plated on 12 -well culture plates at density of $1 \times 10^{5}$ cells $/ \mathrm{mL}$. After $24 \mathrm{hr}$ incubation, the media was removed and cells were rinsed with 1X PBS. Fresh serum free media with/without $25 \mu \mathrm{M}$ fluorescent probe $\mathrm{C}$ was added to cells and the cells were incubated for 2 hours at $37^{\circ} \mathrm{C}$ before rinsing again with $1 \mathrm{X}$ PBS. Fresh serumfree media containing $0,50,100$, or $200 \mu \mathrm{M} \mathrm{ZnCl} 2$ and/or sodium pyrithione salt $(1: 1)$ was added to the cells and then imaged after $30 \mathrm{~min}$ of incubation. The images were

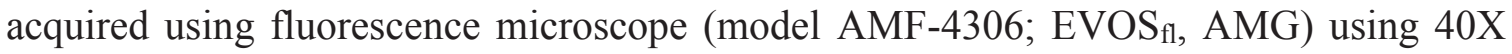
magnification objective. The exposure times for each fluorescence channel (green (GFP light cube) or red (RFP light cube)) were kept constant for all samples. There was no signal observed for blue channel (DAPI light cube) in any of the samples (results not shown). To confirm effect of zinc on fluorescence of cells in presence of the fluorescent probe $\mathrm{C}$, zinc chelator $\mathrm{N}, \mathrm{N}, \mathrm{N}^{\prime}, \mathrm{N}^{\prime}$-tetrakis(2-pyridylmethyl)ethylenediamine (TPEN) was used. After acquiring the images for cells incubated with $100 \mu \mathrm{M}$ each of zinc (II) and pyrithione, cells were washed with PBS and $100 \mu \mathrm{M}$ zinc chelator TPEN dissolved in 
serum-free media was added to the cells. The cells were incubated for 10 minutes before taking fluorescence images using both green and red channels.

\subsection{Results and discussions}

\subsubsection{Synthetic approaches}

Recently, our group has reported a series of highly water-soluble neutral BODIPY dyes with different emissions ranging from green to near-infrared regions by introducing branched oligo(ethylene glycol)methyl ether residues to BODIPY dyes at all positions such as the meso, 2,6-positions, 3-position, 3,5-positions, 1,7-positions, 1,3,5,7-positions or 4,4-positions, and systematically studied their optical behaviors with controllable fluorescence quantum yields in PBS solutions. ${ }^{30}$ In addition, we developed an approach to prepare 2,6-diformyl-BODIPY dyes, employed formyl groups at 2- or 2,6-positions to control Knoevenagel reaction sequence of methyl groups of 1,3,5,7-tetramethyl BODIPY dyes, ${ }^{33}$ and obtained a series of unique 2,6-diformyl-BODIPY dyes with near-infrared emissions. In this paper, ${ }^{33}$ we employed our unique approaches to prepare highly watersoluble BODIPY-based fluorescent probes for $\mathrm{Zn}^{2+}$ ions.

In order to prepare highly water-soluble BODIPY dyes for fluorescent sensing of zinc (II) ions, we introduced branched oligo(ethylene glycol)methyl ether residues to BODIPY dyes at the meso or 1,7-positions (Schemes 6.1 and 6.2) and incorporated zinc (II) chelator bis(pyridin-2-ylmethyl)amine moiety into BODIPY dyes at 2- and 2,6positions. BODIPY dye bearing branched oligo(ethylene glycol)methyl ether at the meso position (6.2) was prepared according to our reported procedure, starting from benzaldehyde derivative (6.1) (Scheme 6.2). ${ }^{30}$ A formyl group was introduced to BODIPY dye 6.2 at the 2-position via the Vilsmeier-Haack reaction, affording 2-formylBODIPY dye (6.3). ${ }^{33}$ BODIPY dye bearing dipicolylamine residue at 2-position (6-A) was prepared by reductive amination of 2-formyl-BODIPY dye $\mathbf{6 . 3}$ with dipicolylamine using sodium acetoxyborohydride in 74\% yield (Scheme 2). BODIPY dye bearing two dipicolylamine residues at 2,6-positions (6-B) was obtained in the same way to prepare fluorescent probe 6-A by using 2,6-diformyl-BODIPY dye (6.5), ${ }^{33}$ which was prepared by further conducting the Vilsmeier-Haack reaction of 2-formyl-BODIPY dye (6.3) (Scheme 6.2). ${ }^{33}$ Fluorescent probes 6-A and 6-B are highly soluble in aqueous solution 
with at least $50 \mathrm{mg} / \mathrm{mL}$ because of hydrophilic feature of branched oligo(ethylene glycol)methyl ether residues at the meso positions.

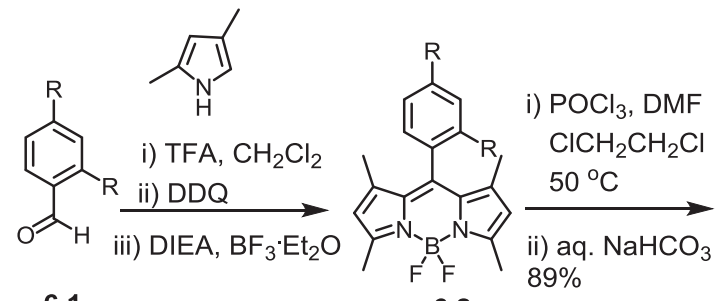

6.1

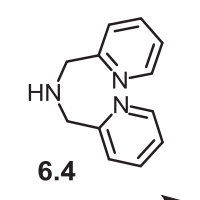

$\mathrm{NaHB}(\mathrm{OAc})_{3}$ $\mathrm{ClCH}_{2} \mathrm{CH}_{2} \mathrm{Cl}$ $52 \%$
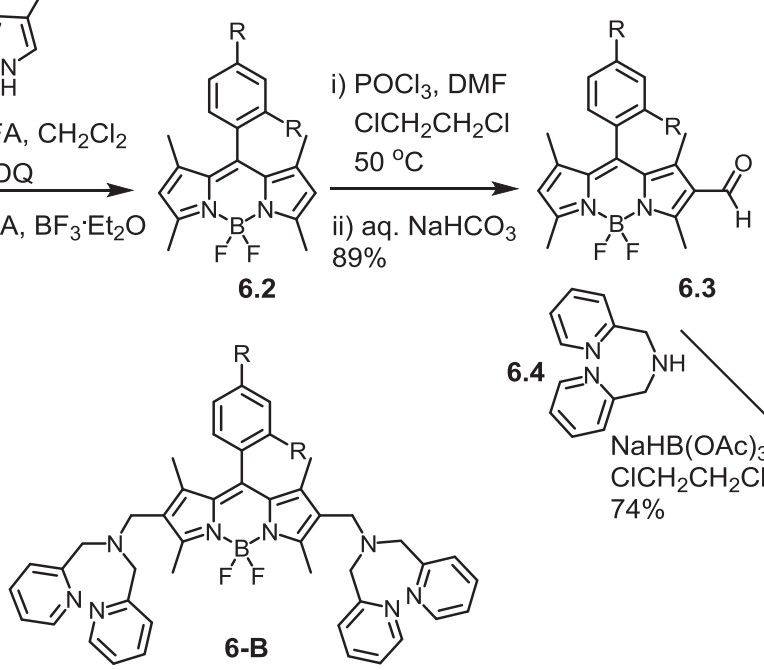

6.3

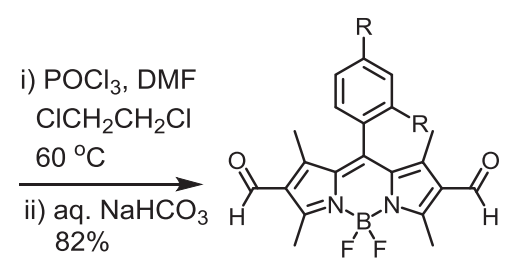

6.5
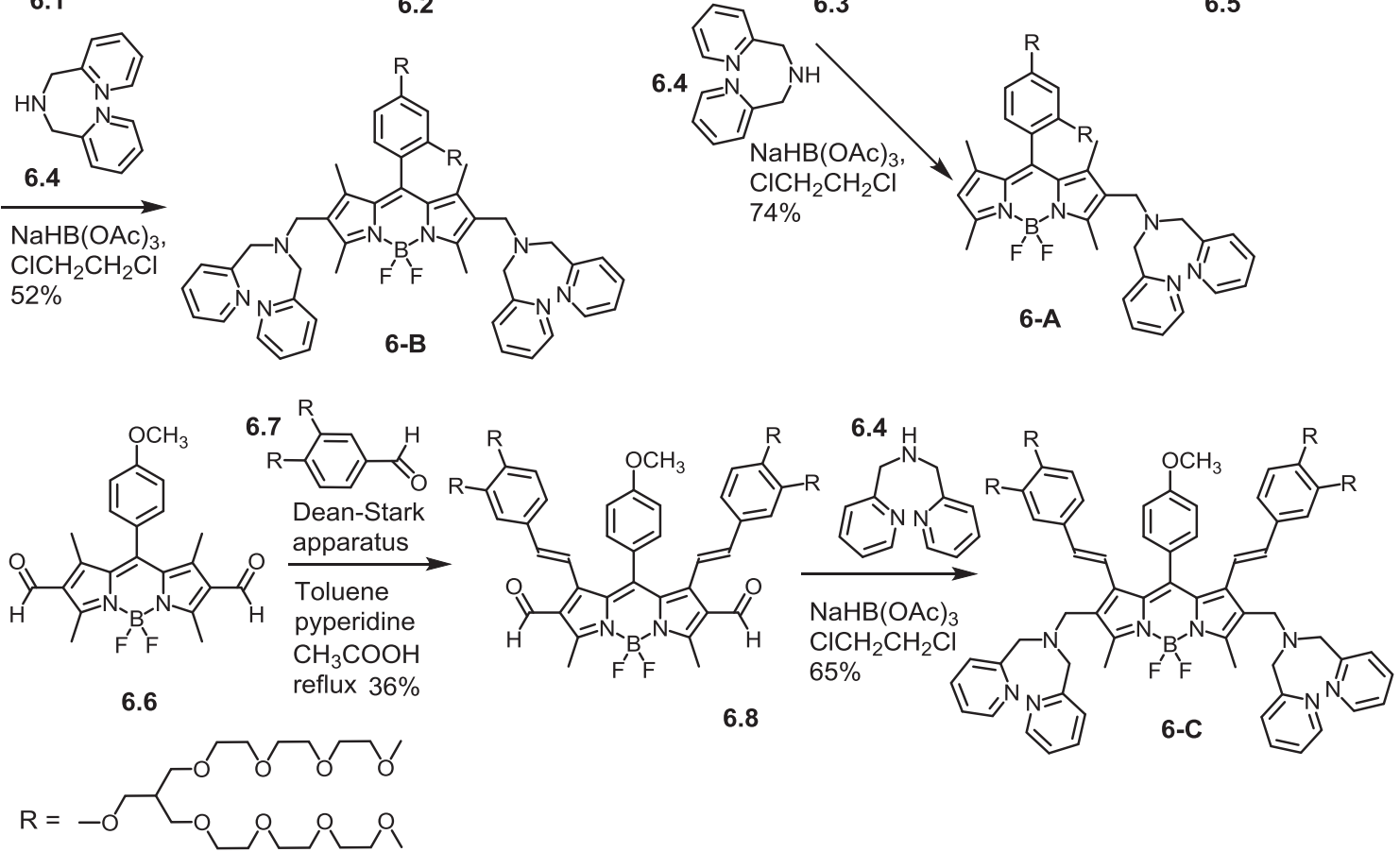

Scheme 6.2. Synthetic route to fluorescent probes 6-A, 6-B and 6-C.

In order to tune fluorescence of BODIPY dye for longer emission wavelength, we conducted the Knoevenagel condensation of 2,6-diformyl-BODIPY dye (6.6) with benzaldehyde derivative (6.7), affording 2,6-diformyl-1,7-distyryl-BODIPY dye (6.8) as the presence of formyl groups at 2,6-positions allows for initial Knoevenagel reactions of methyl groups at 1,7-positions of 1,3,5,7-tetramethyl BODIPY dye (6.6) (Scheme 2). ${ }^{33}$ 1,7-Distyryl-BODIPY dye bearing dipicolylamine residues at 2,6-positions (6-C) was obtained with yield of $52 \%$ by reductive amination of 2,6-diformyl-BODIPY dye (6.8) with dipicolylamine using sodium acetoxyborohydride (Scheme 6.2). Fluorescent probe 6-C also shows excellent solubility with at least $50 \mathrm{mg} / \mathrm{mL}$ in aqueous solution as branched oligo(ethylene glycol)methyl ether residues at 1,7-positions facilitate the 
solubility of BODIPY dye in aqueous solution. The excellent water solubility of fluorescent probe 6-C may arise from bulky hydrophilic dipicolylamine moieties at 2,6positions that may prevent $\pi-\pi$ stacking interactions between BODIPY cores in aqueous solution.

\subsubsection{Spectroscopic responses to zinc (II) and concentration dependency}

The zinc (II)-binding behaviors of fluorescent probes $\mathbf{6 - A}, \mathbf{6 - B}$, and 6-C were investigated by absorption and fluorescence spectroscopic studies in HEPES buffer solution. Fluorescent probe 6-A displays weak fluorescence with fluorescence quantum yield of $7.2 \%$ in HEPES buffer ( $\mathrm{pH}$ 7.0). But this probe shows significant enhancement of fluorescence upon the addition of zinc (II) $(12 \mu \mathrm{M})$, which leads to a fluorescent increase by 2 -fold accompanying with a slightly blue shift in emission spectrum (Figure 6.1a). No significant changes were observed in the absorption spectra in the presence of zinc (II) ions (Figure D.17 in Appendix D). The titration curve of fluorescent probe 6-A to the concentration of zinc (II) ions (Figure 6.1a inset) showed that there are significant increases in the fluorescence intensity with the increase in concentration of zinc (II) ions from $0.5 \mu \mathrm{M}$ to $5 \mu \mathrm{M}$, after that there is negligible increaes in fluorescent intensity at zinc (II) ion concentration of $6 \mu \mathrm{M}$ and higher (Figure 6.1a inset). This suggests that binding of $6 \mu \mathrm{M}$ zinc (II) ions to 2,2'-dipicolylamine residue ( $5 \mu \mathrm{M}$ ) is close to saturation, and that fluorescent probe 6-A interacts with zinc (II) in equivalent molar ratio. 

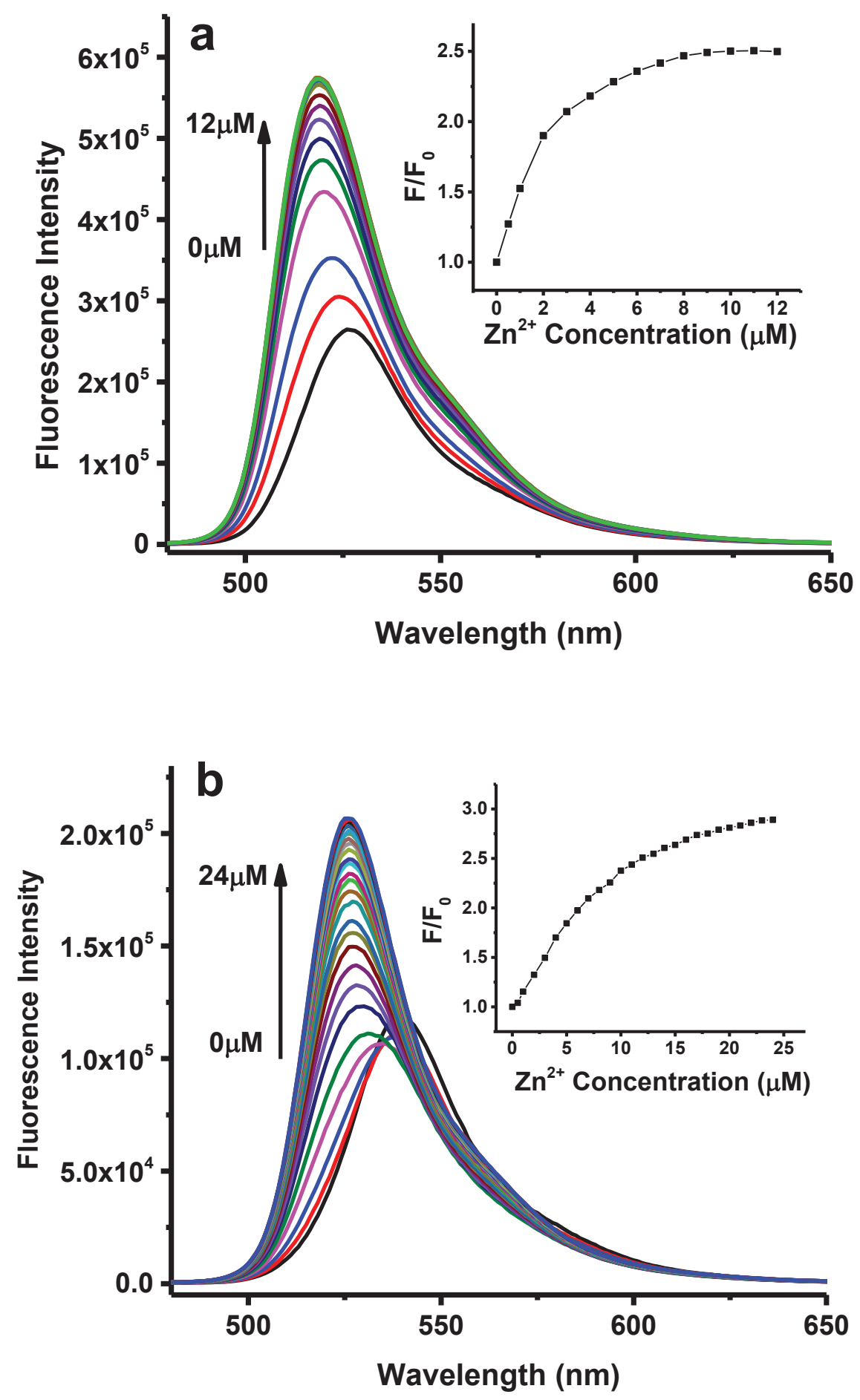

Figure 6.1. Fluorescence spectra of $5 \mu \mathrm{M}$ fluorescent probe 6-A (a) and probe 6-B (b) in $10 \mathrm{mM}$ HEPES buffer ( $\mathrm{pH}$ 7.0) in the absence and presence of different concentrations of 
$\mathrm{ZnCl}_{2}$. Inset: fluorescence response curves at $520 \mathrm{~nm}$ (probe 6-A) and $527 \mathrm{~nm}$ (probe 6B).

Fluorescent probe 6-B displays weaker fluorescence than the probe 6-A with fluorescence quantum yield of $4.7 \%$ in HEPES buffer at $\mathrm{pH} 7.0$. This is due to more efficient fluorescence quenching by photoinduced electron transfer from two tertiary amine groups of dipicolylamine moieties at 2,6-positions to the BODIPY core of the probe 6-B compared with one tertiary amine group of the probe 6-A. It displays similar responses to zinc (II) ions in its absorption and fluorescence spectra as fluorescent probe 6-A. Fluorescent probe 6-B shows increased blue shifts in absorption and emission spectra compared to fluorescent probe 6-A and the addition of $24 \mathrm{mM}$ zinc (II) ions to fluorescent probe 6-B led to $15 \mathrm{~nm}$ blue shifts in emission spectrum (Figure 6.1b) and 6 $\mathrm{nm}$ blue shifts in the absorption spectrum (Figure D.18 in Appendix D). Compared with probe 6-A, fluorescent probe 6-B has larger response range to the concentration of zinc (II) ions (Figure 6.1b inset) with quasilinear sensing range from $0 \mathrm{mM}$ to $10 \mathrm{mM}$ zinc (II) ion.

The obsereved weak fluorescence of fluorescent probe 6-C in HEPES buffer at $\mathrm{pH}$ 7.0 is also attributed to photo-induced electron transfer from tertiary amine groups of dipicolylamine moieties at 2,6-positions to the BODIPY core. Fluorescent probe 6-C displays ratiometric responses to zinc (II) ions and addition of zinc (II) ions to fluorescent probe 6-C causes fluorescence enhancement at $521 \mathrm{~nm}$ and fluorescence quenching at $661 \mathrm{~nm}$. The binding of zinc (II) ions to dipicolylamine moieties at 2,6-positions may disrupt $\pi$-conjugation of distyryl groups at 1,7-positions with BODIPY core, resulting in fluorescence quenching at $661 \mathrm{~nm}$, and effectively stops the photo-induced electron transfer from tertiary amine groups of dipicolylamine moieties at 2,6-positions to the BODIPY core, leading to fluorescence enhancement of fluorescent probe 6-C at $521 \mathrm{~nm}$ (Figure 6.2a). The fluorescent probe 6-C shows sensitive responses to zinc (II) ions with the same response range as fluorescent probe 6-B (Figure 6.2b) 

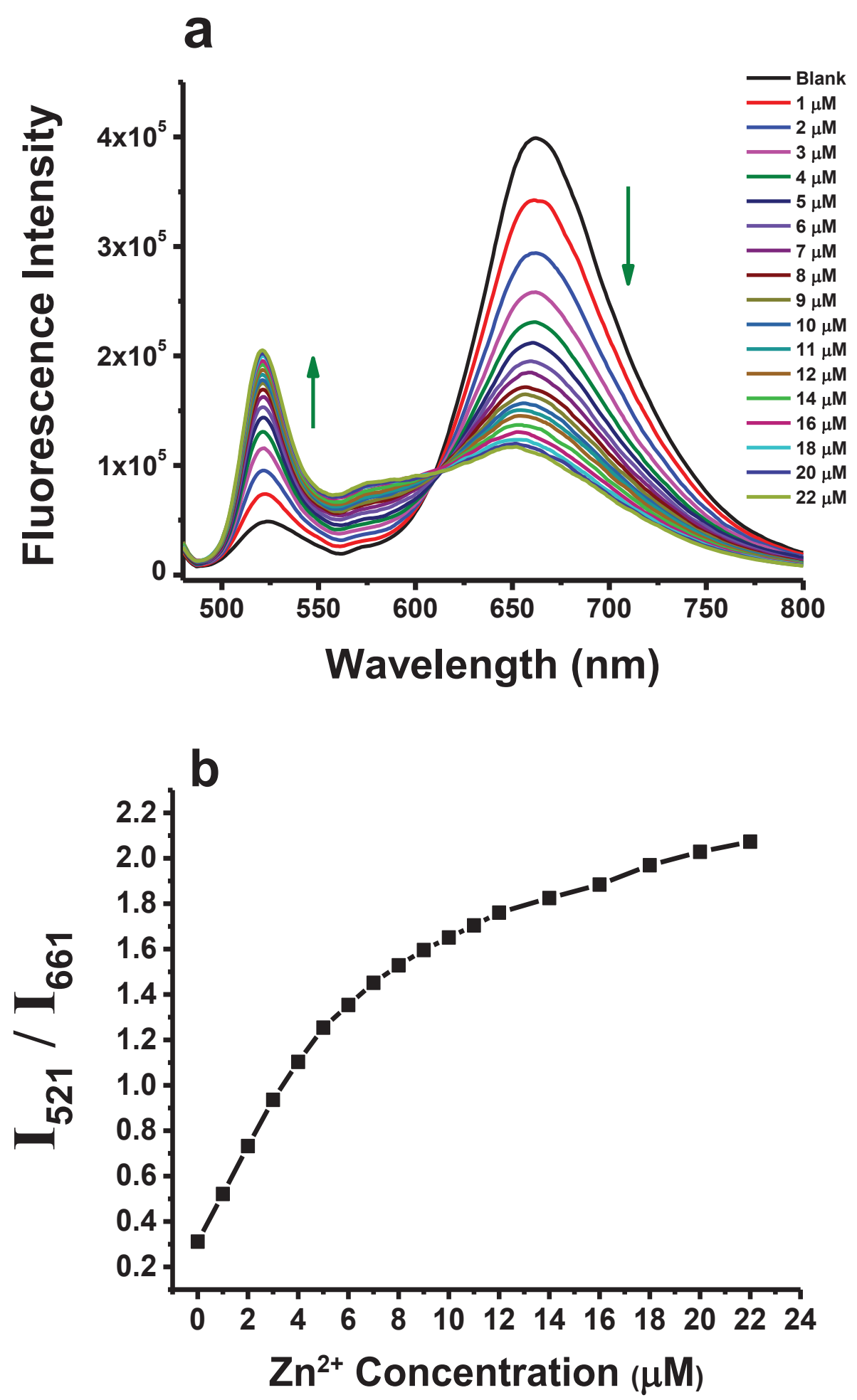

Figure 6.2. Fluorescence spectra (a) and fluorescence retiometric response curve (521 $\mathrm{nm} / 661 \mathrm{~nm}$ ) (b) of $5 \mu \mathrm{M}$ fluorescent probe 6-C in $10 \mathrm{mM}$ HEPES buffer ( $\mathrm{pH} 7.0)$ in the absence and presence of different concentrations of $\mathrm{ZnCl}_{2}$. 


\subsubsection{Selectivity}

The fluorescence response of fluorescent probes $\mathbf{6 - A}, \mathbf{6 - B}$, and 6-C to different metal ions in $10 \mathrm{mM}$ HEPES buffer $(\mathrm{pH}$ 7.0) were also investigated (Figures 6.3). No significant response of fluorescent probe 6-A was observed in the presence of $40 \mathrm{mM}$ alkali-, alkaline-earth metal ions such as $\mathrm{Na}^{+}, \mathrm{K}^{+}, \mathrm{Ca}^{2+}, \mathrm{Mg}^{2+}$ and $\mathrm{Al}^{3+}$ ions, and some transitional metallic ions such as $\mathrm{Mn}^{2+}$ and $\mathrm{Fe}^{3+}$ ions (Figure 6.3a). The presence of 40 $\mu \mathrm{M} \mathrm{Ni}^{2+}, \mathrm{Co}^{2+}$ or $\mathrm{Cu}^{2+}$ ions significantly quenches fluorescence of fluorescent probe 6-A most likely due to metal-to-ligand electron transfer upon excitation; A mechanism similar to previously reported for paramagnetic ions such as $\mathrm{Ni}^{2+}, \mathrm{Co}^{2+}$ or $\mathrm{Cu}^{2+}$ ions for fluorescence quenching of fluorescein-based dyes for $\mathrm{Zn}^{2+}$ ions. ${ }^{20-25}$ Whereas, addition of $20 \mathrm{mM} \mathrm{Zn}^{2+}$ resulted in significant fluorescence enhancement of fluorescent probe 6-A (Figure 6.3a). The presence of $40 \mu \mathrm{M}$ alkali-, alkaline-earth metal ions such as $\mathrm{Na}^{+}, \mathrm{K}^{+}$, $\mathrm{Ca}^{2+}, \mathrm{Mg}^{2+}$ and $\mathrm{Al}^{3+}$ ions, and some transitional metal ions such as $\mathrm{Mn}^{2+}, \mathrm{Fe}^{3+}, \mathrm{Ni}^{2+}, \mathrm{Co}^{2+}$ or $\mathrm{Cu}^{2+}$ ions caused only slight changes in absorption spectra of fluorescent probe 6-A in 10 mM HEPES buffer ( $\mathrm{pH}$ 7.0) (see Appendix D). A similar response to these alkali-, alkaline-earth and transitional metallic ions was observed for fluorescent probe 6-B in 10 mM HEPES buffer at pH 7.0 (Figure 6.3).

Like probe 6-A and 6-B, fluorescent probe 6-C is insensitive to similar alkali-, alkaline earth metal ions and some transitional metal ions like $\mathrm{Mn}^{2+}$ and $\mathrm{Fe}^{3+}$. (Figure 6.3c). However, in the presence of $40 \mu \mathrm{M} \mathrm{Ni}^{2+}, \mathrm{Co}^{2+}$ or $\mathrm{Cu}^{2+}$ ions, significant fluorescence quenching at both $661 \mathrm{~nm}$ and $521 \mathrm{~nm}$ due to the metal-to-ligand electron transfer upon excitation because of the paramagnetic property of those ions. Whereas, presence of $20 \mu \mathrm{M} \mathrm{Zn}{ }^{2+}$ caused significant fluorescence enhancement of fluorescent probe 6-C at $521 \mathrm{~nm}$ and significant fluorescence quenching at $661 \mathrm{~nm}$ (Figure 6.3c). It was observed that the sensitivity and response ranges of fluorescent probes are significantly dependent on their number of binding sites for $\mathrm{Zn}^{2+}$ ions. Fluorescent probes 6-B and 6-C with two binding sites at 2,6-positions show wider response range to $\mathrm{Zn}^{2+}$ ions than fluorescent probe 6-A with one binding site at 2-position. 

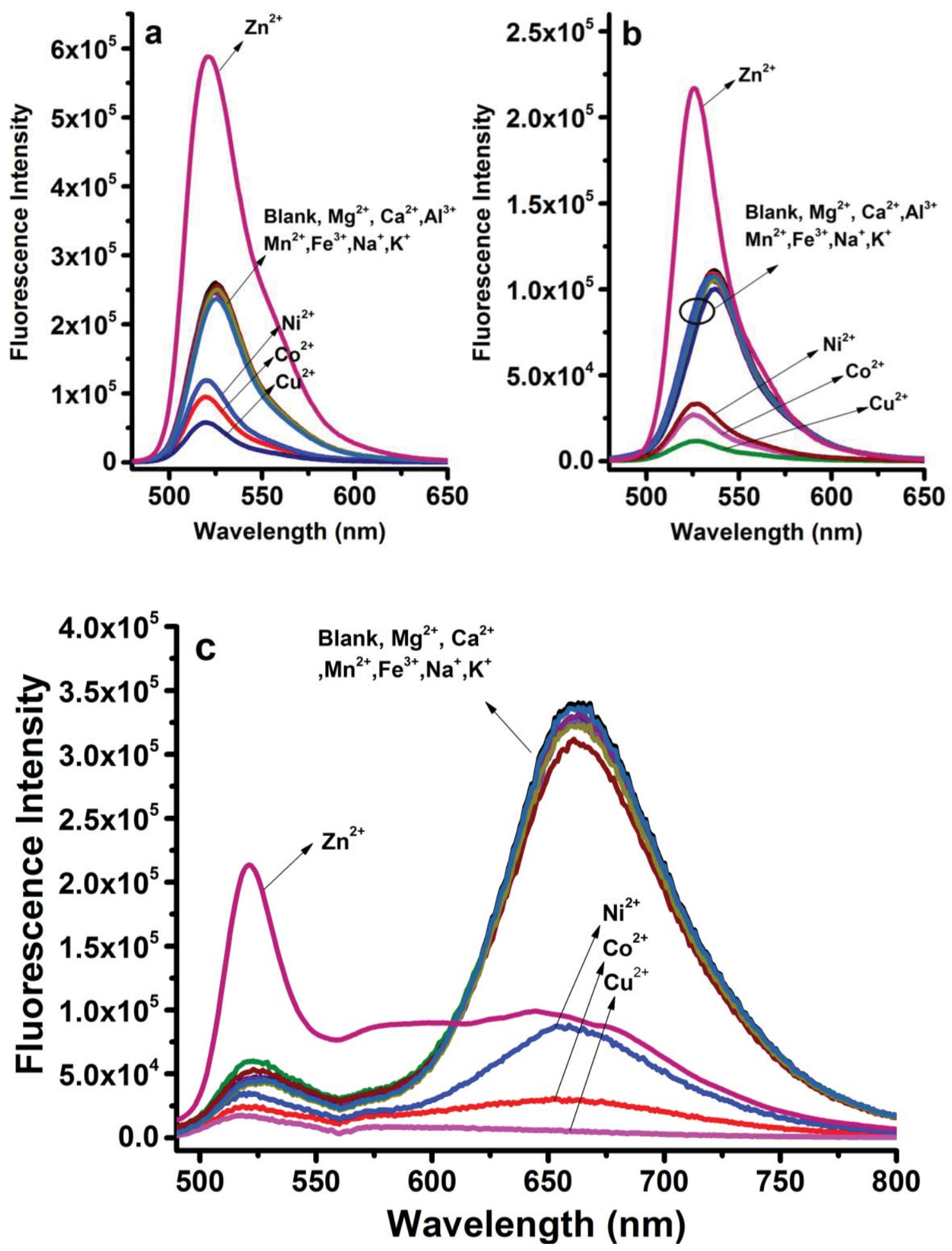

Figure 6.3. Fluorescent responses of $5 \mu \mathrm{M}$ fluorescent probes 6-A (a), 6-B (b) and 6-C (c) to different metal ions $\left(20 \mu \mathrm{M} \mathrm{Zn}^{2+}\right.$, all other ions at $\left.40 \mu \mathrm{M}\right)$ in $10 \mathrm{mM}$ HEPES buffer ( $\mathrm{pH} 7.0)$, respectively. 

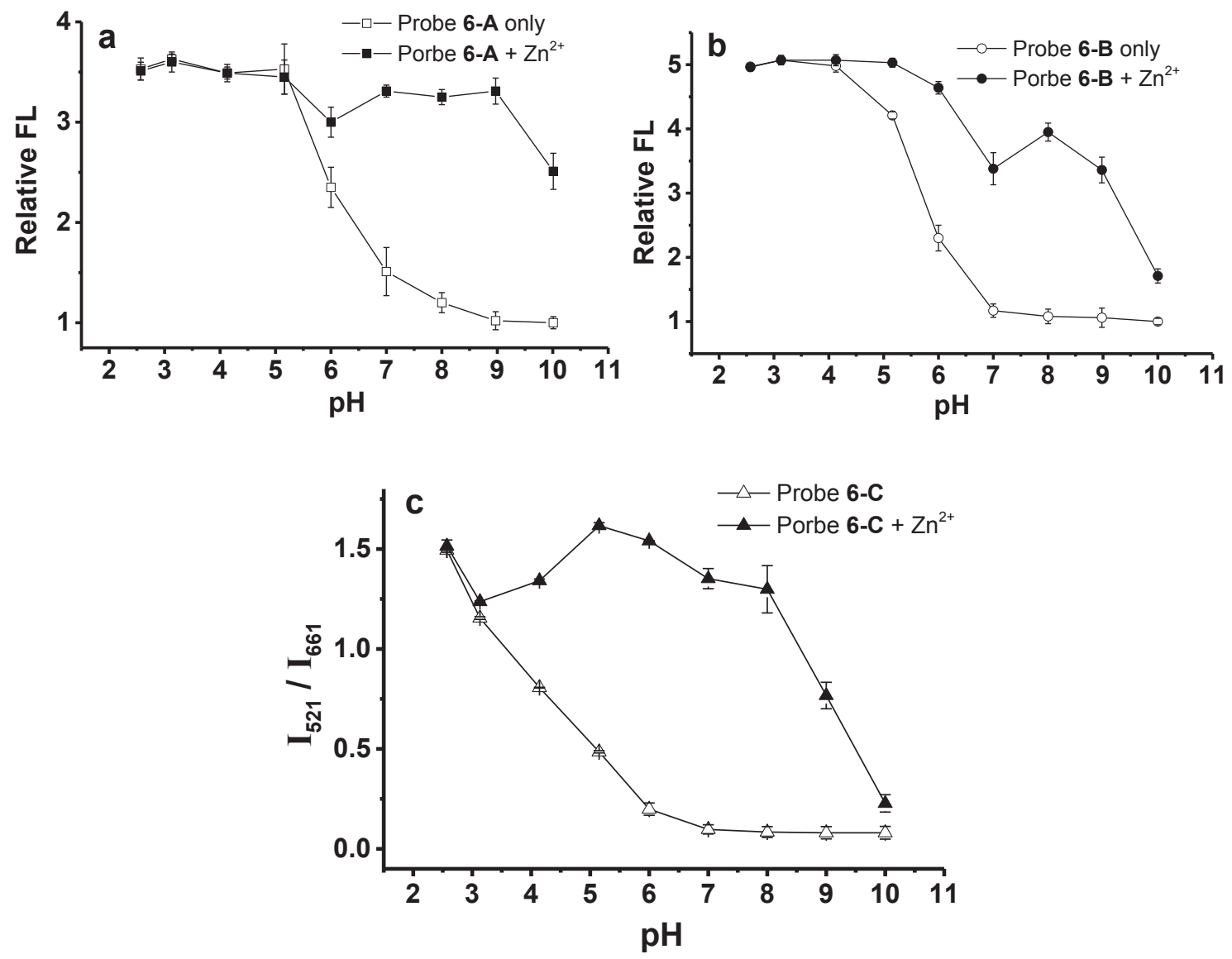

Figure 6.4. Fluorescence responses of $5 \mu \mathrm{M}$ fluorescent probes 6-A (a), 6-B (b) and 6-C (c) at different $\mathrm{pHs}$ in absence and presence of $\mathrm{Zn}^{2+}\left(6 \mu \mathrm{M}\right.$ of $\mathrm{Zn}^{2+}$ for the probes 6-A and $22 \mu \mathrm{M}$ for probe 6-B and 6-C).

\subsection{4 pH responses}

Figures 6.4 shows the effect of $\mathrm{pH}$ on fluorescence of three fluorescent probes 6-A, 6-B and 6-C. The fluorescent probe 6-A undergoes a significant increase in fluorescence intensity between $\mathrm{pH} 2.5$ and 5.0 and a considerable descrease in fluorescence intensity at $\mathrm{pH}$ greater than 5.0 (Figure 6.4a). In addition, the probe 6-A displays no responses to zinc (II) between $\mathrm{pH} 2.5$ and 5.0, which suggests that the tertiary amine of the probe 6-A is almost completely protonated at pH 5.0 (Figure 6.4a). The tertiary amine quenches the fluorescence by a photoinduced electron-transfer mechanism, and results in the reduced emission at higher $\mathrm{pH}$ when it is not bound to a proton or a diamagnetic metal. The fluorescent probes 6-B and 6-C display similar fluorescence responses to $\mathrm{pH}$ values but 
with completely different protonated $\mathrm{pH}$ ranges compared with the probe 6-A. The fluorescent probe 6-B is completely protonated at $\mathrm{pH} 4.0$ (Figure 6.4b) while the fluorescent probe 6-C is almost completely protonated at $\mathrm{pH} 3.0$ (Figure 6.4c). The probes 6-B and 6-C show no response to zinc (II) at pHs 4.0 and 3.0, respectively.

\subsubsection{Fluorescence responses of probe 6-C to zinc (II) in living cells}

Fluorescent probe 6-C also shows very useful ratiometric responses to $\mathrm{Zn}^{2+}$ ions with very good $\mathrm{pH}$ sensitivity and wide response range that may have promising application in biological and biomedical applications. Zinc (II) is an essential micronutrient and plays an important role in the cells such as ionic signaling, as cofactor for several proteins, and its levels regulate several important metabolic functions. ${ }^{34-36}$ Therefore, any drastic change in zinc (II) concentration inside the cell can cause injury and affect key cellular processes leading to disease. ${ }^{37-39}$ Thus, having a fluorescent probe that can be used to monitor the level of $\mathrm{Zn}^{2+}$ inside live cells can prove to be a valuable tool. In this study, we tested fluorescent probe 6-C for zinc sensing in SW620 colorectal adenocarcinoma. We used fluorescence microscopy to monitor and detect mobile $\mathrm{Zn}^{2+}$ ions in live cells (Figures 6.5 and 6.6). SW 620 cells incubated with $25 \mu \mathrm{M}$ probe 6-C for $2 \mathrm{hr}$ at $37^{\circ} \mathrm{C}$ were washed with PBS before imaging to remove any free probe. Cells showed fluorescence signal at longer wavelength (RFP light cube; emission in red region) but no signal for shorter wavelength region (GFP light cube, emission in green region) (Figure 6.5B). It is expected that when probe 6-C enters the cells it will bind to free $\mathrm{Zn}^{2+}$ inside the cell and give fluorescence signal at lower wavelength (green). However, in the current experimental conditions, in presence of fluorescent probe 6-C alone, strong fluorescence signal at the longer wavelength (red) region was observed (Figure 6.5B). This may be either due to low concentration of $\mathrm{Zn}^{2+}$ ions in cytosol (zinc is present in picomolar range inside cells) or due to unavailability of free zinc ions for dye binding. ${ }^{40-}$ ${ }^{41}$ SW620 cells having probe 6-C were further incubated with up to $200 \mu \mathrm{M} \mathrm{Zn}{ }^{2+}$ ions for 30 min but did not show any noticeable difference in fluorescence compared to the cells incubated with probe 6-C alone (Figure 6.5B and 6.5C). This is because the gated channels on the cell membrane do not allow entry of excess $\mathrm{Zn}^{2+}$ inside the cells. ${ }^{42}$ 
However, the cells incubated with $200 \mu \mathrm{M}$ pyrithione only showed decrease in fluorescence signal for longer wavelength (red region) and a noticeable increase in fluorescence signal for shorter wavelength (green region) (Figure 6.5D). This is because pyrithione is a $\mathrm{Zn}^{2+}$ ionophore and facilitates import of excess $\mathrm{Zn}^{2+}$ from the media inside the cells, making more free zinc ions available for binding with the probe 6-C. ${ }^{43}$ When $200 \mu \mathrm{M}$ of both $\mathrm{Zn}^{2+}$ and pyrithione were added (ratio 1:1) cells showed much higher increase in fluorescence signal at lower wavelength (green region) whereas a drastic decrease in fluorescence signal in the longer wavelength (red) region (Figure 6.5E). This increase in fluorescence emission at the shorter wavelength (green region) after incubation of cells with both $\mathrm{Zn}^{2+}$ and pyrithione is due to import of excess free $\mathrm{Zn}^{2+}$ inside the cells facilitated by pyrithione, hence more zinc availability for dye binding. The fluorescence signal at lower wavelength (green region) increases as the concentration of free $\mathrm{Zn}^{2+}$ and pyrithione increases from $50 \mu \mathrm{M}$ to $200 \mu \mathrm{M}$ (Figure 6.5E, and Figures 6.6C, 6.6D). There was no fluorescence signal observed in blue region (DAPI light cube) for any of the cells incubation conditions (results not shown). Control wells containing cells incubated only with $\mathrm{Zn}^{2+}$ ions and pyrithione but no fluorescent probe 6-C showed no signal in any wavelength region (Figure 6.5A). When N,N,N',N'-tetrakis(2pyridylmethyl)ethylenediamine (TPEN), a cell permeable high-affinity heavy metal chelator was added to the cells (cells already incubated with $\mathrm{Zn}^{2+}$ ions and pyrithione); ${ }^{44}$ the fluorescence signal for green channel decreased significantly and an increase in fluorescence signal for red channel was observed due to chelation of zinc ions (Figure 6.6E). These results underscore the importance and usefulness of fluorescent probe 6-C for monitoring changes in intracellular level of zinc ions. 


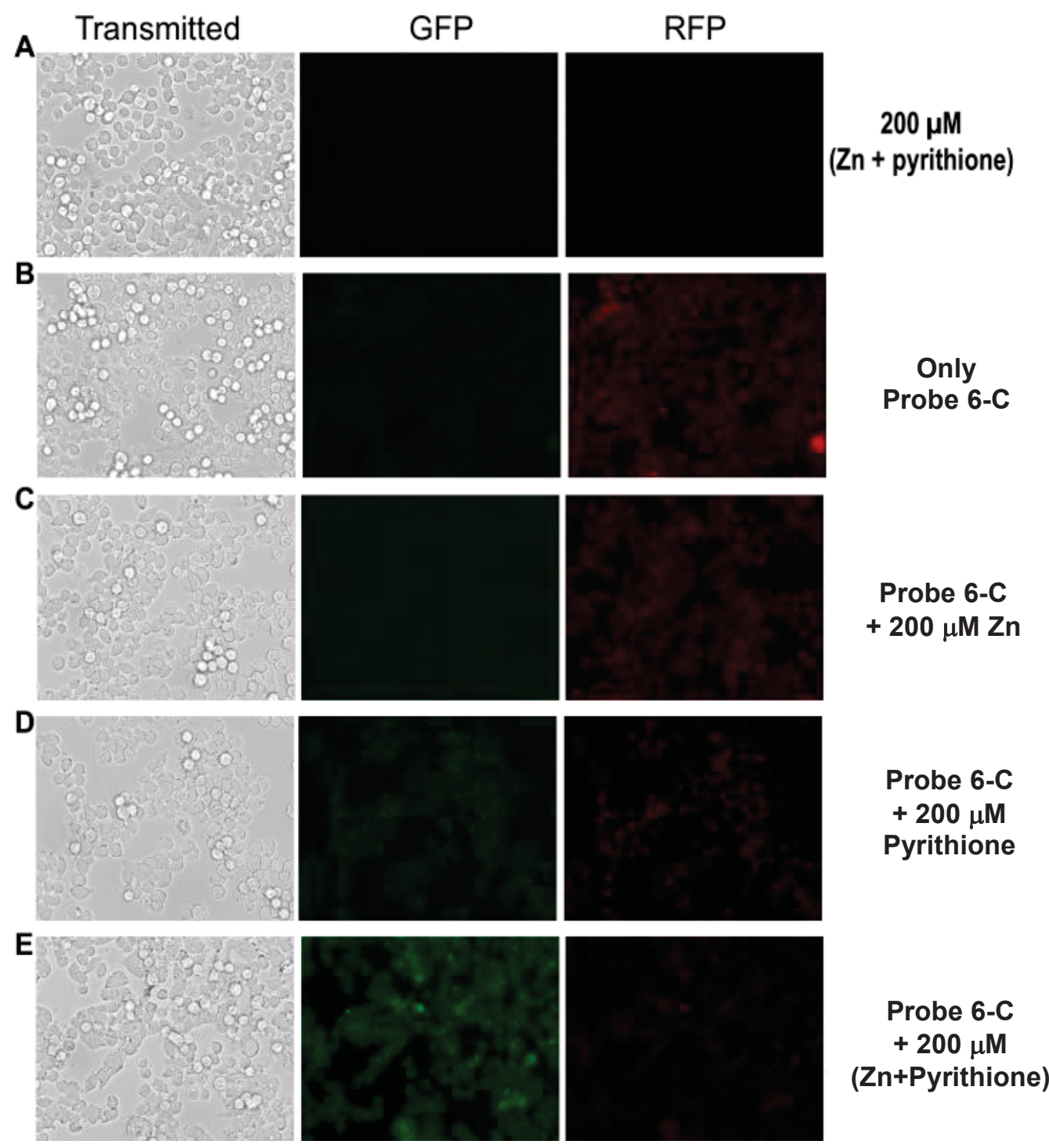

Figure 6.5. Fluorescence images of SW 620 cells with fluorescent probe 6-C.

(The cells incubated with $200 \mu \mathrm{M}$ zinc and pyrithione but without probe 6-C imaged as control (A), images of cells incubated with probe 6-C alone in serum-free media for $2 \mathrm{hr}$ (B), cells in (B) rinsed with PBS and then further incubated with $200 \mu \mathrm{M} \mathrm{Zn}^{2+}$ for $30 \mathrm{~min}$ before imaging (6-C), cells incubated with $200 \mu \mathrm{M}$ pyrithione in presence of fluorescent probe 6-C (D) and cells incubated with $200 \mu \mathrm{M}$ each of $\mathrm{Zn}^{2+}$ and pyrithione in presence of fluorescent probe 6-C (E). The bright field images were acquired at 40X magnification with transmitted light and the fluorescence images were acquired using green (GFP) and red (RFP) light cubes) 


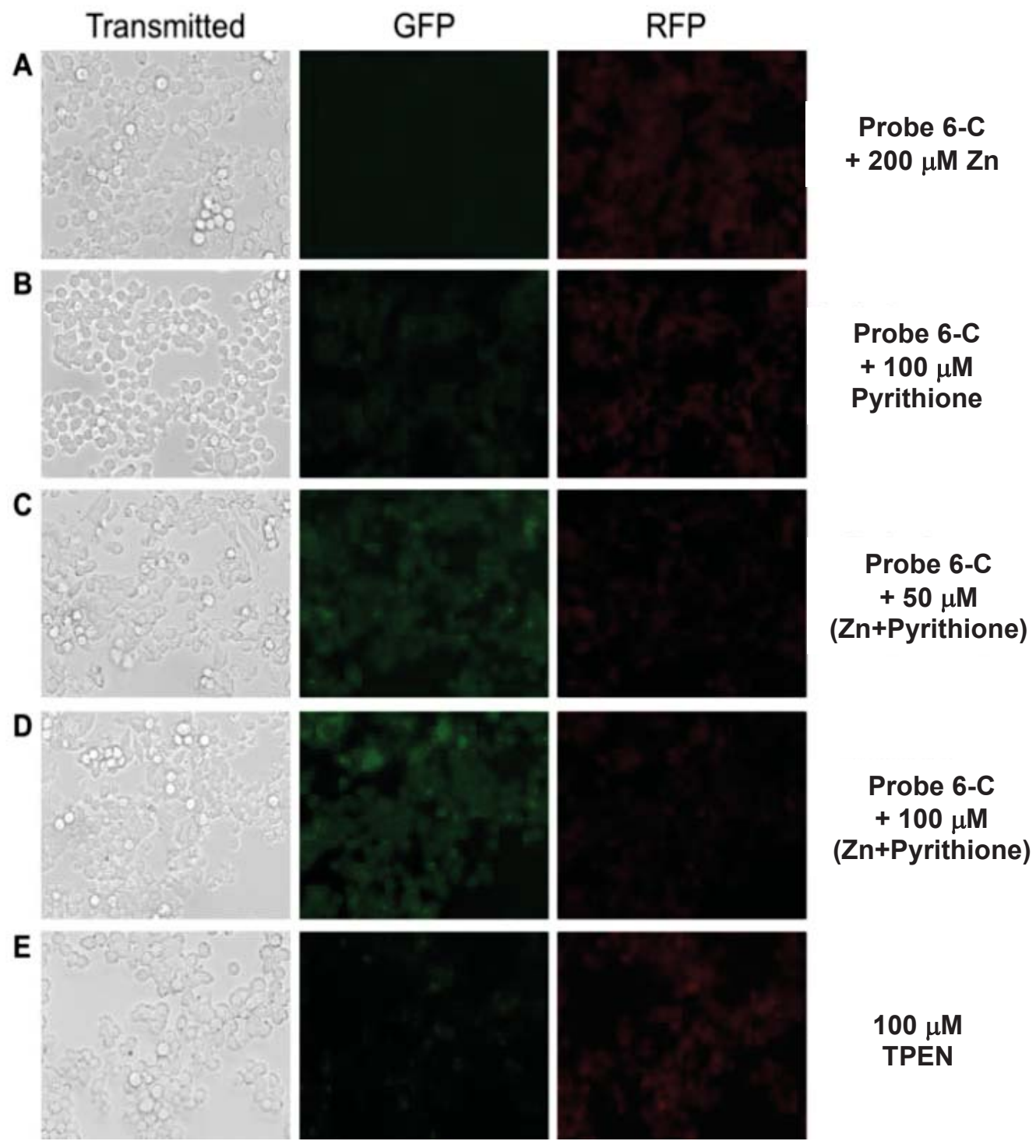

Figure 6.6. Effect of zinc and pyrithione concentrations on fluorescence probe 6-C in SW620 cells.

(The SW620 cells incubated with probe 6-C and indicated concentrations of zinc and pyrithione before imaging (A-D). The cells imaged in (D) were further incubated with $100 \mu \mathrm{M}$ of TPEN a zinc chelator for $10 \mathrm{~min}$ before re-imaging (E). The bright field images were acquired at $40 \mathrm{X}$ magnification with transmitted light and the fluorescence images were acquired using green (GFP) and red (RFP) light cubes) 


\subsection{Conclusion}

Three highly water-soluble BODIPY-based fluorescent probes (6-A, 6-B and 6-C) have been prepared by incorporating zinc (II) chelator bis(pyridin-2-ylmethyl)amine moiety into 2-formyl-BODIPY dye, 2,6-diformyl BODIPY dye, and 2,6-diformyl-1,7distyryl-BODIPYdye at 2-position and 2,6-positions. These probes exhibit excellent sensitive and selective responses to zinc (II) ions. The ratiometric fluorescent probe 6-C has been successfully applied in detection of intracellular zinc (II) in cancer cells because of its unique ratiometric fluorescent responses to zinc (II).

\section{References:}

1. Pluth, M. D., Tomat, E., Lippard, S. J., Biochemistry of Mobile Zinc and Nitric Oxide Revealed by Fluorescent Sensors. In Annual Review of Biochemistry, Kornberg, R. D.; Raetz, C. R. H.; Rothman, J. E.; Thorner, J. W., Eds. 2011; Vol. 80, pp 333-355.

2. Kay, A. R., Toth, K., Is Zinc A Neuromodulator? Science Signaling 2008, 1 (19).

3. Sensi, S. L., Paoletti, P., Bush, A. I., Sekler, I., Zinc in The Physiology and Pathology of The CNS. Nature Reviews Neuroscience 2009, 10 (11), 780-U38.

4. Frederickson, C. J., Klitenick, M. A., Manton, W. I., Kirkpatrick, J. B., Cytoarchitectonic Distribution of Zinc in The Hippocampus of Man and The Rat. Brain Res. 1983, 273 (2), 335-339.

5. Li, Y., Hough, C. J., Suh, S. W., Sarvey, J. M., Frederickson, C. J., Rapid Translocation of $\mathrm{Zn} 2+$ from Presynaptic Terminals into Postsynaptic Hippocampal Neurons after Physiological Stimulation. J. Neurophysiol. 2001, 86 (5), 2597-2604.

6. Li, Y., Hough, C. J., Frederickson, C. J., Sarvey, J. M., Induction of Mossy Fiber $>$ CA3 Long-term Potentiation Requires Translocation of Synaptically Released Zn2+. J. Neurosci. 2001, 21 (20), 8015-8025.

7. Outten, C. E., O'Halloran, T. V., Femtomolar Sensitivity of Metalloregulatory Proteins Controlling Zinc Homeostasis. Science 2001, 292 (5526), 2488-2492. 
8. Finney, L. A., O'Halloran, T. V., Transition Metal Speciation in The Cell: Insights from The Chemistry of Metal Ion Receptors. Science 2003, 300 (5621), 931-936.

9. Komatsu, K., Kikuchi, K., Kojima, H., Urano, Y., Nagano, T., Selective Zinc Sensor Molecules with Various Affinities for Zn2+, Revealing Dynamics and Regional Distribution of Synaptically Released Zn2+ in Hippocampal Slices. Journal of the American Chemical Society 2005, 127 (29), 10197-10204.

10. Park, J. G., Qin, Y., Galati, D. F., Palmer, A. E., New Sensors for Quantitative Measurement of Mitochondrial Zn2+. ACS Chem. Biol. 2012, 7 (10), 1636-1640.

11. Dean, K. M., Qin, Y., Palmer, A. E., Visualizing Metal Ions in Cells: An Overview of Analytical Techniques, Approaches, and Probes. Biochim. Biophys. Acta-Mol. Cell Res. 2012, 1823 (9), 1406-1415.

12. Dittmer, P. J., Miranda, J. G., Gorski, J. A., Palmer, A. E., Genetically Encoded Sensors to Elucidate Spatial Distribution of Cellular Zinc. J. Biol. Chem. 2009, 284 (24), 16289-16297.

13. Watnick, R. S., Herring, S. C., Palmer, A. G., Gottesman, M. E., The Carboxyl Terminus of Phage HK022 Nun Includes A Novel Zinc-Binding Motif and A Tryptophan Required for Transcription Termination. Genes Dev. 2000, 14 (6), 731-739.

14. Jiang, W., Fu, Q., Fan, H., Wang, W., An NBD Fluorophore-based Sensitive and Selective Fluorescent Probe for Zinc Ion. Chemical Communications 2008, (2), 259-261.

15. Zhang, Y., Guo, X., Si, W., Jia, L., Qian, X., Ratiometric and Water-soluble Fluorescent Zinc Sensor of Carboxamidoquinoline with An Alkoxyethylamino Chain as Receptor. Organic Letters 2008, 10 (3), 473-476.

16. Hennrich, G., Sonnenschein, H., Resch-Genger, U., Redox Switchable Fluorescent Probe Selective for Either $\mathrm{Hg}(\mathrm{II})$ or $\mathrm{Cd}(\mathrm{II})$ and $\mathrm{Zn}(\mathrm{II})$. Journal of the American Chemical Society 1999, 121 (21), 5073-5074.

17. Komatsu, K., Urano, Y., Kojima, H., Nagano, T., Development of An Iminocoumarin-based Zinc Sensor Suitable for Ratiometric Fluorescence Imaging of Neuronal Zinc. Journal of the American Chemical Society 2007, 129 (44), 13447-13454. 
18. Mizukami, S., Okada, S., Kimura, S., Kikuchi, K., Design and Synthesis of Coumarin-Based $\mathrm{Zn}(2+)$ Probes for Ratiometric Fluorescence Imaging. Inorganic Chemistry 2009, 48 (16), 7630-7638.

19. Xu, Z., Qian, X., Cui, J., Zhang, R., Exploiting The Deprotonation Mechanism for The Design of Ratiometric and Colorimetric $\mathrm{Zn}(2+)$ Fluorescent Chemosensor with A Large Red-shift in Emission. Tetrahedron 2006, 62 (43), 10117-10122.

20. Nolan, E. M., Burdette, S. C., Harvey, J. H., Hilderbrand, S. A., Lippard, S. J., Synthesis and Characterization of Zinc Sensors Based on A Monosubstituted Fluorescein Platform. Inorganic Chemistry 2004, 43 (8), 2624-2635.

21. Buccella, D., Horowitz, J. A., Lippard, S. J., Understanding Zinc Quantification with Existing and Advanced Ditopic Fluorescent Zinpyr Sensors. Journal of the American Chemical Society 2011, 133 (11), 4101-4114.

22. Nolan, E. M., Jaworski, J., Okamoto, K. I., Hayashi, Y., Sheng, M., Lippard, S. J., QZ1 and QZ2: Rapid, Reversible Quinoline-derivatized Fluoresceins for Sensing Biological Zn(II). Journal of the American Chemical Society 2005, 127 (48), 16812-16823.

23. Nolan, E. M., Jaworski, J., Racine, M. E., Sheng, M., Lippard, S. J., Midrange Affinity Fluorescent $\mathrm{Zn}$ (II) Sensors of The Zinpyr Family: Syntheses, Characterization, and Biological Imaging Applications. Inorganic Chemistry 2006, 45 (24), 9748-9757.

24. Nolan, E. M., Ryu, J. W., Jaworski, J., Feazell, R. P., Sheng, M., Lippard, S. J., Zinspy Sensors with Enhanced Dynamic Range for Imaging Neuronal Cell Zinc Uptake and Mobilization. Journal of the American Chemical Society 2006, 128 (48), 15517-15528.

25. Du, P., Lippard, S. J., A Highly Selective Turn-On Colorimetric, Red Fluorescent Sensor for Detecting Mobile Zinc in Living Cells. Inorganic Chemistry 2010, 49 (23), 10753-10755.

26. Han, Z.-X., Zhang, X.-B., Zhuo, L., Gong, Y.-J., Wu, X.-Y., Zhen, J., He, C.-M., Jian, L.-X., Jing, Z., Shen, G.-L., Yu, R.-Q., Efficient Fluorescence Resonance Energy Transfer-based Ratiometric Fluorescent Cellular Imaging Probe for 
$\mathrm{Zn}(2+)$ using A Rhodamine Spirolactam as A Trigger. Analytical Chemistry 2010, $82(8), 3108-3113$.

27. Tornat, E., Lippard, S. J., Ratiometric and Intensity-based Zinc Sensors Built on Rhodol and Rhodamine Platforms. Inorganic Chemistry 2010, 49 (20), 91139115.

28. Ojida, A., Sakamoto, T., Inoue, M.-a., Fujishima, S.-h., Lippens, G., Hamachi, I., Fluorescent BODIPY-based Zn(II) Complex as A Molecular Probe for Selective Detection of Neurofibrillary Tangles in The Brains of Alzheimer's Disease Patients. Journal of the American Chemical Society 2009, 131 (18), 6543-6548.

29. Xiao-an, Z., Lovejoy, K. S., Jasanoff, A., Lippard, S. J., Water-Soluble Porphyrins as A Dual-Function Molecular Imaging Platform for MRI and Fluorescence Zinc Sensing. Proceedings of the National Academy of Sciences of the United States of America 2007, 104 (26).

30. Zhu, S., Zhang, J., Vegesna, G., Luo, F.-T., Green, S. A., Liu, H., Highly Watersoluble Neutral BODIPY Dyes with Controllable Fluorescence Quantum Yields. Organic Letters 2011, 13 (3), 438-441.

31. Kubin, R. F., Fletcher, A. N., Fluorescence Quantum Yields of Some Rhodamine Dyes. Journal of Luminescence 1982, 27 (4), 455-462.

32. Du, H., Fuh, R. C. A., Li, J. Z., Corkan, L. A., Lindsey, J. S., Photochemcad: A Computer-aided Design and Research Tool in Photochemistry. Photochemistry and Photobiology 1998, 68 (2), 141-142.

33. Zhu, S., Zhang, J., Vegesna, G., Tiwari, A., Luo, F.-T., Zeller, M., Luck, R., Li, H., Green, S., Liu, H., Controlled Knoevenagel Reactions of Methyl Groups of 1,3,5,7-Tetramethyl BODIPY Dyes for Unique BODIPY Dyes. RSC Adv., 2012, 2, 404-407.

34. Vallee, B. L., Falchuk, K. H., The Biochemical Basis of Zinc Physiology. Physiological Reviews 1993, 73 (1), 79-118.

35. Ohalloran, T. V., Transition-Metals in Control of Gene-Expression. Science 1993, 261 (5122), 715-725.

36. King, J. C., Shames, D. M., Woodhouse, L. R., Zinc Homeostasis in Humans. Journal of Nutrition 2000, 130 (5), 1360S-1366S. 
37. Frederickson, C. J., Koh, J. Y., Bush, A. I., The Neurobiology of Zinc in Health and Disease. Nature Reviews Neuroscience 2005, 6 (6), 449-462.

38. Hedberg, K. K., Birrell, G. B., Mobley, P. L., Griffith, O. H., Transition-Metal Chelator TPEN Counteracts Phorbol Ester-induced Actin Cytoskeletal Disruption in C6 Rat Glioma-Cells without Inhibiting Activation or Translocation of ProteinKinase-C. J. Cell. Physiol. 1994, 158 (2), 337-346.

39. Link, T. A., Vonjagow, G., Zinc Ions Inhibit The Q(P) Center of Bovine Heart Mitochondrial BC(1) Complex by Blocking A Protonatable Group. J. Biol. Chem. 1995, 270 (42), 25001-25006.

40. Krezel, A., Maret, W., Zinc-buffering Capacity of A Eukaryotic Cell at Physiological pZn. J. Biol. Inorg. Chem. 2006, 11 (8), 1049-1062.

41. Bozym, R. A., Thompson, R. B., Stoddard, A. K., Fierke, C. A., Measuring Picomolar Intracellular Exchangeable Zinc in PC-12 Cells using A Ratiometric Fluorescence Biosensor. ACS Chem. Biol. 2006, 1 (2), 103-111.

42. Marin, P., Israel, M., Glowinski, J., Premont, J., Routes of Zinc Entry in Mouse Cortical Neurons: Role in Zinc-Induced Neurotoxicity. Eur. J. Neurosci. 2000, 12 (1), 8-18.

43. Jasim, S., Tjalve, H., Effect of Sodium Pyridinethione on The Uptake and Distribution of Nickel, Cadmium and Zinc in Pregnant and Nonpregnant Mice. Toxicology 1986, 38 (3), 327-350.

44. Matias, C. M., Sousa, J. M., Quinta-Ferreira, M. E., Arif, M., Burrows, H. D., Validation of TPEN as A Zinc Chelator in Fluorescence Probing of Calcium in Cells with The Indicator Fura-2. Journal of Fluorescence 2010, 20 (1), 377-380. 


\section{Chapter 7. Near-infrared Fluorescent Probes Based on Piperazine- Functionalized BODIPY Dyes for Sensitive Detection of Lysosomal $\mathrm{pH}^{1}$}

Jingtuo Zhang, ${ }^{\text {a }} \mathrm{Mu}$ Yang, ${ }^{\mathrm{a}}$ Cong Li, ${ }^{\mathrm{a}}$ Nethaniah Dorh, ${ }^{\mathrm{a}}$ Fei Xie, ${ }^{\mathrm{a}}$ Fen-Tair Luo, ${ }^{* b}$ Ashutosh Tiwari*a and Haiying Liu*a

a Department of Chemistry, Michigan Technological University, 1400 Townsend Drive, Houghton, MI 49931, USA

${ }^{\mathrm{b}}$ Institute of Chemistry, Academia Sinica, Taipei, Taiwan 11529, Republic of China

\footnotetext{
${ }^{1}$ The material included in this chapter was previously published in [Journal of Materials Chemistry B 2015, 3 (10), 2173-2184. DOI: 10.1039/C3TB00249G Publication Date (Web): Jan 19, 2015]-Reproduced by permission of The Royal Society of Chemistry http://pubs.rsc.org/en/content/articlelanding/2015/tb/c4tb01878h\#!divAbstract.
} 


\subsection{Introduction}

Lysosomes, membrane-delimited, acidic organelles present in essentially all eukaryotic cells, employ more than 70 hydrolytic enzymes to degrade biological macromolecules taken up by endocytosis, phagocytosis and autophagy. ${ }^{1-3}$ Significant disruption in the lysosomal $\mathrm{pH}$ can cause lysosome malfunction and thus cause lysosomal storage diseases. ${ }^{4}$ Therefore, it is very important to precisely detect lysosomal $\mathrm{pH}$ in living cells in order to study cellular functions for insightful understanding of physiological and pathological processes. Fluorescence spectroscopy employing $\mathrm{pH}-$ sensitive fluorescent probes is becoming one of the most powerful tools for monitoring intracellular $\mathrm{pH}$, and possesses many technical and practical advantages over other methods because it can detect the intracellular $\mathrm{pH}$ of intact cells and subcellular regions with operational simplicity, high sensitivity, and excellent spatial and temporal resolution. ${ }^{5-15}$ Only a few of them have been applied to detect lysosomal $\mathrm{pH}$ inside living cells even though many fluorescent probes for $\mathrm{pH}$ have been prepared. ${ }^{16}$ Most fluorescent probes are selectively accumulated in acidic lysosomes via lysosomotropism where their tertiary amine residues are protonated in a cellular acidic environment. ${ }^{12}$ The protonation of ionizable tertiary amine groups on the fluorophores in acidic lysosomes enhances the probe fluorescence through the suppression of photo-induced electron transfer from the tertiary amine to the probe fluorophores. ${ }^{9}$ 17-21 The potential drawback for these fluorescent probes is their broad $\mathrm{pH}$ responses and relative high fluorescent background at $\mathrm{pH}$ 7.4. Recent approach to address this high fluorescent background at $\mathrm{pH} 7.4$ is to use fluorescein and Rhodamine dyes by taking advantages of low fluorescence background at $\mathrm{pH} 7.4$ because of the "ring-closed" state of spirolactam rings in the fluorophores at $\mathrm{pH} 7.4 .^{6-7,14,22}$ However, most fluorescent probes are not soluble in aqueous solution, and some of them can cause cell damage due to their short absorption and emission wavelengths with less than $600 \mathrm{~nm}$ as a very few near-infrared fluorescent probes for lysosomal $\mathrm{pH}$ in living cells were reported..$^{23-25}$ In order to address these issues of the high fluorescent background and potential cell damage, we reported Rhodamine dye counterparts with near-infrared excitation and emission wavelengths as fluorescent probes for lysosomal $\mathrm{pH}$ in living cells. ${ }^{26} \mathrm{We}$ have demonstrated the feasibility of near- 
infrared fluorescent probes with spirocyclic structures for sensitive detection of lysosomal $\mathrm{pH}$ in living cells with advantages of excellent photostability, low cytotoxicity, deep tissue light penetration and low autofluorescence background. ${ }^{26}$ Under neutral or basic conditions, the fluorescent probes retain the spirocyclic form that is non-fluorescent and colorless. Acidic environment effectively leads to ring opening of the spirocylic form in fluorescent probes, and results in strong fluorescence. However, these fluorescent probes are still insoluble in aqueous solution. ${ }^{26}$

The search for readily accessible near-infrared fluorescent probes with excellent water solubility, large dynamic range and high specificity is still a challenging task for near-infrared imaging of lysosomal $\mathrm{pH}$ in living cells. In this paper, we design and prepare near-infrared fluorescent probes (7-A, 7-B and 7-C) for lysosomal $\mathrm{pH}$ by using piperazine residues to manipulate the fluorescent responses of the probes to $\mathrm{pH}$ via modulation of intramolecular charge transfer effect of piperazine to BODIPY dyes at different $\mathrm{pH}$ values. Piperazine residues were incorporated into BODIPY dyes at 3,5positions as parts of BODIPY cores while tri(ethylene glycol)methyl ether residues attached to piperazine were used to enhance hydrophilic properties of the probes and improve water solubility of the probes. Fluorescent probes display very weak fluorescence because of strong intramolecular charge transfer from piperazine residues to BODIPY cores at neutral $\mathrm{pH}$. Decrease of $\mathrm{pH}$ significantly enhances fluorescence intensity because the pronation of piperazine residues at acidic $\mathrm{pH}$ considerably reduces intramolecular charge transfer effect of the piperazine residues to BODPY cores. These fluorescent probes have advantages such as high photostability, sensitive and selective near-infrared imaging of lysosomal $\mathrm{pH}$ in living cells with potential intact in vivo imaging and deep tissue penetration without autofluorescence and unintended cellular damage issues. 

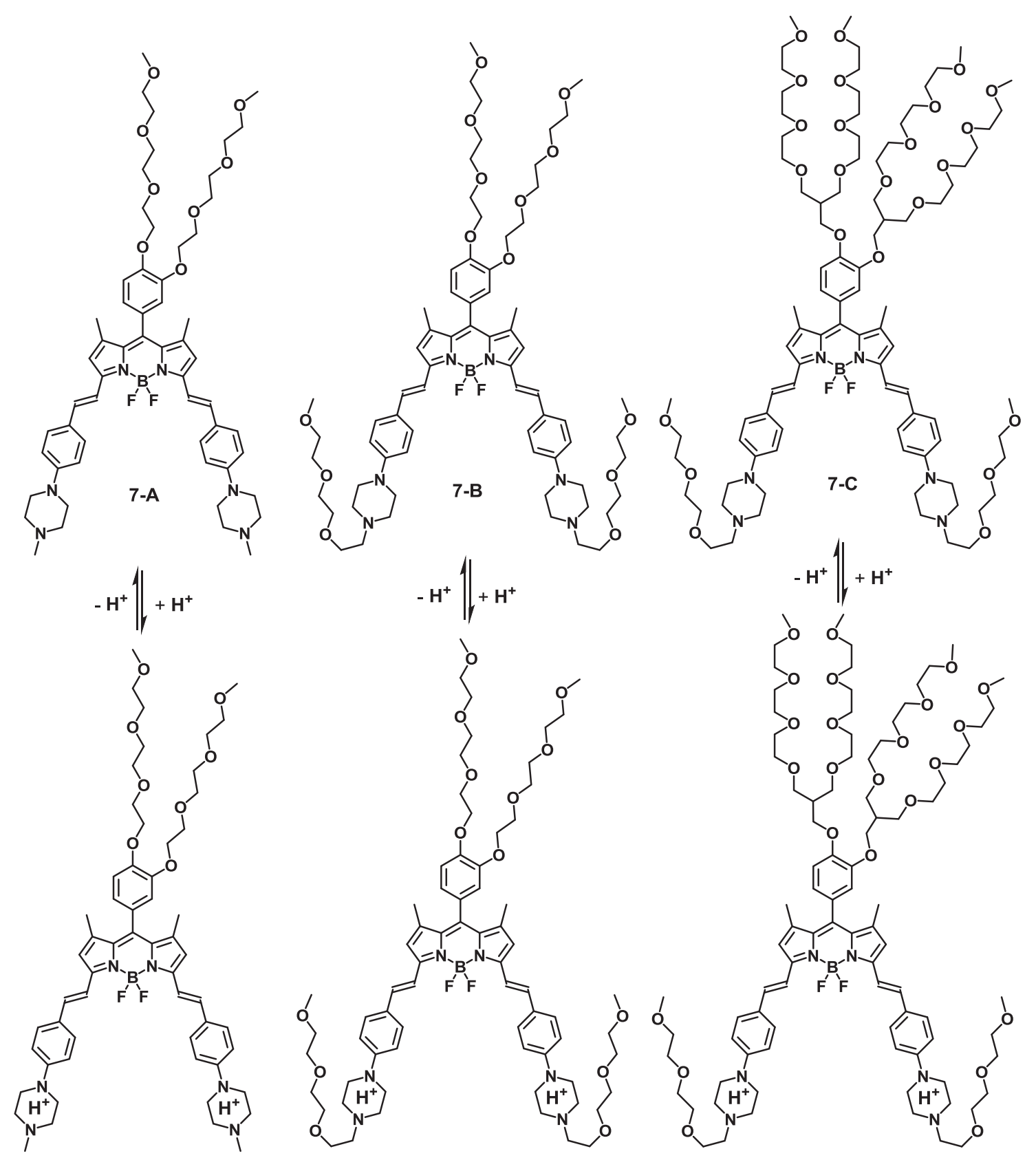

Scheme 7.1. Chemical structures of near-infrared fluorescent probes and their responses to $\mathrm{pH}$. 


\subsection{Experimental section}

\subsubsection{Instrumentations}

${ }^{1} \mathrm{H}$ NMR and ${ }^{13} \mathrm{C}$ NMR spectra were collected by $400 \mathrm{MHz}$ Varian Unity Inova NMR spectrophotometer instrument. ${ }^{1} \mathrm{H}$ and ${ }^{13} \mathrm{C}$ NMR spectra were recorded in $\mathrm{CDCl}_{3}$ and DMSO- $d_{6}$. Chemical shifts $(\delta)$ are given in ppm relative to solvent residual peaks $\left({ }^{1} \mathrm{H}\right.$ : $\delta 7.26$ for $\mathrm{CDCl}_{3}, \delta 2.50$ for DMSO- $d_{6} ;{ }^{13} \mathrm{C}: \delta 77.3$ for $\mathrm{CDCl}_{3}$ ) as internal standard. HRMS were measured with electrospray ionization (ESI) mass spectrometer and electron impact ionization (EI) mass spectrometer. Absorption spectra were taken on a Perkin Elmer Lambda 35 UV/VIS spectrometer. Fluorescence spectra were recorded on a Jobin Yvon Fluoromax-4 spectrofluorometer.

\subsubsection{Materials}

Unless specific indicated, all reagents and solvents were obtained from commercial suppliers and used without further purification. BODIPY dye (7.7) and BODIPY dye (7.9) were prepared from compounds (7.6) and (7.8) respectively, according to our previous reported procedures ${ }^{27-28}$ Compound 7.4 was prepared according the reported literature/patent. ${ }^{29}$

4-(4-methylpiperazin-1-yl)benzaldehyde (7.3): 4-fluorobenzaldehyde (1 $\mathrm{g}, \quad 8.0$ mmol), 1-methylpiperazine (2.4 g, $24.0 \mathrm{mmol})$ and $\mathrm{K}_{2} \mathrm{CO}_{3}(3.3 \mathrm{~g}, 24 \mathrm{mmol})$ was added into $18 \mathrm{~mL}$ DMF in a $50 \mathrm{~mL}$ round flask. The mixture was stirred for 14 hours at $80{ }^{\circ} \mathrm{C}$ and then washed with water and extracted by $100 \mathrm{~mL} \mathrm{CH}_{2} \mathrm{Cl}_{2}$. The organic layer was collected and dried over anhydrous $\mathrm{Na}_{2} \mathrm{SO}_{4}$ and filtered. The filtrate was concentrated in vacuum and the crude product was purified by column chromatograph with eluent $\left(\mathrm{CH}_{2} \mathrm{Cl}_{2} / \mathrm{EtOH}, 9 / 0.8, \mathrm{v} / \mathrm{v}\right)$ to get compound 7.3 as yellow solid $(1.41 \mathrm{~g}, 86 \%) .{ }^{1} \mathrm{H}$ NMR $\left(400 \mathrm{MHz}, \mathrm{CDCl}_{3}\right) \delta 9.76(\mathrm{~s}, 1 \mathrm{H}), 7.73(\mathrm{~d}, J=8 \mathrm{~Hz}, 2 \mathrm{H}), 6.90(\mathrm{~d}, J=8 \mathrm{~Hz}, 2 \mathrm{H}), 3.40(\mathrm{t}$, $J=4.4 \mathrm{~Hz}, 4 \mathrm{H}), 2.54(\mathrm{t}, J=4.4 \mathrm{~Hz}, 4 \mathrm{H}), 2.34(\mathrm{~s}, 3 \mathrm{H}) \cdot{ }^{13} \mathrm{C} \mathrm{NMR}\left(100 \mathrm{MHz}, \mathrm{CDCl}_{3}\right) \delta$ 190.6, 155.2, 132.1, 127.4, 113.8, 54.9, 47.3, 46.3. HRMS (EI+) Calculated for $\mathrm{C}_{12} \mathrm{H}_{16} \mathrm{~N}_{2} \mathrm{O}[\mathrm{M}]^{+}$204.1263, found 204.1262.

4-(4-(2-(2-(2-methoxyethoxy)ethoxy)ethyl)piperazin-1-yl)benzaldehyde $\quad$ (7.5): $\quad 4-$ fluorobenzaldehyde (0.4 g, $3.2 \mathrm{mmol})$, compound $7.2(1.2 \mathrm{~g}, 4.8 \mathrm{mmol})$ and $\mathrm{K}_{2} \mathrm{CO}_{3}(0.66$ $\mathrm{g}, 4.8 \mathrm{mmol}$ ) was added into $7 \mathrm{~mL}$ DMF in a $25 \mathrm{~mL}$ round flask. The mixture was stirred 
for 12 hours at $80{ }^{\circ} \mathrm{C}$ and then washed with water and extracted by $100 \mathrm{~mL} \mathrm{CH}_{2} \mathrm{Cl}_{2}$. The organic layer was collected and dried over anhydrous $\mathrm{Na}_{2} \mathrm{SO}_{4}$ and filtered. The filtrate was concentrated in vacuum and the crude product was purified by column chromatograph with eluent $\left(\mathrm{CH}_{2} \mathrm{Cl}_{2} / \mathrm{EtOH}, 9 / 0.6, \mathrm{v} / \mathrm{v}\right)$ to get compound 7.5 as yellow oil (0.60 g, 55\%). ${ }^{1} \mathrm{H}$ NMR (400 MHz, $\left.\mathrm{CDCl}_{3}\right) \delta 9.76$ (s, 1H), 7.73 (d, J=8 Hz, 2H), 6.89 (d, $J=8 \mathrm{~Hz}, 2 \mathrm{H}), 3.77-3.30$ (m, 17H), 2.67 (brs, $6 \mathrm{H}) .{ }^{13} \mathrm{C} \mathrm{NMR}\left(100 \mathrm{MHz}, \mathrm{CDCl}_{3}\right)$ $\delta 190.3,154.9,131.7,126.9,113.3,71.9,70.3,68.8,59.0,57.6,53.1,46.9$. HRMS (EI+) Calculated for $\mathrm{C}_{18} \mathrm{H}_{28} \mathrm{~N}_{2} \mathrm{O}_{4}[\mathrm{M}]^{+} 336.2049$, found 336.2045

General procedure to prepare fluorescent probes 7-A, 7-B and 7-C: When BODIPY dye (1 eq.), compound $\mathbf{7 . 3}$ or 7.5 (6 eq.) were dissolved in benzene, a small amount of piperidine and acetic acid were added into the solution and the reaction mixture was refluxed for $10-16$ hours at $100{ }^{\circ} \mathrm{C}$. Any water formed during the reaction was removed azeotropically by using a Dean-Stark apparatus. After the reaction was quenched by water at room temperature, the mixture was concentrated under reduced pressure and re-dissolved in $100 \mathrm{~mL}$ dichloromethane. It was sequentially washed with water $(2 \times 100 \mathrm{~mL})$ and brine $(100 \mathrm{~mL})$, dried over $\mathrm{Na}_{2} \mathrm{SO}_{4}$ and filtered. The filtrate was concentrated under reduced pressure and the crude product was purified by column chromatography with silica gel (32-63 microns, 230-400 mesh) by using $\mathrm{CH}_{2} \mathrm{Cl}_{2} / \mathrm{MeOH}$ $(7 / 1, v / v)$ as eluent to obtain fluorescent probe 7-A, 7-B or 7-C:

Fluorescent probe 7-A: According to the general procedure, fluorescent probe 7-A was prepared from BODIPY dye 7.7 (50 mg, $0.077 \mathrm{mmol})$, compound 7.3 (95 mg, 0.465 $\mathrm{mmol})$, benzene $(8 \mathrm{~mL})$, piperidine $(0.1 \mathrm{~mL}, 1.012 \mathrm{mmol})$ and acetic acid $(0.1 \mathrm{~mL}, 1.747$ mmol). The mixture was refluxed for 10 hours. After work-up and purifying by column chromatography with elute solution: $\left(\mathrm{CH}_{2} \mathrm{Cl}_{2} / \mathrm{MeOH} / \mathrm{Et}_{3} \mathrm{~N}, 7 / 1 / 0.01, \mathrm{v} / \mathrm{v} / \mathrm{v}\right)$, the probe 7A was obtained as green solid (33 mg, 42\%). ${ }^{1} \mathrm{H}$ NMR (400 MHz, $\left.\mathrm{CDCl}_{3}\right) \delta 7.67-7.49$ (m, 6H), $7.25-7.12$ (m, 2H), 6.99 (d, $J=8 \mathrm{~Hz}, 1 \mathrm{H}), 6.95-6.87(\mathrm{~m}, 5 \mathrm{H}), 6.84(\mathrm{~d}, J=8$ $\mathrm{Hz}, 1 \mathrm{H}), 6.60$ (br s, 2H), 4.22 (t, $J=4.8 \mathrm{~Hz}, 2 \mathrm{H}), 4.15$ (t, $J=4.8 \mathrm{~Hz}, 2 \mathrm{H}), 3.92$ (t, $J=4.8$ $\mathrm{Hz}, 2 \mathrm{H}), 3.86(\mathrm{t}, J=4.8 \mathrm{~Hz}, 2 \mathrm{H}), 3.81-3.49$ (m, 16H), $3.42-3.30$ (m, 14H), 2.68 (br s, 8H), 2.43 (s, 6H), 1.51 (s, 6H); ${ }^{13} \mathrm{C}$ NMR (100 MHz, $\left.\mathrm{CDCl}_{3}\right) \delta 152.9,151.4,149.8$, 149.7, 141.7, 136.1, 133.6, 129.2, 128.4, 128.3, 122.0, 117.6, 116.8, 115.7, 115.2, 114.7, 
$72.2,72.2,71.2,71.0,70.9,70.1,70.0,69.4,69.0,59.3,54.9,48.1,46.0,15.0$. HRMS (ESI): calculated for $\mathrm{C}_{57} \mathrm{H}_{75} \mathrm{BF}_{2} \mathrm{~N}_{6} \mathrm{O}_{8}[\mathrm{M}]^{+}, 1020.5702$; found, 1020.5736 .

Fluorescent probe 7-B: According to the general procedure, fluorescent probe 7-B was prepared from BODIPY dye $7.7(50 \mathrm{mg}, 0.077 \mathrm{mmol})$, compound 7.5 (157 mg, $0.467 \mathrm{mmol})$, benzene $(10 \mathrm{~mL})$, piperidine $(0.1 \mathrm{~mL}, 1.012 \mathrm{mmol})$ and acetic acid $(0.1$ $\mathrm{mL}, 1.747 \mathrm{mmol})$. The mixture was refluxed for 10 hours. After work-up and purifying by column chromatography with elute solution: $\left(\mathrm{CH}_{2} \mathrm{Cl}_{2} / \mathrm{MeOH}, 7 / 1\right.$, v/v), the probe 7-B was obtained as green oil (51 mg, 52\%). ${ }^{1} \mathrm{H}$ NMR (400 MHz, $\left.\mathrm{CDCl}_{3}\right) \delta 7.60-7.47$ (m, $6 \mathrm{H}), 7.16(\mathrm{~d}, J=16 \mathrm{~Hz}, 2 \mathrm{H}), 6.97(\mathrm{~d}, J=8 \mathrm{~Hz}, 1 \mathrm{H}), 6.91-6.85(\mathrm{~m}, 5 \mathrm{H}), 6.81(\mathrm{~d}, J=8$ Hz, 1H), 6.59 (s, 2H), 4.20 (t, $J=4.8 \mathrm{~Hz}, 2 \mathrm{H}), 4.12(\mathrm{t}, J=4.8 \mathrm{~Hz}, 2 \mathrm{H}), 3.89(\mathrm{t}, J=4.8$ $\mathrm{Hz}, 2 \mathrm{H}), 3.84(\mathrm{t}, J=4.8 \mathrm{~Hz}, 2 \mathrm{H}), 3.79-3.47(\mathrm{~m}, 36 \mathrm{H}), 3.38-3.36(\mathrm{~m}, 9 \mathrm{H}), 3.32(\mathrm{~s}$, 3H), 3.30 (br s, 8H), $2.75-2.64$ (m, 12H), 1.49 (s, 6H); $\left.{ }^{13} \mathrm{C} \mathrm{NMR} \mathrm{(100} \mathrm{MHz,} \mathrm{CDCl}_{3}\right) \delta$ 152.8, 151.6, 149.7, 149.6, 142.8, 141.5, 137.1, 136.1, 133.5, 130.1, 129.0, 128.4, 127.9, 125.6, 121.9, 117.5, 116.5, 115.4, 115.2, 115.0, 114.6, 72.2, 72.1, 71.1, 70.9, 70.8, 70.7, 70.6, 70.0, 69.9, 69.3, 69.1, 68.9, 59.3, 59.2, 48.3, 47.9, 14.9. HRMS (ESI): calculated for $\mathrm{C}_{69} \mathrm{H}_{99} \mathrm{BF}_{2} \mathrm{~N}_{6} \mathrm{O}_{14}[\mathrm{M}]^{+}, 1284.7274$; found, 1284.7261 .

Fluorescent probe 7-C: According to the general procedure, fluorescent probe 7-C was prepared from BODIPY dye $7.9(50 \mathrm{mg}, 0.045 \mathrm{mmol})$, compound 5 (90 $\mathrm{mg}, 0.268$ $\mathrm{mmol})$, benzene $(8 \mathrm{~mL})$, piperidine $(0.1 \mathrm{~mL}, 1.012 \mathrm{mmol})$ and acetic acid $(0.1 \mathrm{~mL}, 1.747$ mmol). The mixture was refluxed for 16 hours. After work-up and purifying by column chromatography with elute solution: $\left(\mathrm{CH}_{2} \mathrm{Cl}_{2} / \mathrm{MeOH}, 7 / 1\right.$, v/v).; The probe 7-C was obtained as green oil (30 mg, 38\%); ${ }^{1} \mathrm{H}$ NMR (400 MHz, DMSO- $\left.d_{6}\right) \delta 7.50-7.41 \quad(\mathrm{~m}$, $6 \mathrm{H}), 7.35$ (d, $J=16 \mathrm{~Hz}, 2 \mathrm{H}), 7.13$ (d, $J=8 \mathrm{~Hz}, 1 \mathrm{H}), 7.04-6.97$ (m, 5H), $6.91-6.87$ (m, $3 \mathrm{H}), 4.06(\mathrm{~d}, J=8 \mathrm{~Hz}, 2 \mathrm{H}), 4.01(\mathrm{~d}, J=8 \mathrm{~Hz}, 2 \mathrm{H}), 3.62-3.36(\mathrm{~m}, 76 \mathrm{H}), 3.34-3.18$ (m, 26H), 2.59 (br s, 12H), 2.31 (sextet, $J=5.6 \mathrm{~Hz}, 1 \mathrm{H}$ ), 2.25 (sextet, $J=5.6 \mathrm{~Hz}, 1 \mathrm{H}$ ) $1.50(\mathrm{~s}, 6 \mathrm{H}) ;{ }^{13} \mathrm{C} \mathrm{NMR}\left(100 \mathrm{MHz}, \mathrm{CDCl}_{3}\right) \delta 152.9,151.5,149.9,149.8,142.7,141.7$, 137.6, 136.0, 133.6, 130.1, 129.1, 128.3, 127.9, 121.5, 117.5, 116.7, 115.6, 114.3, 113.9, $72.2,72.2,71.1,70.9,70.9,70.8,70.7,70.7,69.7,69.6,67.8,67.4,59.3,59.2,57.9,48.1$, 32.2, 23.0, 15.1, 14.4. HRMS (ESI): calculated for $\mathrm{C}_{91} \mathrm{H}_{143} \mathrm{BF}_{2} \mathrm{~N}_{6} \mathrm{O}_{24}[\mathrm{M}]^{+}, 1753.0209$; found, 1753.0194 . 


\subsubsection{Optical measurements}

All the absorption and emission spectra were recorded by using standard $1 \mathrm{~cm}$ path length quartz fluorescence cuvette at room temperature. The slit width of excitation and emission were set to $3 \mathrm{~nm}$ and $5 \mathrm{~nm}$ respectively and the excitation wavelength is set at $620 \mathrm{~nm}$ for fluorescence spectroscopies. 1.0 mM DMSO solutions of probes 7-A and 7-B and $1.0 \mathrm{mM}$ aqueous solution of probe $\mathbf{7 - C}$ were used as stock solutions. All optical spectra of probes 7-A, 7-B and 7-C were measured in the $50 \mathrm{mM}$ citrate-phosphate buffer solutions (contain 0.5\% DMSO from stocks for probe 7-A and 7-B samples).

$50 \mathrm{mM}$ citrate-phosphate buffer was used for $\mathrm{pH}$ dependence and photostability measurements of the fluorescent probes. Five times diluted citrate-phosphate buffer (10 $\mathrm{mM}, \mathrm{pH}$ 7.4) was used for preparing the mixed solvent with different percentage of ethanol for the study of solvent effects of the fluorescent probes. In order to avoid the interference of metal-phosphate and metal-citrate binding interactions (forming precipitate of divalent cation phosphate and forming complex of the metal-citrate), 10 $\mathrm{mM}$ KHP buffer ( $\mathrm{pH} 4.5)$ and $10 \mathrm{mM}$ HEPES (pH 7.4) buffer were used for selectivity measurements of fluorescent probe 7-A, 7-B and 7-C.

The UV-Vis spectra of probes 7-A, 7-B and 7-C for $\mathrm{pH}$ dependency, selectivity, photostability and solvent effect measurements were collected in the range of $300-850$ $\mathrm{nm}$ with increments of $1 \mathrm{~nm}$. Their corresponding fluorescence spectra were collected with excitation wavelength of $620 \mathrm{~nm}$ with increments of $1 \mathrm{~nm}$. The excitation and emission slit widths were set up to $3 \mathrm{~nm}$ and $5 \mathrm{~nm}$ respectively. The concentration of the dye in each sample is $5 \mu \mathrm{M}$. The fluorescence quantum of BODIPY dyes 7.7 and 7.9 were calculated by using fluorescein $\left(\Phi_{\mathrm{f}}=0.95\right.$ in $01 \mathrm{~N} \mathrm{NaOH}$ a.q. with excitation at 490 $\mathrm{nm})^{30}$ as standard. Sulforhodamine 101 dye $\left(\Phi_{f}=95 \%\right.$ with excitation wavelength at 577 $\mathrm{nm}$ in ethanol) ${ }^{31}$ was used as a reference standard to determine the fluorescence quantum

yields of fluorescent probes 7-A, 7-B, 7-C as well as their protonated derivatives in dichloromethane, ethanol, DMSO and aqueous solutions. Both samples and references were freshly prepared under identical conditions. The quantum yields were calculated using the following equation:

$$
\Phi_{X}=\Phi_{s t}\left(\operatorname{Grad}_{X} / \operatorname{Grad}_{s t}\right)\left(\eta_{X}^{2} / \eta_{s t}^{2}\right) \ldots \ldots(\text { eq. 7.1) }
$$


Where the subscripts ' $s t$ ' and ' $X$ ' stand for standard and test, respectively, $\Phi$ is the fluorescence quantum yield, "Grad" represents the gradient from the plot of integrated fluorescence intensity versus absorbance and $\eta$ is the refractive index of the solvent.

\subsubsection{Determination of $\mathrm{p} K_{\mathrm{a}}$ by fluorometric titration}

The constant $K_{\boldsymbol{a}}$ of fluorescent probes 7-A, 7-B or 7-C was determined in aqueous buffered solution by fluorometric titration as a function of $\mathrm{pH}$ using the fluorescence spectra. The expression of the steady-state fluorescence intensity $F$ as a function of the proton concentration has been extended for the case of a n: 1 complex between $\mathrm{H}^{+}$and a fluorescent probe, which expressed by the equation as below. ${ }^{32}$

$$
F=\frac{F_{\max }\left[\mathrm{H}^{+}\right]^{n}+F_{\min } K_{a}}{K_{a}+\left[\mathrm{H}^{+}\right]^{n}} \ldots \ldots \ldots . .(\text { eq. } 7.2)
$$

$F_{\max }$ and $F_{\min }$ stand for the fluorescence intensity at maximal and minimal $\mathrm{H}^{+}$ concentration, respectively, and $\mathrm{n}$ is apparent stoichiometry of $\mathrm{H}^{+}$binding to the probe that affect the fluorescent change. Nonlinear fitting of equation expressed above to the fluorescence titration data recoded as a function of $\mathrm{H}^{+}$concentration with $K_{a}$ and $\mathrm{n}$ as free adjustable parameter yields the estimated apparent constant of $K_{\boldsymbol{a}}$.

\subsubsection{Cell culture and fluorescence imaging}

Breast cancer cells MDA-MB-231 and normal endothelial cells HUVEC-C (from ATCC) were cultured as previously described. ${ }^{33}$ In brief, cells were plated on 12-well culture plates at $1 \times 10^{5}$ cells/well and incubated at $37^{\circ} \mathrm{C}$ in $5 \% \mathrm{CO}_{2}$ incubator overnight. Next day, the media was removed and cells were rinsed twice with $1 \mathrm{X}$ PBS after which fresh serum-free media was added and cells were incubated for $2 \mathrm{~h}$ at $37{ }^{\circ} \mathrm{C}$ in $\mathrm{CO}_{2}$ incubator. Following $2 \mathrm{~h}$ serum starvation, fresh serum free media with/without 2, 5, 10 $\mu \mathrm{M}$ probe 7-A or 5, 15, $25 \mu \mathrm{M}$ probe 7-B or 7-C were added and incubated further with cells for $2 \mathrm{~h}$. Live cell images were acquired using an inverted fluorescence microscope (AMF-4306, EVOS $\mathrm{fl}, \mathrm{AMG}$ ) with DAPI filter for Hoechst 33342 (Sigma-Aldich), GFP

filter for LysoSensor Green DND-189 (Invitrogen), and CY5 filter for fluorescent probes 7-A, 7-B, and 7-C. The fluorescence images were obtained at 40x and 60x magnification for HUVEC-C and at 60x magnification for MDA-MB-231 cells. The exposure times for 
each filter were kept constant. Co-localization analysis based on Pearson's coefficient was done using JACoP plugin from ImageJ. ${ }^{34}$

Live cell fluorescence imaging at different intracellular pH: Normal endothelial cells HUVEC-C (from ATCC) were cultured as previously described. In brief, cells were plated on 12-well culture plates at $1 \times 10^{5}$ cells/well and incubated at $37{ }^{\circ} \mathrm{C}$ in $5 \% \mathrm{CO}_{2}$ incubator overnight. Next day, the media was removed and cells were rinsed twice with 1 $\mathrm{X}$ PBS ( $\mathrm{pH}$ 7.4) after which fresh serum free media with $5 \mu \mathrm{M}$ probe 7-A or $15 \mu \mathrm{M}$ probe 7-B or $15 \mu \mathrm{M}$ probe 7-C were added and incubated further with cells for $2 \mathrm{~h}$. After incubation, the medium was removed and cells were gently rinsed with PBS (pH 7.4) three times. It was then treated with nigericin $(5 \mu \mathrm{g} / \mathrm{mL})$ in $2 \mathrm{~mL}$ potassium rich PBS at different $\mathrm{pH}(5.0,5.5,6.5$, and 7.5) and incubated further for $15 \mathrm{~min}$. Live cell images were acquired using an inverted fluorescence microscope (AMF-4306, EVOSfl, AMG) with DAPI filter for Hoechst 33342 (Sigma-Aldich), GFP filter for LysoSensor Green DND-189 (Invitrogen), and CY5 filter for fluorescent probes 7-A, 7-B, and 7-C. The fluorescence images were obtained at 40x magnification. The exposure times for each filter were kept constant.

\subsubsection{Determination of cellular uptake efficiency}

HUVEC-C cells were plated at a density of $1 \times 10^{4}$ cells/well on a 96-well cell culture plate and incubated at $37^{\circ} \mathrm{C}$ in $5 \% \mathrm{CO}_{2}$ incubator overnight. Next day, the culture medium was removed and cells were rinsed twice with PBS ( $\mathrm{pH} 7.4)$. Fresh $100 \mu \mathrm{L}$ media with $2,5,10 \mu \mathrm{M}$ of probe $7-\mathbf{A}$, or $5,15,25 \mu \mathrm{M}$ of probe 7-B or $5,15,25 \mu \mathrm{M}$ probe 7-C were added to the wells in triplicate and incubated for $2 \mathrm{~h}$. Controls and blanks were also set in triplicate at the same time. Controls had culture media and dyes but no cells. Blanks used for background subtraction had cells and media but no dyes. After $2 \mathrm{~h}$ incubation the media $(100 \mu \mathrm{L})$ was pipetted out from the plate and put in a fresh $96-w e l l$ plate. Each well was rinsed with $100 \mu \mathrm{L}$ of fresh PBS ( $\mathrm{pH}$ 7.4) and the solution was added to the respective wells in the fresh 96-well plate. Then absorbance of wells was measured using an ELISA plate reader (BioTek Instruments, Inc.) at $712 \mathrm{~nm}, 710 \mathrm{~nm}$, and $687 \mathrm{~nm}$ (absorbance peak of probes 7-A, 7-B, and 7-C, respectively). Values of 
respective blanks were subtracted from samples $\left(A_{d y e}\right)$ and controls $\left(A_{c t r l}\right)$. Cellular uptake efficiency was calculated as $\left[1-\left(A_{d y e} / A_{c t r l}\right)\right] \times 100 \%$

\subsubsection{MTS assay}

MTS assay were performed with MDA-MB-231 cells (ATCC) as previously described. ${ }^{35}$ Briefly, the cells were plated at a density of 5,000 cells/well on a 96-well cell culture plate and incubated at $37{ }^{\circ} \mathrm{C}$ in $5 \% \mathrm{CO}_{2}$ incubator overnight. After incubation, the media was removed and the cells were washed with 1 X PBS. Fresh media with 0, 5, 15, 25, and $50 \mu \mathrm{M}$ of fluorescent probes 7-A, 7-B, and 7-C dissolved in DMSO (with less than $<0.5 \%$ DMSO final concentration in media) were added to the wells and measured in replicates of 6 for each dye concentration. Blanks that had everything else except the cells were prepared at the same time. The plates were incubated at $37{ }^{\circ} \mathrm{C}$ in $5 \% \mathrm{CO}_{2}$ incubator for $72 \mathrm{~h}$. After the $72 \mathrm{~h}$ incubation, $20 \mu \mathrm{L}$ of MTS solution (from CellTiter 96 Aqueous Non-Radioactive Cell proliferation Assay (MTS) kit, Promega) was added to each well. The absorbance at $490 \mathrm{~nm}$ was acquired after $4 \mathrm{~h}$ incubation at $37^{\circ} \mathrm{C}$, using an ELISA plate reader (BioTek Instruments, Inc.) Plots were normalized to control wells containing media and cells only.

\subsection{Results and discussions}

\subsubsection{Synthesis approach}

In order to conjugate piperazine moiety into BODIPY dyes as parts of the fluorophores to modulate intramolecular charge transfer from the piperazine moiety to BODIPY cores (Scheme 7.2), we prepared 4-(4-methylpiperazin-1-yl)benzaldehyde (7.3) and 4-(4-(2-(2-(2-methoxyethoxy)ethoxy)ethyl)piperazin-1-yl)benzaldehyde (7.5) by reacting 4-fluorobenzaldehyde (7.1) with 1-methylpiperazine (7.2) and 1-(2-(2-(2methoxyethoxy)ethoxy)ethyl)piperazine (7.4) in DMF solution under a basic condition at $80{ }^{\circ} \mathrm{C}$, respectively. BODPY-based near-infrared fluorescent probe (7-A) was prepared by incorporating piperazine residues into BODIPY cores at 3,5-positions via the Knoevenagel condensation reaction of our previous reported BODIPY dye 7 with 4-(4- 

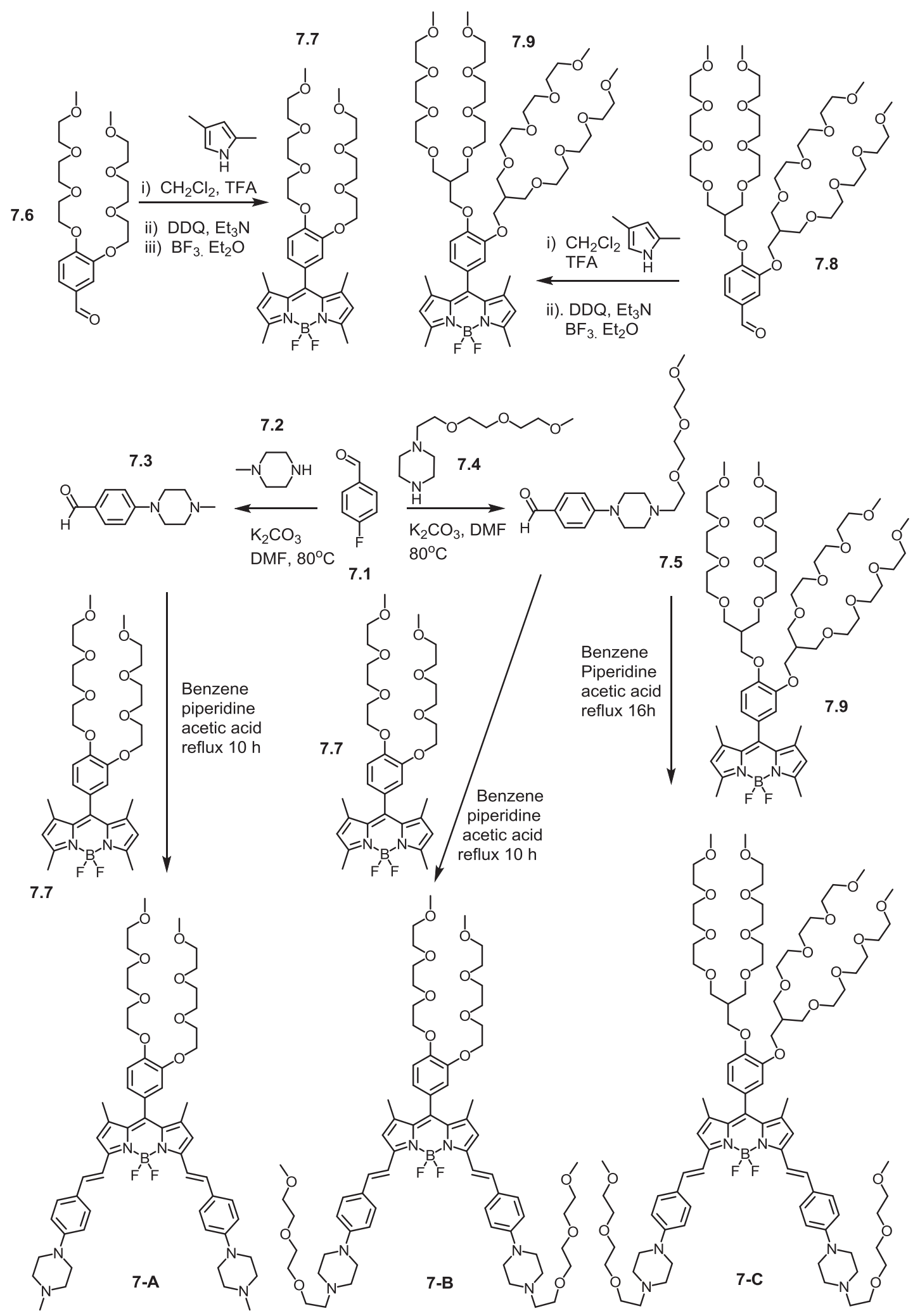

Scheme 7.2. Synthetic route to near-infrared fluorescent probes 7-A, 7-B and 7-C. 
methylpiperazin-1-yl)benzaldehyde (7.3). In order to enhance hydrophilic feature and further improve water solubility of the fluorescent probes, we prepared near-infrared probe (7-B and 7-C) by using hydrophilic 4-(4-(2-(2-(2methoxyethoxy)ethoxy)ethyl)piperazin-1-yl)benzaldehyde (7.5) to conduct the Knoevenagel condensation reaction with BODIPY dye 7.7 and our previously reported water-soluble BODIPY dye 7.9, respectively. The probes $\mathbf{7 - A}$ and 7-B are slightly soluble in aqueous solution. However, the probe 7-C shows excellent water solubility because of its numerous hydrophilic oligo(ethylene glycol)methyl ether residues at mesoand 3,5-positions which significantly facilitate the strong enthalpic interactions with water.

\subsubsection{Optical properties of fluorescent probes}

The absorption properties of BODIPY dye (7.7) in ethanol is characterized by a strong $\mathrm{S}_{0} \rightarrow \mathrm{S}_{1}\left(\pi-\pi^{*}\right)$ transition at $499 \mathrm{~nm}$ and a weaker broad band around $350 \mathrm{~nm}$ attributed to the $\mathrm{S}_{0} \rightarrow \mathrm{S}_{2}\left(\pi-\pi^{*}\right)$ transition. It shows fluorescent peak at $509 \mathrm{~nm}$ in ethanol solution. Fluorescent probe 7-A displays significant red shifts in absorption and emission in ethanol solution with absorption and emission peaks at $668 \mathrm{~nm}$ and $715 \mathrm{~nm}$, respectively because of significantly enhanced $\pi$-conjugation and intramolecular charge transfer effect from piperazine moieties at 3,5-positions to the BODIPY core compared with BODIPY dye 7.7. The similar spectroscopic properties of probe 7-A was obtained in dichloromethane and DMSO solution. However, in aqueous solution the intramolecular charge transfer effect of the probe 7-A becomes more significant because of polar solvent effect and potential aggregation may occur, which results in very low fluorescence and further red shifts absorption and emission of probe 7-A with absorption and emission peaks at $712 \mathrm{~nm}$ and $770 \mathrm{~nm}$ in buffer (pH 7.4), respectively (Table 7.1). Fluorescent probe 7-B exhibits similar optical properties to those of the probe 7-A in organic solvents. But the absorption peak $(710 \mathrm{~nm})$ and emission peak $(755 \mathrm{~nm})$ of probe 7-B in buffer solution ( $\mathrm{pH}$ 7.4) were found slightly blue shifted compared with probe 7-A (Table 7.1). This may be attributed to the reduced aggregation in aqueous solution with introduction of hydrophilic oligo(ethylene glycol)methyl ether on each of piperazine moiety at 3,5-positions. Highly water-soluble BODIPY dye (7.9) displays absorption and 
emission peaks at $500 \mathrm{~nm}$ and $509 \mathrm{~nm}$ in aqueous solution. Fluorescent probe 7-C shows considerable red shifts in absorption and emission with absorption peaks at $669 \mathrm{~nm}$ and $687 \mathrm{~nm}$, and emission peaks at $720 \mathrm{~nm}$ and $750 \mathrm{~nm}$ in ethanol and buffer solution $(\mathrm{pH}$ 7.4), respectively (Table 7.1) because of its significant extended $\pi$-conjugation and intramolecular charge transfer effect from piperazine moieties at 3,5-positions to the BODIPY core compared with those of its precursor BODIPY dye 7.9. Compared with the properties of probes 7-A and 7-B, the highly water-soluble character of probe 7-C may further reduce its self-aggregation effect in buffer solution as it shows the shortest absorption and emission wavelength among three probes in buffer solution ( $\mathrm{pH}$ 7.4).

\subsubsection{Optical responses of fluorescent probes to $\mathrm{pH}$}

We investigated $\mathrm{pH}$ effect on optical properties of fluorescent probes and their BODIPY precursors $(\mathbf{7 . 7}, \mathbf{7 . 9})$ in buffer solutions to verify our hypothesis that incorporation of piperazine moieties into BODIPY dyes at 3,5-positions as parts of the fluorophores can effectively modulate sensitive optical responses of BODIPY dyes to $\mathrm{pH}$. BODIPY dyes (7.7 and 7.9) display no responses to $\mathrm{pH}$ in absorption and emission in buffer solution, which are usual optical properties of typical BODIPY dyes (see Appendix E). However, fluorescent probes 7-A, 7-B and 7-C display very sensitive responses to $\mathrm{pH}$ in both absorption and emission (Figure 7.1). Decreases of $\mathrm{pH}$ values from 9.98 to 2.20 cause significant increases in fluorescent intensities at $715 \mathrm{~nm}$ of fluorescent probes 7-A, 7-B and 7-C with 75, 88 and 102-fold increases, respectively (Figure 7.1). In addition, there are significant blue shifts of $55 \mathrm{~nm}, 41 \mathrm{~nm}$ and $35 \mathrm{~nm}$ in emission spectra of the probes 7-A, 7-B and 7-C, respectively, because of significantly reduced intramolecular charge transfer effect from piperazine moieties to BODIPY cores through protonation of nitrogen atoms in piperazine moieties in acidic conditions (Scheme 7.1), which is further confirmed by significant blue shifts of $46 \mathrm{~nm}, 53 \mathrm{~nm}$ and $23 \mathrm{~nm}$ in absorption spectra of probes 7-A, 7-B and 7-C when $\mathrm{pH}$ values of the buffer were changed from 9.98 to 2.20 .

Figure 7.2 shows more detailed fluorescent intensity change at $715 \mathrm{~nm}$ of probes 7A, 7-B and 7-C at different $\mathrm{pH}$ conditions in buffer solution. From basic to neutral condition, all probes show very weak fluorescence without any obvious change. Their 
fluorescence intensities gradually increase when $\mathrm{pH}$ decreases from 7.0 to 2.2. The $\mathrm{p} K_{\mathrm{a}}$ values of probes 7-A, 7-B and 7-C are 2.91, 3.19 and 3.57 respectively. Obviously, probe 7-C exhibits highest fluorescence responses with highest $\mathrm{p} K_{\mathrm{a}}$ value among all three probes. This indicates that the highly water-soluble probe 7-C has less aggregation and it is protonated much more easily in aqueous solution which gives the higher sensitivity to $\mathrm{pH}$ than probes 7-A and 7-B. In the $\mathrm{pH}$ window between physiological $\mathrm{pH}$ and lysosomal $\mathrm{pH}$ ( $\mathrm{pH} 7.4$ to $\mathrm{pH} 4.2$ ), very good linear fluorescence responses were found for all probes in which probe 7-C gives highest sensitivity (Figure 7.2 inset). From $\mathrm{pH} 7.4$ to $\mathrm{pH} 4.2$, the fluorescence intensities increased by 7, 17 and 22 folds for probes 7-A, 7-B and 7-C, respectively and their fluorescence quantum yields $(\mathrm{pH} 4.5)$ also significantly increase compared with those in neutral condition $(\mathrm{pH}$ 7.4) (Table 7.1). These linear, high dynamic range and sensitive responses to $\mathrm{pH}$ indicate the feasibility of intracellular $\mathrm{pH}$ $\left(\mathrm{pH}_{\mathrm{i}}\right)$ detection by using these probes. Further decreases of $\mathrm{pH}$ values from 2.20 to 1.5 results in significant fluorescence quenching of the probes (Figure 7.2). We have eliminated the possibility of decomposition of the probes as the probes display stable reversible responses to $\mathrm{pH}$ between 1.5 and 10. However, the fluorescence quenching mechanism of the probes at extremely low $\mathrm{pH}$ needs further study.

In addition, we investigated the optical properties of the probes after protonation in organic solvents. After adding two equivalents of trifluoroacetic acid, the absorption and emission spectra of probe 7-A immediately changed in ethanol and dichloromethane solution. The dramatic blue shifts both on absorption and emission were present with new absorption and emission peaks at $652 \mathrm{~nm}$ and $685 \mathrm{~nm}$ in ethanol, and $658 \mathrm{~nm}$ and $690 \mathrm{~nm}$ in dichloromethane solution, respectively. The fluorescence quantum yield of probe 7-A is also enhanced by 2.71 fold in ethanol and 2.67 fold in dichloromethane solution. The disappearance of original peaks and presence of new blue shifted peaks indicate that probe 7-A were fully protonated and the intramolecular charge transfer from nitrogen to BODIPY core was prohibited which enhanced the fluorescence. Probe 7-B and probe 7-C exhibit similar change after protonation in ethanol and dichloromethane. Surprisingly, there is no change after adding trifluoroacetic acid in DMSO solution of probes. It may be due to the relative high $\mathrm{p} K_{\mathrm{a}}$ value of trifluoroacetic acid in DMSO $\left(\mathrm{p} K_{\mathrm{a}}=3.45\right){ }^{36}$ 

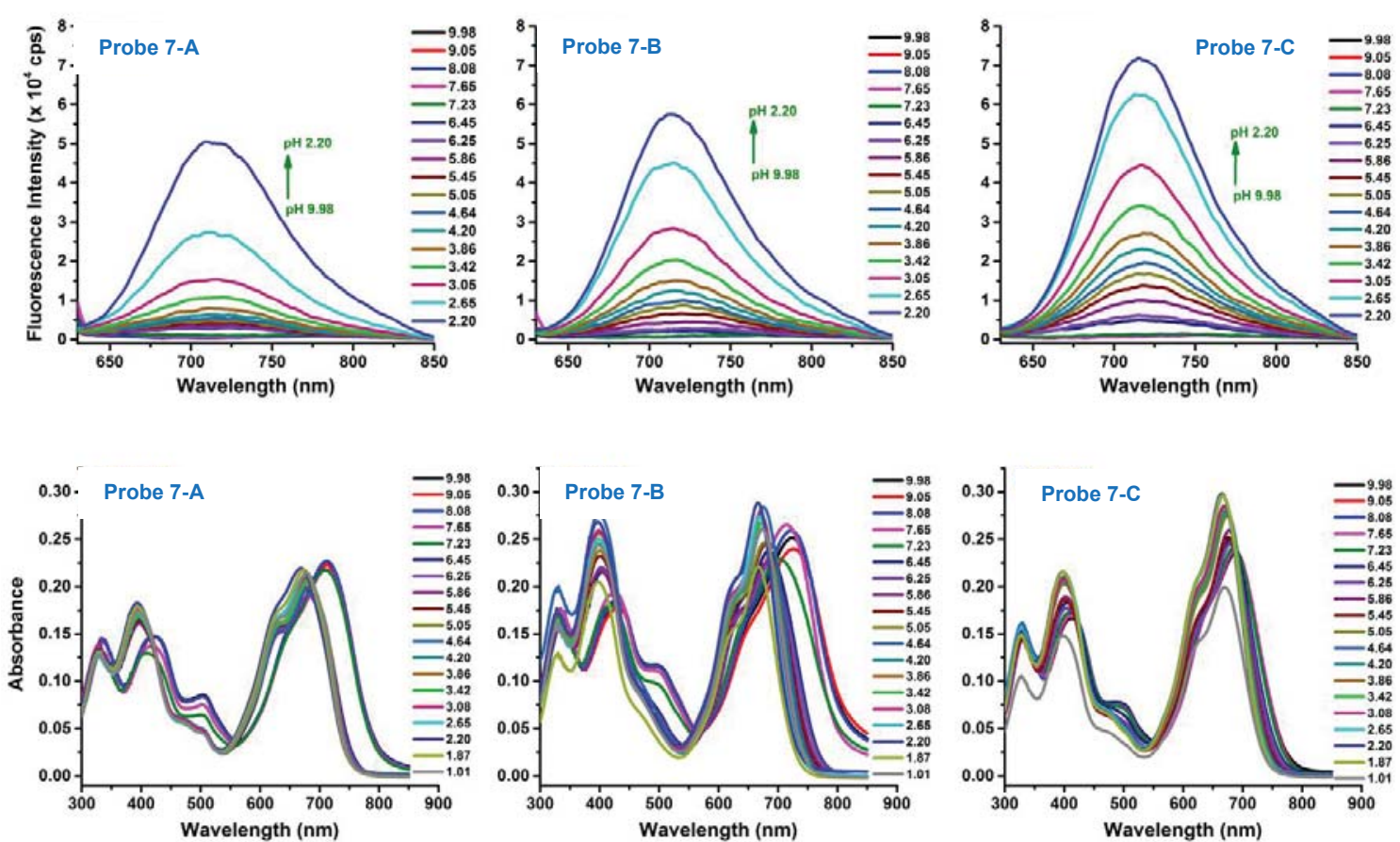

Figure 7.1. Emission (upper row) and absorption (lower row) spectra of probes 7-A, 7-B and 7-C $(5 \mu \mathrm{M})$ in buffer solution at different $\mathrm{pH}$ values.

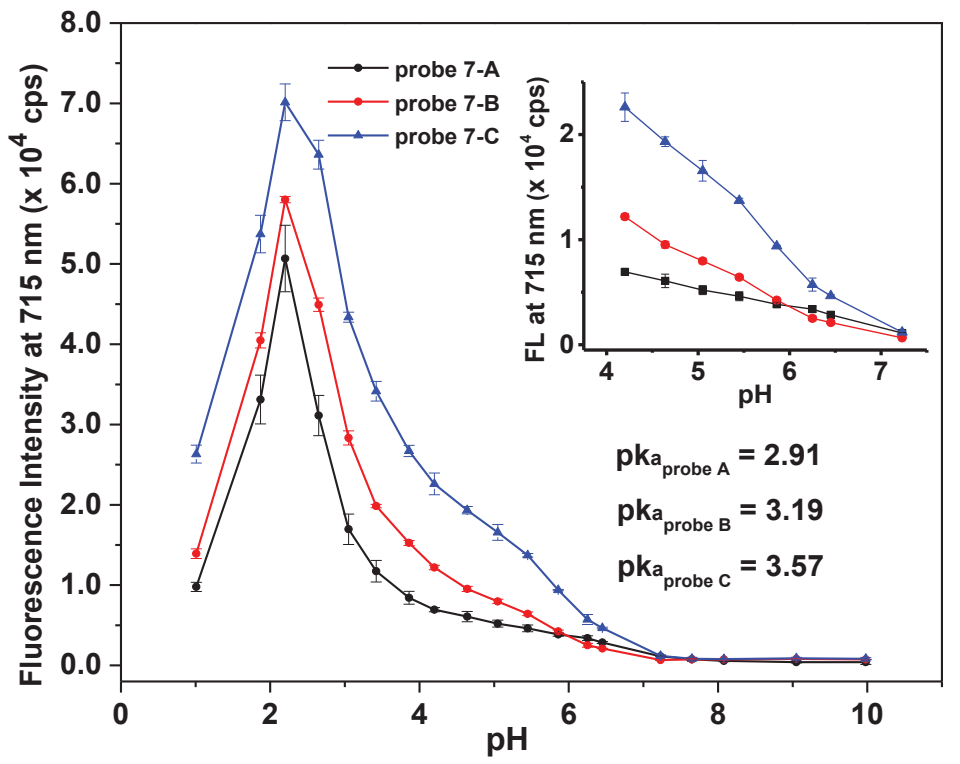

Figure 7.2. Fluorescent responses of probes 7-A, 7-B and 7-C to different $\mathrm{pH}$ values in buffer solution. Inset: zoomed fluorescence intensity responses of probes 7-A, 7-B and 7$\mathbf{C}$ to $\mathrm{pH}$ in the window between cytoplasm $\mathrm{pH}$ to lysosome $\mathrm{pH}$. 
Table 7.1. Photophysical properties of probes 7-A, 7-B and 7-C (protonation and nonprotonation form). The protonation source in organic solvent is trifluoroacetic acid.

\begin{tabular}{|c|c|c|c|c|c|}
\hline & solvent & $\begin{array}{c}\lambda_{\mathrm{abs}} \\
(\mathrm{nm})\end{array}$ & $\begin{array}{c}\lambda \mathrm{em} \\
(\mathrm{nm})\end{array}$ & $\begin{array}{c}\varepsilon_{\max } \\
\left(10^{4} \mathrm{M}^{-1} \mathrm{~cm}^{-1}\right) \\
\end{array}$ & $\begin{array}{c}\Phi_{\mathrm{f}} \\
(\%)\end{array}$ \\
\hline 7.7 & Ethanol & 499 & 509 & 9.10 & 72.1 \\
\hline 7.9 & Ethanol & 500 & 509 & 7.14 & 61.0 \\
\hline \multirow{4}{*}{$7-\mathrm{A}$} & $\mathrm{CH}_{2} \mathrm{Cl}_{2}$ & 675 & 722 & 7.10 & 4.3 \\
\hline & Ethanol & 668 & 715 & 6.88 & 3.8 \\
\hline & DMSO & 674 & 721 & 7.05 & 3.4 \\
\hline & Buffer (pH 7.4) & 712 & 770 & 4.36 & $<0.01$ \\
\hline \multirow{4}{*}{$7-\mathbf{A}+\mathbf{H}^{+}$} & $\mathrm{CH}_{2} \mathrm{Cl}_{2}$ & 658 & 690 & 7.58 & 11.5 \\
\hline & Ethanol & 652 & 685 & 7.86 & 10.3 \\
\hline & Buffer (pH 2.6) & 675 & 715 & 4.35 & 0.033 \\
\hline & Buffer (pH 4.5) & 680 & 716 & 3.94 & 0.01 \\
\hline \multirow{4}{*}{ 7-B } & $\mathrm{CH}_{2} \mathrm{Cl}_{2}$ & 679 & 729 & 9.31 & 3.4 \\
\hline & Ethanol & 670 & 720 & 8.56 & 2.8 \\
\hline & DMSO & 676 & 724 & 9.27 & 3.0 \\
\hline & Buffer (pH 7.4) & 710 & 755 & 5.20 & $<0.01$ \\
\hline \multirow{4}{*}{ 7-B $+\mathbf{H}^{+}$} & $\mathrm{CH}_{2} \mathrm{Cl}_{2}$ & 662 & 696 & 10.4 & 9.2 \\
\hline & Ethanol & 653 & 683 & 11.2 & 10.3 \\
\hline & Buffer (pH 2.6) & 669 & 715 & 5.60 & 0.06 \\
\hline & Buffer (pH 4.5) & 675 & 715 & 4.60 & 0.02 \\
\hline \multirow{4}{*}{ 7-C } & $\mathrm{CH}_{2} \mathrm{Cl}_{2}$ & 675 & 722 & 7.76 & 4.5 \\
\hline & Ethanol & 669 & 720 & 7.88 & 2.8 \\
\hline & DMSO & 675 & 724 & 8.11 & 3.3 \\
\hline & Buffer (pH 7.4) & 687 & 750 & 4.80 & $<0.01$ \\
\hline \multirow{4}{*}{ 7-C+ $+\mathbf{H}^{+}$} & $\mathrm{CH}_{2} \mathrm{Cl}_{2}$ & 661 & 694 & 8.55 & 9.9 \\
\hline & Ethanol & 653 & 687 & 9.22 & 11.0 \\
\hline & Buffer (pH 2.6) & 665 & 715 & 5.92 & 0.16 \\
\hline & Buffer (pH 4.5) & 671 & 716 & 4.46 & 0.07 \\
\hline
\end{tabular}




\subsubsection{NMR study}

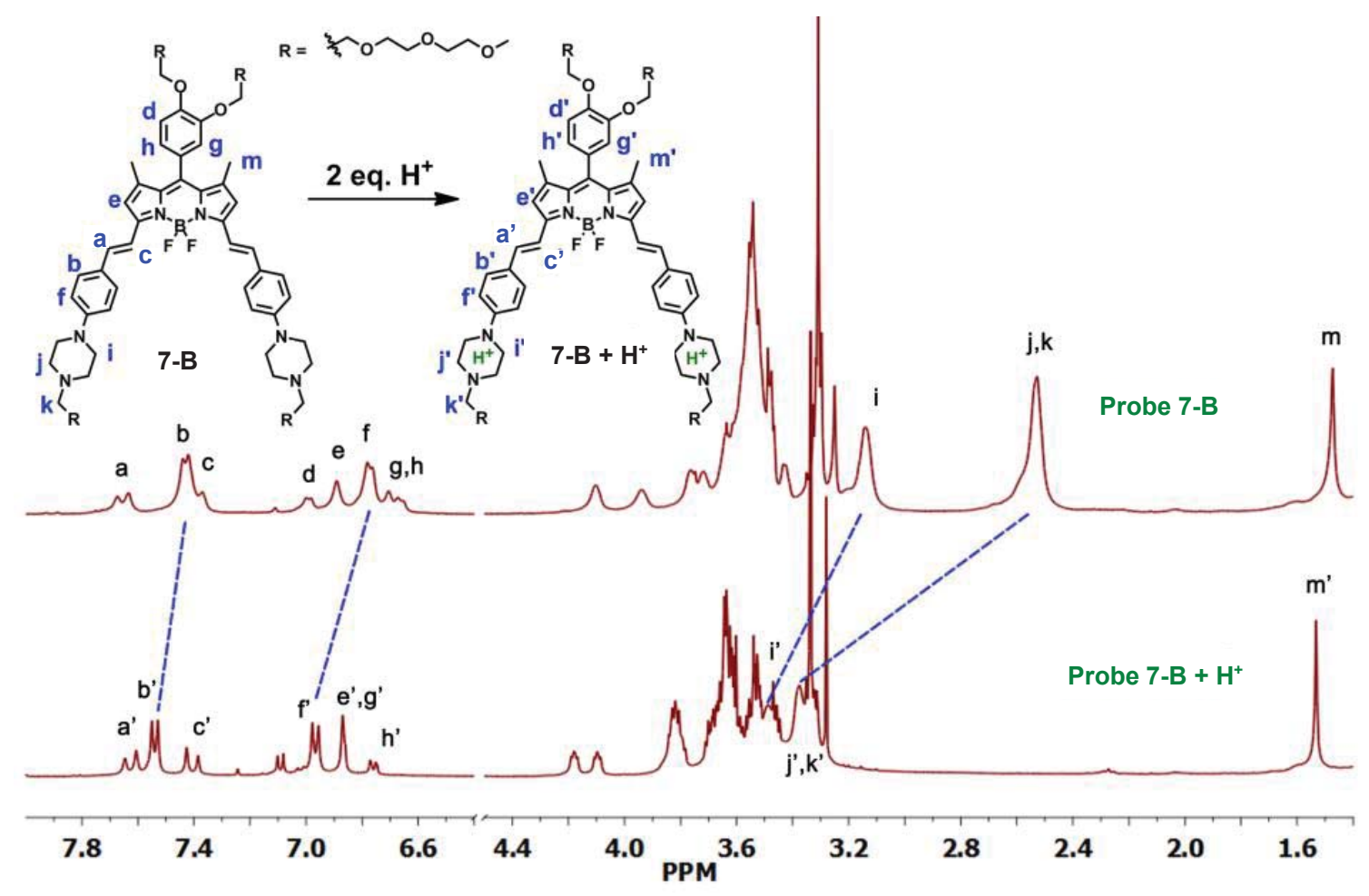

Figure 7.3. NMR spectra of probe 7-B before and after addition of two equivalents of trifluoroacetic acid in $\mathrm{CD}_{3} \mathrm{OD}$ solution.

In order to further study intramolecular charge transfer effect of piperazine moieties to BODIPY cores, we chose probe 7-B and use ${ }^{1} \mathrm{H}$ NMR to investigate the structure change before and after protonation of the probe. Figure 7.3 shows the comparison of ${ }^{1} \mathrm{H}$ NMR spectra of probe 7-B before and after its protonation in deuterated methanol $\left(\mathrm{CD}_{3} \mathrm{OD}\right)$ solution. Broad peaks were observed on the spectra of probe 7-B due to the fast relaxation from its high polarity character and potential hydrogen bonding on the nitrogen atoms of piperazine moiety. After adding two equivalents of trifluoroacetic acid, the spectra of the probe immediately changed. All peaks turned to be very sharp shape and protons $\mathbf{j}$ and $\mathbf{k}$ significantly shifted to down-filed (from 2.53 to $3.38 \mathrm{ppm}$ ) which indicates the protonation of the lower nitrogen on piperazine moiety as it has more lonepair availability to be protonated than the upper nitrogen which conjugated with fluorophore. The neighboring proton $\mathbf{i}$ also down-field shifted after protonation but with 
smaller change of $0.35 \mathrm{ppm}$ ( 3.14 to $3.49 \mathrm{ppm}$ ). In addition, the aromatic protons $\mathbf{f}$ and $\mathbf{b}$ also undergo down-field shifts after protonation and these protons did not further shift with addition of more equivalents of acid source. These results further confirm our previous hypothesis that the proton is transferred or delocalized between the two nitrogens of the piperazine moiety after protonation of the probes, which significantly reduces the electron donation ability of conjugated nitrogen atoms to the fluorophore.

\subsubsection{Solvent effect}
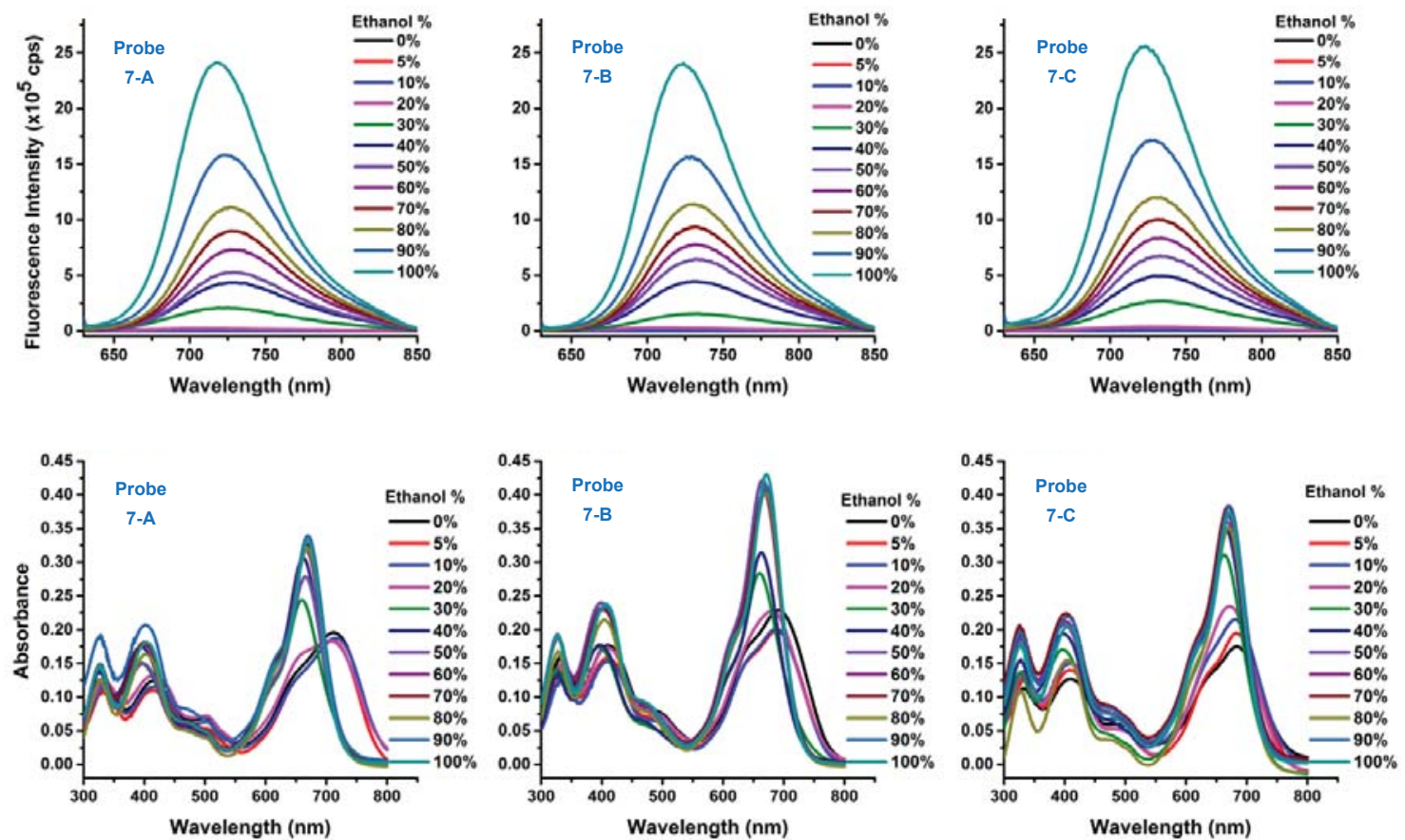

Figure 7.4. Emission (upper row) and absorption (lower row) spectra of probes 7-A, 7-B and 7-C $(5 \mu \mathrm{M})$ in mixed solvent (ethanol/buffer $\mathrm{pH} 7.4)$ with different percentage of ethanol.

Considering the nature of the intramolecular charge transfer (ICT) and potential selfaggregations on fluorophores, we investigated the solvent polarity dependence of fluorescent probes 7-A, 7-B and 7-C (Figure 7.4). Optical properties of probes are highly dependent on solvent polarity and they exhibit the same changing patterns in their absorption and emission spectra in different polarity environments. Decreases of the 
solvent polarity by raising the percentage of ethanol in buffer solutions result in slight blue shifts of the fluorescent peaks, and large fluorescence intensity enhancements of the probes. These changes may result from the reduced self-aggregation effect causing fluorescence quenching, and more importantly from reduced intramolecular charge transfer (ICT) excited states of the fluorophores by nonpolar character of alcohol compared with water which recovers fluorescence given by more dominant local excited (LE) states of the probes. Similar blue shifts in absorption spectra of the probes 7-A, 7-B and 7-C were observed with increase percentage of alcohol in the mixed buffer. However, the probes 7-A and 7-B show more blue shifts (44 nm and $40 \mathrm{~nm}$ ) in their absorption peaks compared with probe 7-C which only gives an 18-nm shift. The more blue shifts in their absorption spectra indicate that the probes $\mathbf{7 - A}$ and 7-B undergo potential aggregation in aqueous solution while the probe 7-C has much weaker aggregation effect because of its highly water-soluble character as the bulky and hydrophilic branched oligo(ethylene glycol)methyl ether chains on the fluorophore could effectively prevent aggregation between BODIPY cores in aqueous solution.
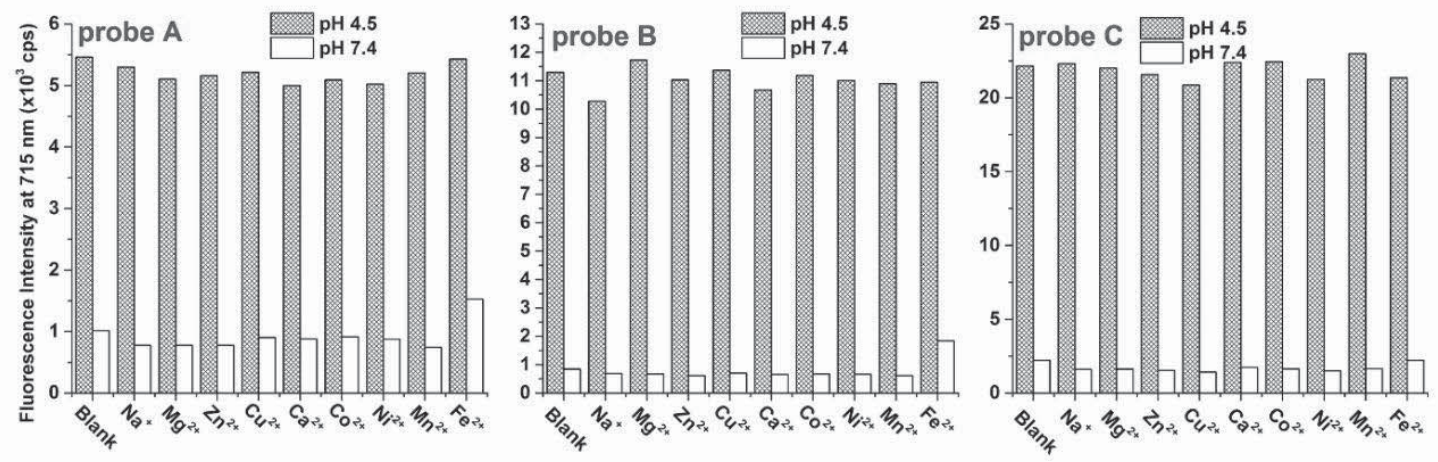

Figure 7.5. Fluorescence responses of probe 7-A, 7-B and 7-C $(5 \mu \mathrm{M})$ to different cations in $\mathrm{pH} 4.5$ and 7.4 buffer solution.

\subsubsection{Selectivity}

We studied effect of different metal ions on fluorescent responses of fluorescent probes to $\mathrm{pH}$ and investigated potential coordination of fluorescent probes with heavy, 
transition, and main group metal ions. The fluorescent probes display high selectivity to $\mathrm{pH}$ over these alkali, alkaline-earth metal ions, and transitional metal ions because fluorescent probes 7-A, 7-B and 7-C display no responses to $200 \mu \mathrm{M}$ alkali and alkalineearth metal ions as such $\mathrm{Na}^{+}, \mathrm{Mg}^{2+}$ and $\mathrm{Ca}^{2+}$, as well as some transitional metal ions (200 $\mu \mathrm{M})$ such as $\mathrm{Zn}^{2+}, \mathrm{Cu}^{2+}, \mathrm{Co}^{2+}, \mathrm{Ni}^{2+}, \mathrm{Mn}^{2+}$ and $\mathrm{Fe}^{2+}$, at $\mathrm{pH} 7.4$ and 4.5 (Figure 7.5).

\subsubsection{Photostability}

The photostability measurement was carried in buffer solution at $\mathrm{pH} 4.5$. Fluorescent probes were excited continuously at $620 \mathrm{~nm}$ for 5 hours and fluorescence intensity was measured every 5 minutes. The result clearly showed that there was no significant decrease of the fluorescence intensity under 5-hour excitation for all three probes (Figure 7.6). The fluorescence intensities of probes $7-\mathbf{A}$ and $7-\mathbf{B}$ increased by $20 \%$ and $7 \%$ in first 80 and 30 minutes respectively, and keep almost constant level after that. These increases may arise from the slow protonation of probes 7-A and 7-B because of their relative low solubility in aqueous solution. Probe 7-C exhibited very stable fluorescent emission in first 2 hours under excitation and only $2 \%$ of fluorescence intensity decreased after 5-hour excitation.

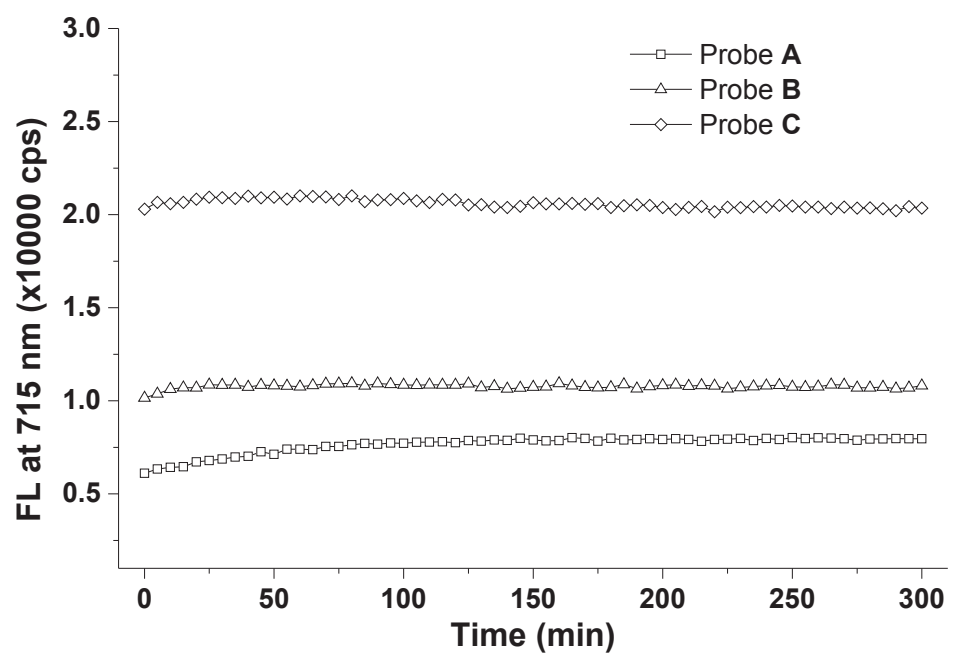

Figure 7.6. Fluorescence intensities at $715 \mathrm{~nm}$ of probe $\mathbf{7 - A}, \mathbf{7 - B}$ and $\mathbf{7 - C}(5 \mu \mathrm{M})$ as function of time in 5 hours under excitation $(620 \mathrm{~nm})$ in citrate-phosphate buffer $(\mathrm{pH}$ 4.5). 


\subsubsection{Fluorescence responses in living cells}

To find out if probes 7-A, 7-B, and 7-C could selectively stain lysosomes or acidic organelles in cells, all three probes were compared with commercial probe LysoSensor Green DND-189, a known lysosome probe that specifically fluorescently stains acidic organelles in cells. Live cell imaging in varying concentrations of probe 7-A, 7-B, and 7C were conducted using two cell lines: breast cancer cell line MDA-MB-231 and normal endothelial cell line HUVEC-C (Figure 7.7 and 7.8). In both cell types, probes 7-A and 7B showed very strong fluorescence signals and probe 7-C showed weak fluorescence signal inside the cell. The area in the cell stained with probe 7-A, 7-B and $\mathbf{C}$ matched those stained with LysoSensor Green DND-189. This suggests that these new probes are able to target lysosomes or other acidic cellular compartments in cells in a manner similar to probe DND-189 and distinguish between different regions inside cells based on $\mathrm{pH}$. Co-localization analysis based on the Pearson's coefficient showed value of 0.85 or higher for probes 7-A, 7-B, and 7-C with LysoSensor Green DND-189 indicating presence of both probes in the same cellular compartment (Figure E.32 in Appendix E). Probe 7-A showed the highest fluorescent intensity among all three probes and displayed good signal even at $2 \mu \mathrm{M}$ concentration (Figure E.24, E.27 in Appendix E). The fluorescent signals from probe 7-B was not as strong as probe 7-A, but it showed measurable signal at $5 \mu \mathrm{M}$ concentration and at higher concentrations (Figure E.25, S28 in Appendix E). However, probe 7-C, the most water-soluble compound among the three probes, showed poor fluorescent signal even at high concentration of $25 \mu \mathrm{M}$ (Figure E.26, E.29 in Appendix E). The possible explanation for this difference could be that probe 7-A and 7-B are more hydrophobic and less steric hindered than probe 7-C, so they could easier bind or interact with lipophilic structures like micelles, lipososomes, membranes in lysosomes which further activate the fluorescence by depressing ICT effect of the fluorophores. ${ }^{37}$ The enlarged 60x magnification images from both cell lines (Figure E.30-E.31 in Appendix E) show high fluorescent area localized in the perinuclear region, which is in line with a previous report that shows lysosomes localized in the perinuclear region in clusters after serum starvation. ${ }^{26,38}$ 


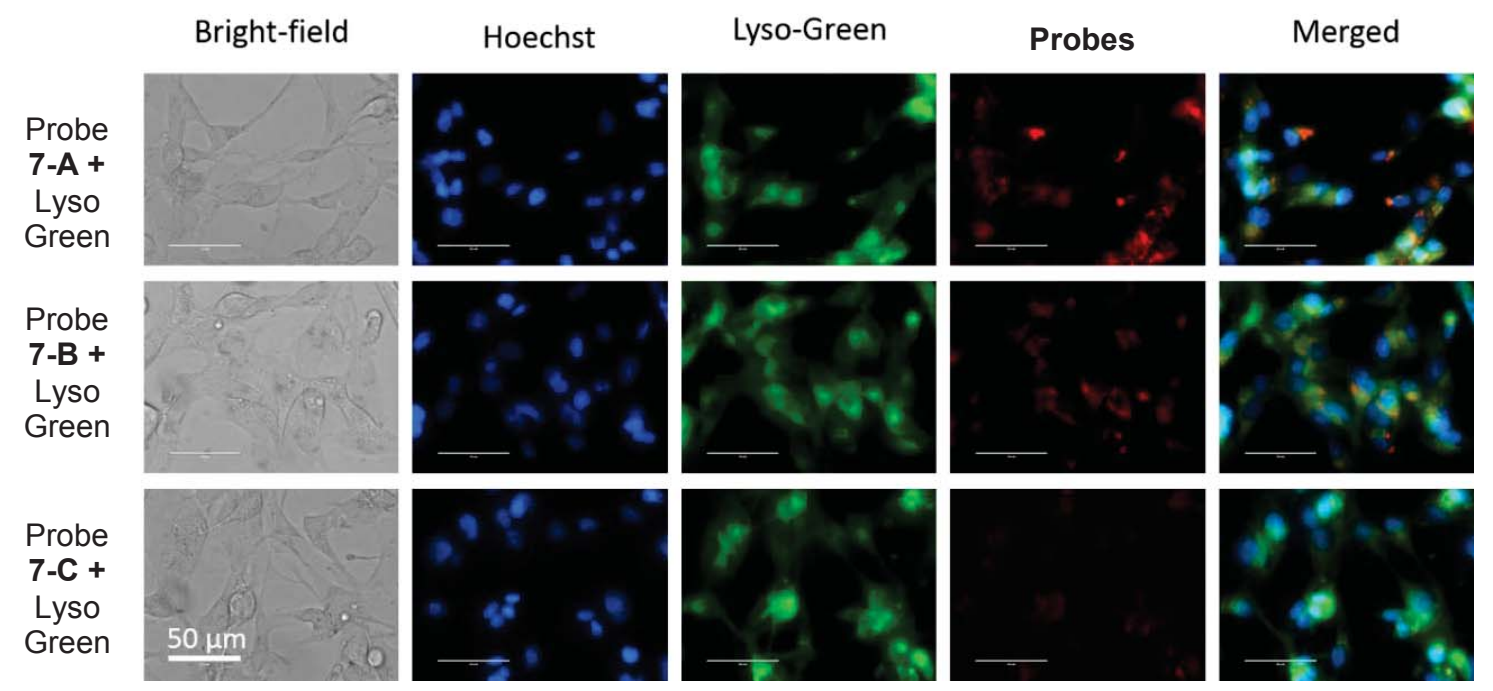

Figure 7.7. Fluorescence images of MDA-MB-231 cells incubated with fluorescent probes 7-A, 7-B, and 7-C. (Cells incubated with $5 \mu \mathrm{M}$ of respective probes for $2 \mathrm{~h}$, post serum starvation ( $2 \mathrm{~h}$ ) and imaged for co-localization with $1 \mu \mathrm{M}$ LysoSensor Green and $(1 \mu \mathrm{g} / \mathrm{mL})$ Hoechst 33342 stains. All images were acquired at 60x magnification using inverted fluorescence microscope (AMF-4306, EVOS $\mathrm{fl}, \mathrm{AMG})$ )

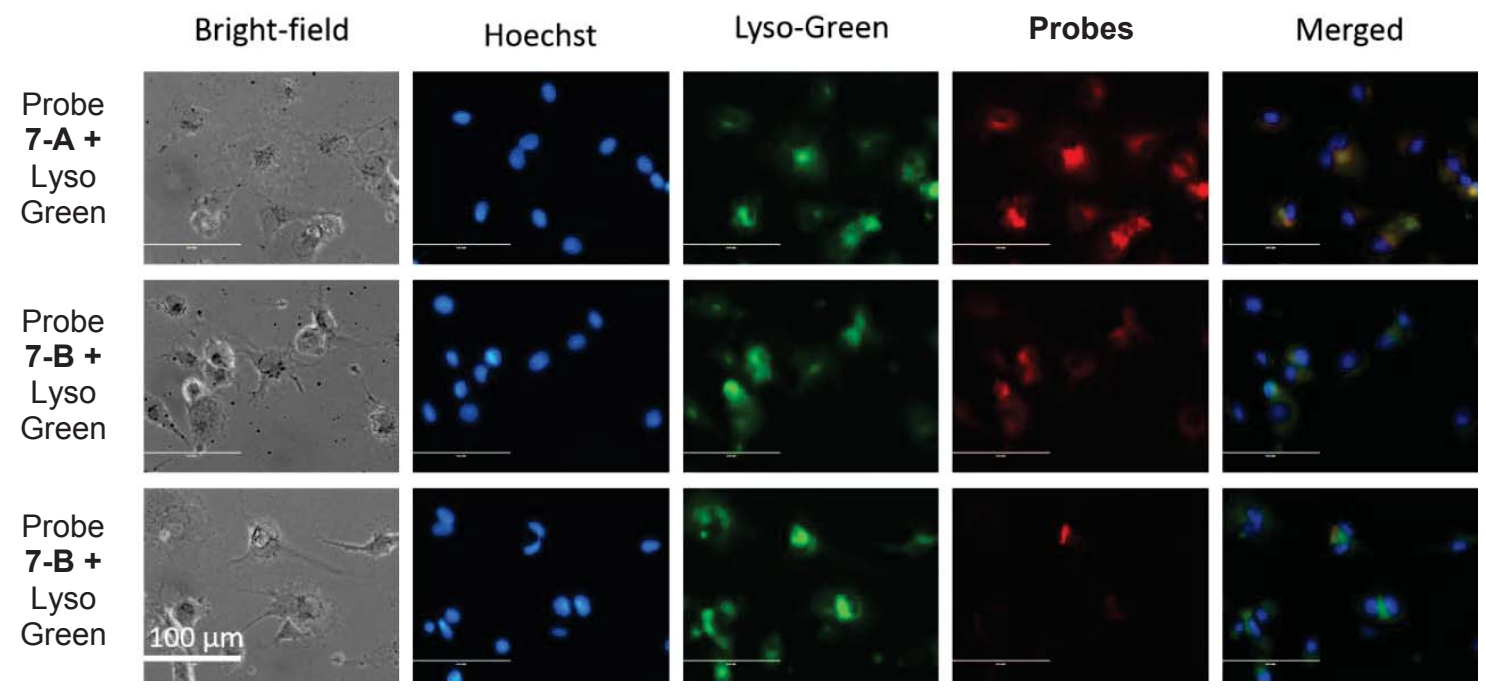

Figure 7.8. Fluorescence images of HUV-EC-C cells incubated with fluorescent probes 7-A, 7-B, and 7-C. (HUV-EC-C cells were incubated with $5 \mu \mathrm{M}$ of respective probes for $2 \mathrm{~h}$, post serum starvation $(2 \mathrm{~h})$ and imaged for co-localization with $1 \mu \mathrm{M}$ LysoSensor 
Green and $(1 \mu \mathrm{g} / \mathrm{mL})$ Hoechst 33342 stains. Images were acquired using the using inverted fluorescence microscope (AMF-4306, EVOS $\mathrm{fl}, \mathrm{AMG})$ at $40 \mathrm{X}$ magnification)

It should be noted that the intensity of fluorescence in living cells is also dependent on the cellular uptake. Therefore, we further investigated the cellular uptake of HUVEC$\mathrm{C}$ cells with different incubation concentrations of probes 7-A, 7-B and 7-C (Figure 7.9). The cellular uptake efficiency was calculated as the percentage of probe taken up by cells out of total amount of probe in initial incubation solution. The results showed probe 7-A has much higher cellular uptake (more than 30\%) at all three incubation concentrations compared with probes 7-B and 7-C. Probe 7-B has moderate cellular uptake efficiency of $18 \%$ at incubation concentration of $5 \mu \mathrm{M}$ but higher concentrations of $15 \mu \mathrm{M}$ and $25 \mu \mathrm{M}$ only give $8 \%$ and 4\% uptake efficiency, respectively. Probe 7-C showed the lowest cellular uptake efficiency with $10 \%, 3 \%$ and $3 \%$ for incubation concentration of $5 \mu \mathrm{M}, 15$ $\mu \mathrm{M}$ and $25 \mu \mathrm{M}$, respectively. This low cellular uptake of probe 7-C could be the main reason for its relatively weak signals of the fluorescence images in living cells compared with probes 7-A and 7-B.

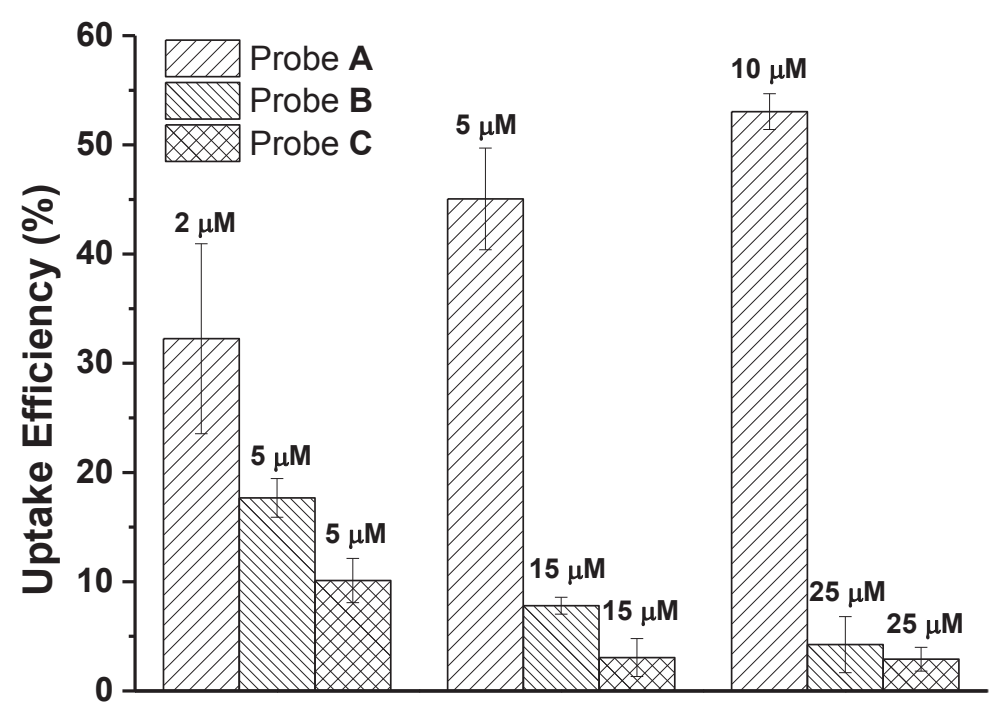

Figure 7.9. The HUVEC-C cells cellular uptake efficiency of probes $\mathbf{A}, \mathbf{B}$ and $\mathbf{C}$ at different concentrations after two hour incubations. The cellular uptake efficiency was calculated as the percentage of probe taken up by cells out of total amount of probe in initial incubation solution. Cell density $=1 \times 10^{4}$ cells $/$ well. 


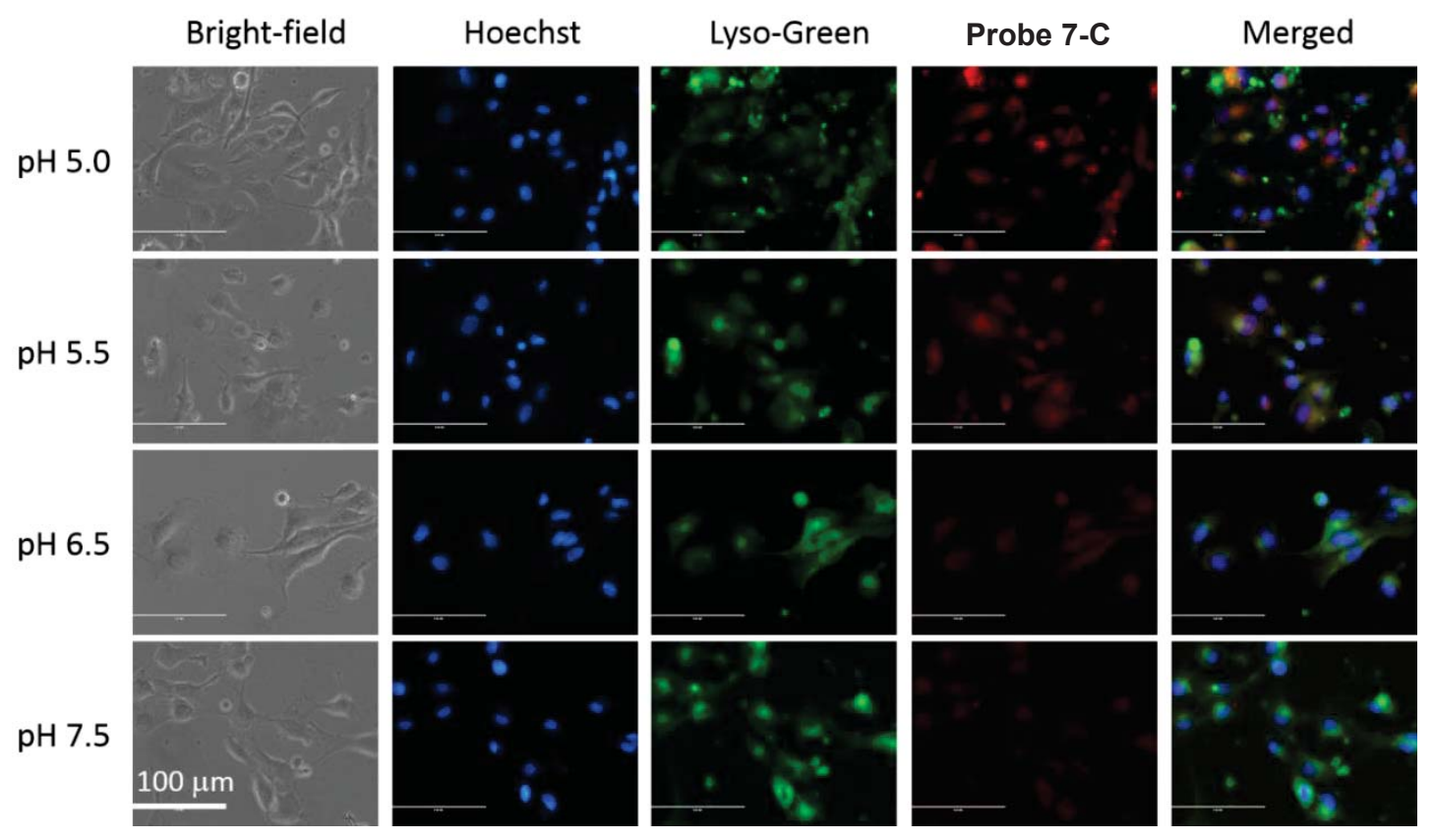

Figure 7.10. Fluorescence images of HUVEC-C cells incubated with $15 \mu \mathrm{M}$ probes 7-C at different $\mathrm{pH}$ values. $1 \mu \mathrm{M}$ LysoSensor Green and $1 \mu \mathrm{g} / \mathrm{mL}$ Hoechst 33342 were used as co-stains. Images were acquired using the using inverted fluorescence microscope $\left(\mathrm{AMF}-4306, \mathrm{EVOS}_{\mathrm{fl}}, \mathrm{AMG}\right)$ at $40 \mathrm{X}$ magnification.

In order to examine the $\mathrm{pH}$ dependency of the probes inside the living cells, we conducted live cell fluorescence imaging at different intracellular $\mathrm{pH}$ values. In this study, HUVEC-C cells were incubated with $5 \mu \mathrm{M}$ probe 7-A, $15 \mu \mathrm{M}$ probe 7-B or $15 \mu \mathrm{M}$ probe 7-C, respectively, and then with nigericin $\left(5 \mu \mathrm{g} \mathrm{mL}^{-1}\right)$ in buffer solution at different $\mathrm{pH}$ values $(\mathrm{pH} 5.0,5.5,6.0$ and 7.5) to equilibrate the intracellular and extracellular $\mathrm{pH}$. This is a standard approach that has been widely used to calibrate the intracellular $\mathrm{pH}$ $\left(\mathrm{pH}_{\mathrm{i}}\right)$. The probe 7-C displayed very weak fluorescence in cells near physiological $\mathrm{pH}$ ( $\mathrm{pH}$ 7.5) while its fluorescence intensity was significantly enhanced as $\mathrm{pH}$ decreased from 7.5 to 5.0 (Figure 7.10). ${ }^{37,39-41}$ This turn-on response of probe 7-C to acidic $\mathrm{pH}_{\mathrm{i}}$ is in line with the trend of its $\mathrm{pH}$ response in buffer solution, which indicates that probe 7-C is sensitive to $\mathrm{pH}$ not only in buffer solution, but also inside the living cells because of reduced ICT effect and potential photo-induced electron transfer effect from piperazine moiety to BODIPY core through protonation. Compared with probe $\mathbf{7 - C}$, commercial 
probe LysoSensor Green did not display any obvious change of fluorescence at different $\mathrm{pH}_{\mathrm{i}}$ values. Probes 7-A and 7- $\mathbf{B}$ exhibited similar response to different $\mathrm{pH}_{\mathrm{i}}$ as probe 7-C, but with smaller fluorescence enhancements when $\mathrm{pH}_{\mathrm{i}}$ decreased from 7.5 to 5.0 (See Figure. E.36-E.37 in Appendix E). This difference may be due to the fact that probe 7-C has higher $\mathrm{p} K_{\mathrm{a}}$ value and better water solubility than probes 7-A and 7-B.

MTS assay were performed to investigate the toxicity of these probes to cells (Figure 7.9). Incubating the cells with the probes for $72 \mathrm{~h}$ shows that probe 7-A has the highest cytotoxicity among the three probes with $\sim 50 \%$ viability observed for cells incubated with $5 \mu \mathrm{M}$ that dropped down to less than $10 \%$ viability at $15 \mu \mathrm{M}$ and higher probe concentration (Figure 7.11). In comparison, probe 7-B and 7-C showed very mild toxicity with greater than $70 \%$ cell viability even at $50 \mu \mathrm{M}$ concentration (Figure 7.9).

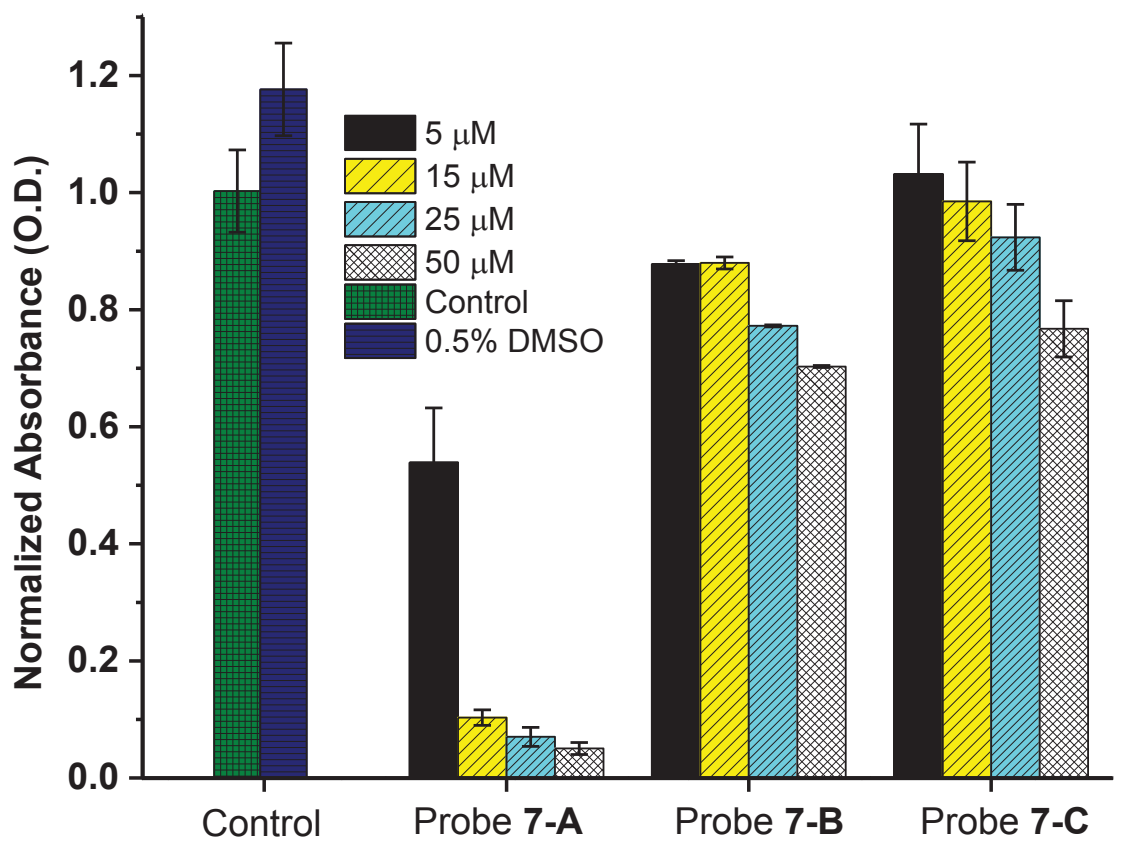

Figure 7.11. Cytotoxicity and cell proliferation effect of probes 7-A, 7-B and 7-C tested by MTS assay. (The MDA-MB-231 cells were incubated with 5, 15, 25, and $50 \mu \mathrm{M}$ of probes 7-A, 7-B and 7-C for $72 \mathrm{~h}$ and cell viability was measured by adding MTS reagent and measuring the formation of formazon at $490 \mathrm{~nm}$. Cell viability is directly 
proportional to the absorbance measured at $490 \mathrm{~nm}$ and was normalized to control cells (no probe dded). The error bars indicate \pm S.D. from six replicates)

\subsection{Conclusion}

We have prepared three BODIPY-based near-infrared fluorescent probes for $\mathrm{pH}$ (7A-C). The response mechanism of the fluorescent probes to $\mathrm{pH}$ value relies on modulation of intramolecular charge transfer of piperazine moieties to BODIPY cores. These probes displays extremely low fluorescent at neutral $\mathrm{pH}$ because of intramolecular charge transfer from piperazine moieties to BOBIDPY cores, and become highly fluorescent at low $\mathrm{pH}$ due to the significantly reduced intramolecular charge transfer effect. The fluorescent probes 7-A, 7-B and 7-C are cell-permeable and provide selective and sensitive labeling of lysosomes for potential non-invasive monitoring of lysosomal $\mathrm{pH}$ changes during physiological and pathological processes.

\section{References:}

1. Johansson, A. C., Appelqvist, H., Nilsson, C., Kagedal, K., Roberg, K., Ollinger, K., Regulation of Apoptosis-Associated Lysosomal Membrane Permeabilization. Apoptosis 2010, 15 (5), 527-540.

2. Turk, B., Turk, V., Lysosomes as "Suicide Bags" in Cell Death: Myth or Reality? J. Biol. Chem. 2009, 284 (33), 21783-21787.

3. Stinchcombe, J., Bossi, G., Griffiths, G. M., Linking Albinism and Immunity: The Secrets of Secretory Lysosomes. Science 2004, 305 (5680), 55-59.

4. Blott, E. J., Griffiths, G. M., Secretory Lysosomes. Nature Reviews Molecular Cell Biology 2002, 3 (2), 122-131.

5. Diwu, Z. J., Chen, C. S., Zhang, C. L., Klaubert, D. H., Haugland, R. P., A Novel Acidotropic $\mathrm{pH}$ Indicator and Its Potential Application in Labeling Acidic Organelles of Live Cells. Chemistry \& Biology 1999, 6 (7), 411-418.

6. Lv, H. S., Liu, J., Zhao, J., Zhao, B. X., Miao, J. Y., Highly Selective and Sensitive pH-Responsive Fluorescent Probe in Living Hela and HUVEC Cells. Sens. Actuator B-Chem. 2013, 177, 956-963. 
7. Zhu, H., Fan, J. L., Xu, Q. L., Li, H. L., Wang, J. Y., Gao, P., Peng, X. J., Imaging of Lysosomal pH Changes with A Fluorescent Sensor Containing A Novel Lysosome-locating Group. Chemical Communications 2012, 48 (96), 11766-11768.

8. Li, Z., Song, Y. L., Yang, Y. H., Yang, L., Huang, X. H., Han, J. H., Han, S. F., Rhodamine-deoxylactam functionalized poly styrene-alter-(maleic acid) $\mathrm{s}$ as lysosome activatable probes for intraoperative detection of tumors. Chem. Sci. 2012, 3 (10), 2941-2948.

9. Ying, L. Q., Branchaud, B. P., Selective Labeling and Monitoring pH Changes of Lysosomes in Living Cells with Fluorogenic pH Sensors. Bioorganic \& Medicinal Chemistry Letters 2011, 21 (12), 3546-3549.

10. Smith, D. G., McMahon, B. K., Pal, R., Parker, D., Live Cell Imaging of Lysosomal $\mathrm{pH}$ Changes with $\mathrm{pH}$ Responsive Ratiometric Lanthanide Probes. Chemical Communications 2012, 48 (68), 8520-8522.

11. Ma, L. J., Cao, W. G., Liu, J. L., Deng, D. Y., Wu, Y. Q., Yan, Y. H., Yang, L. T., A Highly Selective and Sensitive Fluorescence Dual-Responsive $\mathrm{pH}$ Probe in Water. Sens. Actuator B-Chem. 2012, 169, 243-247.

12. Galindo, F., Burguete, M. I., Vigara, L., Luis, S. V., Kabir, N., Gavrilovic, J., Russell, D. A., Synthetic Macrocyclic Peptidomimetics as Tunable pH Probes for The Fluorescence Imaging of Acidic Organelles in Live Cells. Angewandte Chemie-International Edition 2005, 44 (40), 6504-6508.

13. DePedro, H. M., Urayama, P., Using Lysosensor Yellow/Blue DND-160 to Sense Acidic pH under High Hydrostatic Pressures. Anal. Biochem. 2009, 384 (2), 359361.

14. Hasegawa, T., Kondo, Y., Koizumi, Y., Sugiyama, T., Takeda, A., Ito, S., Hamada, F., A Highly Sensitive Probe Detecting Low pH Area of Hela Cells Based on Rhodamine B Modified Beta-cyclodextrins. Bioorganic \& Medicinal Chemistry 2009, 17 (16), 6015-6019.

15. Lin, H. J., Herman, P., Kang, J. S., Lakowicz, J. R., Fluorescence Lifetime Characterization of Novel Low-pH Probes. Anal. Biochem. 2001, 294 (2), 118 125. 
16. Han, J. Y., Burgess, K., Fluorescent Indicators for Intracellular pH. Chemical Reviews 2010, 110 (5), 2709-2728.

17. Li, G. P., Zhu, D. J., Xue, L., Jiang, H., Quinoline-Based Fluorescent Probe for Ratiometric Detection of Lysosomal pH. Organic Letters 2013, 15 (19), 50205023.

18. Li, P., Zhou, H., Tang, B., A Lysosomal-targeted Fluorescent Probe for Detecting Cu2+. Journal of Photochemistry and Photobiology a-Chemistry 2012, 249, 3640 .

19. Wang, X. B., Ma, X. Y., Yang, Z., Zhang, Z., Wen, J. H., Geng, Z. R., Wang, Z. L., An NBD-armed Tetraaza Macrocyclic Lysosomal-targeted Fluorescent Probe For Imaging Copper(II) Ions. Chemical Communications 2013, 49 (96), 11263 11265 .

20. Wu, Z. G., Tang, M. L., Tian, T., Wu, J. G., Deng, Y. L., Dong, X. H., Tan, Z., Weng, X. C., Liu, Z. H., Wang, C. J., Zhou, X., A Specific Probe for Two-photon Fluorescence Lysosomal Imaging. Talanta 2011, 87, 216-221.

21. Xue, L., Li, G. P., Zhu, D. J., Liu, Q., Jiang, H., Rational Design of a Ratiometric and Targetable Fluorescent Probe for Imaging Lysosomal Zinc Ions. Inorganic Chemistry 2012, 51 (20), 10842-10849.

22. Hu, Z. Q., Li, M., Liu, M. D., Zhuang, W. M., Li, G. K., A Highly Sensitive Fluorescent Acidic pH Probe Based on Rhodamine B Diethyl-2aminobutenedioate Conjugate and Its Application in Living Cells. Dyes and Pigments 2013, 96 (1), 71-75.

23. Lee, H., Akers, W., Bhushan, K., Bloch, S., Sudlow, G., Tang, R., Achilefu, S., Near-infrared pH-Activatable Fluorescent Probes for Imaging Primary and Metastatic Breast Tumors. Bioconjugate Chemistry 2011, 22 (4), 777-784.

24. Li, C., Greenwood, T. R., Glunde, K., Glucosamine-bound Near-infrared Fluorescent Probes with Lysosomal Specificity for Breast Tumor Imaging. Neoplasia 2008, 10 (4), 389-398.

25. Wang, X. H., Nguyen, D. M., Yanez, C. O., Rodriguez, L., Ahn, H. Y., Bonder, M. V., Belfield, K. D., High-fidelity Hydrophilic Probe for Two-Photon 
Fluorescence Lysosomal Imaging. Journal of the American Chemical Society 2010, 132 (35), 12237-12239.

26. Vegesna, G. K., Janjanam, J., Bi, J. H., Luo, F.-T., Zhang, J. T., Olds, C., Tiwaria, A., Liu, H. Y., pH-Activatable Near-Infrared Fluorescent Probes for Detection of Lysosomal pH inside Living Cells. Journa of Materials Chemistry B 2014, 2 (28), 4500-4508.

27. Zhu, S., Zhang, J., Vegesna, G., Luo, F.-T., Green, S. A., Liu, H., Highly Watersoluble Neutral BODIPY Dyes with Controllable Fluorescence Quantum Yields. Organic Letters 2011, 13 (3), 438-441.

28. Zhu, S., Zhang, J., Vegesna, G. K., Pandey, R., Luo, F.-T., Green, S. A., Liu, H., One-pot Efficient Synthesis of Dimeric, Trimeric, and Tetrameric BODIPY Dyes for Panchromatic Absorption. Chemical Communications 2011, 47 (12), 35083510

29. Chung, B.-Y., Lee, I.-S., Park, B.-J., Kim, Y.-K., Kim, S.-J., Yoon, S.-H. Preparation of Polyethoxylated Pyrazolo-Pyrimidinone Derivatives and Their Pharmaceutical Compositions for The Treatment of Impotence. 2002.

30. Gabe, Y., Urano, Y., Kikuchi, K., Kojima, H., Nagano, T., Highly Sensitive Fluorescence Probes for Nitric Oxide Based on Boron Dipyrromethene Chromophore-rational Design of Potentially Useful Bioimaging Fluorescence Probe. Journal of the American Chemical Society 2004, 126 (10), 3357-3367.

31. Velapoldi, R. A., Tonnesen, H. H., Corrected Emission Spectra and Quantum Yields for A Series of Fluorescent Compounds in The Visible Spectral Region. Journal of Fluorescence 2004, 14 (4), 465-472.

32. Cielen, E., Tahri, A., Heyen, K. V., Hoornaert, G. J., De Schryver, F. C., Boens, N., Synthesis and Spectroscopic Characterisation of Fluorescent Indicators for $\mathrm{Na}+$ and K+. Journal of the Chemical Society-Perkin Transactions 2 1998, (7), $1573-1580$.

33. Zhu, S. L., Zhang, J. T., Janjanam, J., Bi, J. H., Vegesna, G., Tiwari, A., Luo, F. T., Wei, J. J., Liu, H. Y., Highly Water-soluble, Near-infrared Emissive BODIPY Polymeric Dye Bearing RGD Peptide Residues for Cancer Imaging. Anal. Chim. Acta 2013, 758, 138-144. 
34. Bolte, S., Cordelieres, F. P., A Guided Tour into Subcellular Colocalization Analysis in Light Microscopy. Journal of Microscopy-Oxford 2006, 224, 213232.

35. Ding, X. C., Janjanam, J., Tiwari, A., Thompson, M., Heiden, P. A., PeptideDirected Self-Assembly of Functionalized Polymeric Nanoparticles Part I: Design and Self-Assembly of Peptide-Copolymer Conjugates into Nanoparticle Fibers and 3D Scaffolds. Macromolecular Bioscience 2014, 14 (6), 853-871.

36. Bordwell, F. G., Equilibrium Acidities in Dimethyl-Sulfoxide Solution. Accounts Chem. Res. 1988, 21 (12), 456-463.

37. Thomas, J. A., Buchsbaum, R. N., Zimniak, A., Racker, E., Intracellular pH Measurements in Ehrlich Ascites Tumor-Cells Utilizing Spectroscopic Probes Generated Insitu. Biochemistry 1979, 18 (11), 2210-2218.

38. Pous, C., Codogno, P., Lysosome Positioning Coordinates mTOCR1 Activity and Autophagy. Nature Cell Biology 2011, 13 (4), 342-344.

39. Han, J., Loudet, A., Barhoumi, R., Burghardt, R. C., Burgess, K., A Ratiometric $\mathrm{pH}$ Reporter for Imaging Protein-dye Conjugates in Living Cells. Journal of the American Chemical Society 2009, 131 (5), 1642-1643.

40. Song, P., Chen, X., Xiang, Y., Huang, L., Zhou, Z., Wei, R., Tong, A., A Ratiometric Fluorescent $\mathrm{pH}$ Probe Based on Aggregation-induced Emission Enhancement and Its Application in Live-cell Imaging. Journal of Materials Chemistry 2011, 21 (35), 13470-13475.

41. Fan, L., Liu, Q., Lu, D., Shi, H., Yang, Y., Li, Y., Dong, C., Shuang, S., A Novel Far-Visible and Near-Infrared $\mathrm{pH}$ Probe for Monitoring Near-Neutral Physiological $\mathrm{pH}$ Changes: Imaging in Live Cells. Journal of Materials Chemistry B 2013, 1 (34), 4281-4288. 


\section{Chapter 8. Highly Water-soluble, Near-infrared Emissive Copolymeric BODIPY Dye Bearing RGD Peptide Residues for Cancer Imaging ${ }^{1}$}

Shilei Zhu, ${ }^{\text {a }}$ Jingtuo Zhang, ${ }^{a}$ Jagadeesh Janjanam, ${ }^{a}$ Jianheng $\mathrm{Bi}^{\text {a }}{ }^{\mathrm{a}}$ Giri Vegesna, ${ }^{a}$ Ashutosh Tiwari, ${ }^{a}$ Fen-Tair Luo, ${ }^{b}$ Jianjun Wei, ${ }^{* c}$ and Haiying Liu, ${ }^{* a}$

a Department of Chemistry, Michigan Technological University, 1400 Townsend Drive, Houghton, MI 49931, USA

${ }^{\mathrm{b}}$ Institute of Chemistry, Academia Sinica, Taipei, Taiwan 11529, Republic of China

c Biomedical Technology Branch, CFD Research Corporation, Huntsville, AL 35805, USA

\footnotetext{
${ }^{1}$ The material included in this chapter was previously published in Analytica Chimica Acta 2013, 758, 138-144. DOI: 10.1016/j.aca.2012.10.026 Publication Date (Web): January 3, 2013 Copyright (C) 2014 Elsevier B.V http://www.sciencedirect.com/science/article/pii/S0003267012014973?v=s5
} 


\subsection{Introduction.}

Fluorescence-based optical imaging offers a convenient approach to monitor multiple biological processes simultaneously and in real time with high sensitivity, low tissue autoabsorption and autofluorescence, low light scattering, operational simplicity, relatively low cost, potential miniaturization and mobility. ${ }^{1-4}$ The demand for nearinfrared (NIR) fluorescent dyes for noninvasive and simple diagnostic techniques such as in vivo imaging has been drastically growing as NIR dyes with emission wavelengths in the region between $700 \mathrm{~nm}$ and $900 \mathrm{~nm}$ can propagate through several centimeters of living tissues and may enable deep tissue imaging. ${ }^{1-4}$ Functional bio-conjugated quantum dots $(\mathrm{CdSe})$ could lead to a new generation of nanoparticle imaging probes for in vivo tumor imaging at high sensitivity and specificity, ${ }^{5}$ but their limited tissue penetration, lack of spatial resolution in tumor depth and potential toxicity concerns still restrict their clinical application. As a result, it is very important to develop a novel, highly watersoluble, near-infrared emissive agent with low cost, good biocompatibility, high intensity and photostability. In addition, high specificity for tumor cells, and tissue penetration and quantitative visualization for real time, in vivo detection of tumor will be highly desirable features in such a fluorescent dye. Numerous long-wavelength fluorophores such as 4,4'difluoro-4-bora-3a,4a-diaza-s-indacene (BODIPY) dyes, ${ }^{6}$ rhodamine, ${ }^{7}$ squaraine, ${ }^{8}$ or cyanine dyes ${ }^{9}$ have been recently reported. BODIPY dyes have received renewed interest for promising applications as imaging agents because of their many distinctive and desirable properties such as high absorption coefficients, narrow absorption bands, sharp emissions, high fluorescence quantum yields, and excellent chemical and photostabilities. ${ }^{6}$ Functionalization of BODIPY dyes by the number and nature of the substituents at different positions has been used to tune to near-infrared emission. However, most reported studies have focused on small molecules of BODIPY dyes and only a few reported near-infrared emissive BODIPY dyes are soluble in aqueous solution. ${ }^{6,10-11}$ Very recently, we and other groups reported some deep-red and nearinfrared emissive polymeric BODIPY dyes. ${ }^{12-16}$ However, all these BODIPY polymeric dyes are insoluble in aqueous solution. ${ }^{12-16}$ 
In this paper, we report a facile, convenient and versatile approach to prepare highly water-soluble, near-infrared emissive BODIPY polymeric dye bearing RGD cancerhoming peptide residues for cancer imaging application (Figure 8.1). We prepared highly water-soluble BODIPY polymeric dye (polymer 8-A) through the palladium-catalyzed Suzuki polymerization of highly water-soluble 2,6-diiodo-3,5-distyryl-BODIPY dye bearing bromide residue at the meso-position with benzene-1,4-diboronic acid because this polymer is expected to display the relatively weak $\pi-\pi$ stacking interactions among different polymer backbones. Incorporation of new branched oligo(ethylene glycol)methyl ether residues to distyryl units of BODIPY core at 3,5-positions effectively enhances enthalpic interactions of BODIPY dye with water, significantly increases water solubility of BODIPY polymeric dyes and prevents potential non-specific interactions of BODIPY dyes with other proteins through intermolecular interactions. RGD cancerhoming peptide residues were covalently attached to BODIPY cores at the meso positions through tetra(ethylene glycol) tethered spacers. This BODIPY polymeric dye functions as intrinsic near-infrared fluorophore with high water solubility, good photostability, biocompatibility and resistance to nonspecific interactions to biomolecules, targeting efficacy to tumor cells and easy-to-use during imaging detection. 


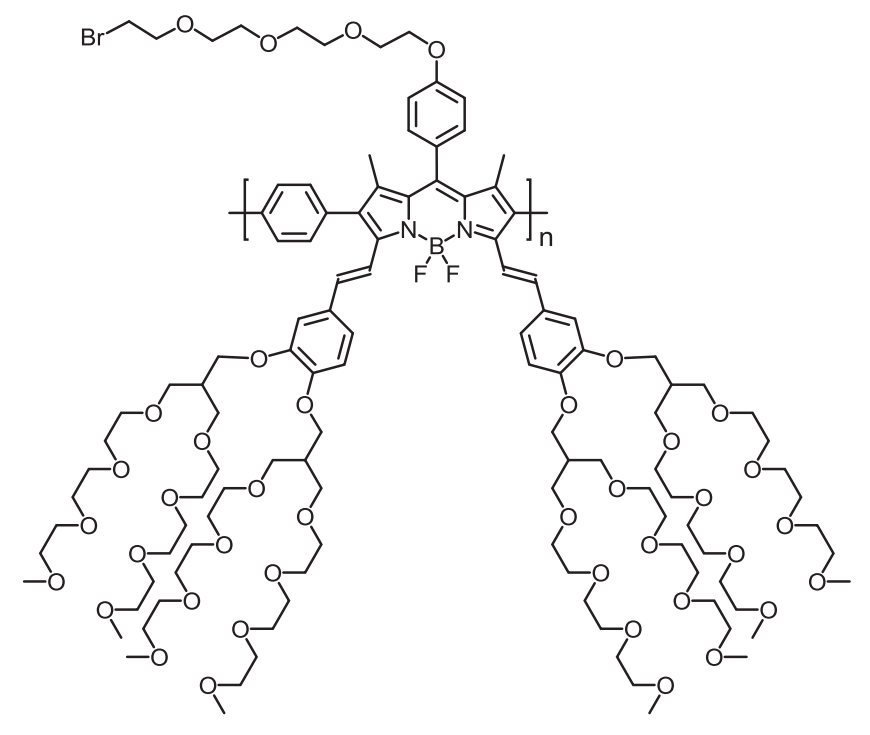

Polymer 8-A

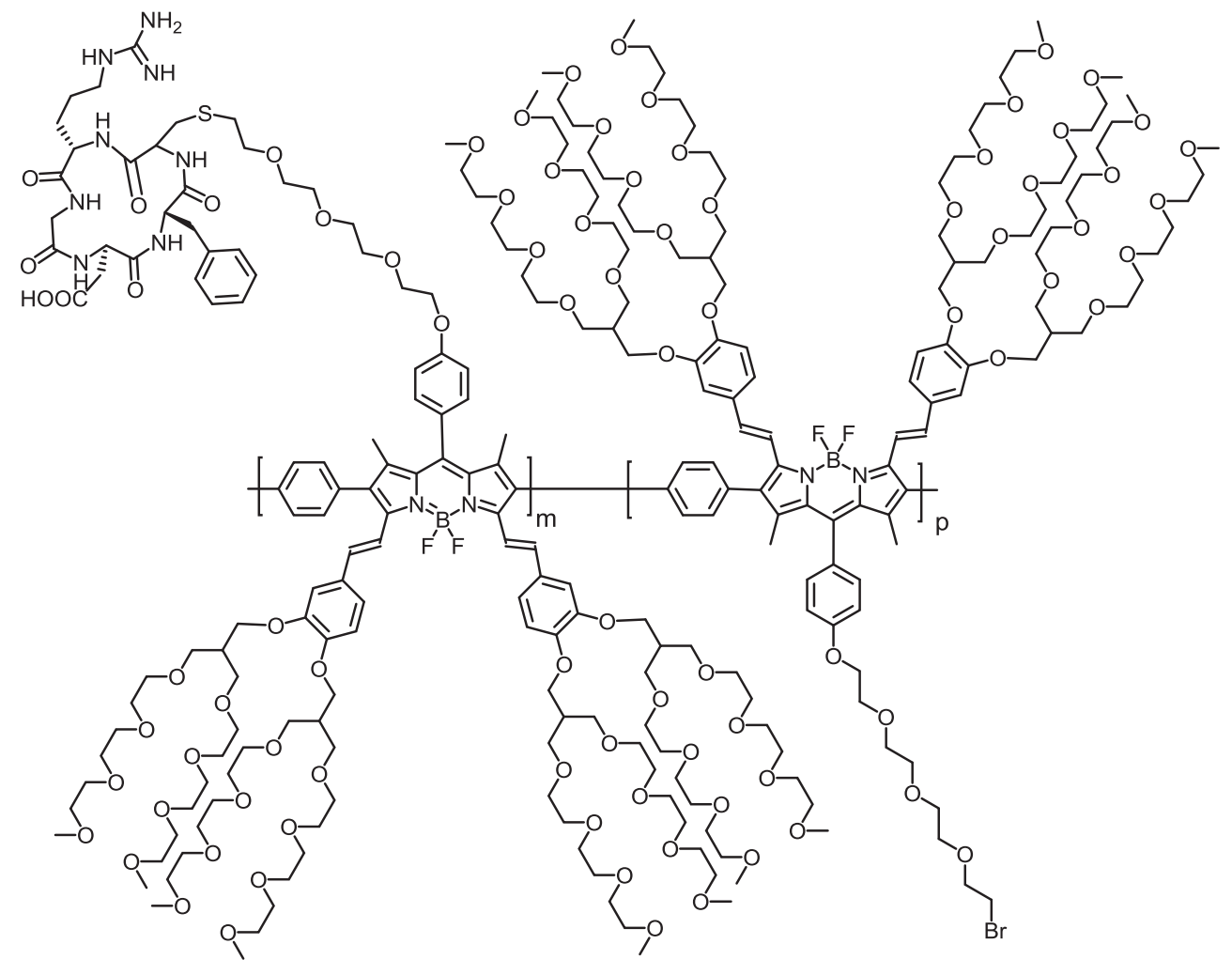

Polymer 8-B

Figure 8.1. Chemical structures of highly water-soluble, near-infrared polymeric BODIPY dyes. 


\subsection{Experimental section}

\subsubsection{Instrumentation}

${ }^{1} \mathrm{H}$ NMR and ${ }^{13} \mathrm{C}$ NMR spectra were taken on a $400 \mathrm{MHz}$ Varian Unity Inova spectrophotometer instrument. ${ }^{1} \mathrm{H}$ and ${ }^{13} \mathrm{C}$ NMR spectra were recorded in $\mathrm{CDCl}_{3}$, chemical shifts $(\delta)$ are given in ppm relative to solvent peaks $\left({ }^{1} \mathrm{H}: \delta 7.26 ;{ }^{13} \mathrm{C}: \delta 77.3\right)$ as internal standard. Absorption spectra were taken on a Perkin Elmer Lambda 35 UV-Vis spectrometer. Fluorescence spectra were recorded on a Jobin Yvon Fluoromax-4 spectrofluorometer. The excitation and emission slit width was set to $1 \mathrm{~nm}$ and all the samples were scanned with an increment of $1 \mathrm{~nm}$. Fluorescence lifetimes were measured on GL-3300 Nitrogen Laser laserstrobe, PTI instrument and analyzed using FeliX32 software. Fluorescein dye ( $\phi_{\mathrm{n}}=85 \%$ in $0.1 \mathrm{~N} \mathrm{NaOH}$ aqueous solution) was used as a standard to determine the fluorescence quantum yields of BODIPY dye 8.4 and 8.5. ${ }^{17}$ Sulforhodamine 101 dye ( $\phi_{\mathrm{n}}=95 \%$ with excitation wavelength at $577 \mathrm{~nm}$ in ethanol) was used as fluorescence standard to determine fluorescence quantum yields of BODIPY dye 8.7, 8.A and polymers 8-A and 8-B. ${ }^{18}$ Both samples and reference dye were prepared under identical conditions and the absorption, excitation and emission spectra were acquired under same experimental conditions. The areas for all emission spectra were calculated by integration of peak. Each plot of integrated area versus absorbance contained the corresponding reference data at that wavelength as well as the data for the test sample. A linear fit was then employed to determine the slope of the line (gradient) for the reference and the sample. The fluorescence quantum yield of each sample was obtained by using the following equation: ${ }^{18}$

$$
\Phi_{\mathrm{x}}=\Phi_{\mathrm{st}}\left(\operatorname{Grad}_{\mathrm{x}} / \operatorname{Grad}_{\mathrm{st}}\right)\left(\eta_{\mathrm{x}}{ }^{2} / \eta_{\mathrm{st}}{ }^{2}\right) \ldots . .(\text { eq. 8.1) }
$$

Where the subscripts ' $s t$ ' and ' $x$ ' represent standard and test samples, respectively, $\Phi$ is the fluorescence quantum yield, Grad stands for the gradient from the plot of integrated fluorescence intensity versus absorbance, and $\eta$ is the refractive index of the solvent.

Molecular weights of the polymers were determined by gel permeation chromatography (GPC) by using a Waters Associates Model 6000A liquid 
chromatograph. Mobile phase was HPLC grade THF which was filtered and degassed by vacuum filtration through a $0.5 \mu \mathrm{m}$ Fluoropore filter prior to use. The polymer was detected by a Waters Model 2410 refractive index detector. Molecular weight was measured relative to polystyrene standards.

\subsubsection{Materials and synthesis}

Unless otherwise indicated, all reagents and solvents were obtained from commercial suppliers (Aldrich, Sigma, Fluka, Acros Organics, Fisher Scientific, Lancaster) and used without further purification. Air- and moisture-sensitive reactions were conducted in oven-dried glassware using a standard Schlenk line or drybox techniques under an inert atmosphere of dry nitrogen.

BODIPY dye 8.4. The aldehyde $8.3(2.0 \mathrm{~g}, 5.56 \mathrm{mmol})$ and 2,4-dimethylpyrrole $(1.16 \mathrm{~g}, 12.23 \mathrm{mmol})$ were dissolved in $1000 \mathrm{~mL}$ of dry $\mathrm{CH}_{2} \mathrm{Cl}_{2}$ in a $2000-\mathrm{mL}$ three-neck flask. Five drops of trifluoroacetic acid (TFA) were added to the reaction mixture, and the resulting mixture was stirred in the dark for $12 \mathrm{~h}$ under a nitrogen atmosphere at room temperature. After the complete consumption of aldehyde $\mathbf{3}$ (which was monitored by TLC), DDQ (2,3-dichloro-5,6-dicyano-1,4-benzoquinone) (1.26 g, $5.56 \mathrm{mmol}$ ) was added to the reaction mixture. When the mixture was stirred for $40 \mathrm{~min}, 15 \mathrm{~mL}$ of diisopropylethylamine (DIPEA) and $15 \mathrm{~mL}$ of $\mathrm{BF}_{3} \cdot \mathrm{OEt}_{2}$ were added to the mixture. After the mixture was further stirred for $40 \mathrm{~min}$, it was concentrated to $200 \mathrm{~mL}$ and filtered. The filtrate was washed twice with water and brine solution, dried over anhydrous $\mathrm{MgSO}_{4}$, and concentrated under reduced pressure. The crude product was purified by column chromatography to obtain 8.4 as a red-orange oil (1.35 g, 42\%). ${ }^{1} \mathrm{H}$ NMR (400 $\left.\mathrm{MHz}, \mathrm{CDCl}_{3}\right): \delta 7.12(\mathrm{~d}, J=8.8 \mathrm{~Hz}, 2 \mathrm{H}), 6.99(\mathrm{~d}, J=8.8 \mathrm{~Hz}, 2 \mathrm{H}), 5.94(\mathrm{~s}, 2 \mathrm{H}), 4.16(\mathrm{t}, J$ $=4.4 \mathrm{~Hz}, 2 \mathrm{H}), 3.88(\mathrm{t}, J=4.8 \mathrm{~Hz}, 2 \mathrm{H}), 3.1-3.65(\mathrm{~m}, 10 \mathrm{H}), 3.45(\mathrm{t}, J=6.4 \mathrm{~Hz}, 2 \mathrm{H}), 2.52$ $(\mathrm{s}, 6 \mathrm{H}), 1.39$ (s, 6H). ${ }^{13} \mathrm{C} \mathrm{NMR}\left(100 \mathrm{MHz}, \mathrm{CDCl}_{3}\right): \delta$ 159.6, 155.4, 143.4, 142.0, 132.0, $129.4,127.4,121.3,115.4,71.4,71.1,70.9,70.8,70.7,69.9,67.7,30.5,14.8 . \mathrm{IR}\left(\mathrm{cm}^{-1}\right)$ : $2867,1609,1542,1508,1468,1409,1363,1304,1285,1245,1191,1155,1106,1083$, 1047, 973, 833, 810, 764, 704. $\mathrm{HRMS}(\mathrm{FAB})$ calcd for $\mathrm{C}_{27} \mathrm{H}_{34} \mathrm{BBrF}_{2} \mathrm{~N}_{2} \mathrm{O}_{4}[\mathrm{M}]^{+}$, 578.1763 ; found, 578.1757. 
2,6-Diiodo-BODIPY dye (8.5). When iodic acid $(0.54 \mathrm{~g}, 3.065 \mathrm{mmol})$ in $3 \mathrm{~mL}$ of water was added dropwise (30 mins) to the ethanol solution $(20 \mathrm{~mL})$ containing compound 8.4 (896 $\mathrm{mg}, 1.55 \mathrm{mmol})$ and iodine $(0.43 \mathrm{~g}, 3.36 \mathrm{mmol})$, the mixture was stirred for $4 \mathrm{~h}$. After the completion of the reaction, the mixture was concentrated under reduced pressure, dissolved in $\mathrm{CH}_{2} \mathrm{Cl}_{2}$, and washed twice with water and saturated saline solution. The organic layer was collected, dried over anhydrous $\mathrm{Na}_{2} \mathrm{SO}_{4}$, and concentrated under reduced pressure. The crude product was purified by column chromatography to yield 5 as red crystals $(1.20 \mathrm{~g}, 93 \%) .{ }^{1} \mathrm{H}$ NMR $\left(400 \mathrm{MHz}, \mathrm{CDCl}_{3}\right): \delta$ $7.11(\mathrm{~d}, J=8.8 \mathrm{~Hz}, 2 \mathrm{H}), 7.02(\mathrm{~d}, J=8.8 \mathrm{~Hz}, 2 \mathrm{H}), 4.18(\mathrm{t}, J=4.4 \mathrm{~Hz}, 2 \mathrm{H}), 3.91(\mathrm{t}, J=4.8$ $\mathrm{Hz}, 2 \mathrm{H}), 3.81$ (t, $J=6.4 \mathrm{~Hz}, 2 \mathrm{H}), 3.77-3.68(\mathrm{~m}, 8 \mathrm{H}), 3.46$ (t, $J=6.4 \mathrm{~Hz}, 2 \mathrm{H}), 2.62$ (s, $6 \mathrm{H}), 1.42(\mathrm{~s}, 6 \mathrm{H}) .{ }^{13} \mathrm{C}$ NMR $\left(100 \mathrm{MHz}, \mathrm{CDCl}_{3}\right): \delta$ 160.0, 156.8, 145.6, 141.8, 131.9, 129.3, 127.1, 115.7, 71.4, 71.1, 70.9, 70.8, 70.7, 69.9, 67.8, 30.5, 17.4, 16.2. IR $\left(\mathrm{cm}^{-1}\right)$ : 2862, 1607, 1522, 1454, 1396, 1342, 1304, 1247, 1173, 1114, 1091, 1059, 994, 961, 913, 834, 763, 704. HRMS(FAB) calcd for $\mathrm{C}_{27} \mathrm{H}_{32} \mathrm{BBrF}_{2} \mathrm{I}_{2} \mathrm{~N}_{2} \mathrm{O}_{4}[\mathrm{M}]^{+}$, 829.9696; found, 829.9690 .

2,5-Diiodo-3,5-distyryl BODIPY dye (8.7). 2,6-Diiodo-BODIPY dye 8.5 (1.66 g, $2.0 \mathrm{mmol})$, and compound $8.6(5.4 \mathrm{~g}, 6.0 \mathrm{mmol})$ were refluxed in a mixture of benzene $(150 \mathrm{~mL})$, piperidine $(3.5 \mathrm{~mL})$ and $\mathrm{AcOH}(3.2 \mathrm{~mL})$. Any water formed during the reaction, was removed azeotropically by heating the mixture in a Dean-Stark apparatus. After $2.5 \mathrm{~h}$, the mixture was concentrated in vacuo, diluted with EtOAc, and then washed with water and brine, respectively. The organic phase was dried over $\mathrm{Na}_{2} \mathrm{SO}_{4}$ and concentrated in vacuo. The crude product was simply purified by silica gel column chromatography (hexane/EtOAc/ $\mathrm{CH}_{2} \mathrm{Cl}_{2} / \mathrm{EtOH}, \quad 5 / 1 / 3 / 0.5, \quad \mathrm{v} / \mathrm{v}$ to hexane/EtOAc $/ \mathrm{CH}_{2} \mathrm{Cl}_{2} / \mathrm{EtOH}, 5 / 1 / 3 / 1$, v/v) to obtain distyryl BODIPY 7 (1.23 g, 24\%) as green oil. ${ }^{1} \mathrm{H}$ NMR (400 MHz, $\left.\mathrm{CDCl}_{3}\right): \delta 8.00$ (d, $\left.J=16.8 \mathrm{~Hz}, 2 \mathrm{H}\right), 7.46$ (d, $J=16.4 \mathrm{~Hz}$, 2H), $7.25(\mathrm{~d}, J=5.6 \mathrm{~Hz}, 2 \mathrm{H}), 7.10(\mathrm{~d}, J=8.4 \mathrm{~Hz}, 2 \mathrm{H}), 7.02(\mathrm{~s}, 2 \mathrm{H}), 6.99$ (d, $J=5.2 \mathrm{~Hz}$, 4H), $6.87(\mathrm{~d}, J=8.4 \mathrm{~Hz}, 2 \mathrm{H}), 4.17$ (t, $J=4.4 \mathrm{~Hz}, 2 \mathrm{H}), 4.04$ (t, $J=5.6 \mathrm{~Hz}, 6 \mathrm{H}), 3.88$ (t, $J$ $=5.6 \mathrm{~Hz}, 2 \mathrm{H}), 3.77(\mathrm{t}, J=6.4 \mathrm{~Hz}, 2 \mathrm{H}), 3.73-3.41(\mathrm{~m}, 104 \mathrm{H}), 3.32-3.29\left(\mathrm{~s} \times 8 \mathrm{CH}_{3}, 24 \mathrm{H}\right)$, 2.42-2.36 (m, 4H), $1.45(\mathrm{~s}, 6 \mathrm{H}) .{ }^{13} \mathrm{C}$ NMR (100 MHz, $\left.\mathrm{CDCl}_{3}\right): \delta 160.0,150.6,150.5$, 149.2, 145.8, 139.7, 138.7, 133.5, 130.0, 129.8, 127.6, 120.5, 117.0, 115.6, 113.9, 113.7, $83.3,77.5,72.1,71.4,71.1,70.9,70.8,70.8,70.7,70.7,70.6,69.9,69.5,69.4,67.8,67.3$, 188 
67.1, 63.7, 59.2, 41.4, 40.2, 30.5, 17.9. IR $\left(\mathrm{cm}^{-1}\right): 2867,1594,1509,1463,1409,1350$, 1265, 1250, 1178, 1095, 1012, 958, 849, 806, 769, 711. HRMS(MAIDL) calcd for $\mathrm{C}_{113} \mathrm{H}_{184} \mathrm{BBrF}_{2} \mathrm{I}_{2} \mathrm{~N}_{2} \mathrm{O}_{40}[\mathrm{M}+\mathrm{Na}]^{+}, 2613.9656$; found, 2613.9688 .

BODIPY dye 8.A. 2,5-Diiodo-3,5-distyryl BODIPY dye 8.7 (80 mg, $0.03 \mathrm{mmol}$ ), phenylboronic acid $(12.2 \mathrm{mg}, 0.1 \mathrm{mmol}), \mathrm{Pd}(\mathrm{dppf})_{2} \mathrm{Cl}_{2}(1.1 \mathrm{mg})$ and $\mathrm{Na}_{2} \mathrm{CO}_{3}(32 \mathrm{mg}$, $0.30 \mathrm{mmol})$ were added to a $50-\mathrm{mL}$ flask under a nitrogen atmosphere. When toluene (6 $\mathrm{mL})$, EtOH $(4 \mathrm{~mL})$ and $\mathrm{H}_{2} \mathrm{O}(3 \mathrm{~mL})$ were added to the flask, the mixture was stirred at 80 ${ }^{\circ} \mathrm{C}$ under a nitrogen atmosphere overnight. After removal of the solvent, the residue was dissolved in ethyl acetate and washed with water twice. The organic layer was collected, dried over anhydrous $\mathrm{Na}_{2} \mathrm{SO}_{4}$, and filtered. The filtrate was concentrated under reduced pressure and purified using TLC plate (hexanes $/ \mathrm{CH}_{2} \mathrm{Cl}_{2} / \mathrm{EtOAc} / \mathrm{MeOH}, 5 / 3 / 1 / 0.7$, v/v) to attain BODIPY dye 8.A as green oil (74 mg, 83\%). ${ }^{1} \mathrm{H}$ NMR (400 MHz, $\left.\mathrm{CDCl}_{3}\right): \delta 7.52$ (d, $J=16.4 \mathrm{~Hz}, 2 \mathrm{H}), 7.41-7.33$ (m, 6H), 7.24-7.21 (m, 6H), 7.03 (dd, $J=8.4,1.6 \mathrm{~Hz}$, 2H), 6.99 (d, $J=8.8 \mathrm{~Hz}, 2 \mathrm{H}), 6.79(\mathrm{~d}, J=8.4 \mathrm{~Hz}, 2 \mathrm{H}), 6.48(\mathrm{~d}, J=1.6 \mathrm{~Hz}, 2 \mathrm{H}), 6.42(\mathrm{~d}, J$ $=16.4 \mathrm{~Hz}, 2 \mathrm{H}), 4.14(\mathrm{t}, J=4.4 \mathrm{~Hz}, 2 \mathrm{H}), 3.98(\mathrm{~d}, J=5.6 \mathrm{~Hz}, 4 \mathrm{H}), 3.91(\mathrm{~d}, J=5.6 \mathrm{~Hz}$, $4 \mathrm{H}), 3.85(\mathrm{t}, J=4.4 \mathrm{~Hz}, 2 \mathrm{H}), 3.76(\mathrm{t}, J=6.0 \mathrm{~Hz}, 2 \mathrm{H}), 3.72-3.47(\mathrm{~m}, 120 \mathrm{H}), 3.42(\mathrm{t}, J=$ $6.0 \mathrm{~Hz}, 2 \mathrm{H},-\mathrm{CH}_{2} \mathrm{Br}$ ), 3.33-3.32 (s, $\left.8 \mathrm{CH}_{3}, 24 \mathrm{H}\right), 2.38-2.34(\mathrm{~m}, 4 \mathrm{H}), 1.24(\mathrm{~s}, 6 \mathrm{H}) .{ }^{13} \mathrm{C}$ NMR (100 MHz, $\left.\mathrm{CDCl}_{3}\right): \delta$ 159.6, 150.3, 150.2, 149.0, 139.6, 138.9, 138.5, 135.6, 133.8, 133.7, 131.4, 130.7, 130.4, 129.9, 128.9, 128.5, 128.4, 128.2, 127.6, 120.0, 117.3, 115.4, 114.0, 113.6, 77.5, 72.1, 71.4, 71.1, 70.9, 70.8, 70.7, 70.6, 69.9, 69.5, 67.7, 67.3, 67.1, 59.2, 40.2, 40.2, 30.5, 12.9. IR ( $\left.\mathrm{cm}^{-1}\right): 2868,1594,1576,1509,1449,1398,1364,1348$, 1268, 1246, 1185, 1090, 1029, 1009, 996, 964, 849, 808, 707. ESI MS(m/z): 1248.0 (M $\left.+2 \mathrm{H}^{+}\right)$.

Polymer 8-A. 2,6-Diiodo3,5-distyryl BODIPY dye 7 (600 mg, $0.23 \mathrm{mmol}$ ), benzene-1,4-diboronic acid (42 $\mathrm{mg}, 0.253 \mathrm{mmol}), \mathrm{Pd}(\mathrm{dppf})_{2} \mathrm{Cl}_{2}(19.0 \mathrm{mg}, 0.023 \mathrm{mmol})$ and $\mathrm{Na}_{2} \mathrm{CO}_{3}(244 \mathrm{mg}, 2.3 \mathrm{mmol})$ were added to a $50 \mathrm{~mL}$ flask under a nitrogen atmosphere. When toluene $(40 \mathrm{~mL})$, EtOH $(10 \mathrm{~mL})$ and $\mathrm{H}_{2} \mathrm{O}(20 \mathrm{~mL})$ were added to the flask, the mixture was stirred at $80{ }^{\circ} \mathrm{C}$ under a nitrogen atmosphere for 2 days. After removal of the solvent by under vacuum, the residue was dissolved in ethyl acetate and washed with water twice. The organic layer was collected, dried over anhydrous $\mathrm{Na}_{2} \mathrm{SO}_{4}$, and filtered. The filtrate was concentrated under reduced pressure and purified using TLC 189 
plate (hexanes $/ \mathrm{CH}_{2} \mathrm{Cl}_{2} / \mathrm{EtOAc} / \mathrm{MeOH}, 5 / 3 / 1 / 1$, v/v) to attain polymer 8-A as green oil (426 mg). ${ }^{1} \mathrm{H}$ NMR (400 MHz, $\left.\mathrm{CDCl}_{3}\right): \delta 7.55$ (d, $\left.J=14.4,2 \mathrm{H}\right), 7.32-7.23(\mathrm{~m}, 6 \mathrm{H}), 7.05$ (br, 2H), 6.91 (br, 2H), 6.75 (br, 2H), 6.63 (s, 2H), 6.60-6.56 (m, 2H), 4.18 (br, 2H), 4.07 (br, 2H), 3.98 (br, 4H), 3.89 (br, 4H), 3.78-3.24 (m, 148H), 2.42-2.34 (m, 4H), 1.23 (s, $6 \mathrm{H})$. IR $\left(\mathrm{cm}^{-1}\right): 2867,1594,1575,1506,1447,1416,1362,1349,1268,1247,1180$, 1087, 1021, 1006, 962, 841, 808, 770, 709. Gel Permeation Chromatograph (GPC): (THF, polystyrene standard), $\mathrm{M}_{\mathrm{n}}: 38,700 \mathrm{~g} / \mathrm{mol}$; polydispersity: 2.1 .

Polymer 8-B: Polymer 8-A (20 mg, $0.00865 \mathrm{mmol}$, repeated unit), c(RGDfC) (5 mg, $0.00865 \mathrm{mmol})$ and $\mathrm{Na}_{2} \mathrm{CO}_{3}(9 \mathrm{mg}, 0.0865 \mathrm{mmol})$ dissolved in absolute solvents of DMF (3 mL) and $\mathrm{H}_{2} \mathrm{O}(0.5 \mathrm{~mL})$. After the mixture was stirred at room temperature under nitrogen atmosphere for 2 days, the solvents were removed under reduced pressure. The residue was dissolved in $\mathrm{CH}_{2} \mathrm{Cl}_{2}$ and filtered. The filtrate was concentrated under a reduce pressure and precipitated by using $\mathrm{Et}_{2} \mathrm{O}$ and $\mathrm{CH}_{2} \mathrm{Cl}_{2}$. The solid was collected and dried under reduced pressure to attain polymer 8-B as green solid $(7 \mathrm{mg})$. IR $\left(\mathrm{cm}^{-1}\right)$ : $3304(\mathrm{~N}-\mathrm{H}), 2869,1747$ (C=O), 1594, 1509, 1449, 1414, 1362, 1349, 1268, 1248, 1183, 1024, 964, 842, 770, 709. Elem. Anal. Found: N, 6.77; S, 0.96. Functionalization degree with RGD peptide residues is $\sim 20 \%$ according to elemental analysis of nitrogen and sulfur atoms.

\subsubsection{Cell Culture and Fluorescence Imaging}

MDA-MB-231 and HUVEC-C cell lines were procured from ATCC ${ }^{\circledR}$. MDA-MB231 cells were cultured in Dulbecco's Modified Eagle's Medium/Nutrient Mixture F12 Ham's Liquid Media (DMEM/F-12) supplemented with 10\% fetal bovine serum (FBS, GIBCO), penicillin (100 U/ml) and streptomycin $(100 \mu \mathrm{g} / \mathrm{ml})(1 \mathrm{X}$ PenStrep, GIBCO), and HUVEC cells were cultured in medium 199 with 2mM L-glutamine, 10\% FBS, heparin $(100 \mu \mathrm{g} / \mathrm{ml})$, endothelial cell growth supplements $(50 \mu \mathrm{g} / \mathrm{ml})$ and $1 \mathrm{X}$ PenStrep at $37{ }^{\circ} \mathrm{C}$ in humidified incubator with $5 \% \mathrm{CO}_{2}$. The cells were plated on 12-well culture plates at a density of $1 \times 10^{5}$ cells/ $\mathrm{ml}$ for live cell imaging. After 24-hour incubation at $37{ }^{\circ} \mathrm{C}, 5 \% \mathrm{CO}_{2}$ incubator, the media was removed and rinsed with $1 \mathrm{X}$ PBS and fresh serum free media with polymer A or polymer B was added at $50 \mu \mathrm{g} / \mathrm{ml}$ concentration. Polymer 8-A (or polymer 8-B) were incubated with cells for 4 hours and then washed 
with $1 \mathrm{X}$ PBS before imaging. The cell imaging was performed with fluorescence microscope (Zeiss Axiovert 200) using DAPI and Cy5 filters for Hoechst stain and polymer $\mathrm{A} / \mathrm{B}$ dye respectively. The fluorescence images were obtained with $20 \mathrm{X}$ magnification objective and the exposure times for each filter were kept constant for each image series.

\subsubsection{Photostability experiment}

To test the photostability (bleaching) of polymer Bin cell culture media polymer 8-B was dissolved at a concentration of 0.5 and $200 \mu \mathrm{g} / \mathrm{mL}$ in $1 \mathrm{~mL}$ media and placed on an inverted microscope (NIKON TE 2000U). In a 96 well plate, a $200 \mu \mathrm{L}$ aliquot of each of the concentrations was dispensed with the control being a $200 \mu \mathrm{L}$ aliquot of media solution. Each concentration was tested one at a time using the Nikon CY5 LP filter to excite the polymer in solution. Fluorescence intensity of the polymer was then quantified. The intensity was then measured (x20 objective) up to 60 minutes without switching off the excitation light with an exposure time of $30 \mathrm{sec}$. Images were captured using a cooled CCD camera (Coolsnap HQ2, Roper Scientific) and post processed using imaging software (NIKON Elements, Melville, NY). ImageJ software (NIH) was used to determine the average pixel intensity, representing the fluorescence emission brightness, of each fluorescent image. The light intensity used is comparable to that used in microscopy for cancer imaging. The absorbance of the polymer solution is between 0 and 0.3 .

\subsection{Results and discussions}

\subsubsection{Synthesis of BODIPY Polymeric Dyes}

We employed a simple, convenient, versatile post-polymerization functionalization method to prepare BODIPY copolymeric dye bearing RGD cancer-homing peptide residues as we have demonstrated its feasibility to functionalize bromide-bearing conjugated fluorescent polymers such as polythiophenes and fluorene-based conjugated polymers with thiol-functionalized carbohydrates through via thioether bonds under a mild basic condition with almost 100\% degree post-polymerization functionalization of the bromide groups with the thiol groups. ${ }^{19-21}$ In order to introduce a bromide group 
through tetra(ethylene glycol) spacer to BODIPY dye at the meso position, we prepared bromide-functionalized aldehyde derivative (8.3) by reaction of 1-bromo-2-(2-(2bromoethoxy)ethoxy)ethoxy)ethane (8.2) with 4-hydroxybenzaldehyde (8.1) in DMF under a basic condition (Scheme 8.1). BODIPY dye bearing bromide group through a tetra(ethylene glycol) tethered spacer at the meso position (8.4) was prepared through the condensation of the benzaldehyde derivative (8.3) with 2,4-dimethylpyrrole in the presence of a catalytic amount of trifluoroacetic acid (TFA), followed by oxidation with 2,3-dichloro-5,6-dicyano-1,4-benzoquinone (DDQ) and chelation with $\mathrm{BF}_{3}$-etherate in the presence of N,N-diisopropylethylamine (DIPEA). ${ }^{11}$ 2,6-Diiodo-BODIPY dye (8.5) was prepared by iodination of BODIPY dye 8.4 at 2,6-positions. In order to prepare highly water-soluble 2,6-diiodo-3,5-distyryl-BODIPY monomeric dye with longer emission (8.7), we incorporated branched oligo(ethylene glycol)methyl etoher into distyryl groups at 3,5-positions by the Knoevenagel condensation of methyl groups of 2,6-diiodo-BODIPY dye (8.5) at 3,5-positions with aldehyde derivative (8.6). ${ }^{11,22}$ In order to confirm the feasibility to prepare distyryl 2,6-diiodo-3,5-distyryl-BODIPY dye (8.7) bearing a bromide group through a tetra(ethylene glycol) tethered spacer at the meso position via the Knoevenagel reaction, we prepared BODIPY dye bearing bromide group through tetra(ethylene glycol) at the meso position (8.A) by the palladium-catalyzed Suzuki coupling of 2,6-diiodo-3,5-distyryl-BODIPY dye (7) with 1-phenylboronic acid (Scheme 8.1). This is because the proton peak of methylene protons adjacent to bromide group in 2,6-diiodo-3,5-distyryl-BODIPY dye 7 was expected to overlap with those proton peaks from branched oligo(ethylene glycol)ether methyl groups in ${ }^{1} \mathrm{H}$ NMR spectrum. Comparison to ${ }^{1} \mathrm{H}$ NMR spectra and high-resolution MS data of BODIPY dyes 8.5, 8.7 and 8.A clearly indicate that bromide group of 2,6-diiodo-BODIPY dye $\mathbf{8 . 5}$ remains intact during the Knoevenagel reaction in the presence of piperidine and $\mathrm{AcOH}$ in benzene solution under a reflux condition (Figure F.1 in Appendix F). Triplet peaks corresponding to methylene protons adjacent to bromide group were observed at 3.46 ppm in 2,6-diiodo-BODIPY dye 8.5 (Figure F.1 in Appendix F). After the Knoevenagel reaction, those peaks corresponding to methylene protons adjacent to bromide group were overlapped with proton peaks of branched oligo(ethylene glycol)methyl ether in 2,6diiodo-3,5-distyryl-BODIPY dye 8.7. 

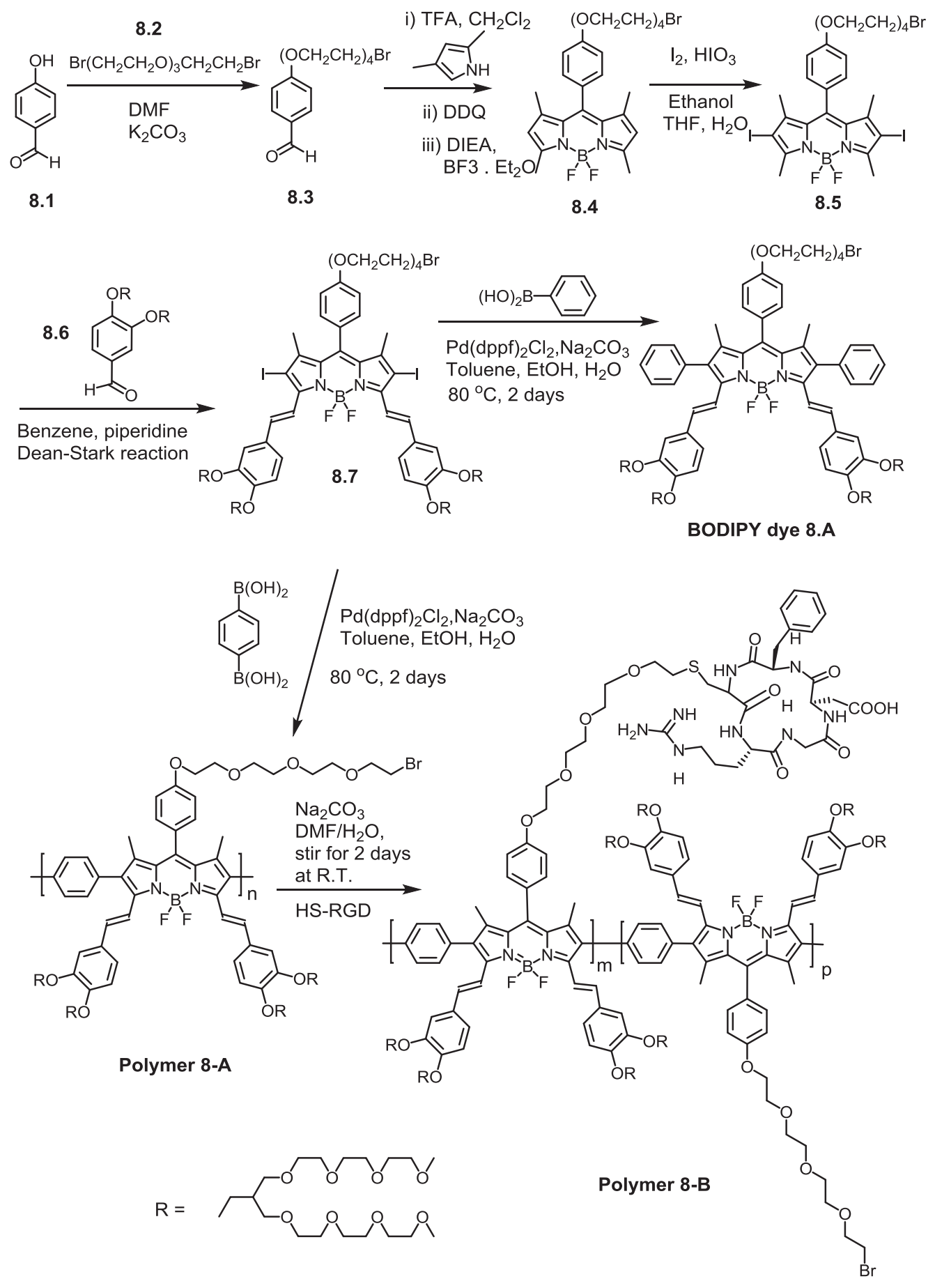

Scheme 8.1. Synthetic route to polymers 8-A and 8-B.

However, after the palladium-catalyzed Suzuki coupling of 2,6-diiodo-3,5-distyrylBODIPY dye (8.7) with 1-phenylboronic acid, triplet peaks corresponding to methylene protons adjacent to bromide group show up at 3.43 ppm in BODIPY dye A (Figure F.1 in 
Appendix F), which indicates that the bromide groups in BODIPY dyes $\mathbf{8 . 5}$ and 8.7 are intact during the Knoevenagel condensation reaction and the palladium-catalyzed Suzuki coupling reaction. Highly water-soluble, near-infrared emissive BODIPY polymeric dye bearing bromide groups (polymer 8-A) was prepared by the palladium-catalyzed Suzuki polymerization of 2,6-diiodo-3,5- distyryl-BODIPY dye 8.7 with benzene-1,4-diboronic acid. Highly water-soluble, near-infrared emissive BODIPY polymeric dye bearing RGD peptide residues (polymer 8-B) was prepared by postpolymerization functionalization of polymer 8-A with thiol-functionalized RGD peptide through thioether bonds under a mild basic condition. The post-polymerization functionalization degree of polymer 8-A with thiol-functionalized RGD peptide was estimated as $20 \%$ according to the elemental analysis (sulfur and nitrogen atoms) of polymer 8-B.

\subsubsection{Optical properties of BODIPY-based polymeric dyes}

BODIPY dye (8.4) in methylene chloride shows two absorption peaks which are attributed to a strong $\mathrm{S}_{0} \rightarrow \mathrm{S}_{1}\left(\pi-\pi^{*}\right)$ transition at $501 \mathrm{~nm}$ and a weaker broad band around $350 \mathrm{~nm}$ resulting from the $\mathrm{S}_{0} \rightarrow \mathrm{S}_{2}\left(\pi-\pi^{*}\right)$ transition (Table 8.1). Introduction of 2,6-diiodo substituents to the BODIPY dye 8.4 to give BODIPY dye 8.5 causes large red shifts (32 $\mathrm{nm}$ and $36 \mathrm{~nm}$ ) in both the absorption and fluorescence maxima, respectively, because 2,6-diiodo substituents serve as auxochromes (Table 8.1). 2,6-Diiodo-3,5-distyrylBODIPY dye 8.7 shows absorption and emission peaks at $675 \mathrm{~nm}$ and $706 \mathrm{~nm}$, respectively, because of its significantly extended $\pi$-conjugation compared with 2,6diiodo-BODIPY dye 8.5. It shows slightly red shifts by $8 \mathrm{~nm}$ and $9 \mathrm{~nm}$ in absorption and emission spectra from methylene chloride to aqueous solution, respectively. BODIPY dye A exhibits absorption and emission peaks at $665 \mathrm{~nm}$ and $702 \mathrm{~nm}$, respectively, indicating that two phenyl units and BODIPY core are not coplanar because of steric hindrance among phenyl protons with vinyl protons at 3,5-positions and methyl protons at 1,7-positions. Polymer 8-A displays slightly red shifts by only $22 \mathrm{~nm}$ and $8 \mathrm{~nm}$ in absorption and emission spectra in aqueous solution compared with BODIPY dye 8.A (Table 8.1), indicating that phenyl units and BODIPY cores in the polymer backbone are not coplanar because of the same kind of steric hindrance as BODIPY dye 8.A. The lack of significant shifts in emission spectra of polymer 8-A and BODIPY dye 8.A could be 
due to phenylene moiety itself without any donor groups such as $\mathrm{OMe}$ or $\mathrm{NMe}_{2}$. However, the weak $\pi$-conjugation of polymer A significantly enhances solubility of the polymer in aqueous solution by preventing potential $\pi-\pi$ stacking interactions among polymer backbones. Polymer 8-B shows slightly red shifts by $2 \mathrm{~nm}$ and $1 \mathrm{~nm}$ in absorption and emission spectra in aqueous solution compared with polymer 8-A. BODIPY dye 8.A, and polymers 8-A and 8-B are highly soluble with at least $20 \mathrm{mg} / \mathrm{mL}$ in aqueous solution (Figure 8.2).

Table 8.1. Absorption and emission peaks, fluorescence quantum yields, and fluorescence lifetime of BODIPY monomeric and polymeric dyes in methylene chloride and aqueous solution.

\begin{tabular}{cccccc}
\hline BODIPY dye & $\begin{array}{c}\text { Absorption } \\
\text { Peak (nm) }\end{array}$ & $\begin{array}{c}\text { Emission } \\
\text { Peak (nm) }\end{array}$ & $\mathbf{t}_{\text {lifetime }}(\mathbf{n s})$ & $\boldsymbol{\Phi}_{\mathbf{f}}(\mathbf{\%})$ & Solvent \\
\hline $\mathbf{8 . 4}$ & 501 & 510 & 3.8 & 81 & $\mathrm{CH}_{2} \mathrm{Cl}_{2}$ \\
\hline $\mathbf{8 . 5}$ & 533 & 546 & 4.5 & 3.6 & $\mathrm{CH}_{2} \mathrm{Cl}_{2}$ \\
\hline \multirow{2}{*}{$\mathbf{8 . 7}$} & 667 & 697 & 3.4 & 2 & $\mathrm{CH}_{2} \mathrm{Cl}_{2}$ \\
\hline \multirow{2}{*}{ 8.A } & 635,675 & 706 & 0.6 & 0.1 & Water \\
\hline \multirow{2}{*}{ Polymer 8-A } & 614,663 & 685 & 7.7 & 18 & $\mathrm{CH}_{2} \mathrm{Cl}_{2}$ \\
& 622,665 & 702 & 2.6 & 0.7 & $\mathrm{Water}^{2}$ \\
\hline \multirow{2}{*}{ Polymer 8-B } & 680 & 691 & 1.0 & 2.3 & $\mathrm{CH}_{2} \mathrm{Cl}_{2}$ \\
& 687 & 711 & 0.4 & 0.8 & $\mathrm{Water}^{2}$ \\
\hline
\end{tabular}

Figure 8.3 shows the fluorescence intensity plot of the polymer at different concentrations. As expected, the average pixel value significantly depends on the different polymer concentration. The measurement of average pixel value was conducted at different times as 5 minutes, 30 minutes, and 60 minutes. The percent change seen among the three time points is less than $5 \%$. The results show that the polymer exhibits very good photostability without any significant decline in intensity due to photobleaching. 


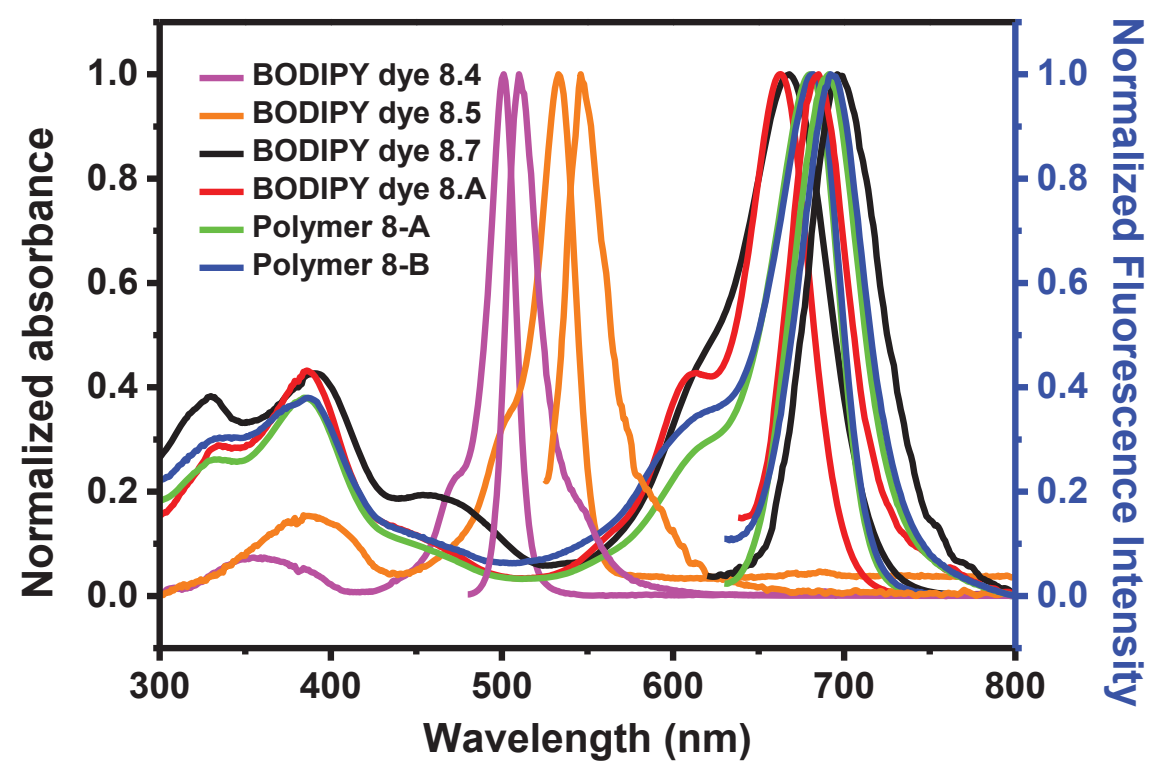

Figure 8.2. Normalized absorption and Emission spectra of BODIPY dyes $(\mathbf{8 . 4}, \mathbf{8 . 5}, \mathbf{8 . 7}$, 8.A) and polymers 8-A and 8-B in methylene chloride solution.

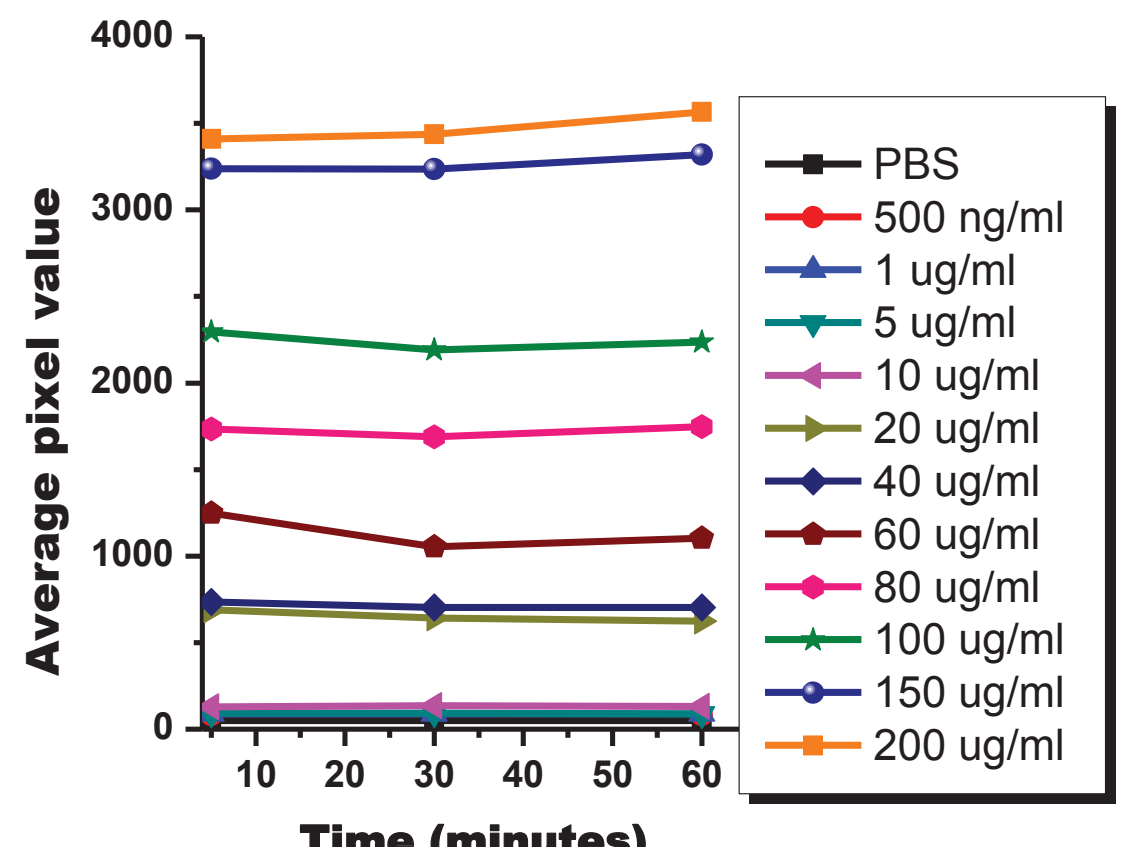

Figure 8.3. Average light intensity versus time of different concentrations of polymer 8$\mathbf{B}$ in media under light illumination. 


\subsubsection{Application of BODIPY polymeric dye bearing cancer-homing RGD peptides in near-infrared imaging of cancer cells}

The specificity of RGD-bearing polymeric BODIPY dye (polymer 8-B) was evaluated for tumor cell targeting using MDA-MB-231 breast cancer cell lines and compared with polymer 8-A (lacking RGD peptide). The normal endothelial cells (HUVEC) were used as control cells. We observed a strong fluorescence signal with polymer 8-B at $100 \mu \mathrm{g} / \mathrm{mL}$ concentration (Figure 8.4) as well as at $50 \mu \mathrm{g} / \mathrm{mL}$ (Figure F.21 in Appendix F) in MDA-MB-231 cells. The fluorescence signal was very poor with polymer 8-A in both MDA-MB-231 and HUVEC cells, suggesting very little or no incorporation of free polymer $\mathrm{A}$ in these cells. Whereas, polymer 8-B showed increased fluorescence signal for MDA-MB-231 cells in the peri-nuclear region (Figure 8.4 and Figure F.22 in Appendix F), indicating that RGD-receptor mediated specific transport and incorporation of polymer $B$ to the breast tumor cells through $\alpha v \beta_{3}$ integrins that are overexpressed in MDA-MB-231 cells but are known to be expressed at very low concentrations on normal cells such as the HUVEC-C cells ${ }^{23}$. The sensitivity of the RGD peptide-bearing BODIPY polymeric dye for targeting MDA-MB-231 cells is $40 \mu \mathrm{g} / \mathrm{mL}$ (Figure F.23 in Appendix F). The high affinity and selectivity of the polymer 8-B arise from the cyclic RGD peptides that bind $\alpha_{v} \beta_{3}$ integrins that are over-expressed in MDAMB-231 breast cells. ${ }^{23}$

\subsection{Conclusion}

Conjugation of cancer-homing cyclic RGD peptide to BODIPY polymeric dye not only further enhances water solubility of the polymer, but also significantly increases binding affinity and specificity of the polymer to breast cancer cells via specific cooperative bindings of RGD peptide residues to $\alpha v \beta_{3}$ integrins on cancer cells. Combination of BODIPY polymeric dye with other specific caner-homing peptides such as NGR, GFE, F3, and LyP-1 instead of RGD peptide is expected to significantly extend this application in in vitro and in vivo detection of different cancer cells and tissues. 


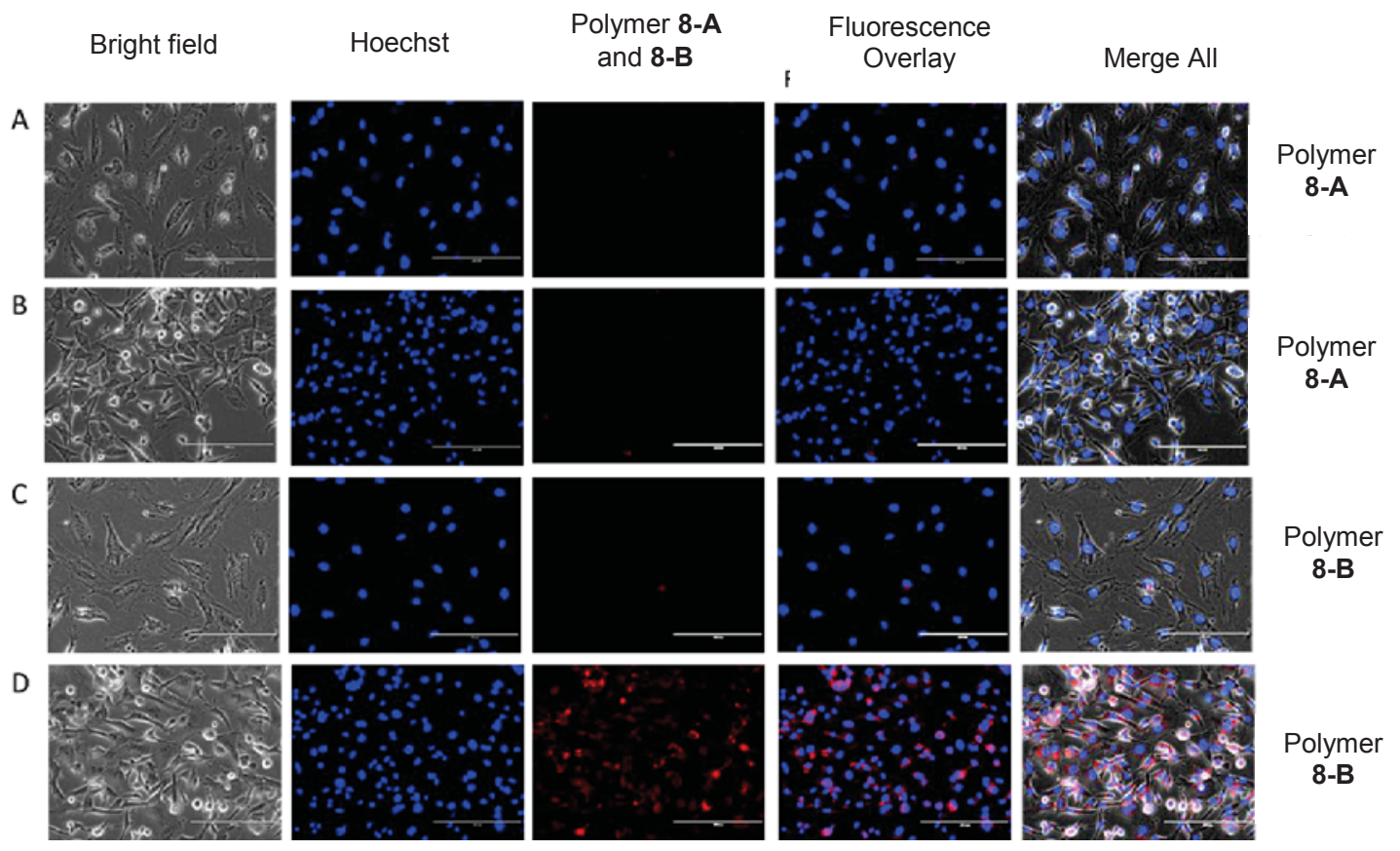

Figure 8.4. Bright-field and fluorescence images of HUVEC-C cells (A) and (C), and MDA-MB-231 cells (B) and (D) after incubation with $100 \mu \mathrm{g} / \mathrm{ml}$ of polymer 8-A (control BODIPY dye) or polymer 8-B (RGD-bearing polymeric BODIPY dye) for 4 hours and then washing the cells with PBS before imaging.

(The bright-field images were acquired at 20X magnification with transmitted light and fluorescence images were acquired using DAPI (for Hoechst stain) and Cy5 filters (for polymers 8-A or 8-B). Hoechst stain was used for nuclear localization and is shown in the middle panel (blue). Fluorescence overlay (Hoechst and polymer 8-A or 8-B) and merged all (Brightfield + fluorescence overlay) shows peri-nuclear localization of polymer 8-B in MDA-MB-231 cells with little or no incorporation in HUVEC cells. Please see detailed close-up image of panels C and D in Appendix F, Figure F.22.) 


\section{References:}

1. Shah, N., Cerussi, A., Eker, C., Espinoza, J., Butler, J., Fishkin, J., Hornung, R., Tromberg, B., Noninvasive Functional Optical Spectroscopy of Human Breast Tissue. Proceedings of the National Academy of Sciences of the United States of America 2001, 98 (8), 4420-4425.

2. Weissleder, R., A Clearer Vision for In Vivo Imaging. Nature Biotechnology 2001, 19 (4), 316-317.

3. Hawrysz, D. J., Sevick-Muraca, E. M., Developments toward Diagnostic Breast Cancer Imaging Using Near-infrared Optical Measurements and Fluorescent Contrast Agents. Neoplasia 2000, 2 (5), 388-417.

4. Luo, S. L., Zhang, E. L., Su, Y. P., Cheng, T. M., Shi, C. M., A Review of NIR Dyes in Cancer Targeting and Imaging. Biomaterials 2011, 32 (29), 7127-7138.

5. Cai, W. B., Shin, D. W., Chen, K., Gheysens, O., Cao, Q. Z., Wang, S. X., Gambhir, S. S., Chen, X. Y., Peptide-labeled Near-Infrared Quantum Dots for Imaging Tumor Vasculature in Living Subjects. Nano Letters 2006, 6 (4), 669676.

6. Loudet, A., Burgess, K., BODIPY Dyes and Their Derivatives: Syntheses and Spectroscopic Properties. Chemical Reviews 2007, 107, 4891-4932.

7. Sakanoue, J., Ichikawa, K., Nomura, Y., Tamura, M., Rhodamine 800 as A Probe of Energization of Cells and Tissues in The Near-infrared Region: A Study with Isolated Rat Liver Mitochondria and Hepatocytes. Journal of Biochemistry 1997, $121(1), 29-37$.

8. Lee, Y. D., Lim, C. K., Kim, S., Kwon, I. C., Kim, J., Squaraine-doped Functional Nanoprobes: Lipophilically Protected Near-infrared Fluorescence for Bioimaging. Advanced Functional Materials 2010, 20 (17), 2786-2793.

9. Berezin, M. Y., Guo, K. V., Akers, W., Livingston, J., Solomon, M., Lee, H., Liang, K. X., Agee, A., Achilefu, S., Rational Approach to Select Small Peptide Molecular Probes Labeled with Fluorescent Cyanine Dyes for In Vivo Optical Imaging. Biochemistry 2011, 50 (13), 2691-2700. 
10. Bura, T., Ziessel, R., Water-soluble Phosphonate-Substituted BODIPY Derivatives with Tunable Emission Channels. Organic Letters 2011, 13 (12), 3072-3075.

11. Zhu, S. L., Zhang, J. T., Vegesna, G., Luo, F. T., Green, S. A., Liu, H. Y., Highly Water-soluble Neutral BODIPY Dyes with Controllable Fluorescence Quantum Yields. Organic Letters 2011, 13 (3), 438-441.

12. Donuru, V. R., Vegesna, G. K., Velayudham, S., Meng, G., Liu, H. Y., Deep-Red Emissive Conjugated Poly(2,6-BODIPY-ethynylene)s Bearing Alkyl Side Chains. J. Polym. Sci., Part A: Polym. Chem. 2009, 47 (20), 5354-5366.

13. Donuru, V. R., Vegesna, G. K., Velayudham, S., Green, S., Liu, H. Y., Synthesis and Optical Properties of Red and Deep-Red Emissive Polymeric and Copolymeric BODIPY Dyes. Chemistry of Materials 2009, 21 (10), 2130-2138.

14. Yoshii, R., Nagai, A., Chujo, Y., Highly Near-infrared Photoluminescence from Aza-Borondipyrromethene-based Conjugated Polymers. J. Polym. Sci., Part A: Polym. Chem. 2010, 48 (23), 5348-5356.

15. Donuru, V. R., Zhu, S. L., Green, S., Liu, H. Y., Near-infrared Emissive BODIPY Polymeric and Copolymeric Dyes. Polymer 2010, 51 (23), 5359-5368.

16. Thivierge, C., Loudet, A., Burgess, K., Brilliant BODIPY-fluorene Copolymers with Dispersed Absorption and Emission Maxima. Macromolecules 2011, 44 (10), 4012-4015.

17. Brannon, J. H., Magde, D., Absolute Quantum Yield Determination by Thermal Blooming - Fluorescein. Journal of Physical Chemistry 1978, 82 (6), 705-709.

18. Velapoldi, R. A., Tonnesen, H. H., Corrected Emission Spectra and Quantum Yields for A Series of Fluorescent Compounds in The Visible Spectral Region. Journal of Fluorescence 2004, 14 (4), 465-472.

19. Xue, C. H., Donuru, V. R. R., Liu, H. Y., Facile, Versatile Prepolymerization and Postpolymerization Functionalization Approaches for Well-Defined Fluorescent Conjugated Fluorene-based Glycopolymers. Macromolecules 2006, 39 (17), 5747-5752. 
20. Xue, C. H., Luo, F. T., Liu, H. Y., Post-polymerization Functionalization Approach for Highly Water-soluble Well-defined Regioregular Head-To-Tall Glycopolythiophenes. Macromolecules 2007, 40 (19), 6863-6870.

21. Xue, C., Velayudham, S., Johnson, S., Saha, R., Smith, A., Brewer, W., Murthy, P., Bagley, S. T., Liu, H., Highly Water-soluble, Fluorescent, Conjugated Fluorene-based Glycopolymers with Poly(ethylene glycol)-tethered Spacers for Sensitive Detection of Escherichia coli. Chemistry-a European Journal 2009, 15 (10), 2289-2295.

22. Zhu, S. L., Zhang, J. T., Vegesna, G. K., Pandey, R., Luo, F. T., Green, S. A., Liu, H. Y., One-pot Efficient Synthesis of Dimeric, Trimeric, and Tetrameric BODIPY Dyes for Panchromatic Absorption. Chemical Communications 2011, 47 (12), 3508-3510.

23. Desgrosellier, J. S., Cheresh, D. A., Integrins in Cancer: Biological Implications and Therapeutic Opportunities. Nature Reviews Cancer 2010, 10 (12), 890-890. 


\section{Summary and Perspectives}

In this thesis, we chose BODIPY as the skeleton for further modifications and probe design because of its excellent chemical and photophysical properties and great potential it has shown for fluorescence sensing applications.

In the beginning of this research, our aims are to enhance the hydrophilicity of BODIPY dye and tune its main spectra wavelength to red, deep red and near infrared (NIR) regions. In order to achieve these goals, we first incorporated oligo(ethylene glycol) methyl ether moieties and our original branched oligo(ethylene glycol) methyl ether moieties to BODIPY dyes at different positions including meso-, 2-, 3-, 4-, 5-, 6positions. A series of highly water-soluble BODIPY dyes with different emission wavelengths and controllable fluorescence quantum yields have been synthesized based on this modification strategy. Then we have employed formal groups to BODIPY dyes at 2, and/or 6-positions to activate the condensation reactions at 1,3,5,7-positions, resulting in facile syntheses of 1,7-di-, 1,3,7-tri- and 1,3,5,7-tetra-styryl BODIPY dyes whose absorption and emission wavelengths were tuned to deep red, and NIR regions.

Based on the foundation of the strategies of hydrophilicity enhancement and wavelength tuning (chapter 3 and 4), we have designed and developed a series of BODIPY-based fluorescent probes for various analytes. We have designed three hybrid BODIPY-based fluorescent probes by incorporating indolium moiety at 6-position of BODIPY core for sensitive detection of cyanide ions which is a highly toxic substance and is very harmful to human health. These probes were fully characterized by UVvis/fluorescence spectroscopies, NMR and theoretical calculations. They showed highly sensitive and ratiometric responses (555 $\mathrm{nm}$ and $600 \mathrm{~nm}$ ) to cyanide ions in the concentration range of $(0-70 \mu \mathrm{M})$ of cyanide ions with detection limit down to $0.5 \mu \mathrm{M}$ of cyanide ions. In addition, one of these three probes is highly water-soluble and displayed potential application in the detection of cyanide ions in aqueous solution.

Later on, we moved to our target analytes to important ion species in biological system like Zn (II) ions and protons (lysosomal pH). In Chapter 6, we have designed and synthesized three highly water-soluble BODIPY fluorescent probes (6-A, 6-B and 6-C) by attaching $\mathrm{Zn}$ (II) chelator, di-2-picolylamine moiety (DPA), to 2-formyal, 2,6- 
diformyal and 2,6-diformyal-1,7-distyryl-BODIPY dyes at 2, and 2,6- positions. All three probes exhibited turn-on fluorescence responses to the increased $\mathrm{Zn}$ (II) concentrations in aqueous solutions because chelation of $\mathrm{Zn}$ (II) ions to the receptors significantly reduced the photoinduced electron transfer (PET) effect which occur from electron rich DPA moieties to the BODIPY core and cause fluorescence quenching. Probes 6-A and 6-B showed excellent sensitivities and selectivities to $\mathrm{Zn}$ (II) ions with more than 2-fold enhancement of fluorescence intensity at $520 \mathrm{~nm}$ and $527 \mathrm{~nm}$, respectively. Probe 6-C absorbs and emits longer wavelengths and it has ratiometric responses to $\mathrm{Zn}$ (II) ions with fluorescence increase at $521 \mathrm{~nm}$ and decrease at $661 \mathrm{~nm}$ upon addition of $\mathrm{Zn}$ (II) ions. Probe 6-C has been successfully applied in detection of intracellular Znic (II) in human breast cancer cells.

In order to detect intracellular $\mathrm{pH}$ (especially lysosomal $\mathrm{pH}$ ) by using fluorescent probe, we have incorporated tertiaryaimne-contained piperazine moieties onto BODIPY dyes at 3,5-position as parts of distyryl-conjugation to manipulate the fluorescent responses of the probes to $\mathrm{pH}$ via modulation of intramolecular charge transfer (ICT) effect of piperazine moieties to BODIPY dyes at different $\mathrm{pH}$, yielding three NIR emissive (>700 $\mathrm{nm}$ ) probes 7-A, 7-B and 7-C. Probes 7-A and 7-B are hydrophobic with low water solubility while probe 7-C is highly water-soluble because it contains most number of hydrophilic chains (oligo(ethylene glycol) methyl ether residues). All three probes are $\mathrm{pH}$-sensitive and showed high fluorescence in acidic condition and extreme low fluorescence in neutral and basic conditions. All three probes have been applied to live cell fluorescence imaging. The bright NIR fluorescence in lysosome areas indicated probes are cell permeable and sensitively turn-on responsive to lysosomal $\mathrm{pH}$, which demonstrates the great potential for the applications of non-invasive lysosomal labeling and intracellular $\mathrm{pH}$ monitoring by using these probes.

At the end, we aimed our analyte to cancer cells. In order to enhance the sensitivity by using multivalent interactions and to tune the emission wavelength to NIR region, we have synthesized polymeric BODIPY dye (polymer 8-B) bearing numbers of cancer homing peptide (cRGD) by Suzuki cross coupling reaction of water-soluble, 2,6-diiodo3,5-distyryl BODIPY monomer with benzene-1,4-diboronic acid. Polymer 8-B is highly water-soluble and it absorbs and emits light at 689 and $712 \mathrm{~nm}$ with excellent 
photostability under excitation. Incubating of polymer 8-B with normal cells showed almost no fluorescence while bright NIR fluorescence was observed in cancer cells with same incubation condition. The high specificity and sensitivity of polymer 8-B to tumor cells is due to the specific cooperative bindings effect of cRGD peptide residues to $\alpha_{v} \beta_{3}$ integrins which is over expressed on the surface of cancer cells. This specificity was also confirmed by the comparison of live cell fluorescence imaging results using polymer 8-B and its analog polymer 8-A that does not contain cRGD peptide reside, in which polymer 8-A did not display fluorescence with cancer cells while polymer 8-B did.

Although we have developed several BODIPY-based fluorescent probes for various types of analytes and they all have showed excited sensing results and have been successfully applied to in vitro fluorescence sensing and imaging in living cells, there are still plenty of space and challenges about research on the BODIPY-based fluorescent probes. So, in perspective, it might useful to apply BODIPY dyes to the photodynamic therapy applications. Because halogenated BODIPY dyes are considered as a type of good sensitizers that generates high yield of singlet oxygen, modifications of this type of BODIPY dyes with other functional bio-receptors like cancer homing peptides could afford novel highly specific photosensitizer to realize the "target-see-and-treat" therapy. It also might useful to incorporate single or multiple cyclodextrin moieties on BODIPY dye to increase the aqueous solubility of the dye. These cyclodextrin moieties also can function as anchor groups so that adamantine-modified receptors, functional moieties and fluorophores can be easily conjugated with BODIPY dyes through self-assembly via host-guest interactions in aqueous solutions to form multifunctional fluorescent probes or energy transfer cassette. 


\section{Appendix A Supporting Information for Chapter 3}

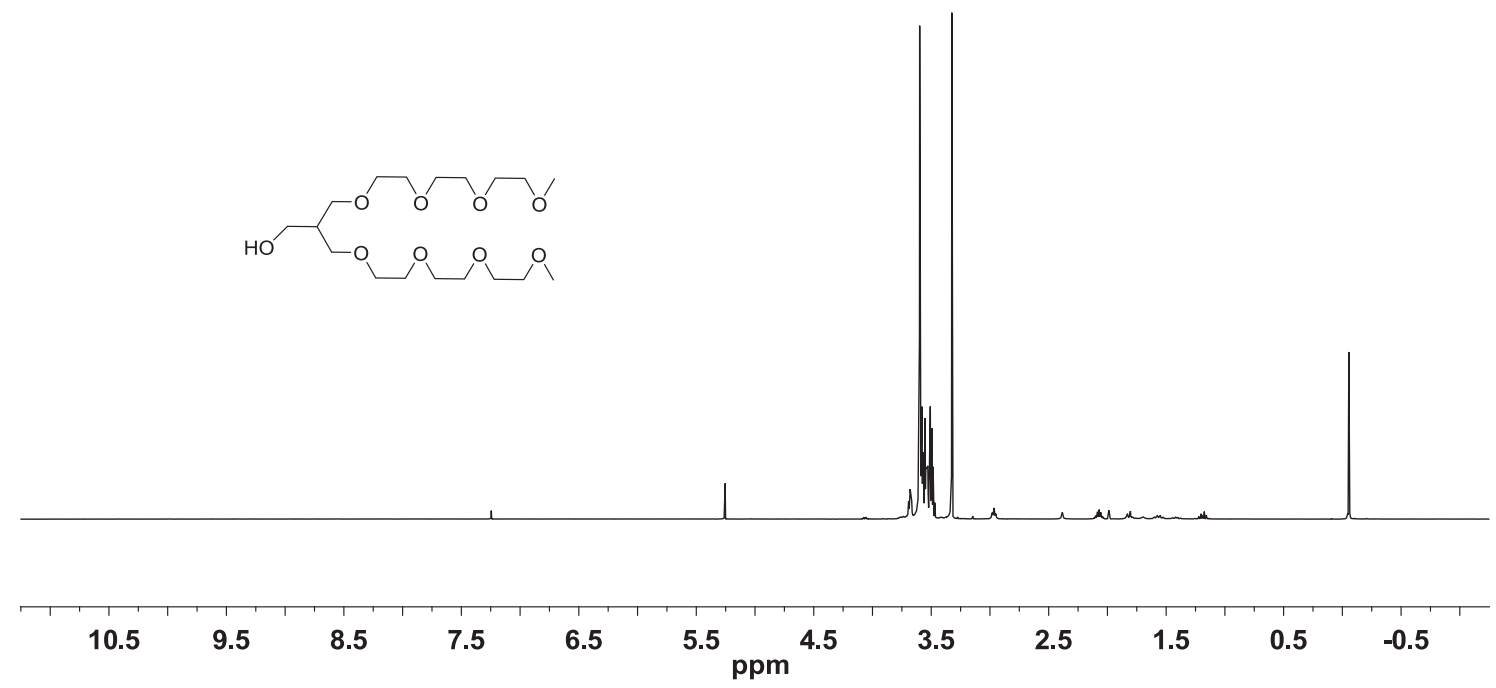

Figure A.1. $\quad{ }^{1} \mathrm{H}$ NMR spectrum of compound $\mathbf{3 . 4}$ in $\mathrm{CDCl}_{3}$ solution.

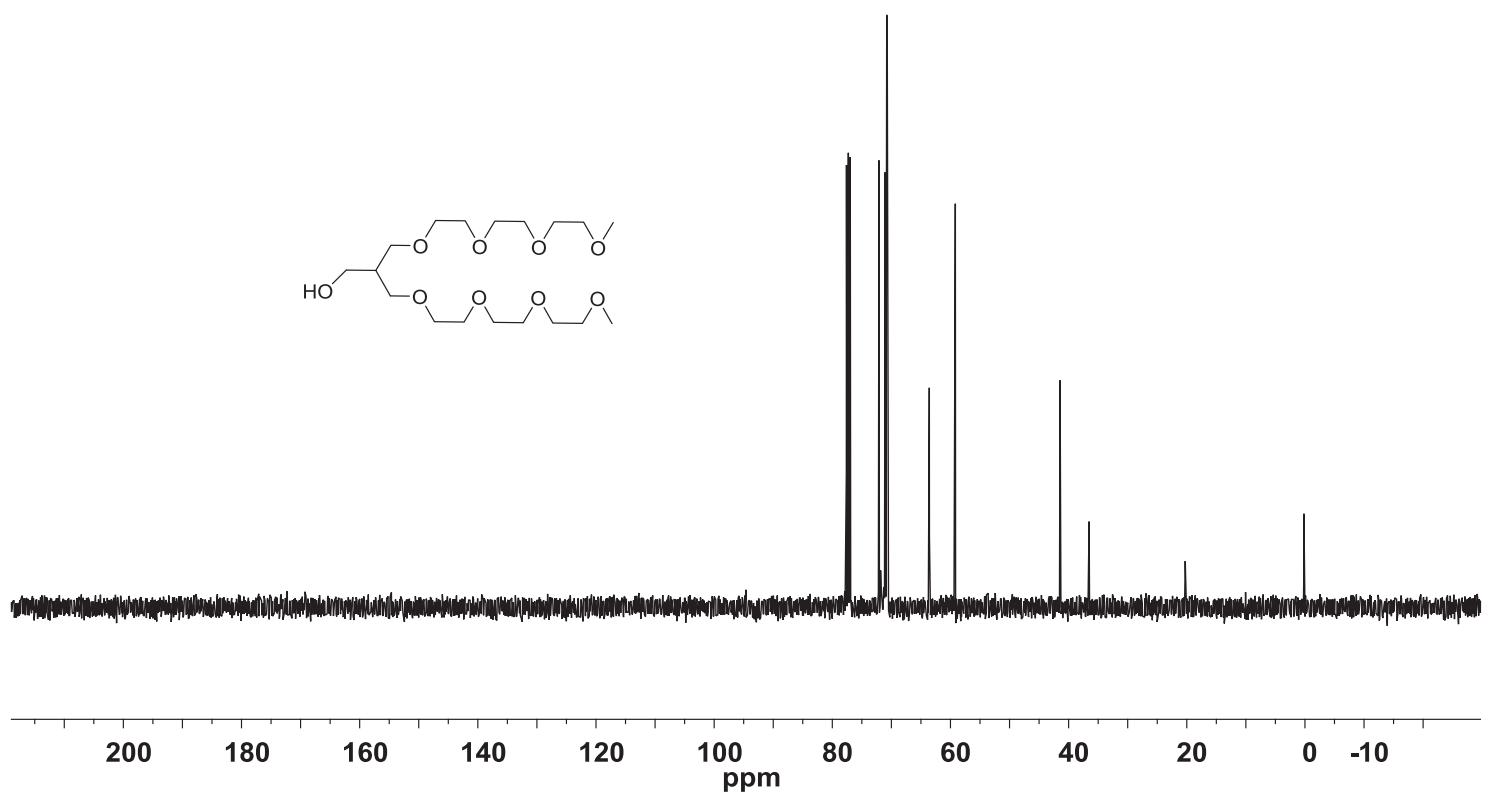

Figure A.2. ${ }^{13} \mathrm{C}$ NMR spectrum of compound 3.4 in $\mathrm{CDCl}_{3}$ solution. 


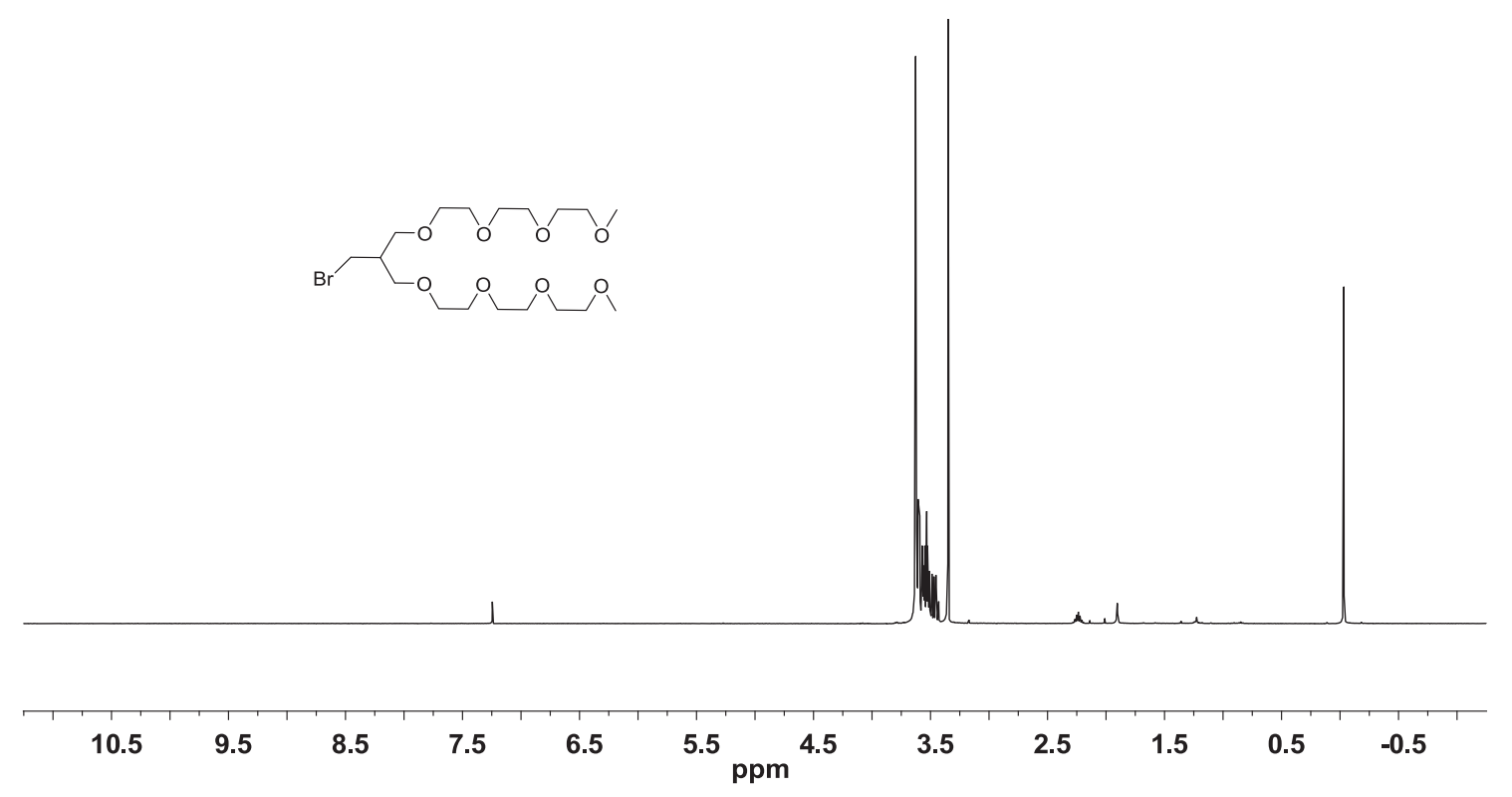

Figure A.3. $\quad{ }^{1} \mathrm{H}$ NMR spectrum of compound $\mathbf{3 . 5}$ in $\mathrm{CDCl}_{3}$ solution.

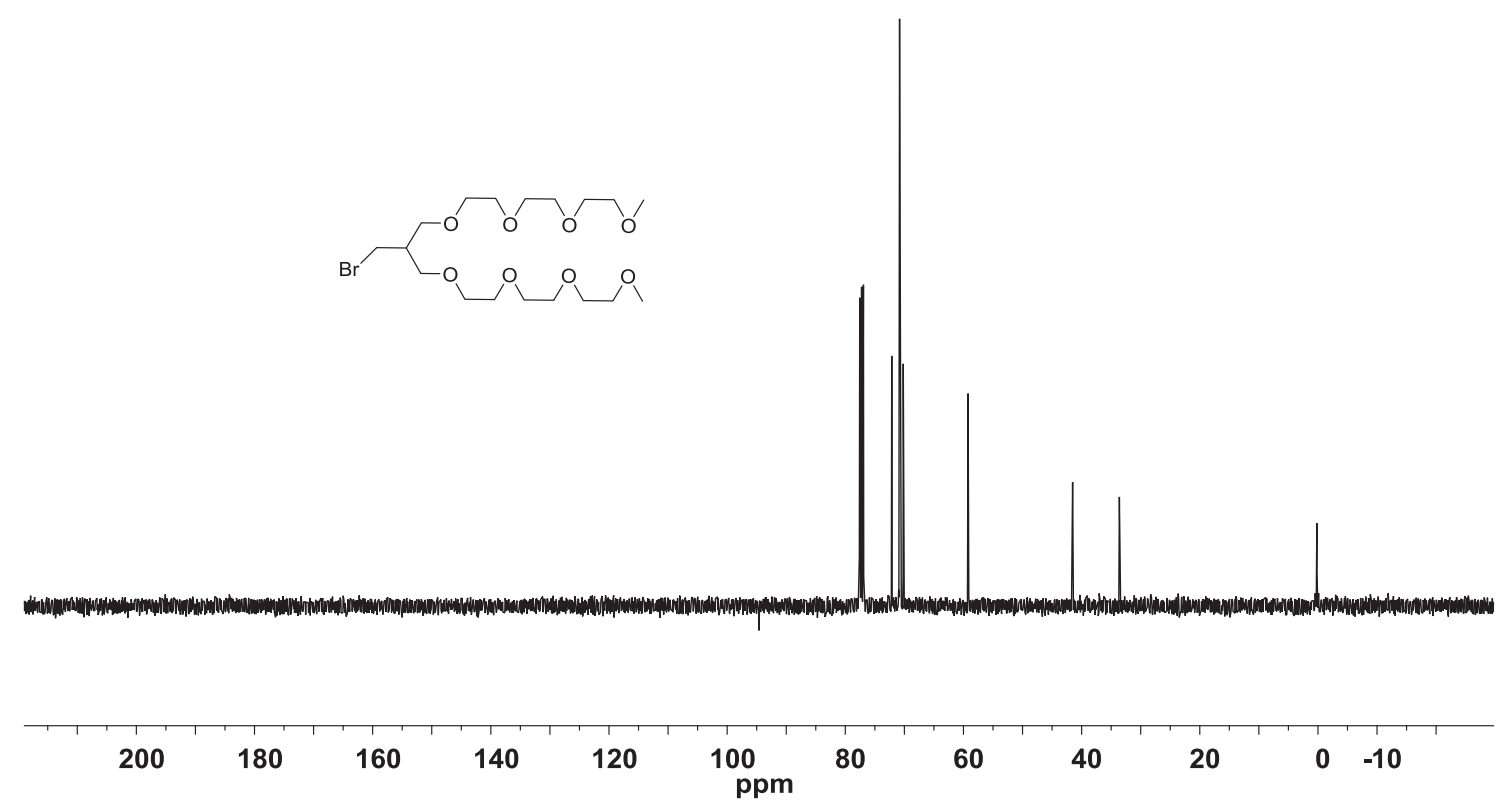

Figure A.4. ${ }^{13} \mathrm{C}$ NMR spectrum of compound 3.5 in $\mathrm{CDCl}_{3}$ solution. 

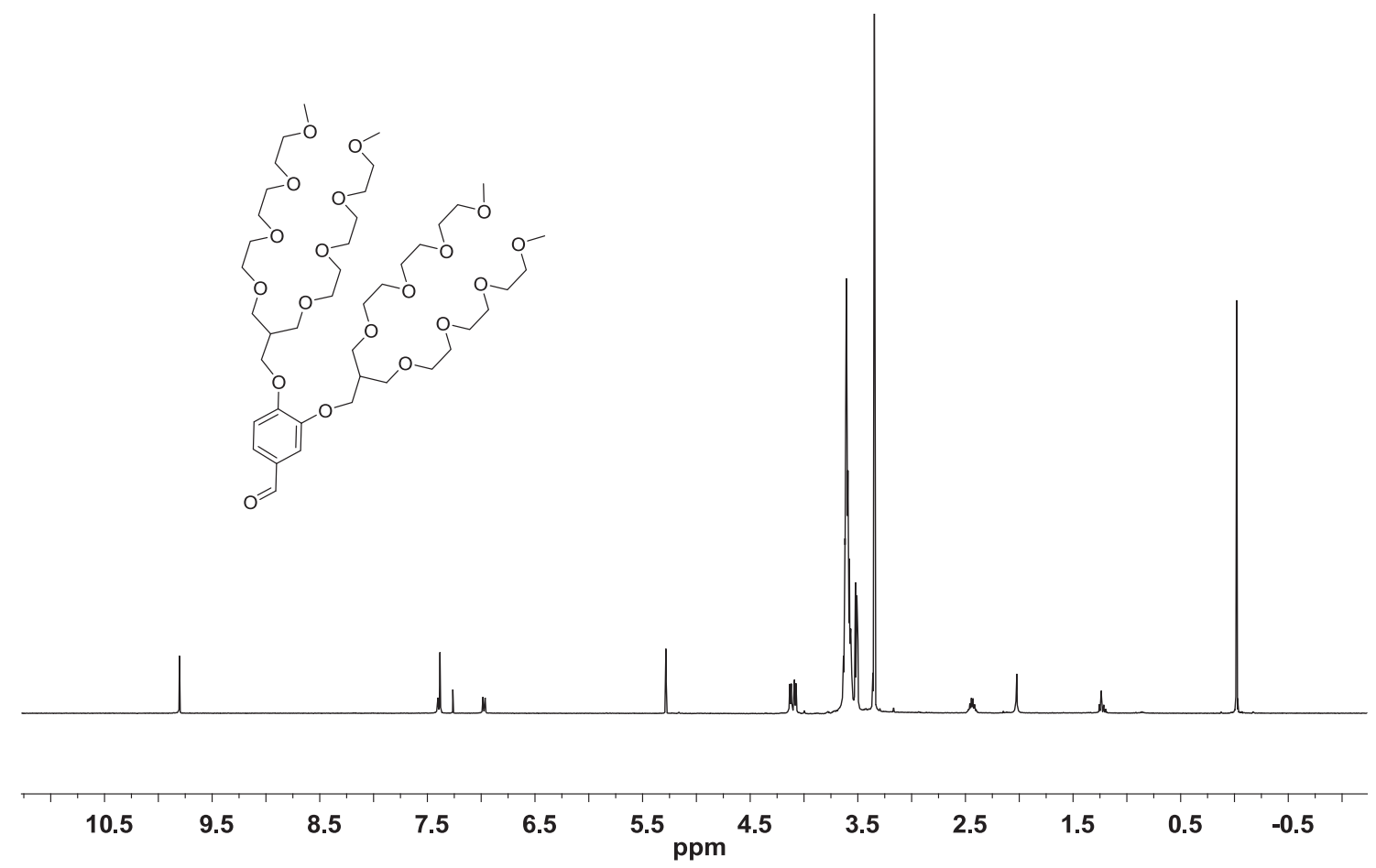

Figure A.5. $\quad{ }^{1} \mathrm{H}$ NMR spectrum of compound 3.7 in $\mathrm{CDCl}_{3}$ solution.

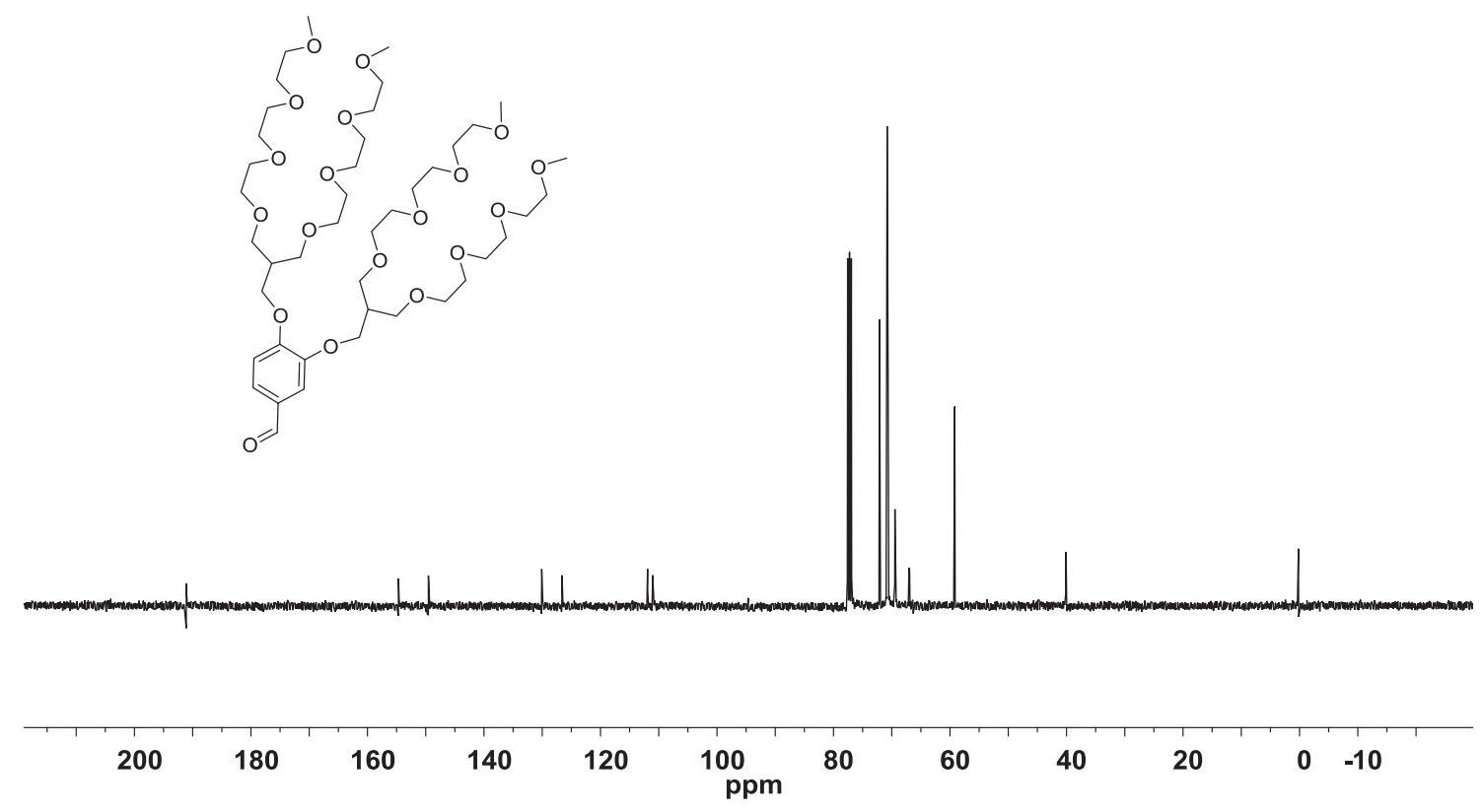

Figure A.6. ${ }^{13} \mathrm{C}$ NMR spectrum of compound 3.7 in $\mathrm{CDCl}_{3}$ solution. 


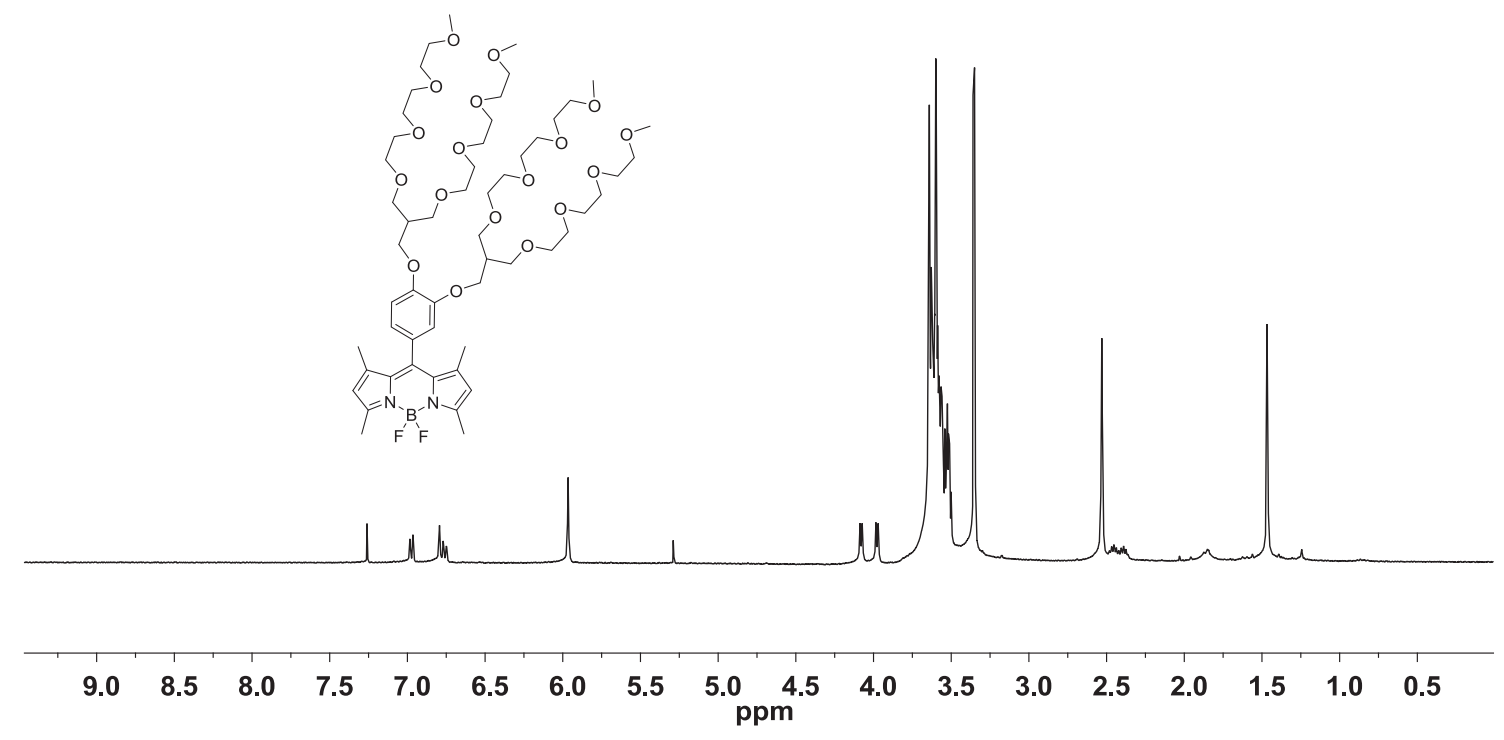

Figure A.7. $\quad{ }^{1} \mathrm{H}$ NMR spectrum of BODIPY dye 3-A in $\mathrm{CDCl}_{3}$ solution.

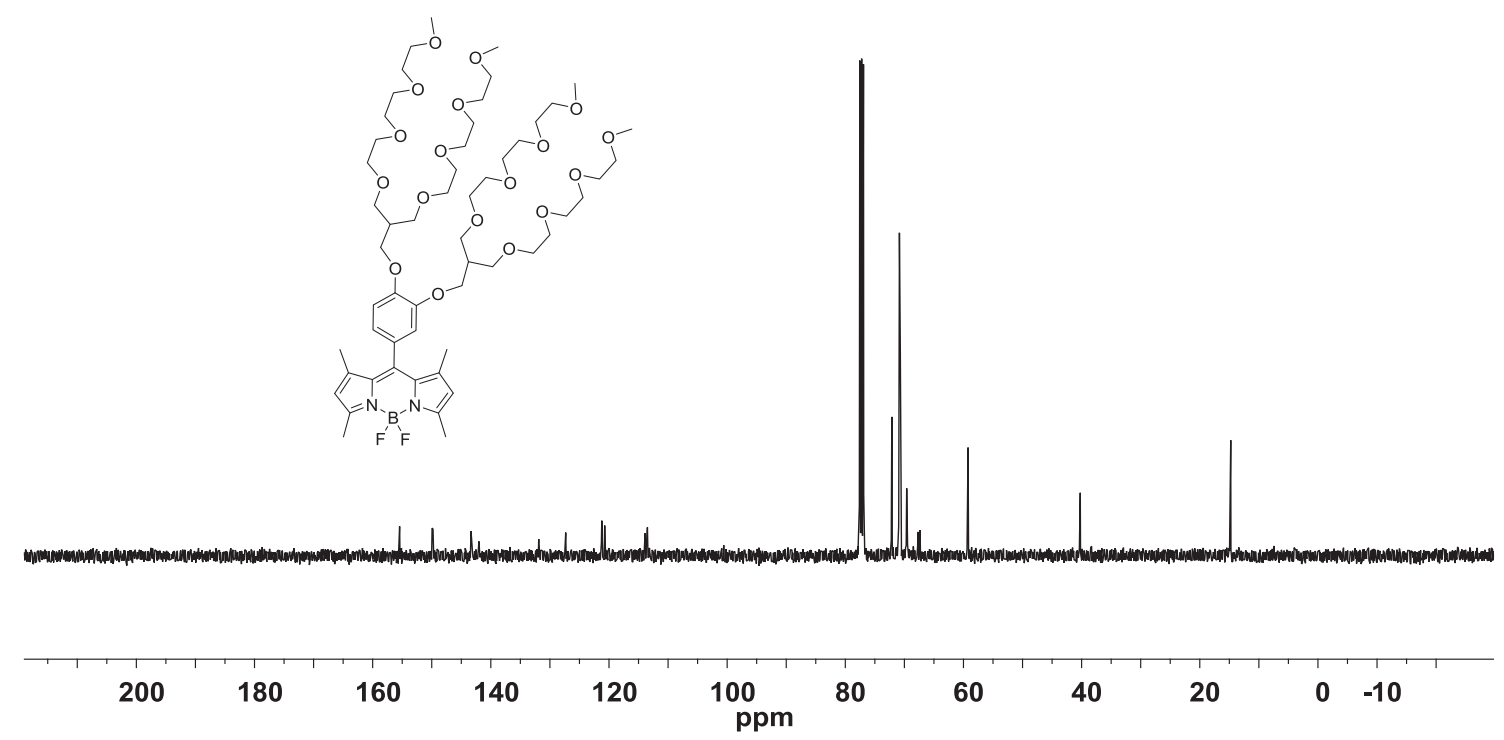

Figure A.8. ${ }^{13} \mathrm{C}$ NMR spectrum of BODIPY dye 3-A in $\mathrm{CDCl}_{3}$ solution. 

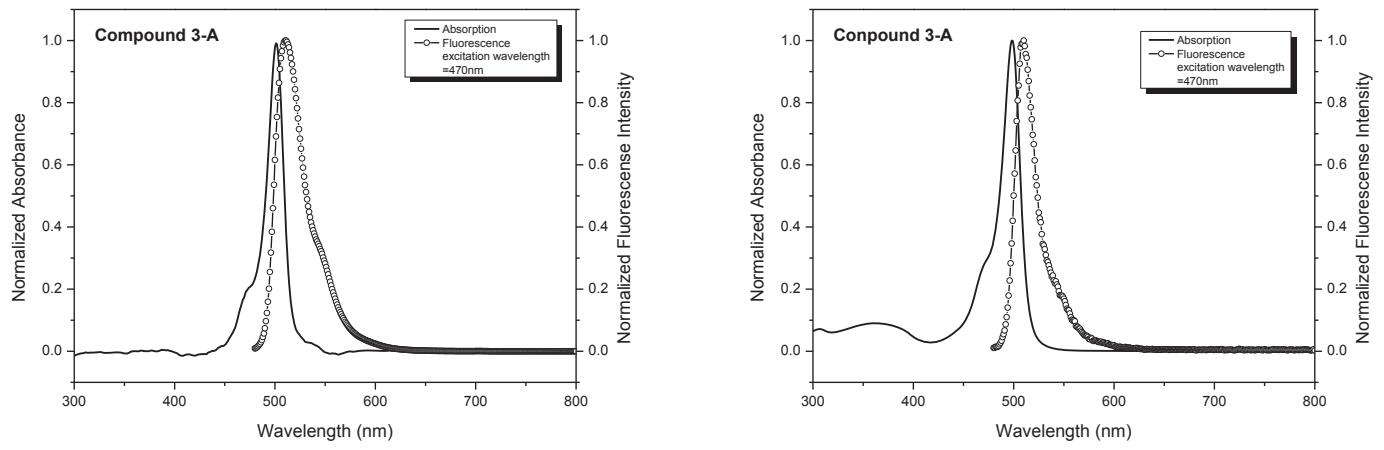

Figure A.9. Absorption and fluorescence spectra of BODIPY dye 3-A in dichloromethane solution (left) and in 0.5 M PBS buffer $(\mathrm{pH}=7.4)$ aqueous solution (right) ), $\lambda_{\mathrm{ex}}=470 \mathrm{~nm}$.

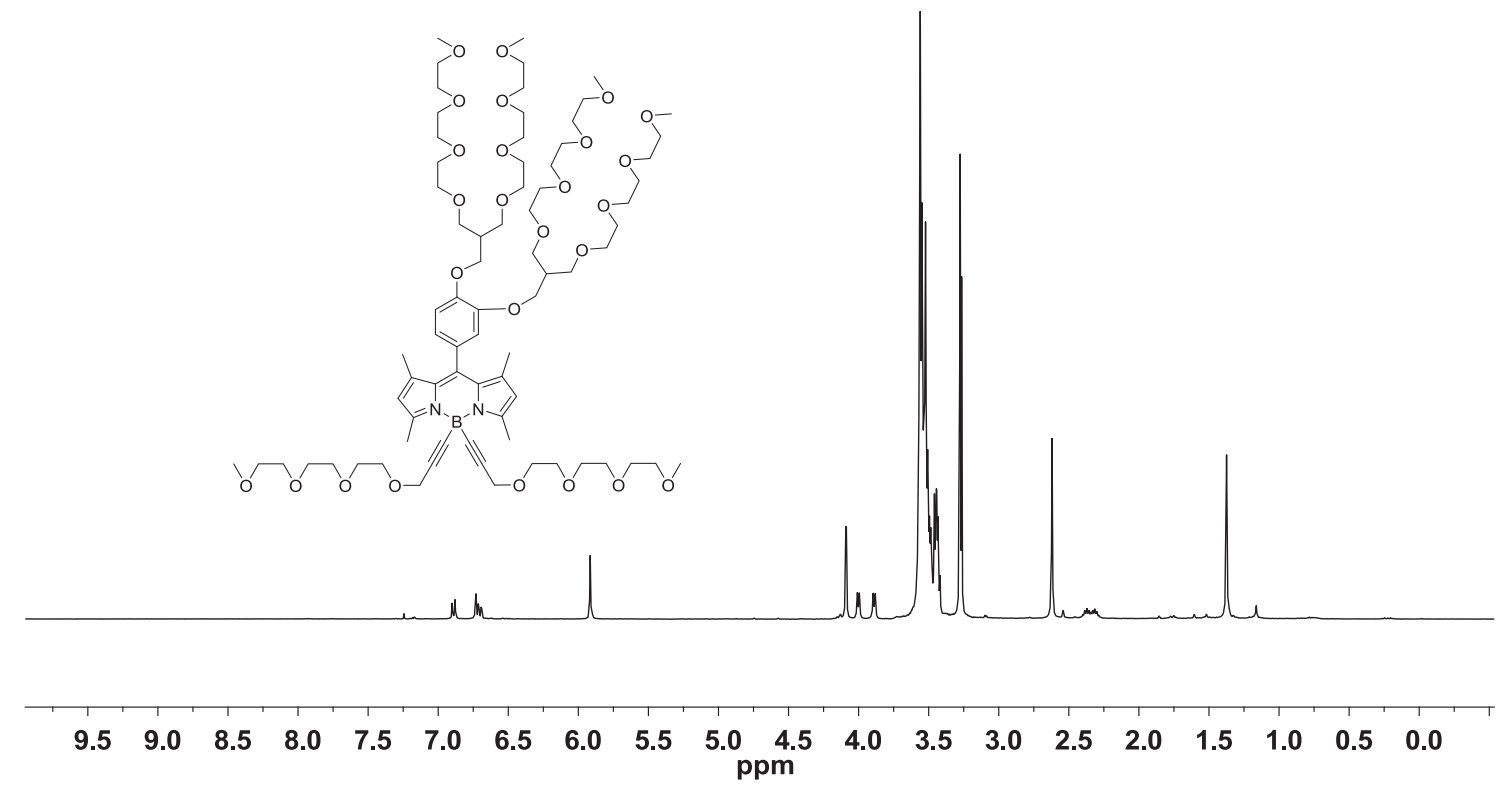

Figure A.10. ${ }^{1} \mathrm{H}$ NMR spectrum of BODIPY dye 3-B in $\mathrm{CDCl}_{3}$ solution. 


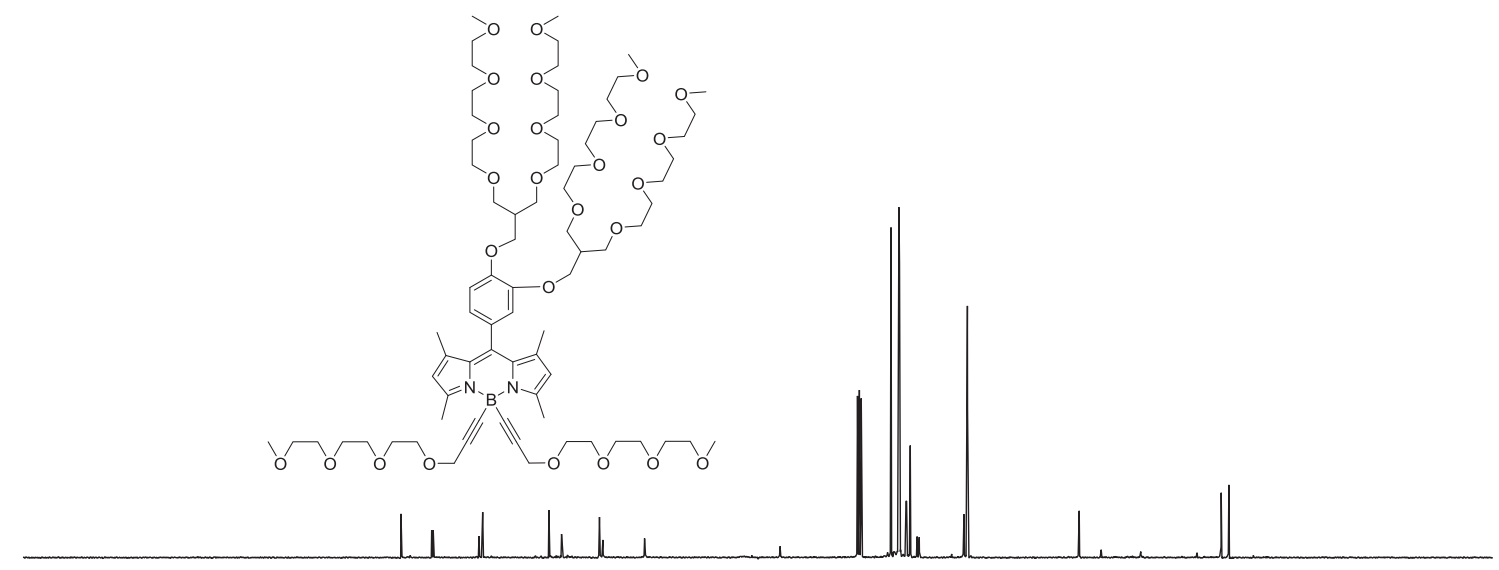

200

$180 \quad 160$

$140 \quad 120 \quad 100$

ppm

Figure A.11. ${ }^{13} \mathrm{C}$ NMR spectrum of BODIPY dye 3-B in $\mathrm{CDCl}_{3}$ solution.
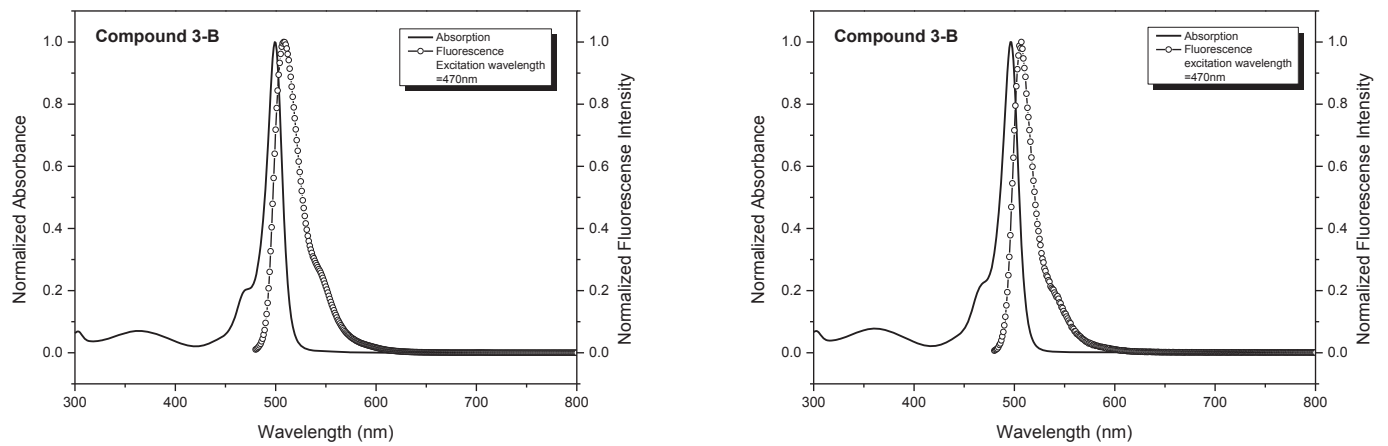

Figure A.12. Absorption and fluorescence spectra of BODIPY dye 3-B in dichloromethane solution (left) and in 0.5 M PBS buffer $(\mathrm{pH}=7.4)$ aqueous solution (right) ), $\lambda_{\mathrm{ex}}=470 \mathrm{~nm}$. 

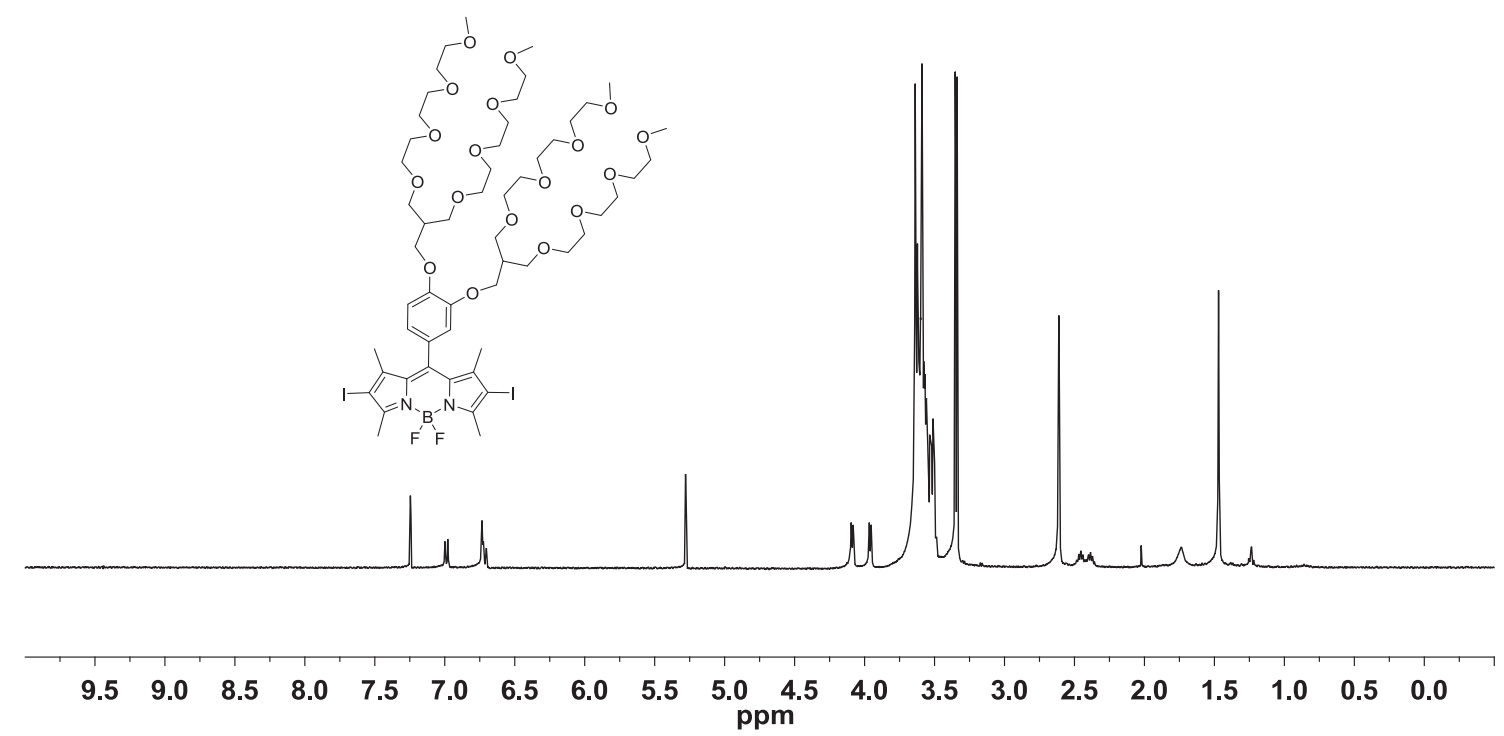

Figure A.13. ${ }^{1} \mathrm{H}$ NMR spectrum of BODIPY dye 3-C in $\mathrm{CDCl}_{3}$ solution.

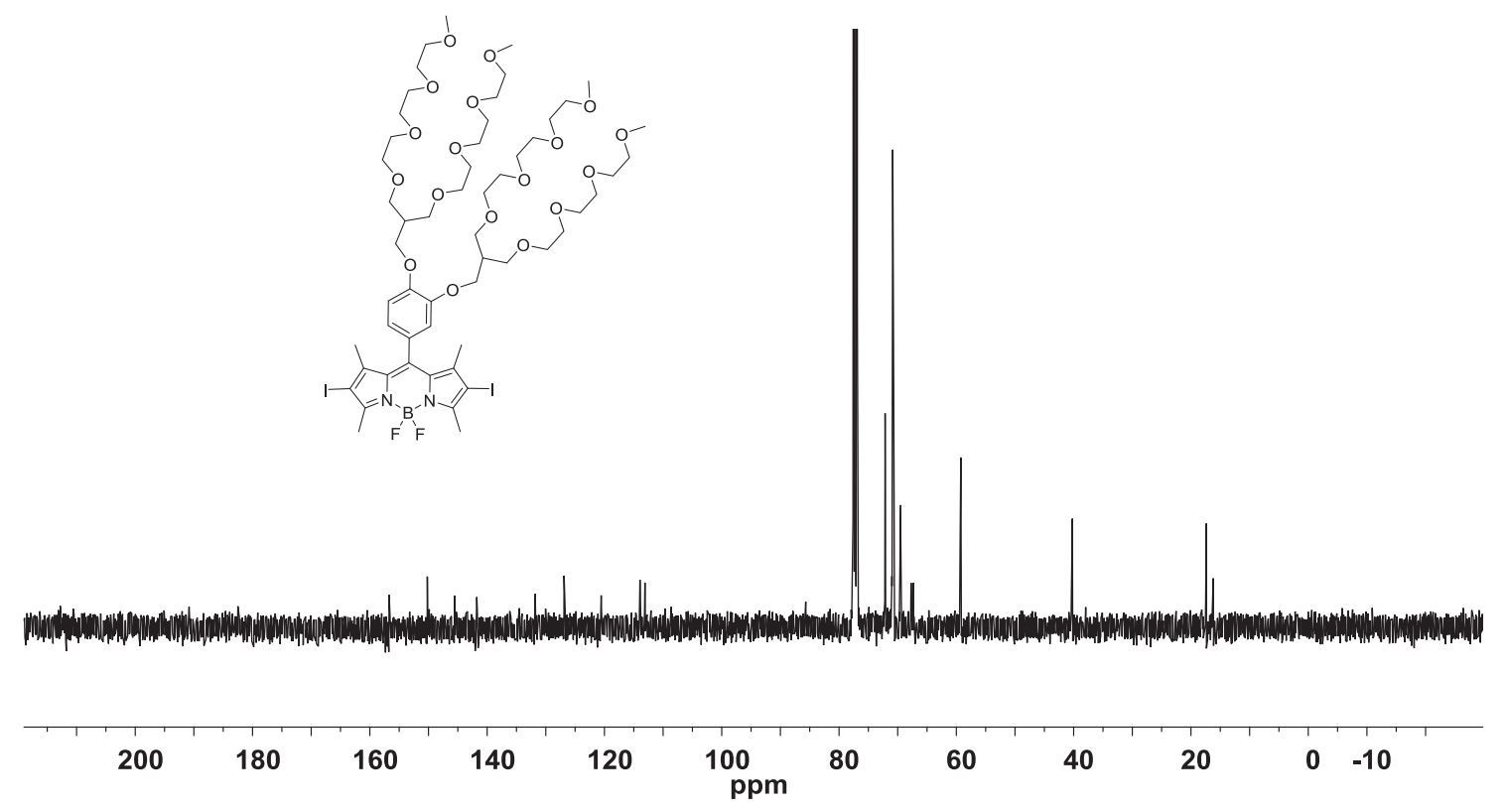

Figure A.14. ${ }^{13} \mathrm{C}$ NMR spectrum of BODIPY dye 3-C in $\mathrm{CDCl}_{3}$ solution. 

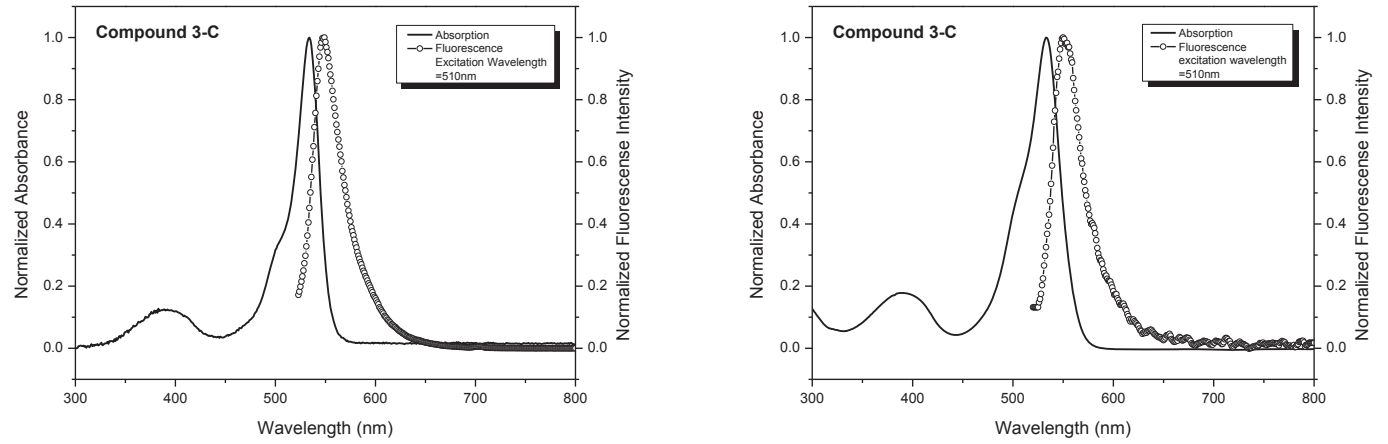

Figure A.15. Absorption and fluorescence spectra of BODIPY dye 3-C in dichloromethane solution (left) and in 0.5 M PBS buffer $(\mathrm{pH}=7.4)$ aqueous solution (right) ), $\lambda_{\mathrm{ex}}=470 \mathrm{~nm}$.
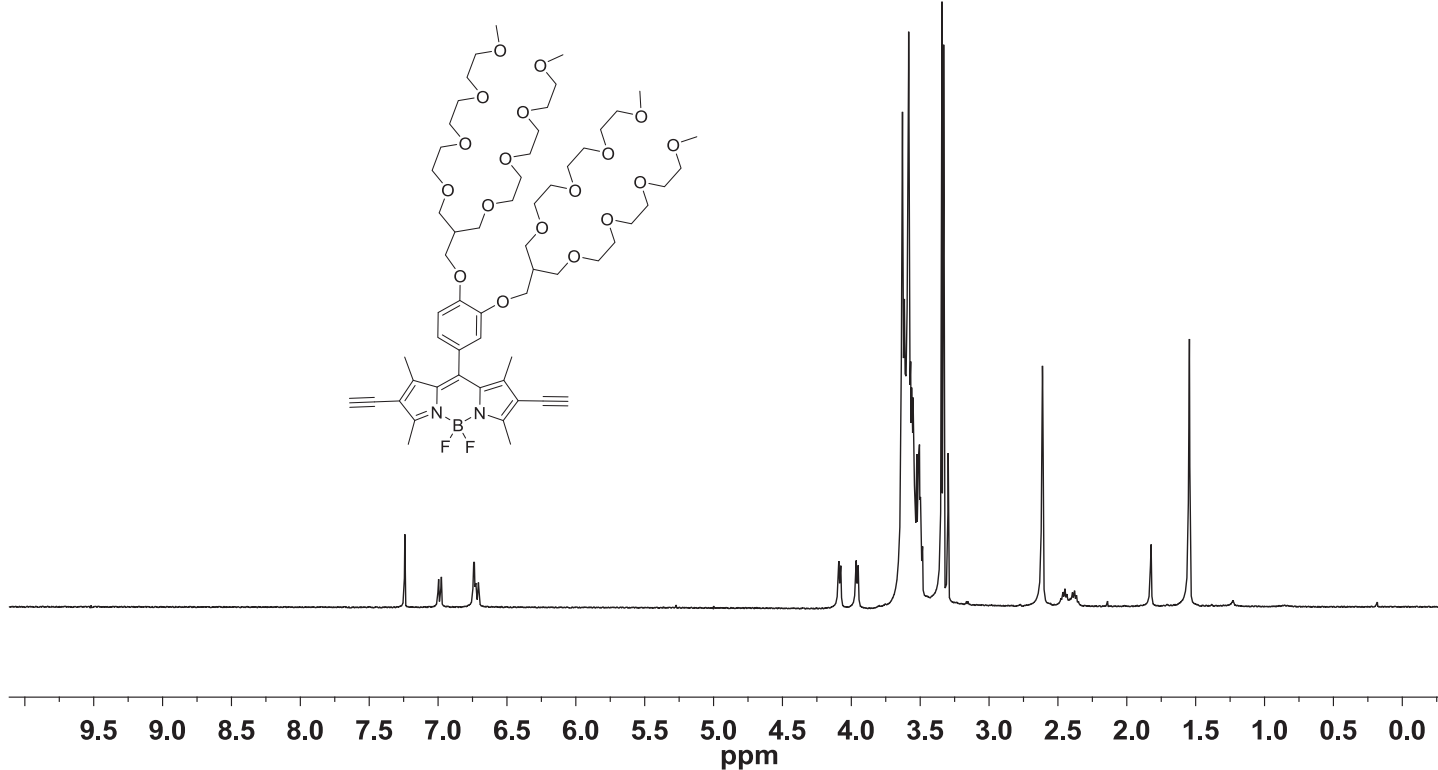

Figure A.16. ${ }^{1} \mathrm{H}$ NMR spectrum of BODIPY dye 3-D in $\mathrm{CDCl}_{3}$ solution. 

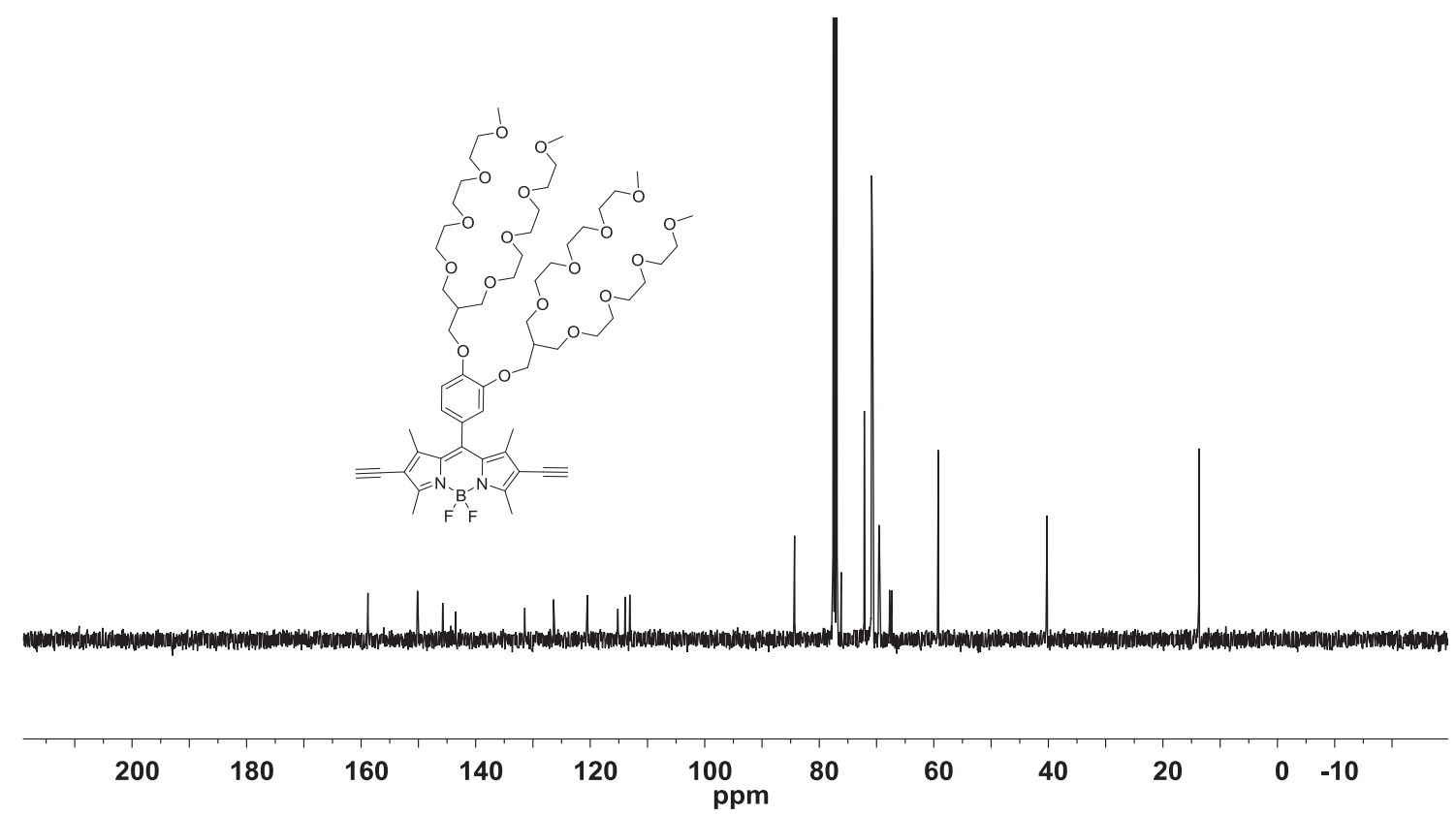

Figure A.17. ${ }^{13} \mathrm{C}$ NMR spectrum of BODIPY dye 3-D in $\mathrm{CDCl}_{3}$ solution.
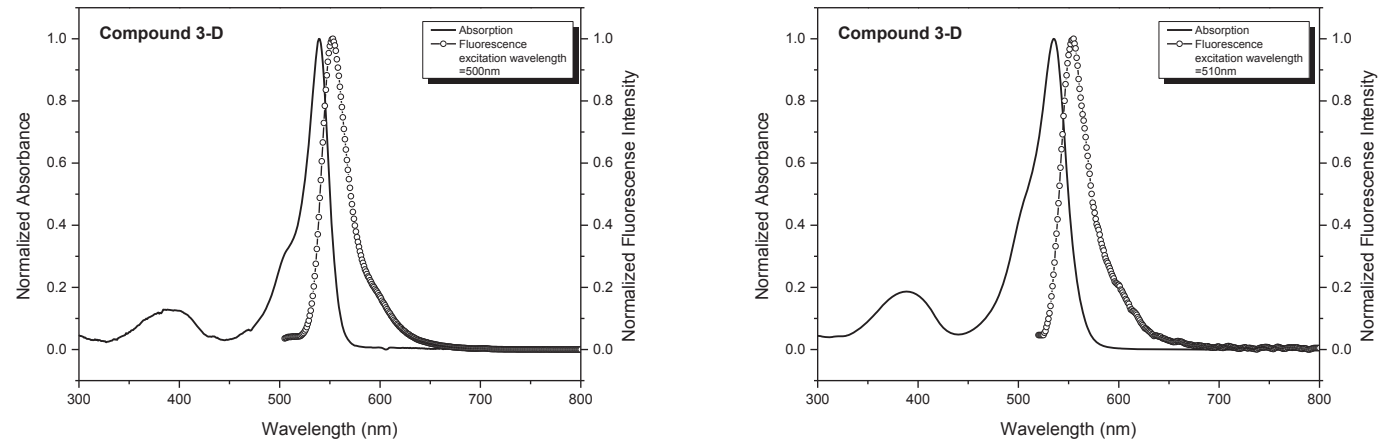

Figure A.18. Absorption and fluorescence spectra of BODIPY dye 3-D in dichloromethane solution (left) and in 0.5 M PBS buffer ( $\mathrm{pH}=7.4)$ aqueous solution (right) ), $\lambda_{\mathrm{ex}}=470 \mathrm{~nm}$. 


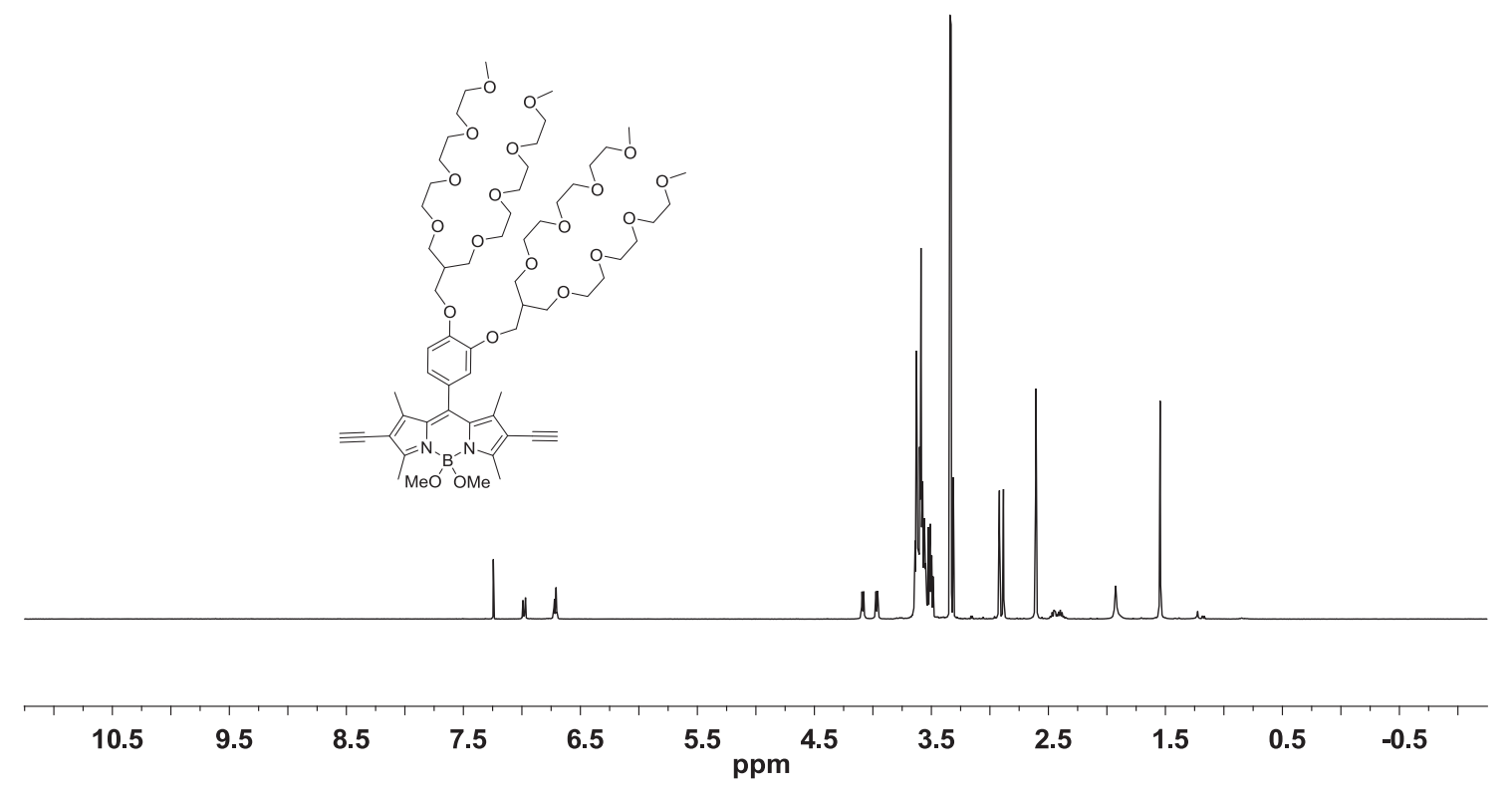

Figure A.19. ${ }^{1} \mathrm{H}$ NMR spectrum of BODIPY dye 3-E in $\mathrm{CDCl}_{3}$ solution.

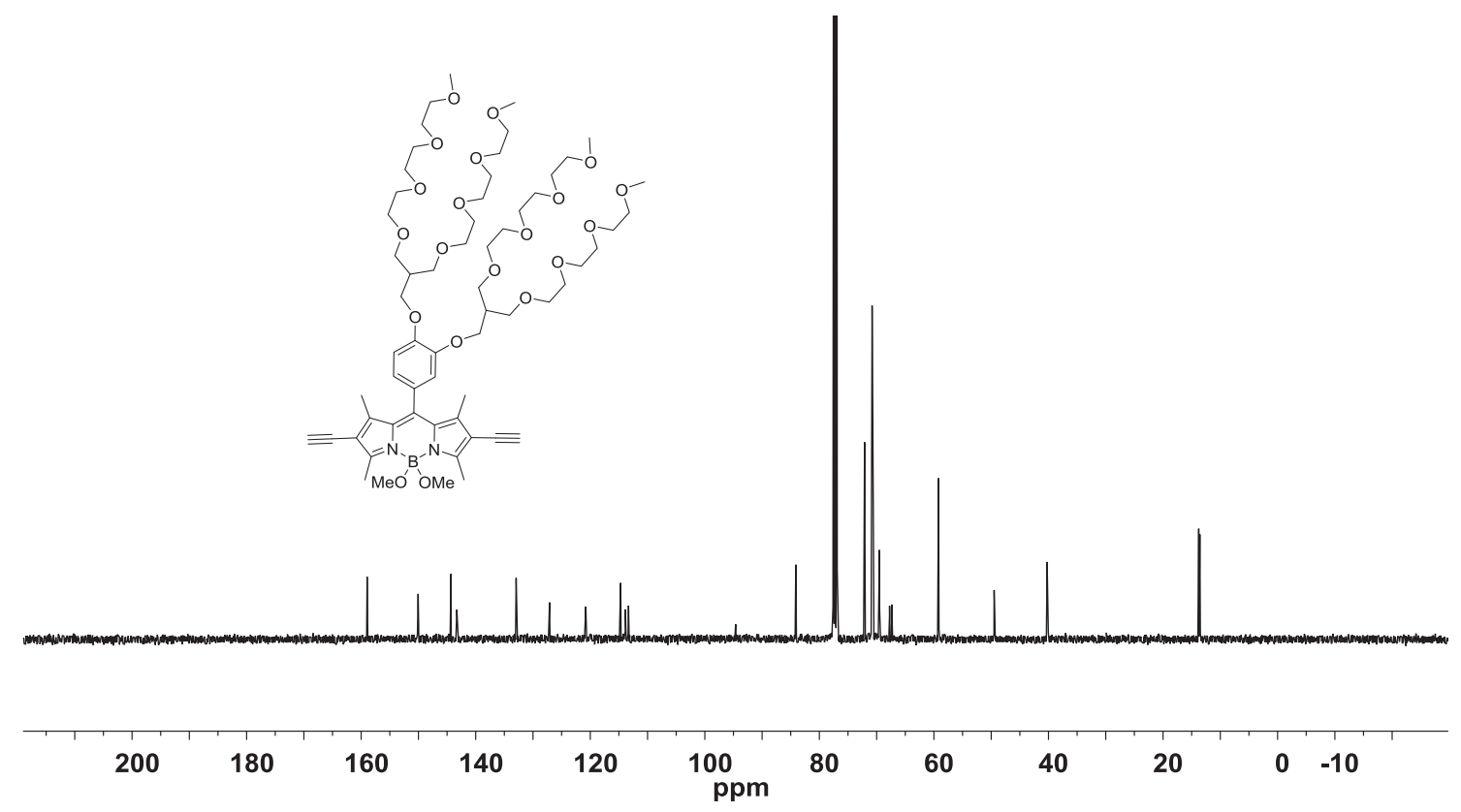

Figure A.20. ${ }^{13} \mathrm{C}$ NMR spectrum of BODIPY dye 3-E in $\mathrm{CDCl}_{3}$ solution. 

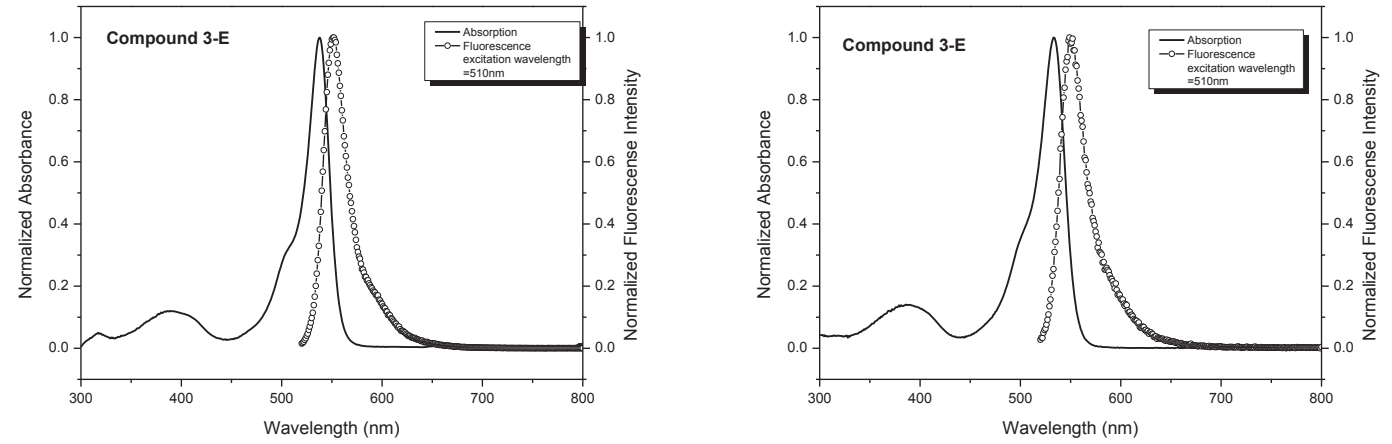

Figure A.21. Absorption and fluorescence spectra of BODIPY dye 3-E in dichloromethane solution (left) and in 0.5 M PBS buffer $(\mathrm{pH}=7.4)$ aqueous solution (right) ), $\lambda_{\mathrm{ex}}=470 \mathrm{~nm}$.

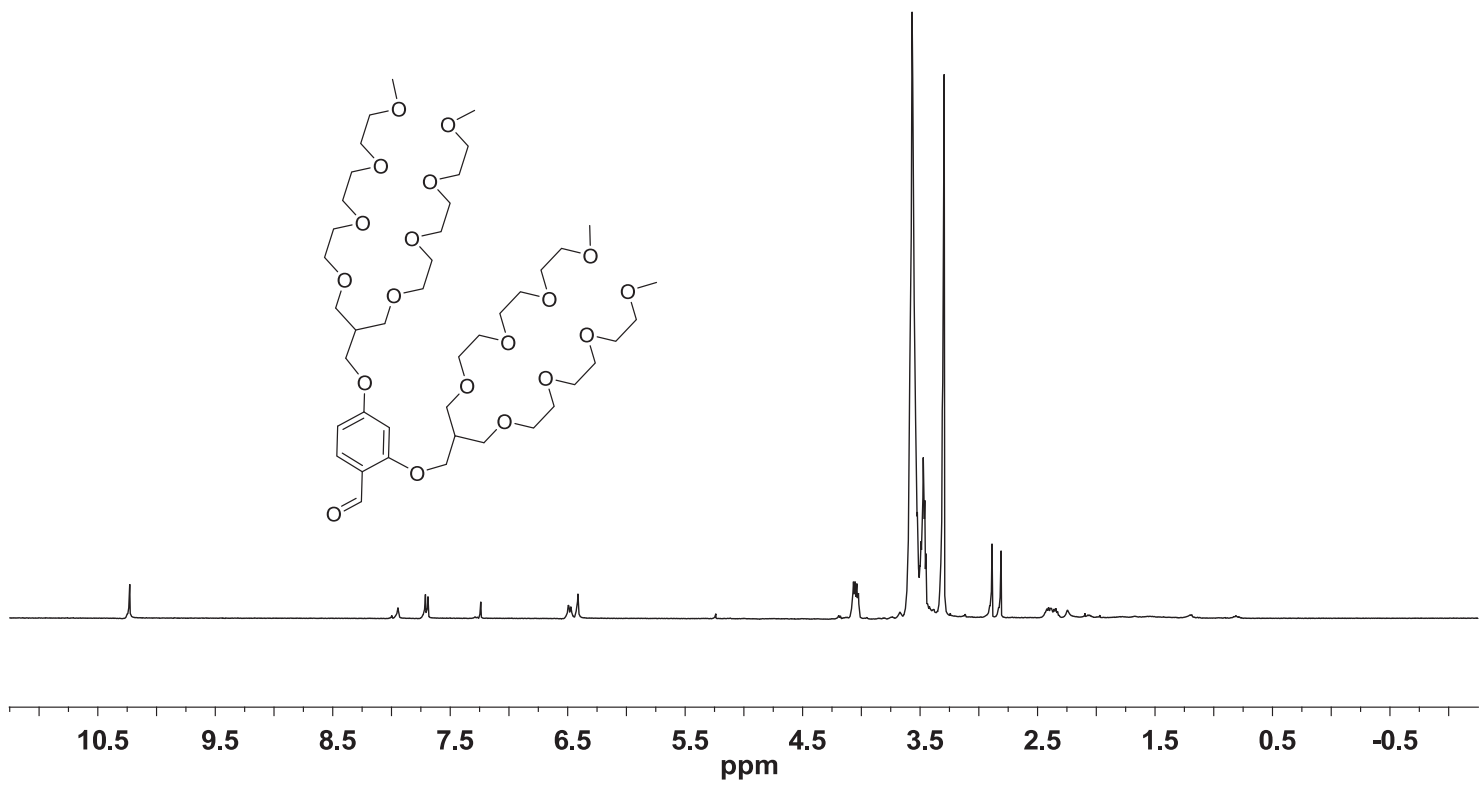

Figure A.22. ${ }^{1} \mathrm{H}$ NMR spectrum of compound $\mathbf{3 . 1 0}$ in $\mathrm{CDCl}_{3}$ solution. 


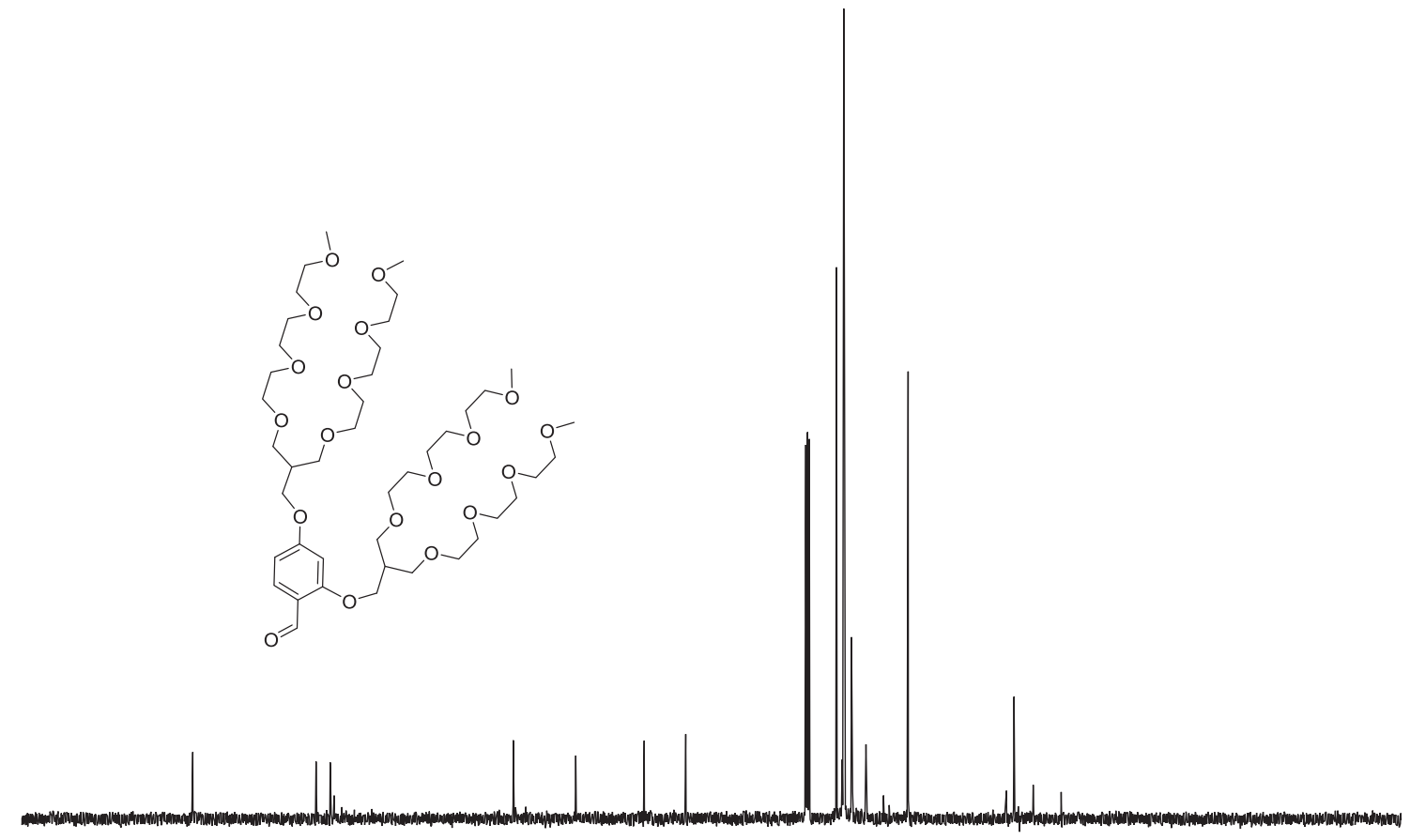

$\begin{array}{llllllllllll}200 & 180 & 160 & 140 & 120 & \underset{\mathrm{ppm}}{100} & 80 & 60 & 40 & 20 & 0 & -10\end{array}$

Figure A.23. ${ }^{13} \mathrm{C} \mathrm{NMR}$ spectrum of compound $\mathbf{3 . 1 0}$ in $\mathrm{CDCl}_{3}$ solution.

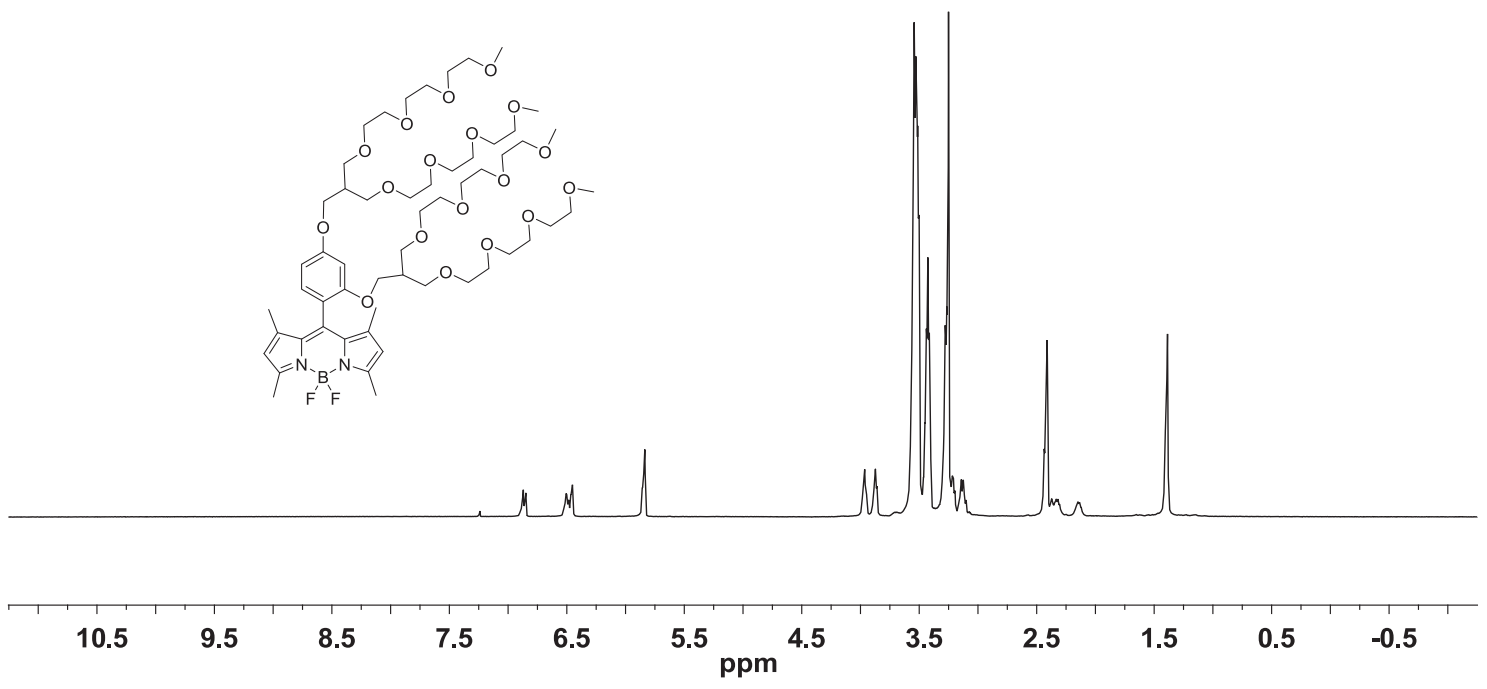

Figure A.24. ${ }^{1} \mathrm{H}$ NMR spectrum of BODIPY dye 3-F in $\mathrm{CDCl}_{3}$ solution. 


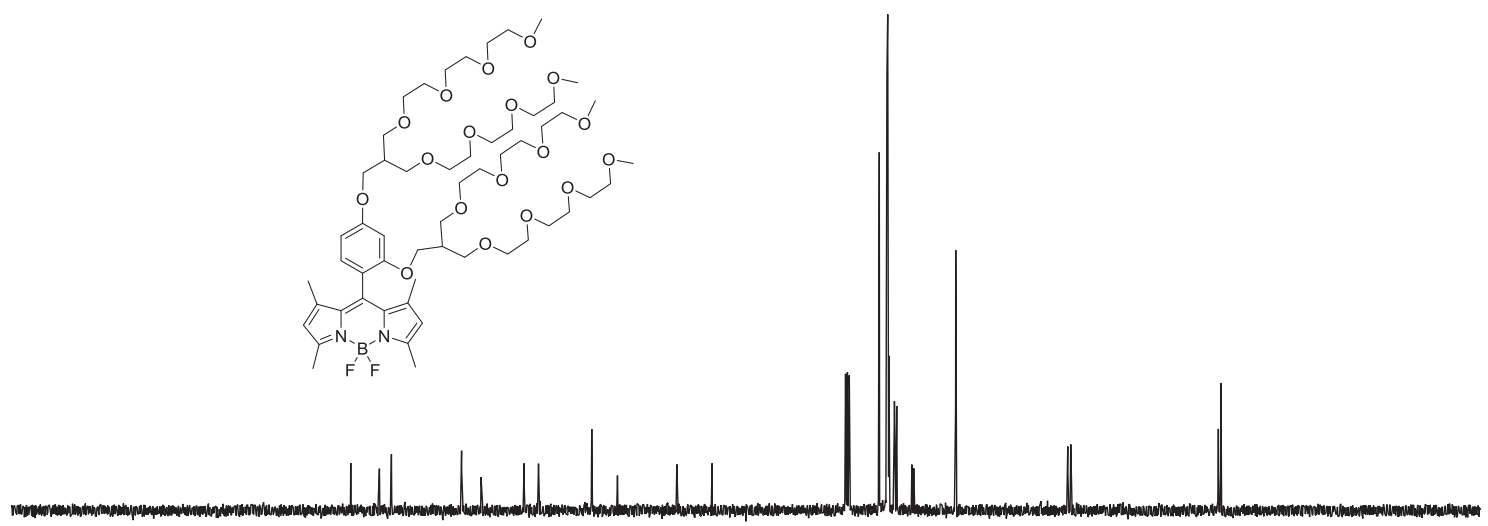

200

180

160

140

120100

ppm

Figure A.25. ${ }^{13} \mathrm{C}$ NMR spectrum of BODIPY dye 3-F in $\mathrm{CDCl}_{3}$ solution.
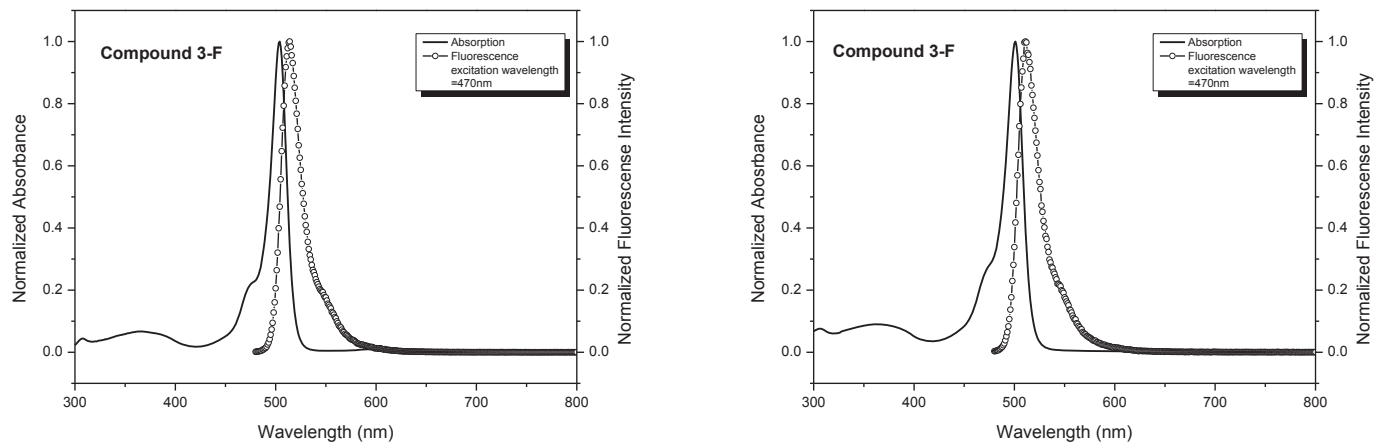

Figure A.26. Absorption and fluorescence spectra of BODIPY dye 3-F in dichloromethane solution (left) and in $0.5 \mathrm{M}$ PBS buffer $(\mathrm{pH}=7.4)$ aqueous solution (right) ), $\lambda_{\mathrm{ex}}=470 \mathrm{~nm}$. 


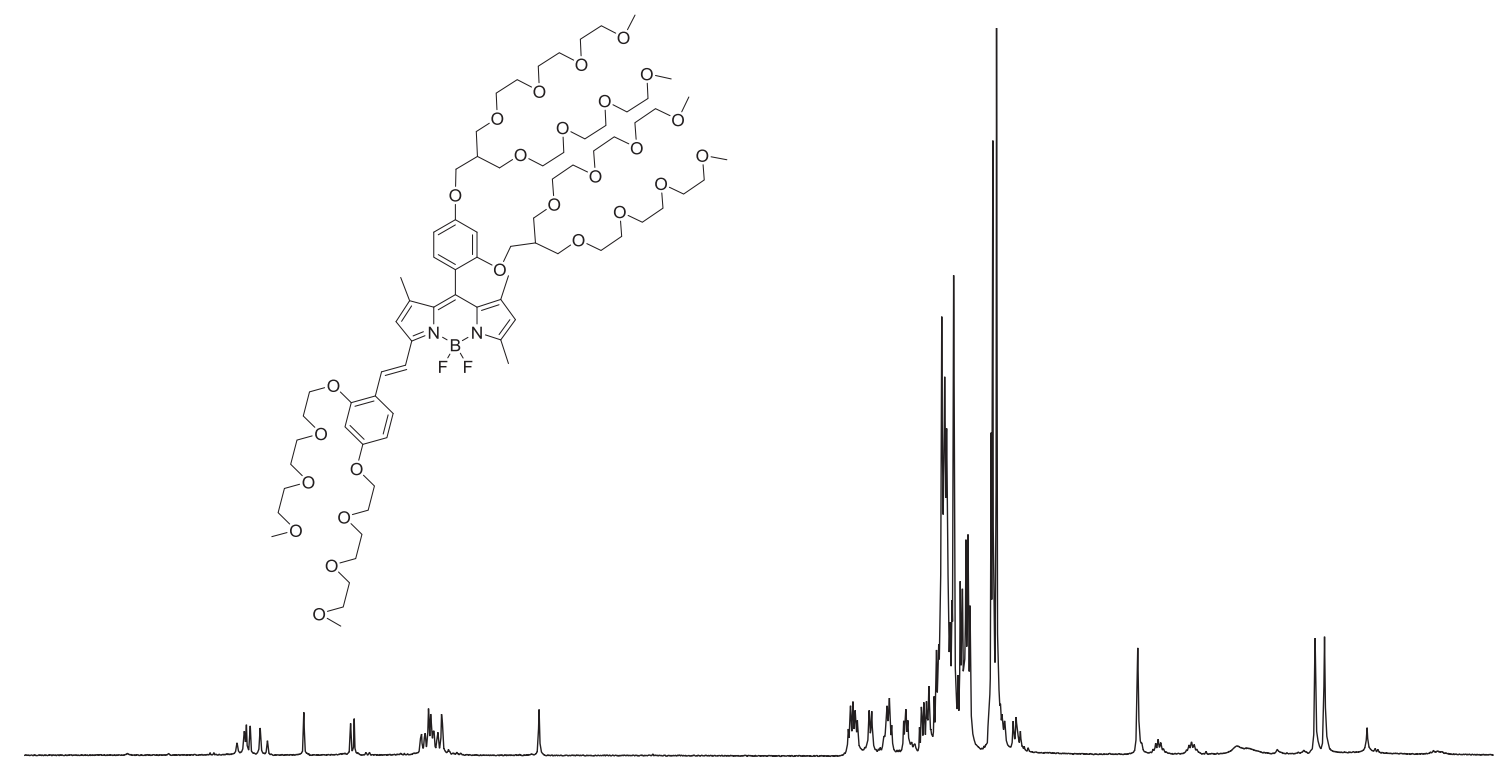

\begin{tabular}{llllllllllllllll}
\hline 8.5 & 8.0 & 7.5 & 7.0 & 6.5 & 6.0 & 5.5 & 5.0 & $\begin{array}{c}4.5 \\
\mathrm{ppm}\end{array}$ & 4.0 & 3.5 & 3.0 & 2.5 & 2.0 & 1.5 & 1.0
\end{tabular}

Figure A.27. ${ }^{1} \mathrm{H}$ NMR spectrum of BODIPY dye 3-G in $\mathrm{CDCl}_{3}$ solution.

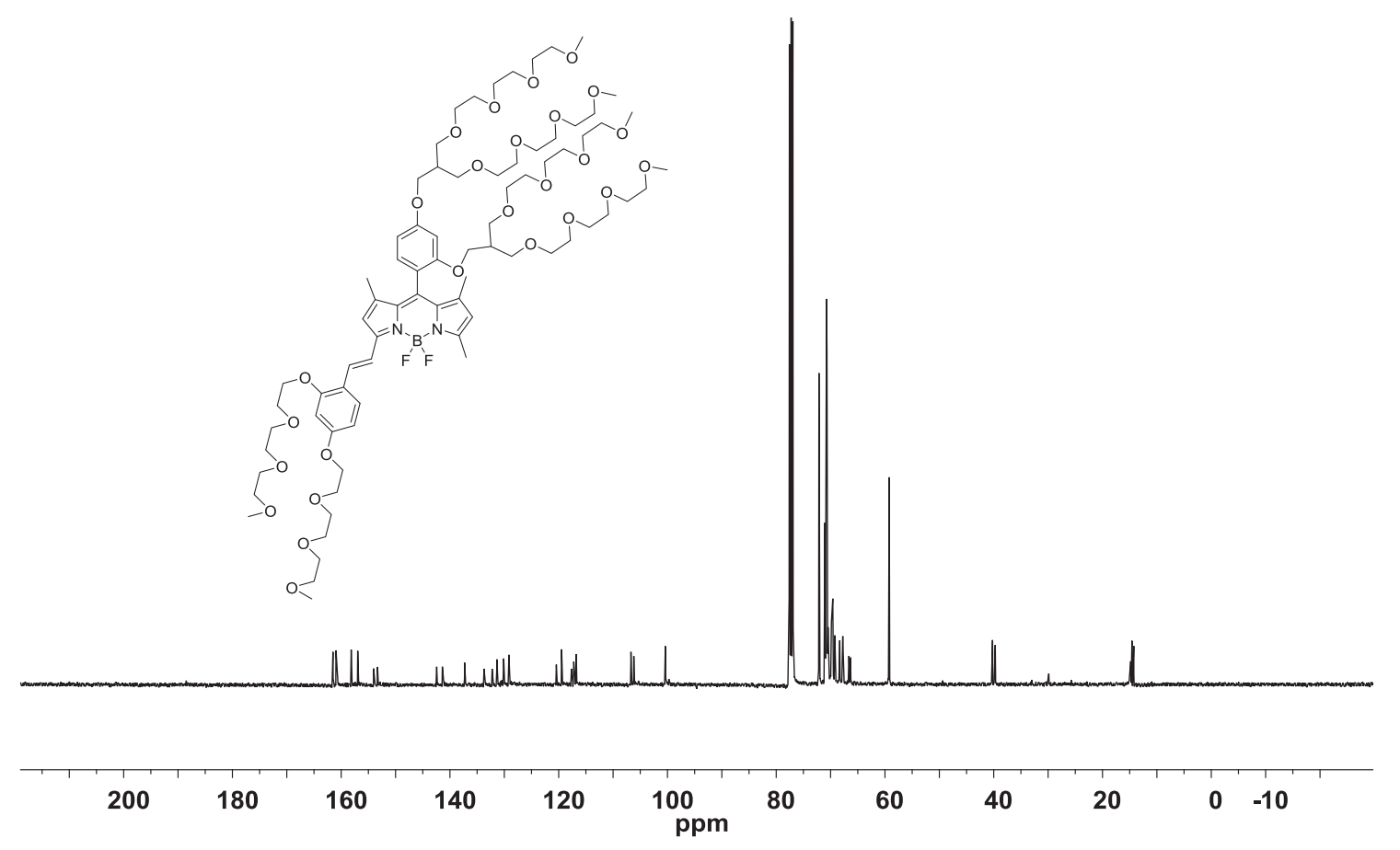

Figure A.28. ${ }^{13} \mathrm{C}$ NMR spectrum of BODIPY dye 3-G in $\mathrm{CDCl}_{3}$ solution. 

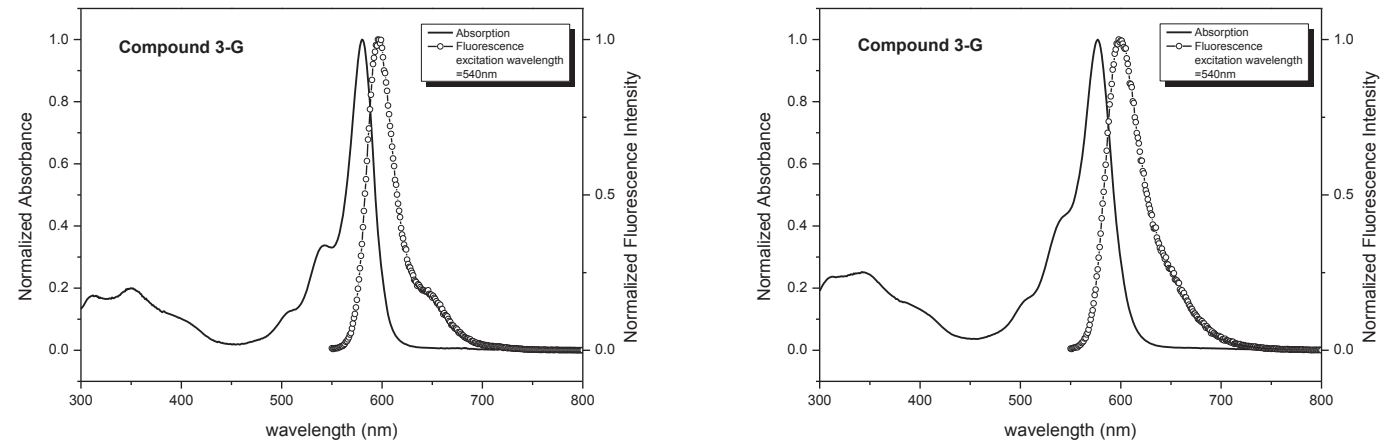

Figure A.29. Absorption and fluorescence spectra of BODIPY dye $\mathbf{3 - G}$ in dichloromethane solution (left) and in 0.5 M PBS buffer $(\mathrm{pH}=7.4)$ aqueous solution (right) ), $\lambda_{\mathrm{ex}}=470 \mathrm{~nm}$.

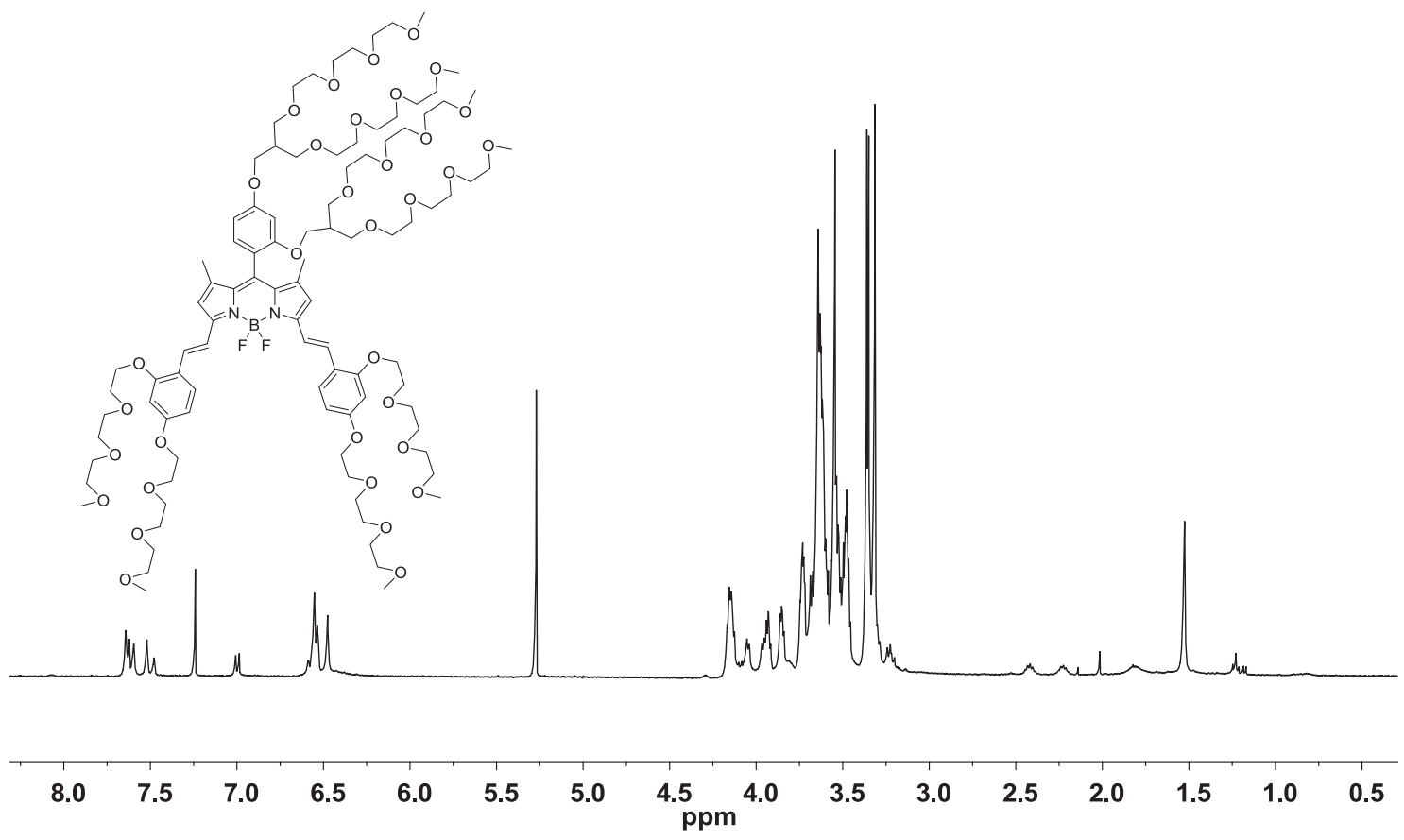

Figure A.30. ${ }^{1} \mathrm{H}$ NMR spectrum of BODIPY dye 3-H in $\mathrm{CDCl}_{3}$ solution. 


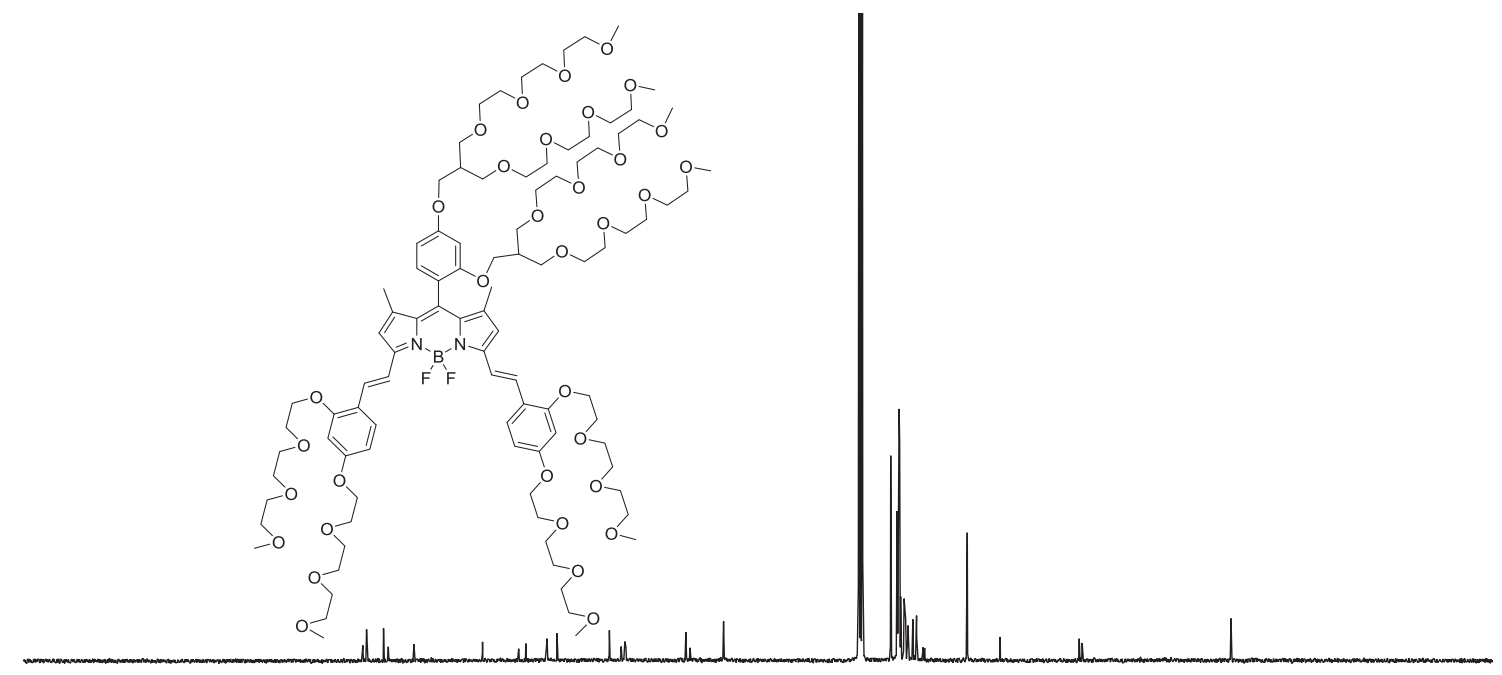

Figure A.31. ${ }^{13} \mathrm{C}$ NMR spectrum of BODIPY dye 3-H in $\mathrm{CDCl}_{3}$ solution.
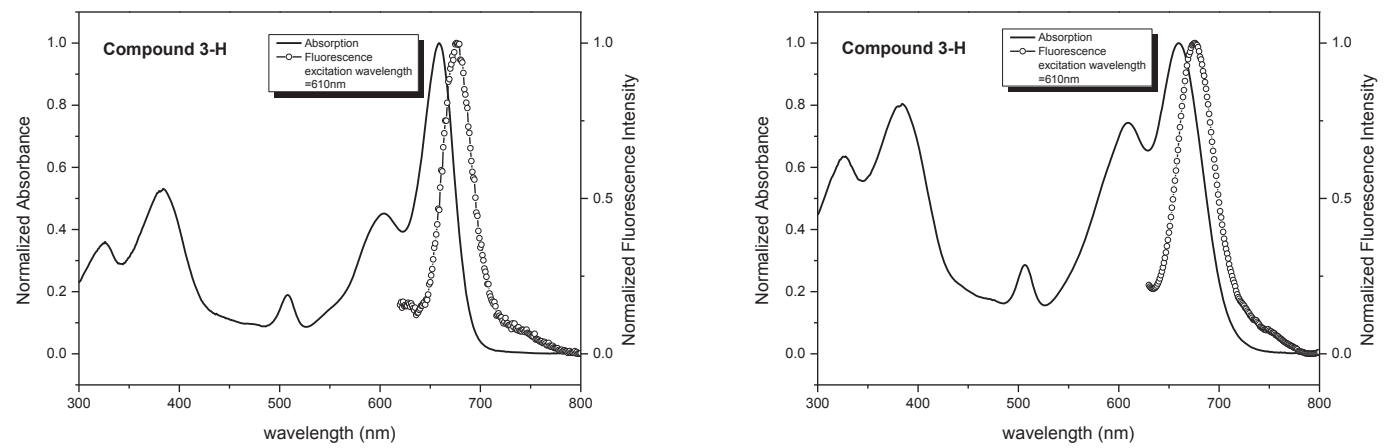

Figure A.32. Absorption and fluorescence spectra of BODIPY dye $\mathbf{3 - H}$ in dichloromethane solution (left) and in $0.5 \mathrm{M}$ PBS buffer $(\mathrm{pH}=7.4)$ aqueous solution (right) ), $\lambda_{\mathrm{ex}}=470 \mathrm{~nm}$. 


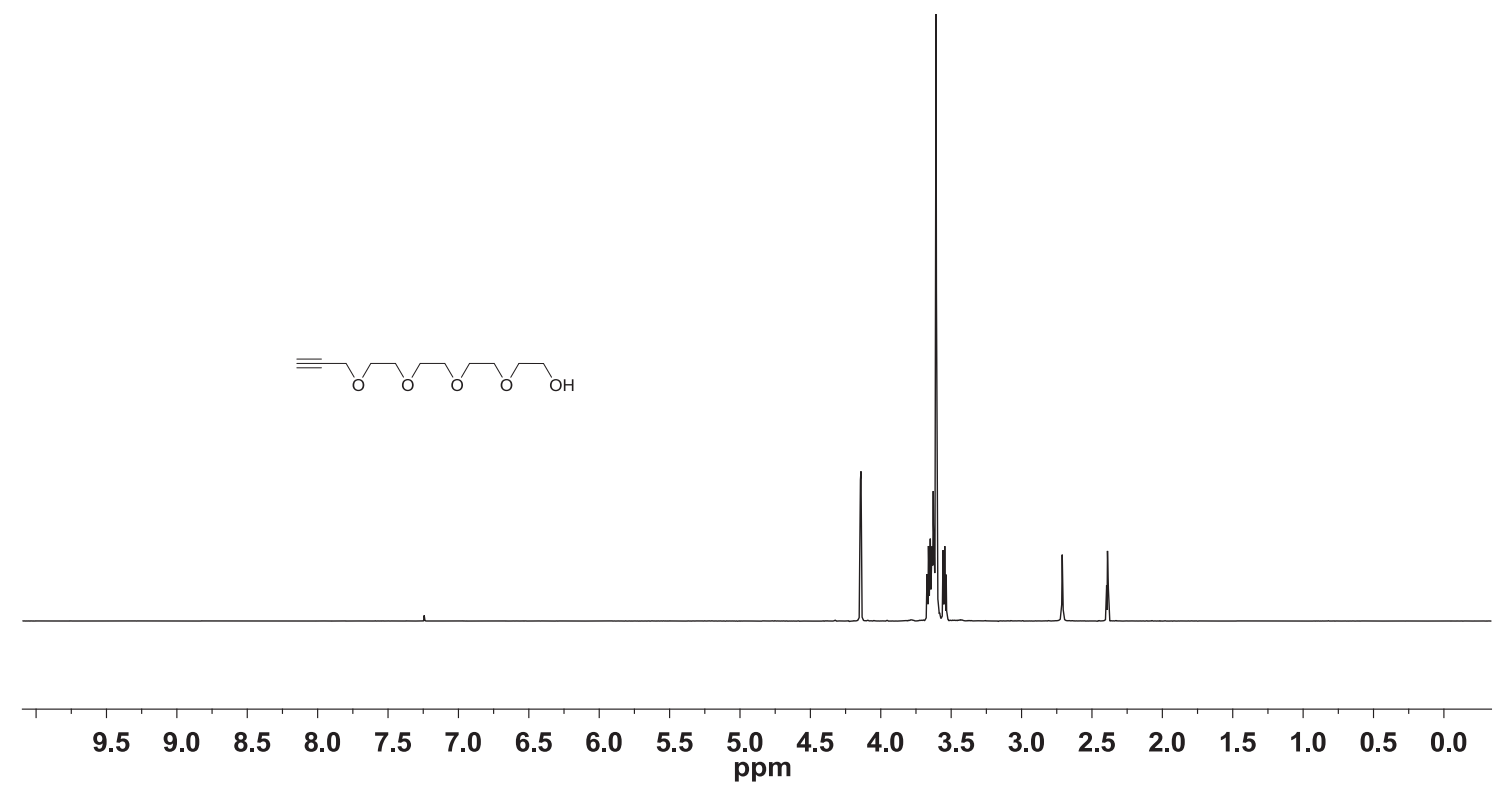

Figure A.33. ${ }^{1} \mathrm{H}$ NMR spectrum of compound 3.12 in $\mathrm{CDCl}_{3}$ solution.

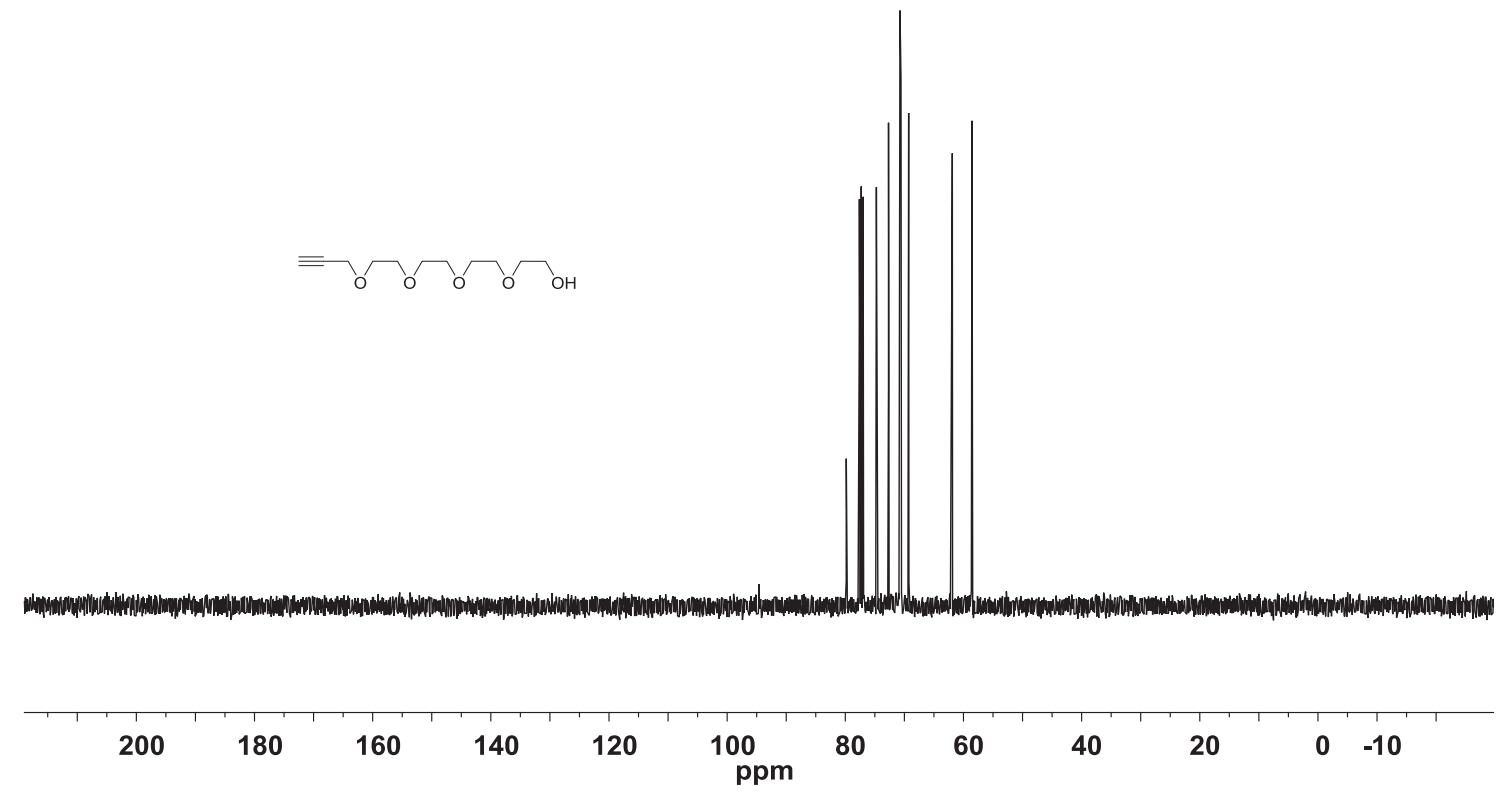

Figure A.34. ${ }^{13} \mathrm{C} \mathrm{NMR}$ spectrum of compound 3.12 in $\mathrm{CDCl}_{3}$ solution. 


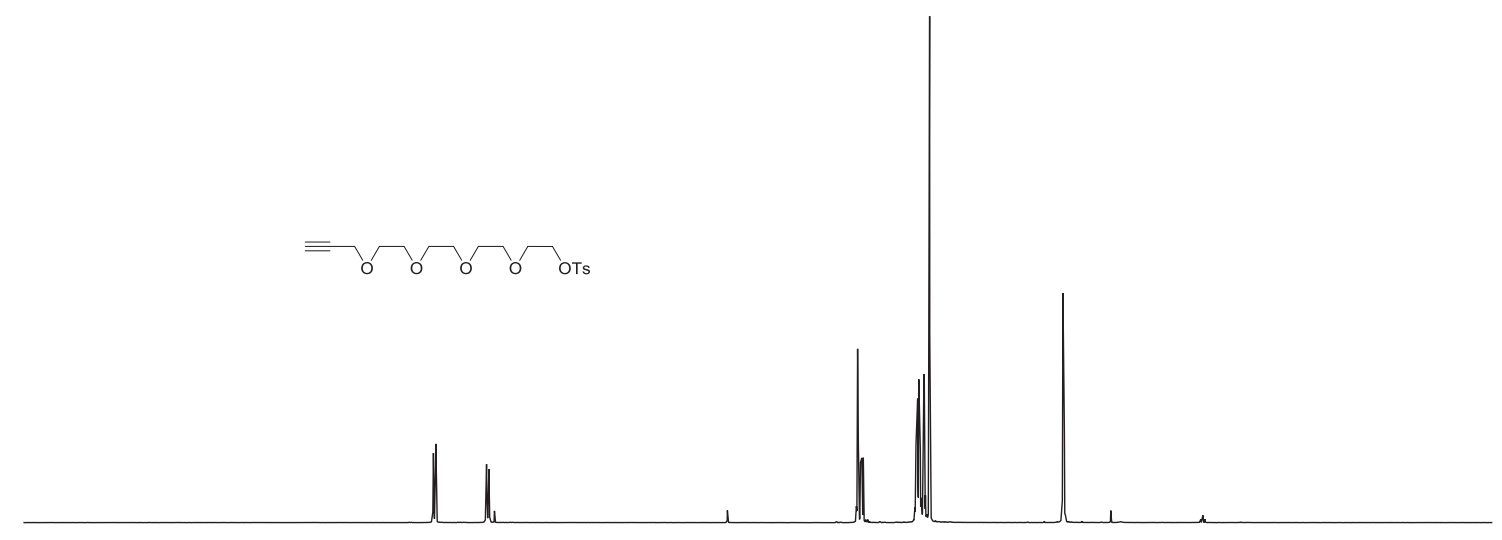

\begin{tabular}{|c|c|c|c|c|c|c|c|c|c|c|c|}
\hline 10.5 & 9.5 & 8.5 & 7.5 & 6.5 & 5.5 & 4.5 & 3.5 & 2.5 & 1.5 & 0.5 & -0.5 \\
\hline
\end{tabular}

Figure A.35. ${ }^{1} \mathrm{H}$ NMR spectrum of compound $\mathbf{3 . 1 3}$ in $\mathrm{CDCl}_{3}$ solution.

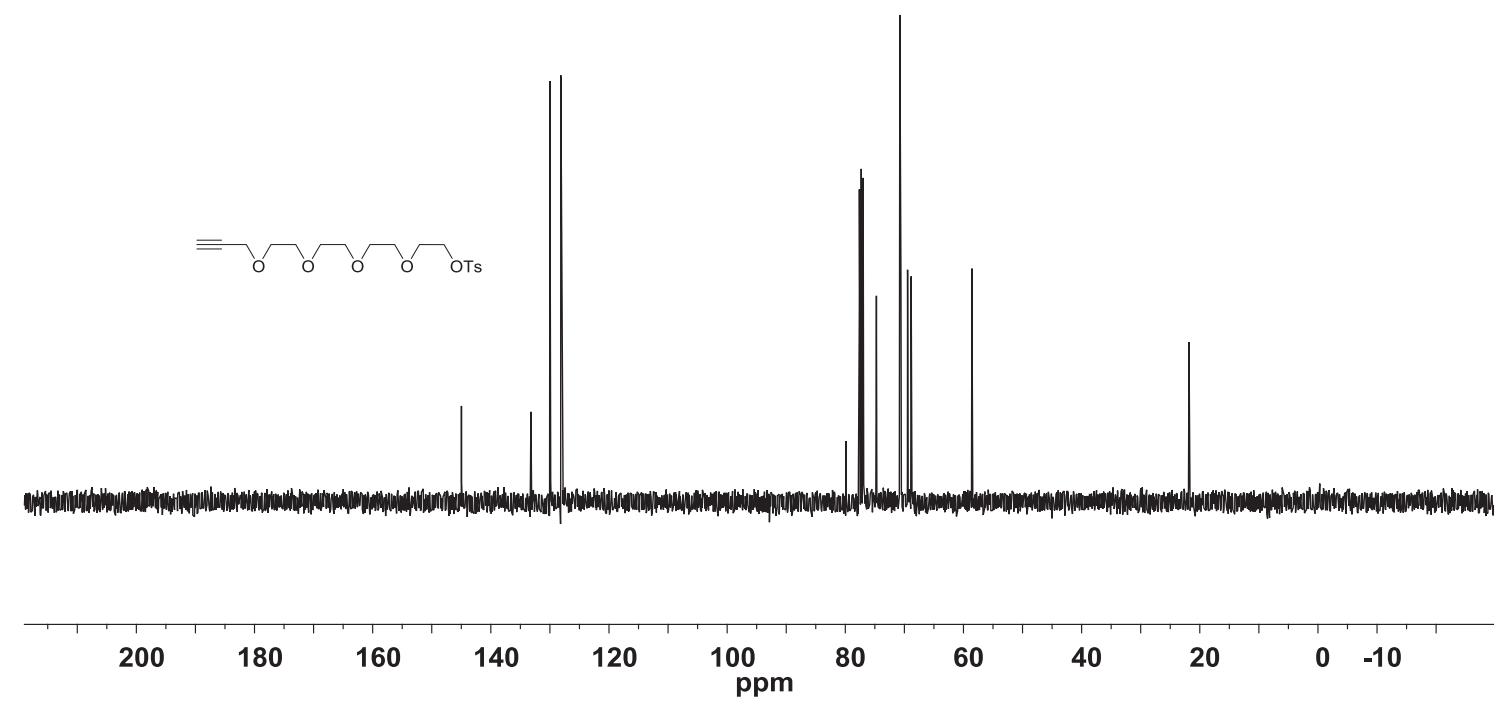

Figure A.36. ${ }^{13} \mathrm{C} \mathrm{NMR}$ spectrum of compound $\mathbf{3 . 1 3}$ in $\mathrm{CDCl}_{3}$ solution. 


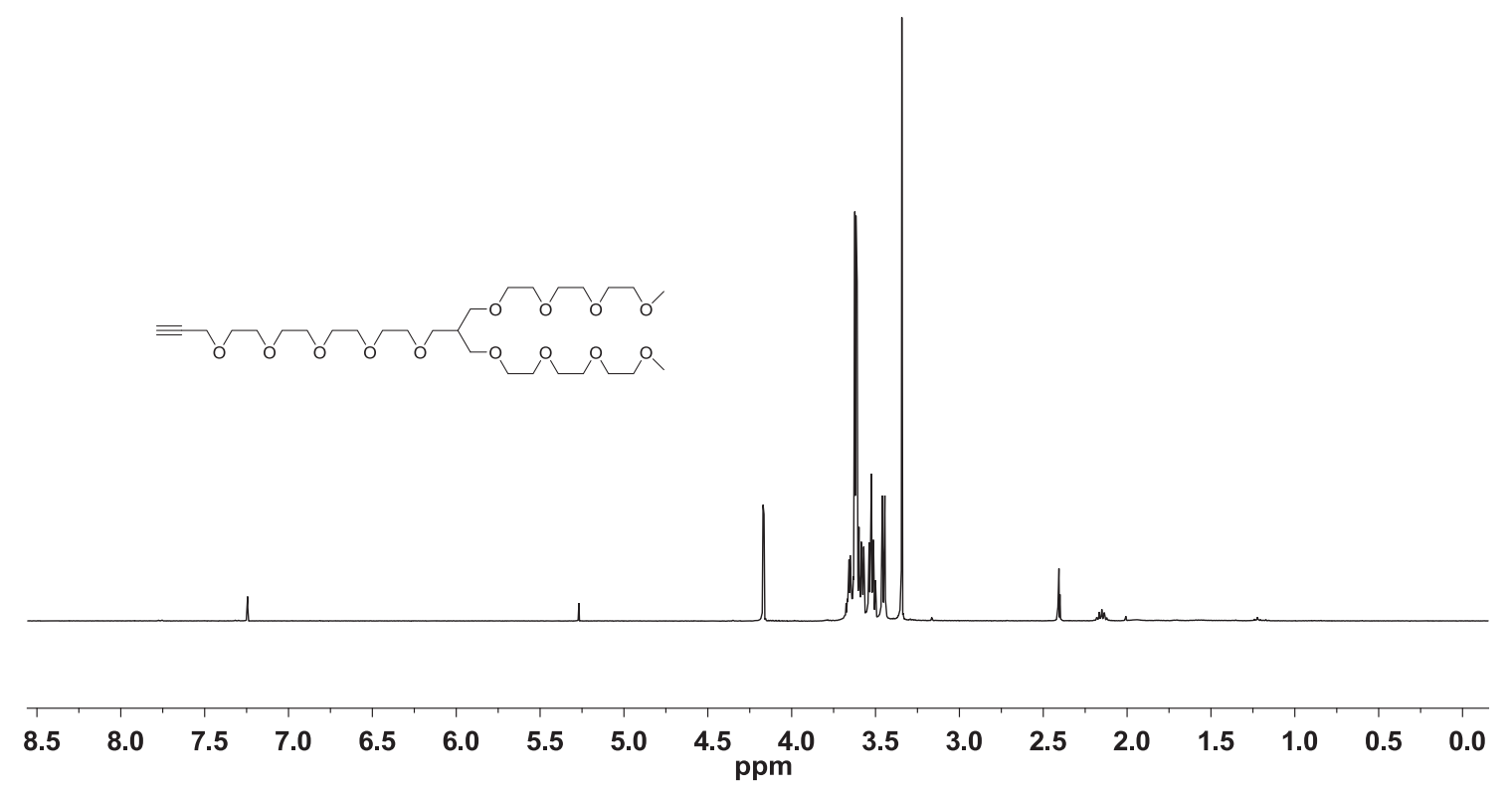

Figure A.37. ${ }^{1} \mathrm{H}$ NMR spectrum of compound 3.14 in $\mathrm{CDCl}_{3}$ solution.

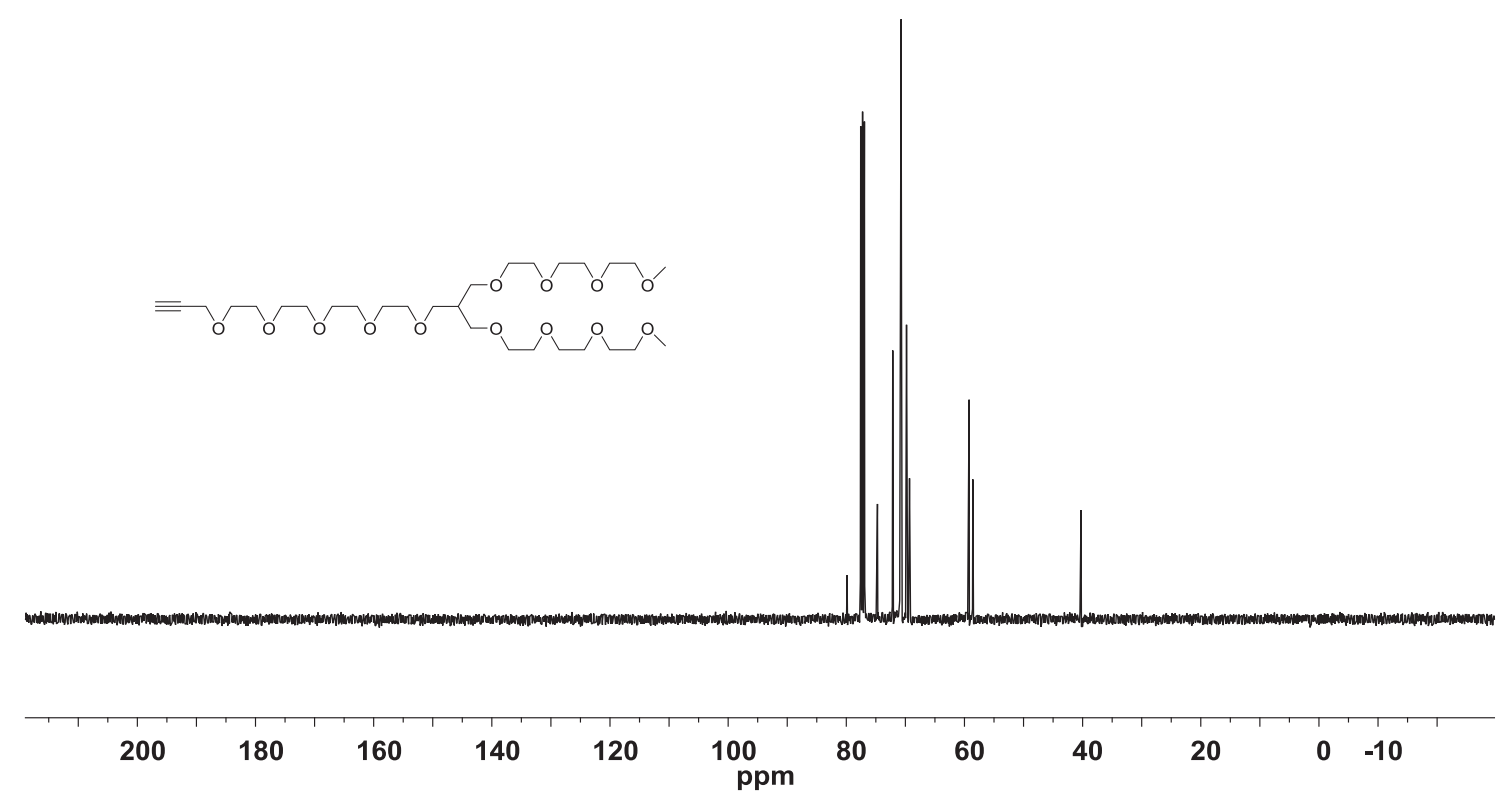

Figure A.38. ${ }^{13} \mathrm{C} \mathrm{NMR}$ spectrum of compound 3.14 in $\mathrm{CDCl}_{3}$ solution. 


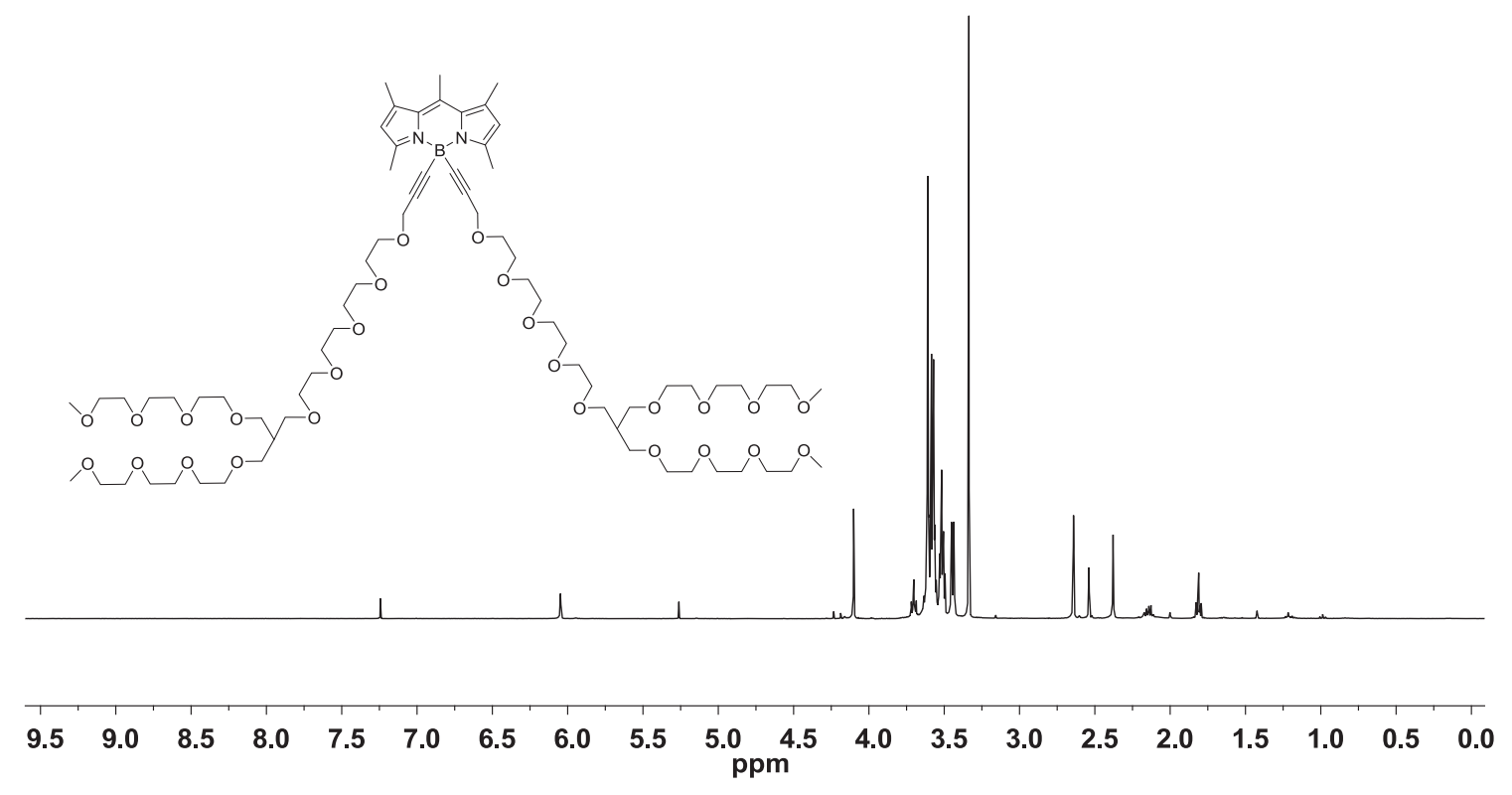

Figure A.39. ${ }^{1} \mathrm{H}$ NMR spectrum of BODIPY dye 3-I in $\mathrm{CDCl}_{3}$ solution.

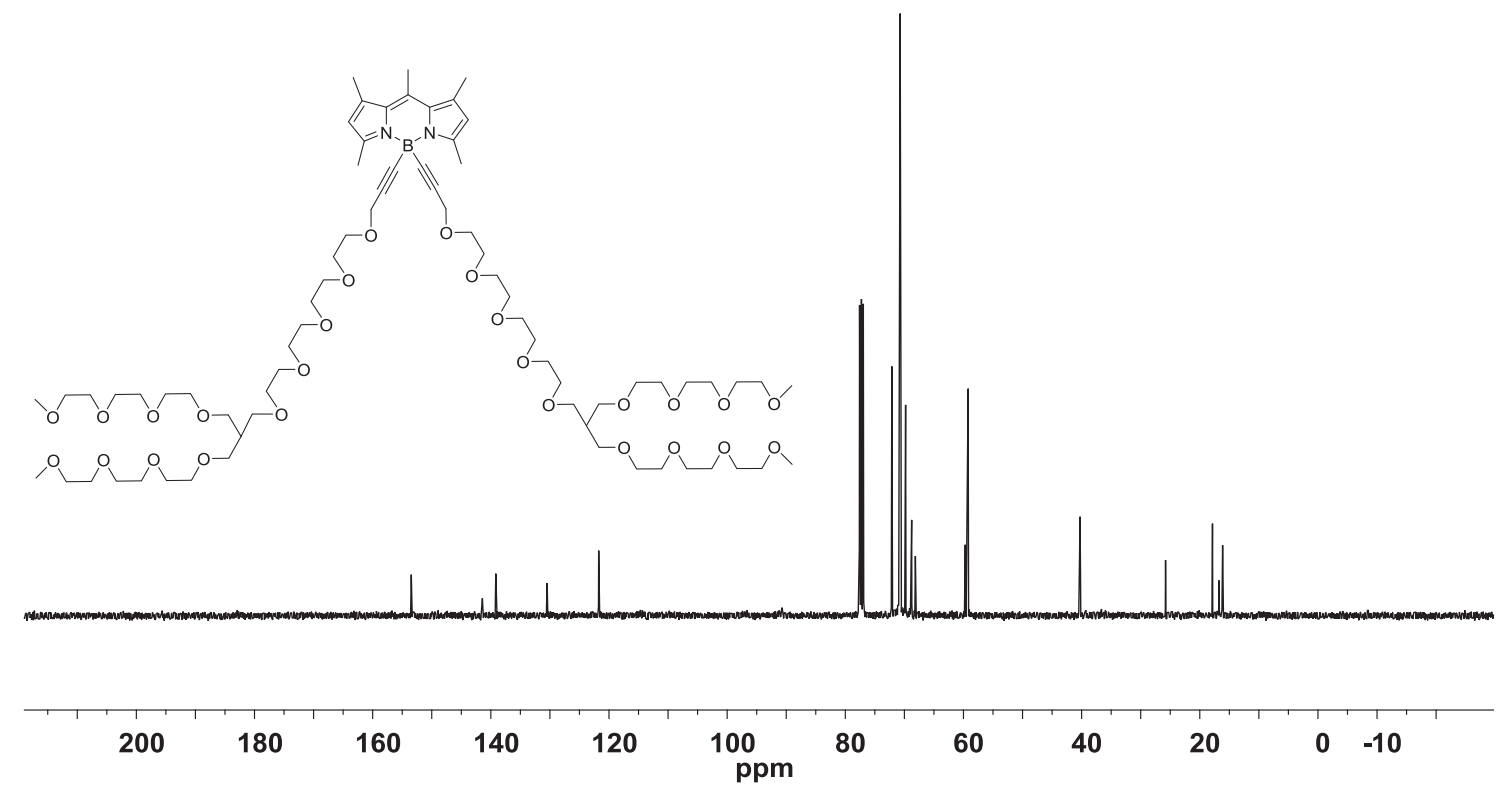

Figure A.40. ${ }^{13} \mathrm{C}$ NMR spectrum of BODIPY dye 3-I in $\mathrm{CDCl}_{3}$ solution. 

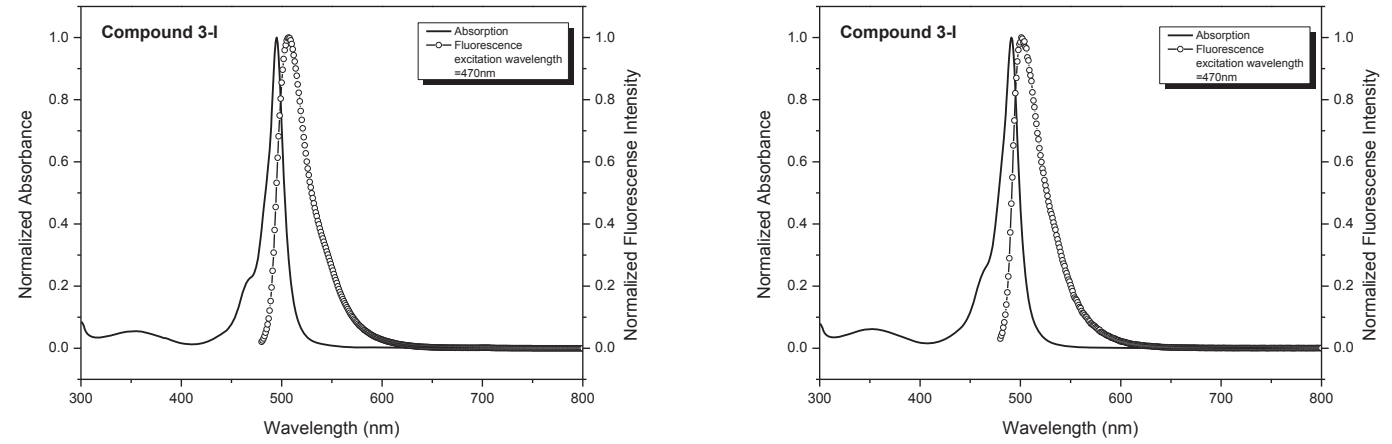

Figure A.41. Absorption and fluorescence spectra of BODIPY dye 3-I in dichloromethane solution (left) and in 0.5 M PBS buffer $(\mathrm{pH}=7.4)$ aqueous solution (right) ), $\lambda_{\mathrm{ex}}=470 \mathrm{~nm}$.

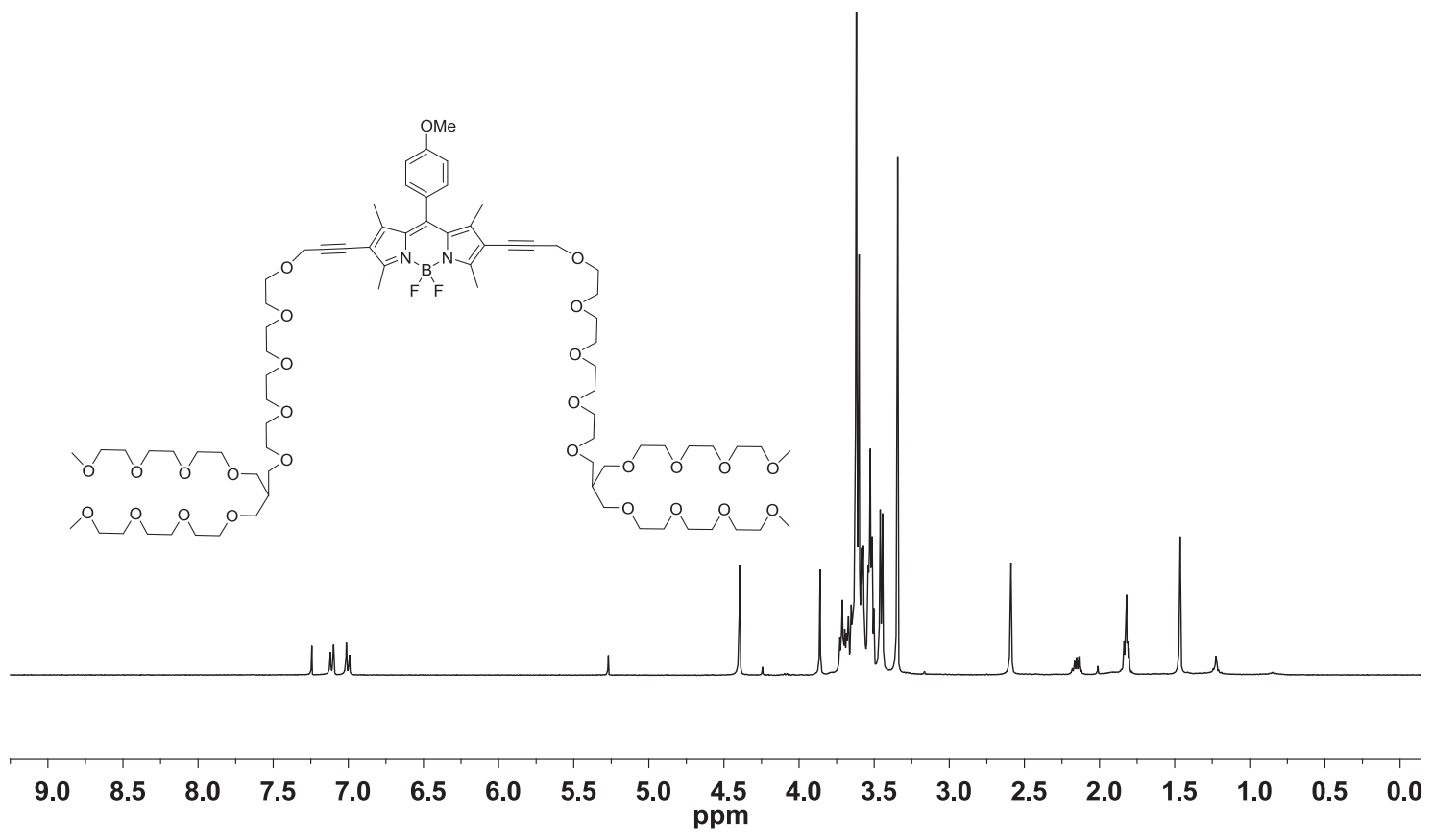

Figure A.42. ${ }^{1} \mathrm{H}$ NMR spectrum of BODIPY dye 3-J in $\mathrm{CDCl}_{3}$ solution. 


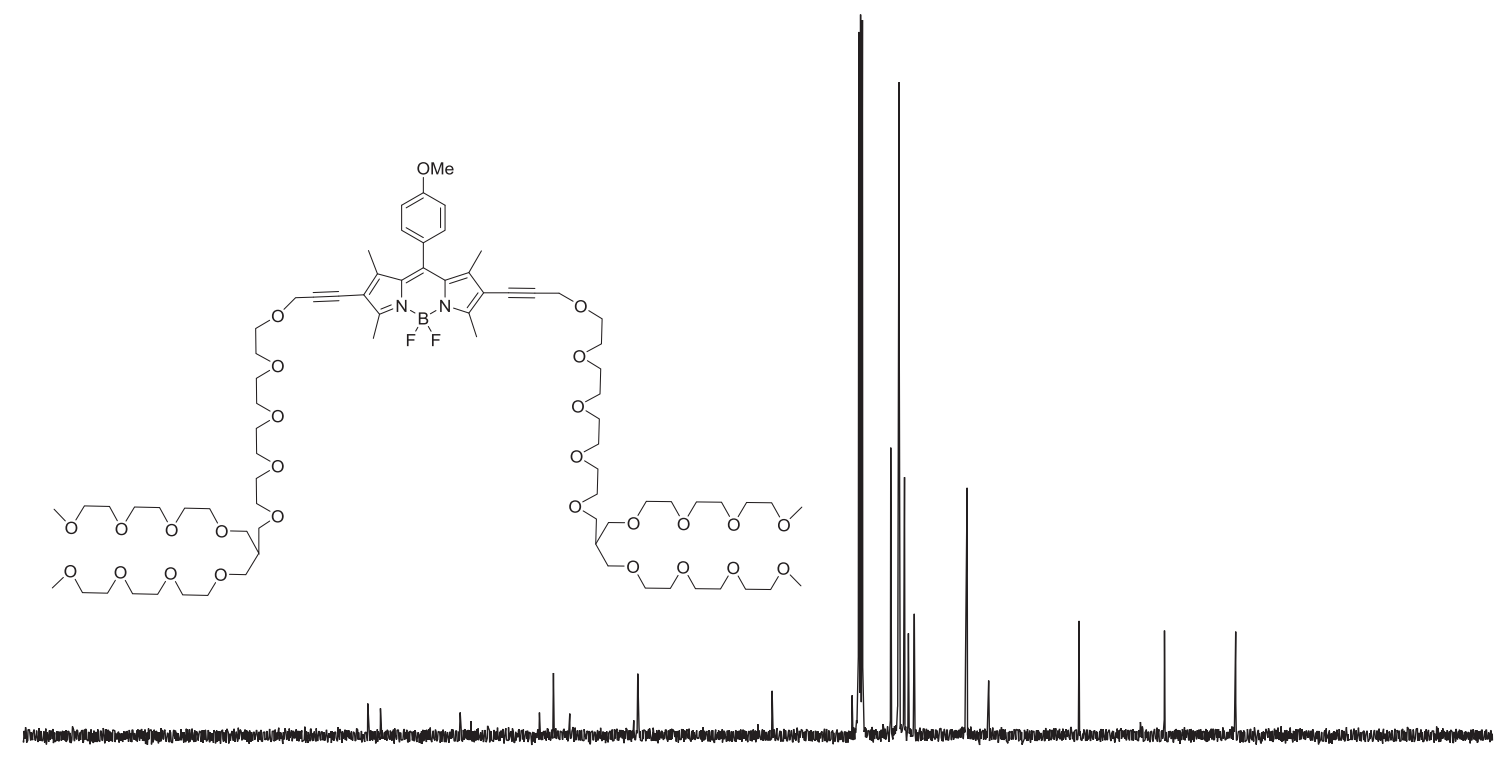

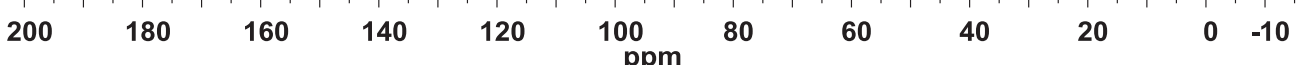

Figure A.43. ${ }^{13} \mathrm{C}$ NMR spectrum of BODIPY dye 3-J in $\mathrm{CDCl}_{3}$ solution.
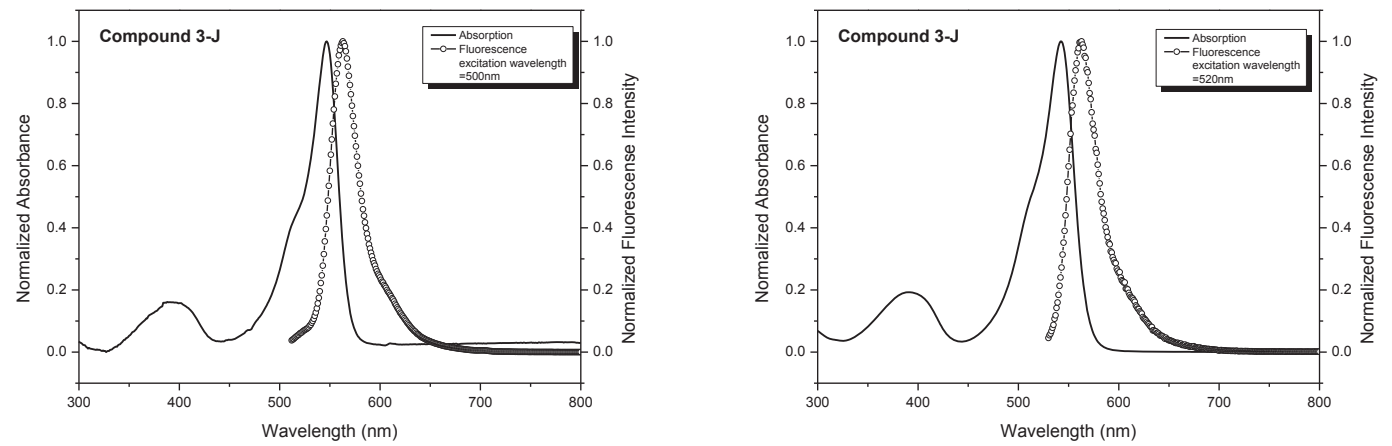

Figure A.44. Absorption and fluorescence spectra of BODIPY dye $3-\mathbf{J}$ in dichloromethane solution (left) and in 0.5 M PBS buffer $(\mathrm{pH}=7.4)$ aqueous solution (right), $\lambda_{\mathrm{ex}}=470 \mathrm{~nm}$. 


\section{Appendix B Supporting Information for Chapter 4}
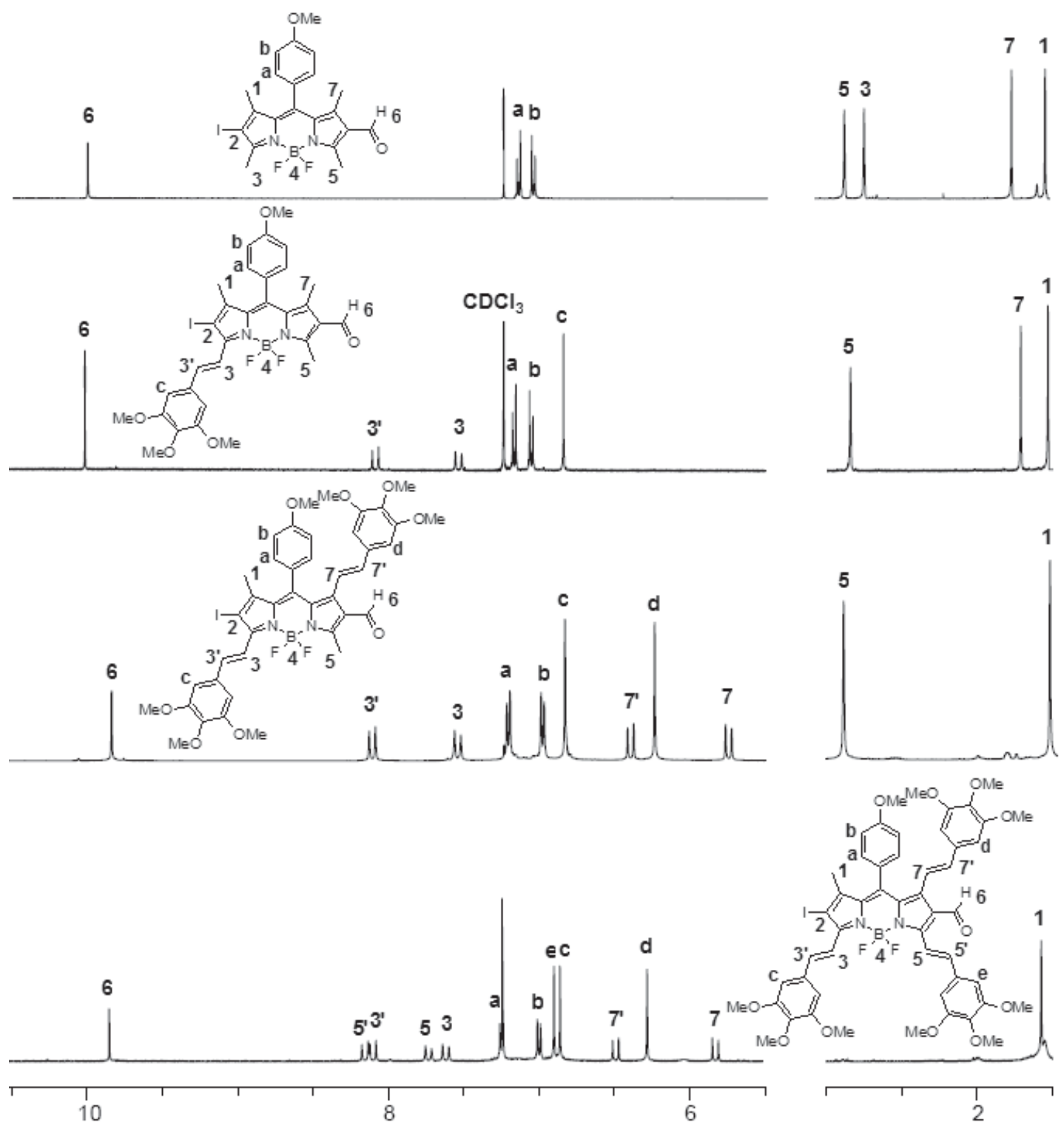

Figure B.1. Comparison of ${ }^{1} \mathrm{H}$ spectra of BODIPY dye 4.4, 4-A, 4-B and 4-C. 


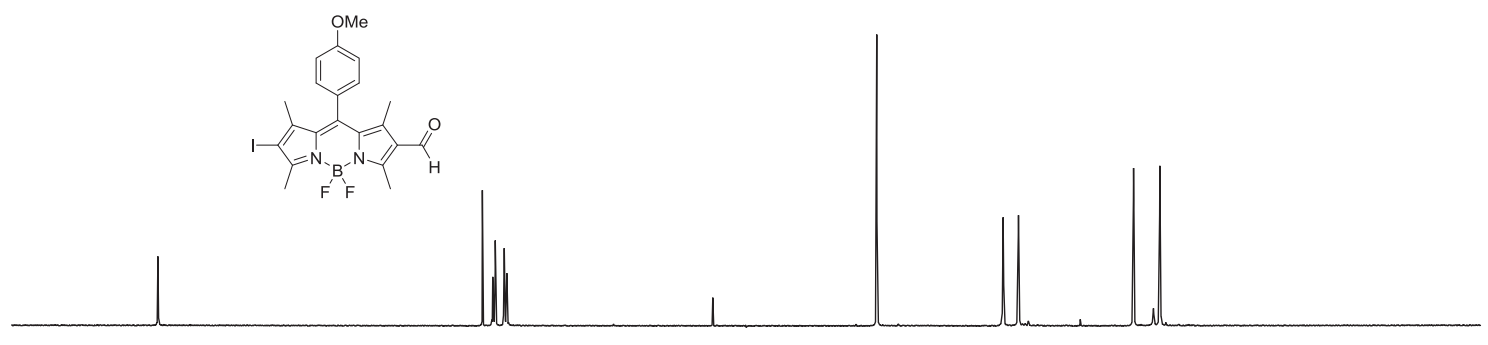

\begin{tabular}{|c|c|c|c|c|c|c|c|c|c|c|c|}
\hline 10.5 & 9.5 & 8.5 & 7.5 & 6.5 & & 4.5 & 3.5 & 2.5 & 1.5 & 0.5 & -0.5 \\
\hline
\end{tabular}

Figure B.2. $\quad{ }^{1} \mathrm{H}$ NMR spectrum of BODIPY dye 4.4 in $\mathrm{CDCl}_{3}$ solution.

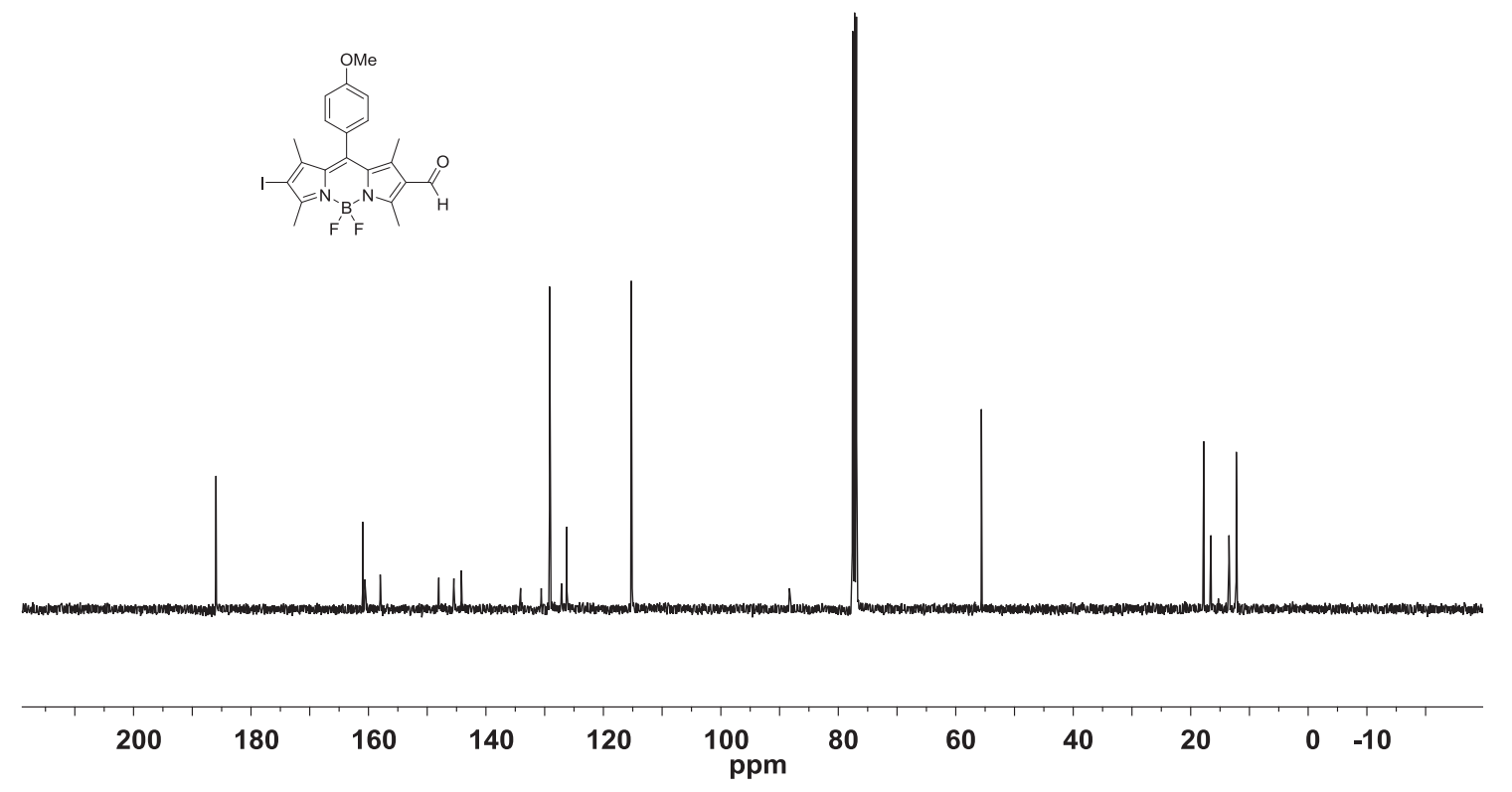

Figure B.3. $\quad{ }^{13} \mathrm{C}$ NMR spectrum of BODIPY dye 4.4 in $\mathrm{CDCl}_{3}$ solution. 


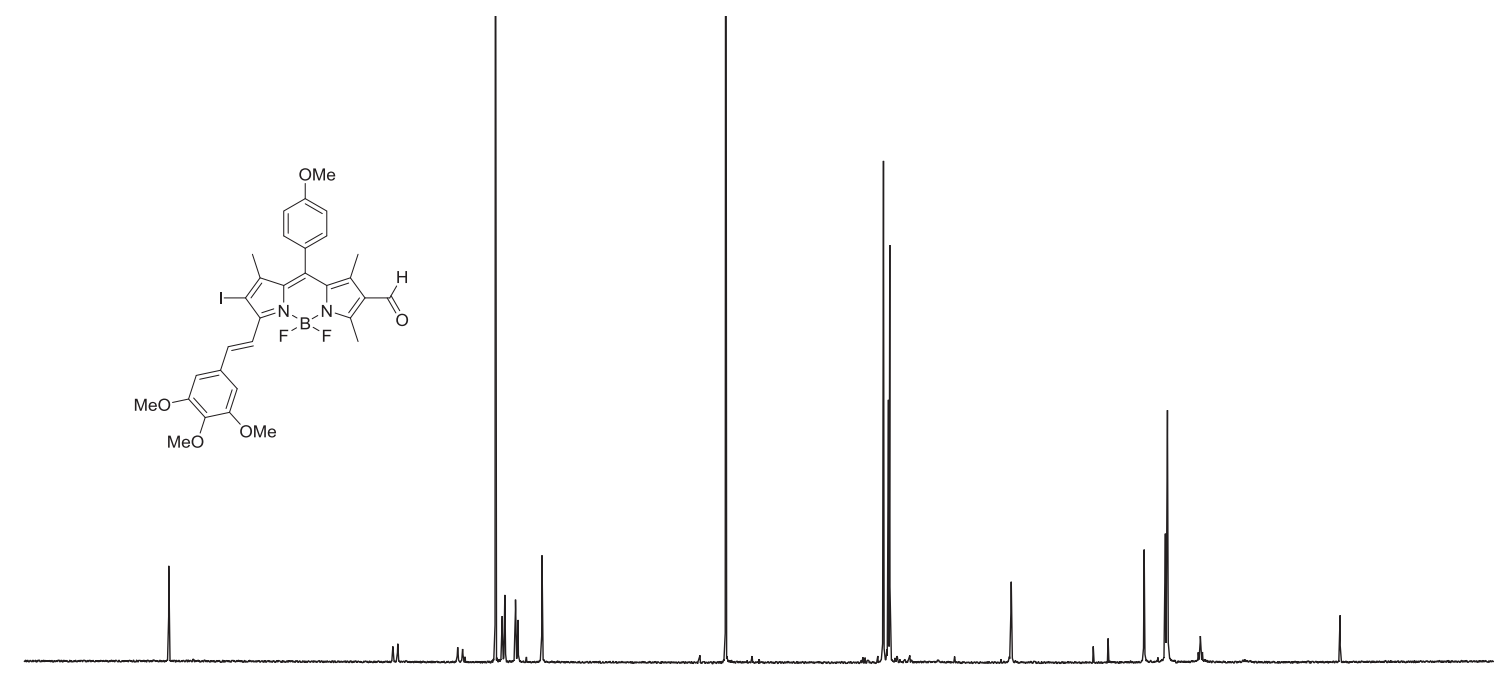

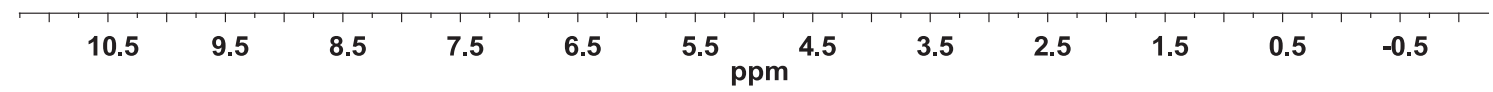

Figure B.4. $\quad{ }^{1} \mathrm{H}$ NMR spectrum of BODIPY dye 4-A in $\mathrm{CDCl}_{3}$ solution.
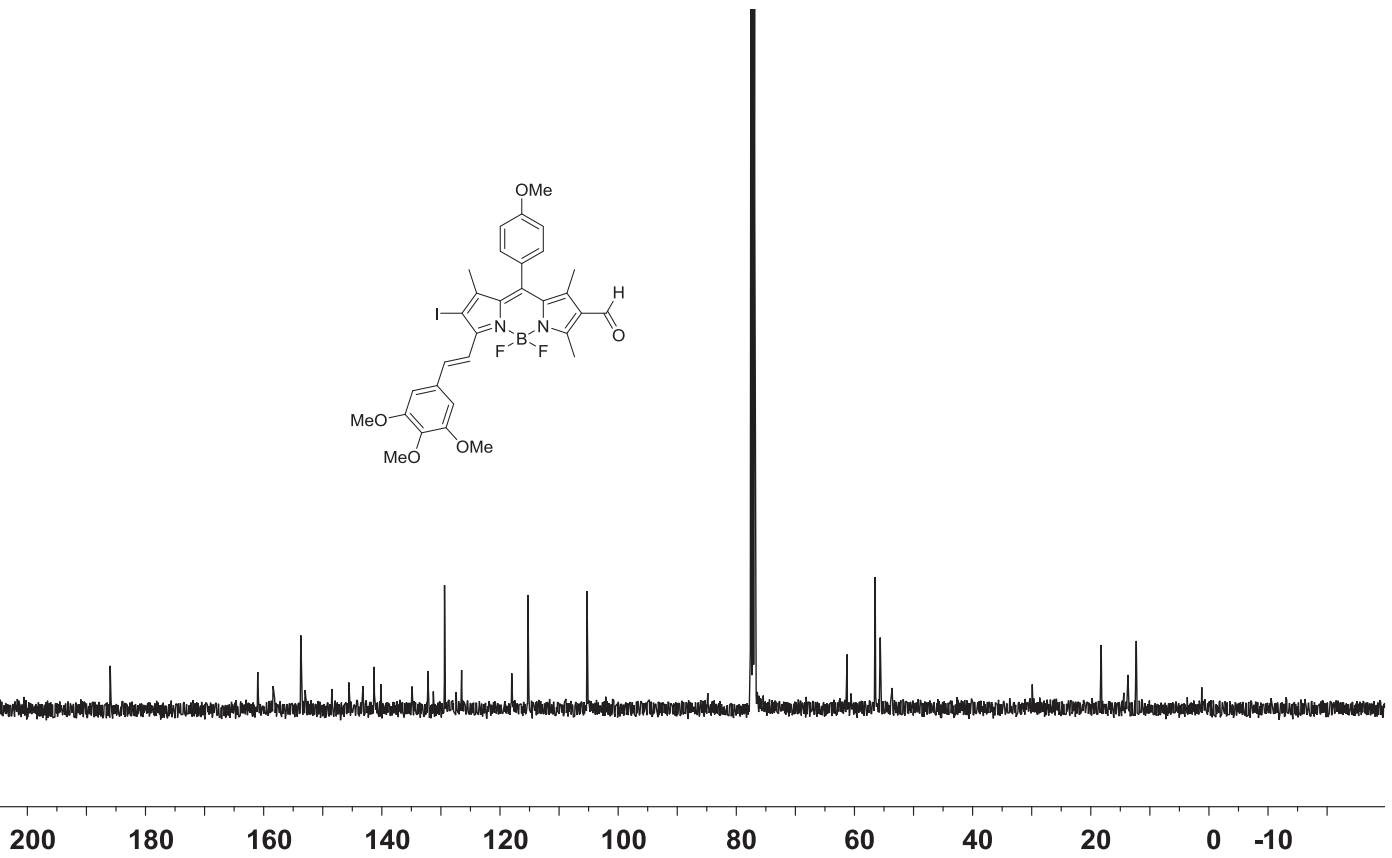

160

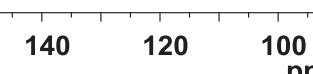
ppm

80 60 $40 \quad 20$ $\begin{array}{ll}0 & -10\end{array}$

Figure B.5. ${ }^{13} \mathrm{C}$ NMR spectrum of BODIPY dye 4-A in $\mathrm{CDCl}_{3}$ solution. 


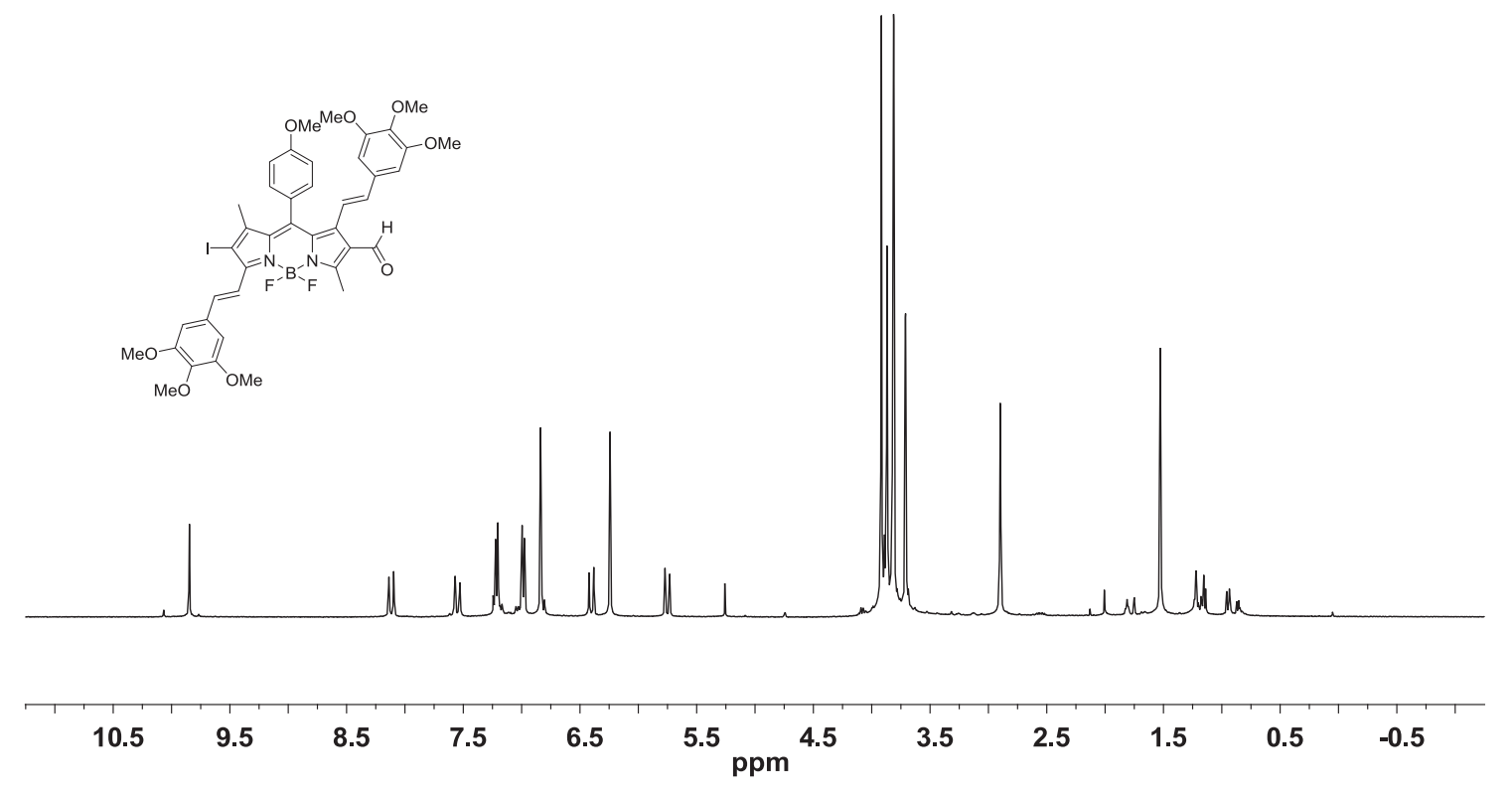

Figure B.6. $\quad{ }^{1} \mathrm{H}$ NMR spectrum of BODIPY dye 4-B in $\mathrm{CDCl}_{3}$ solution.

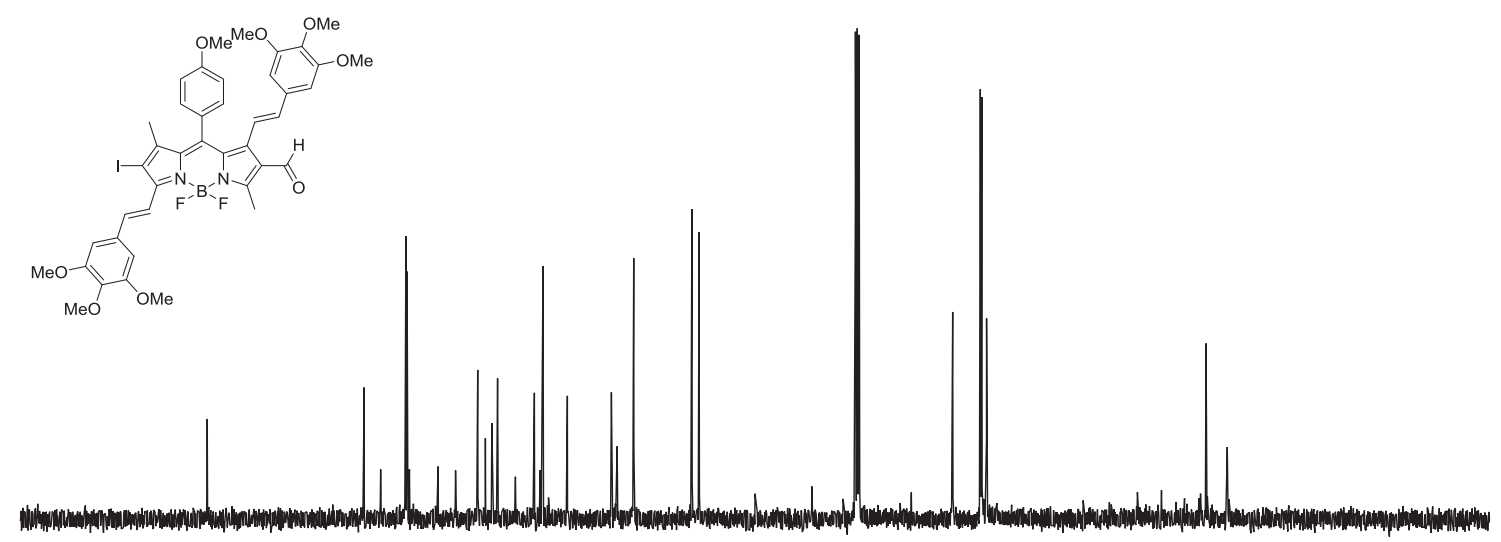

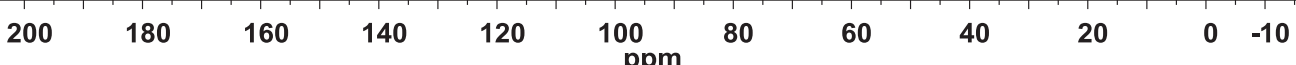

Figure B.7. ${ }^{13} \mathrm{C}$ NMR spectrum of BODIPY dye 4-B in $\mathrm{CDCl}_{3}$ solution. 

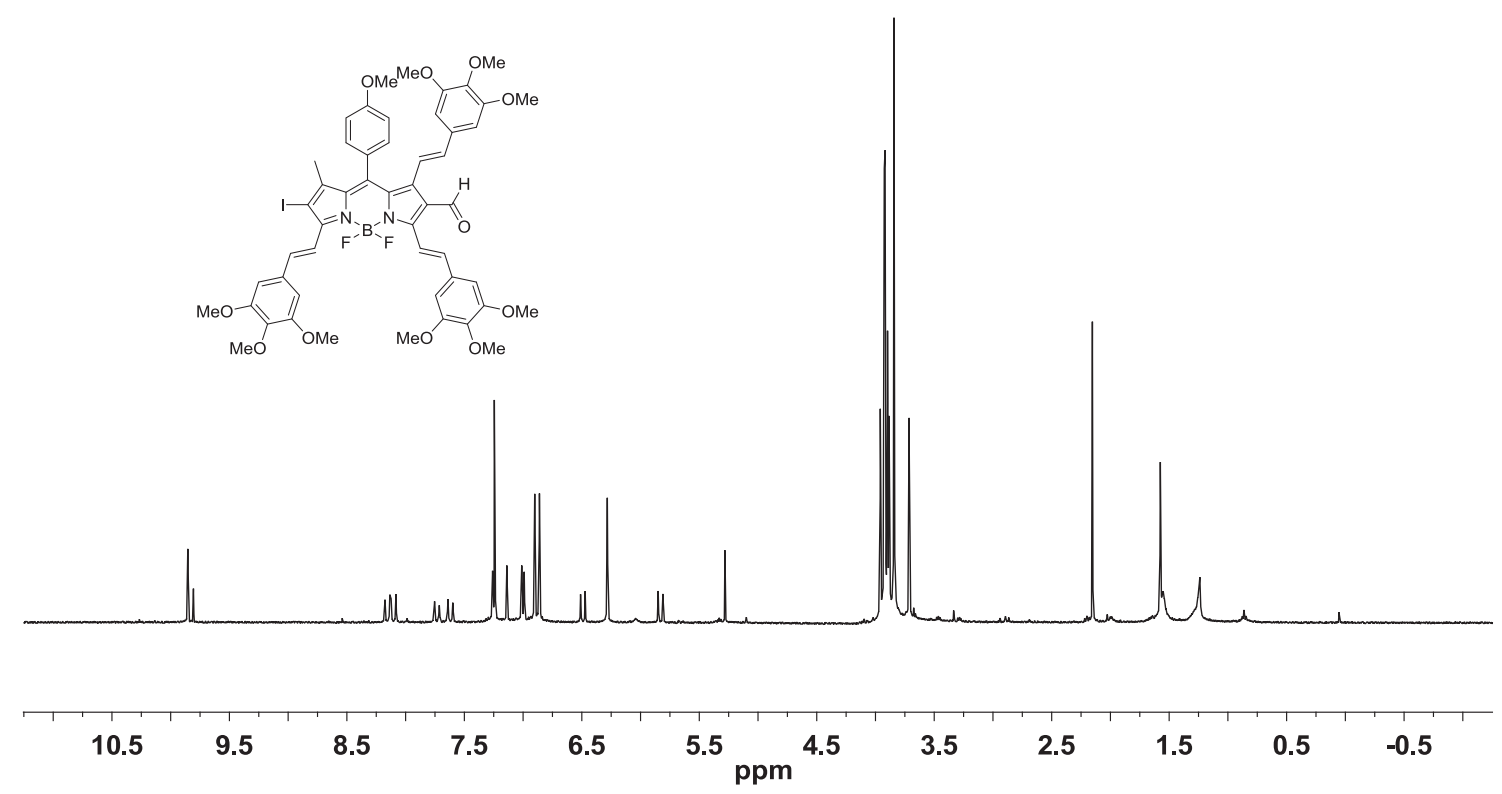

Figure B.8. $\quad{ }^{1} \mathrm{H}$ NMR spectrum of BODIPY dye 4-C in $\mathrm{CDCl}_{3}$ solution.

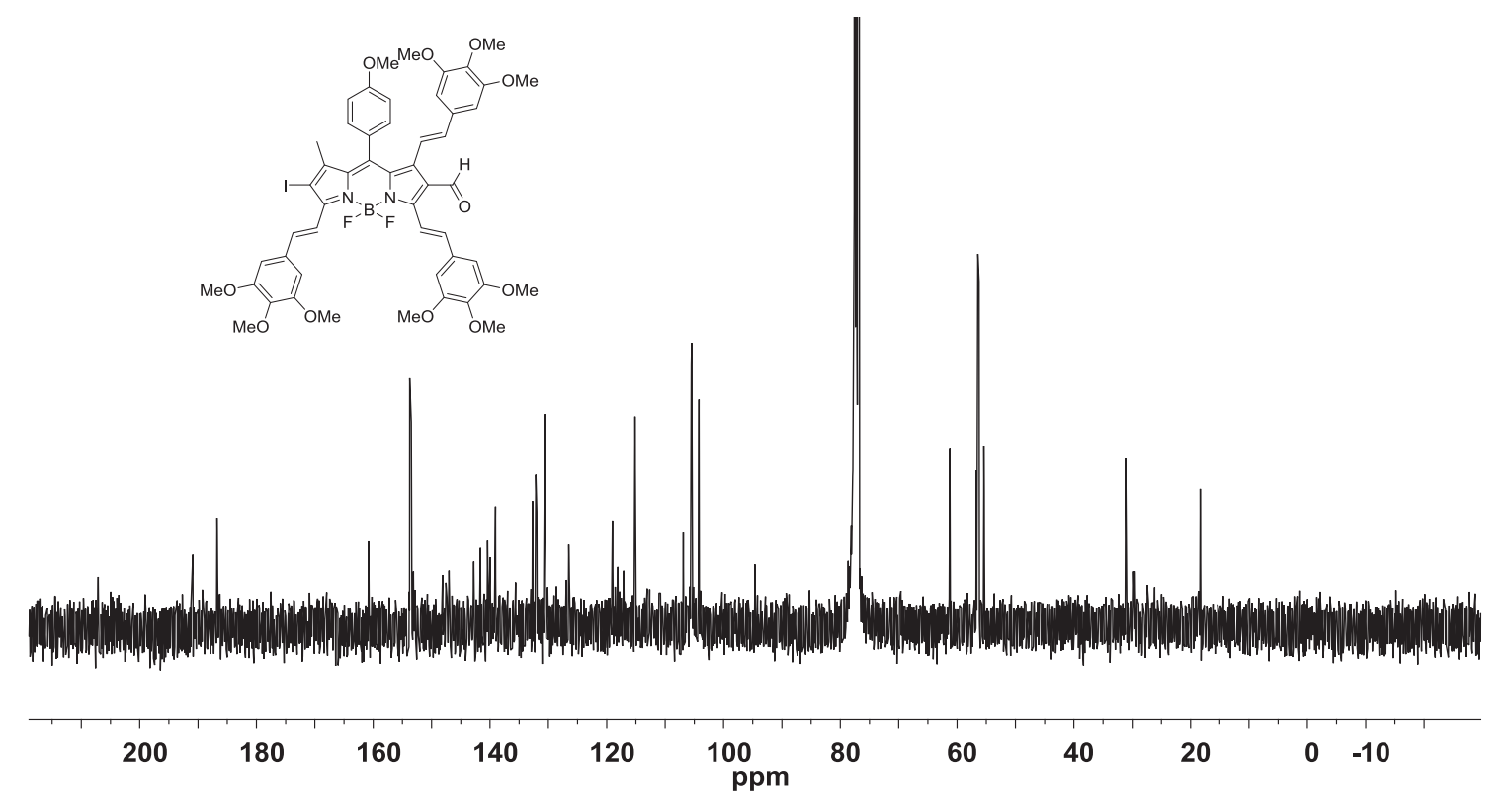

Figure B.9. ${ }^{13} \mathrm{C}$ NMR spectrum of BODIPY dye 4-C in $\mathrm{CDCl}_{3}$ solution. 


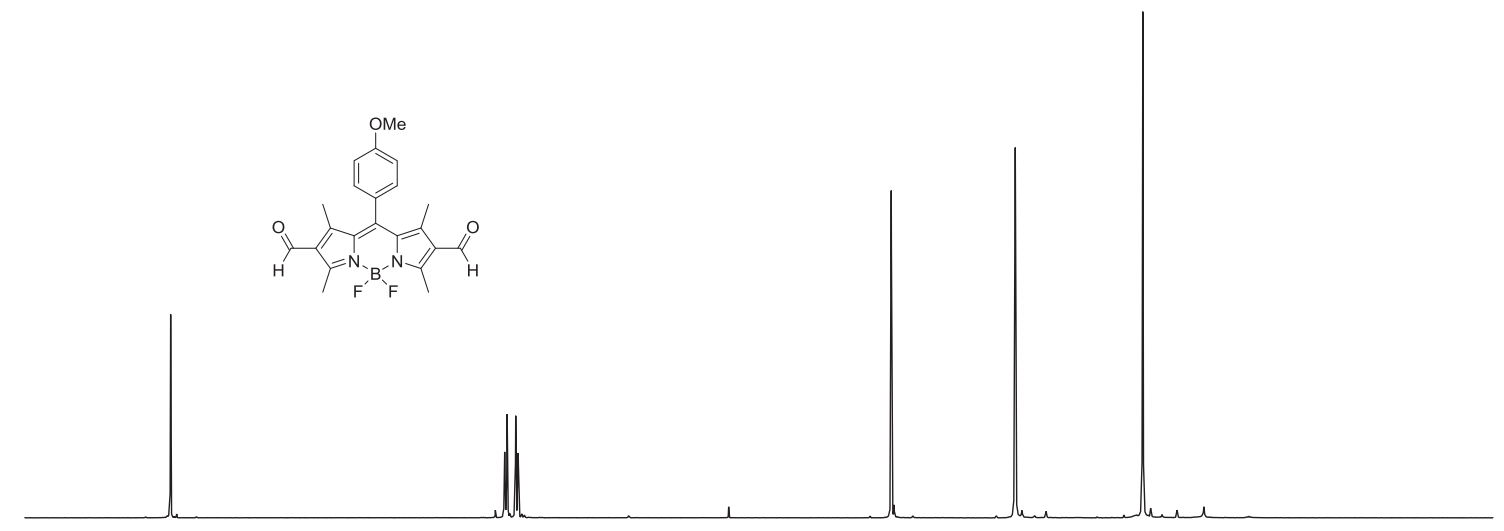

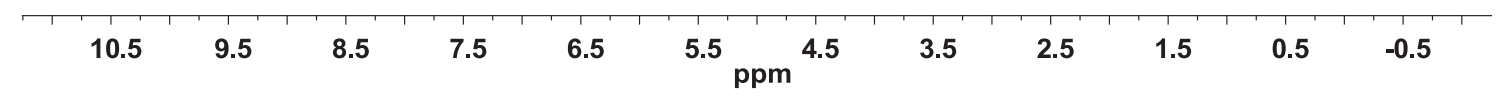

Figure B.10. ${ }^{1} \mathrm{H}$ NMR spectrum of BODIPY dye 4.6 in $\mathrm{CDCl}_{3}$ solution.

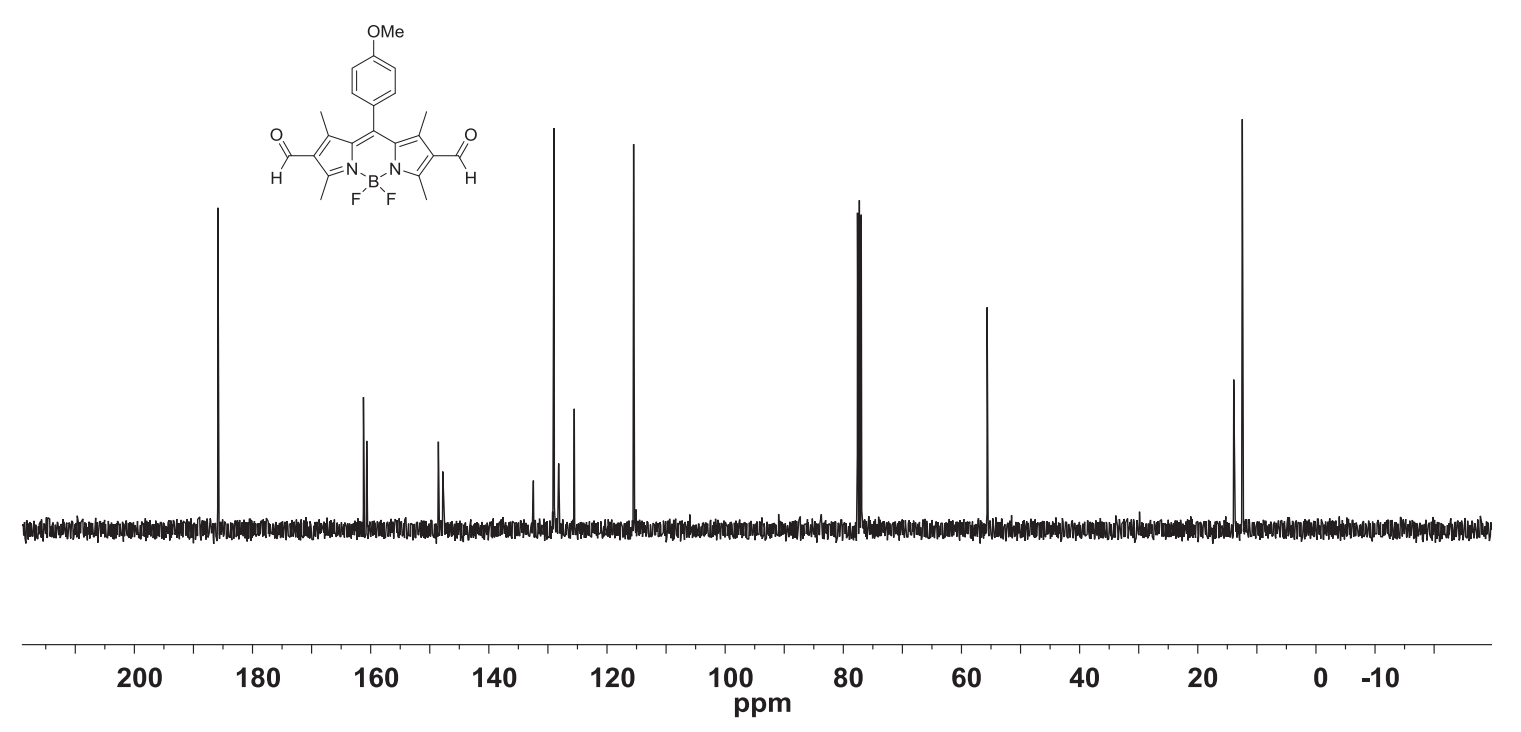

Figure B.11. ${ }^{13} \mathrm{C}$ NMR spectrum of BODIPY dye 4.6 in $\mathrm{CDCl}_{3}$ solution. 


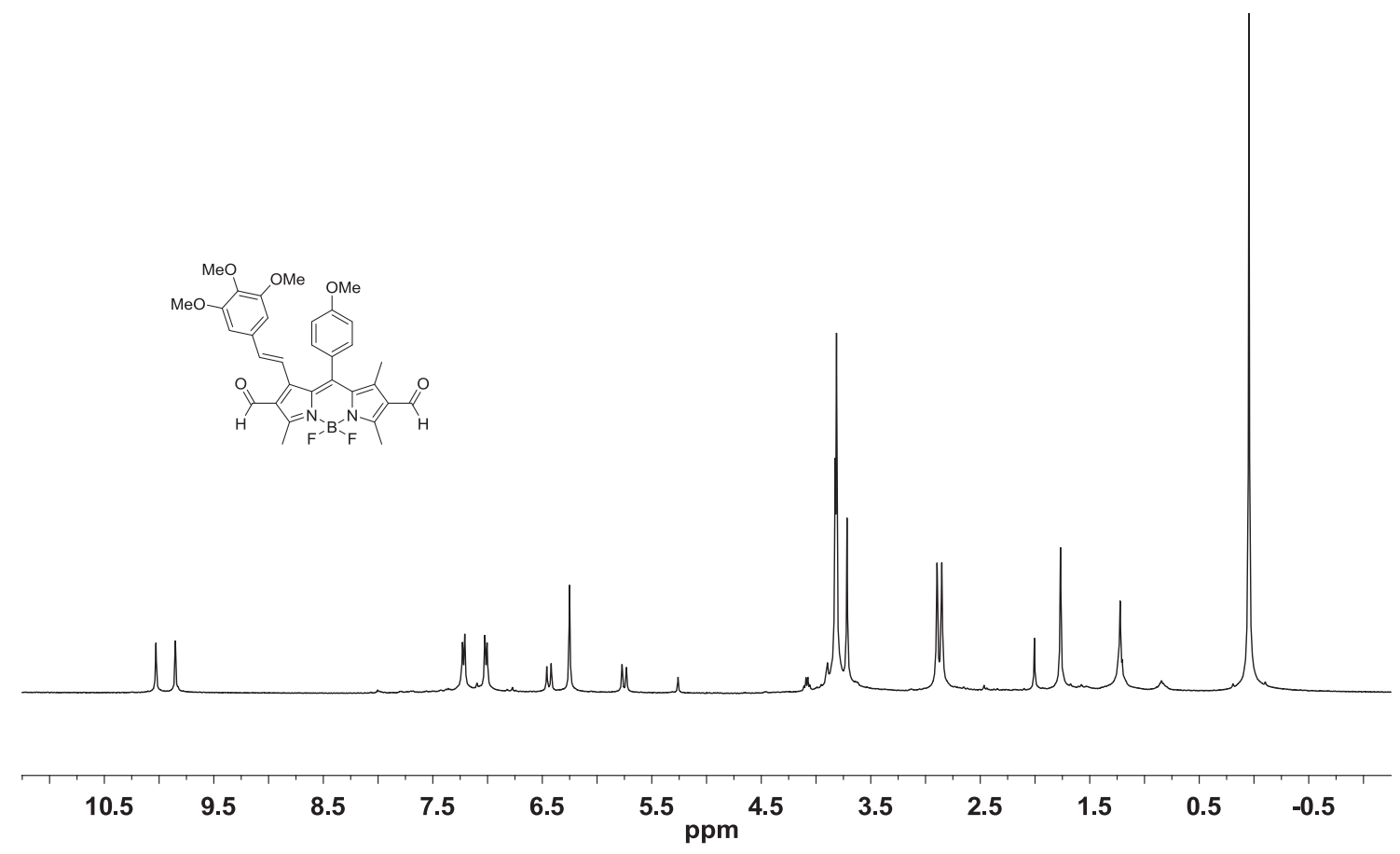

Figure B.12. ${ }^{1} \mathrm{H}$ NMR spectrum of BODIPY dye 4-Da in $\mathrm{CDCl}_{3}$ solution.

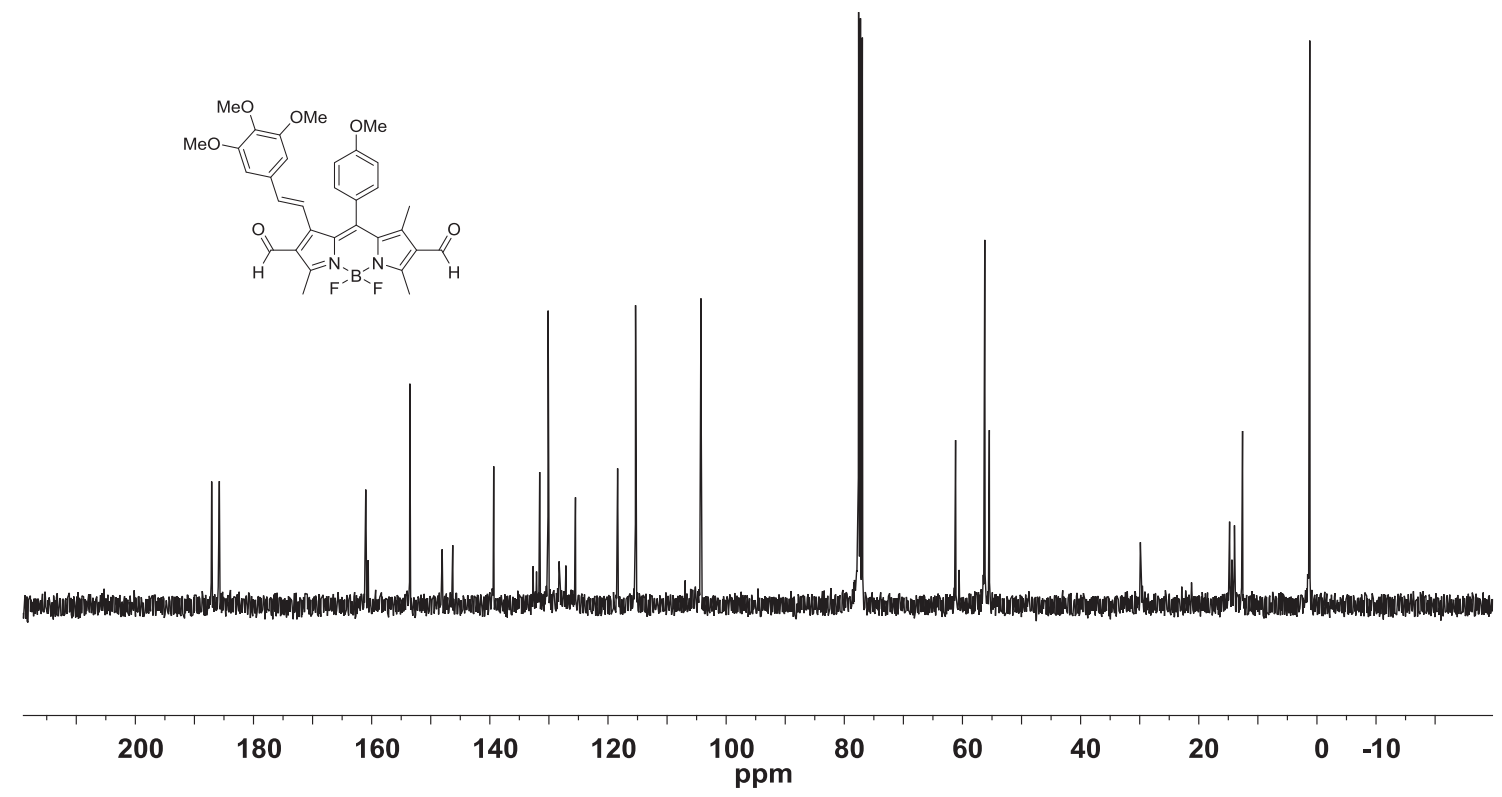

Figure B.13. ${ }^{13} \mathrm{C}$ NMR spectrum of BODIPY dye 4-Da in $\mathrm{CDCl}_{3}$ solution. 


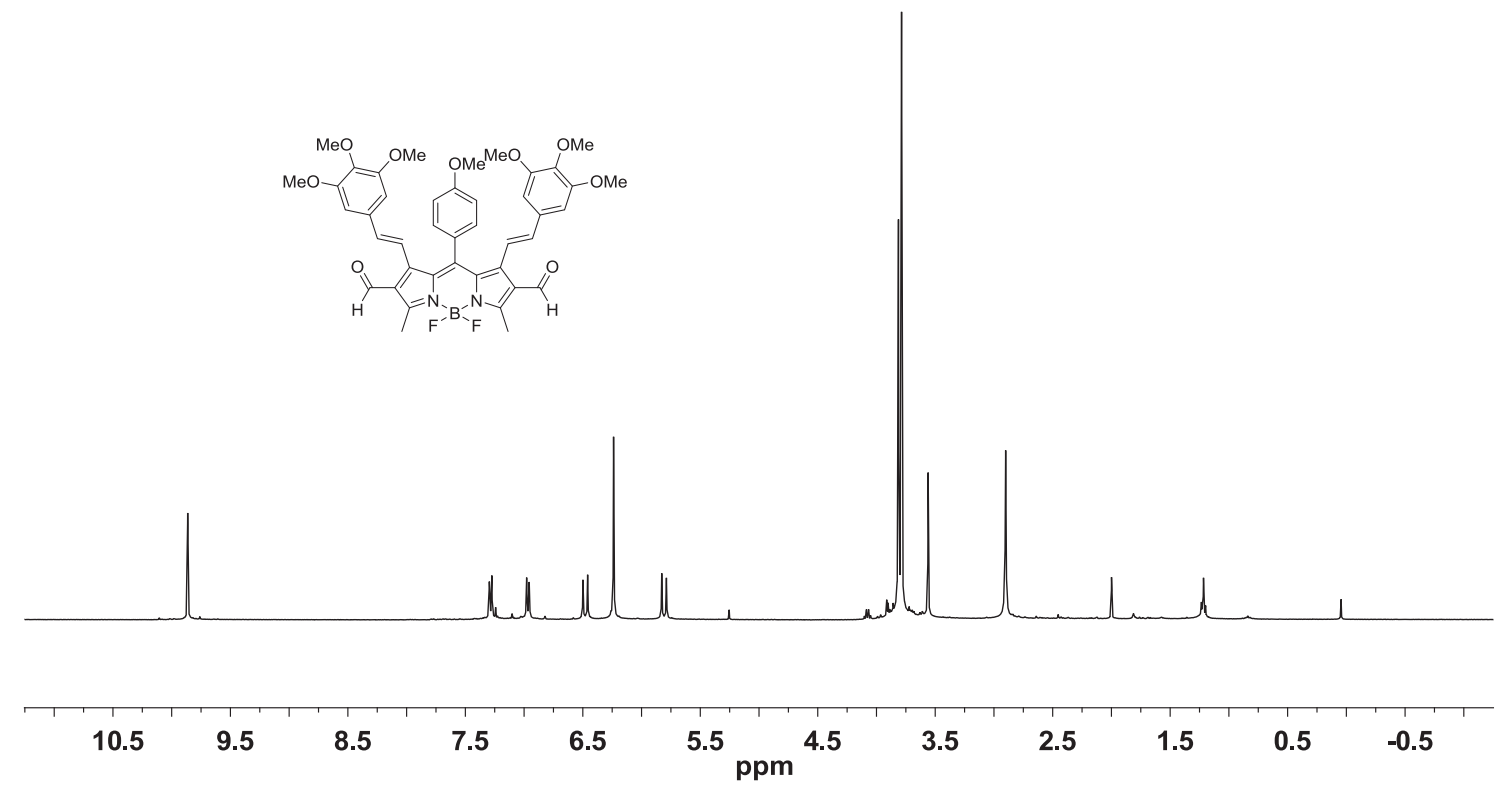

Figure B.14. ${ }^{1} \mathrm{H}$ NMR spectrum of BODIPY dye 4-Ea in $\mathrm{CDCl}_{3}$ solution.

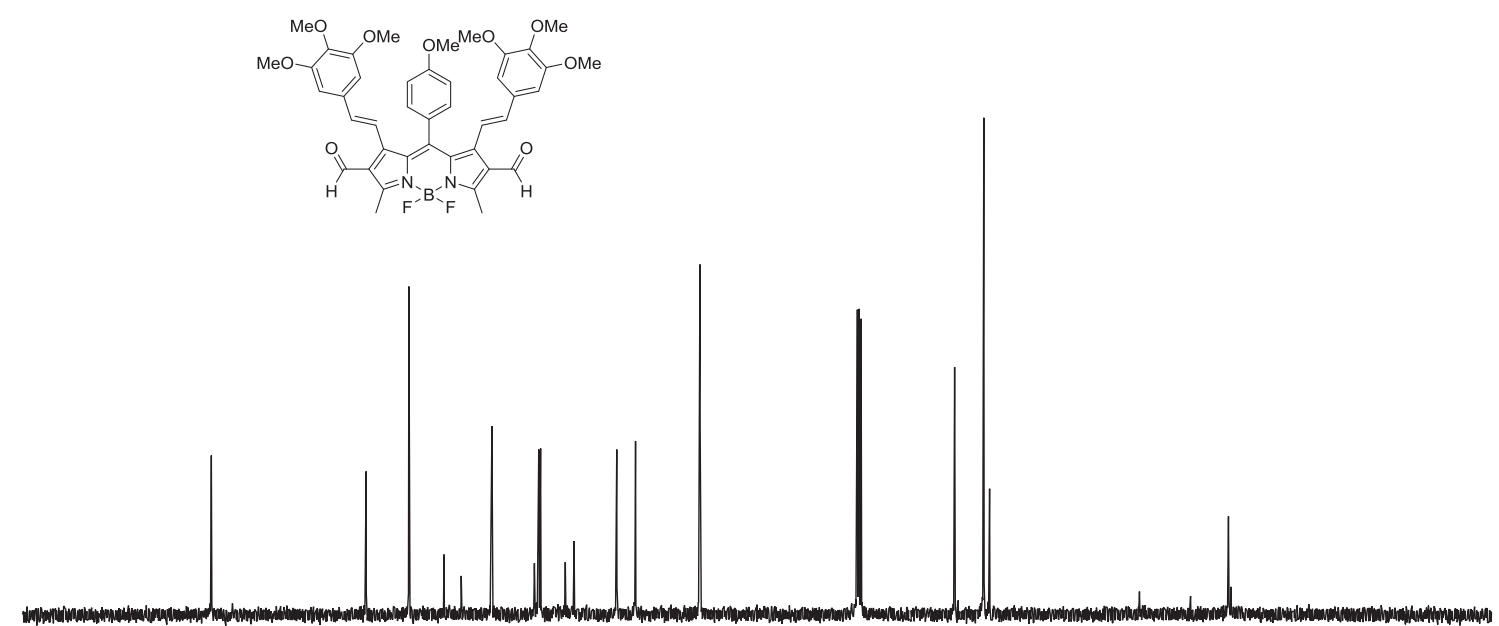

\begin{tabular}{llllllllllll}
\hline 200 & 180 & 160 & 140 & 120 & $\underset{\mathrm{ppm}}{100}$ & 80 & 60 & 40 & 20 & 0 & -10
\end{tabular}

Figure B.15. ${ }^{13} \mathrm{C}$ NMR spectrum of BODIPY dye 4-E $\mathbf{a}$ in $\mathrm{CDCl}_{3}$ solution. 


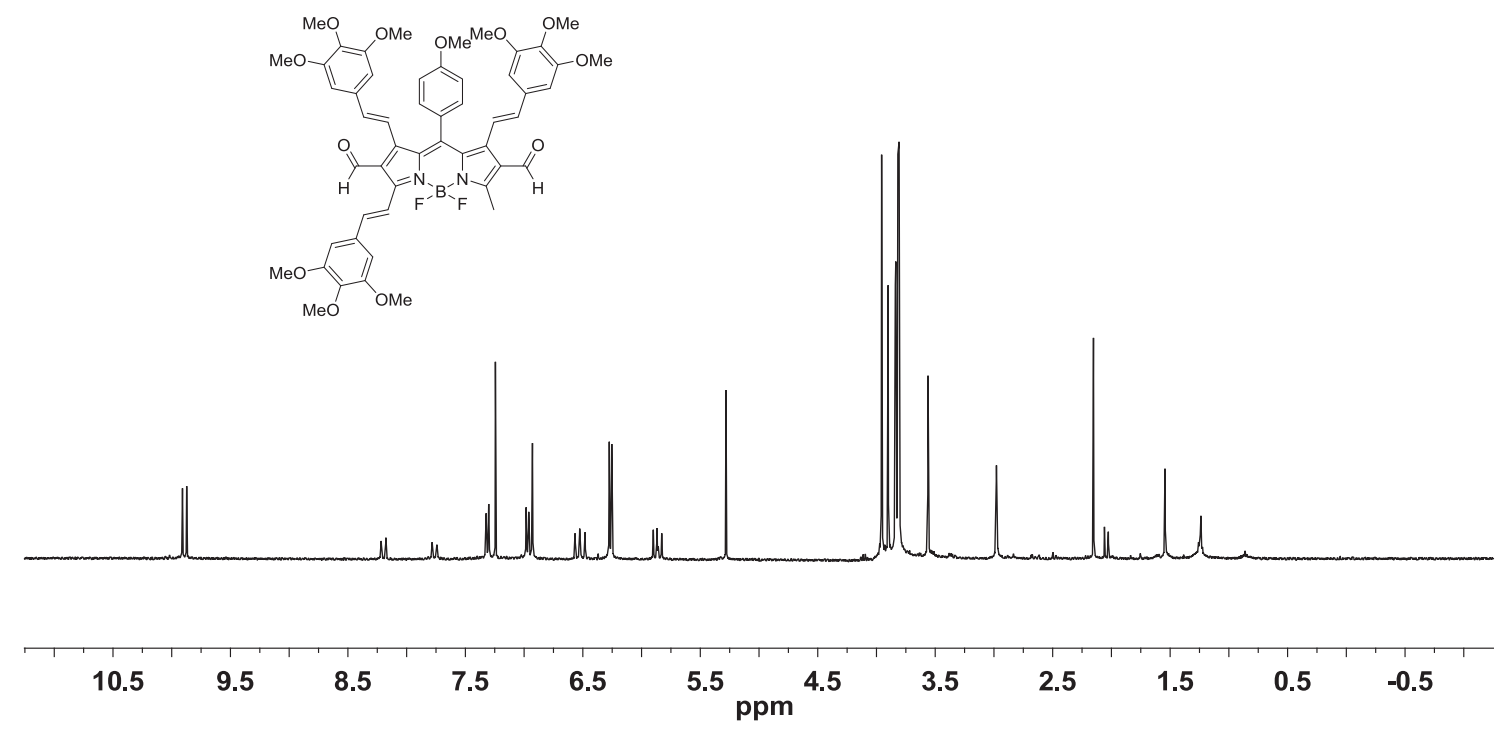

Figure B.16. ${ }^{1} \mathrm{H}$ NMR spectrum of BODIPY dye 4-Fa in $\mathrm{CDCl}_{3}$ solution.

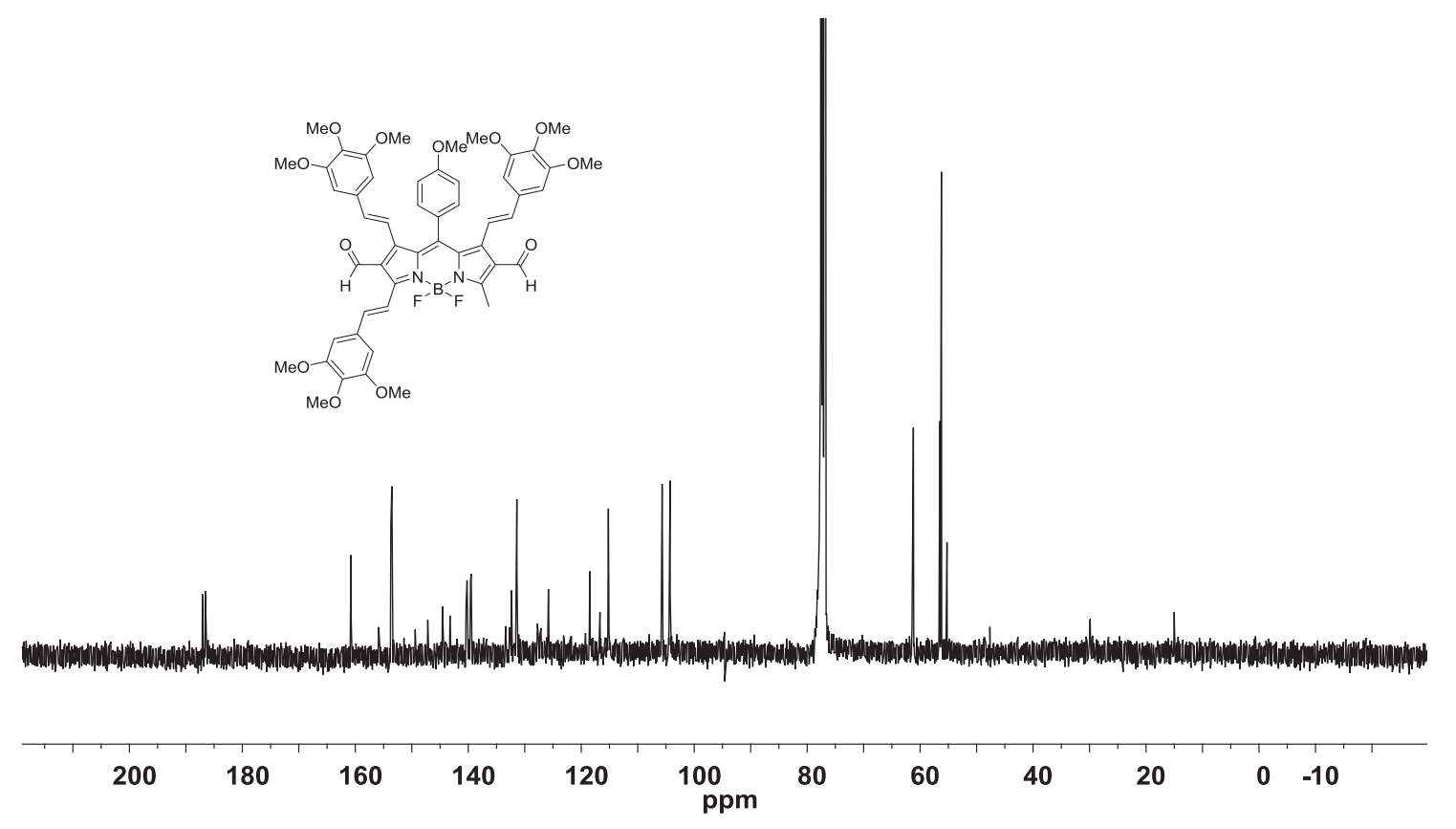

Figure B.17. ${ }^{13} \mathrm{C}$ NMR spectrum of BODIPY dye 4-F $\mathbf{a}$ in $\mathrm{CDCl}_{3}$ solution. 


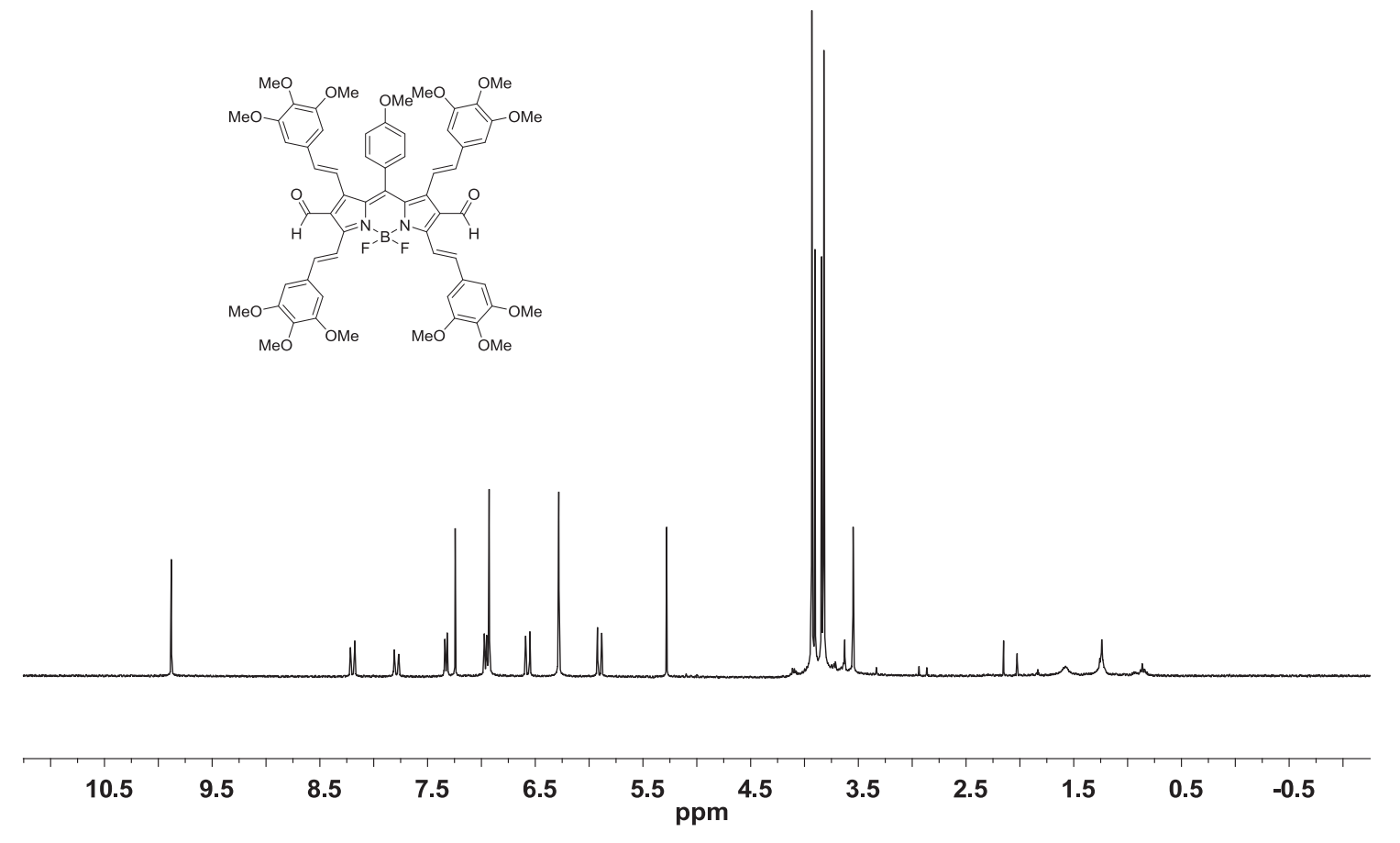

Figure B.18. ${ }^{1} \mathrm{H}$ NMR spectrum of BODIPY dye $\mathbf{4 - \mathbf { G } _ { \mathbf { a } }}$ in $\mathrm{CDCl}_{3}$ solution.
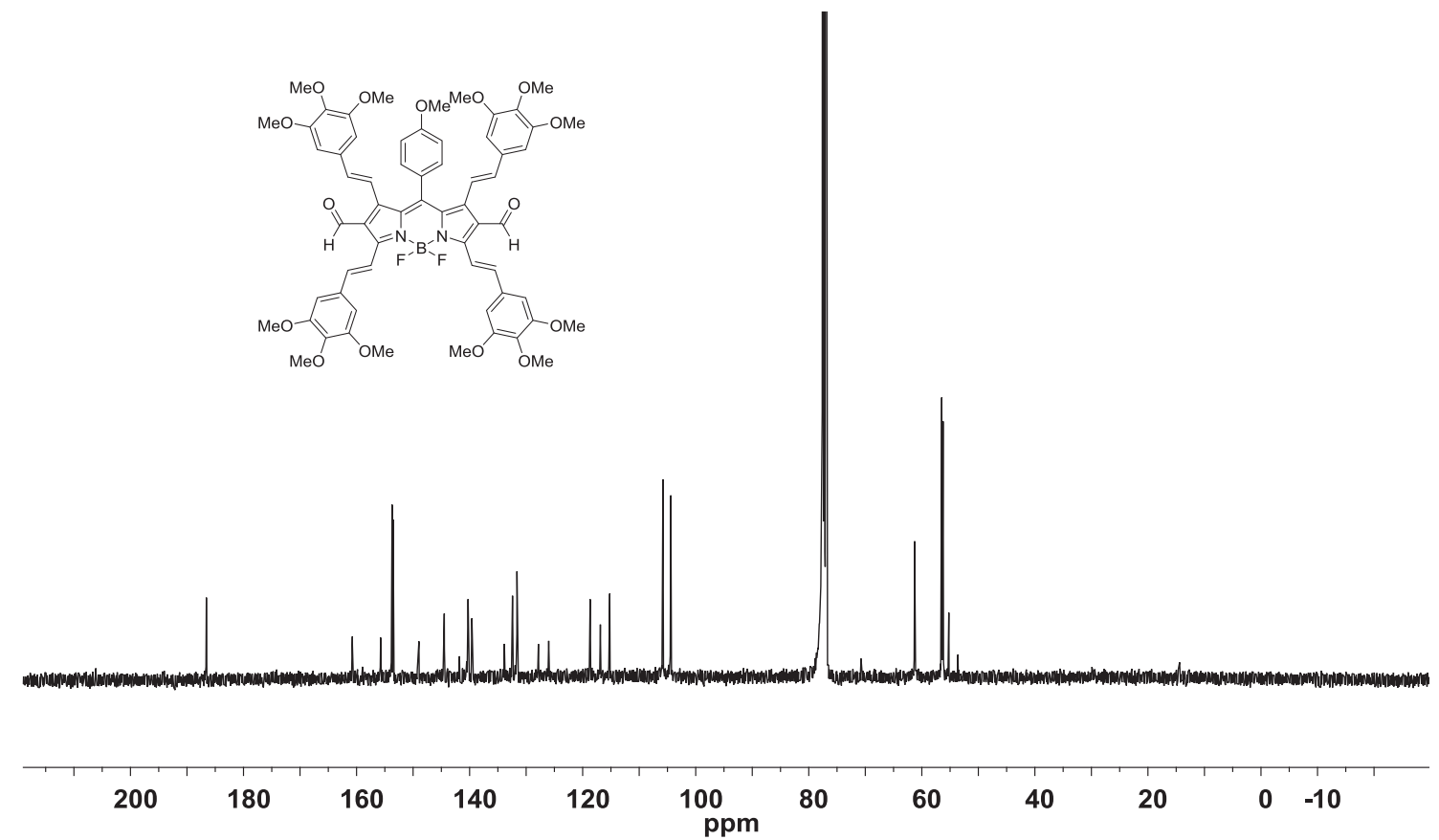

Figure B.19. ${ }^{13} \mathrm{C}$ NMR spectrum of BODIPY dye $\mathbf{4 - G} \mathbf{a}$ in $\mathrm{CDCl}_{3}$ solution. 


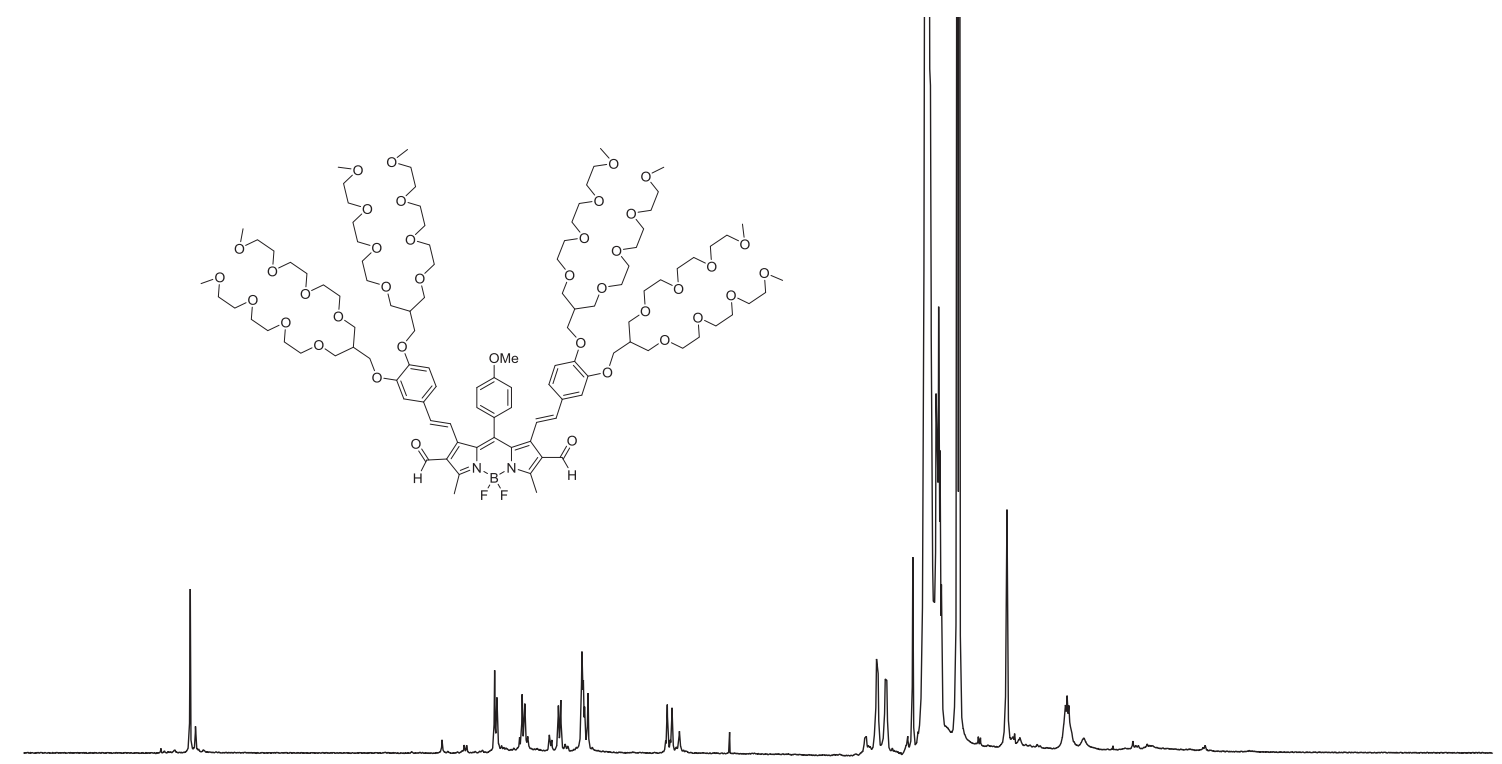

\begin{tabular}{llllllllllll}
\hline 10.5 & 9.5 & 8.5 & 7.5 & 6.5 & 5.5 & & & & & & \\
$\mathrm{ppm}$ & 3.5 & 2.5 & 1.5 & 0.5 & -0.5
\end{tabular}

Figure B.20. ${ }^{1} \mathrm{H}$ NMR spectrum of BODIPY dye 4-E $\mathbf{b}$ in $\mathrm{CDCl}_{3}$ solution.

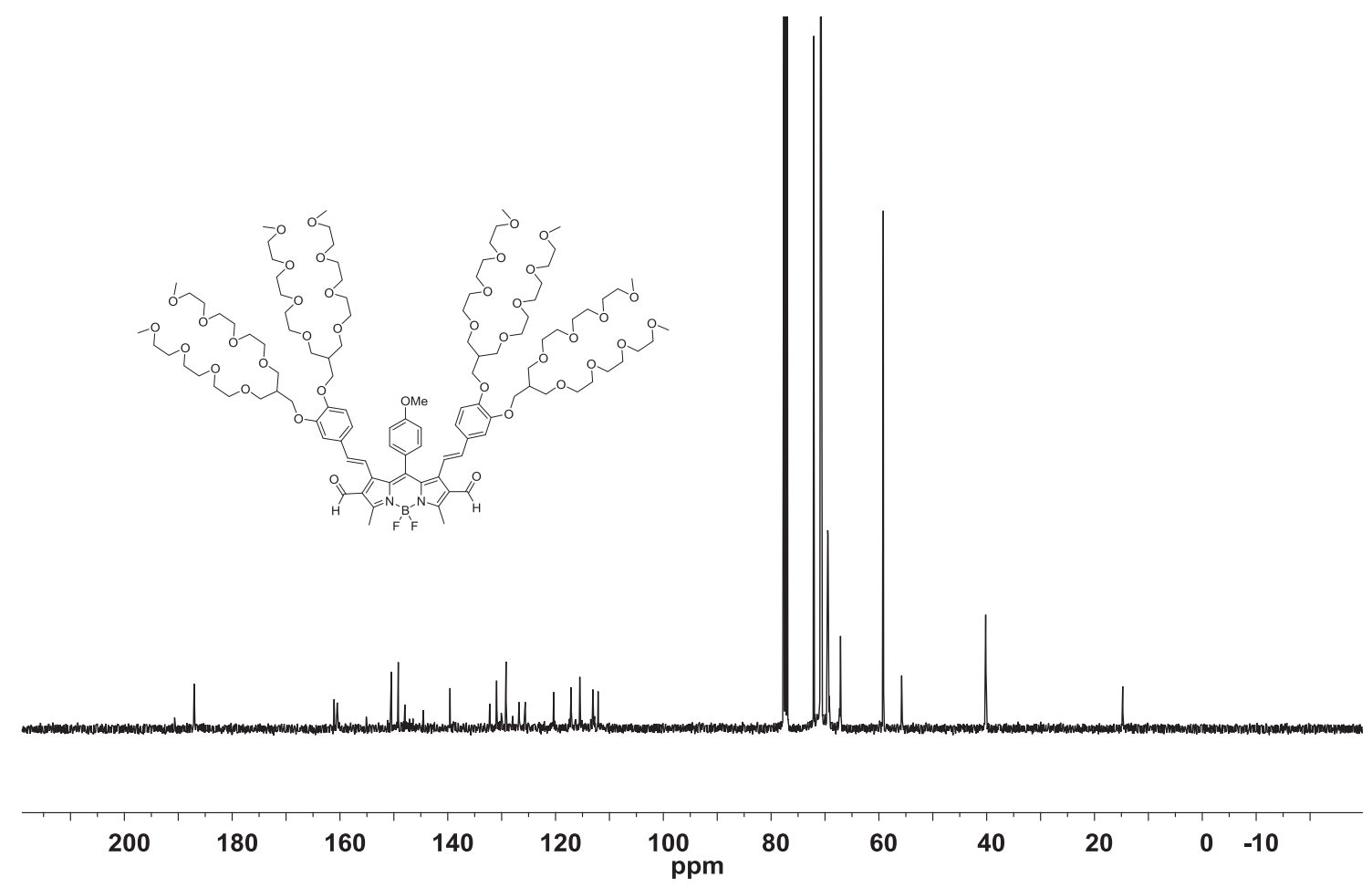

Figure B.21. ${ }^{13} \mathrm{C}$ NMR spectrum of BODIPY dye 4-E $\mathbf{E}$ in $\mathrm{CDCl}_{3}$ solution. 

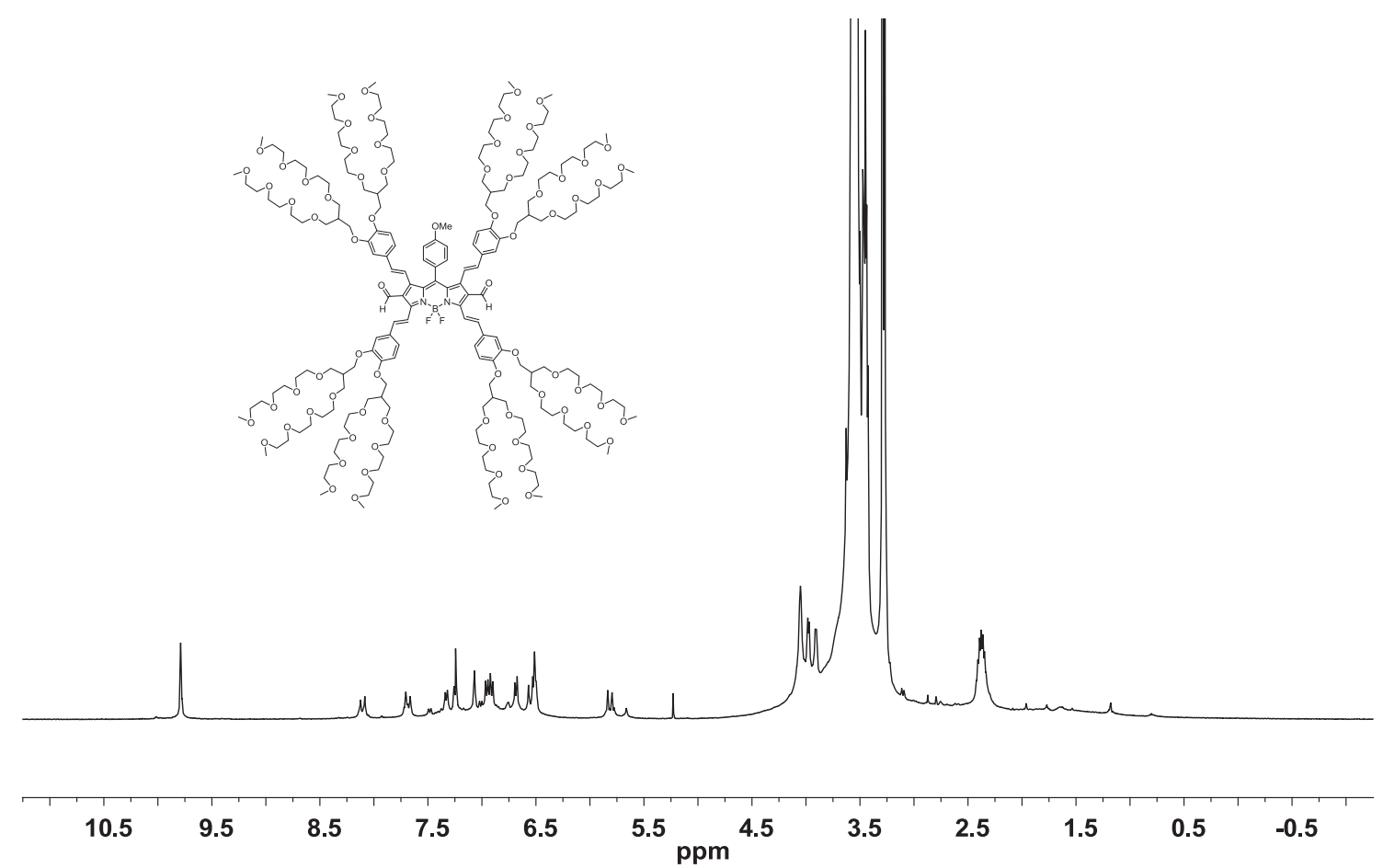

Figure B.22. ${ }^{1} \mathrm{H}$ NMR spectrum of BODIPY dye $\mathbf{4 - \mathbf { G } _ { \mathbf { b } }}$ in $\mathrm{CDCl}_{3}$ solution.

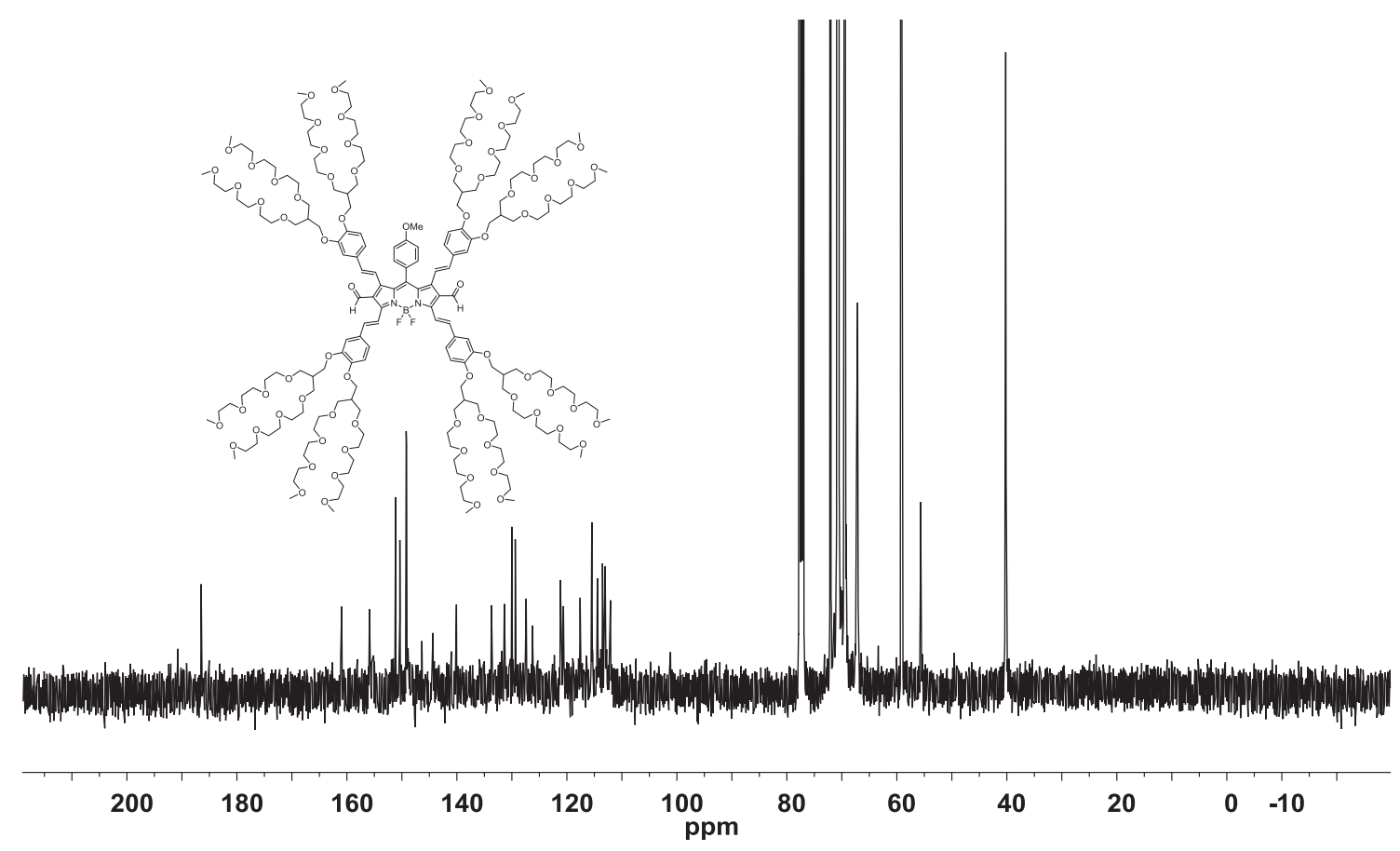

Figure B.23. ${ }^{13} \mathrm{C}$ NMR spectrum of BODIPY dye $\mathbf{4 - G} \mathbf{G}_{\mathbf{b}}$ in $\mathrm{CDCl}_{3}$ solution. 

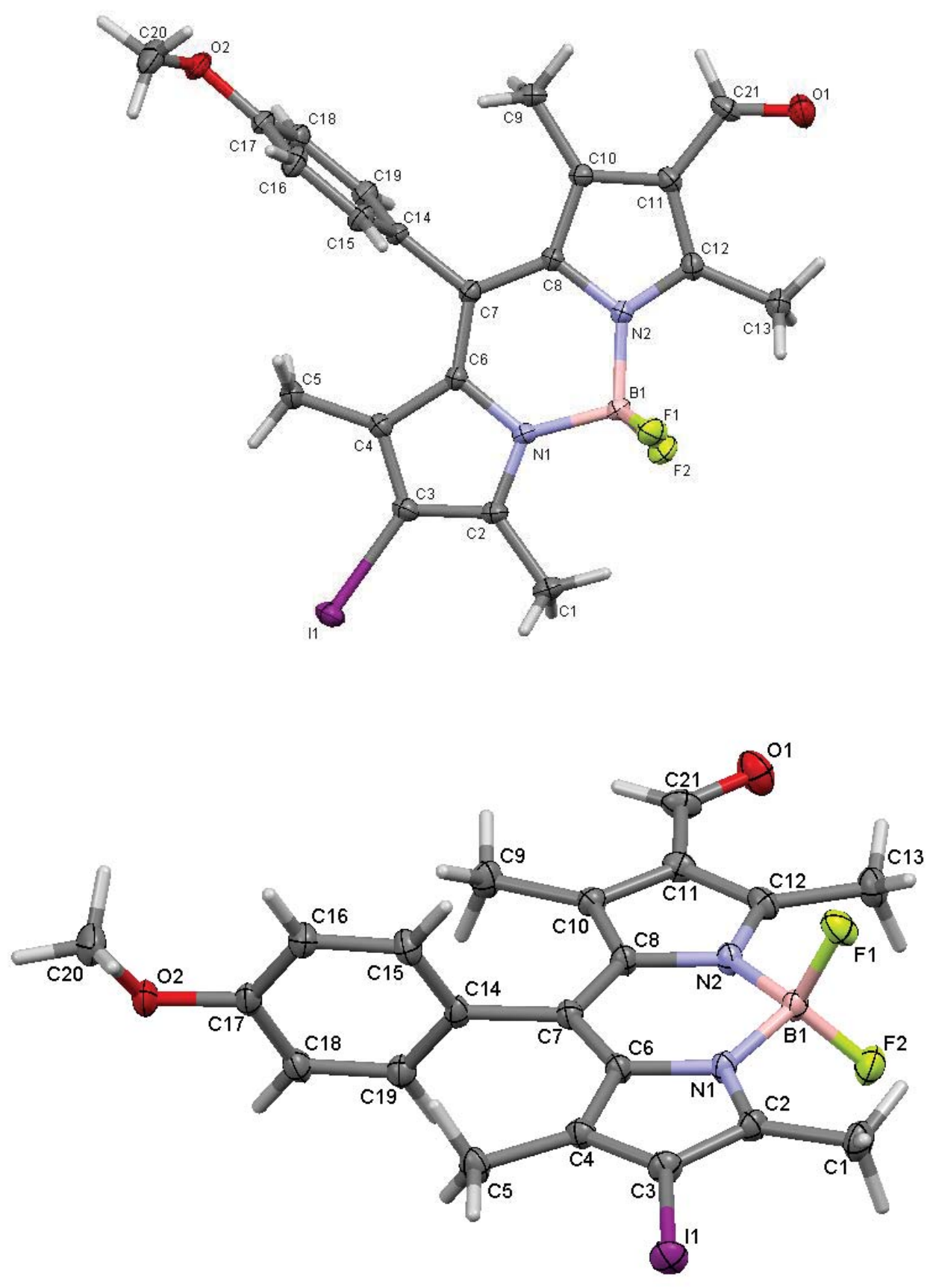

Figure B.24. X-ray structure of BODIPY dye 4.4 


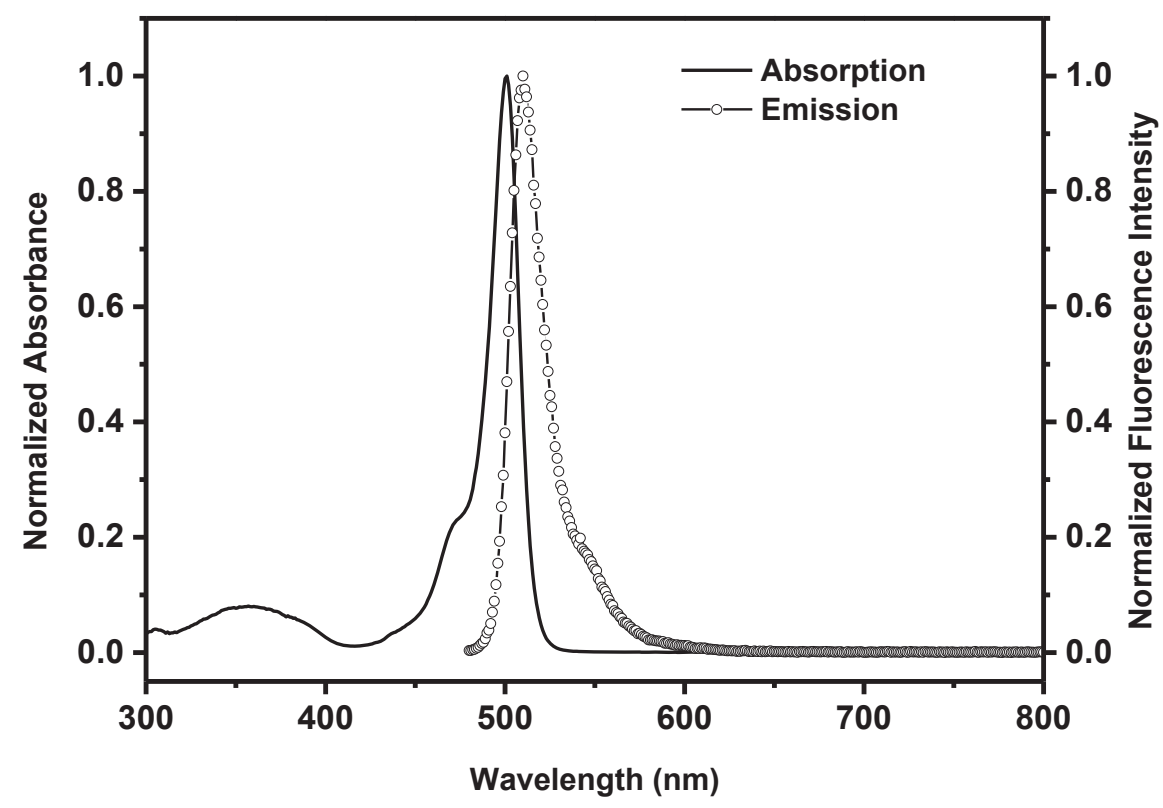

Figure B.25. Normalized absorption and emission spectra of BODIPY dye $\mathbf{4 . 2}$ in methylene chloride solution $\left(\lambda_{\mathrm{ex}}=470 \mathrm{~nm}\right)$.

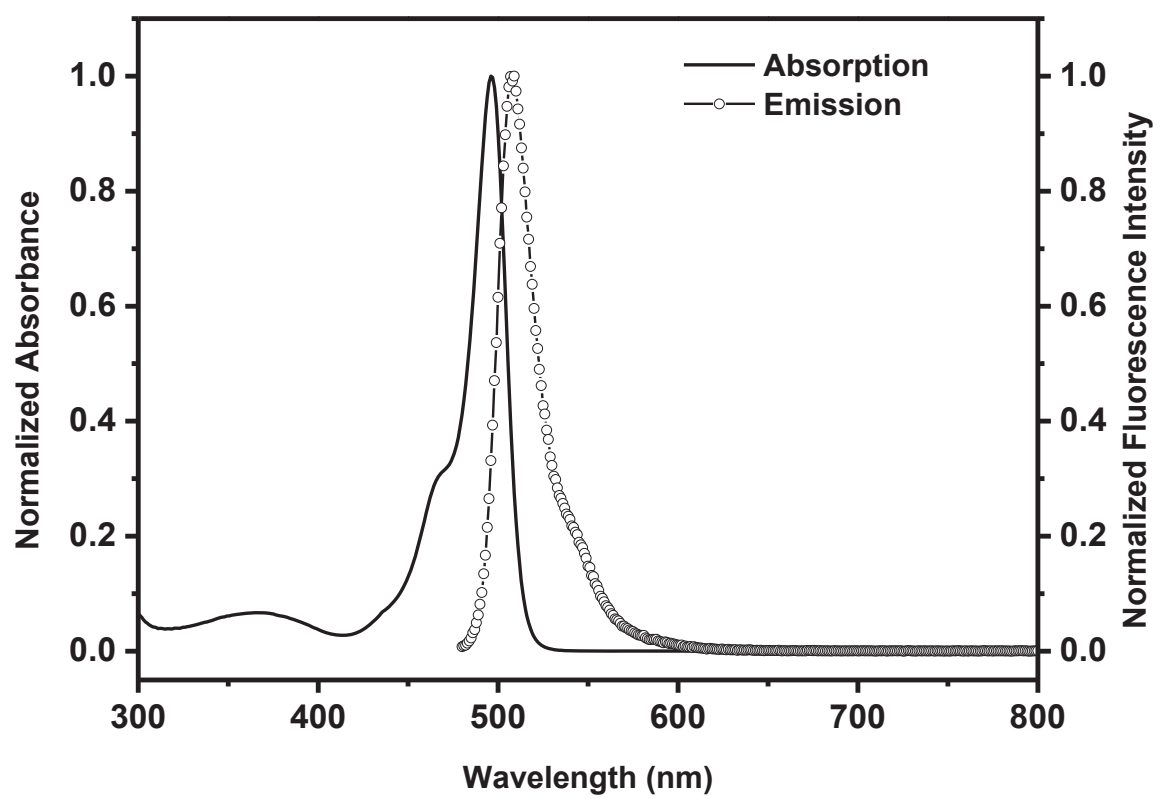

Figure B.26. Normalized absorption and emission spectra of BODIPY dye $\mathbf{4 . 3}$ in methylene chloride solution $\left(\left(\lambda_{\mathrm{ex}}=470 \mathrm{~nm}\right)\right.$. 


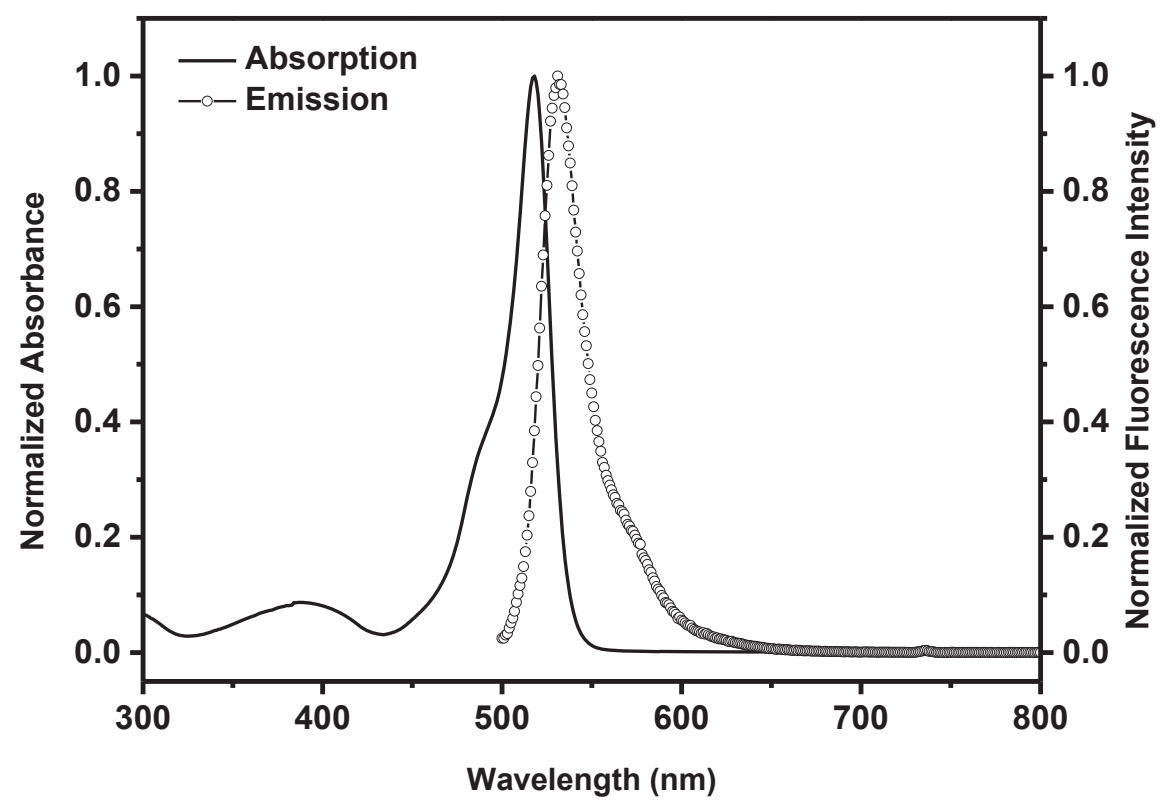

Figure B.27. Normalized absorption and emission spectra of BODIPY dye $\mathbf{4 . 4}$ in methylene chloride solution $\left(\left(\lambda_{\mathrm{ex}}=490 \mathrm{~nm}\right)\right.$

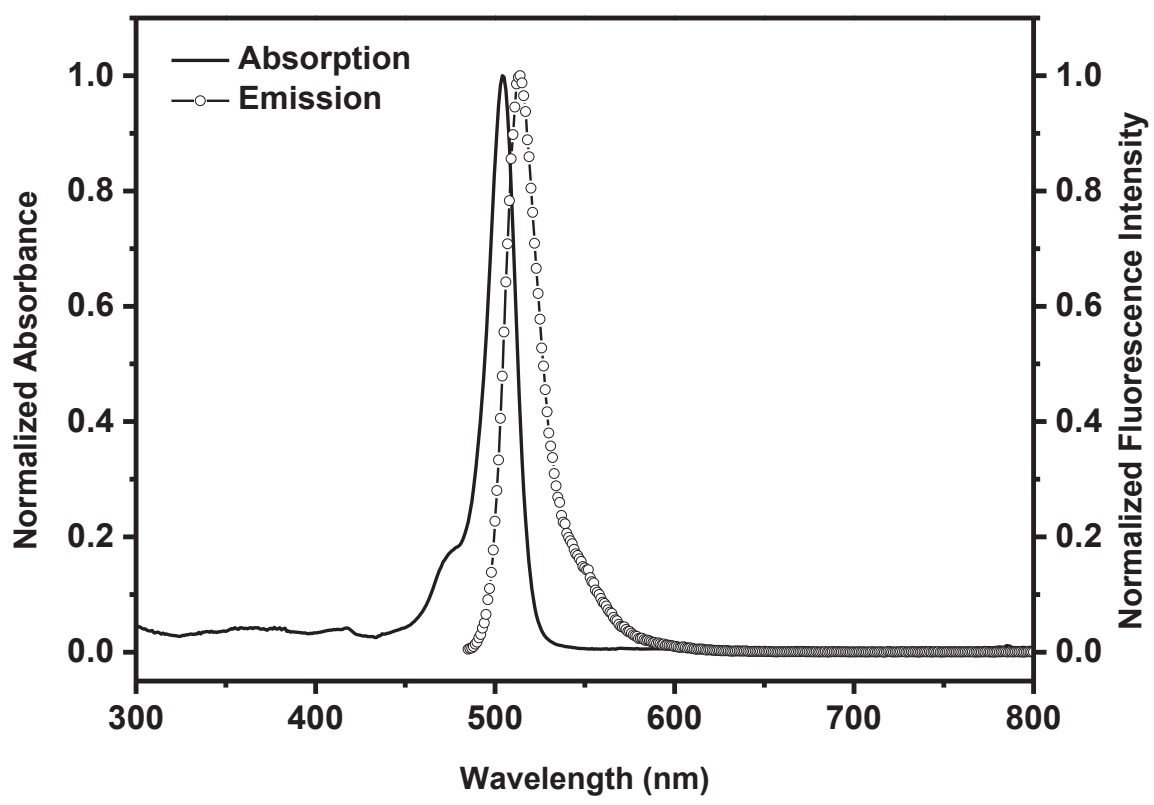

Figure B.28. Normalized absorption and emission spectra of BODIPY dye $\mathbf{4 . 6}$ in methylene chloride solution $\left(\left(\lambda_{\mathrm{ex}}=475 \mathrm{~nm}\right)\right.$ 


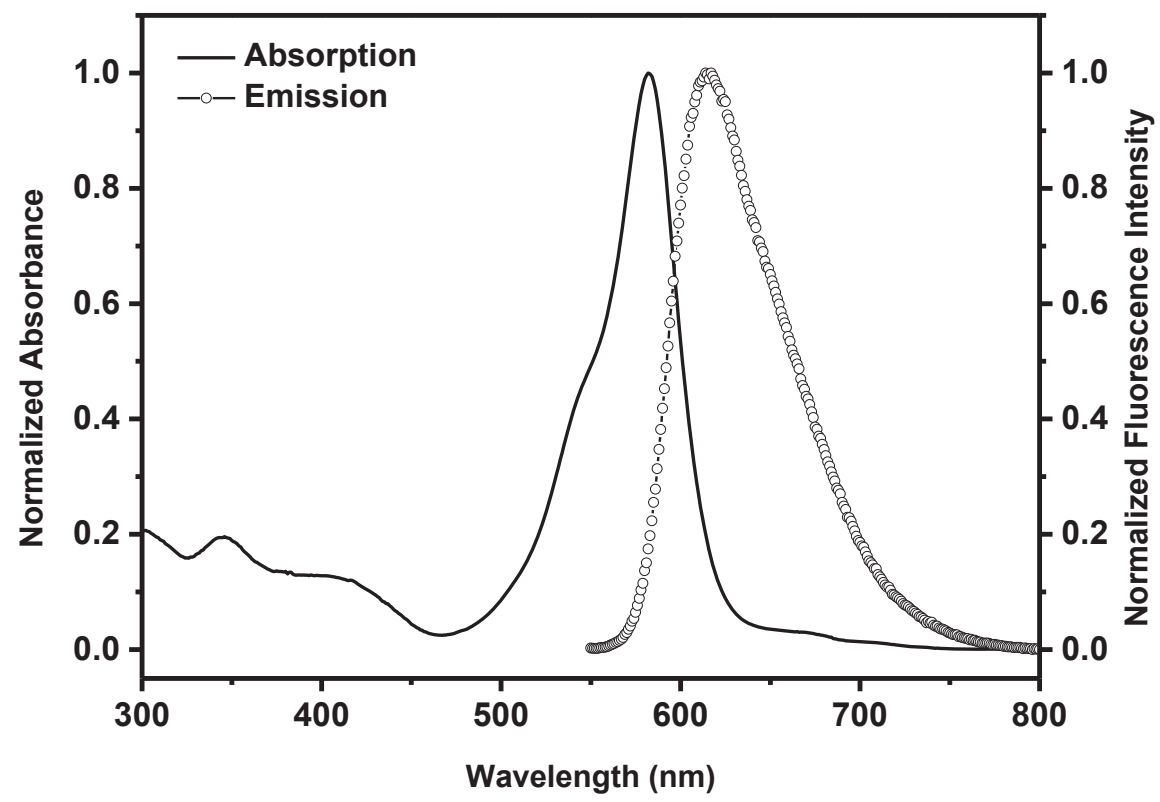

Figure B.29. Normalized absorption and emission spectra of BODIPY dye $\mathbf{4 - A}$ in methylene chloride solution $\left(\left(\lambda_{\mathrm{ex}}=540 \mathrm{~nm}\right)\right.$

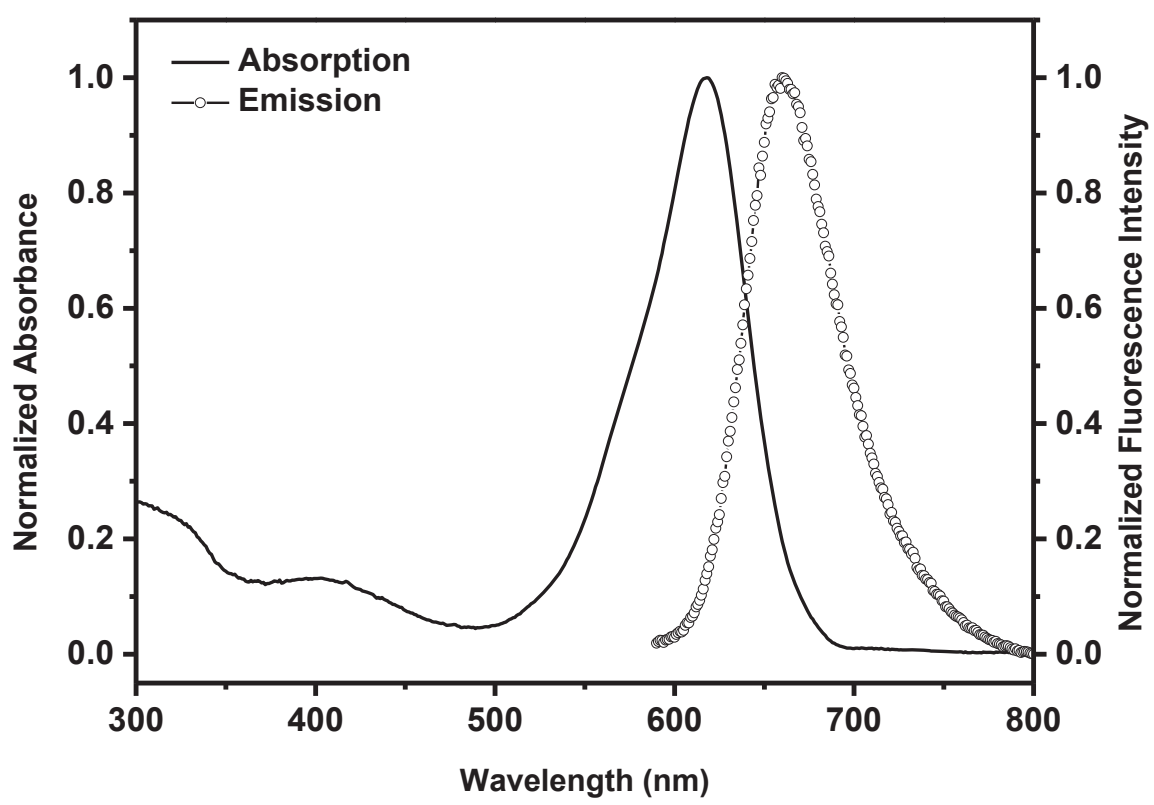

Figure B.30. Normalized absorption and emission spectra of BODIPY dye 4-B in methylene chloride solution $\left(\left(\lambda_{\mathrm{ex}}=580 \mathrm{~nm}\right)\right.$ 


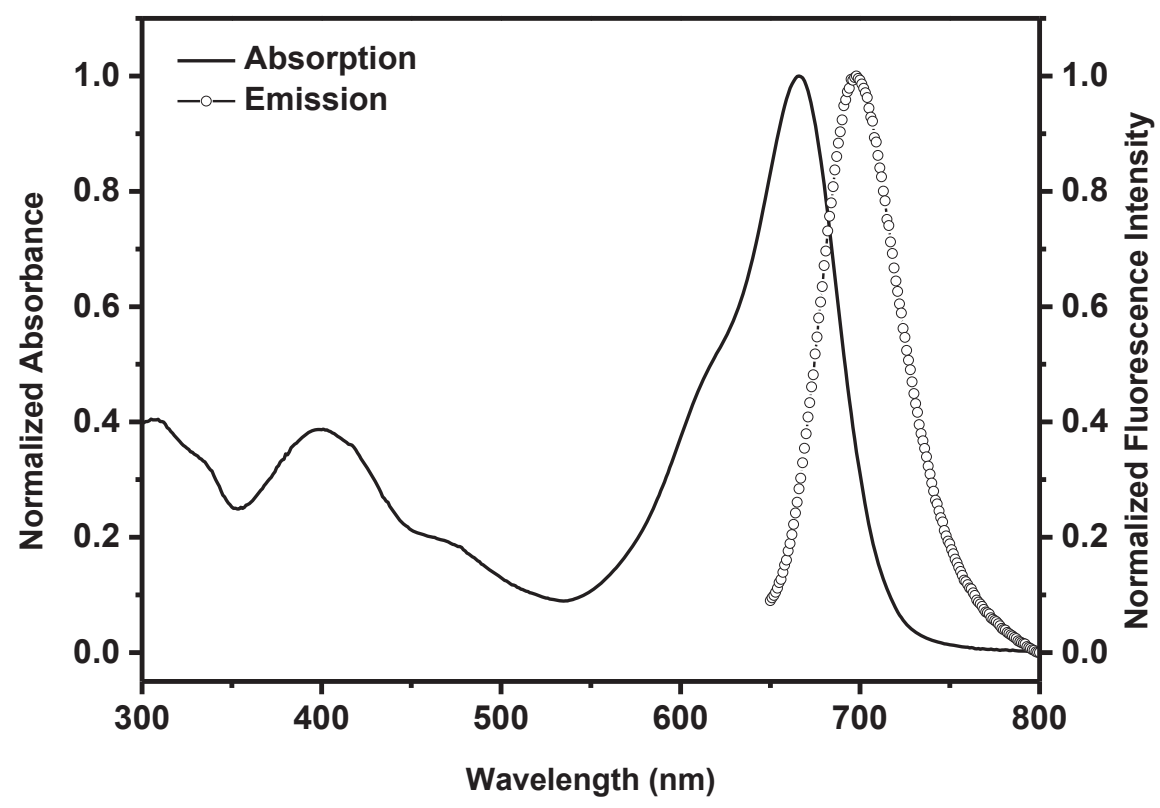

Figure B.31. Normalized absorption and emission spectra of BODIPY dye 4-C in methylene chloride solution $\left(\left(\lambda_{\mathrm{ex}}=640 \mathrm{~nm}\right)\right.$

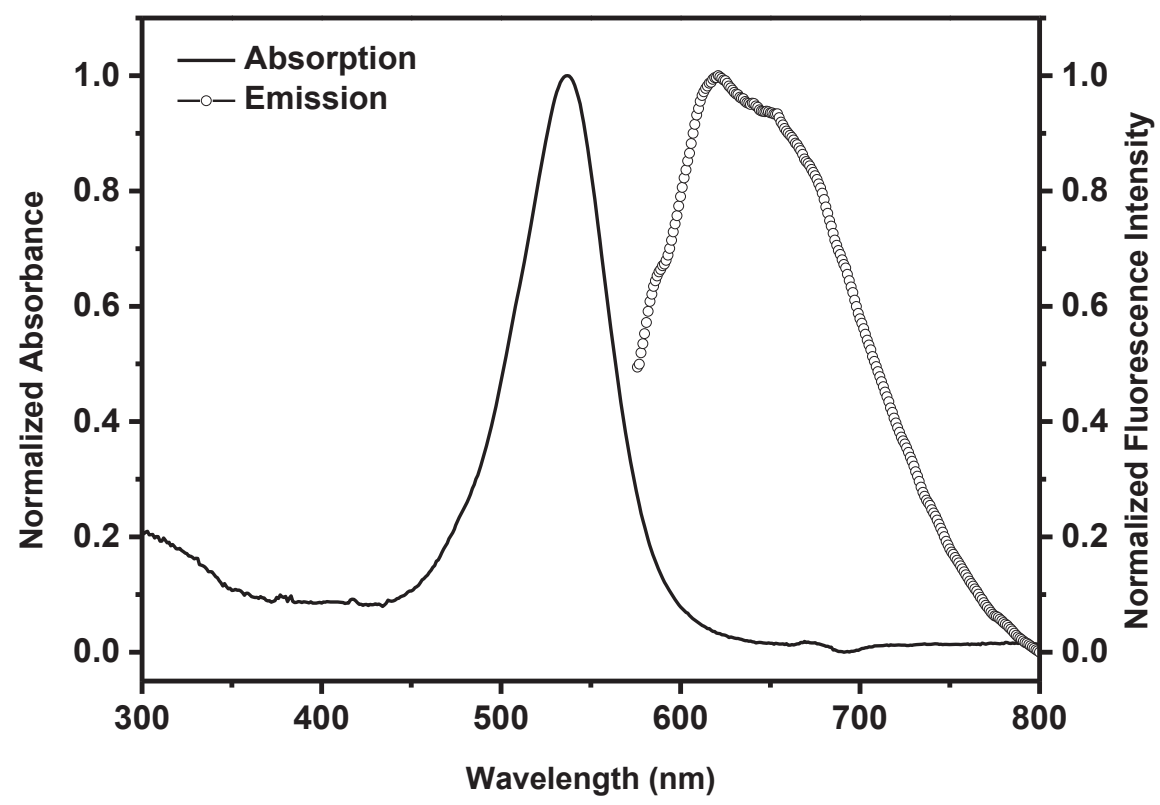

Figure B.32. Normalized absorption and emission spectra of BODIPY dye 4-Da in methylene chloride solution $\left(\lambda_{\mathrm{ex}}=550 \mathrm{~nm}\right)$ 


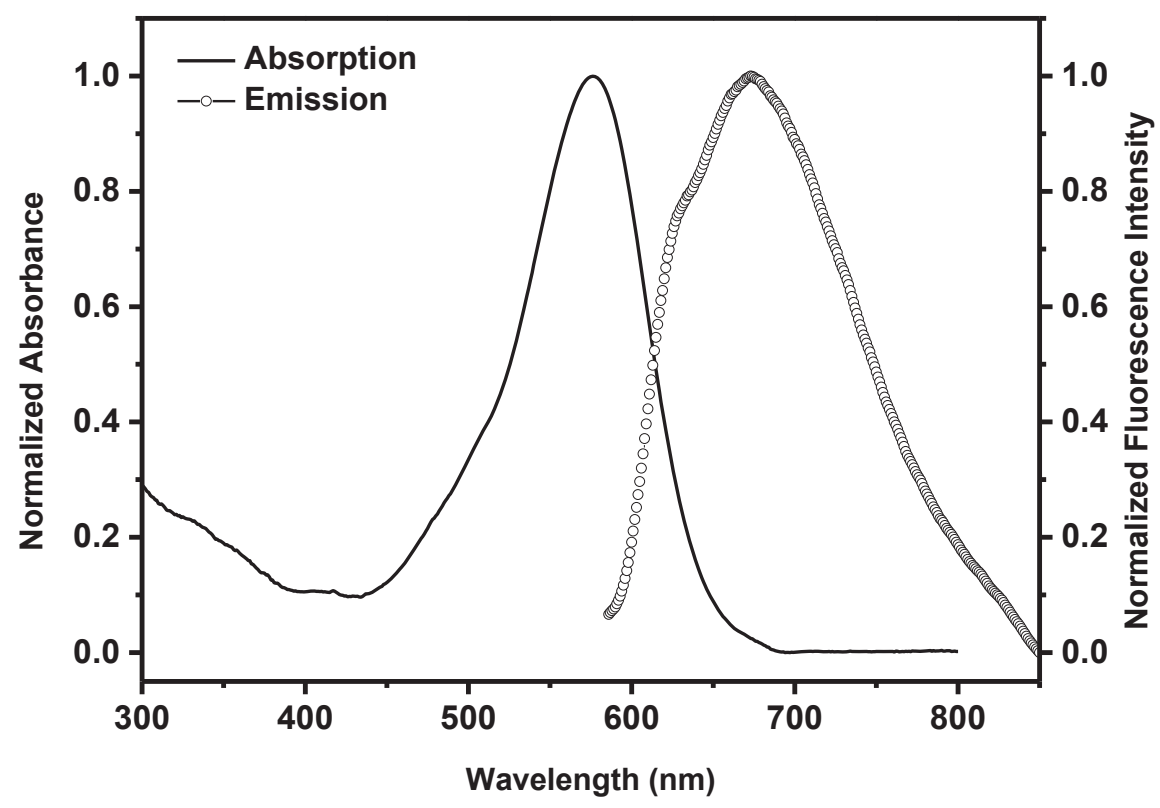

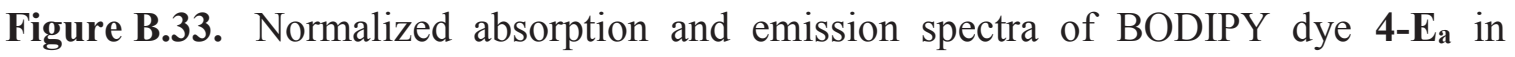
methylene chloride solution $\left(\lambda_{\mathrm{ex}}=560 \mathrm{~nm}\right)$

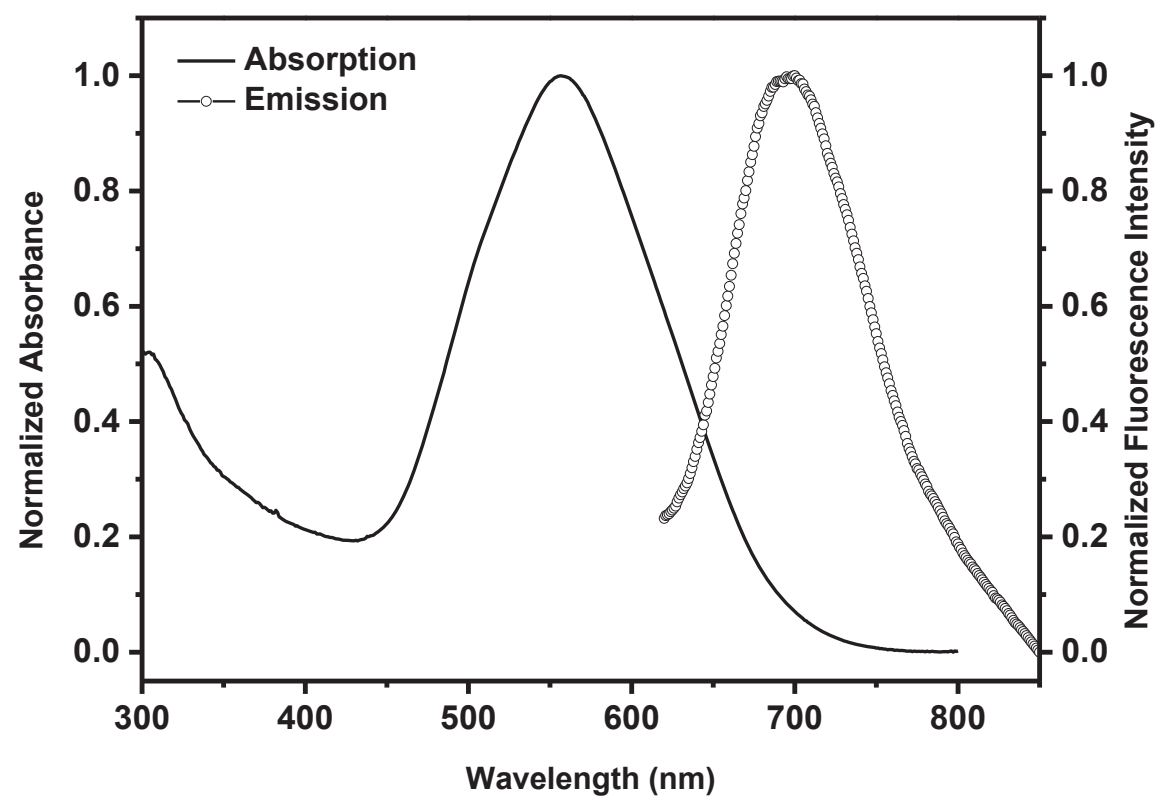

Figure B.34. Normalized absorption and emission spectra of BODIPY dye 4-E $\mathbf{b}(4 \mu \mathrm{M})$ in aqueous solution $\left(\lambda_{\mathrm{ex}}=550 \mathrm{~nm}\right)$. 


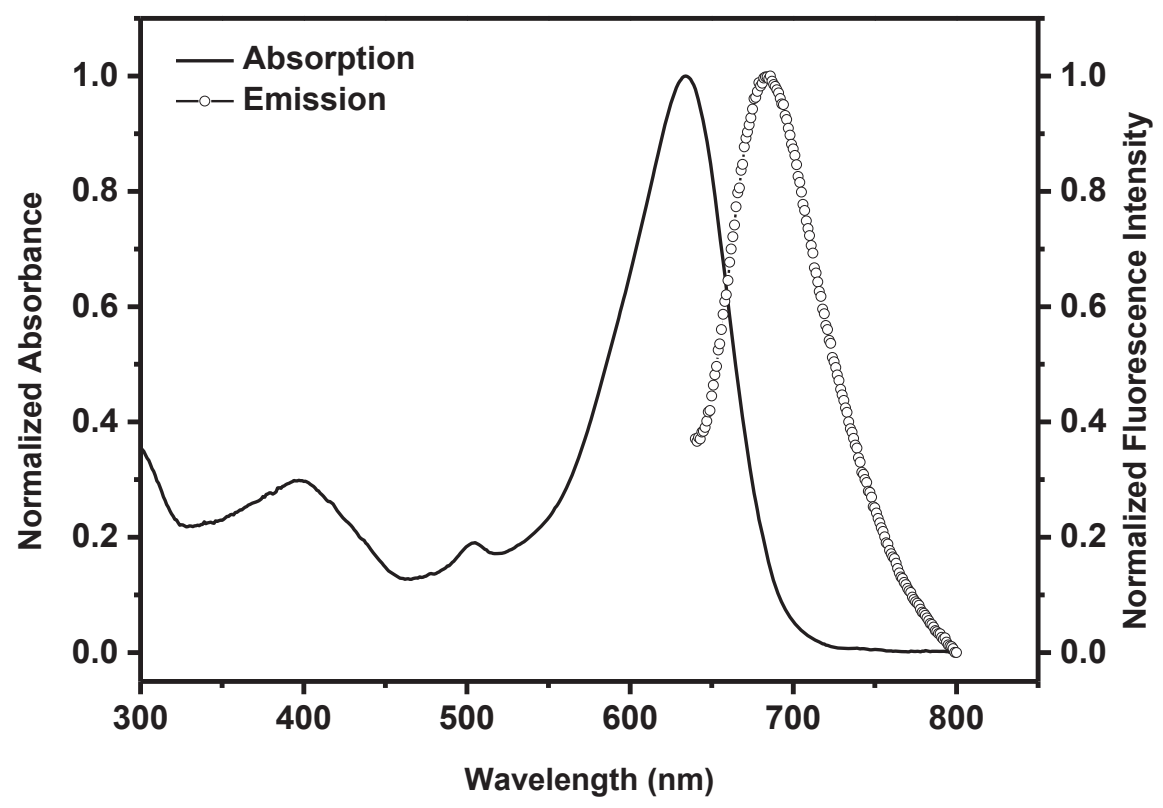

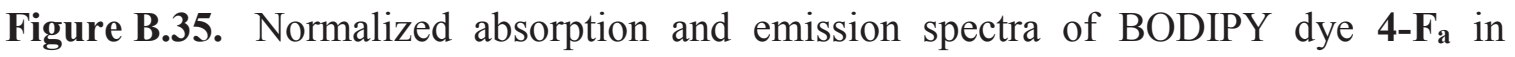
methylene chloride solution $\left(\lambda_{\mathrm{ex}}=630 \mathrm{~nm}\right)$.

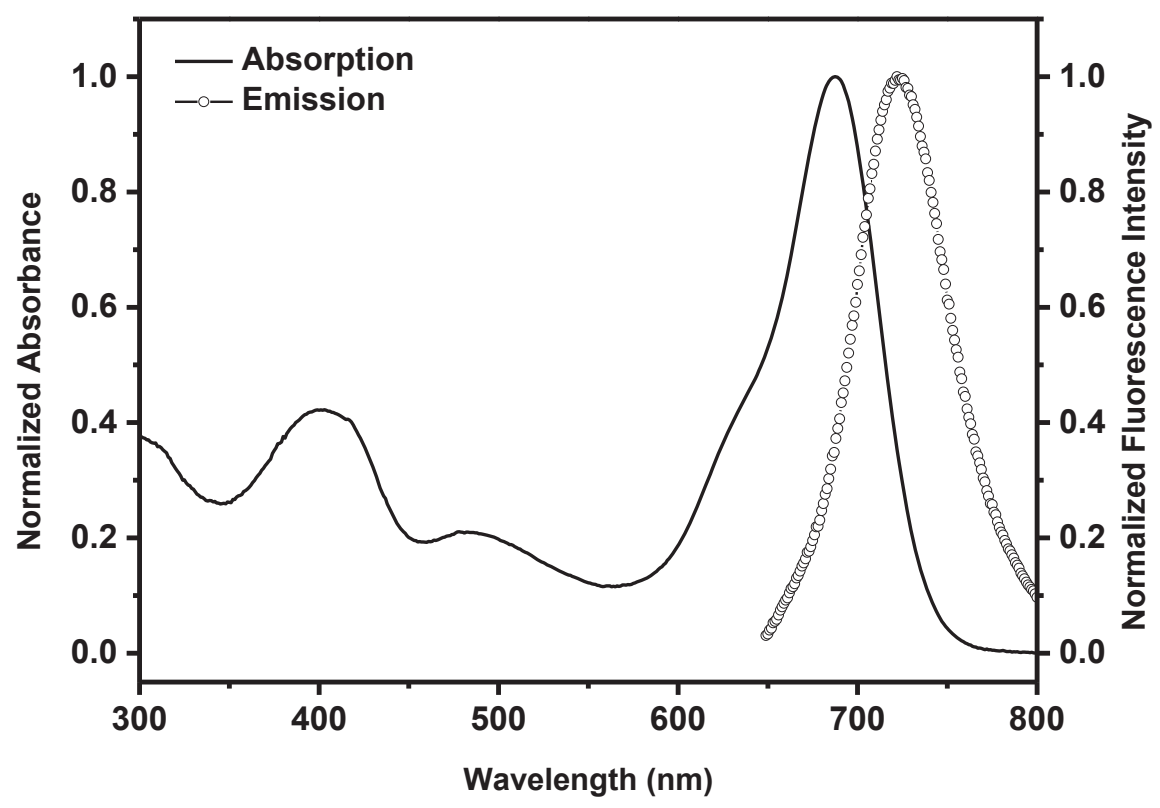

Figure B.36. Normalized absorption and emission spectra of BODIPY dye 4-Ga in methylene chloride solution $\left(\lambda_{\mathrm{ex}}=630 \mathrm{~nm}\right)$. 


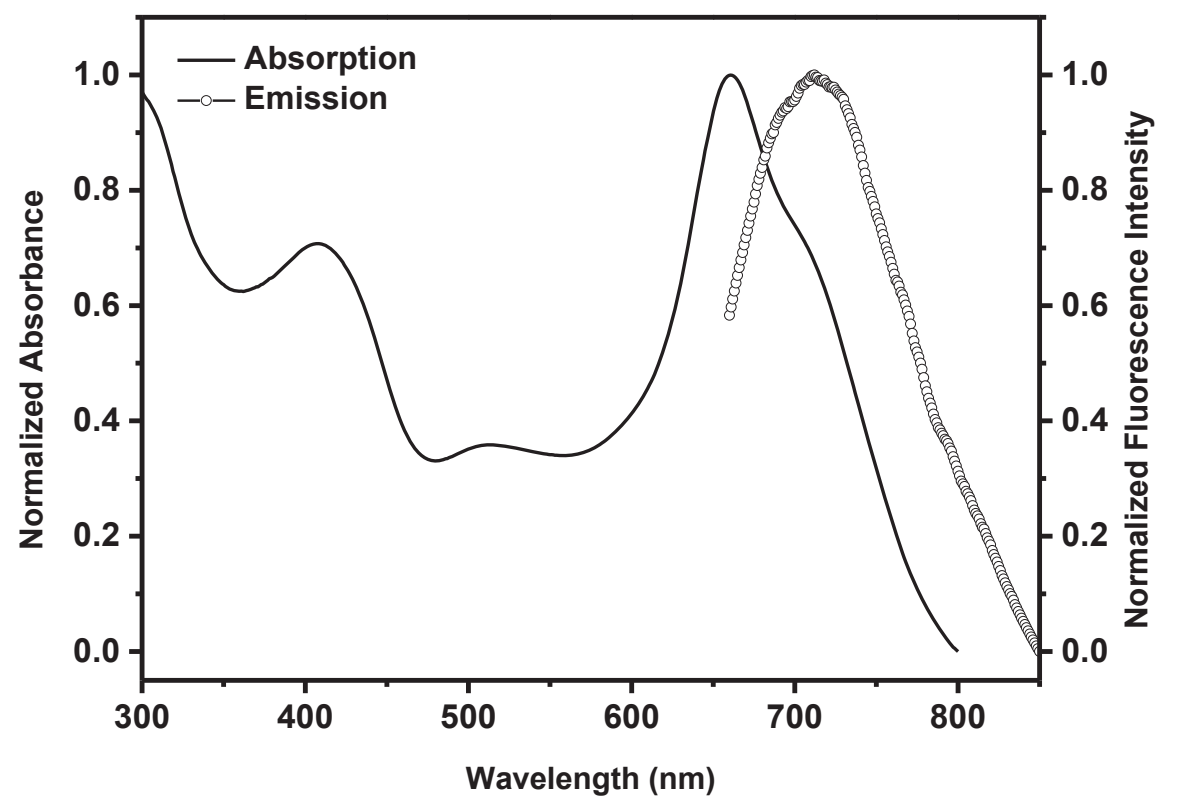

Figure B.37. Normalized absorption and emission spectra of BODIPY dye 4-Gb $(4 \mu \mathrm{M})$ in aqueous solution $\left(\lambda_{\mathrm{ex}}=650 \mathrm{~nm}\right)$. 


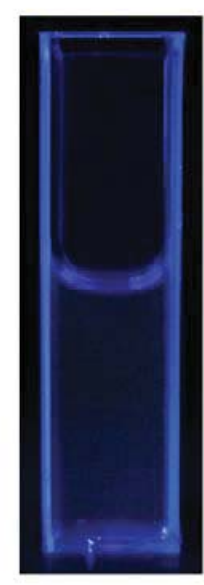

\section{cysteine}

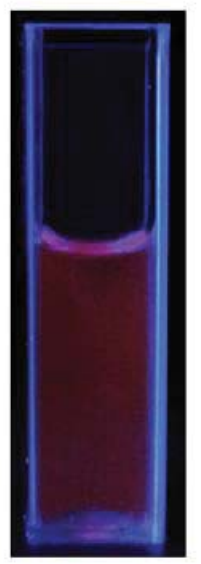

Figure B.38. Visualized Images of BODIPY dye 4-E $\mathbf{E}_{\mathbf{b}}(20 \mu \mathrm{M})$ in $0.01 \mathrm{M}$ PBS buffer $\mathrm{pH} 7.4$ in the absence and presence of L-cysteine $(10 \mathrm{mM})$ at different times. Images were taken under a transilluminator. $\left(\lambda_{\mathrm{ex}}=470 \mathrm{~nm}\right)$
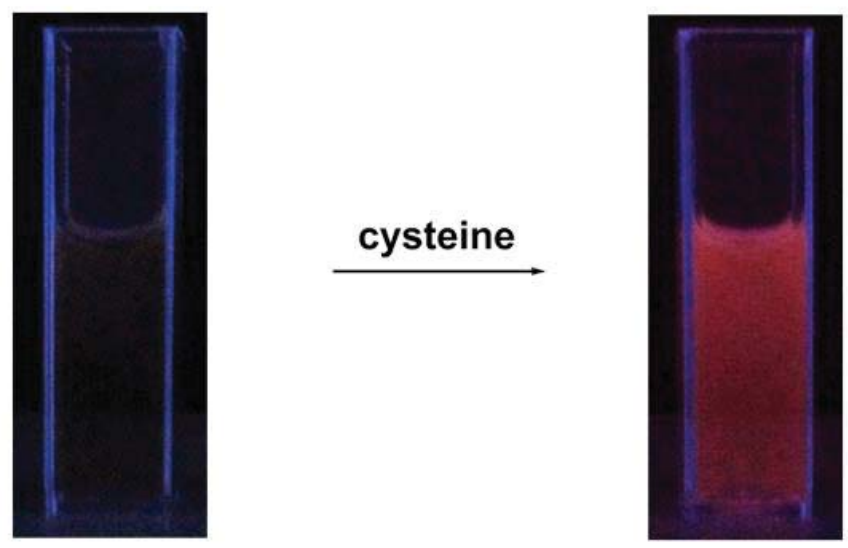

Figure B.39. Visualized images of BODIPY dye 4-Gb $(20 \mu \mathrm{M})$ in $0.01 \mathrm{M}$ PBS buffer $\mathrm{pH} 7.4$ in the absence and presence of L-cysteine $(10 \mathrm{mM})$ at different times. Images were taken under a transilluminator. $\left(\lambda_{\mathrm{ex}}=470 \mathrm{~nm}\right)$ 


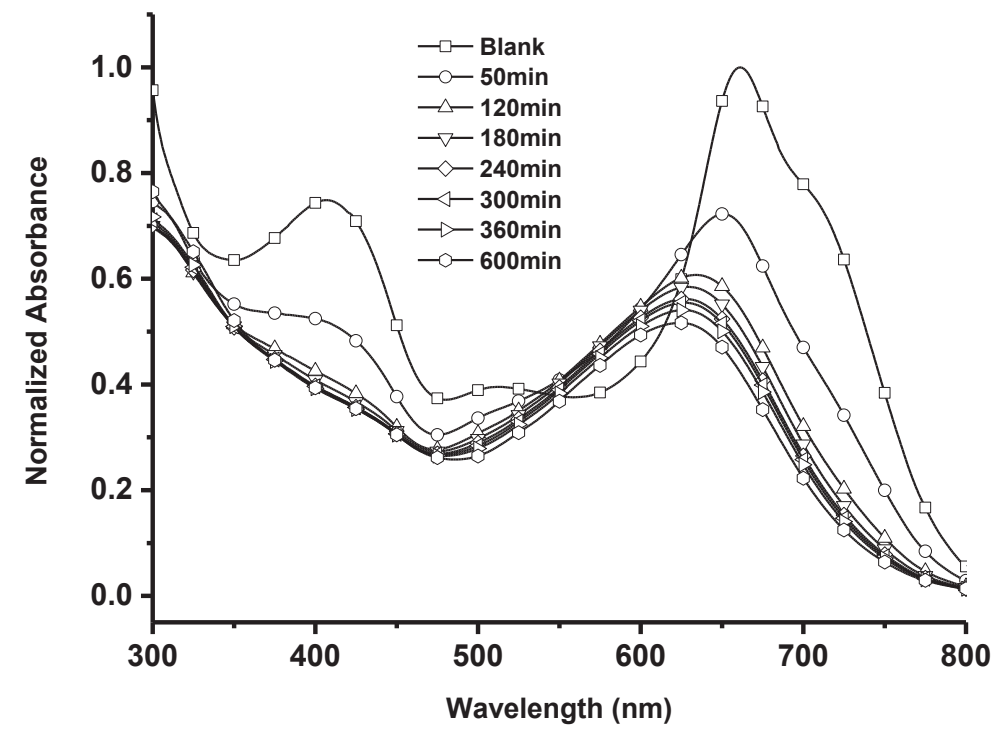

Figure B.40. Normalized absorption spectra of BODIPY dye 4-Gb $(20 \mu \mathrm{M})$ in $0.01 \mathrm{M}$ PBS buffer $\mathrm{pH} 7.4$ in the absence and presence of L-cysteine $(10 \mathrm{mM})$ at different times.

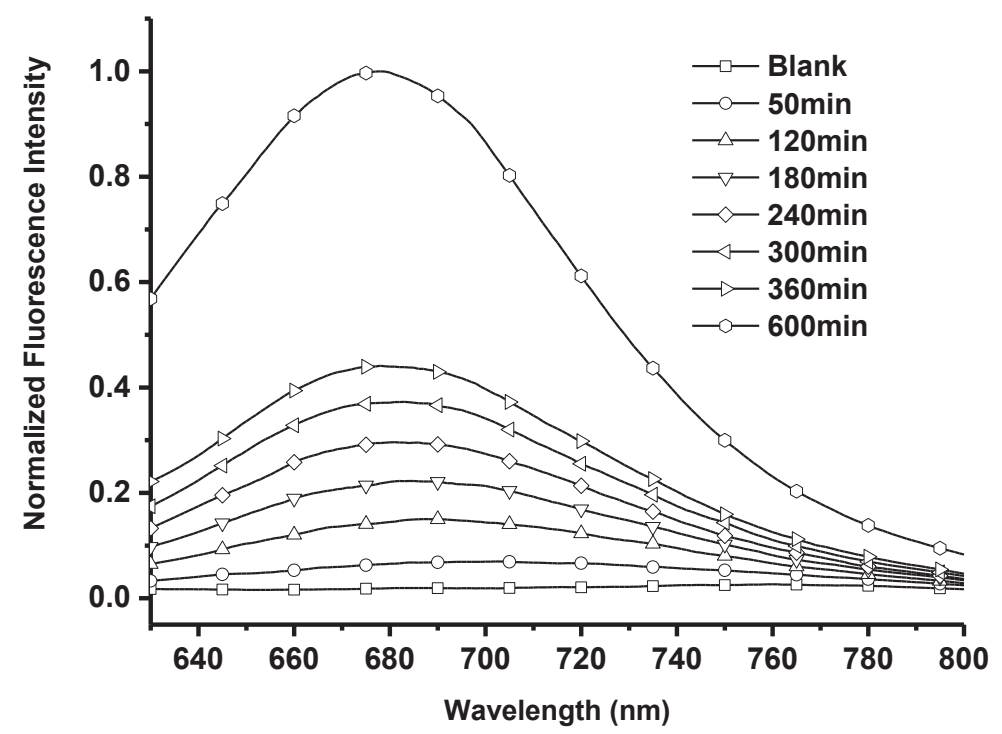

Figure B.41. Normalized fluorescence spectra of BODIPY dye 4-Gb $(20 \mu \mathrm{M})$ in $0.01 \mathrm{M}$ PBS buffer pH 7.4 in the absence and presence of L-cysteine $(10 \mathrm{mM})$ at different times. $\left(\lambda_{\mathrm{ex}}=620 \mathrm{~nm}\right)$ 

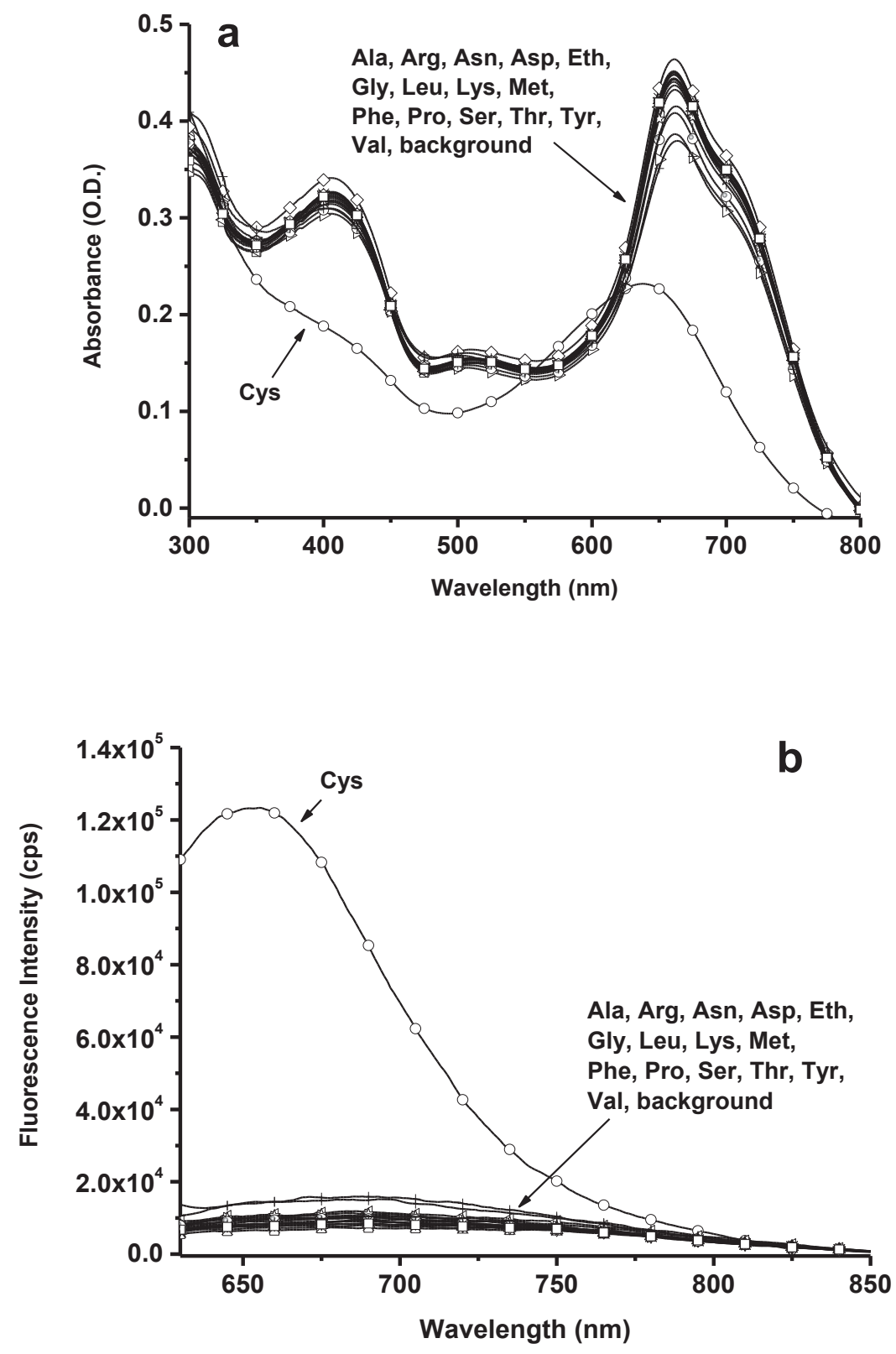

Figure B.42. Absorption (a) and Emission (b) spectra of BODIPY dye 4-Gb $(10 \mu \mathrm{M})$ in the absence and presence of various amino acids $(5 \mathrm{mM}) .\left(\lambda_{\mathrm{ex}}=620 \mathrm{~nm}\right)$. 


\section{Appendix C Supporting Information for Chapter 5}

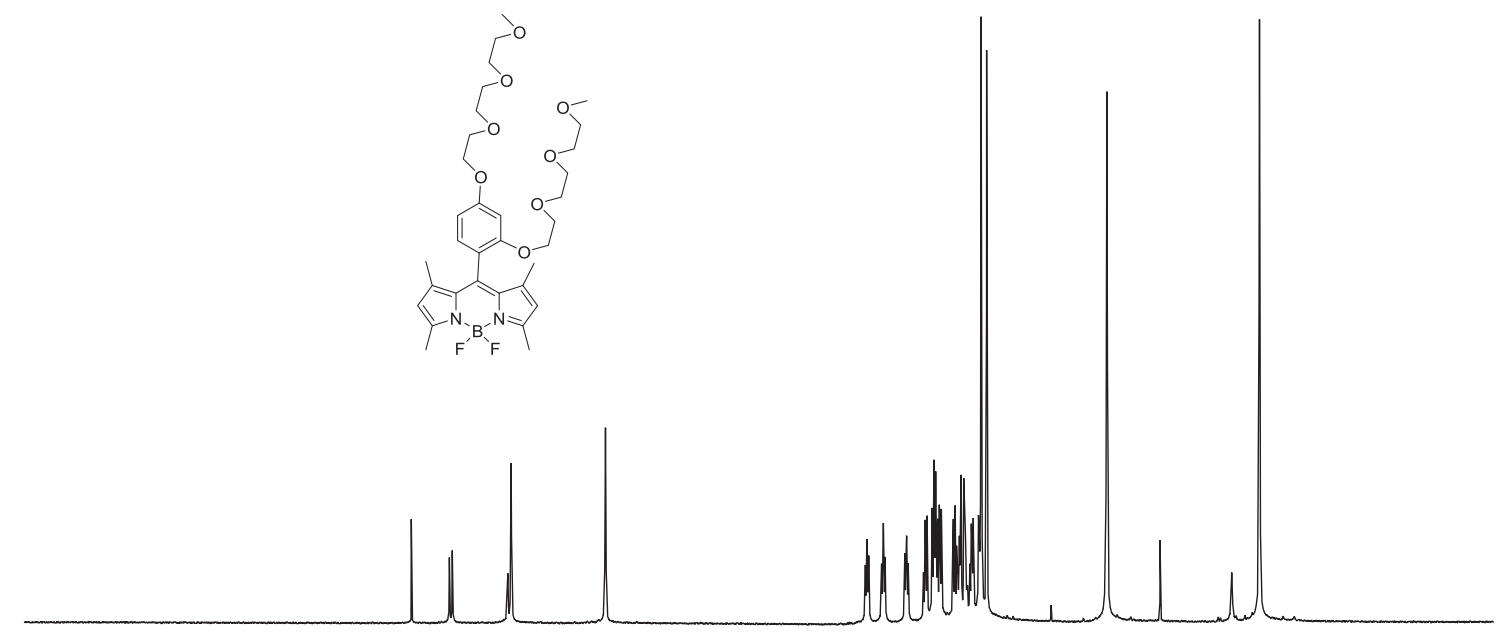

$\begin{array}{llllllllllllllllllll}9.5 & 9.0 & 8.5 & 8.0 & 7.5 & 7.0 & 6.5 & 6.0 & 5.5 & \begin{array}{c}5.0 \\ \mathrm{ppm}\end{array} & 4.5 & 4.0 & 3.5 & 3.0 & 2.5 & 2.0 & 1.5 & 1.0 & 0.5 & 0.0\end{array}$

Figure C.1. $\quad{ }^{1} \mathrm{H}$ NMR spectrum of BODIPY dye 5.2a in $\mathrm{CDCl}_{3}$ solution.

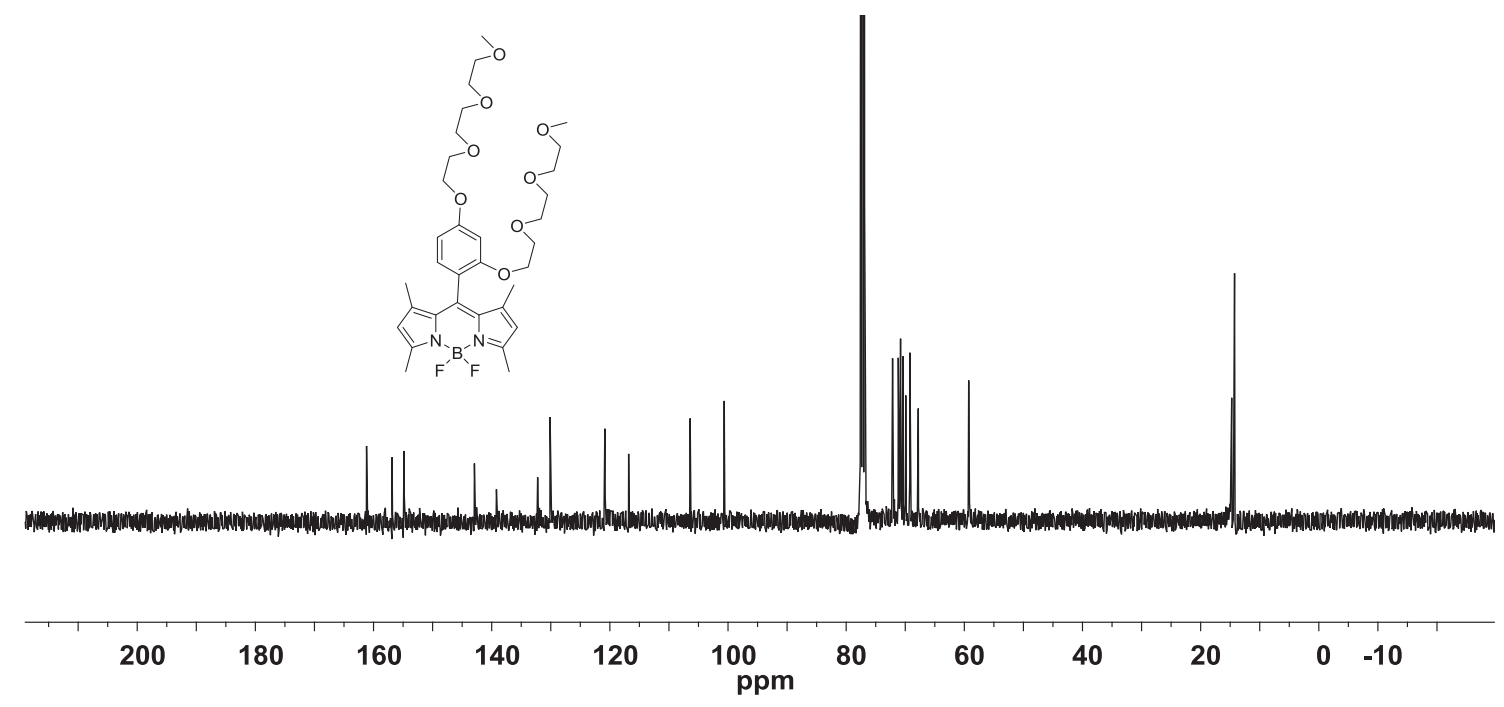

Figure C.2. ${ }^{13} \mathrm{C}$ NMR spectrum of BODIPY dye 5.2a in $\mathrm{CDCl}_{3}$ solution. 

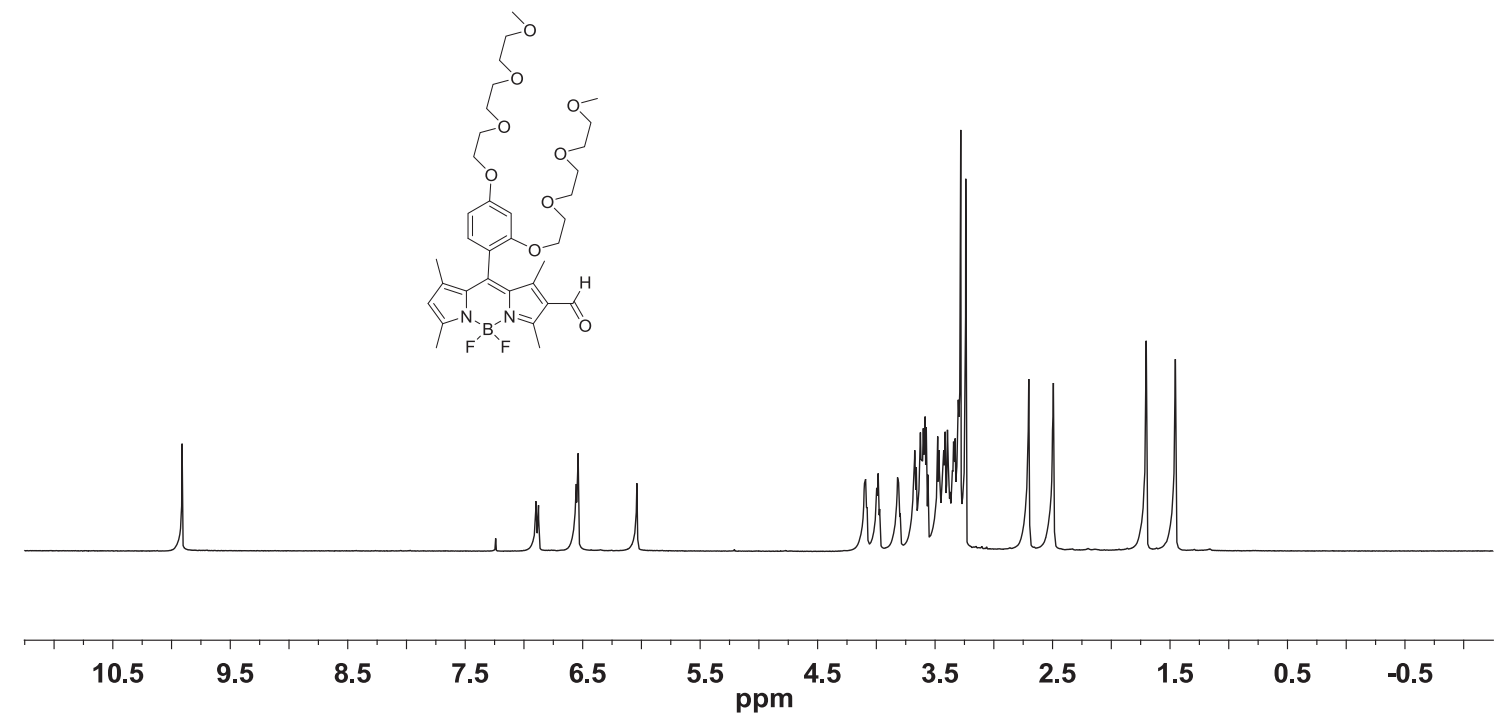

Figure C.3. $\quad{ }^{1} \mathrm{H}$ NMR spectrum of BODIPY dye 5.3a in $\mathrm{CDCl}_{3}$ solution.

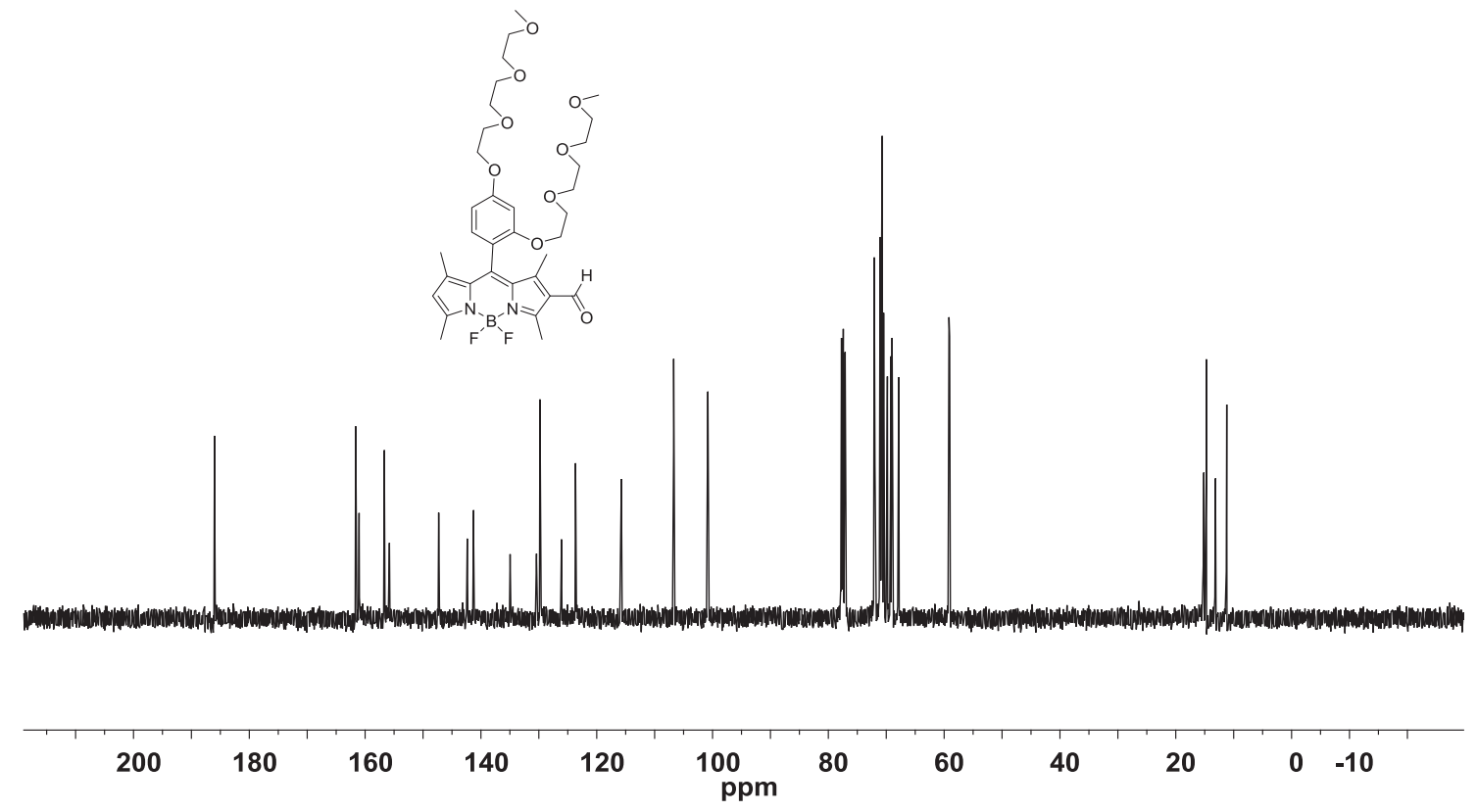

Figure C.4. $\quad{ }^{13} \mathrm{C}$ NMR spectrum of BODIPY dye 5.3a in $\mathrm{CDCl}_{3}$ solution. 


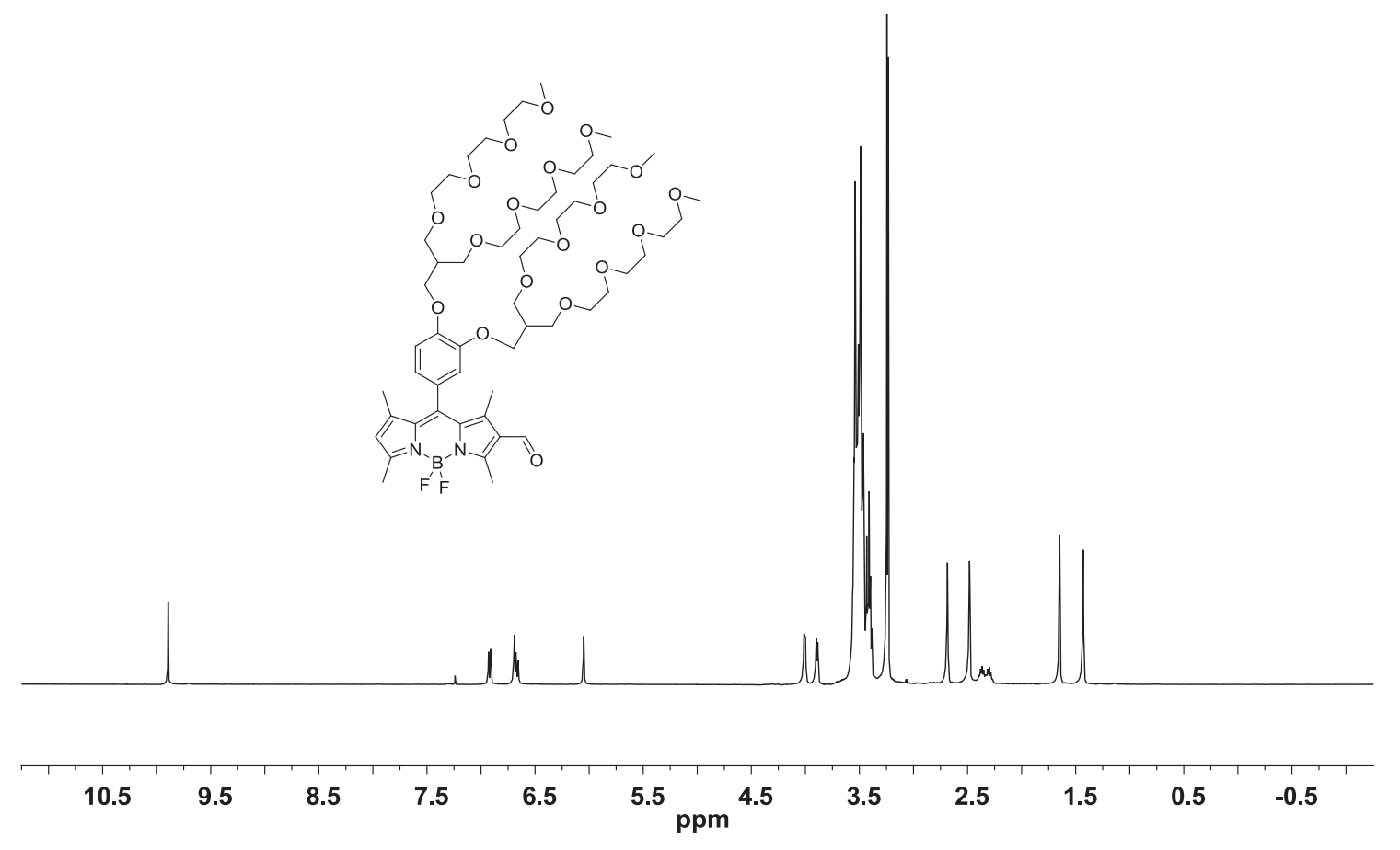

Figure C.5. $\quad{ }^{1} \mathrm{H}$ NMR spectrum of BODIPY dye 5.3b in $\mathrm{CDCl}_{3}$ solution.

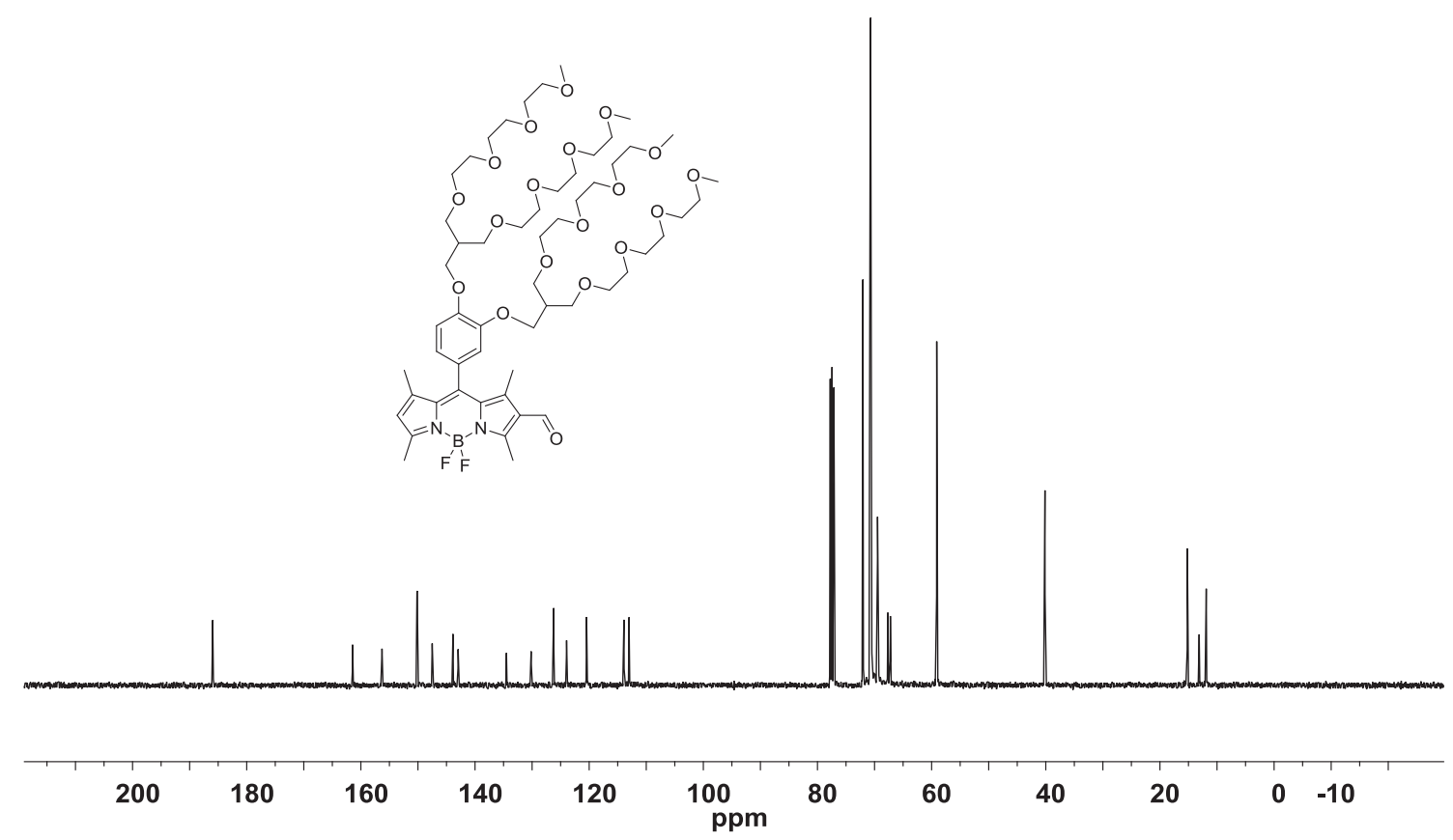

Figure C.6. ${ }^{13} \mathrm{C}$ NMR spectrum of BODIPY dye 5.3b in $\mathrm{CDCl}_{3}$ solution. 

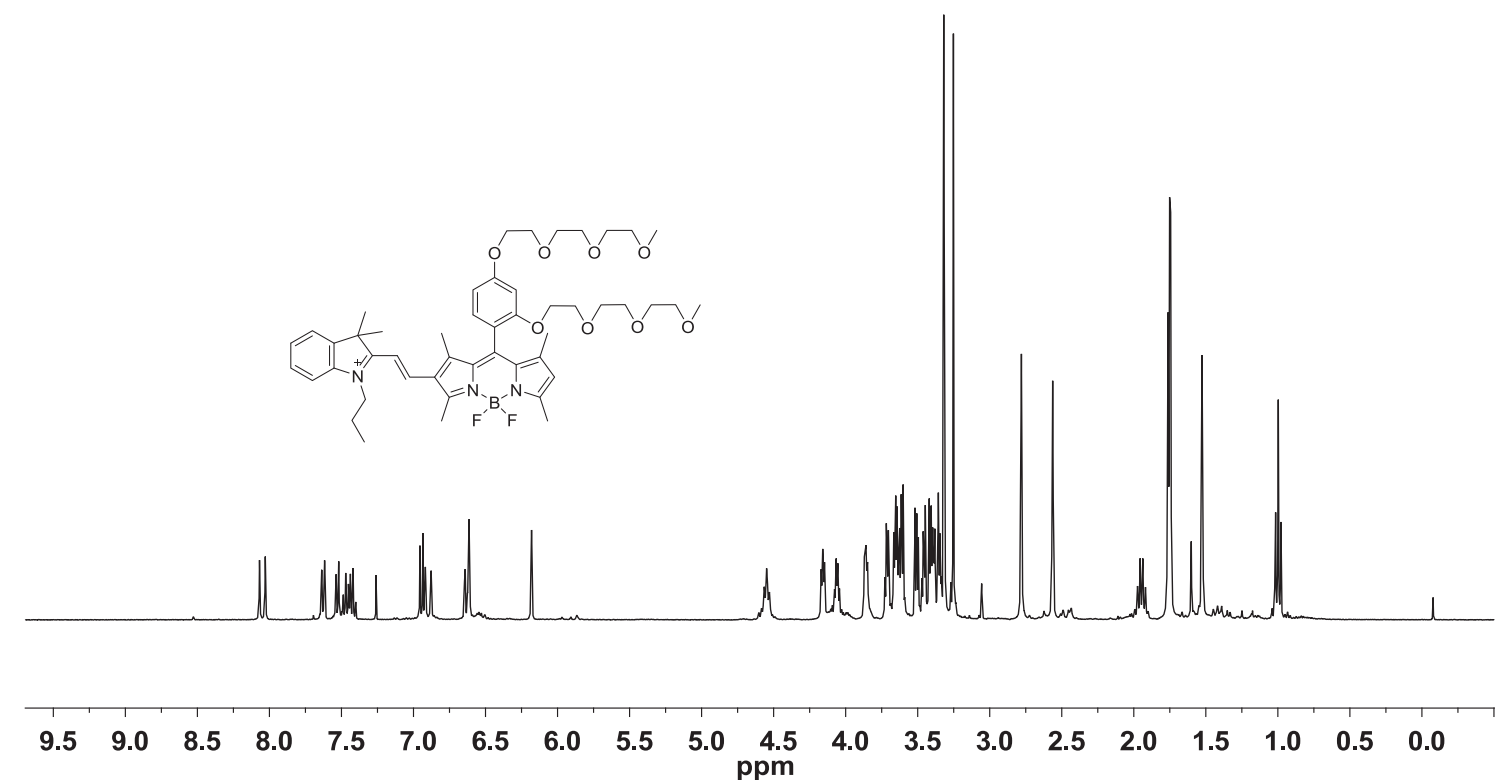

Figure C.7. $\quad{ }^{1} \mathrm{H}$ NMR spectrum of probe $\mathbf{5 - A}$ in $\mathrm{CDCl}_{3}$ solution.

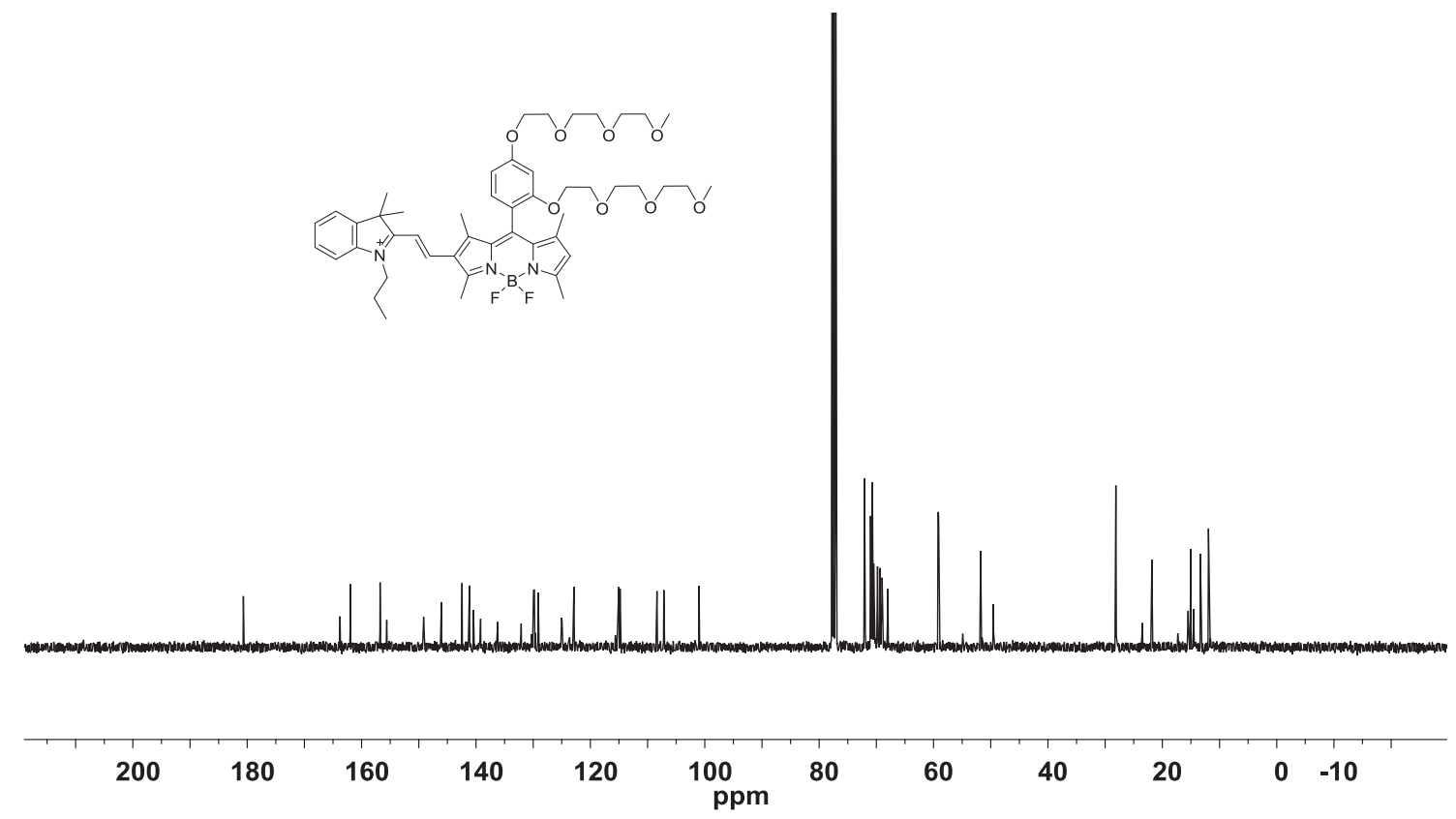

Figure C.8. ${ }^{13} \mathrm{C}$ NMR spectrum of probe 5-A in $\mathrm{CDCl}_{3}$ solution. 


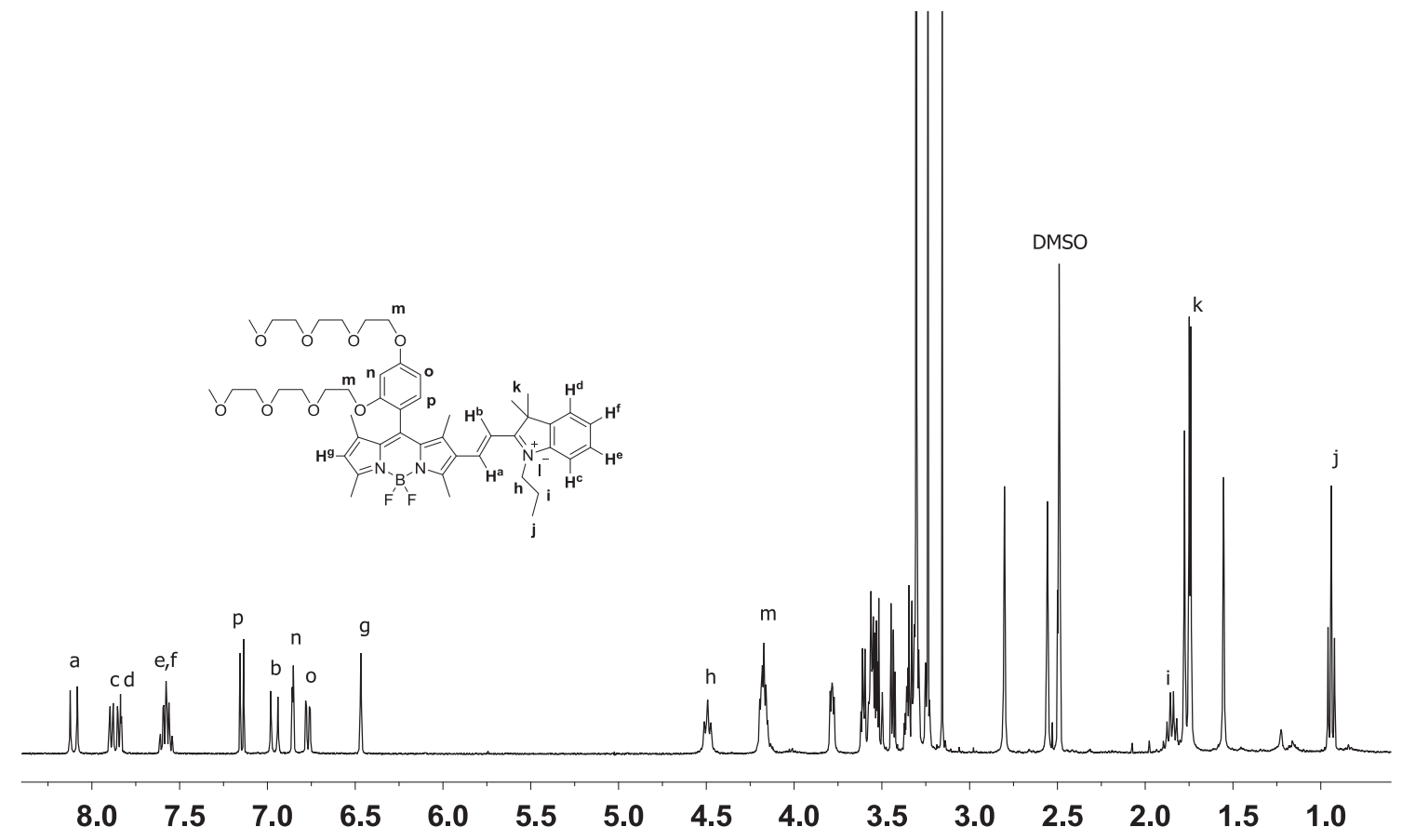

Figure C.9. $\quad{ }^{1} \mathrm{H}$ NMR spectrum of probe 5-A in DMSO- $d_{6}$ solution.

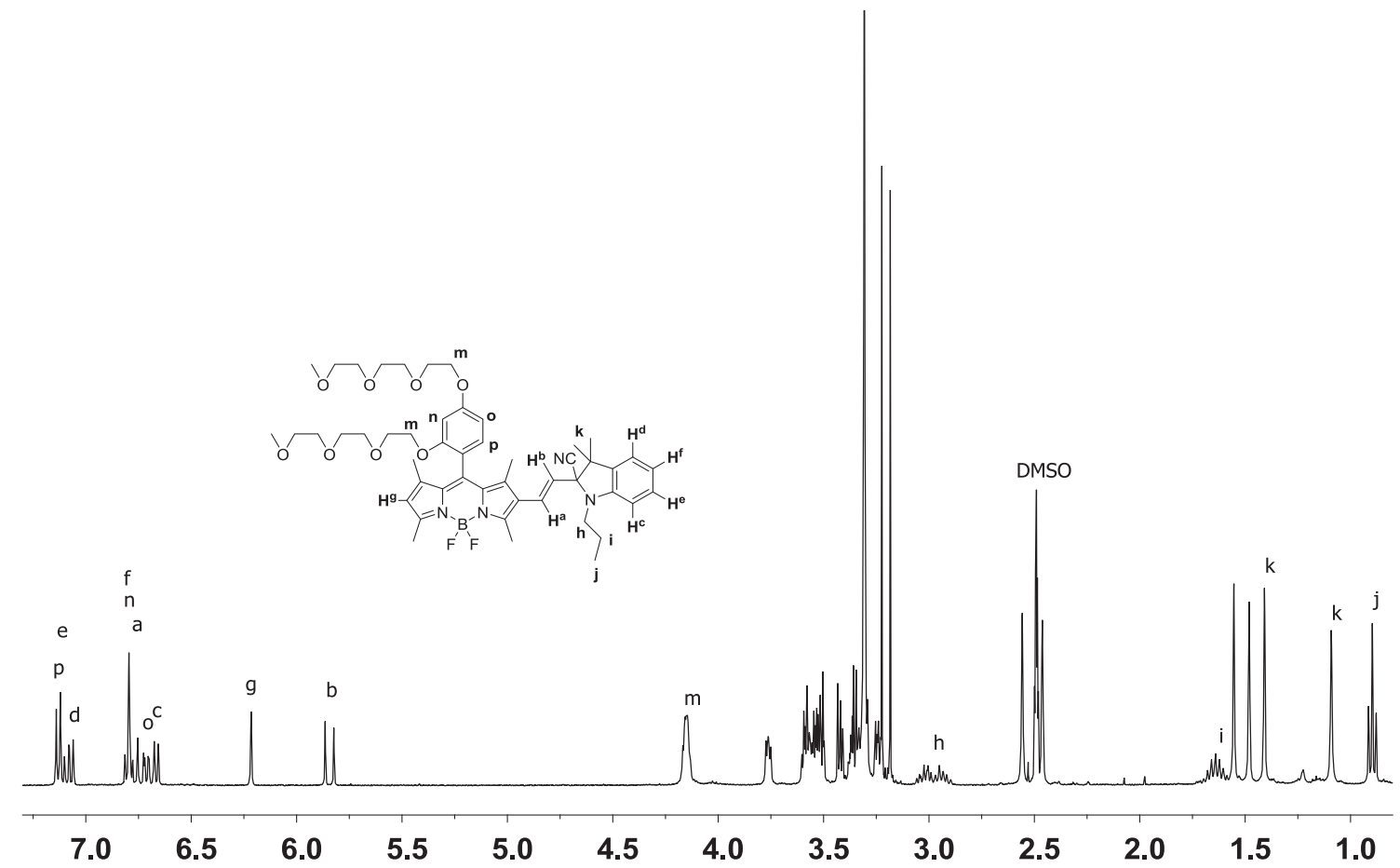

Figure C.10. ${ }^{1} \mathrm{H}$ NMR spectrum of $\mathbf{5 - A - C N}$ adduct in DMSO- $d_{6}$ solution. 

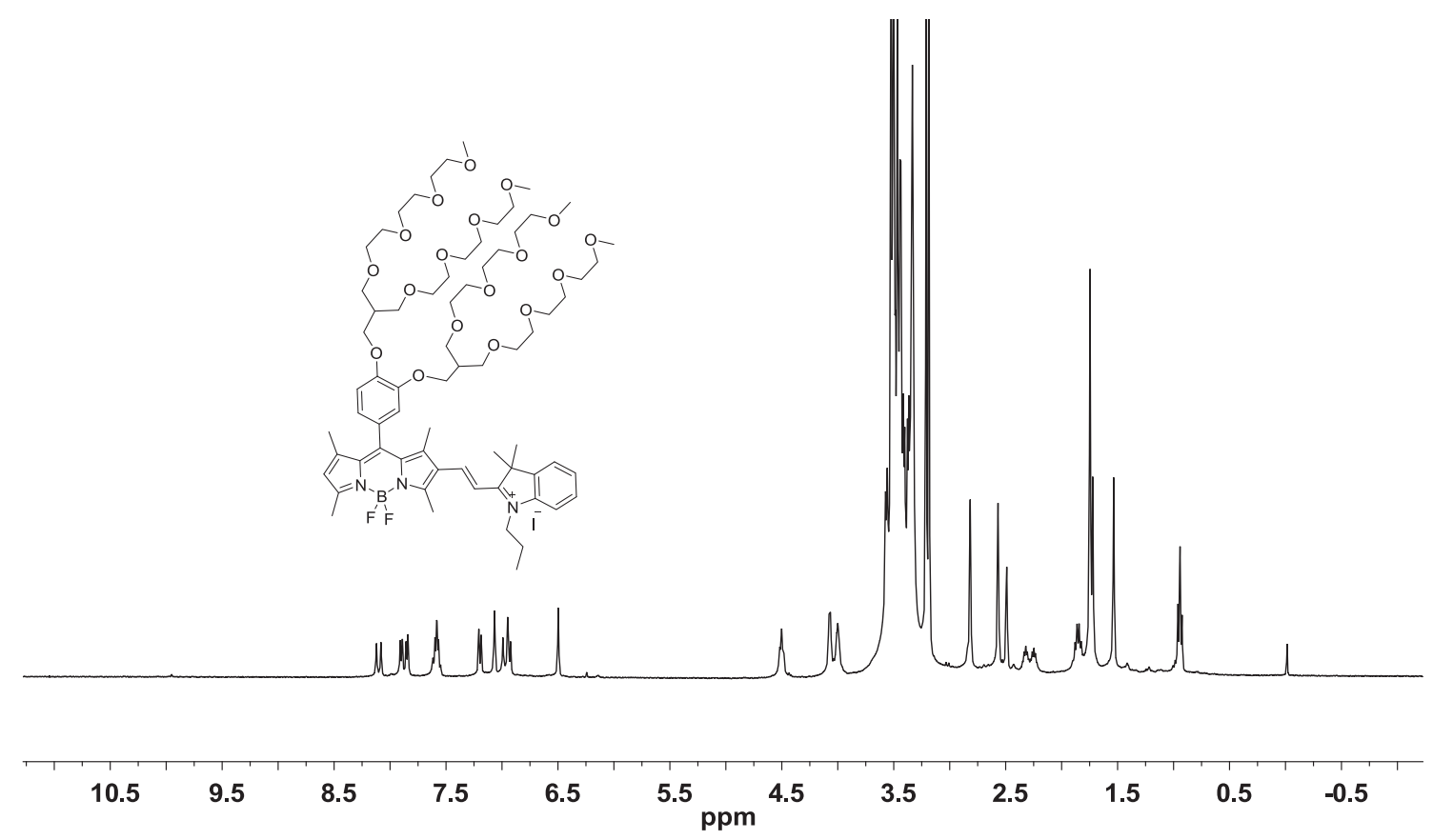

Figure C.11. ${ }^{1} \mathrm{H}$ NMR spectrum of probe 5-B in DMSO- $d_{6}$ solution.

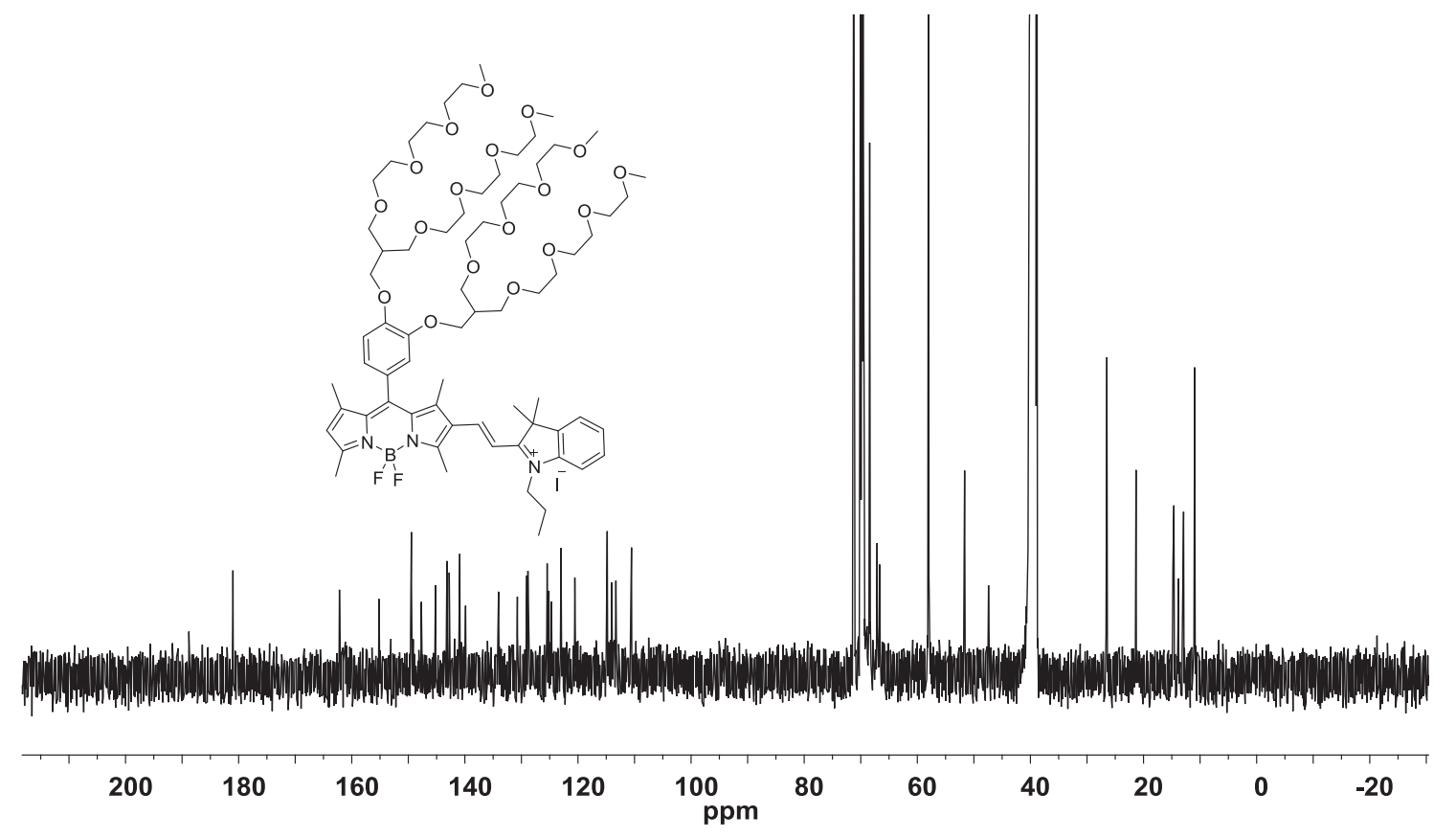

Figure C.12. ${ }^{13} \mathrm{C}$ NMR spectrum of probe 5-B in DMSO- $d_{6}$ solution. 


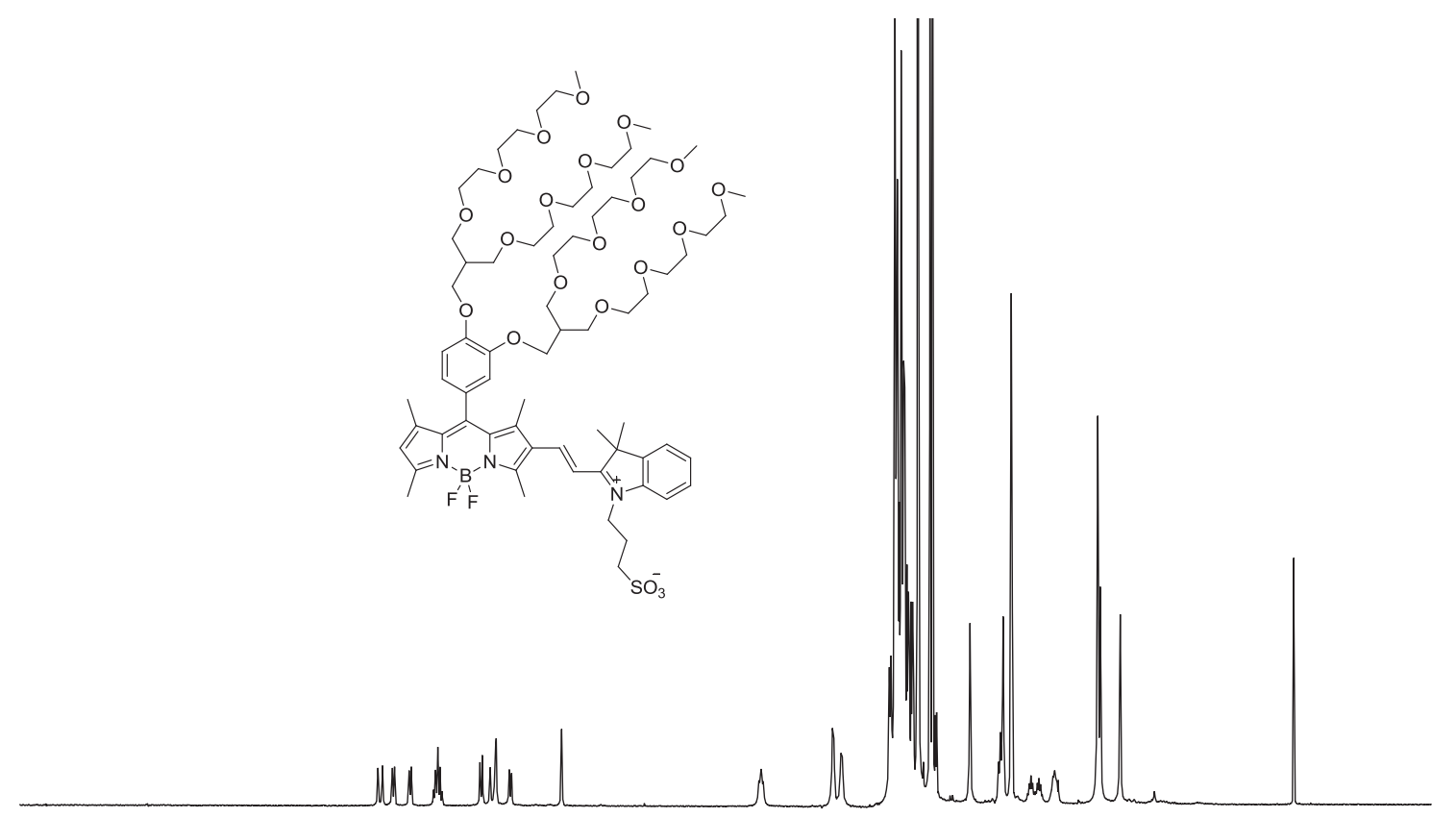

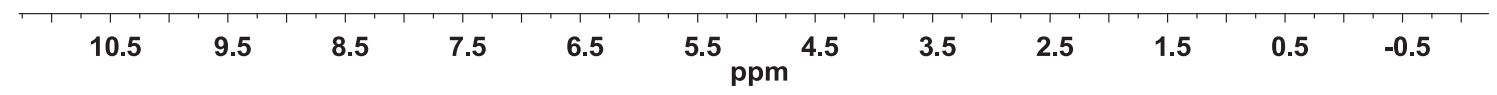

Figure C.13. ${ }^{1} \mathrm{H}$ NMR spectrum of probe 5-C in DMSO- $d_{6}$ solution.

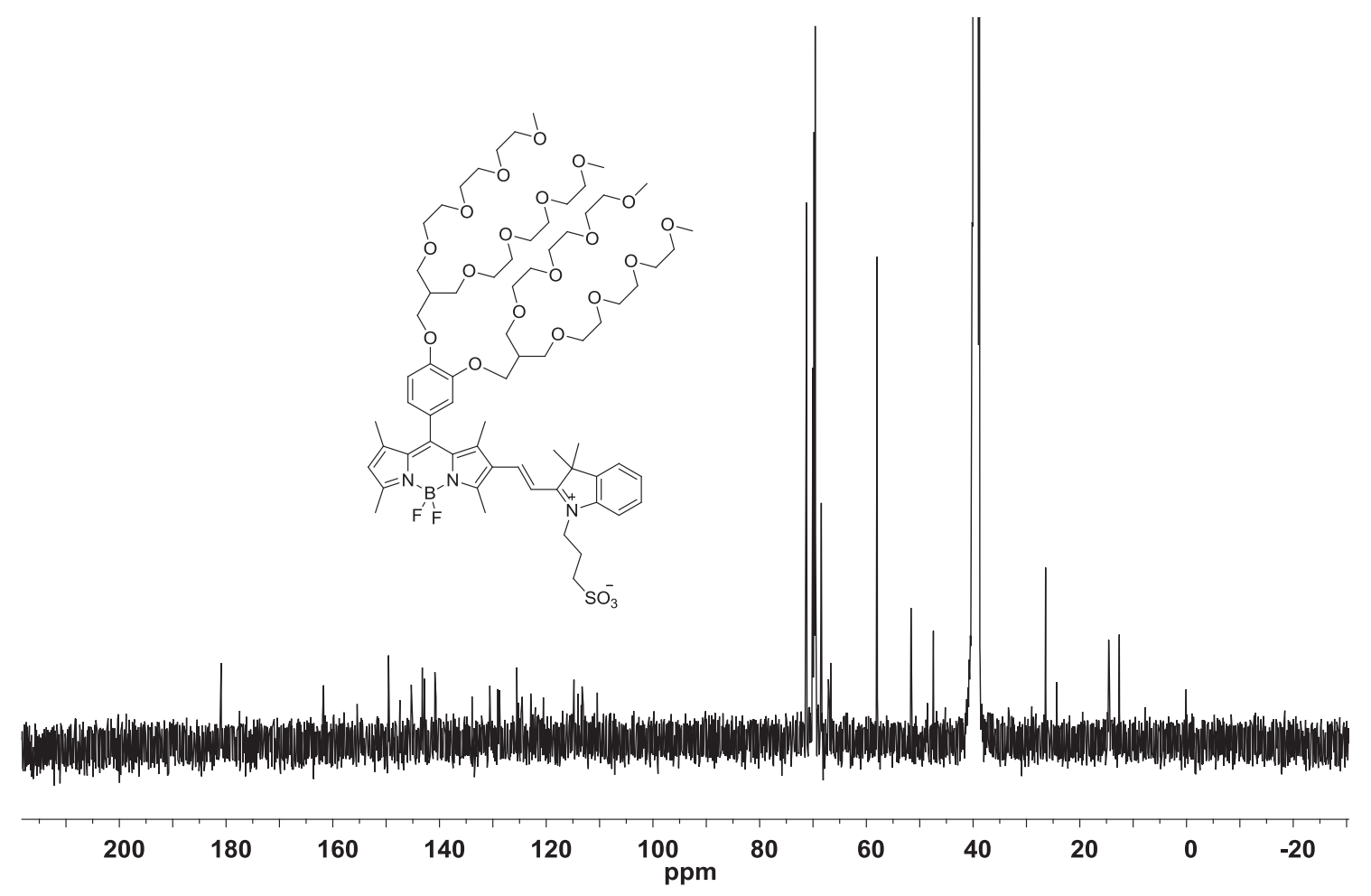

Figure C.14. ${ }^{13} \mathrm{C}$ NMR spectrum of probe 5-C in DMSO- $d_{6}$ solution. 

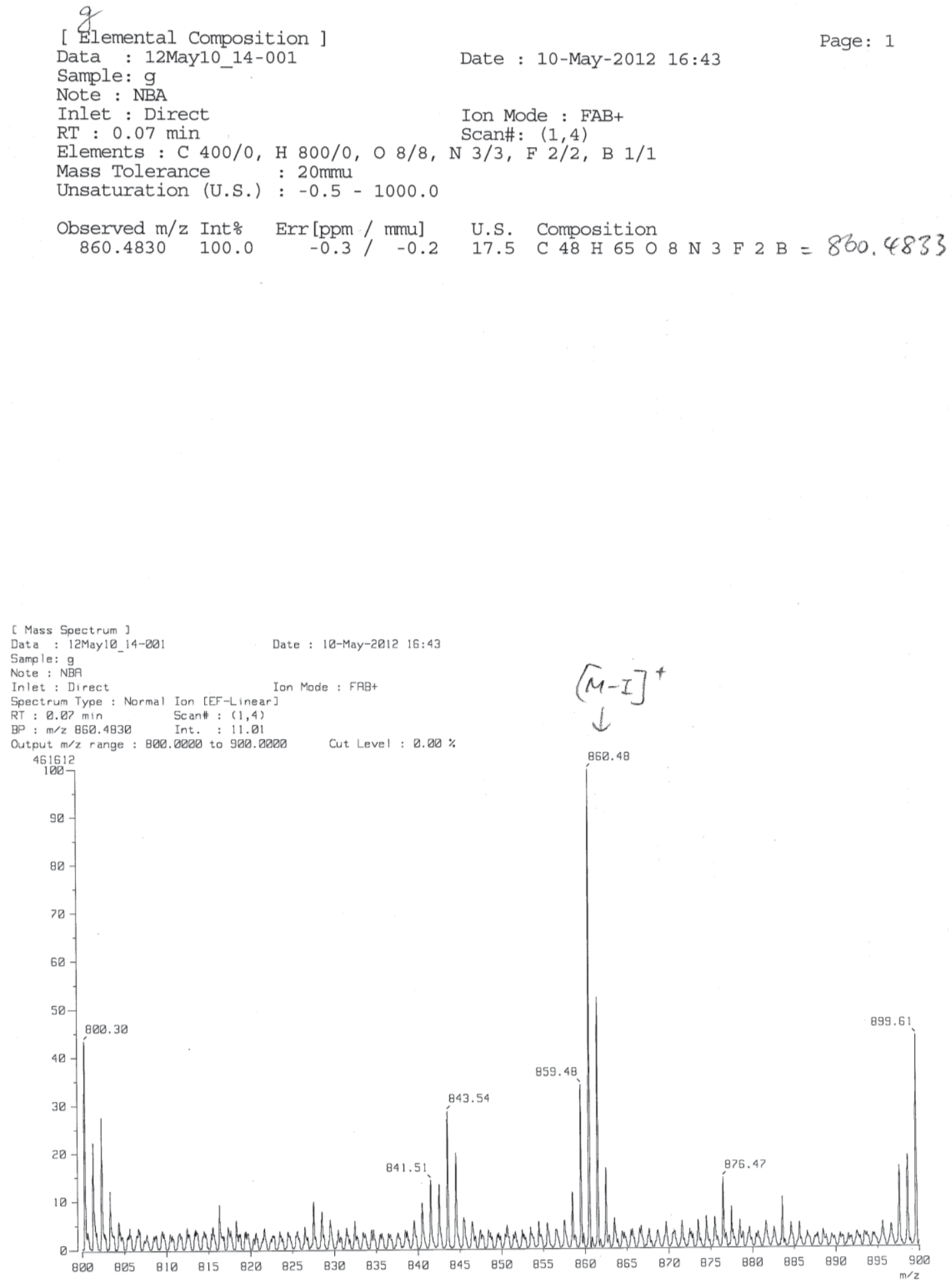

Figure C.15. The HRMS (FAB) of probe 5-A. 

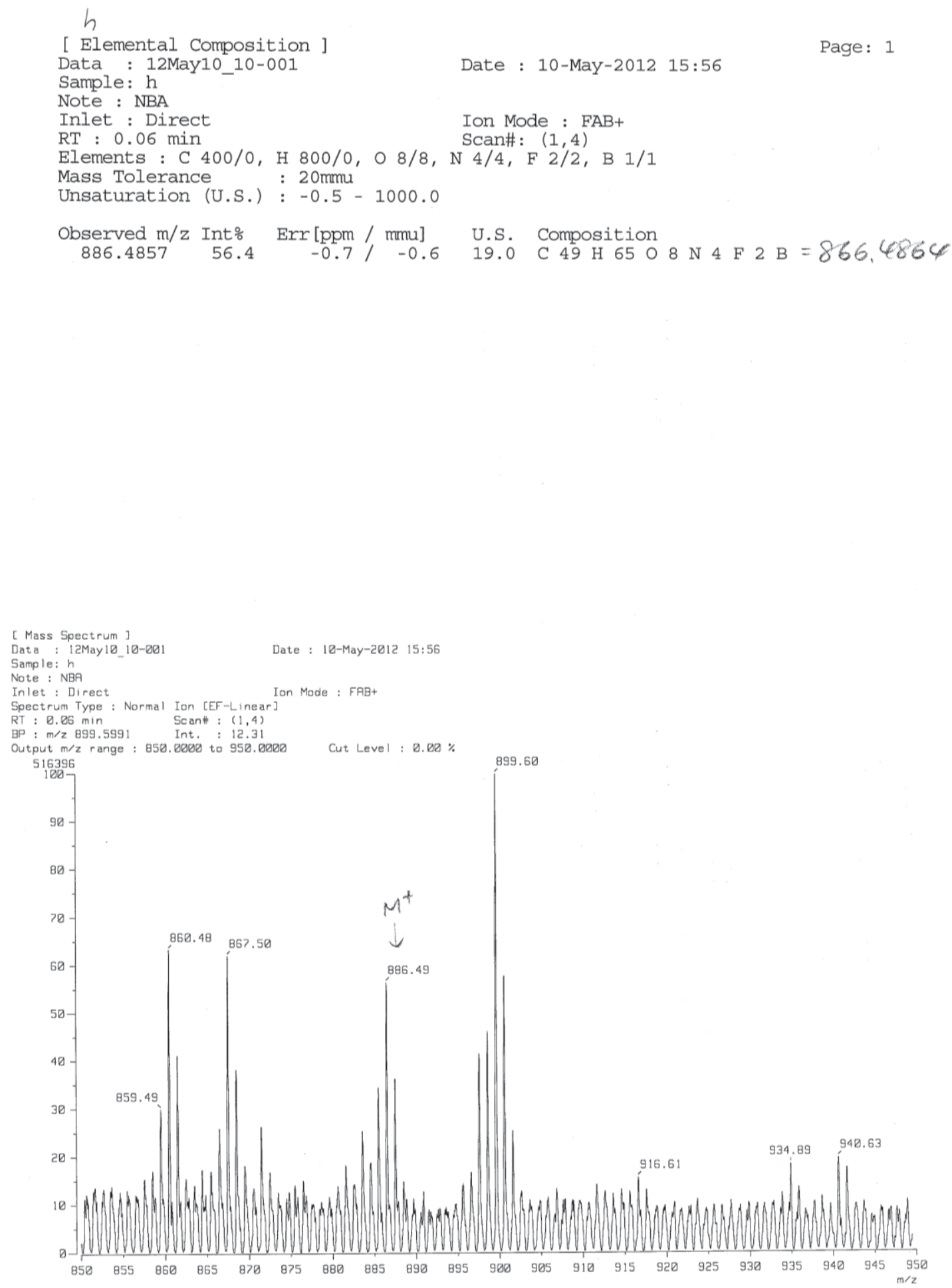

Figure C.16. The HRMS (FAB) of 5-A-CN adduct. 


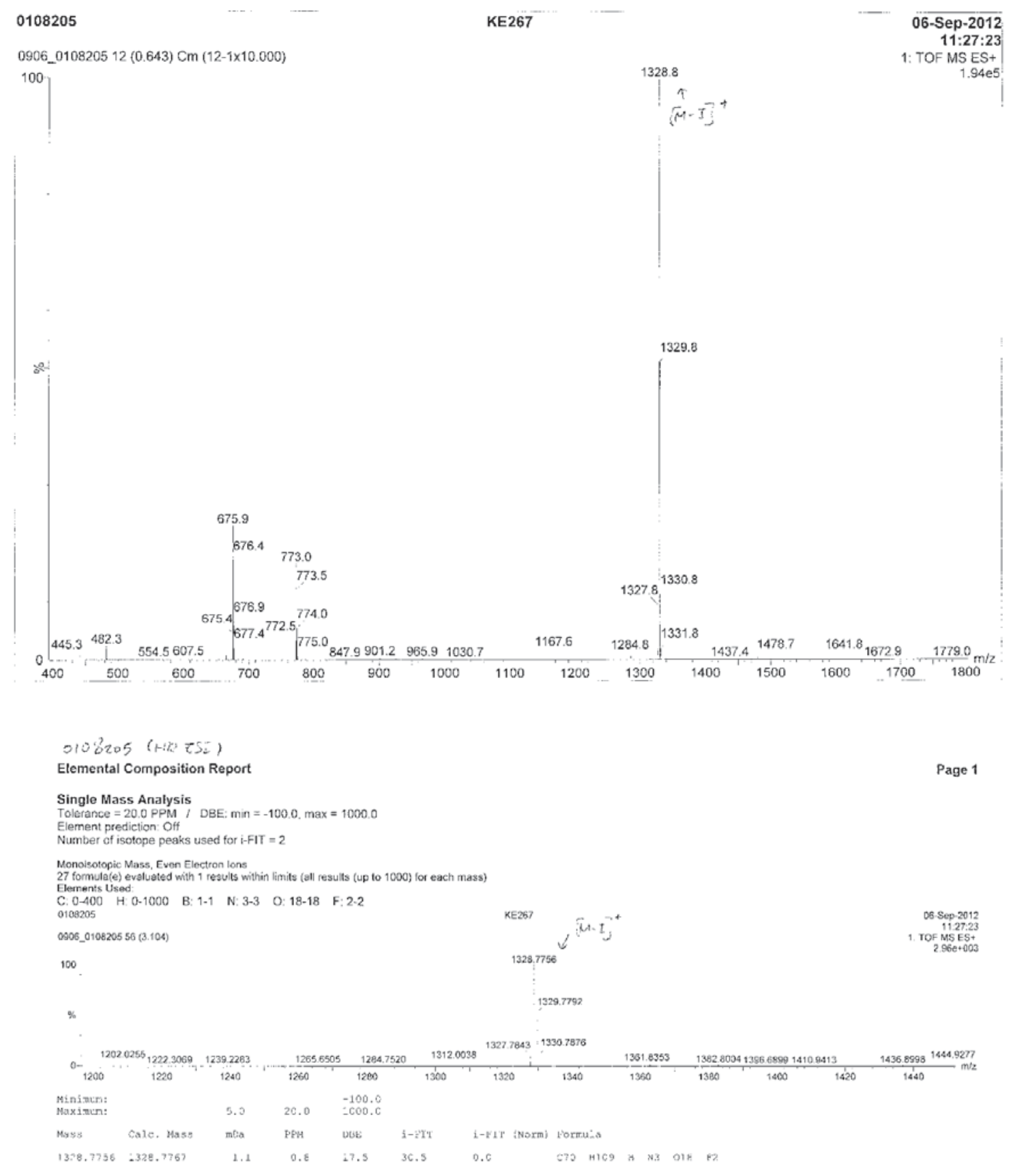

Figure C.17. The LRMS(top) and HRMS(bottom) (ESI) of probe 5-B. 


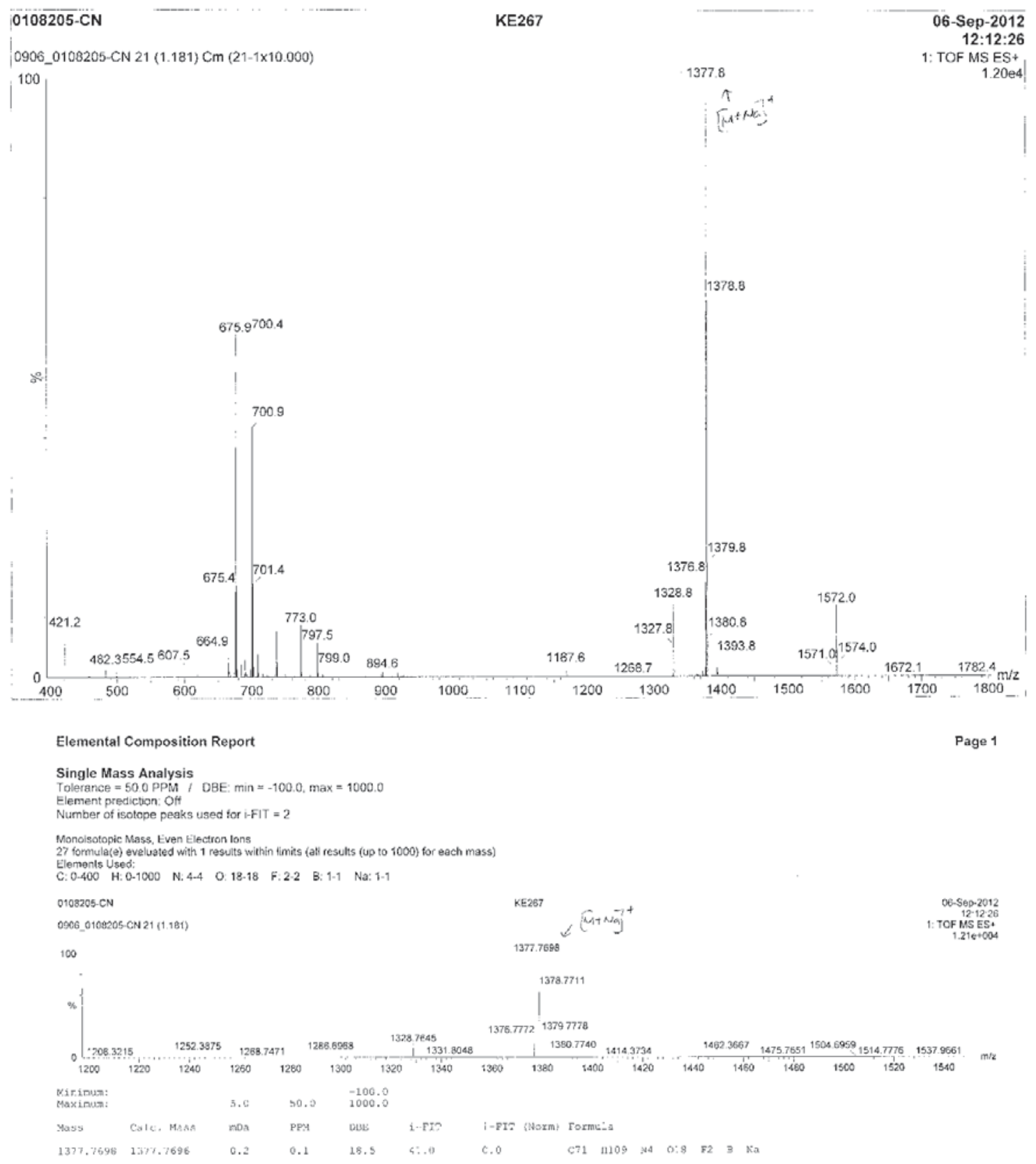

Figure C.18. The LRMS(top) and HRMS(bottom) (ESI) of 5-B-CN. 


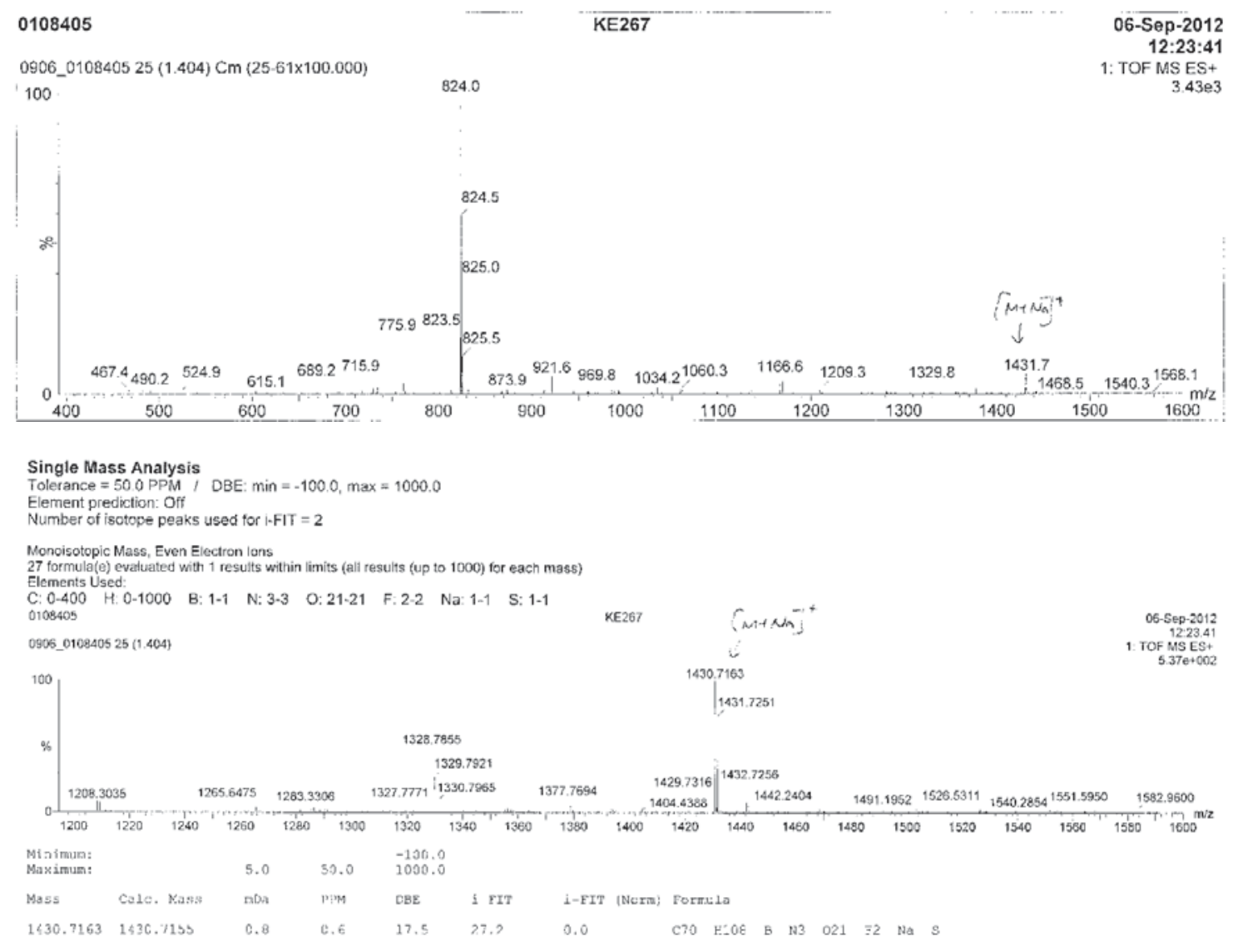

Figure C.19. The LRMS(top) and HRMS(bottom) (ESI) of probe 5-C. 


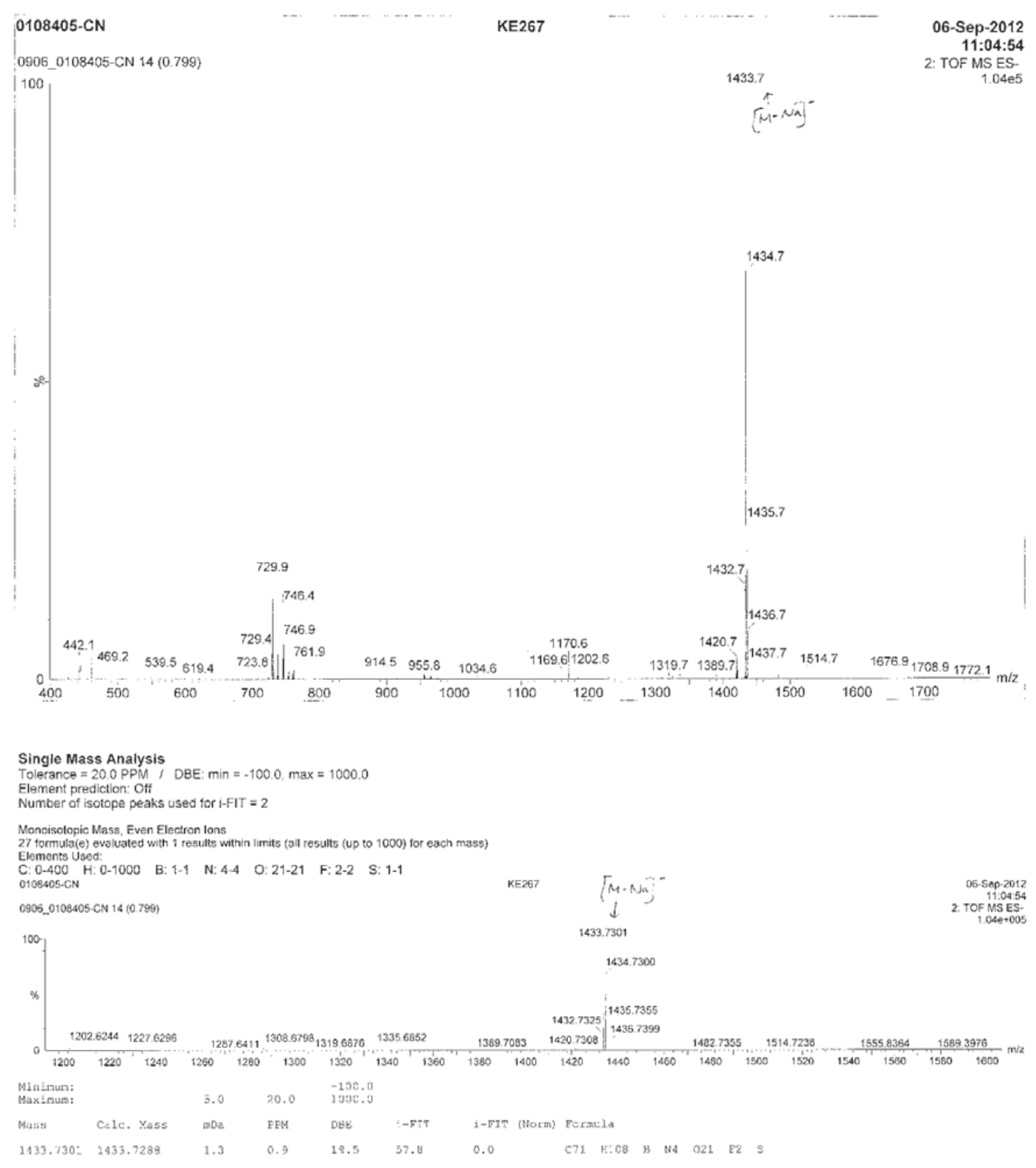

Figure C.20. The LRMS(top) and HRMS(bottom) (ESI) of 5-C-CN 


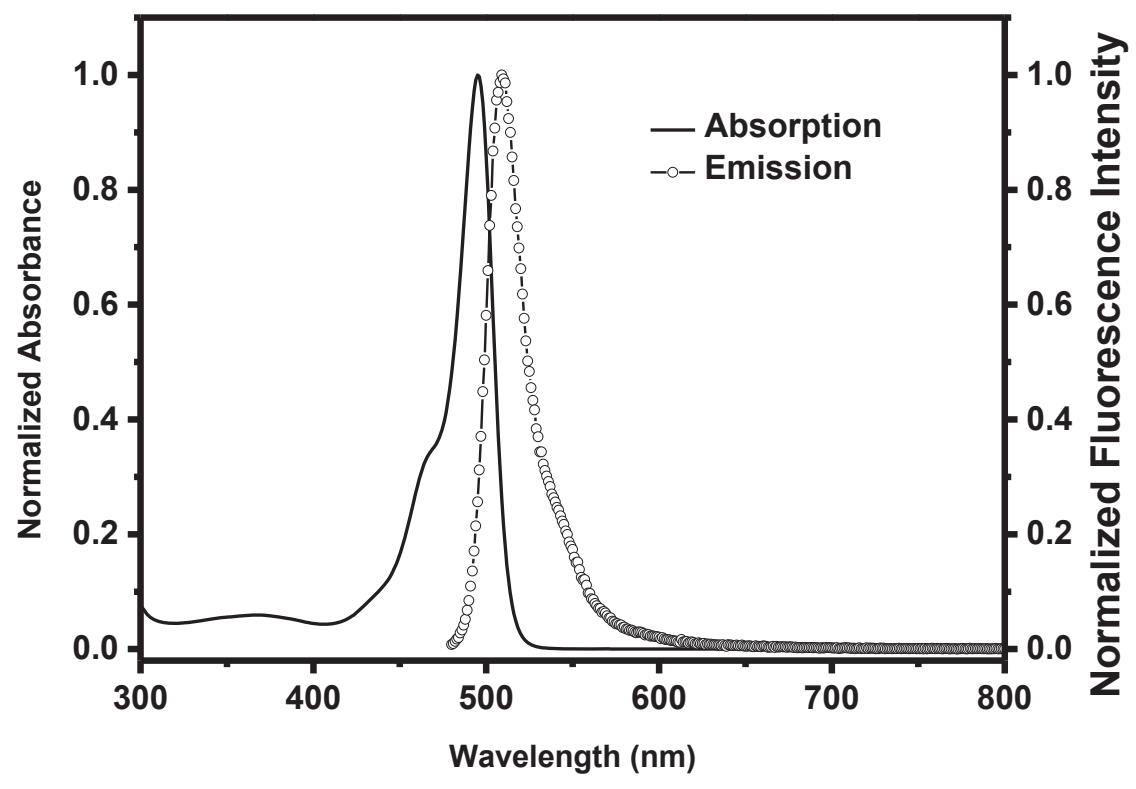

Figure C.21. Normalized absorption and emission spectra of BODIPY dye 5.3a in acetonitrile solution. (excitation wavelength $=470 \mathrm{~nm}$ ) 


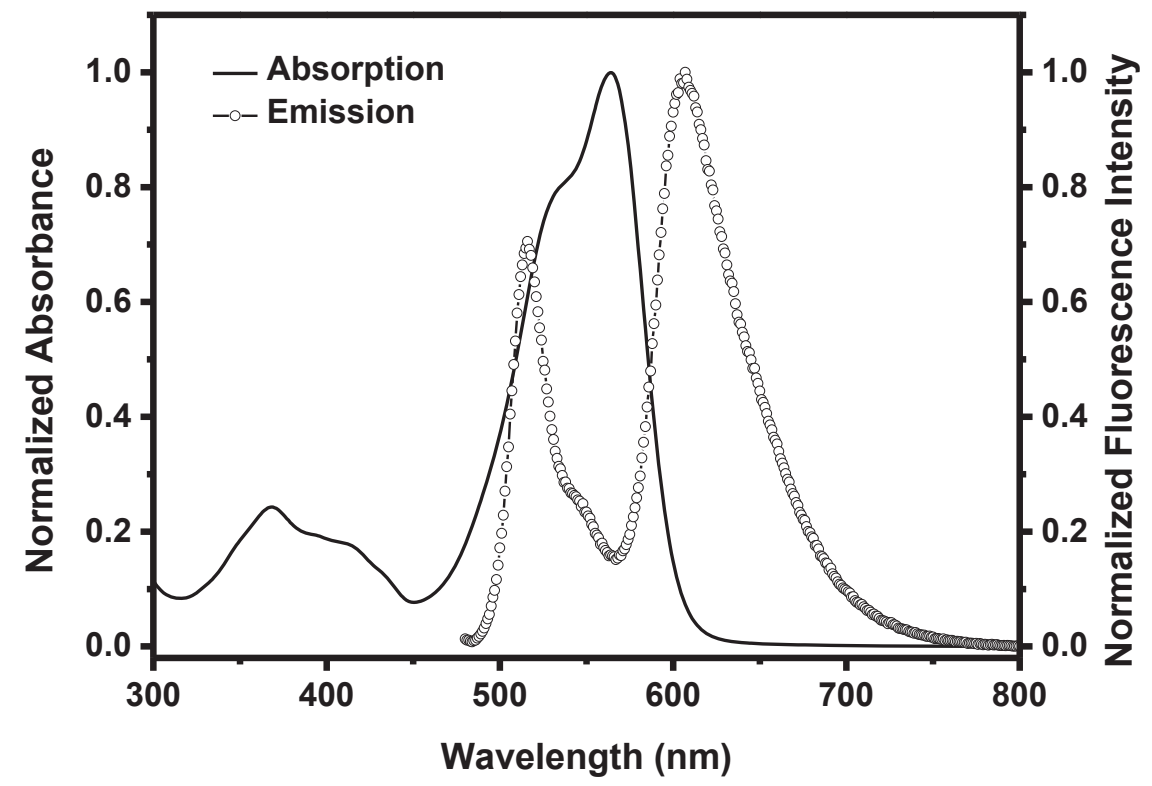

Figure C.22. Normalized absorption and emission spectra of fluorescent probe 5-A in acetonitrile solution. (excitation wavelength $=470 \mathrm{~nm}$ )

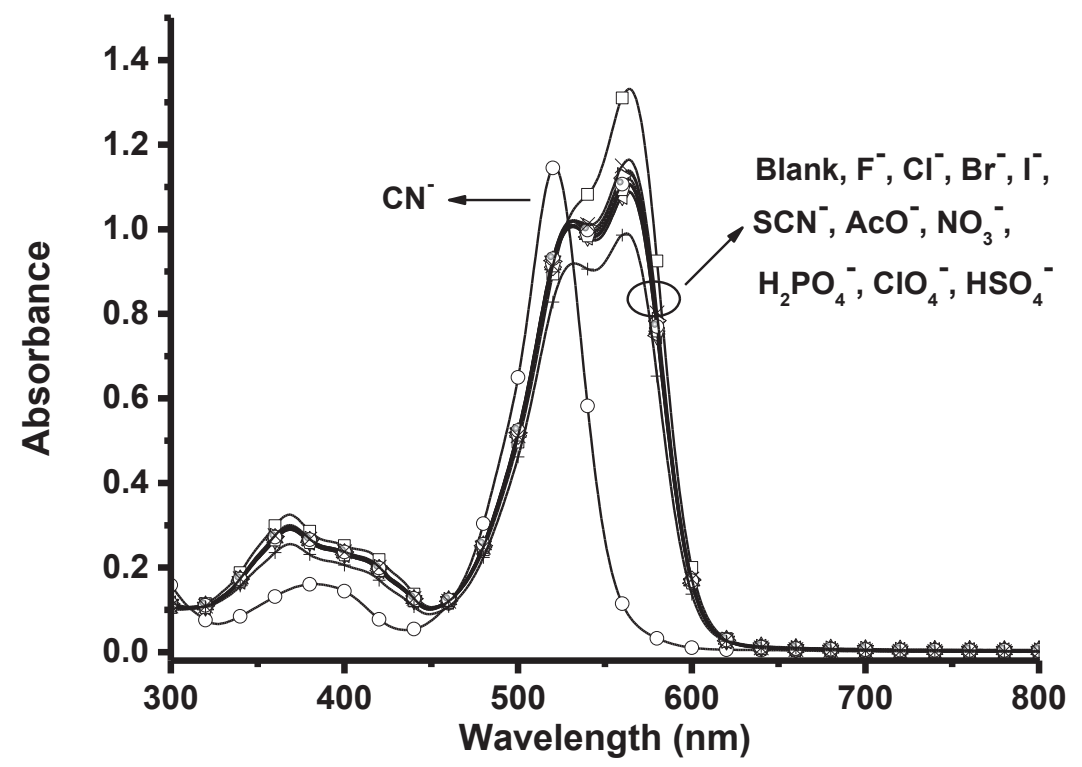

Figure C.23. Absorption spectra of probe 5-A in the presence of different anions (100 $\mu \mathrm{M})$ in a mixed solution of $\mathrm{CH}_{3} \mathrm{CN}$ and Tris $\cdot \mathrm{HCl}$ buffer $(10 \mathrm{mM}, \mathrm{pH}=9.3)(9: 1, \mathrm{v} / \mathrm{v})$. 


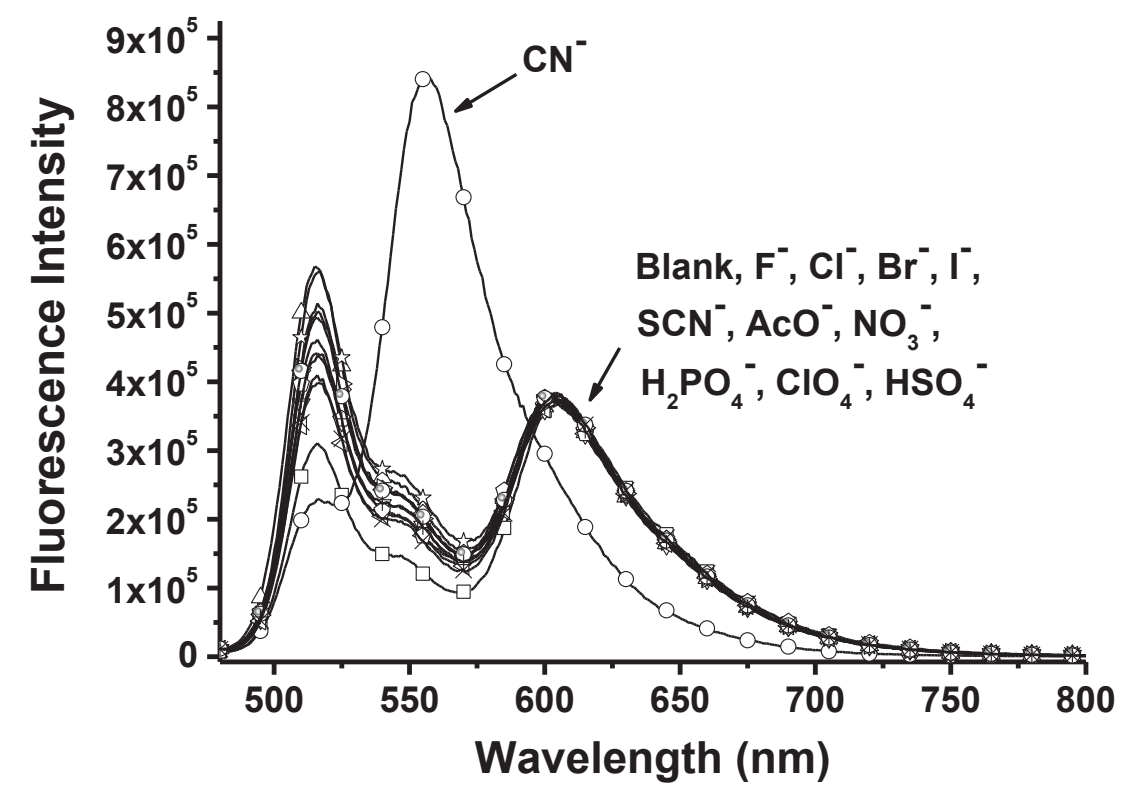

Figure C.24. The fluorescent spectra of probe 5-A to different anions $(100 \mu \mathrm{M})$ in mixed solution of $\mathrm{CH}_{3} \mathrm{CN}$ and Tris buffer $(10 \mathrm{mM}, \mathrm{pH}=9.3)(9: 1, \mathrm{v} / \mathrm{v})$.

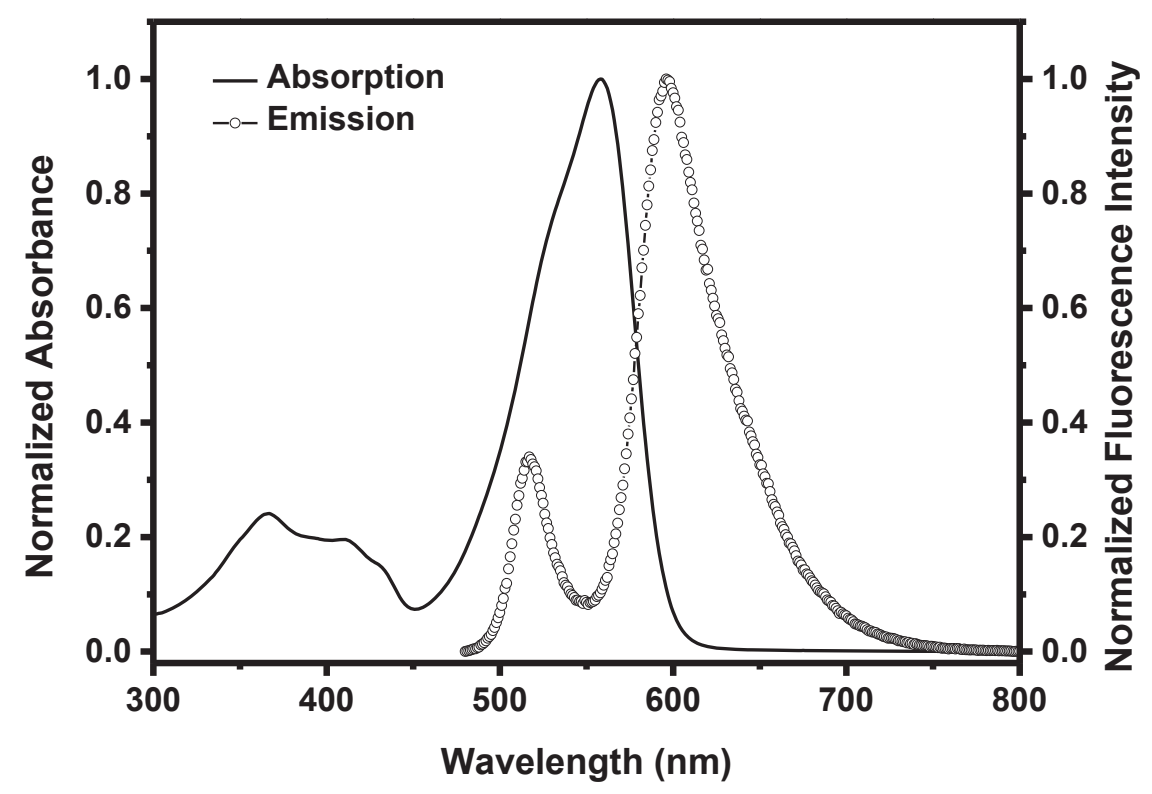

Figure C.25. Normalized absorption and emission spectra of fluorescent probe 5-B in Tris buffer solution $(10 \mathrm{mM}, \mathrm{pH} 9.3)$. (excitation wavelength $=470 \mathrm{~nm}$ ) 


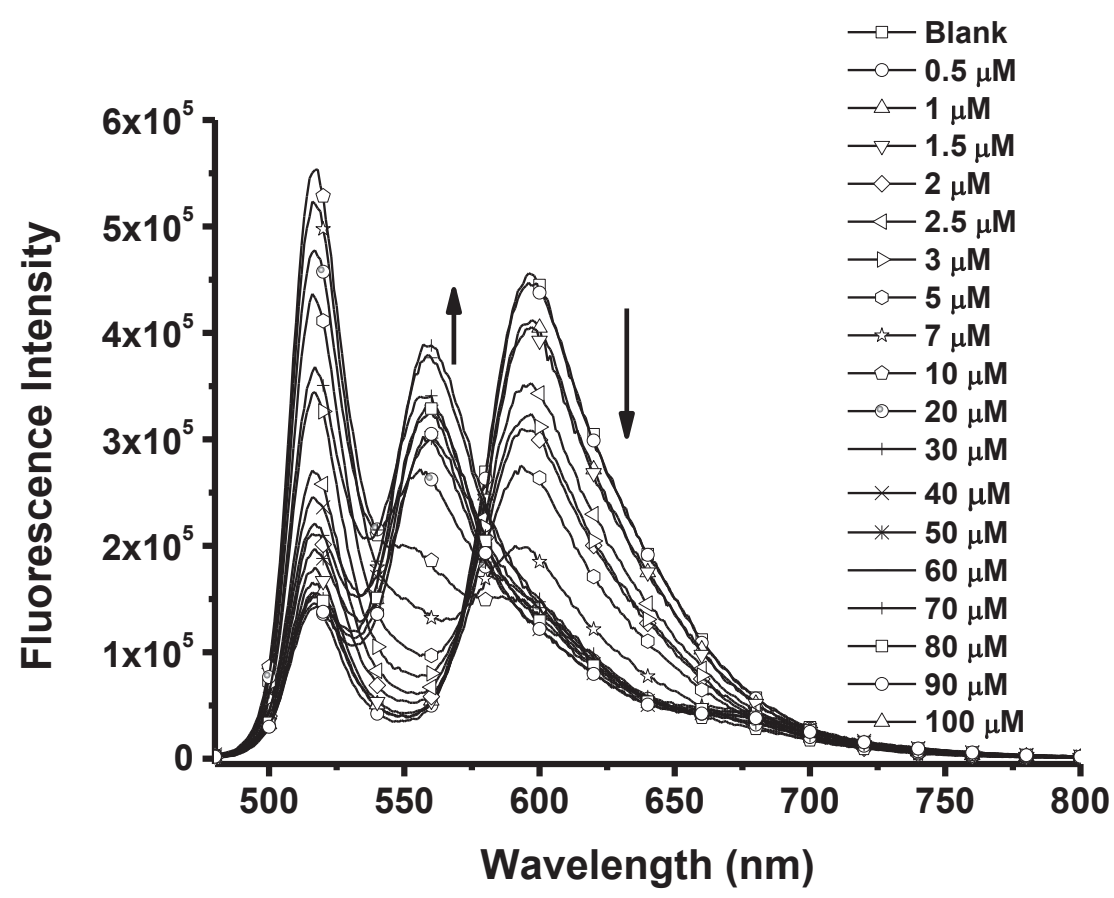

Figure C.26. Fluorescence spectra of the fluorescent probe 5-B $(20 \mu \mathrm{M})$ in the absence and presence of different amounts of cyanide ion in Tris buffer $(10 \mathrm{mM}, \mathrm{pH} 9.3)$ solution.

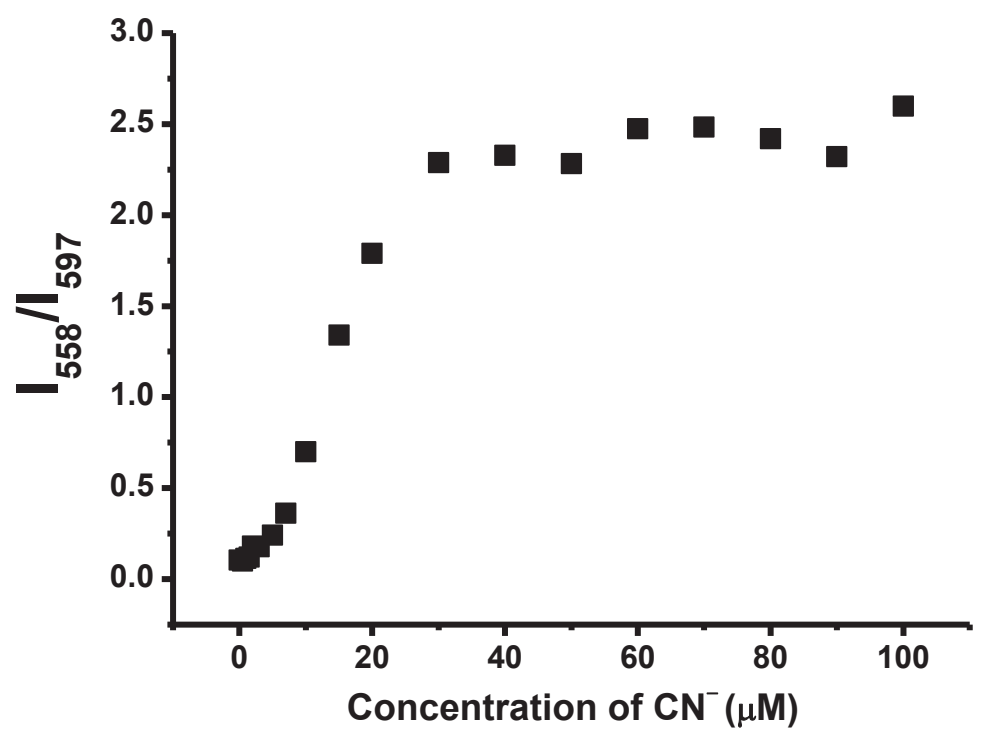

Figure C.27. Fluorescent intensity ratio change at $558 \mathrm{~nm}$ and $597 \mathrm{~nm}\left(\mathrm{I}_{558} / \mathrm{I}_{597}\right)$ of the fluorescent probe 5-B $(20 \mu \mathrm{M})$ upon titration of cyanide ions (range 0-100 $\mu \mathrm{M}$ )in Tris buffer (10 mM, pH 9.3) solution. 


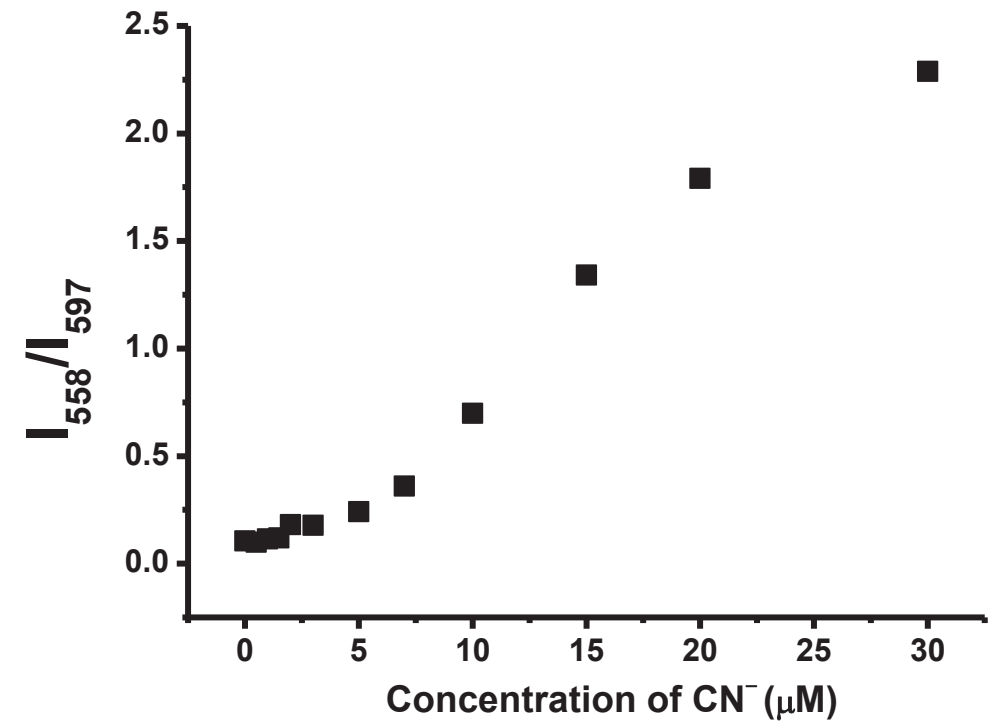

Figure C.28. Fluorescent intensity ratio change at $558 \mathrm{~nm}$ and $597 \mathrm{~nm}\left(\mathrm{I}_{558} / \mathrm{I}_{597}\right)$ of the fluorescent probe 5-B $(20 \mu \mathrm{M})$ upon titration of cyanide ions (range $0-30 \mu \mathrm{M})$ in Tris buffer (10 mM, pH 9.3) solution.

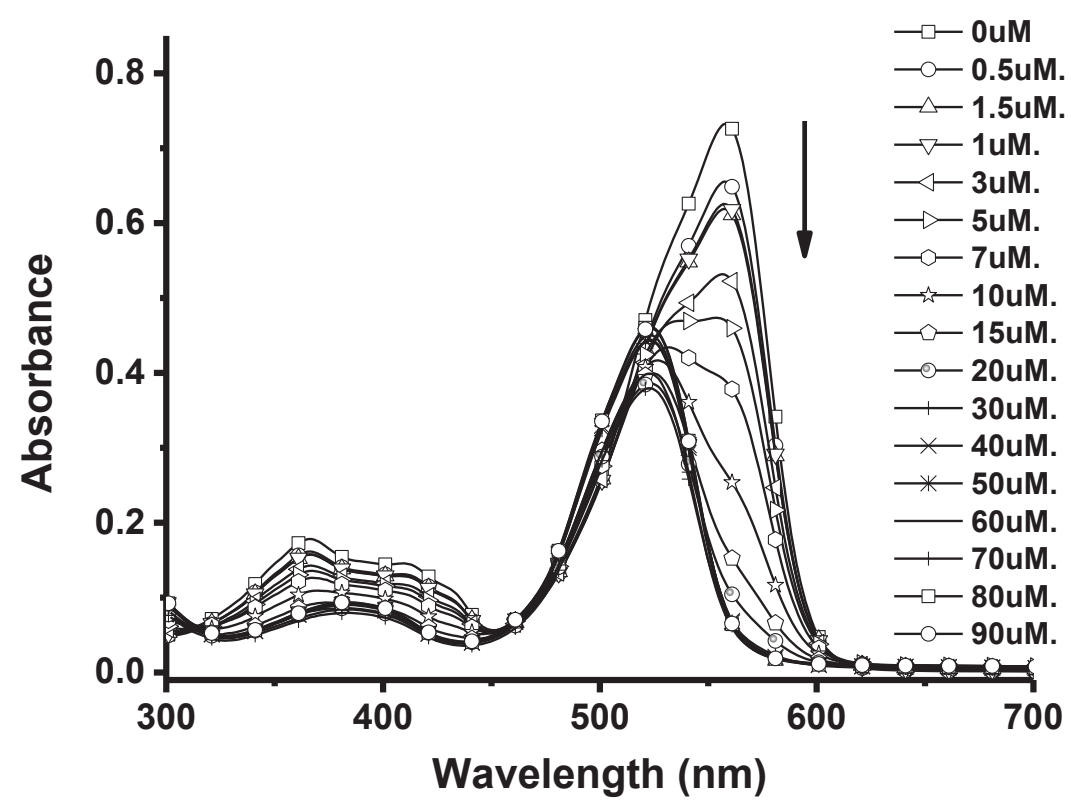

Figure C.29. Absorption spectra of the probe 5-B $(20 \mu \mathrm{M})$ in the absence and presence of different amount of cyanide ion in Tris buffer $(10 \mathrm{mM}, \mathrm{pH} 9.3)$ solution. 


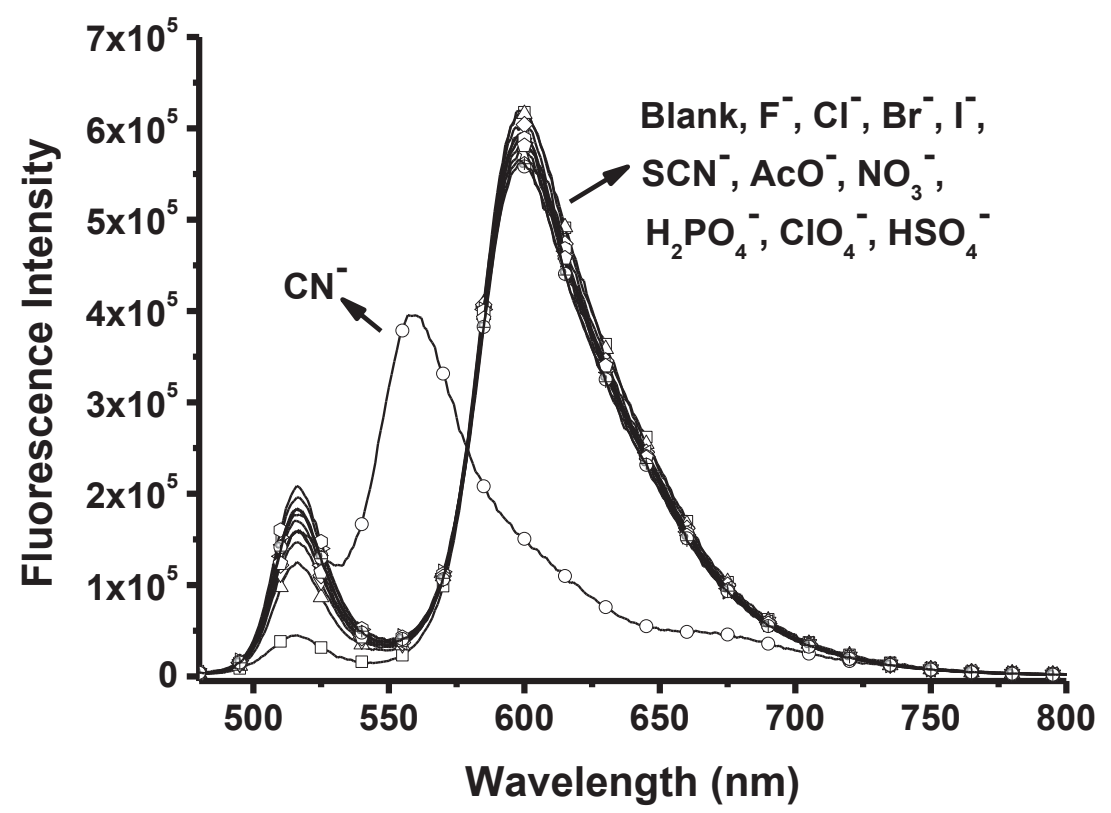

Figure C.30. Emission spectra of probe 5-B in the presence of different anions (100 $\mu \mathrm{M})$ in Tris buffer (10 mM, pH 9.3) solution.

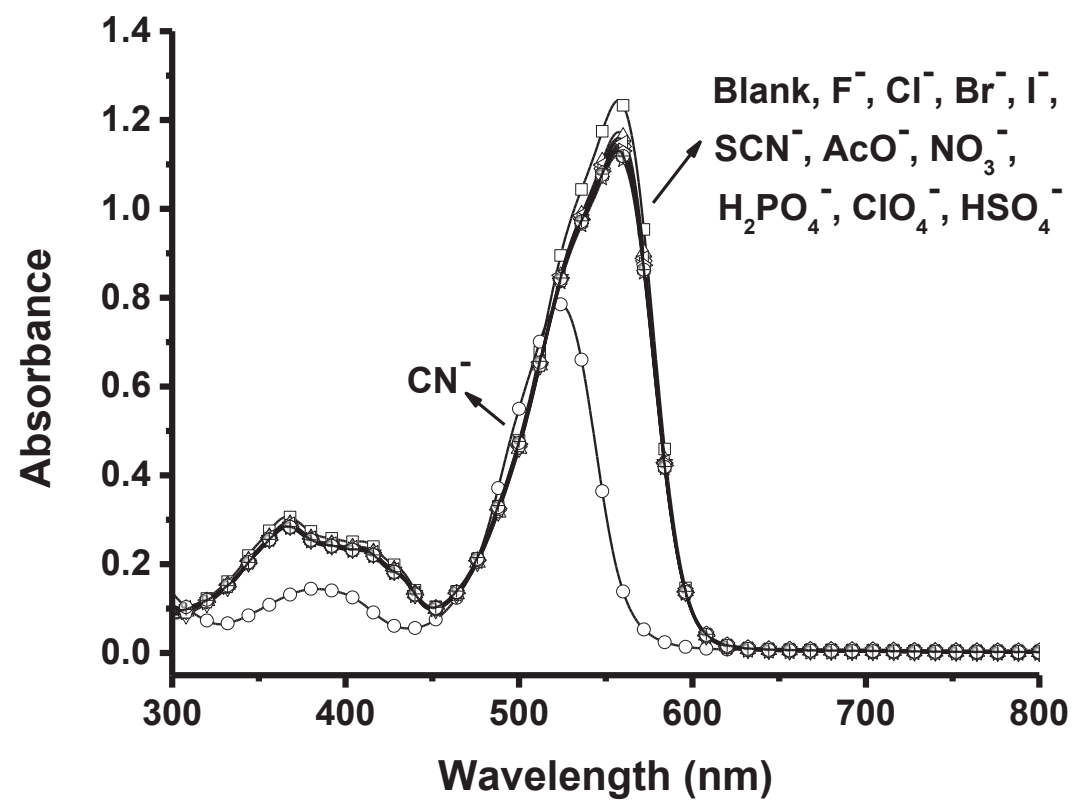

Figure C.31. Absorption spectra of probe 5-B in the presence of different anions (100 $\mu \mathrm{M})$ in Tris buffer $(10 \mathrm{mM}, \mathrm{pH} 9.3)$ solution. 


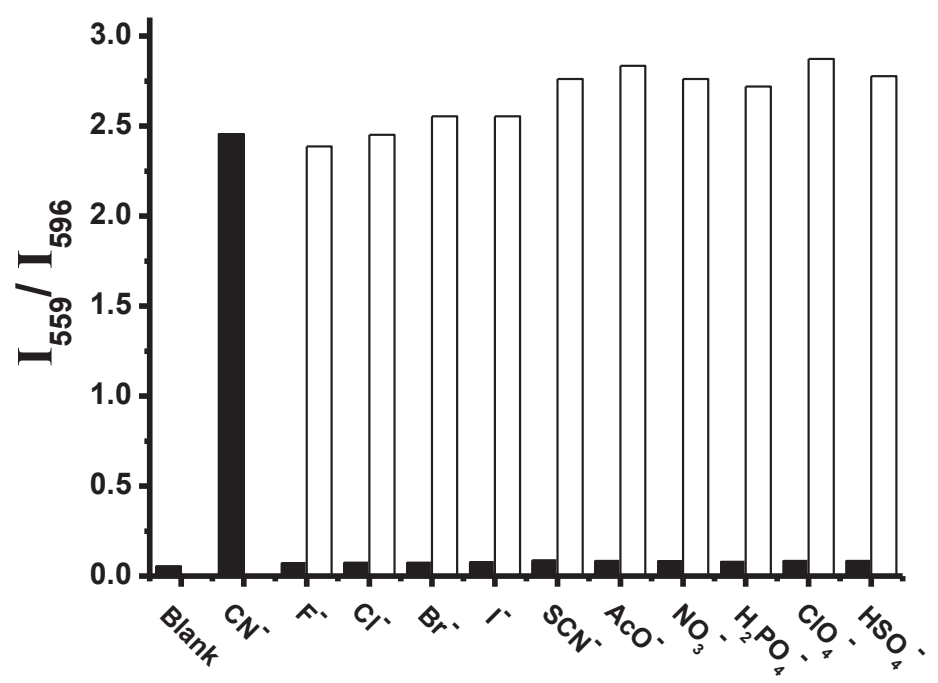

Figure C.32. The fluorescent ratiometric responses of probe 5-B to different anions $(100 \mu \mathrm{M})$ in Tris buffer solution $(10 \mathrm{mM}, \mathrm{pH} 9.3)$ in the presence and presence of 70 $\mu \mathrm{M}$ cyanide ion. Black bars show the fluorescent responses of the probe 5-B to different anions while white bars display the fluorescent responses of the probe to different anions in the presence of cyanide ion.

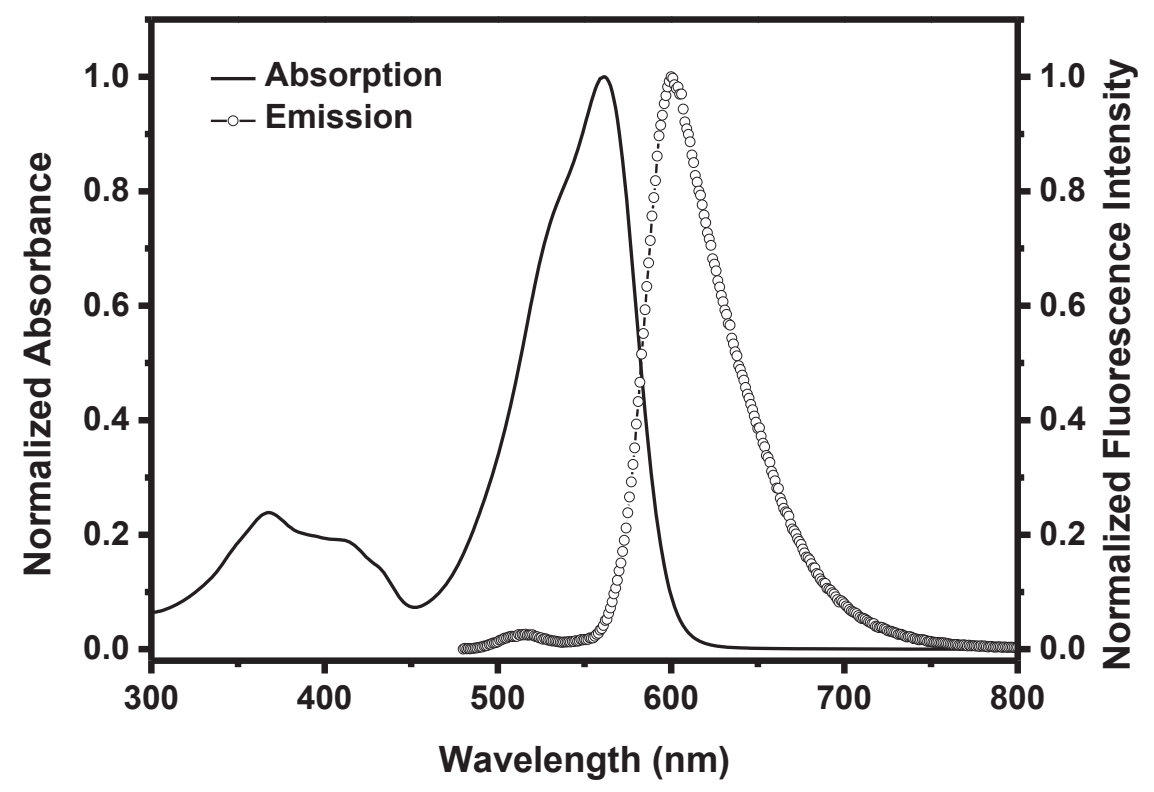

Figure C.33. Normalized absorption and emission spectra of fluorescent probe 5-C in Tris buffer solution (10 mM, pH 9.3) 


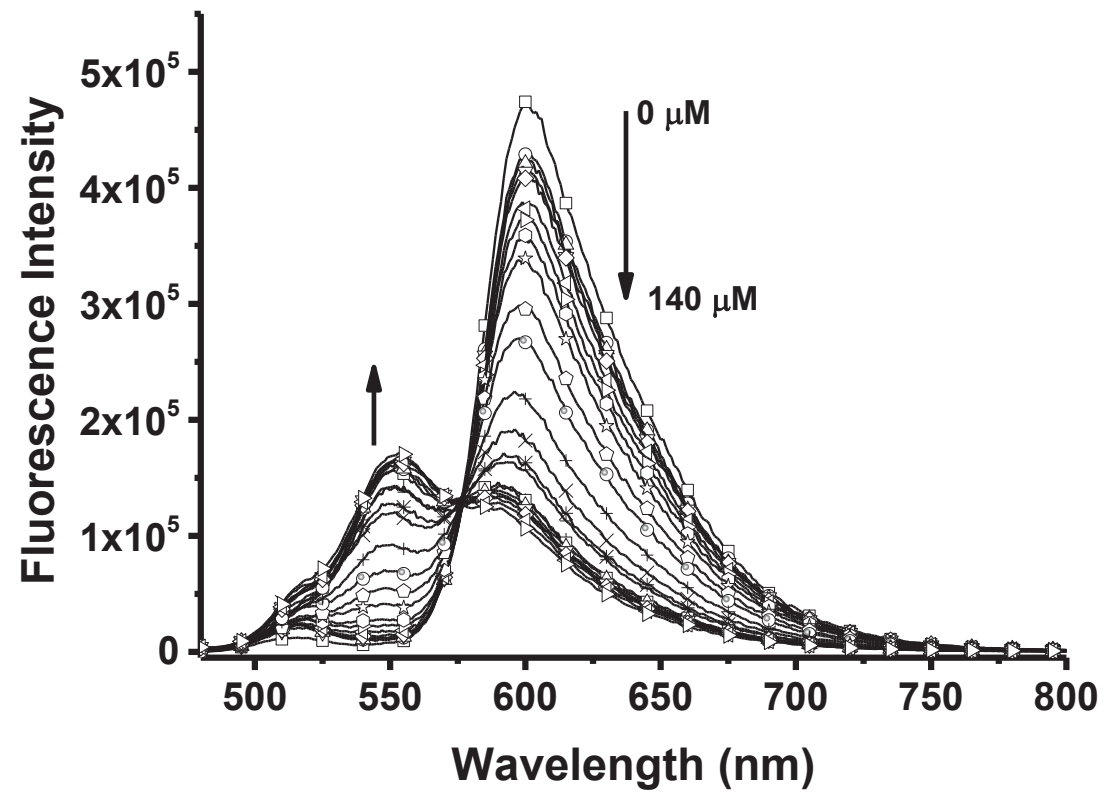

Figure C.34. Fluorescence spectra of the fluorescent probe 5-C $(20 \mu \mathrm{M})$ in the absence and presence of different amounts of cyanide ion in Tris buffer $(10 \mathrm{mM}, \mathrm{pH} 9.3)$ solution.

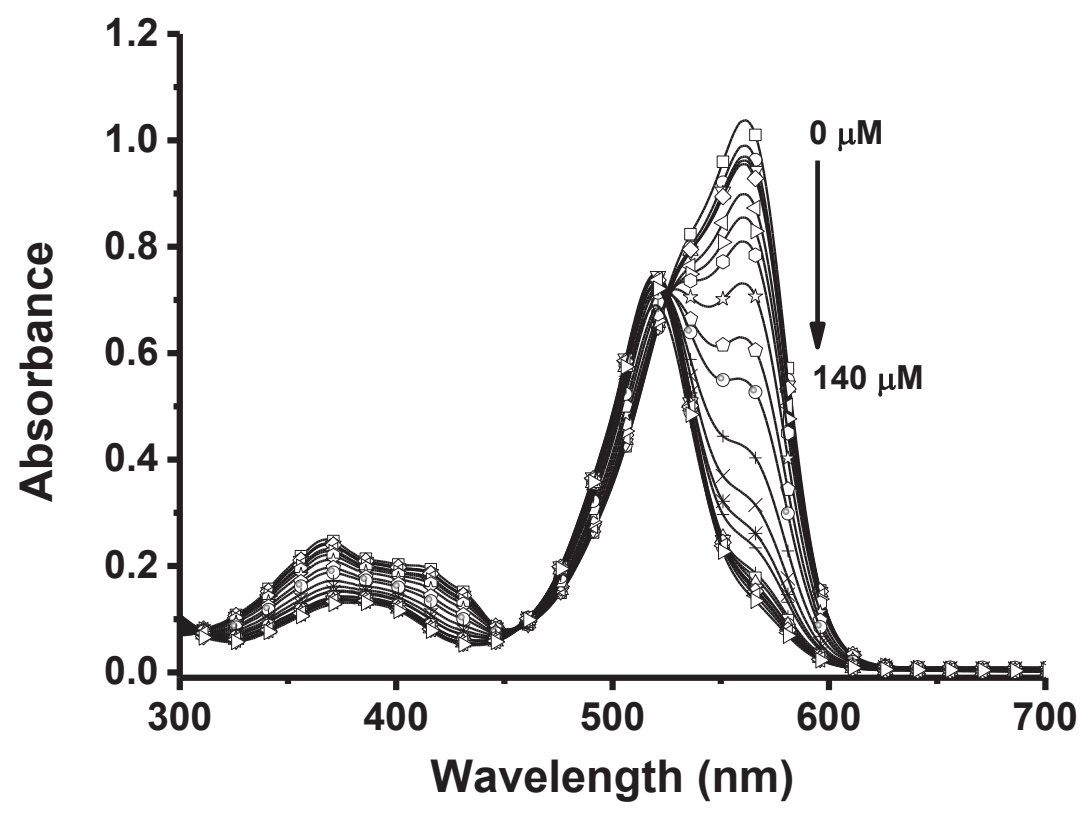

Figure C.35. Absorption spectra of the probe 5-C $(20 \mu \mathrm{M})$ in the absence and presence of different amount of cyanide ion Tris buffer (10 mM, pH 9.3) solution. 


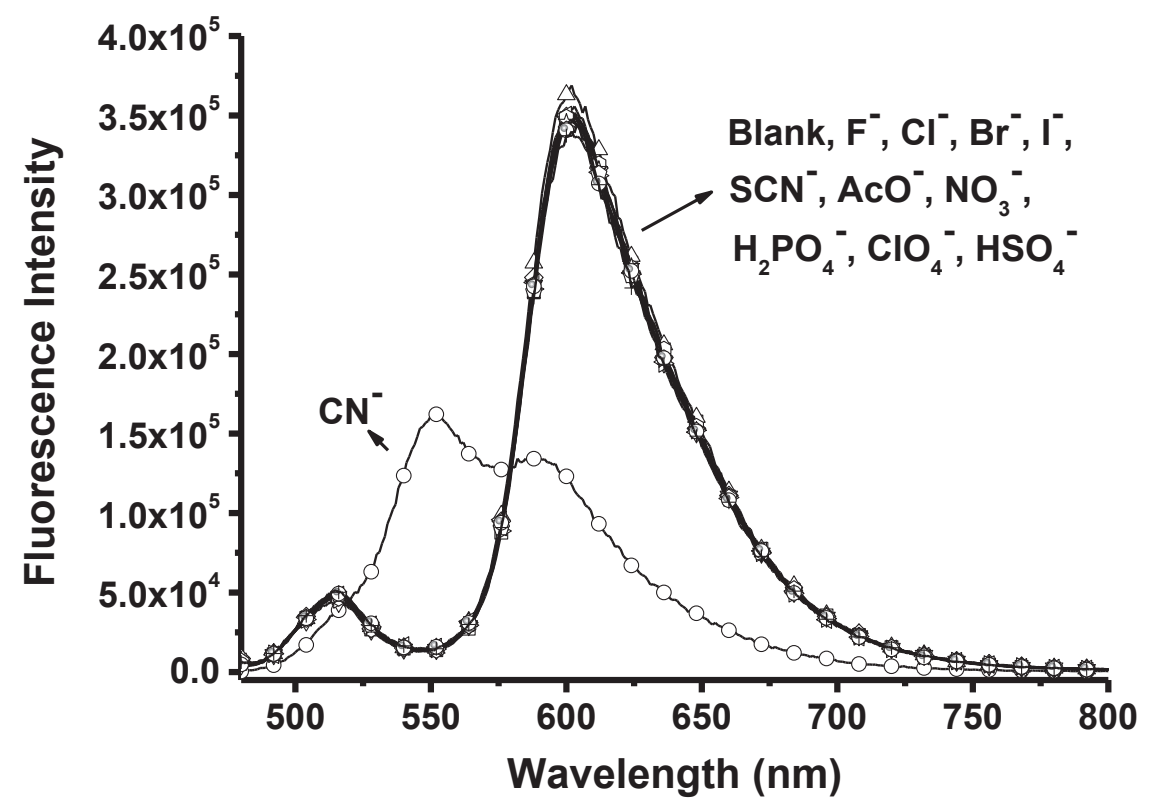

Figure C.36. Emission spectra of probe 5-C in the presence of different anions (100 $\mu \mathrm{M})$ in Tris buffer (10 mM, pH 9.3) solution.

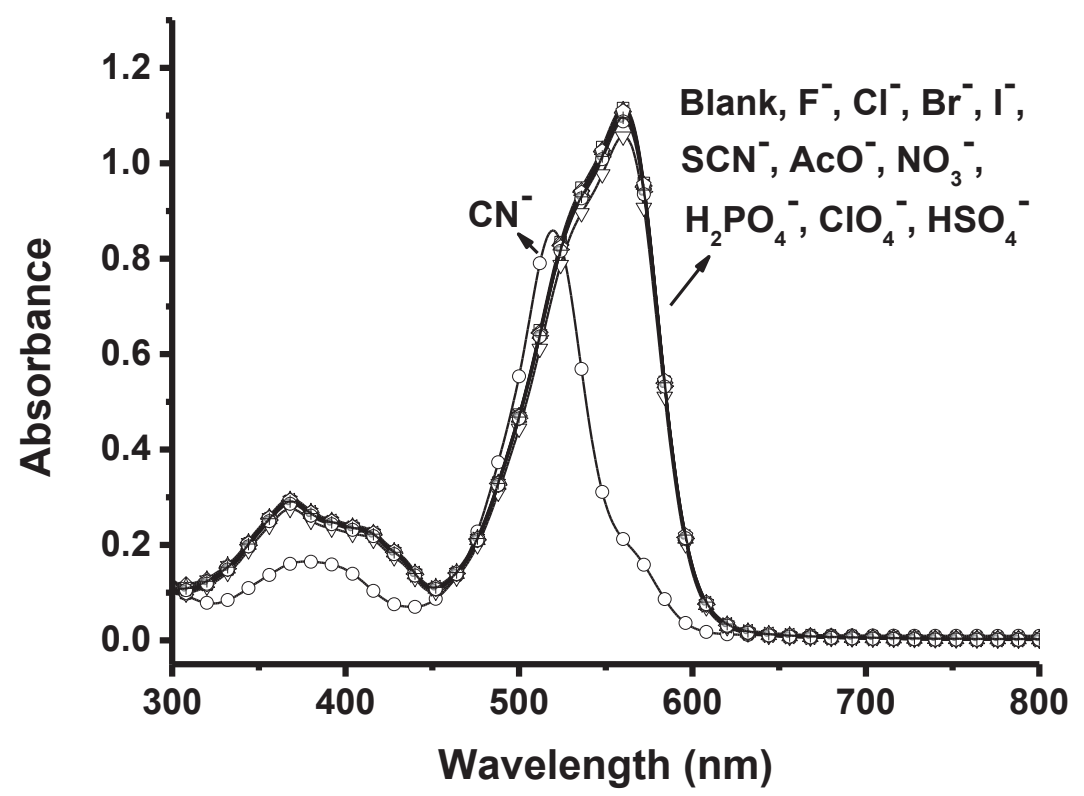

Figure C.37. Absorption spectra of probe 5-C in the presence of different anions (100 $\mu \mathrm{M})$ in Tris buffer $(10 \mathrm{mM}, \mathrm{pH} 9.3)$ solution. 
a

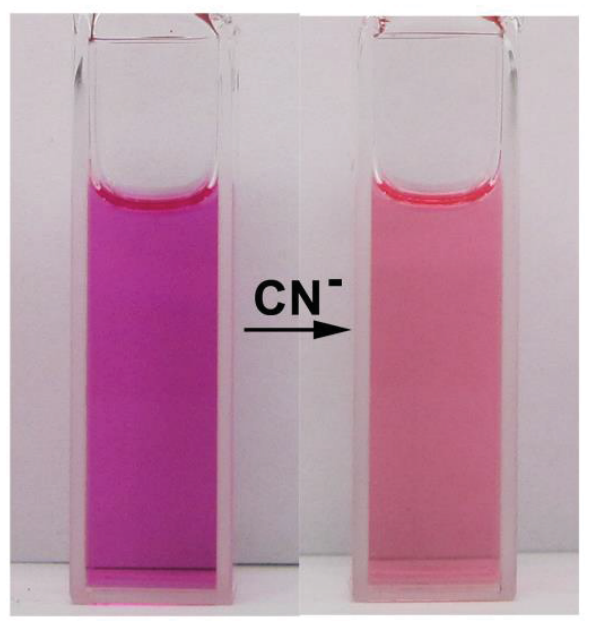
probe 1 b

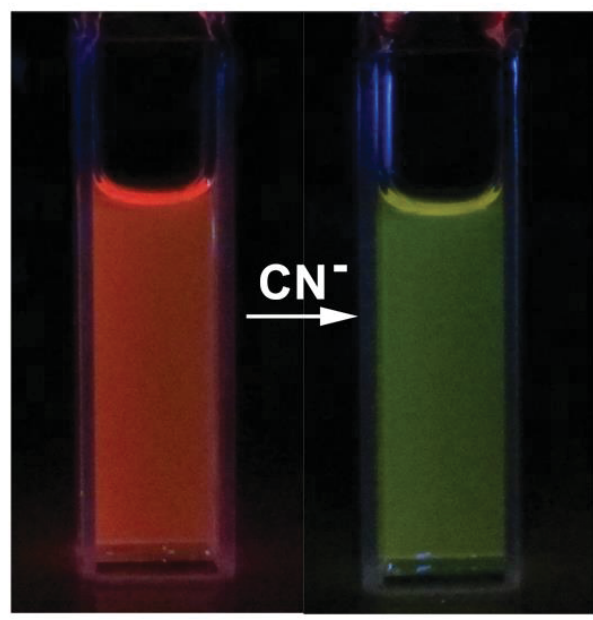

probe 1 1-CN adduct

Figure C.38. Comparison of visible color (a) and luminescence color (b) of probe 5-A in absence and presence of cyanide ions. 


\section{Appendix D Supporting Information for Chapter 6}
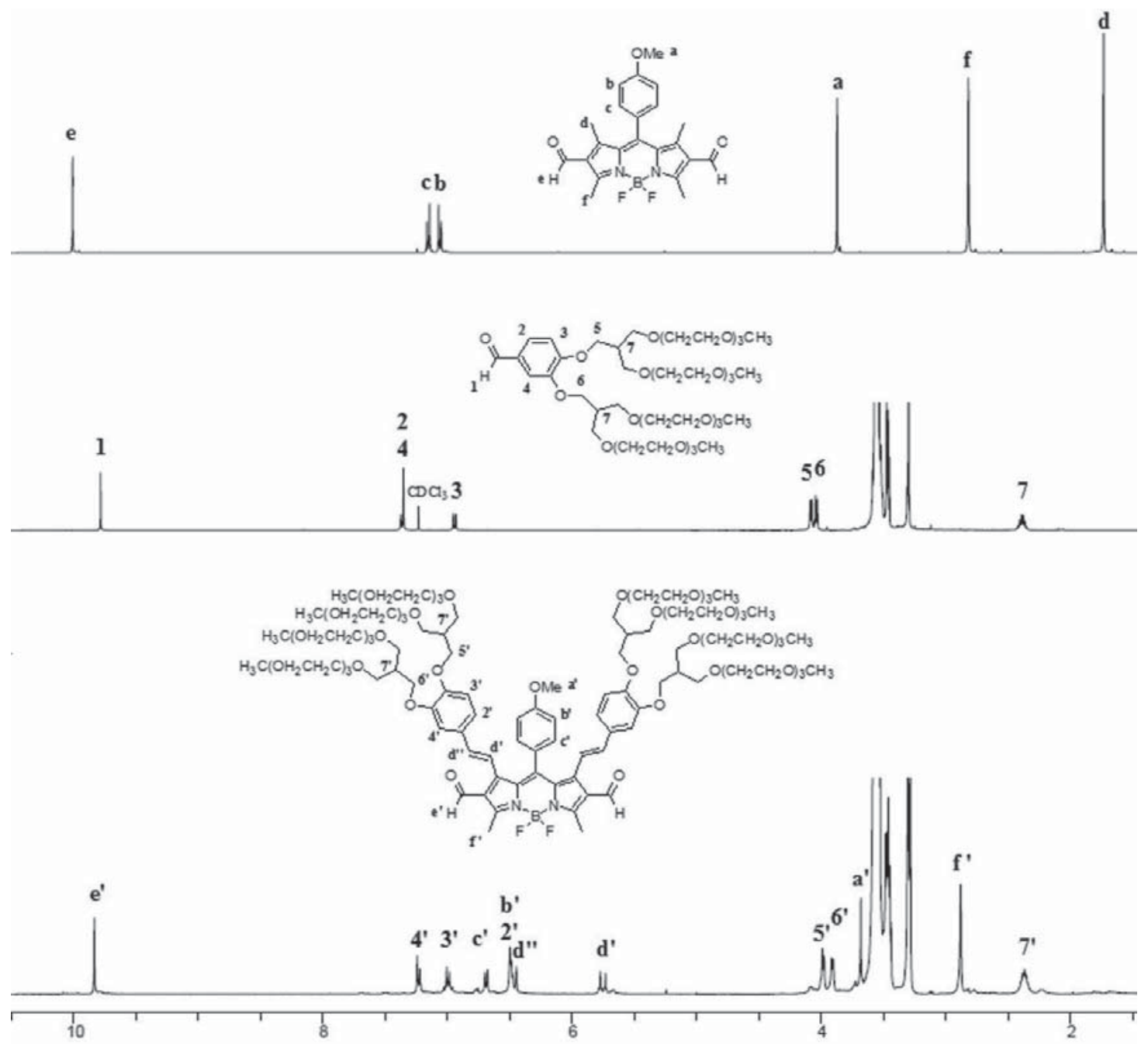

Figure D.1. Comparing of ${ }^{1} \mathrm{H}$ spectra of BODIPY dyes 6.6, and $\mathbf{6 . 8}$ and compound $\mathbf{6 . 7}$ 


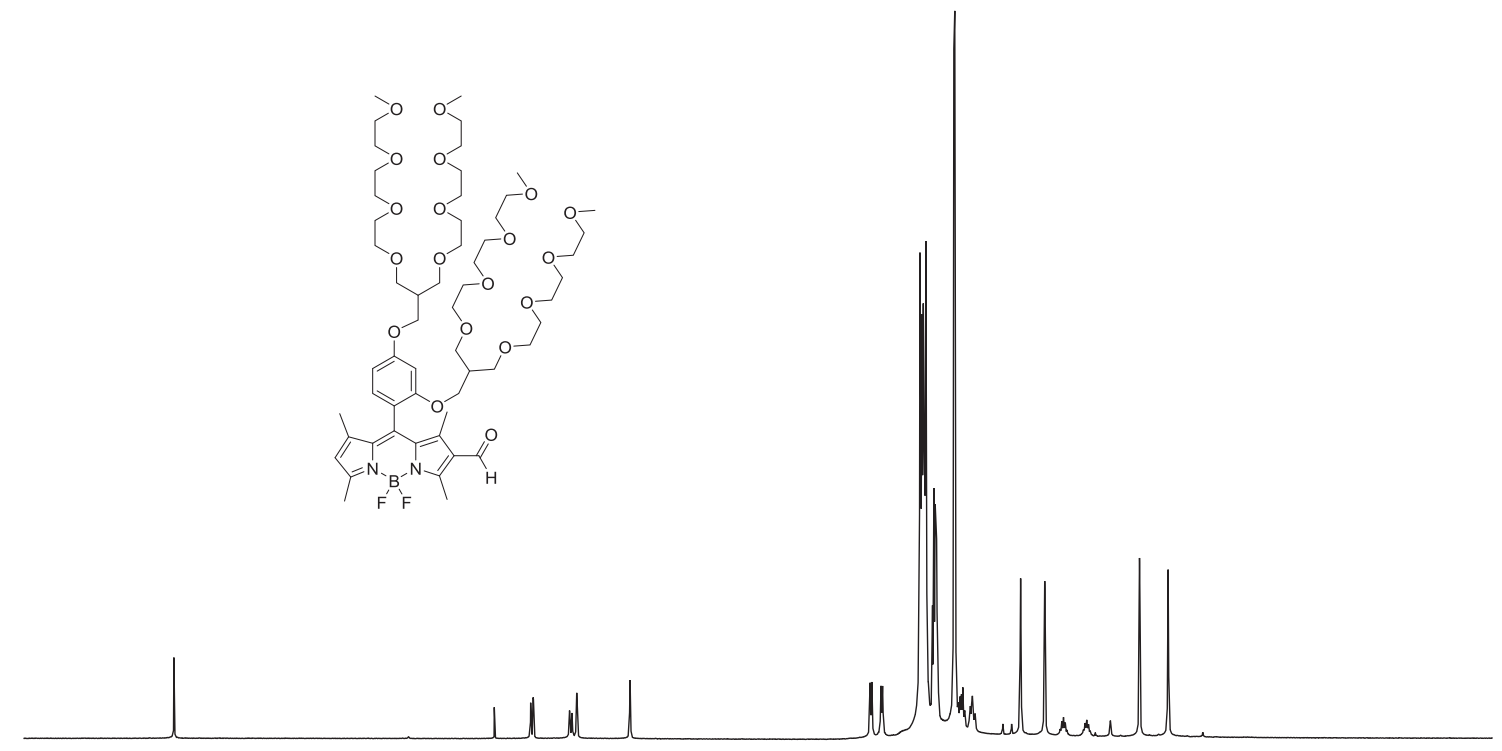

\begin{tabular}{llllllllllll}
\hline 10.5 & 9.5 & 8.5 & 7.5 & 6.5 & 5.5 & 4.5 & 3.5 & 2.5 & 1.5 & 0.5 & -0.5
\end{tabular}

Figure D.2. ${ }^{1} \mathrm{H}$ NMR spectrum of BODIPY dye $\mathbf{6 . 3}$ in $\mathrm{CDCl}_{3}$ solution.

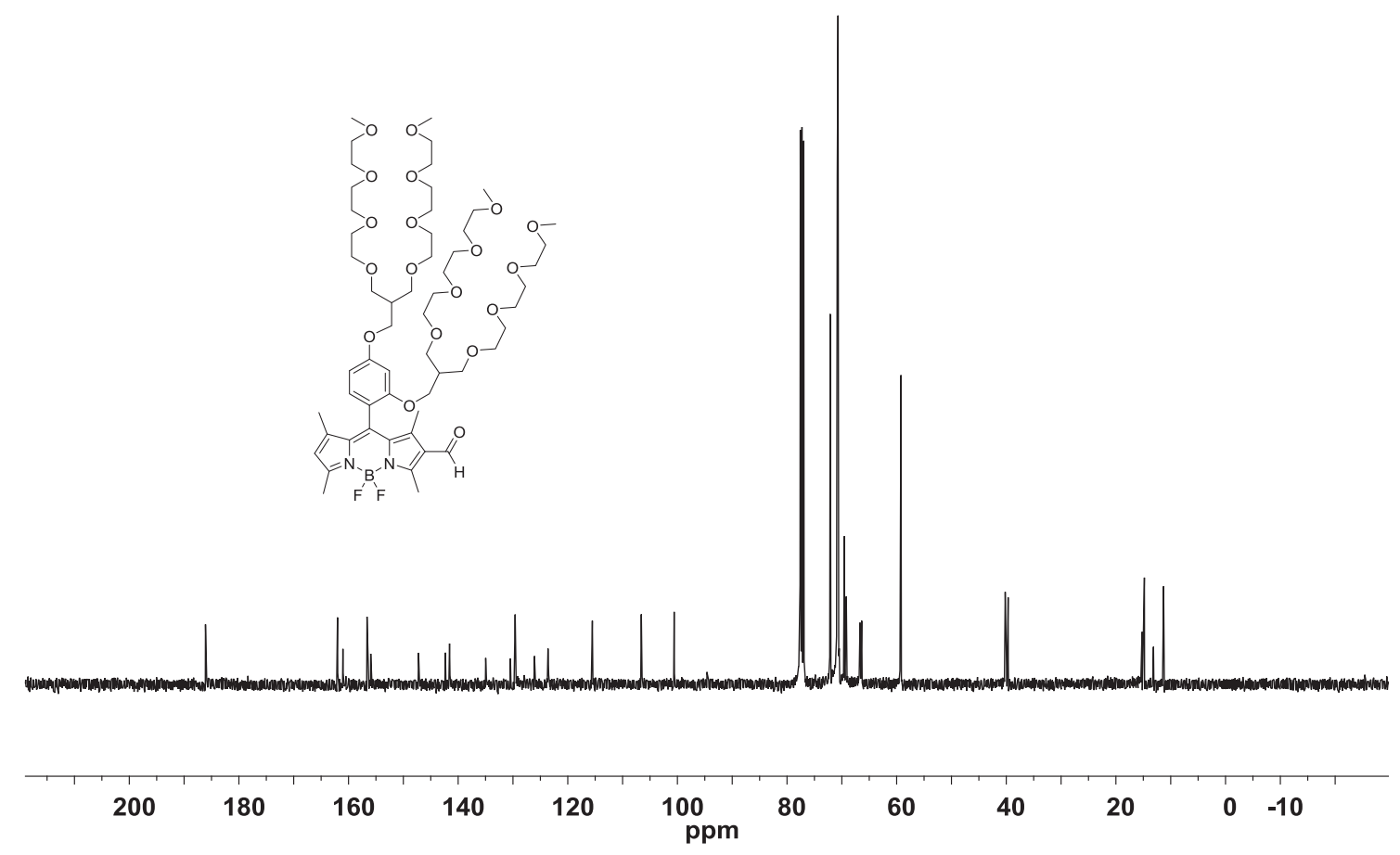

Figure D.3. ${ }^{13} \mathrm{C}$ NMR spectrum of BODIPY dye 6.3 in $\mathrm{CDCl}_{3}$ solution. 


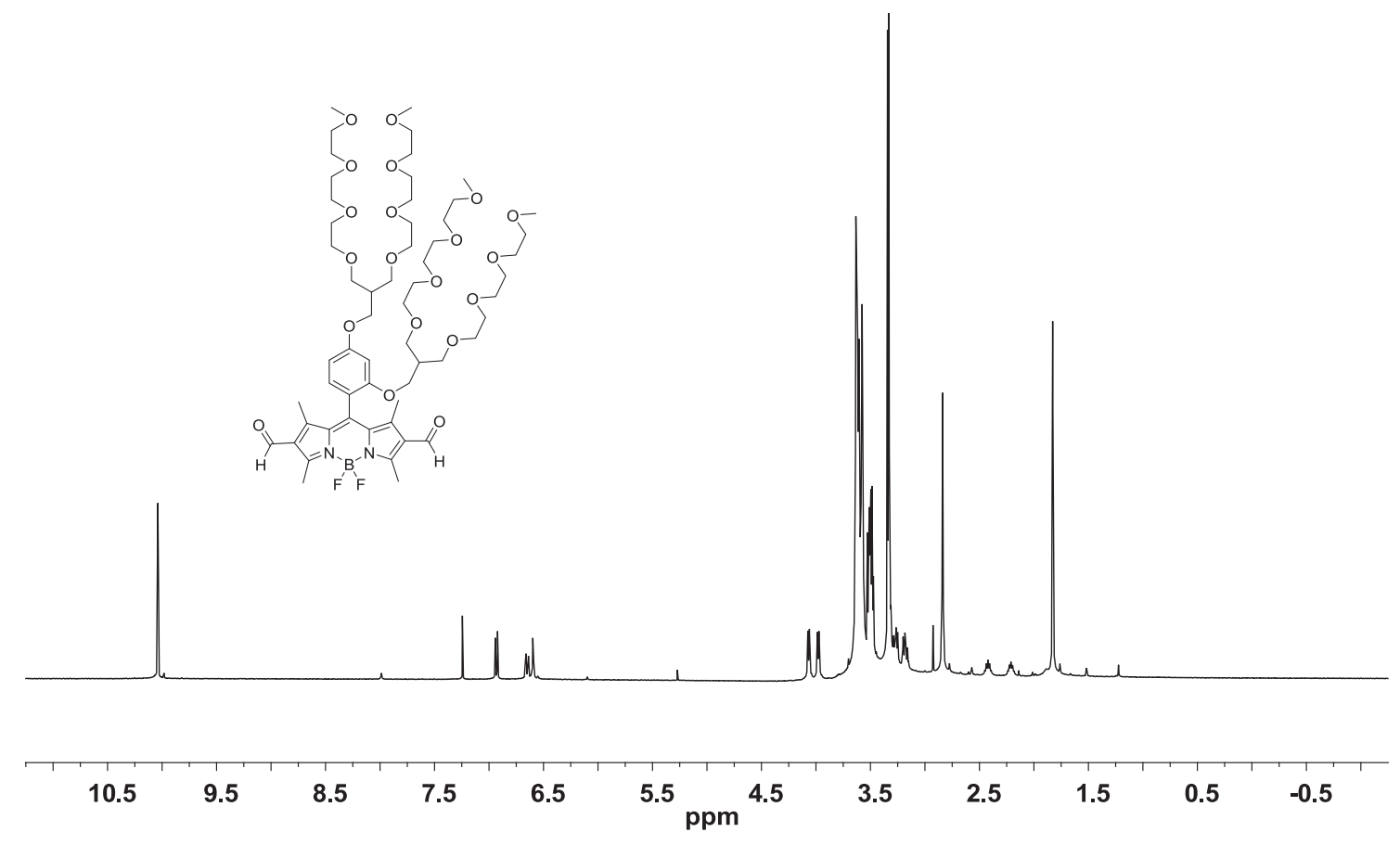

Figure D.4. ${ }^{1} \mathrm{H}$ NMR spectrum of BODIPY dye $\mathbf{6 . 5}$ in $\mathrm{CDCl}_{3}$ solution.

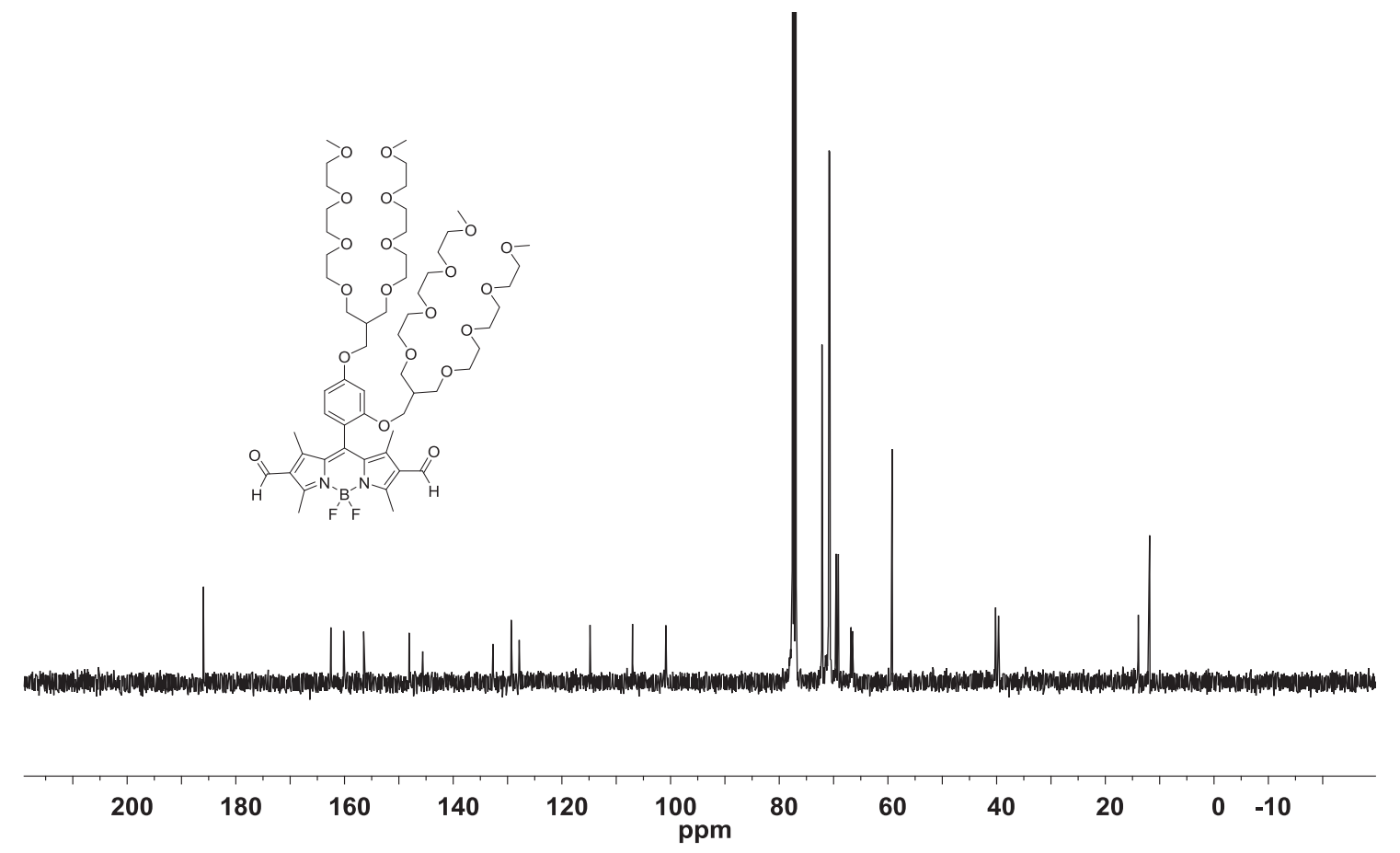

Figure D.5. ${ }^{13} \mathrm{C}$ NMR spectrum of BODIPY dye $\mathbf{6 . 5}$ in $\mathrm{CDCl}_{3}$ solution. 

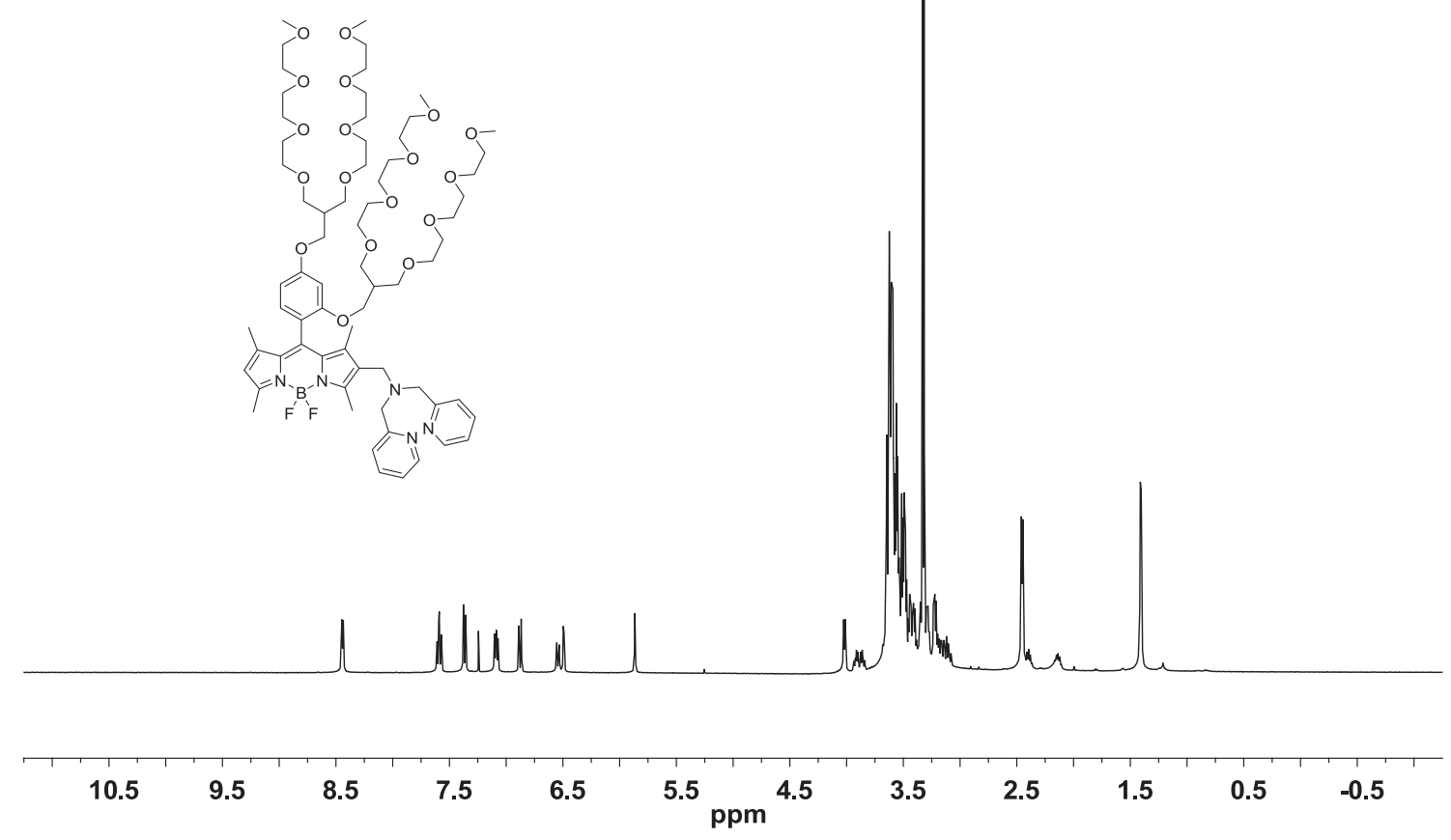

Figure D.6. ${ }^{1} \mathrm{H}$ NMR spectrum of fluorescent probe 6-A in $\mathrm{CDCl}_{3}$ solution.

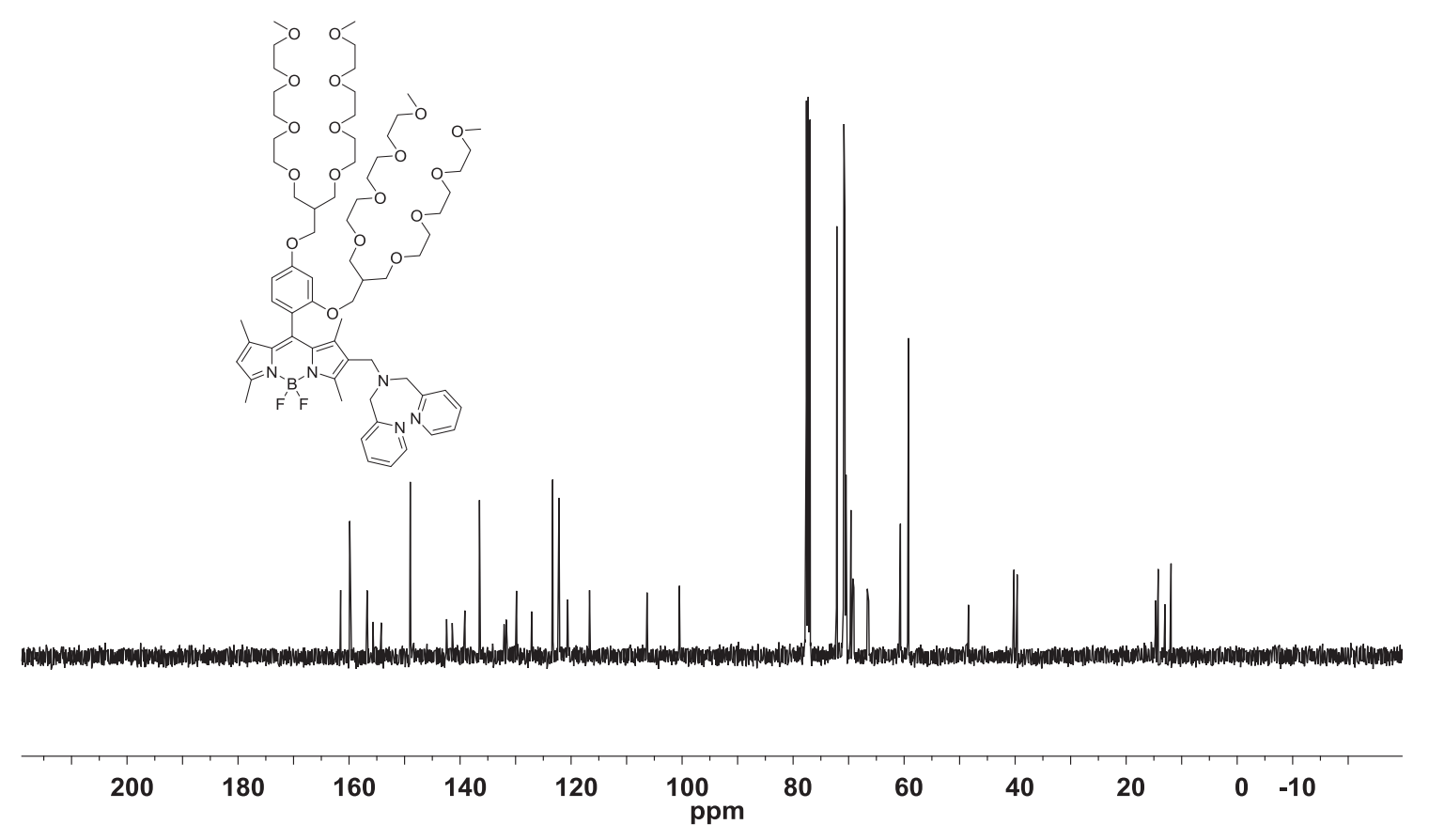

Figure D.7. ${ }^{13} \mathrm{C}$ NMR spectrum of fluorescent probe 6-A in $\mathrm{CDCl}_{3}$ solution. 


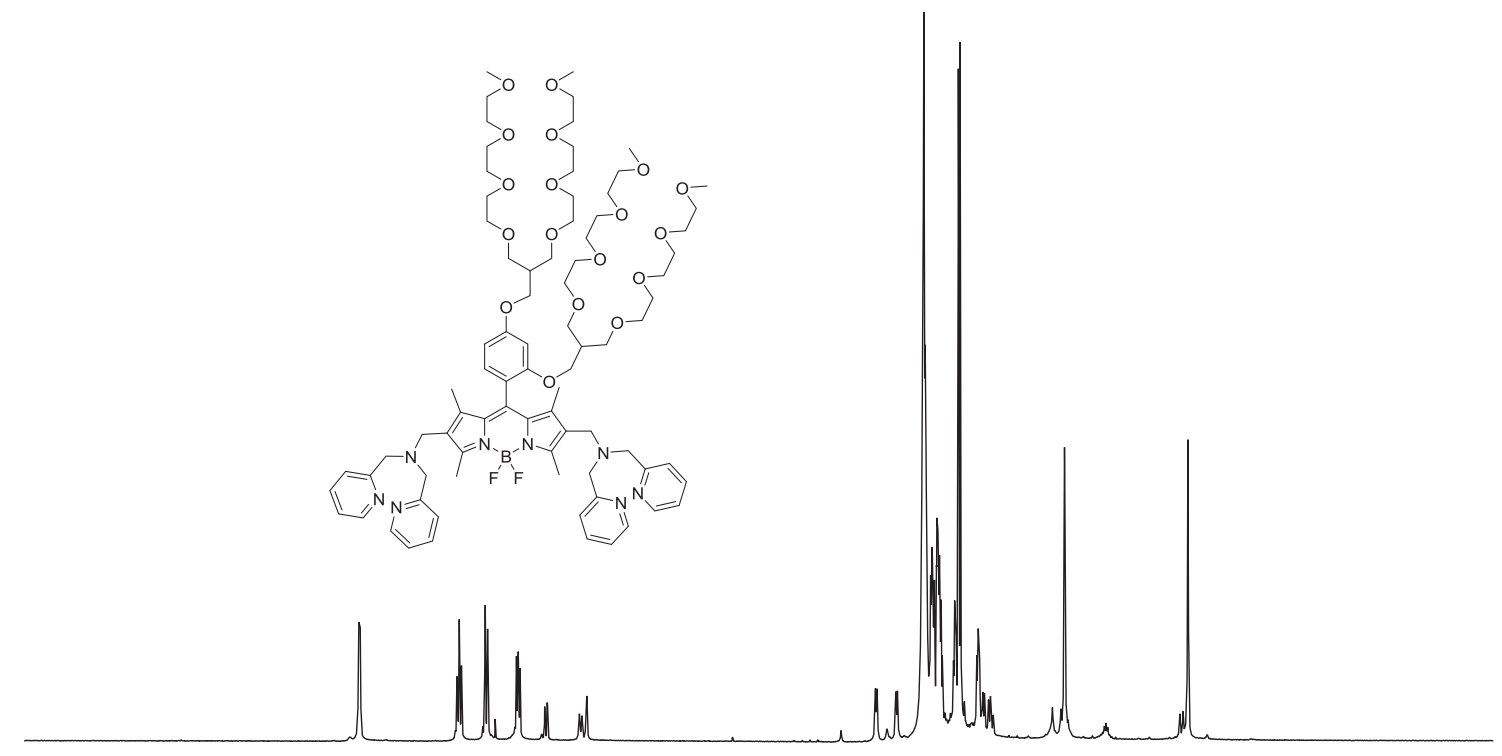

$\begin{array}{llllllllllllll} & 10.5 & 9.5 & 8.5 & 7.5 & 6.5 & 5.5 & 4.5 & 3.5 & 2.5 & 1.5 & 0.5 & -0.5\end{array}$

Figure D.8. ${ }^{1} \mathrm{H}$ NMR spectrum of fluorescent probe 6-B in $\mathrm{CDCl}_{3}$ solution.

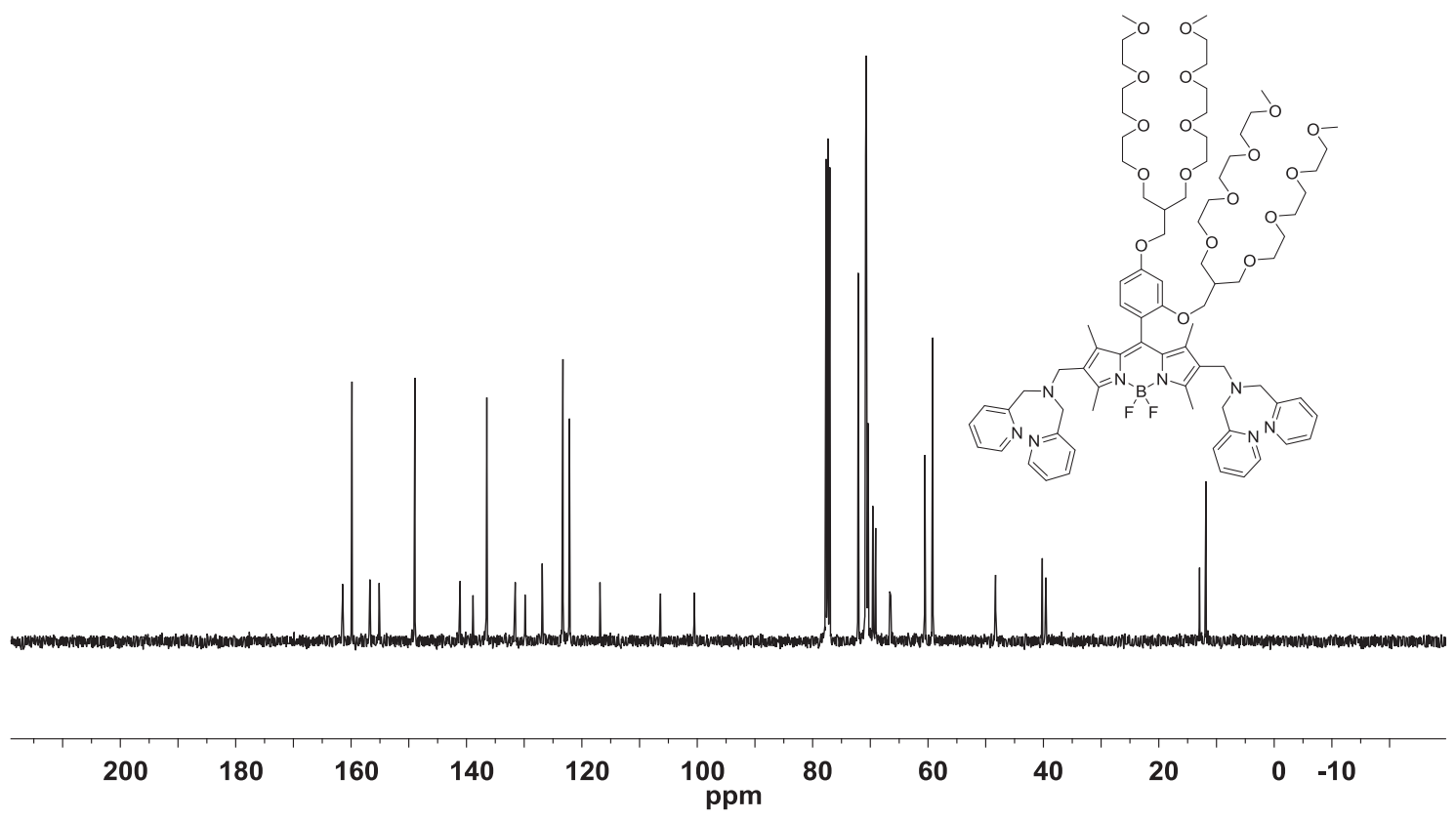

Figure D.9. ${ }^{13} \mathrm{H}$ NMR spectrum of fluorescent probe 6-B in $\mathrm{CDCl}_{3}$ solution. 


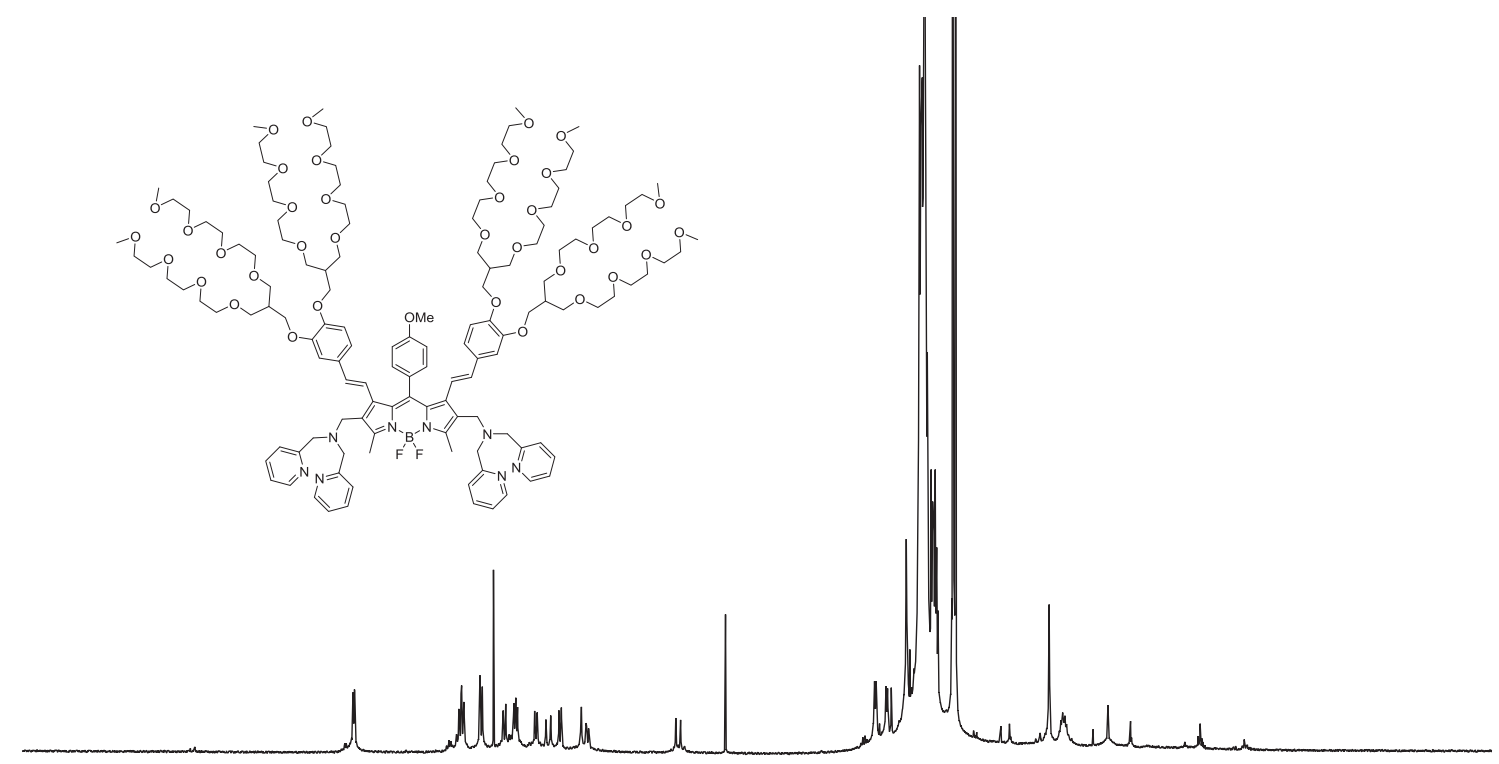

\begin{tabular}{llllllllllll}
\hline 10.5 & 9.5 & 8.5 & 7.5 & 6.5 & 5.5 & 4.5 & 3.5 & 2.5 & 1.5 & 0.5 & -0.5
\end{tabular}

Figure D.10. ${ }^{1} \mathrm{H}$ NMR spectrum of fluorescent probe $6-\mathrm{C}$ in $\mathrm{CDCl}_{3}$ solution.

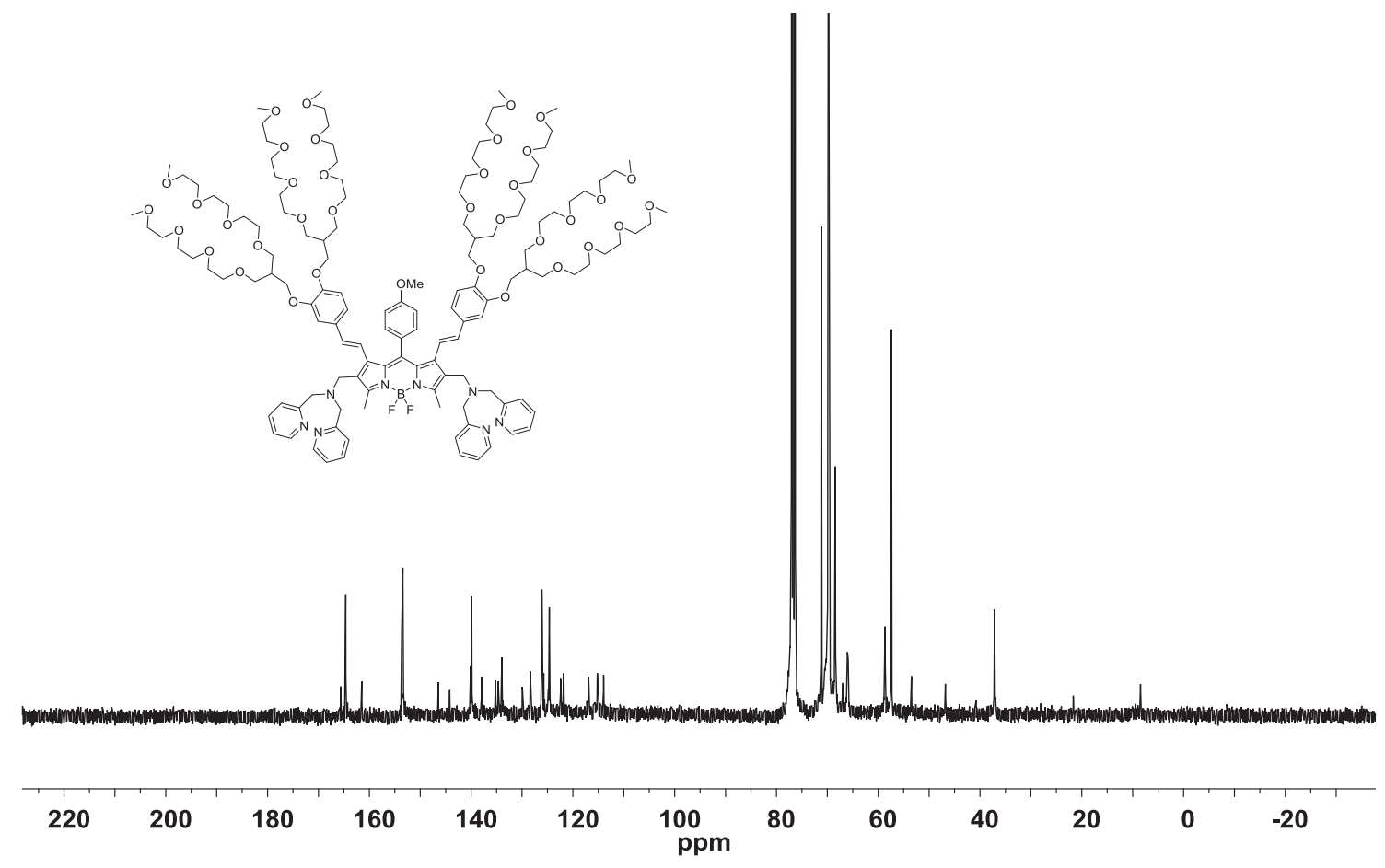

Figure D.11. ${ }^{13} \mathrm{C}$ NMR spectrum of fluorescent probe 6-C in $\mathrm{CDCl}_{3}$ solution. 


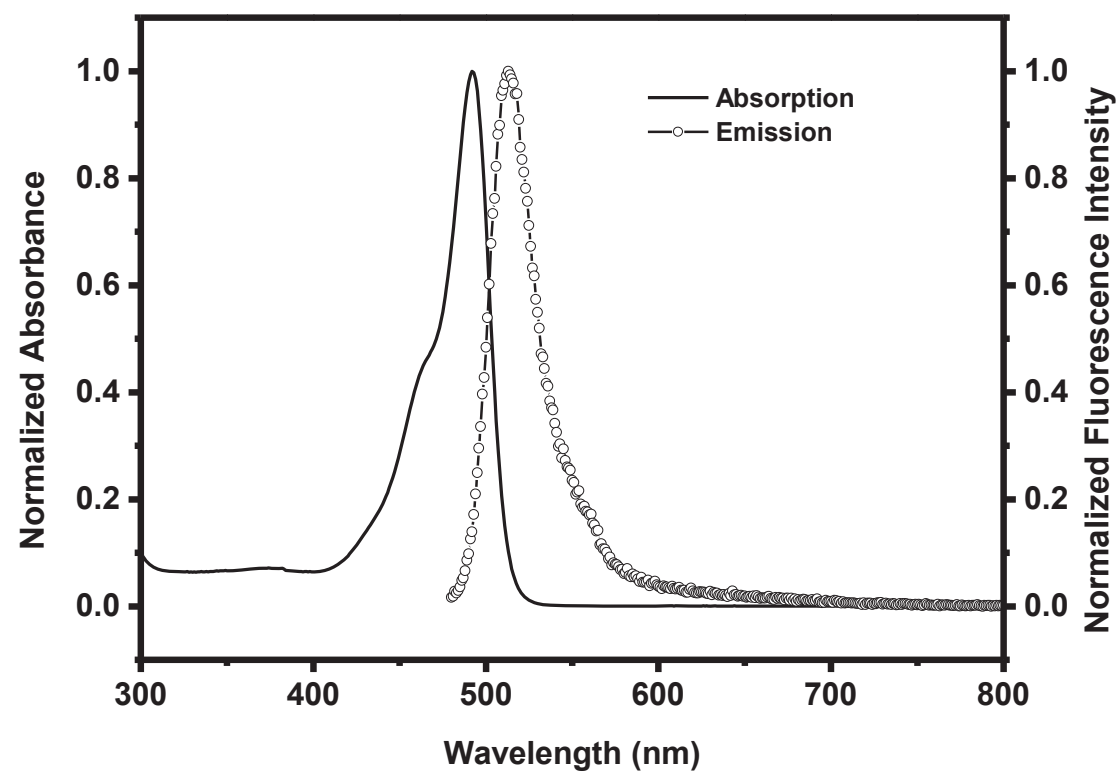

Figure D.12. Normalized absorption and emission spectra of BODIPY dye 6.3 in $10 \mathrm{mM}$ HEPES buffer solution pH 7.0.

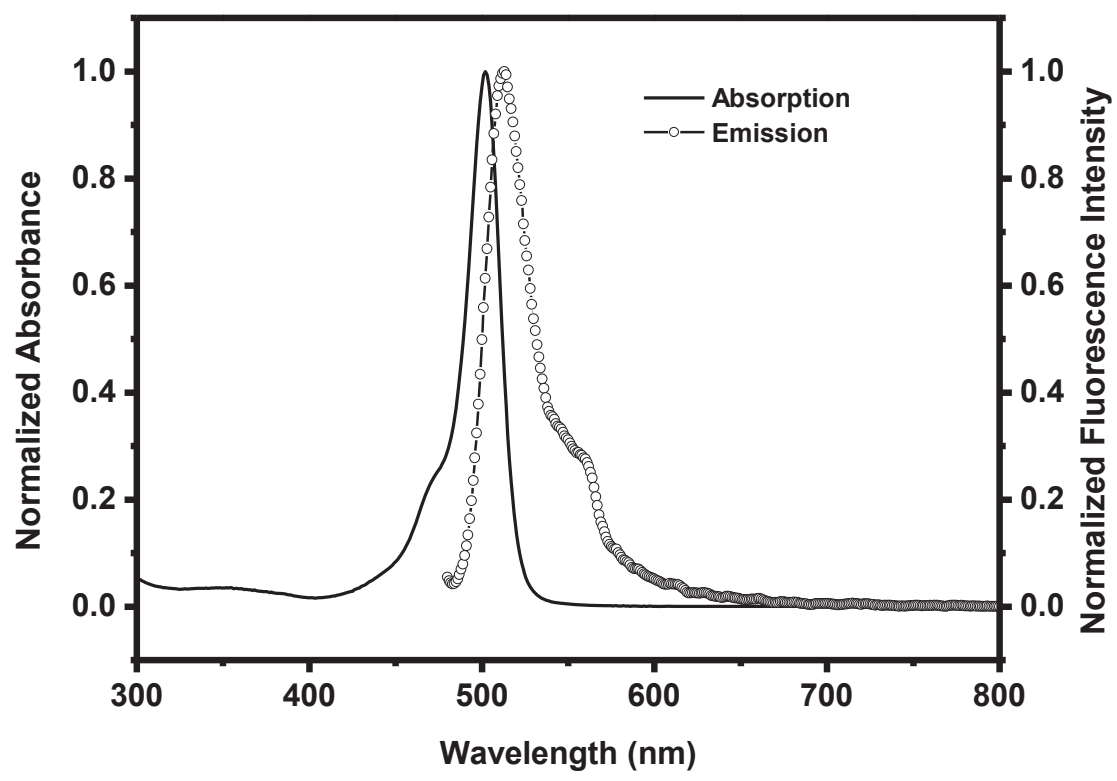

Figure D.13. Normalized absorption and emission spectra of BODIPY dye 6.5 in $10 \mathrm{mM}$ HEPES buffer solution $\mathrm{pH}$ 7.0. 


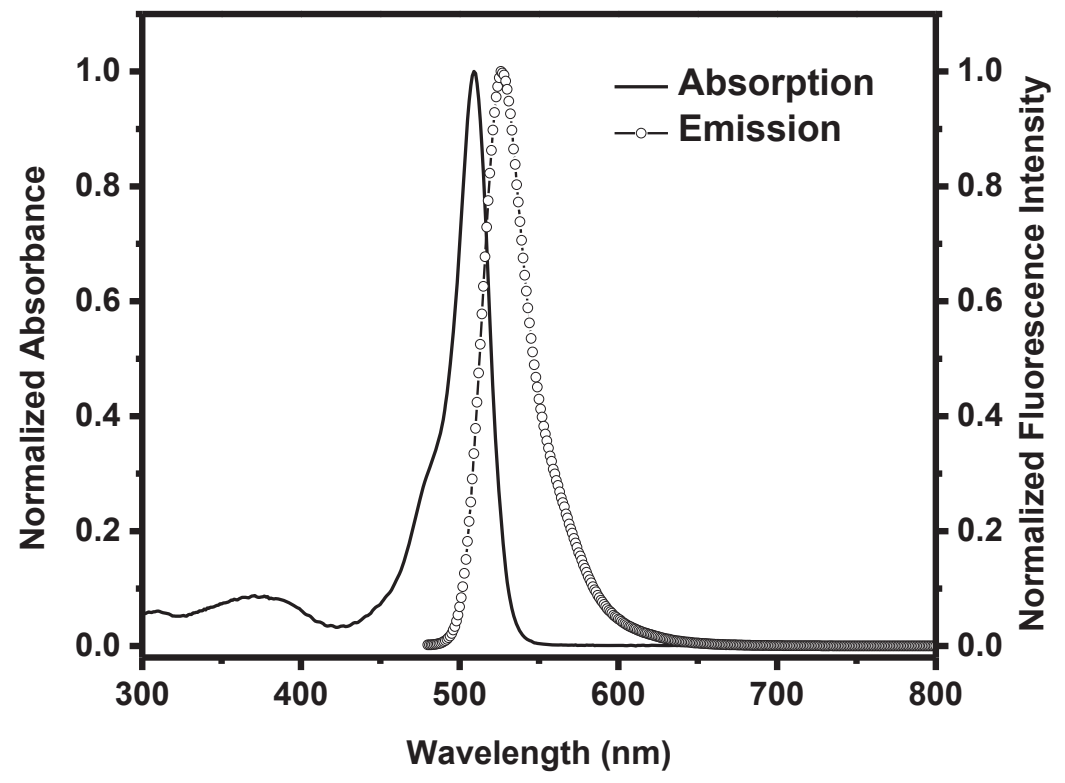

Figure D.14. Normalized absorption and emission spectra of fluorescent probe 6-A in 10 mM HEPES buffer solution $\mathrm{pH}$ 7.0.

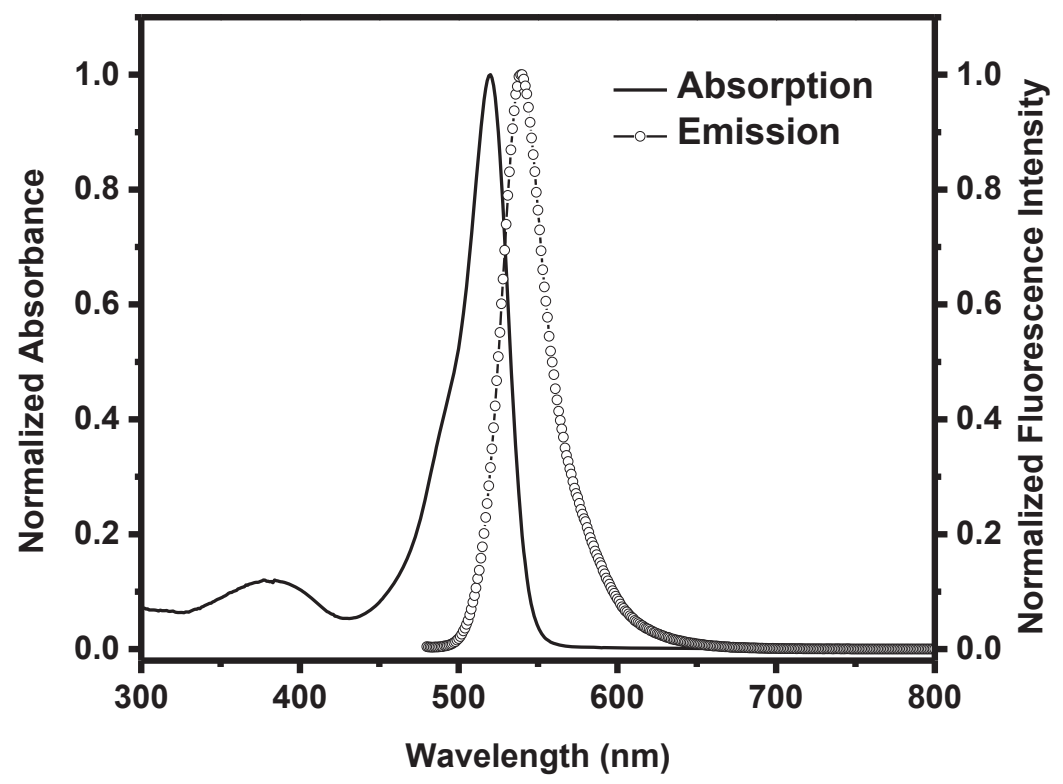

Figure D.15. Normalized absorption and emission spectra of fluorescent probe 6-B in 10 mM HEPES buffer solution $\mathrm{pH}$ 7.0. 


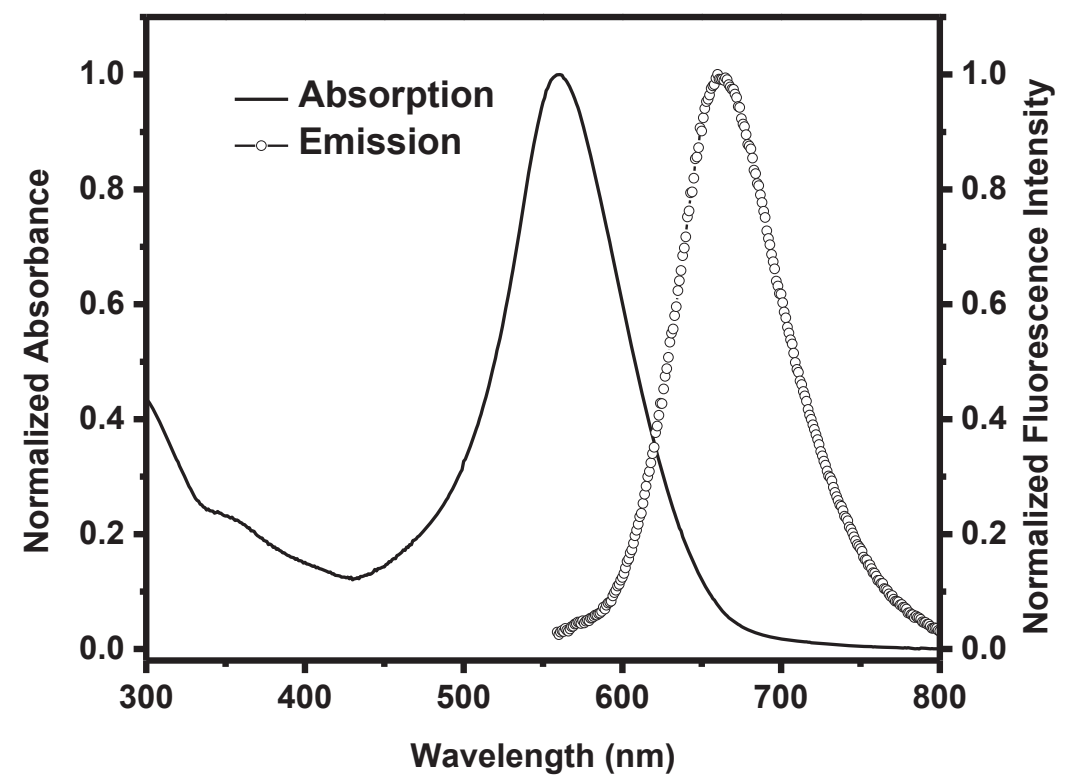

Figure D.16. Normalized absorption and emission spectra of fluorescent probe 6-C in 10 mM HEPES buffer solution $\mathrm{pH}$ 7.0.

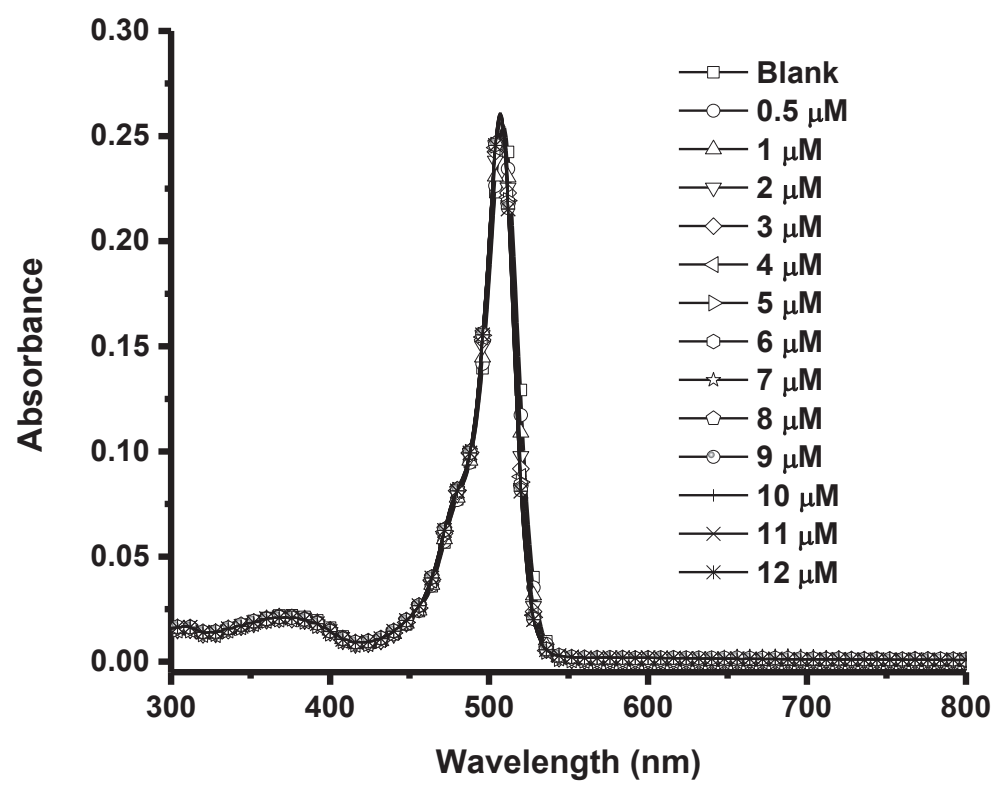

Figure D.17. Absorption spectra of fluorescent probe 6-A upon addition of different amount of $\mathrm{ZnCl}_{2}$ in $10 \mathrm{mM}$ HEPES buffer $\mathrm{pH}$ 7.0. 


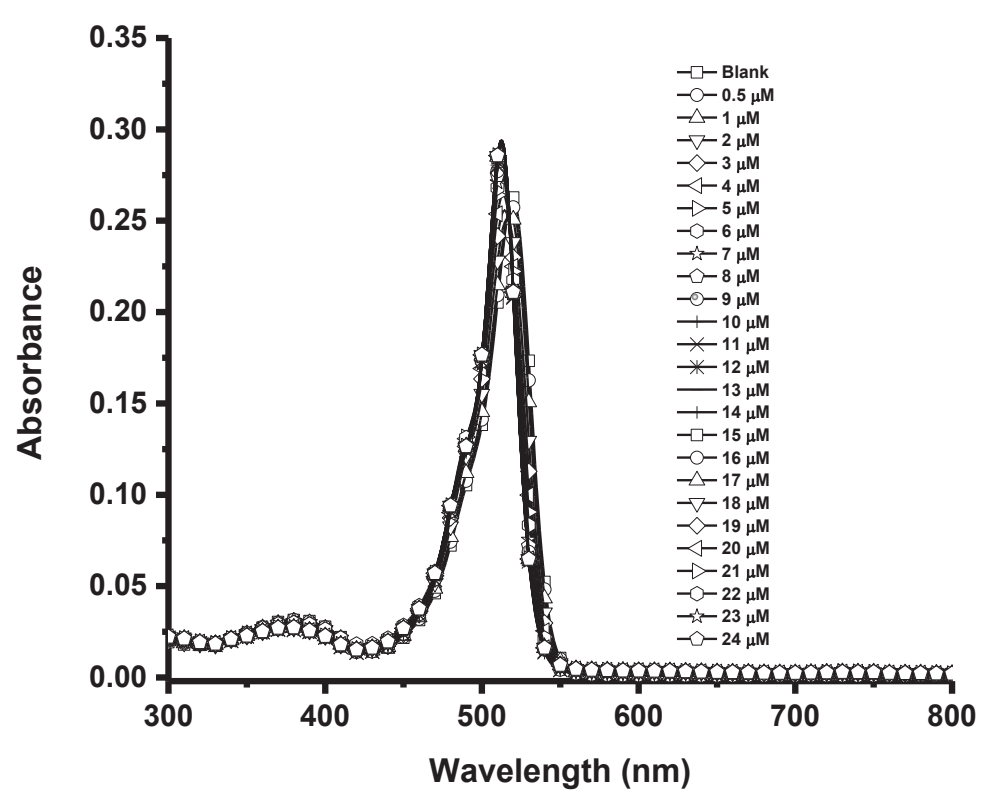

Figure D.18. Absorption spectra of fluorescent probe 6-B upon addition of different amount of $\mathrm{ZnCl}_{2}$ in $10 \mathrm{mM}$ HEPES buffer $\mathrm{pH}$ 7.0.

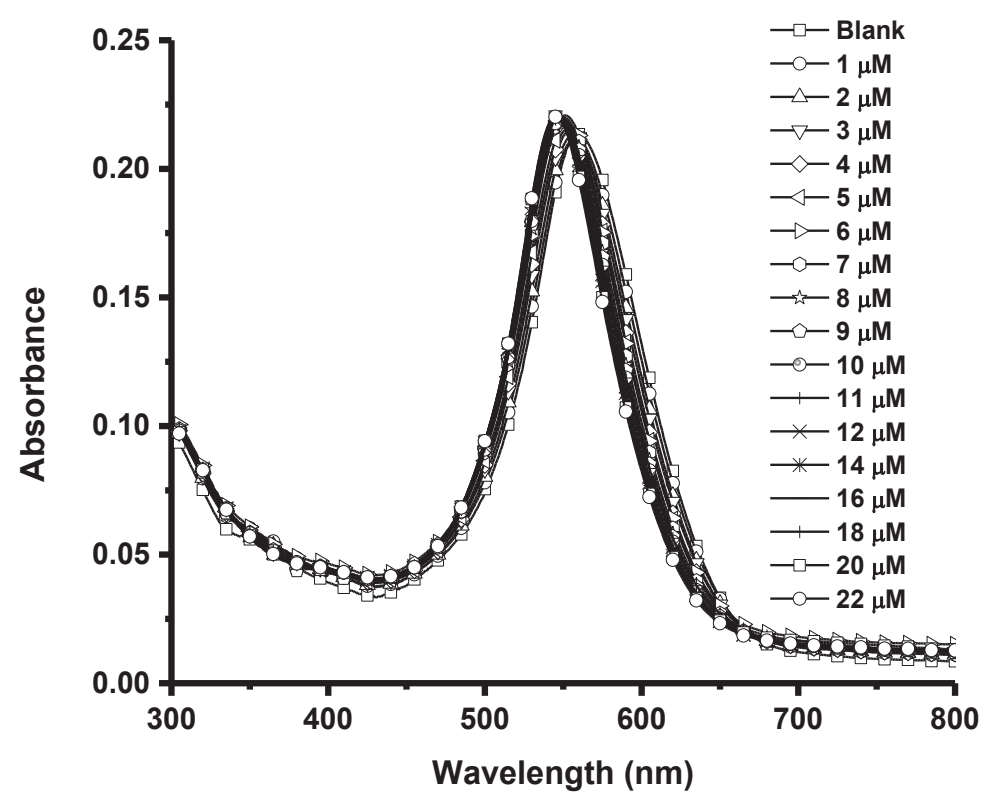

Figure D.19. Absorption spectra of fluorescent probe 6-C upon addition of different amount of $\mathrm{ZnCl}_{2}$ in $10 \mathrm{mM}$ HEPES buffer $\mathrm{pH}$ 7.0. 


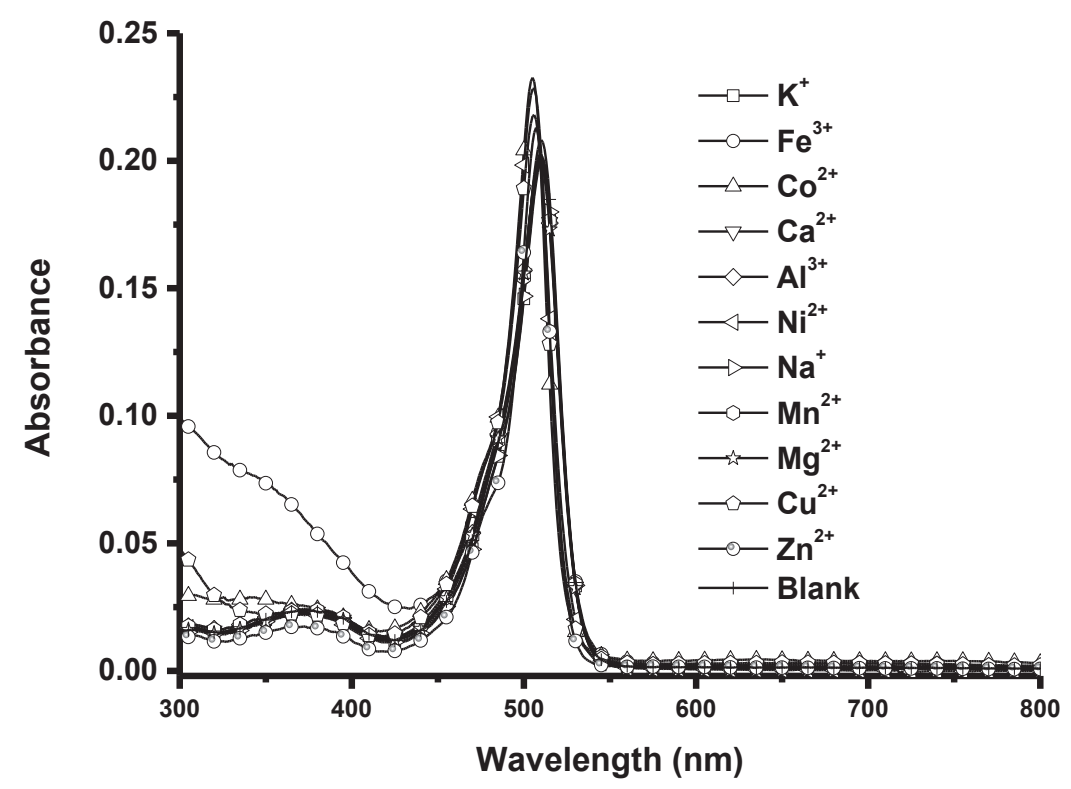

Figure D.20. Absorption spectra of fluorescent probe 6-A upon addition of different cations $\left(20 \mu \mathrm{M} \mathrm{Zn}{ }^{2+}\right.$, other ions at $\left.40 \mu \mathrm{M}\right)$ in $10 \mathrm{mM}$ HEPES buffer $\mathrm{pH}$ 7.0.

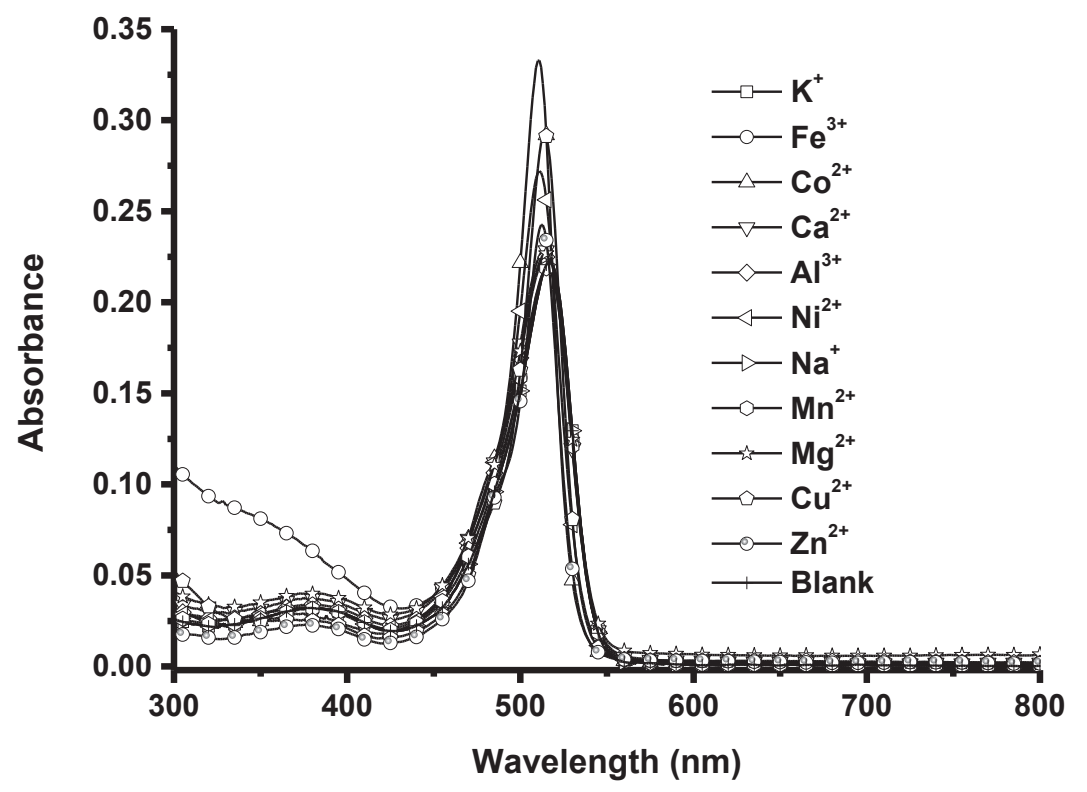

Figure D.21. Absorption spectra of fluorescent probe 6-B upon addition of different cations $\left(20 \mu \mathrm{M} \mathrm{Zn}{ }^{2+}\right.$, other ions at $\left.40 \mu \mathrm{M}\right)$ in $10 \mathrm{mM}$ HEPES buffer $\mathrm{pH}$ 7.0. 


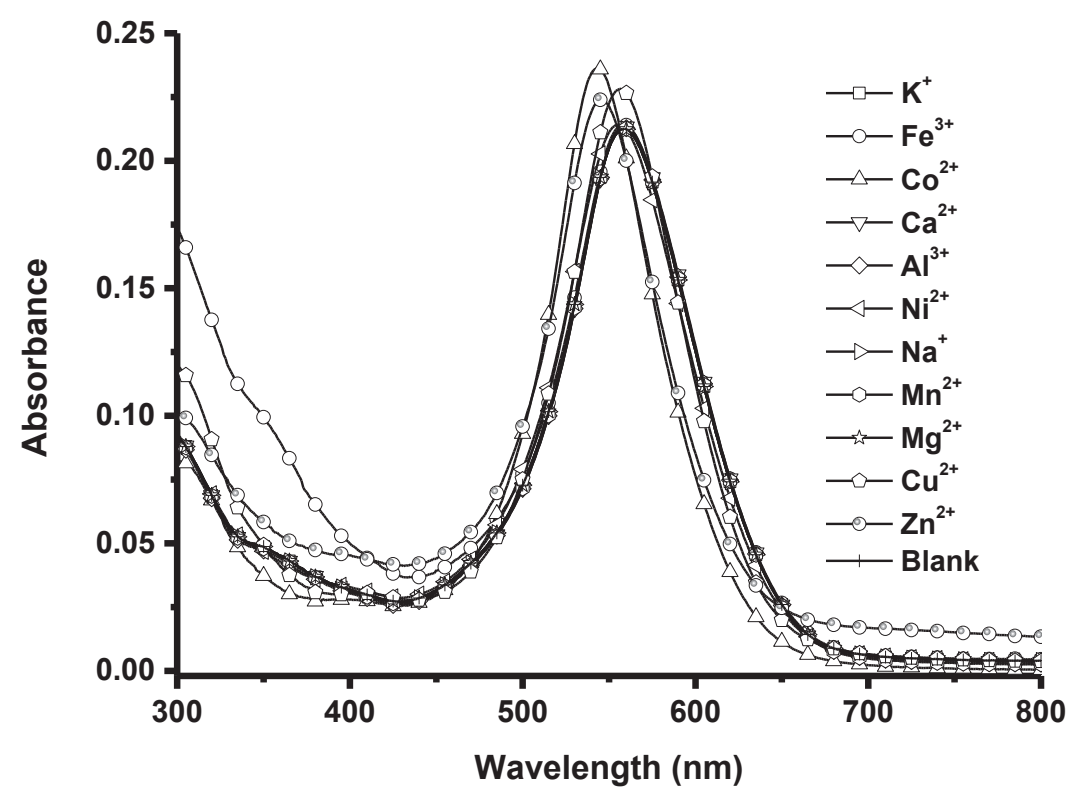

Figure D.22. Absorption spectra of fluorescent probe 6-C upon addition of different cations $\left(20 \mu \mathrm{M} \mathrm{Zn}^{2+}\right.$, other ions at $\left.40 \mu \mathrm{M}\right)$ in $10 \mathrm{mM}$ HEPES buffer $\mathrm{pH}$ 7.0. 


\section{Appendix E Supporting Information for Chapter 7}

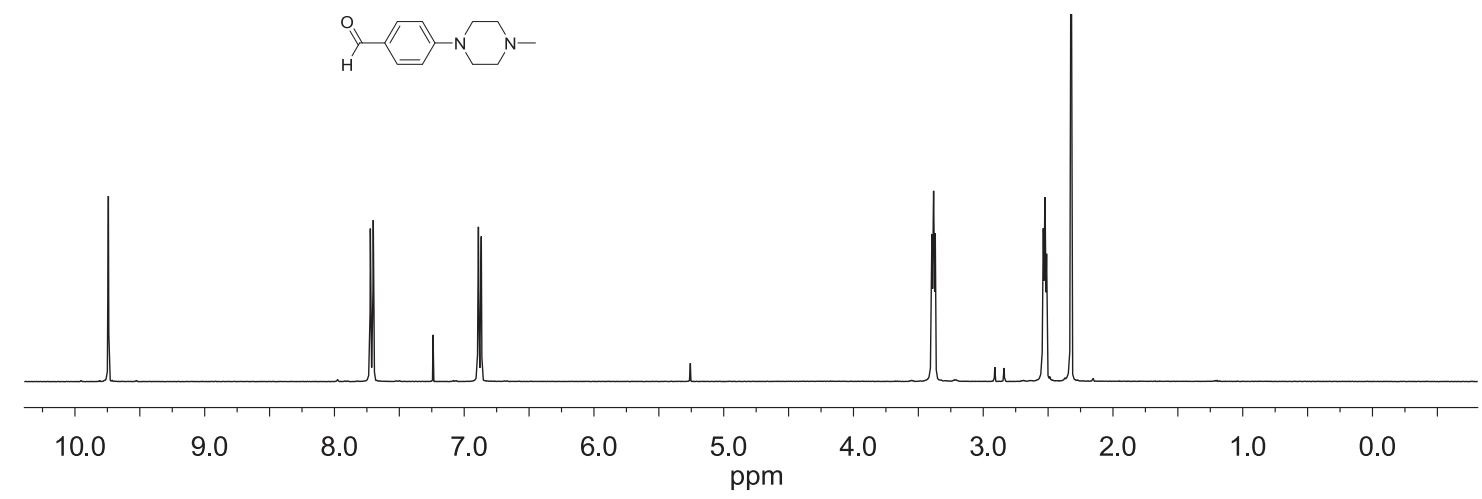

Figure E.1. $\quad{ }^{1} \mathrm{H}$ NMR spectrum of compound 7.3 in $\mathrm{CDCl}_{3}$ solution.

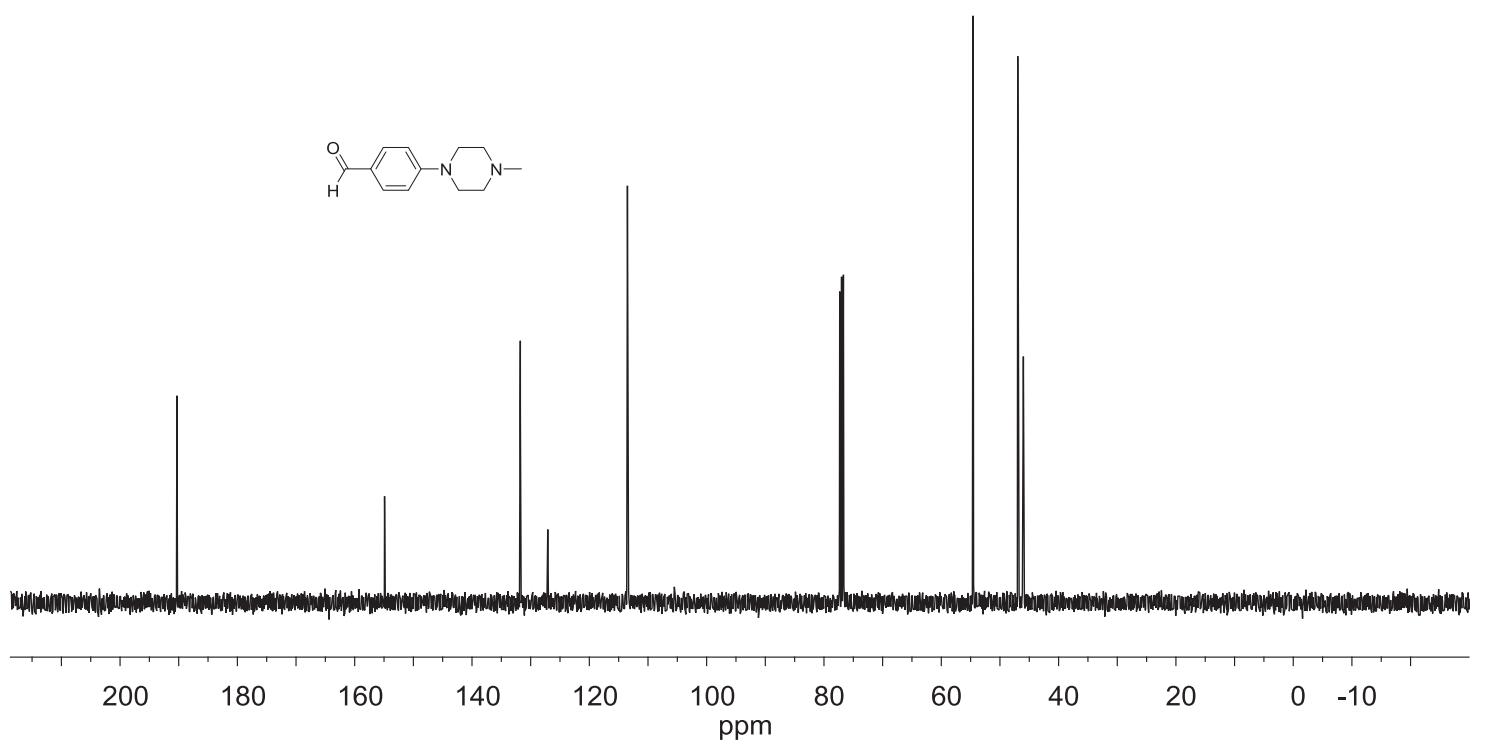

Figure E.2. $\quad{ }^{13} \mathrm{C}$ NMR spectrum of compound 7.3 in $\mathrm{CDCl}_{3}$ solution. 


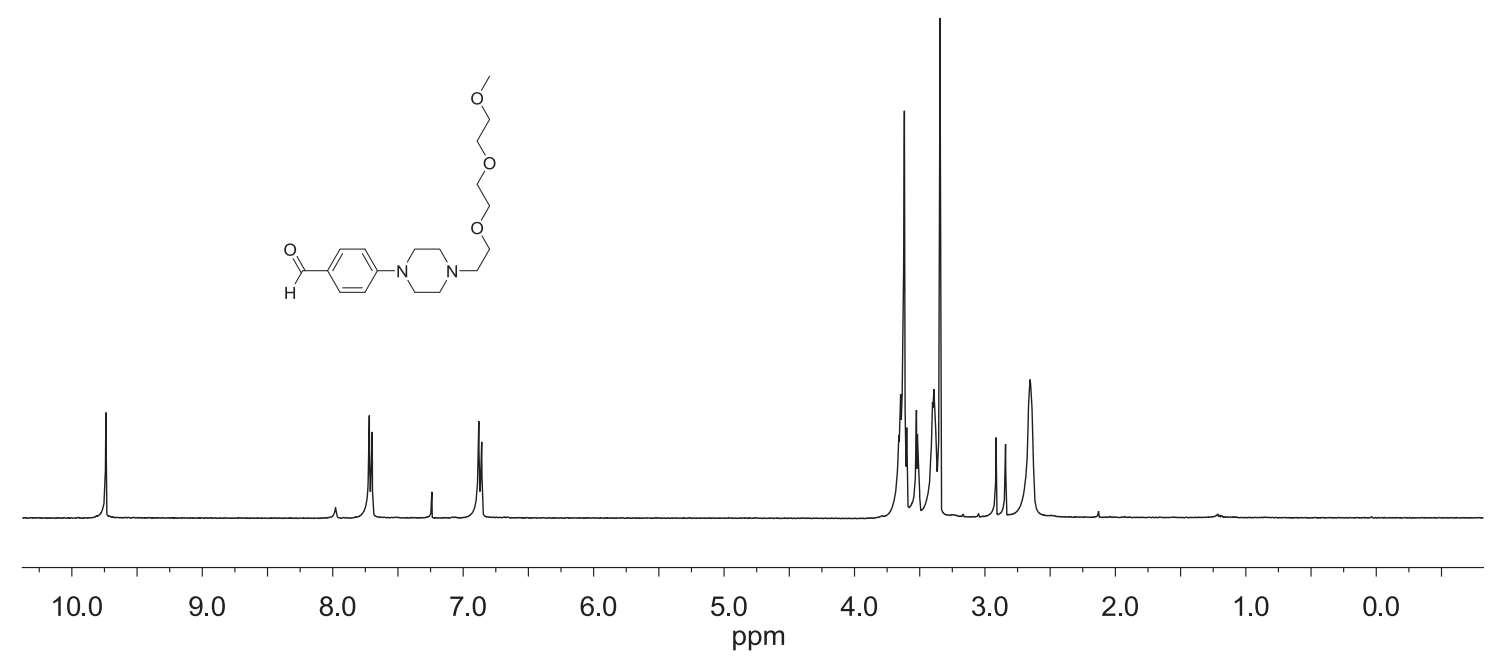

Figure E.3. $\quad{ }^{1} \mathrm{H}$ NMR spectrum of compound 7.5 in $\mathrm{CDCl}_{3}$ solution.

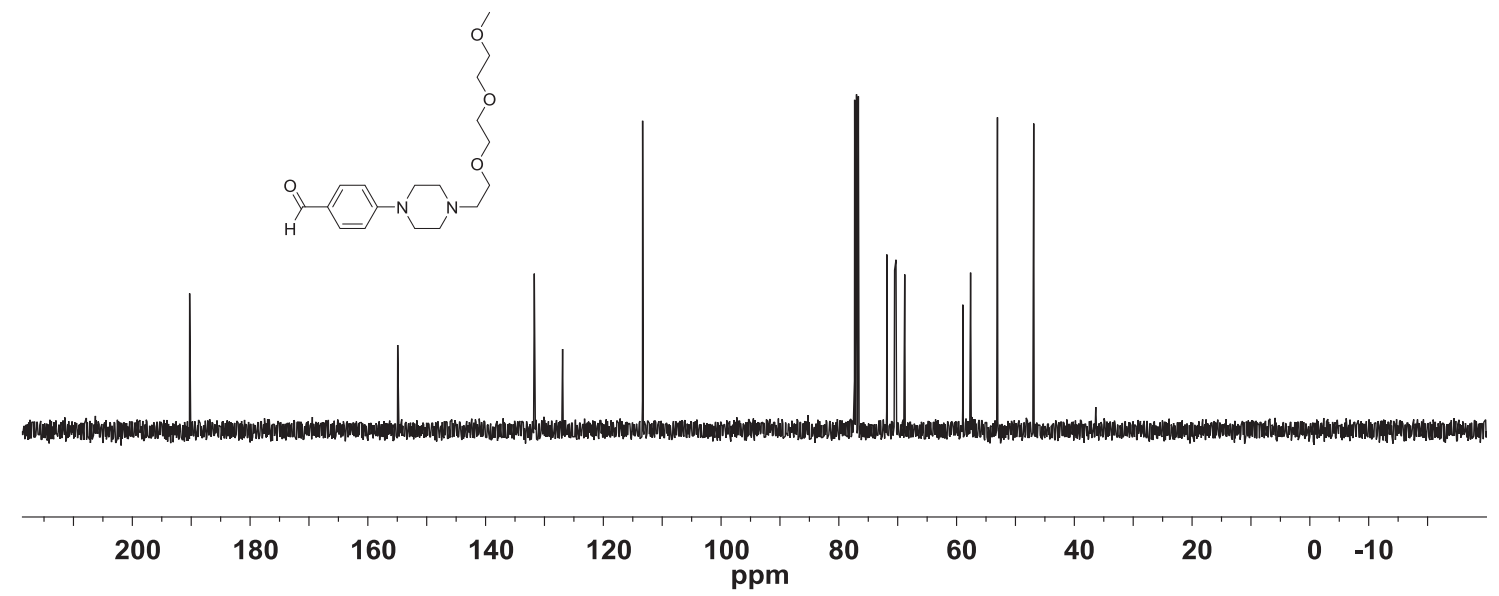

Figure E.4. $\quad{ }^{13} \mathrm{C}$ NMR spectrum of compound 7.5 in $\mathrm{CDCl}_{3}$ solution. 


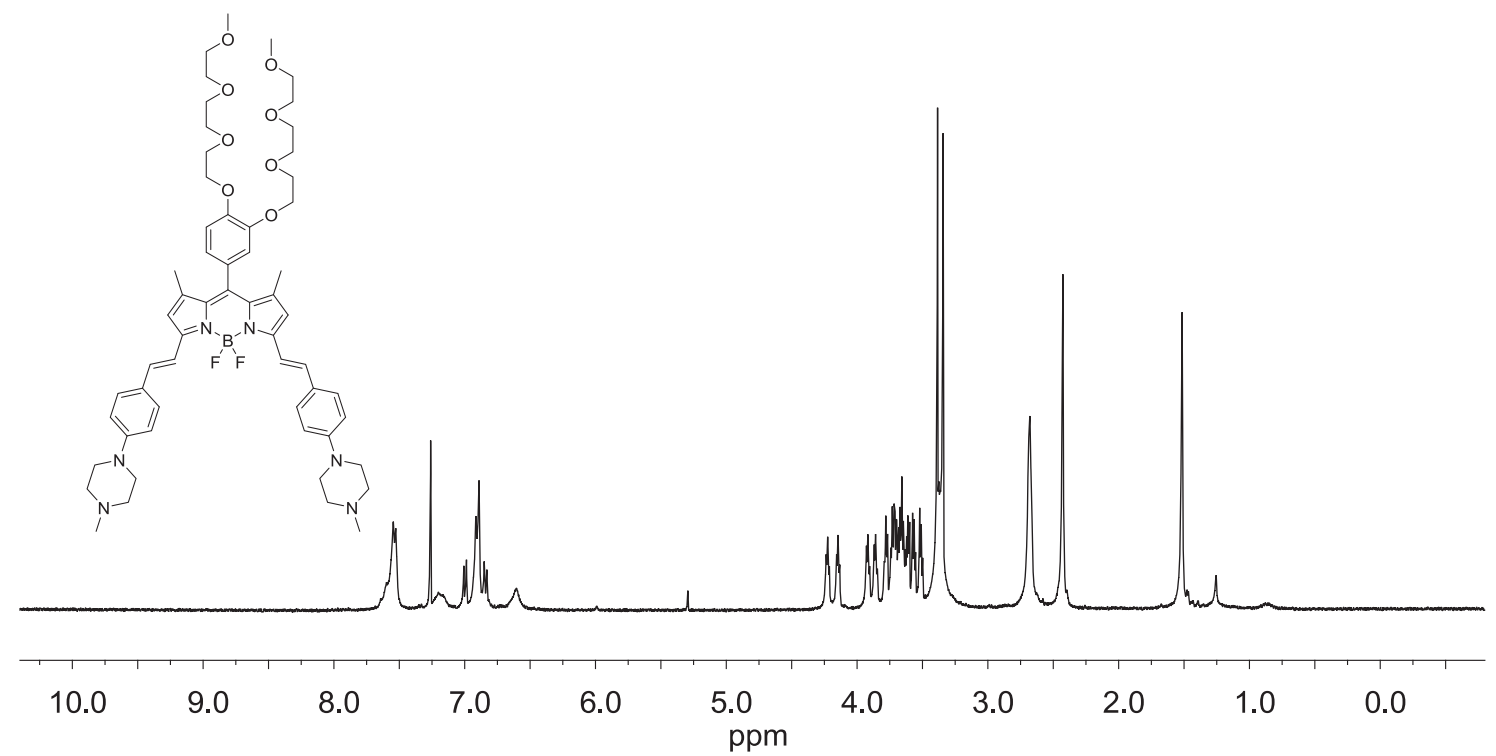

Figure E.5. $\quad{ }^{1} \mathrm{H}$ NMR spectrum of fluorescent probe 7-A in $\mathrm{CDCl}_{3}$ solution.

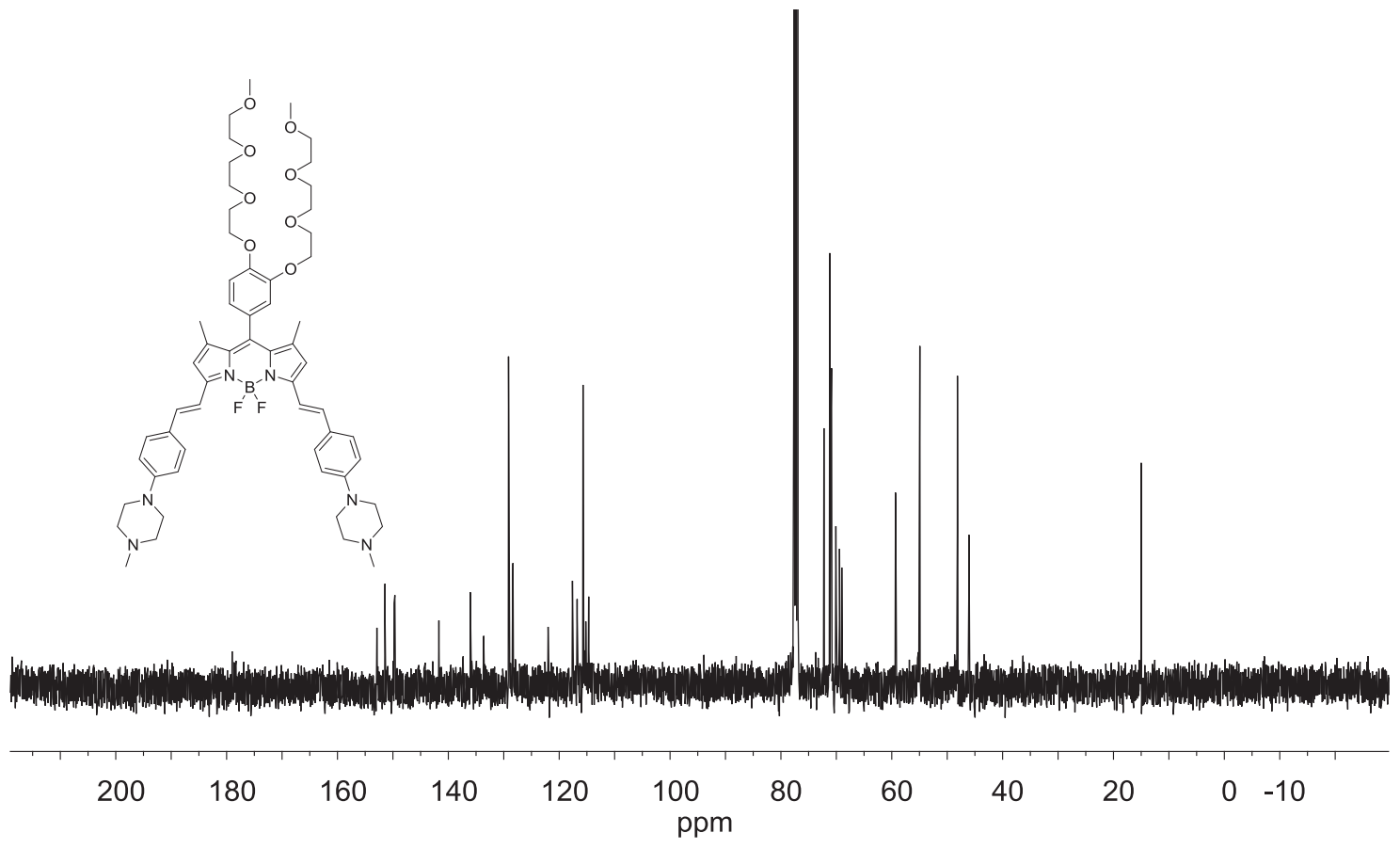

Figure E.6. $\quad{ }^{13} \mathrm{C}$ NMR spectrum of fluorescent probe 7-A in $\mathrm{CDCl}_{3}$ solution 


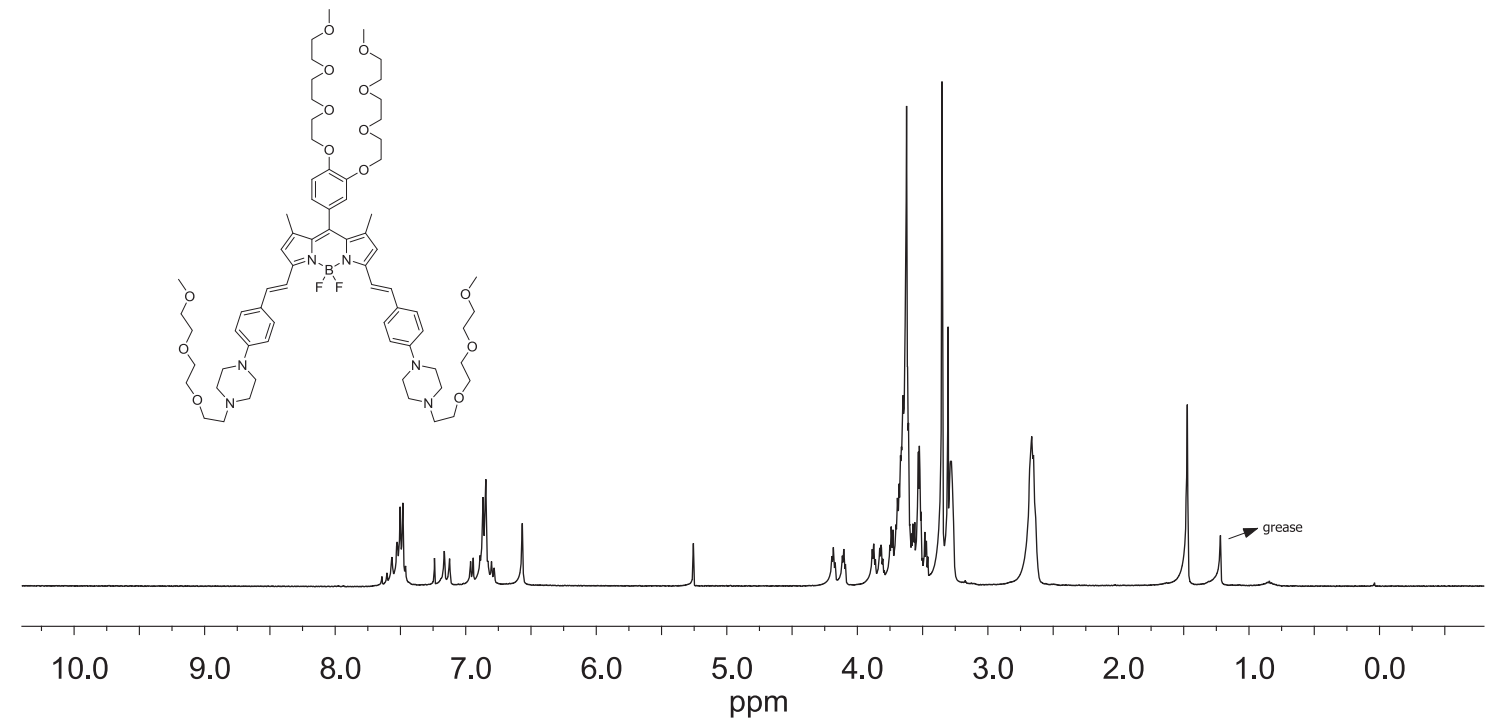

Figure E.7. $\quad{ }^{1} \mathrm{H}$ NMR spectrum of fluorescent probe 7-B in $\mathrm{CDCl}_{3}$ solution.

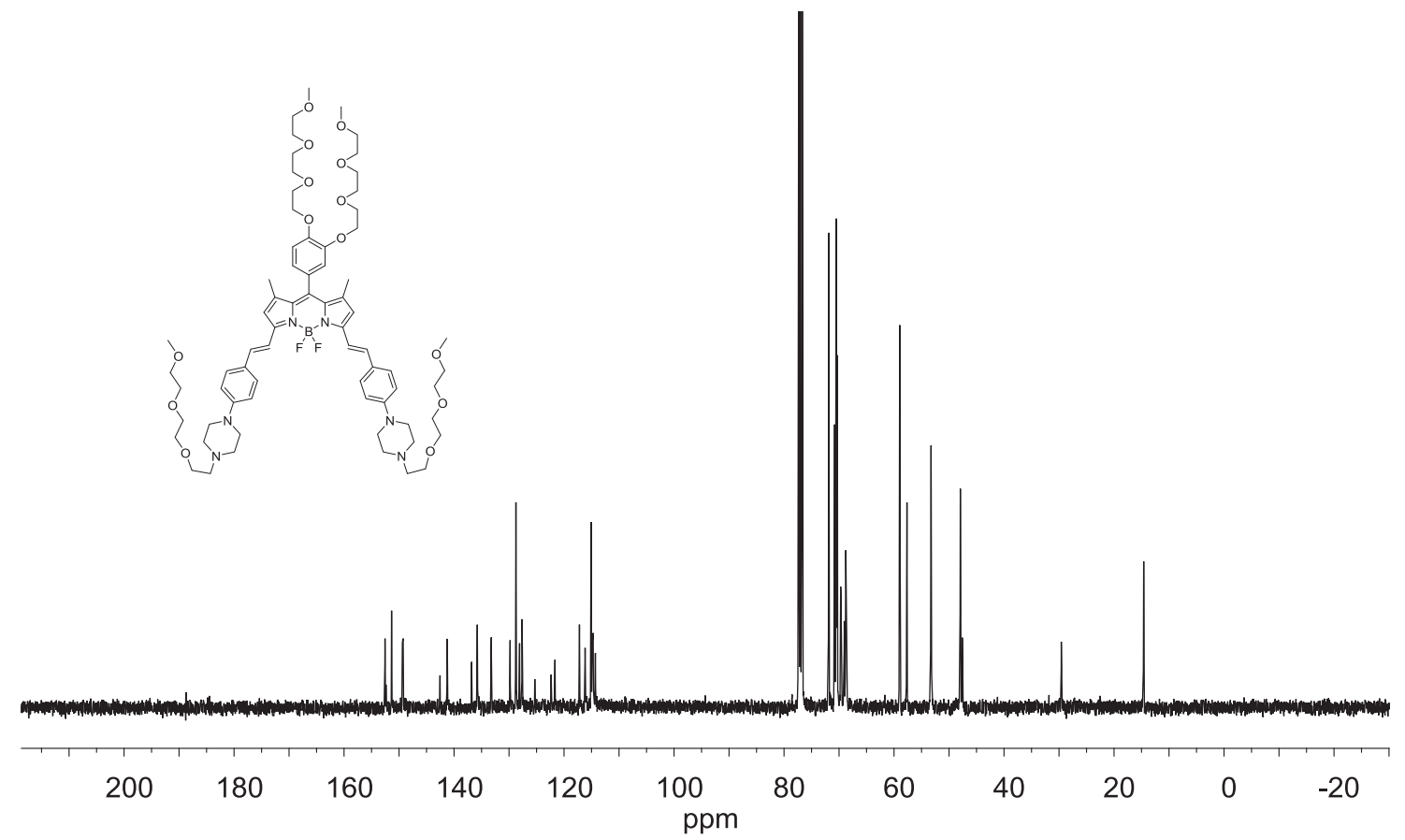

Figure E.8. ${ }^{13} \mathrm{C}$ NMR spectrum of fluorescent probe 7-B in $\mathrm{CDCl}_{3}$ solution. 


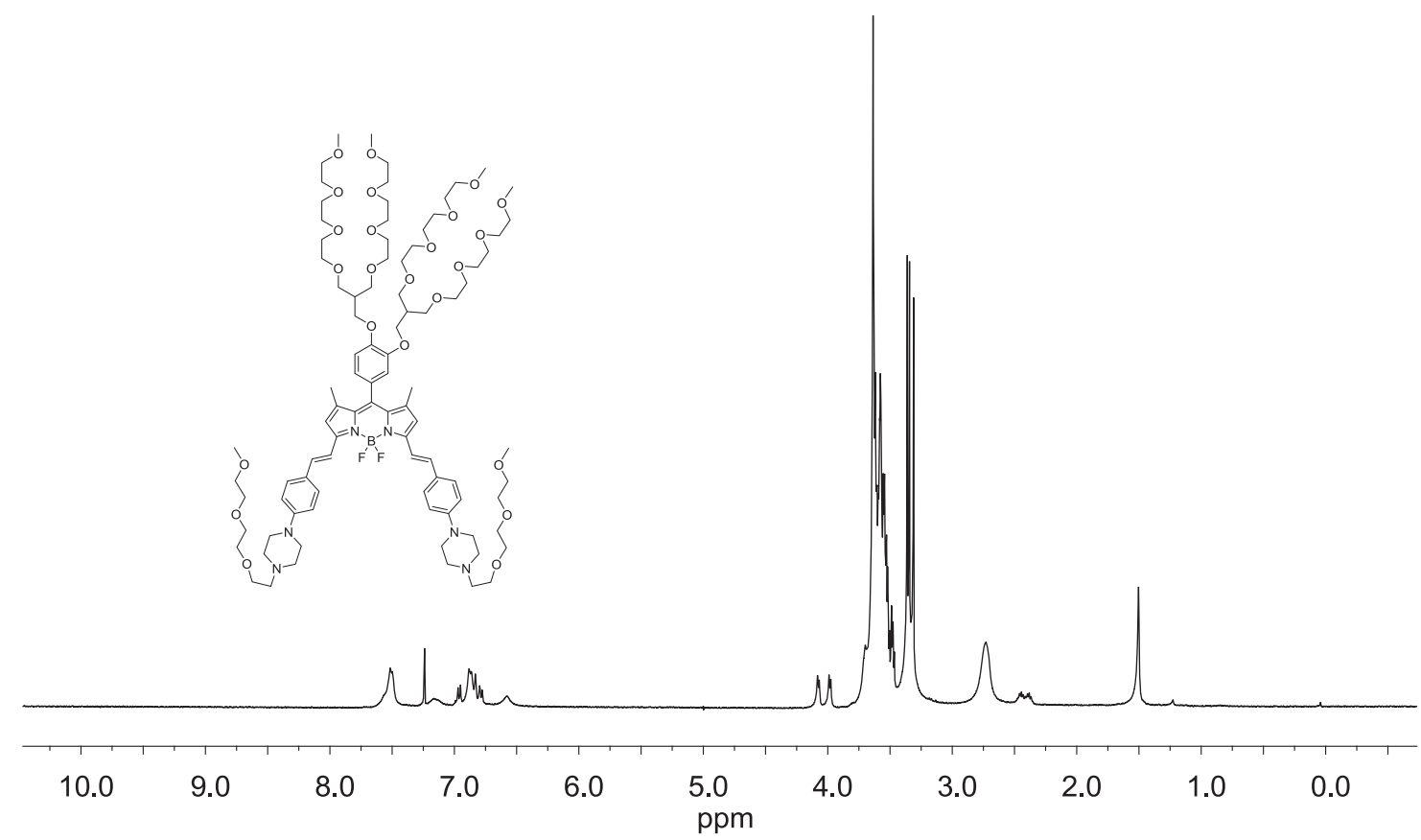

Figure E.9. $\quad{ }^{1} \mathrm{H}$ NMR spectrum of fluorescent probe 7-C in $\mathrm{CDCl}_{3}$ solution.

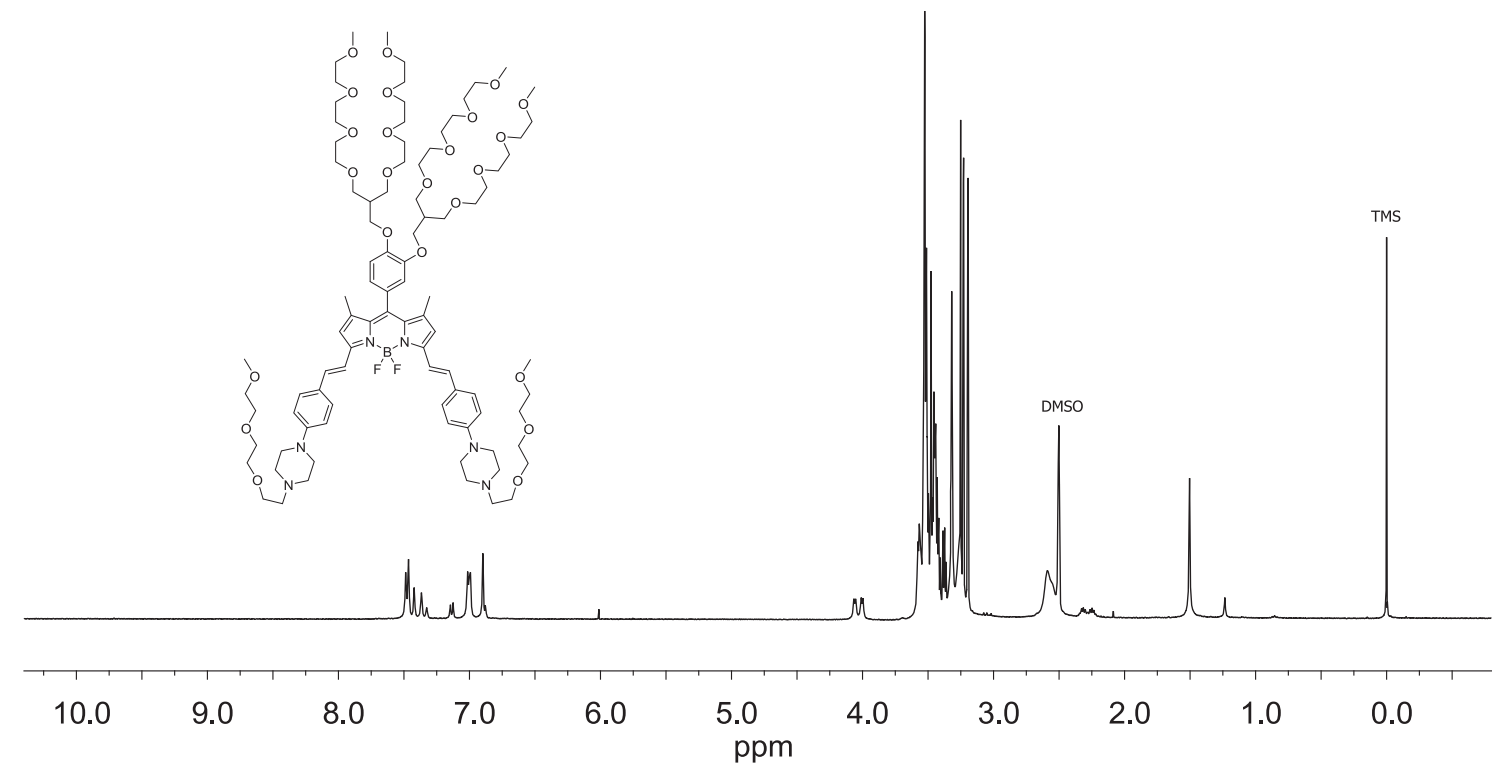

Figure E.10. ${ }^{1} \mathrm{H}$ NMR spectrum of fluorescent probe 7-C in DMSO- $d_{6}$ solution. 


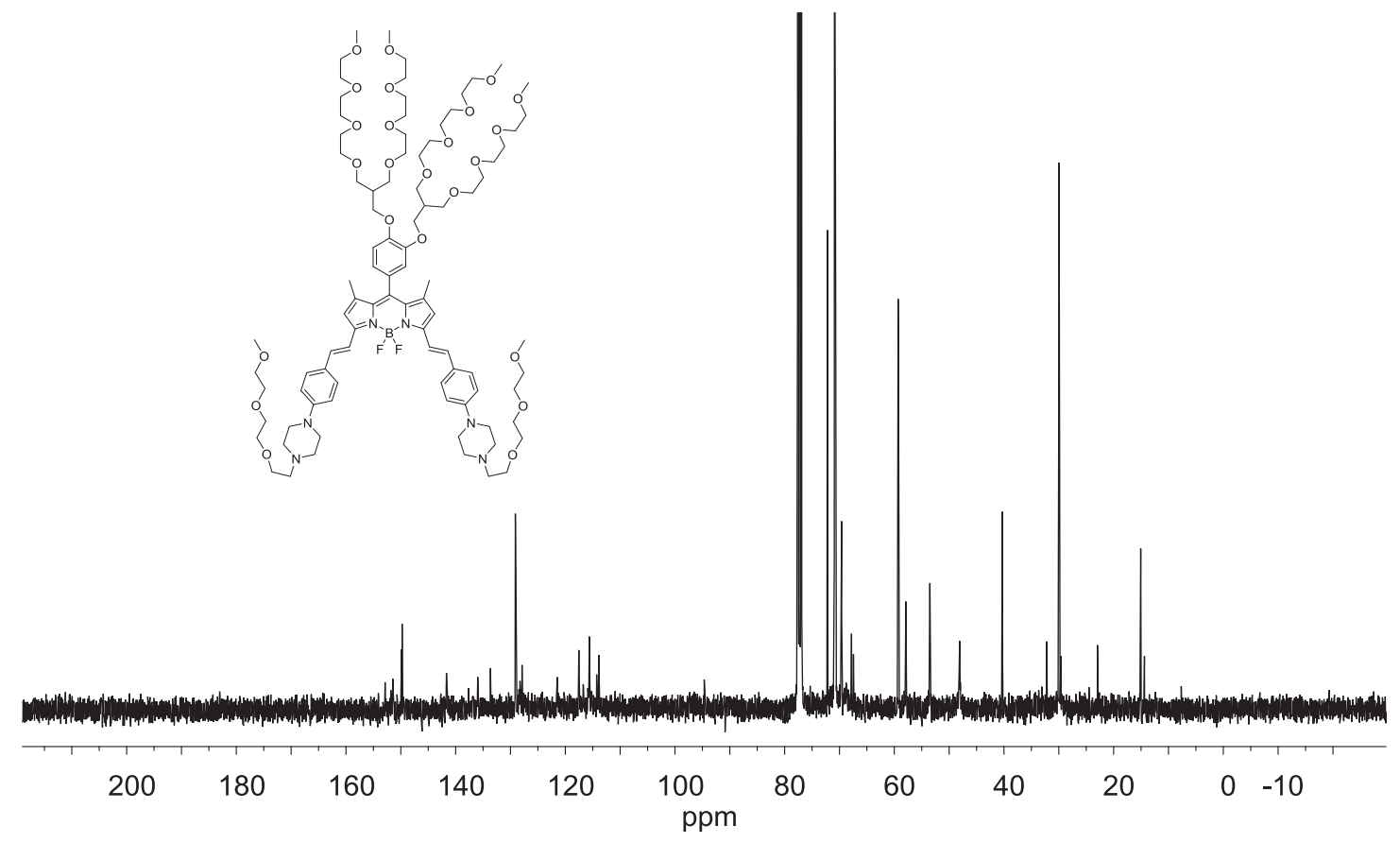

Figure E.11. ${ }^{13} \mathrm{C}$ NMR spectrum of fluorescent probe 7-C in $\mathrm{CDCl}_{3}$ solution 


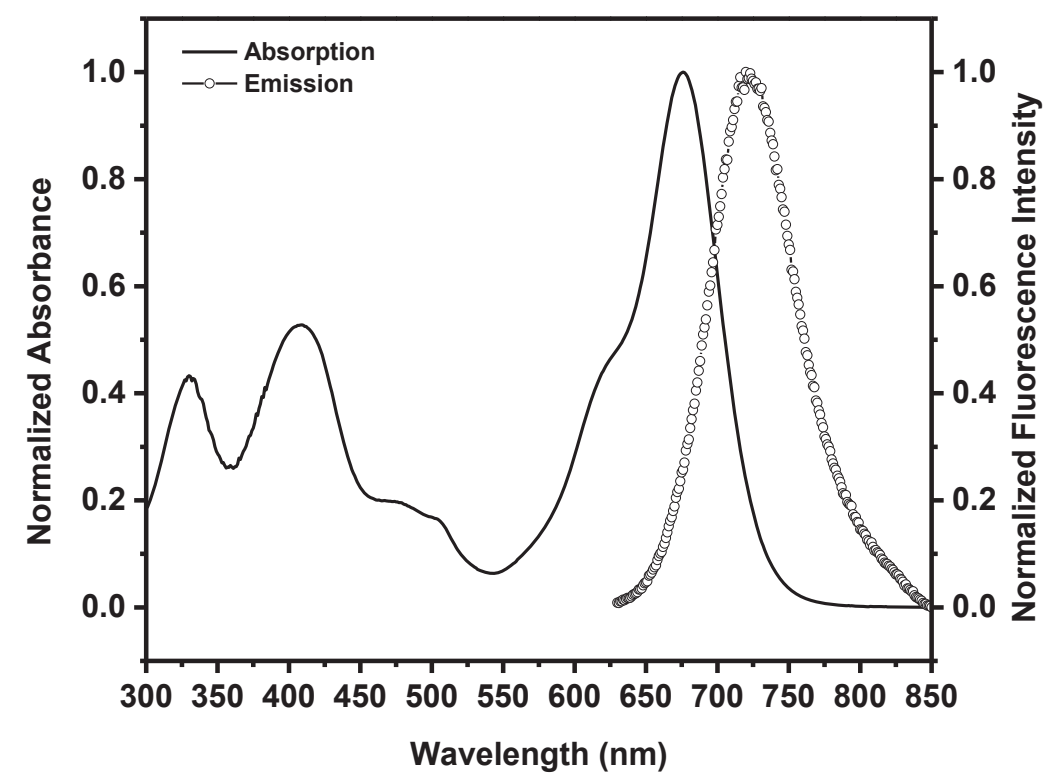

Figure E.12. Normalized absorption and emission spectra of Probe 7-A in dichloromethane solution.

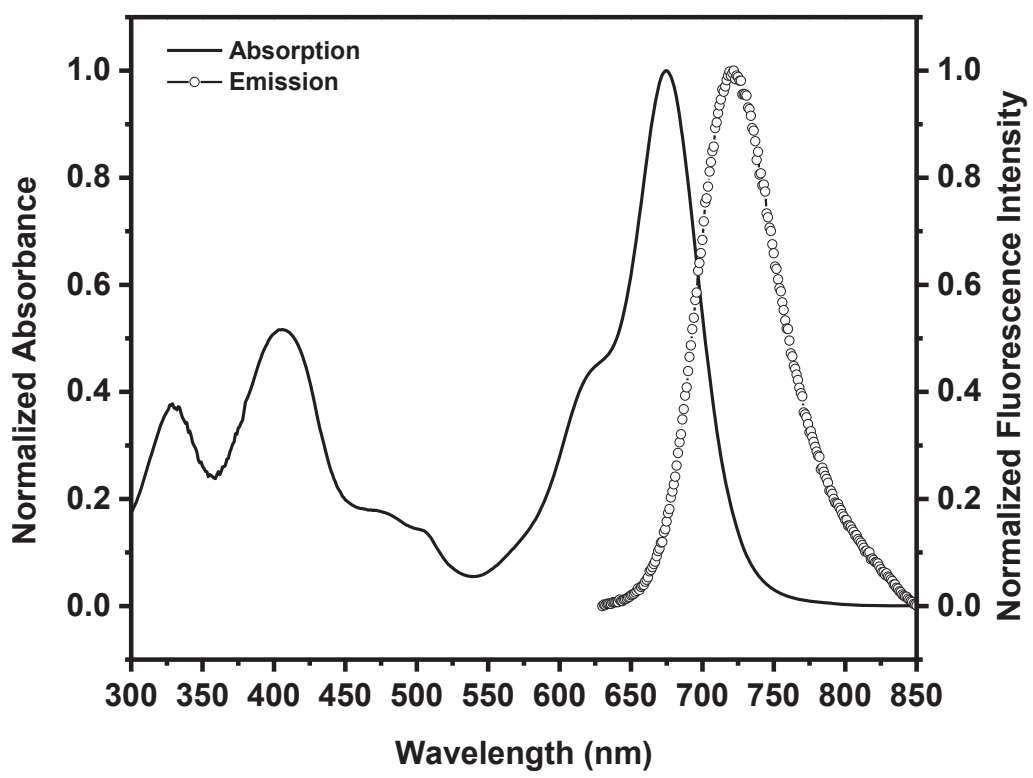

Figure E.13. Normalized absorption and emission spectra of Probe 7-A in DMSO solution. 


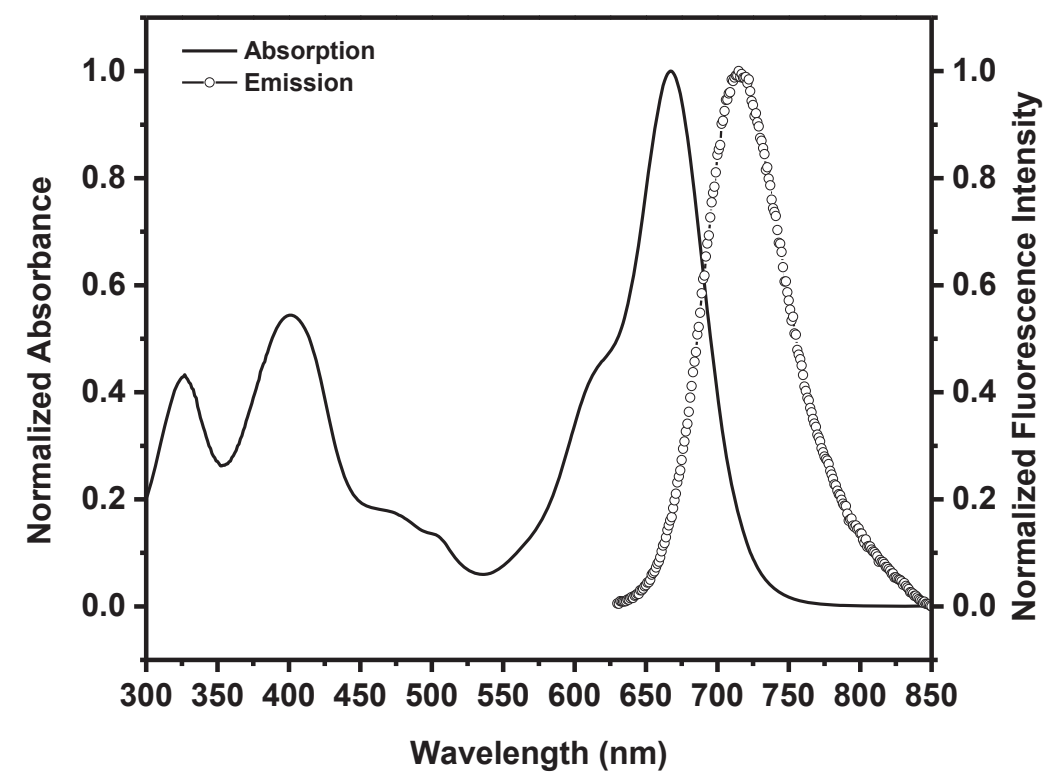

Figure E.14. Normalized absorption and emission spectra of Probe 7-A in ethanol solution.

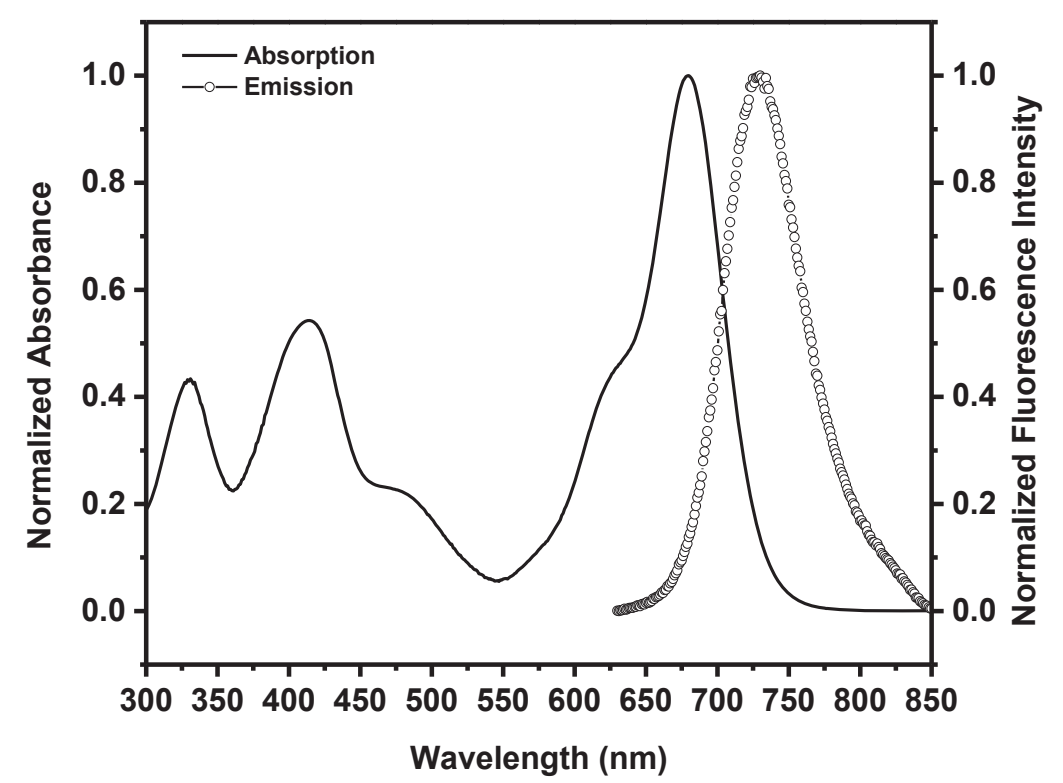

Figure E.15. Normalized absorption and emission spectra of Probe 7-B in dichloromethane solution. 


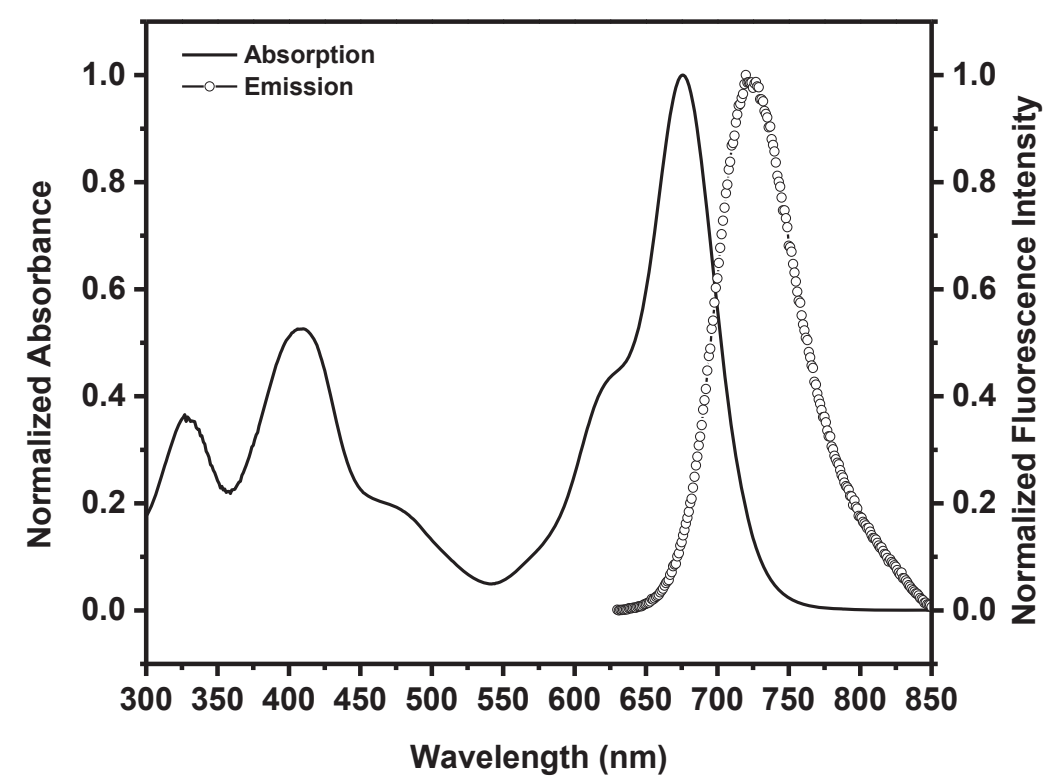

Figure E.16. Normalized absorption and emission spectra of Probe 7-B in DMSO solution.

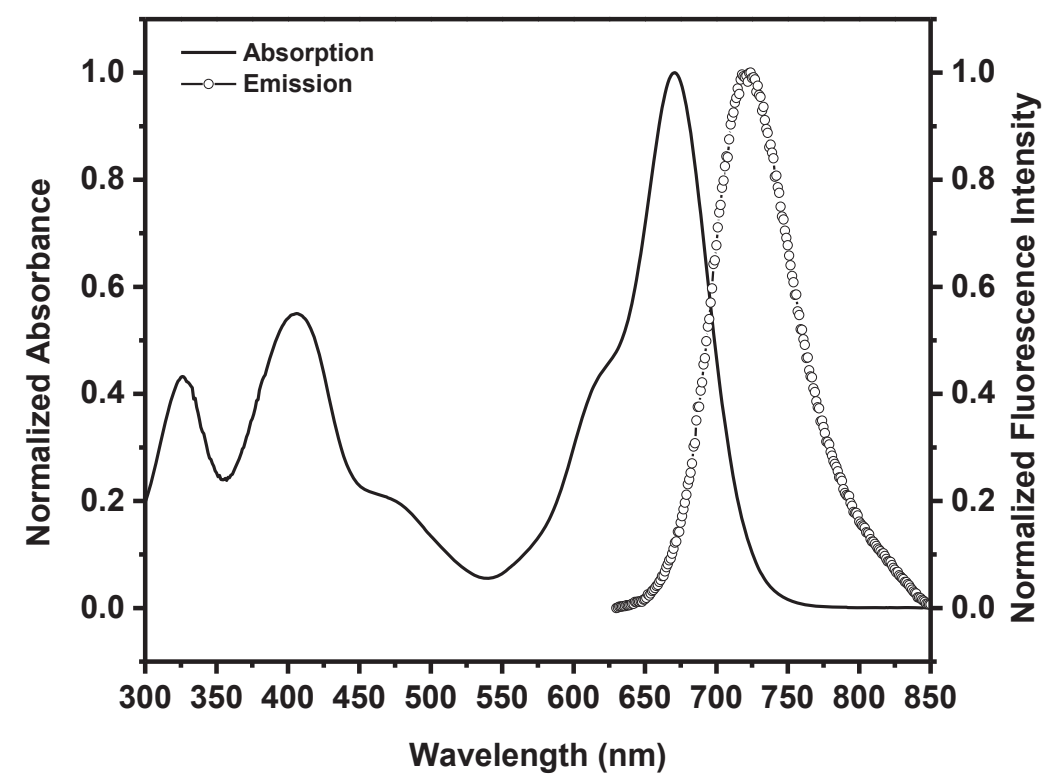

Figure E.17. Normalized absorption and emission spectra of Probe 7-B in ethanol solution. 


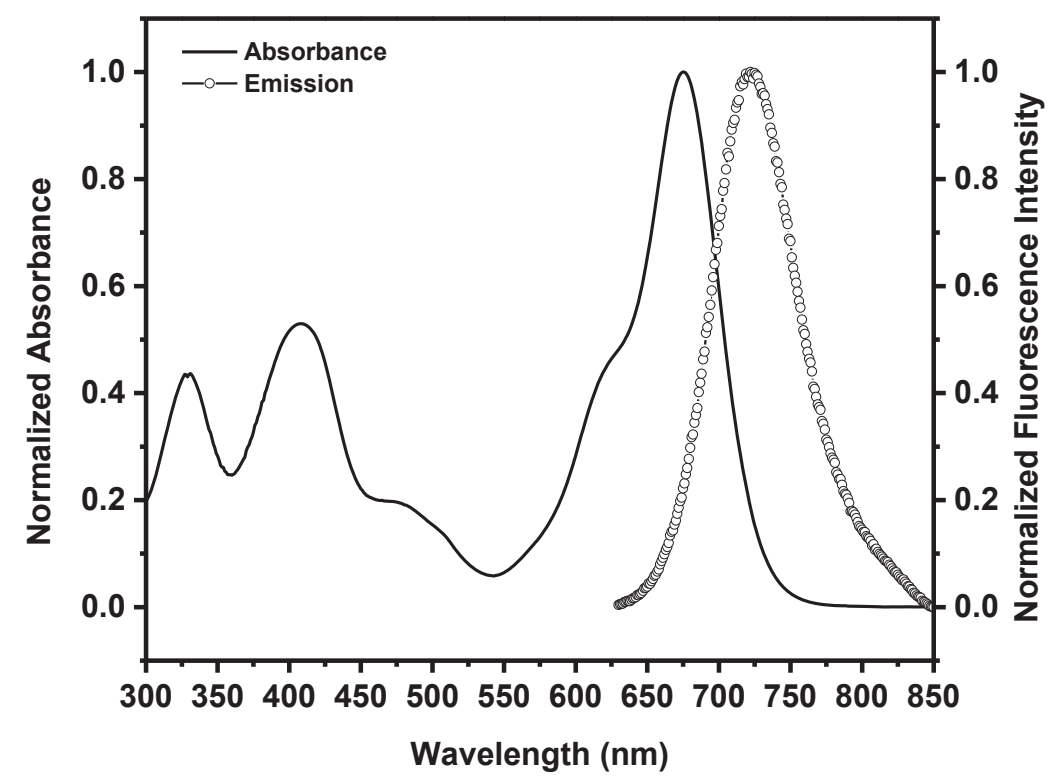

Figure E.18. Normalized absorption and emission spectra of Probe 7-C in dichloromethane solution.

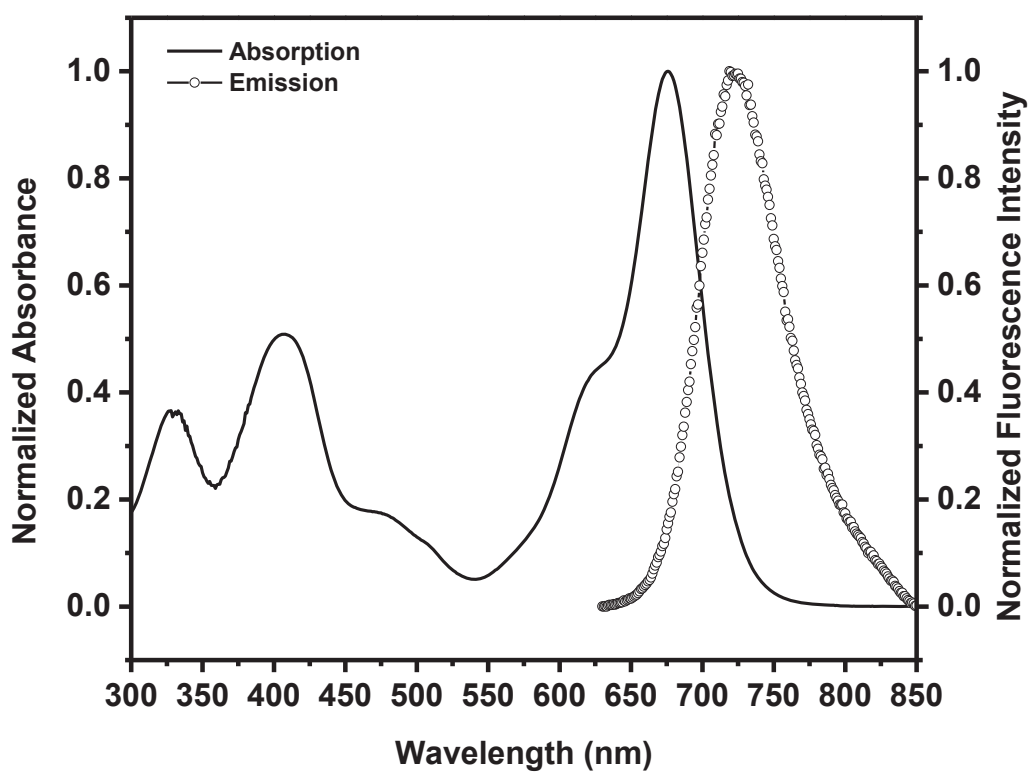

Figure E.19. Normalized absorption and emission spectra of Probe 7-C in DMSO solution. 


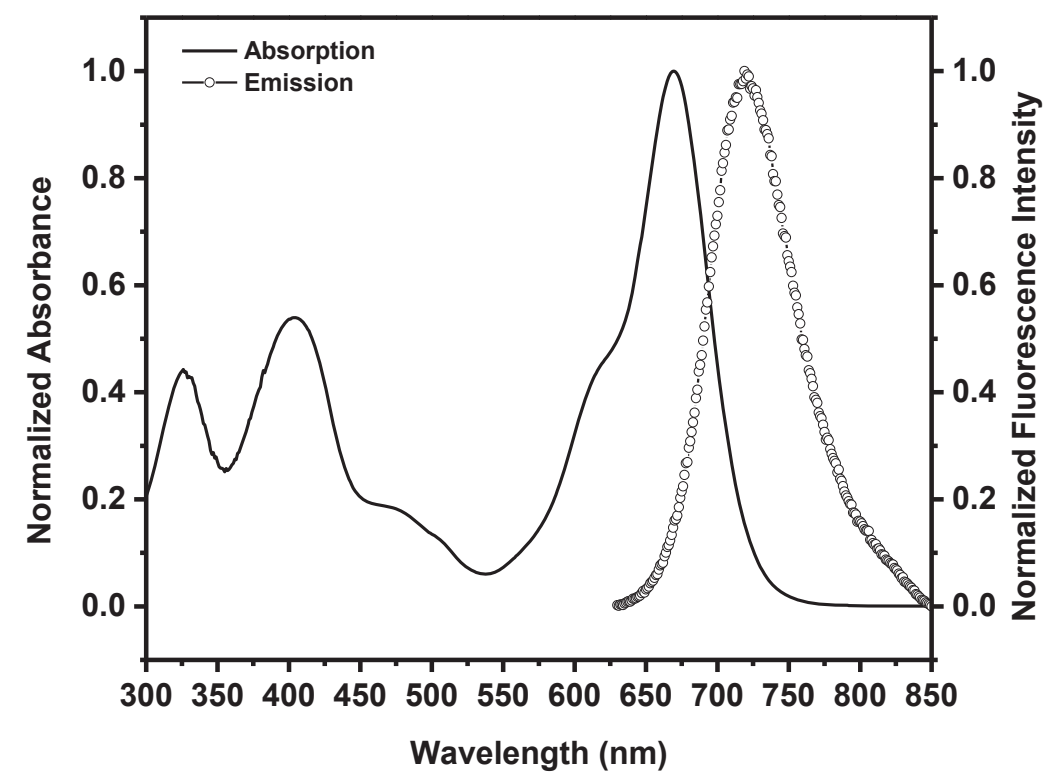

Figure E.20. Normalized absorption and emission spectra of Probe 7-C in ethanol solution.
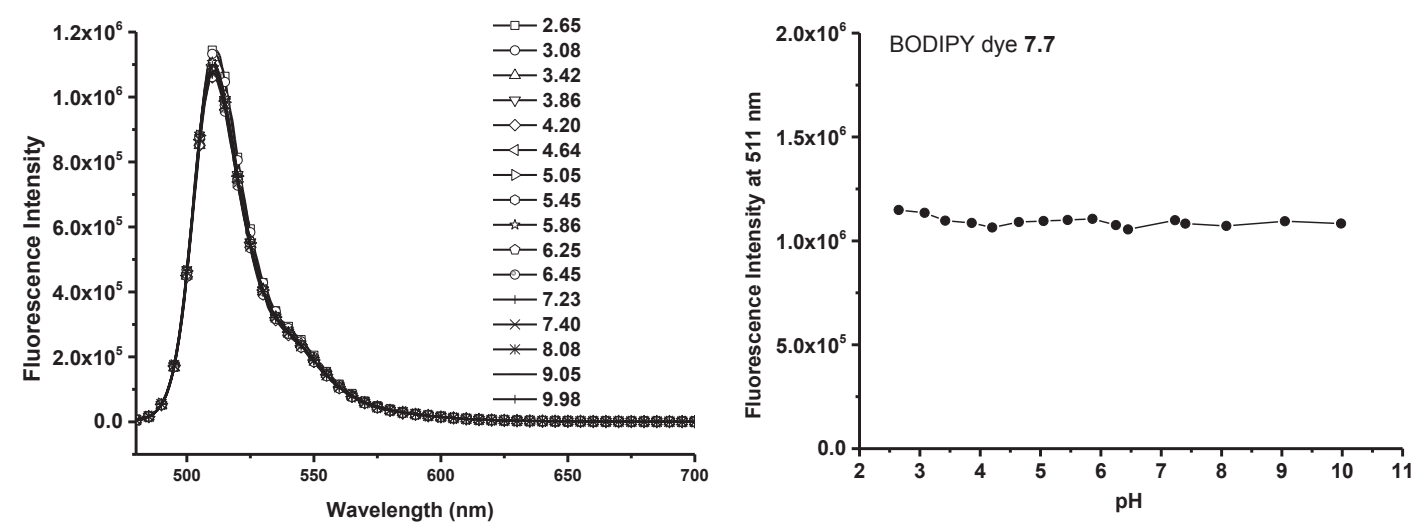

Figure E.21. Left: Fluorescence spectra of BODIPY dye $7.7(5 \mu \mathrm{M})$ under different $\mathrm{pH}$ conditions. Right: fluorescence intensity responses of BODIPY dye $7.7(5 \mu \mathrm{M})$ to different $\mathrm{pH}$ values. 

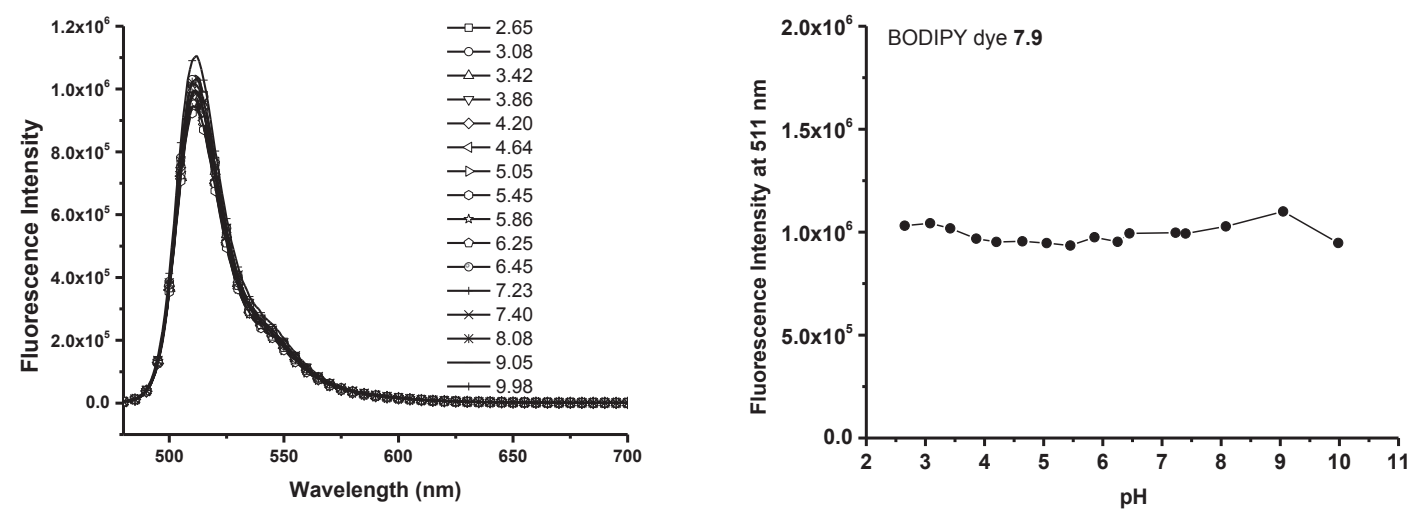

Figure E.22. Left: Fluorescence spectra of BODIPY dye $7.9(5 \mu \mathrm{M})$ under different $\mathrm{pH}$ conditions. Right: fluorescence intensity responses of BODIPY dye 7.9 $(5 \mu \mathrm{M})$ to $\mathrm{pH}$.
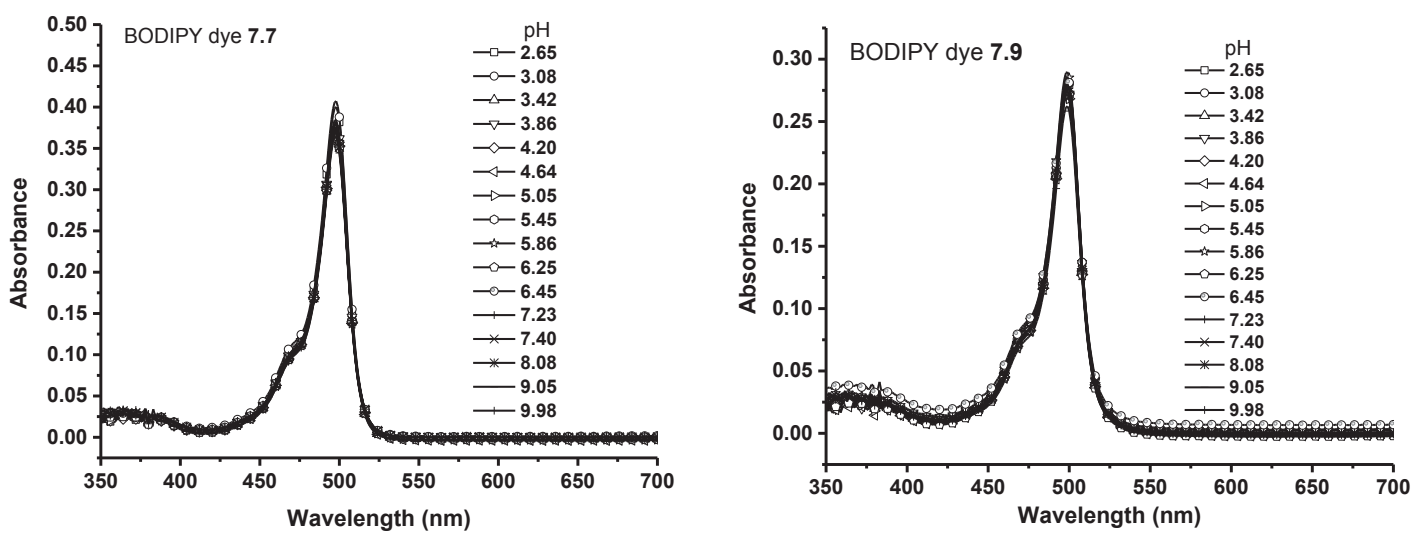

Figure E.23. Absorption spectra of BODIPY dye 7.7 (left) and BODIPY dye $\mathbf{7 . 9}$ (right) at different $\mathrm{pH}$ conditions in buffer solutions. 


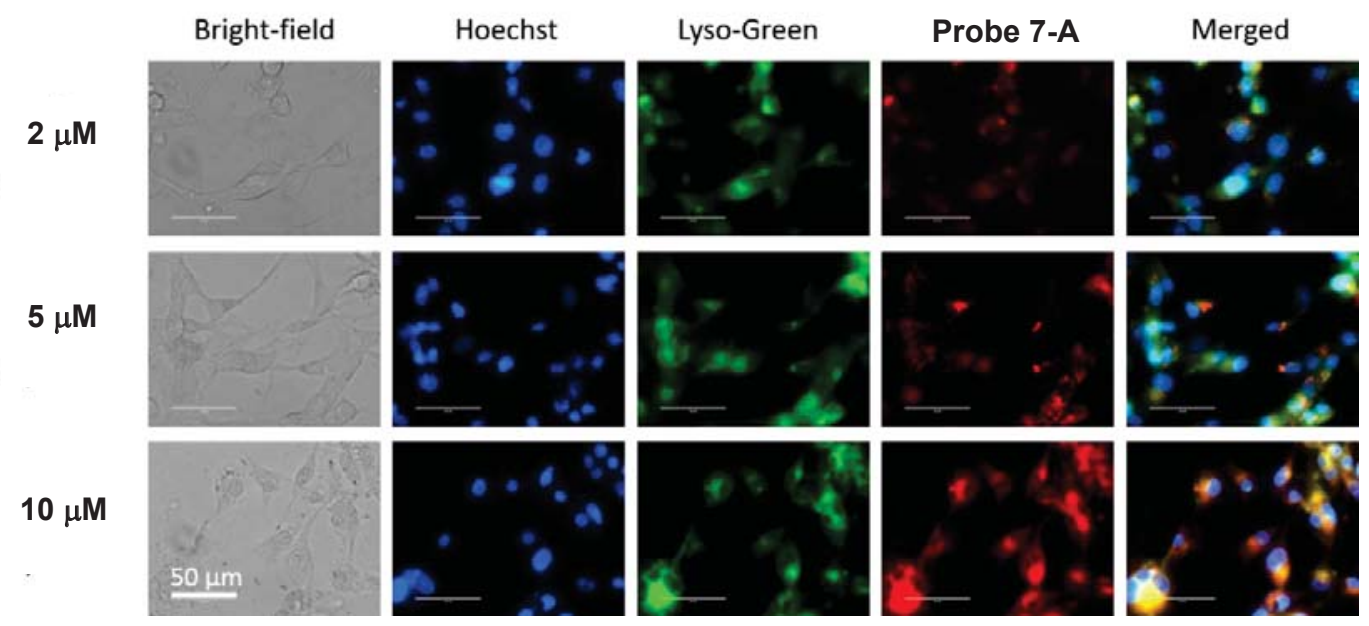

Figure E.24. Fluorescent images of MDA-MB-231 cell incubates with different concentration of probe 7-A. (After $2 \mathrm{~h}$ serum starvation, cells were incubated with 2, 5, 10 $\mu \mathrm{M}$ of fluorescent probe 7-A for $2 \mathrm{~h}$ and imaged for co-localization with lysosomal stain LysoSensor Green $(1 \mu \mathrm{M}) .1 \mu \mathrm{g} / \mathrm{ml}$ of Hoechst were used to stain the nuclear. All images were acquired at 60x magnification using inverted fluorescence microscope (AMF-4306, $\left.\left.\mathrm{EVOS}_{\mathrm{fl}}, \mathrm{AMG}\right)\right)$

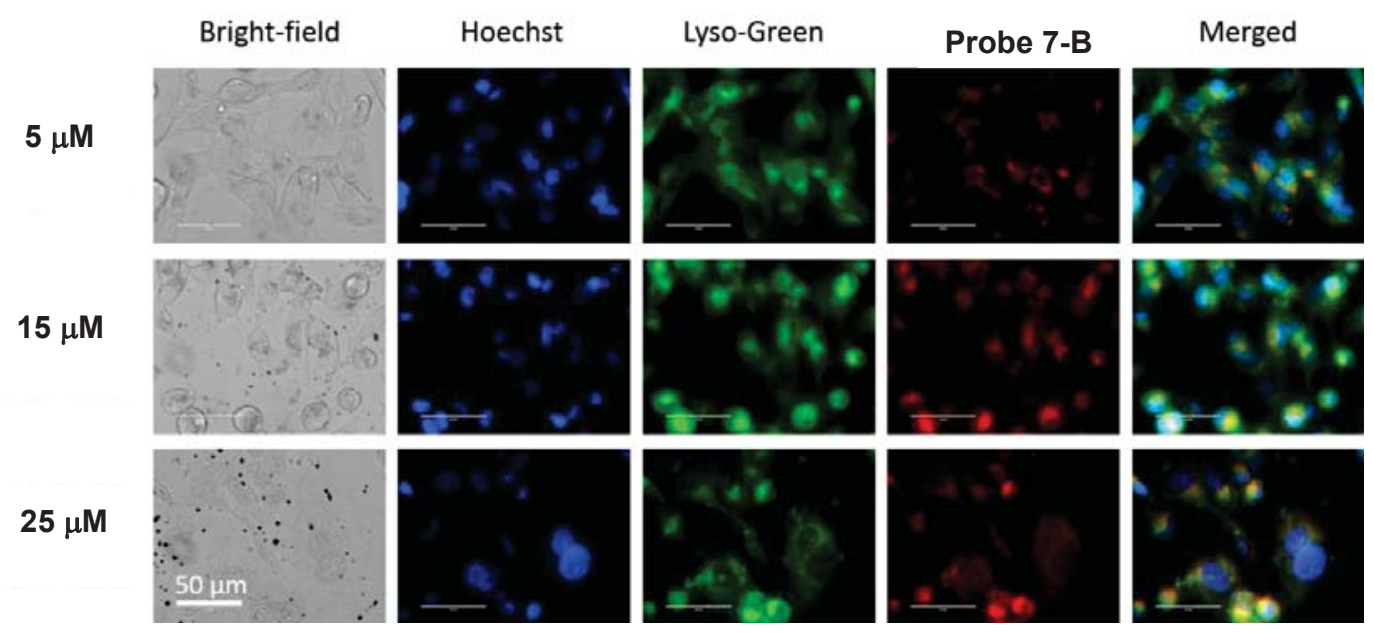

Figure E.25. Fluorescent images of MDA-MB-231 cell incubates with different concentration of probe 7-B. (After $2 \mathrm{~h}$ serum starvation, cells were incubated with 5, 15, $25 \mu \mathrm{M}$ of fluorescent probe 7-B for $2 \mathrm{~h}$ and imaged for co-localization with lysosomal stain LysoSensor Green $(1 \mu \mathrm{M}) .1 \mu \mathrm{g} / \mathrm{ml}$ of Hoechst were used to stain the nuclear. All images were acquired at 60x magnification using inverted fluorescence microscope $\left(\mathrm{AMF}-4306, \mathrm{EVOS}_{\mathrm{fl}}, \mathrm{AMG}\right)$.) 


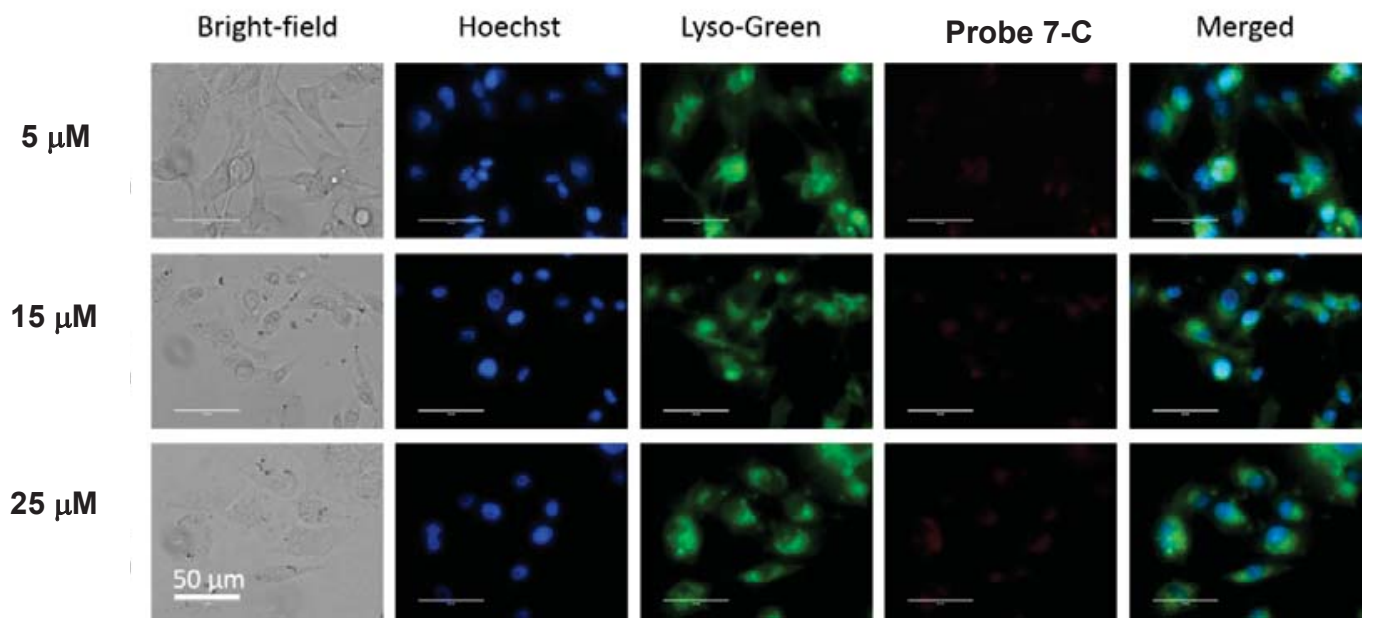

Figure E.26. Fluorescent images of MDA-MB-231 cell incubates with different concentration of probe 7-C. (After $2 \mathrm{~h}$ serum starvation, cells were incubated with 5, 15, $25 \mu \mathrm{M}$ of fluorescent probe 7-C for $2 \mathrm{~h}$ and imaged for co-localization with lysosomal stain LysoSensor Green $(1 \mu \mathrm{M}) .1 \mu \mathrm{g} / \mathrm{ml}$ of Hoechst were used to stain the nuclear. All images were acquired at 60x magnification using inverted fluorescence microscope (AMF-4306, EVOS $f$ l, AMG).)

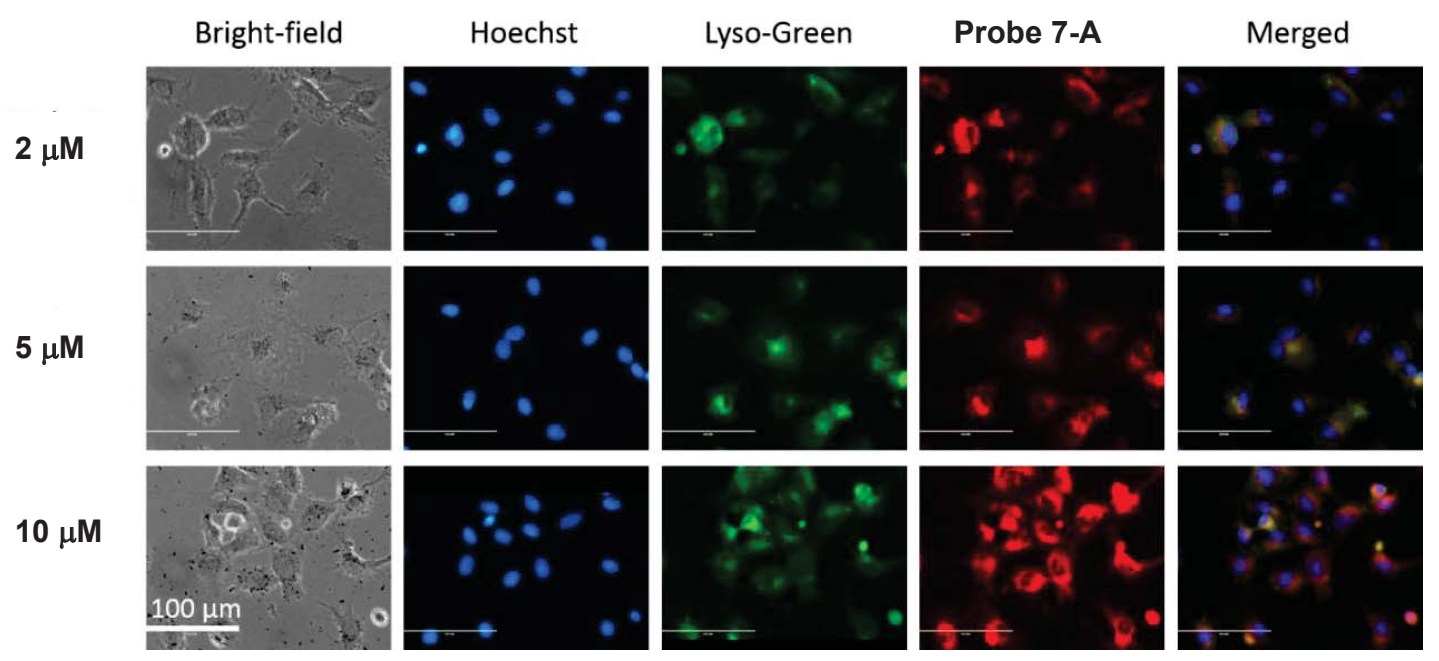

Figure E.27. Fluorescence images of HUV-EC-C cells incubated with fluorescent probe 7-A at 2, 5 and $10 \mu \mathrm{M}$. (HUV-EC-C cells were incubated with probe 7-A for 2 hrs, post serum starvation ( $2 \mathrm{hrs})$ and imaged for co-localization with $(1 \mu \mathrm{M})$ LysoSensor Green and $(1 \mu \mathrm{g} / \mathrm{ml})$ Hoechst 33342 stains. Images were acquired using the EVOS-FL inverted fluorescence microscope at 40X magnification.) 


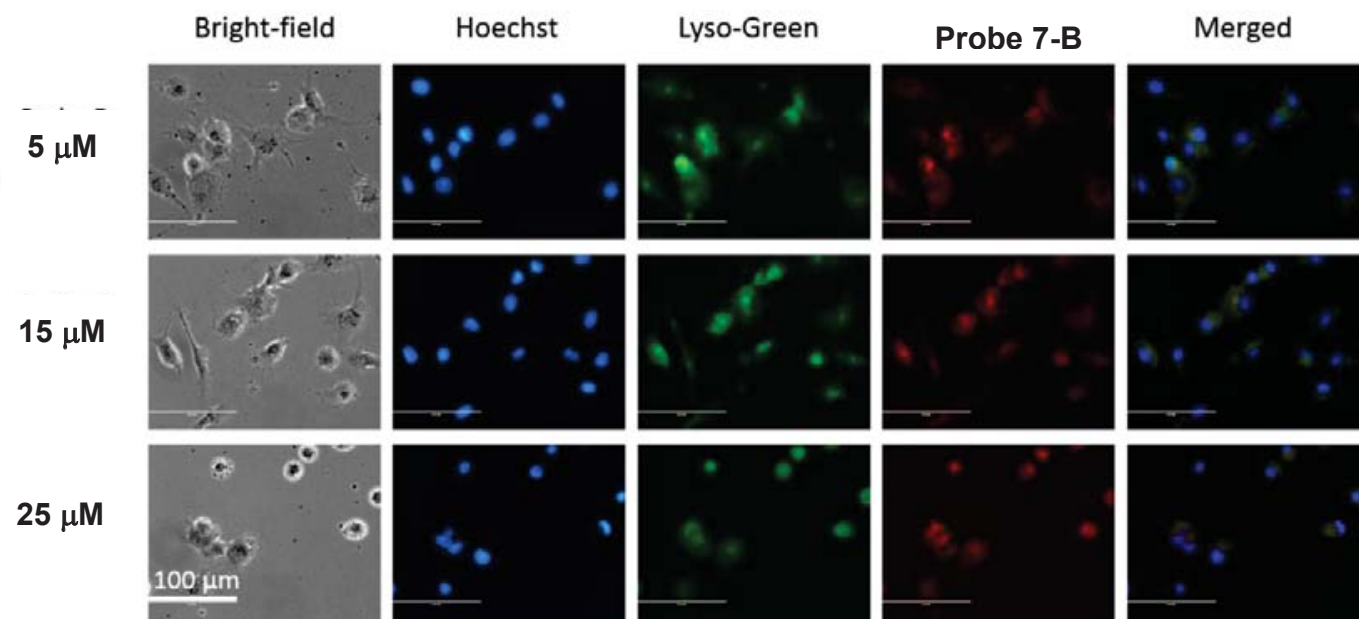

Figure E.28. Fluorescence images of HUV-EC-C cells incubated with fluorescent probe 7-B. (HUV-EC-C cells were incubated with 5, 15 or $25 \mu \mathrm{M}$ of probe 7-B for 2 hrs, post serum starvation (2 hrs) and imaged for co-localization with $1 \mu \mathrm{M}$ LysoSensor Green and (1 $\mu \mathrm{g} / \mathrm{ml})$ Hoechst 33342 stains. Images were acquired using the EVOS-FL inverted fluorescence microscope at 40X magnification.)

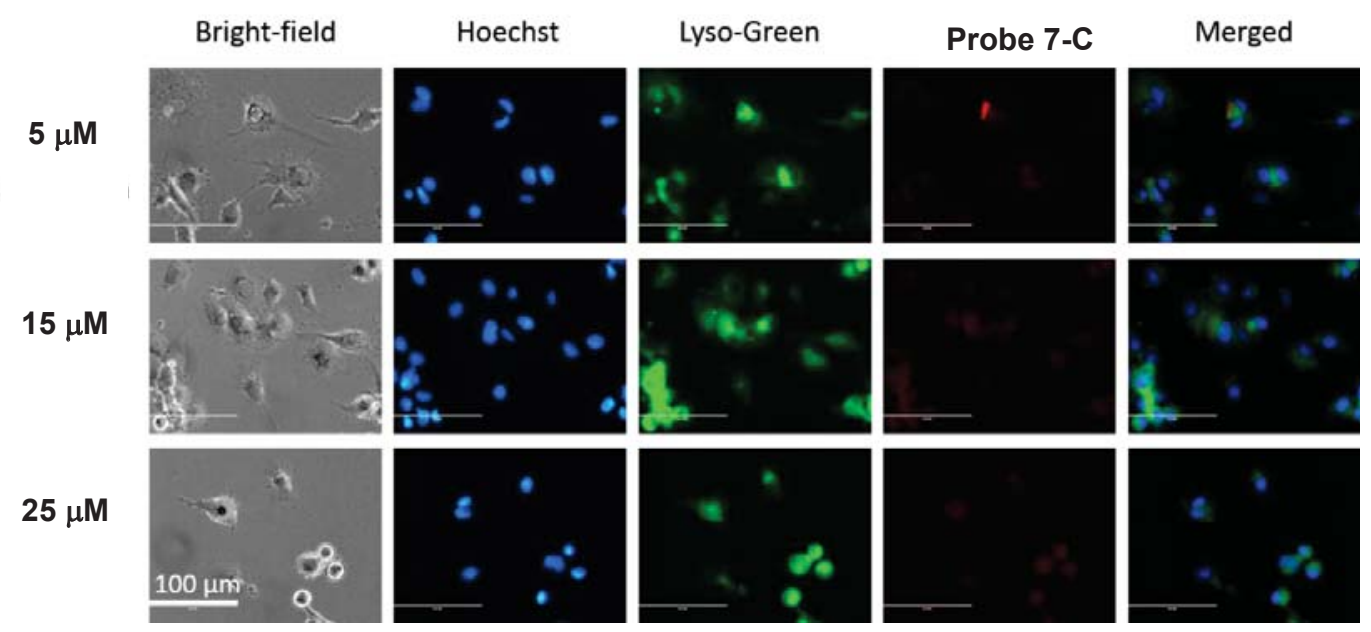

Figure E.29. Fluorescence images of HUV-EC-C cells incubated with fluorescent probe 7-C. (HUV-EC-C cells were incubated with 5, 15 or $25 \mu \mathrm{M}$ of probe 7-C for $2 \mathrm{hrs,} \mathrm{post}$ serum starvation (2 hrs) and imaged for co-localization with $(1 \mu \mathrm{M})$ LysoSensor Green and $(1 \mu \mathrm{g} / \mathrm{ml})$ Hoechst 33342 stains. Images were acquired using the EVOS-FL inverted fluorescence microscope at $40 \mathrm{X}$ magnification.) 


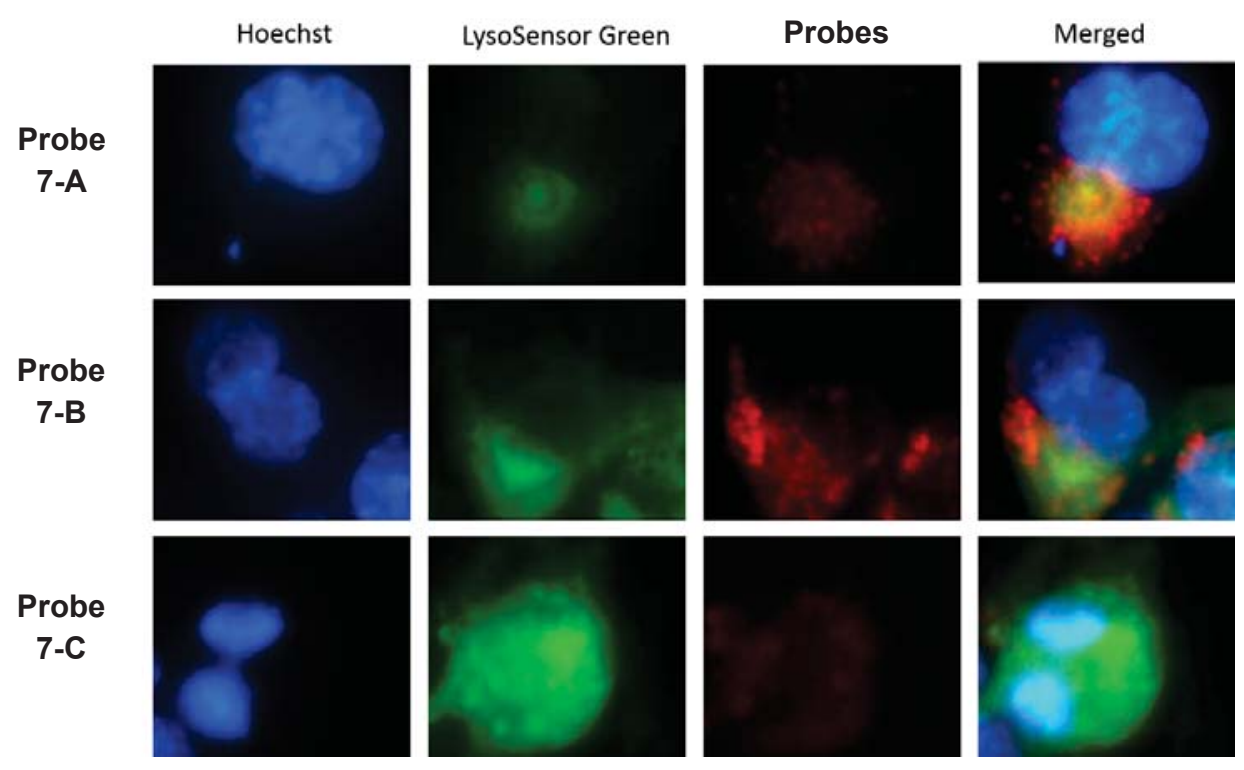

Figure E.30. Enlarged images of MDA-MB-231 cells with fluorescent probes (7-A/7B/7-C) (Images were taken at 60X magnification showing colocalization of probes with LysoSensor green in lysosomes. The probe 7-A shows a much stronger signal compared to probes 7-B/7-C. The lysosomes are mostly perinuclear in cells as they were serum starved for 2 hours before staining.)

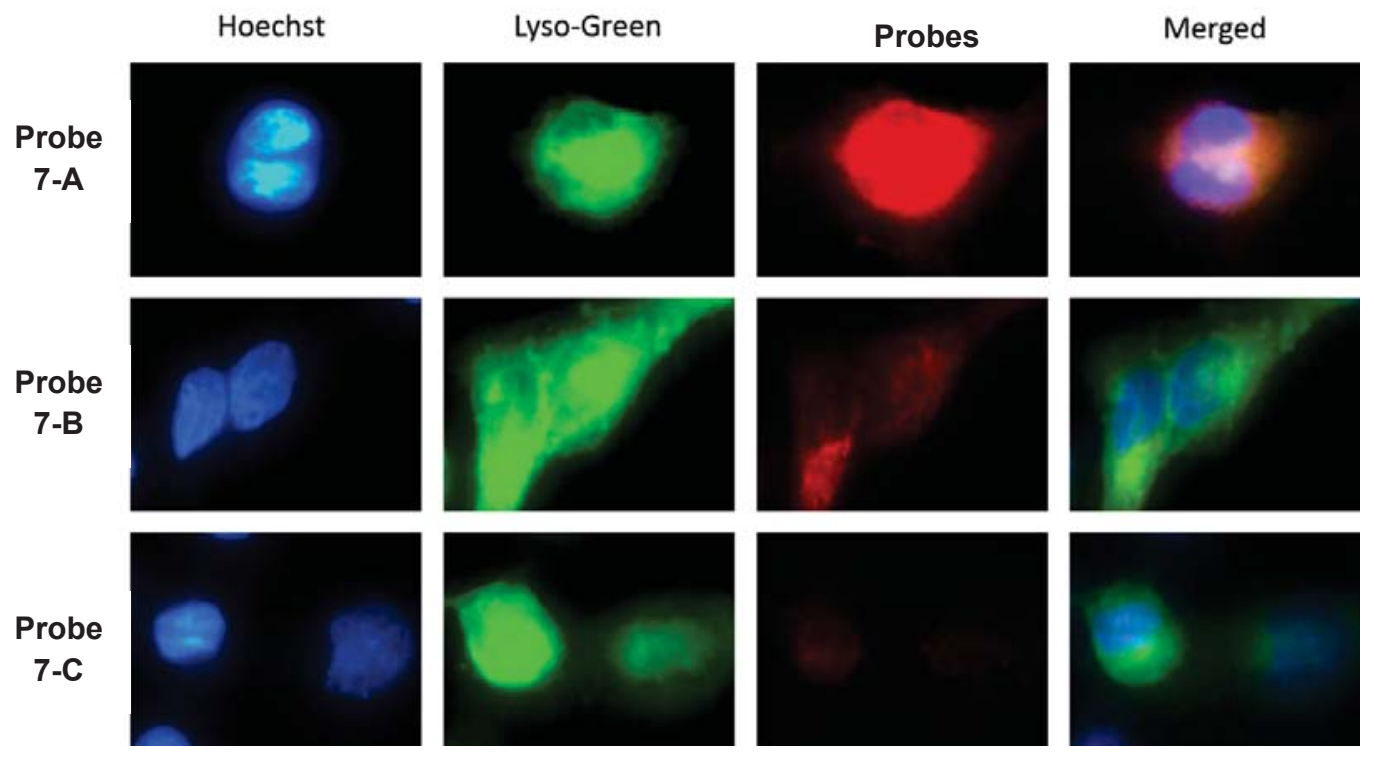


Figure E.31. Enlarged images of HUVEC-C cells with fluorescent probes (7-A/7-B/7C) taken at $60 \mathrm{X}$ magnification showing colocalization of probes with LysoSensor green in lysosomes. (The probe 7-A shows a much stronger signal compared to probes 7-B/7-C. Probe 7-B shows punctate staining under merged image while probe 7-C shows very faint staining. The lysosomes are mostly perinuclear in cells as they were serum starved for 2 hours before staining)

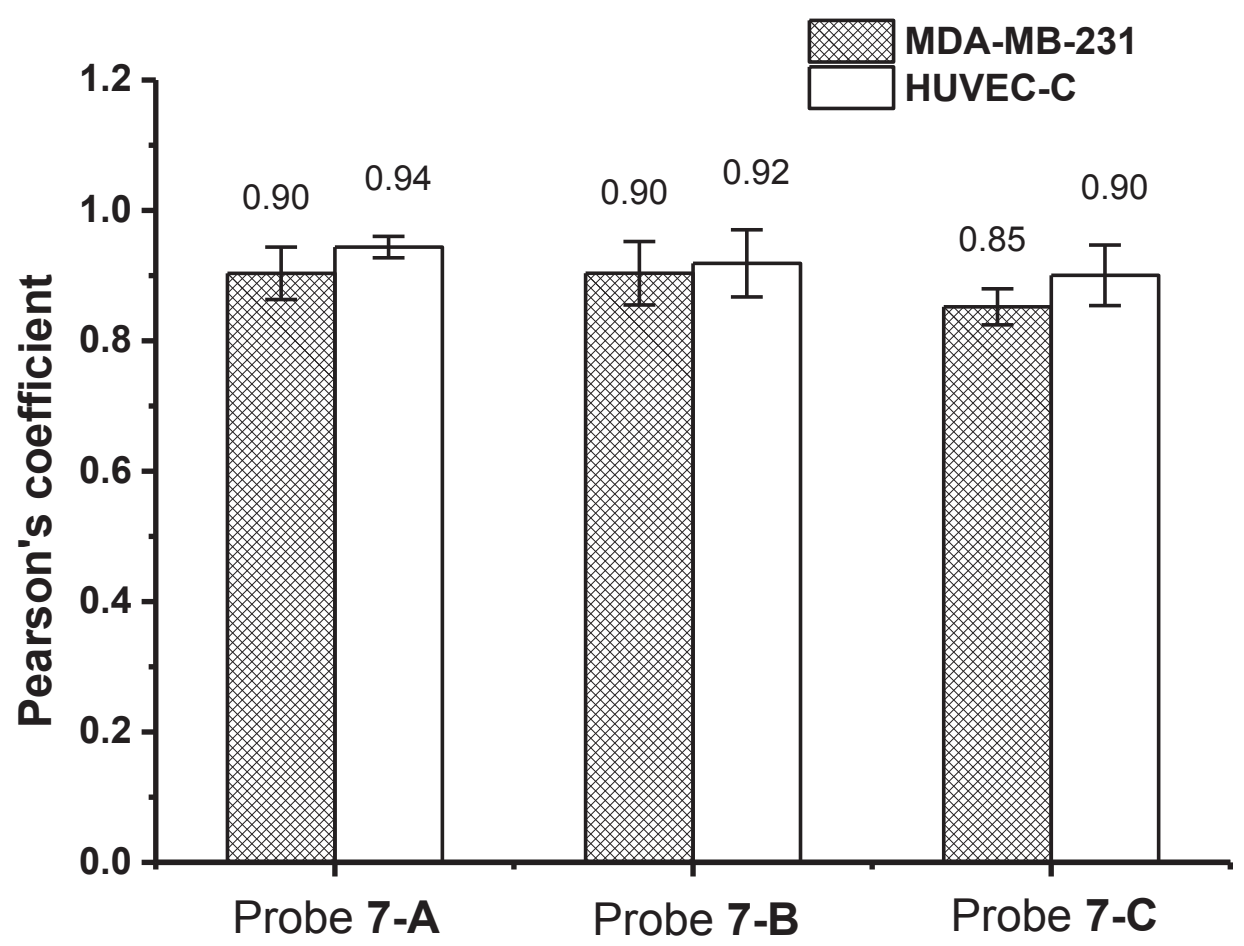

Figure E.32. Pearson's co-localization coefficient of Probes 7-A, 7-B and 7-C with LysoSensor Green. (The co-localization of probes 7-A, 7-B and 7-C with LysoSensor Green were quantified by Perason's coefficient (mean $\pm \mathrm{SD}, \mathrm{n}=6$ ). Methods: Pearson's coefficient was quantified using JACoP plug in from imageJ. (Bolte and Cordelieres, 2006, Journal of microscopy, 224, 213-232)) 
Unimodal Results Summary

\begin{tabular}{|c|c|c|c|c|c|c|}
\hline Angle & $\begin{array}{c}\text { Mean } \\
(\mathbf{n m})\end{array}$ & P.I. & $\begin{array}{c}\text { Diff.Coef } \\
\left(\mathbf{m}^{2} / \mathbf{s}\right)\end{array}$ & Counts/s & $\begin{array}{c}\text { Baseline } \\
\text { Error }\end{array}$ & Overflow \\
\hline $90.0^{\circ}$ & 500.4 & 0.683 & $9.70 \mathrm{e}-13$ & $7.44 \mathrm{e}+04$ & $0.35 \%$ & 0 \\
\hline
\end{tabular}

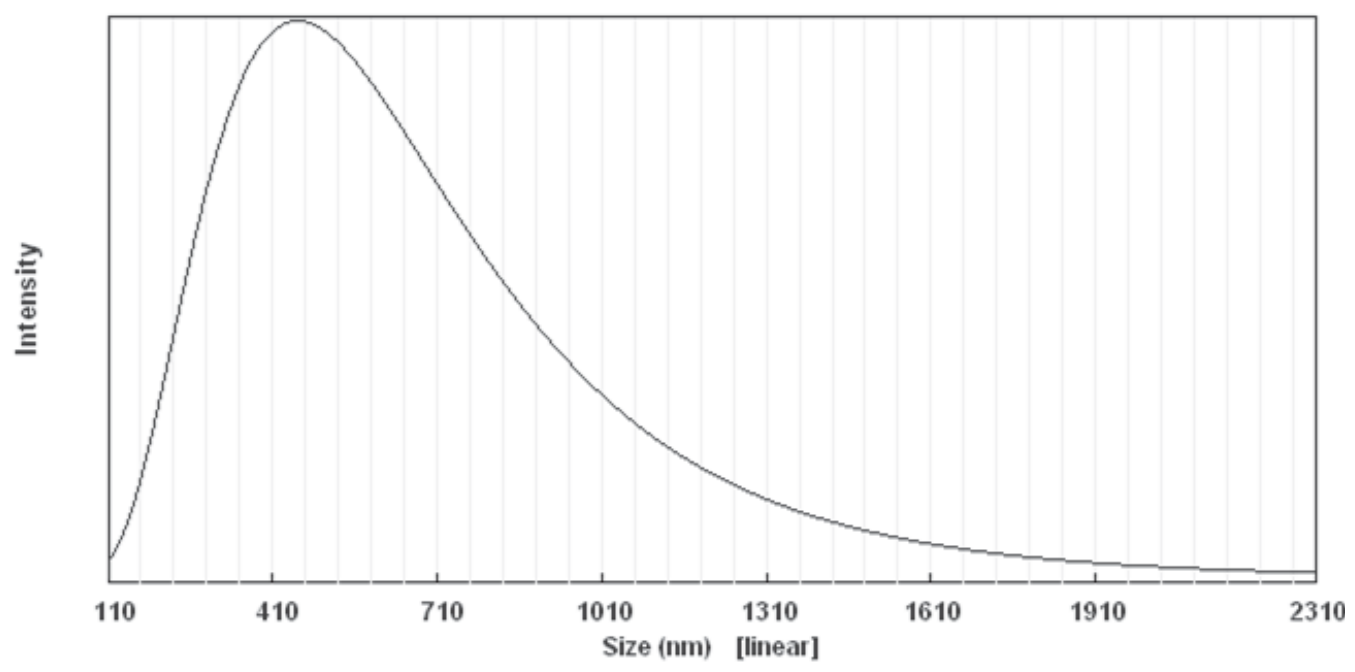

Figure E.33. Dynamic light scattering measurement result of aqueous solution (pH 7.4) of probe $7-\mathrm{A}(5 \mu \mathrm{M})$.

Unimodal Results Summary

\begin{tabular}{|c|c|c|c|c|c|c|}
\hline Angle & $\begin{array}{c}\text { Mean } \\
(\mathrm{nm})\end{array}$ & P.I. & $\begin{array}{c}\text { Diff.Coef } \\
\left(\mathbf{m}^{2} / \mathbf{s}\right)\end{array}$ & Counts/s & $\begin{array}{c}\text { Baseline } \\
\text { Error }\end{array}$ & Overflow \\
\hline $90.0^{\circ}$ & 333.6 & -1.768 & $1.46 \mathrm{e}-12$ & $2.63 \mathrm{e}+04$ & $0.53 \%$ & 0 \\
\hline
\end{tabular}

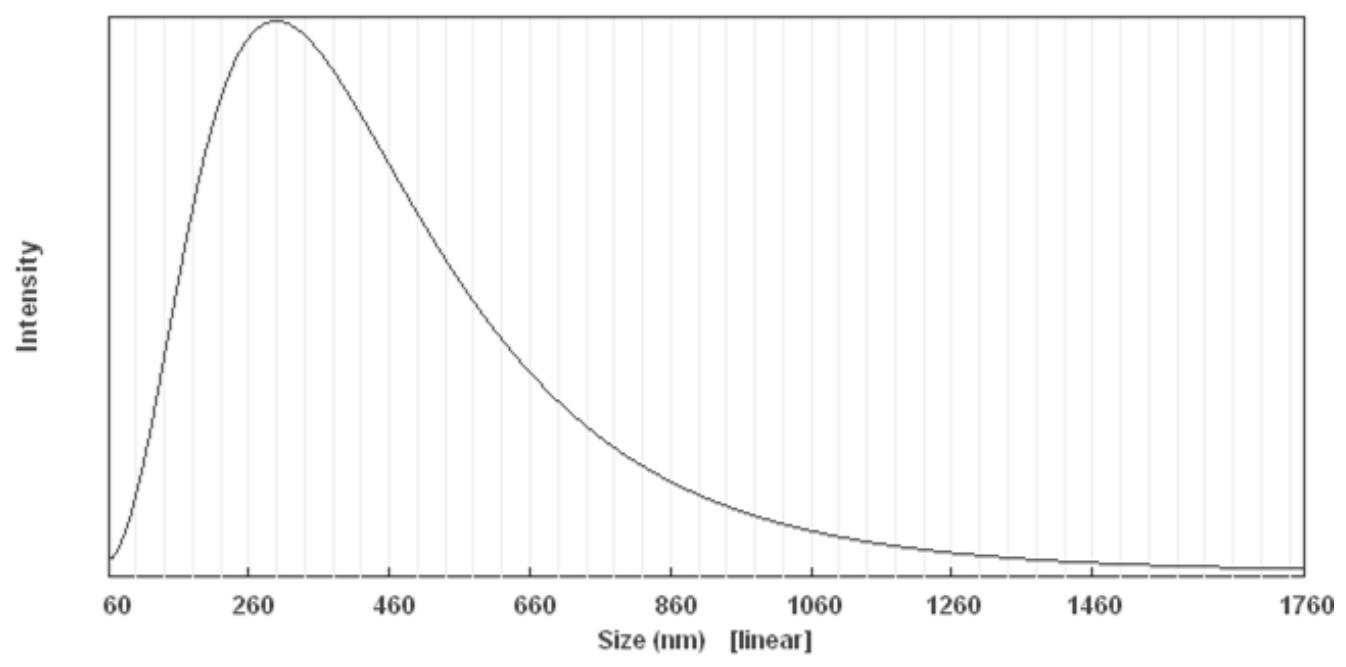

Figure E.34. Dynamic light scattering measurement result of aqueous solution ( $\mathrm{pH}$ 7.4) of probe 7-B $(5 \mu \mathrm{M})$. 


\begin{tabular}{|c|c|c|c|c|c|c|}
\hline \multicolumn{1}{|c|}{ Unimodal Results Summary } \\
\hline Angle & $\begin{array}{c}\text { Mean } \\
(\mathrm{nm})\end{array}$ & P.I. & $\begin{array}{c}\text { Diff.Coef } \\
\left(\mathbf{m}^{2} / \mathrm{s}\right)\end{array}$ & Counts/s & $\begin{array}{c}\text { Baseline } \\
\text { Error }\end{array}$ & Overflow \\
\hline $90.0^{\circ}$ & 271.8 & 1.589 & $1.79 \mathrm{e}-12$ & $2.98 \mathrm{e}+04$ & $0.51 \%$ & 0 \\
\hline
\end{tabular}

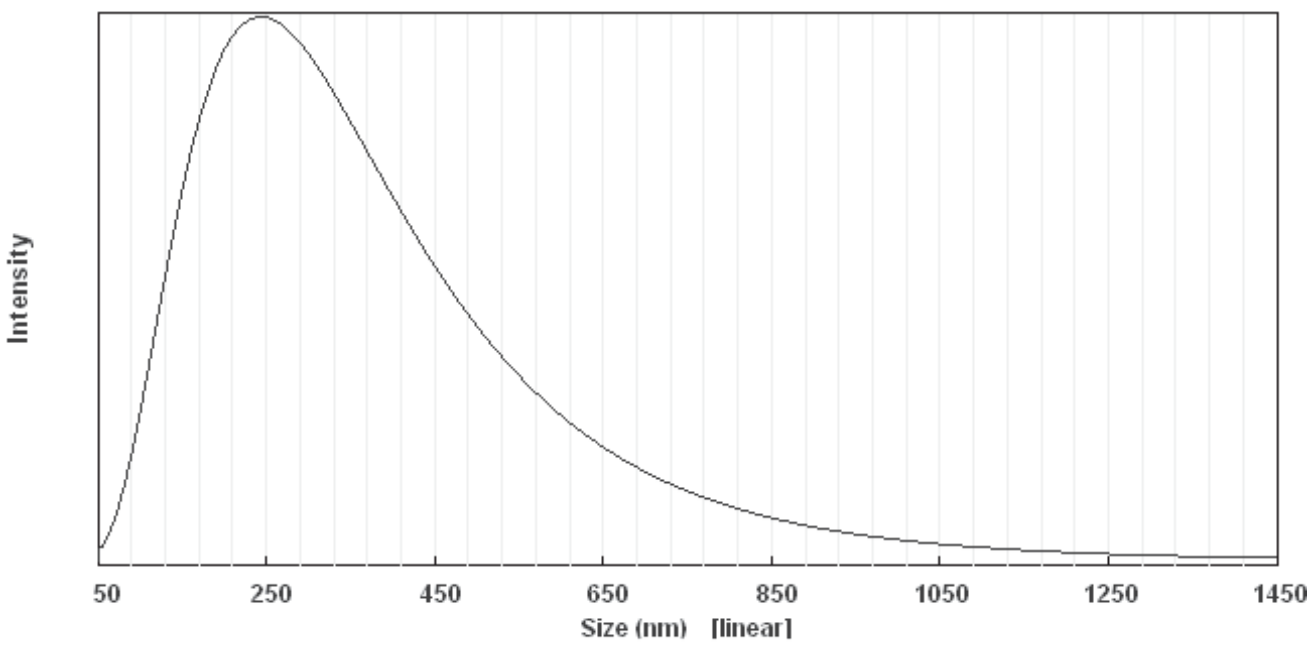

Figure E.35. Dynamic light scattering measurement result of aqueous solution ( $\mathrm{pH}$ 7.4) of probe 7-C $(5 \mu \mathrm{M})$. 


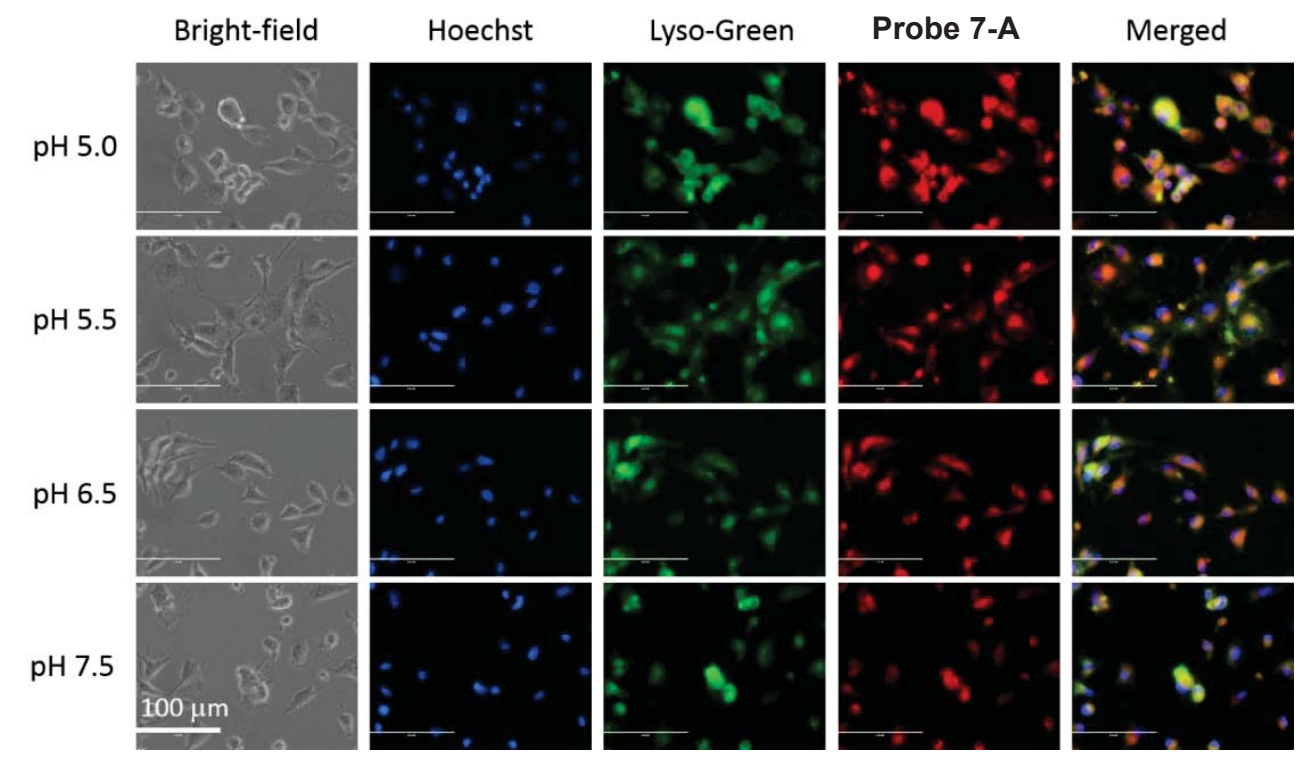

Figure E.36. Fluorescence images of HUVEC-C cells incubated with $5 \mu \mathrm{M}$ probes 7-A at different $\mathrm{pH}$ values. $1 \mu \mathrm{M}$ LysoSensor Green and $1 \mu \mathrm{g} / \mathrm{mL}$ Hoechst 33342 were used as co-stains. Images were acquired using the using inverted fluorescence microscope (AMF-4306, EVOS $\mathrm{fl}, \mathrm{AMG})$ at 40X magnification.

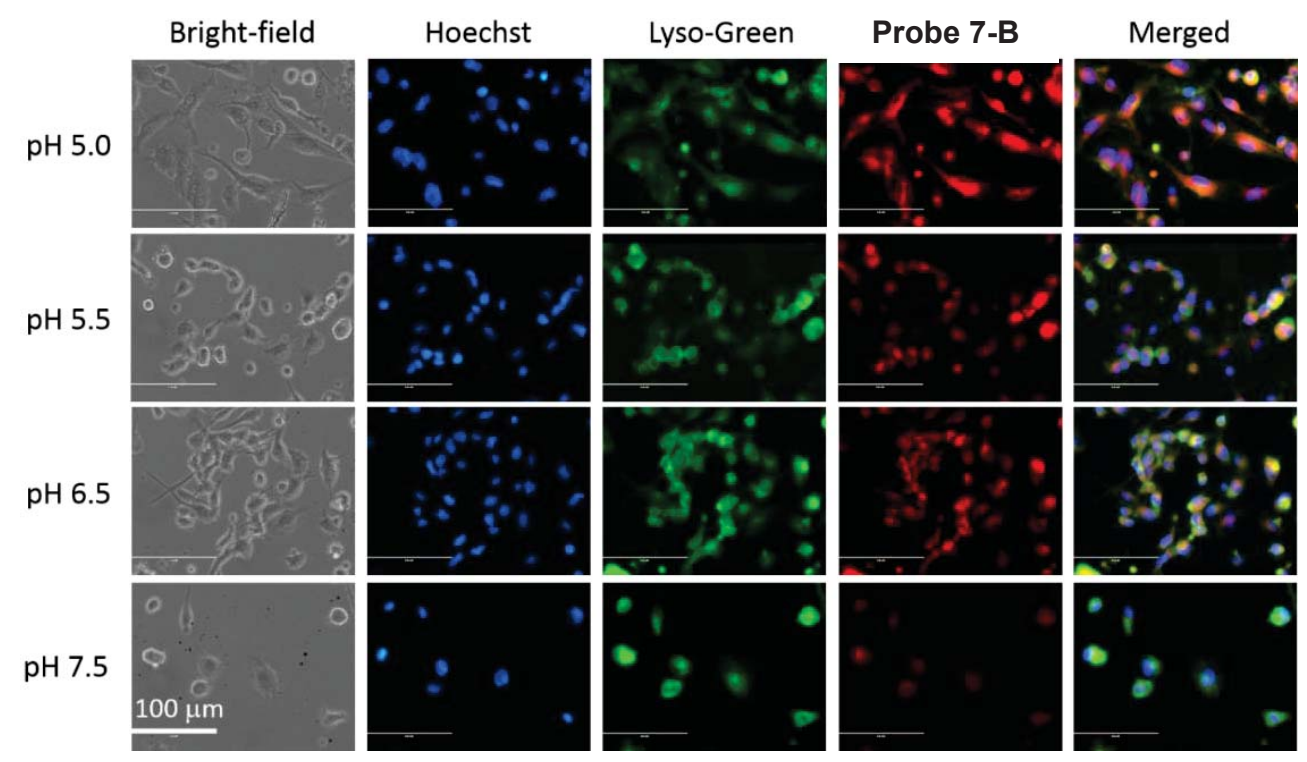

Figure E.37. Fluorescence images of HUVEC-C cells incubated with $15 \mu \mathrm{M}$ probes 7B at different pH values. $1 \mu \mathrm{M}$ LysoSensor Green and $1 \mu \mathrm{g} / \mathrm{mL}$ Hoechst 33342 were used as co-stains. Images were acquired using the using inverted fluorescence microscope (AMF-4306, EVOS $\mathrm{fl}, \mathrm{AMG})$ at 40X magnification. 


\section{Appendix F Supporting Information for Chapter 8}

Figure F.1 shows the comparisons of ${ }^{1} \mathrm{H}$ NMR spectra of BODIPY dyes 8.5, 8.7, 8.A and polymer 8-A. Doublet peaks at $8.00 \mathrm{ppm}$ corresponding to vinyl protons at 3',5'positions in 2,6-diiodo-3,5-distyryl-BODIPY dye 8.7 shift upfield to $6.42 \mathrm{ppm}$ in BODIPY dye 8.A (Figure F.1). This is because the vinyl protons in BOIDPY dye 8.A are in the shielding zone of the phenyl substituents at 2,6-positions and experience a shielding by $1.58 \mathrm{ppm}$. Peaks at $7.02 \mathrm{ppm}$ corresponding to phenyl protons at c-positions in 2,6-diiodo-3,5-distyryl-BODIPY dye $\mathbf{8 . 7}$ also shift upfield to $6.48 \mathrm{ppm}$ in BODIPY

dye 8.A because the phenyl protons at c-positions in BODIPY dye 8.A are in the shielding zone of the phenyl substituents at 2,6-positions (Figure F.1). 


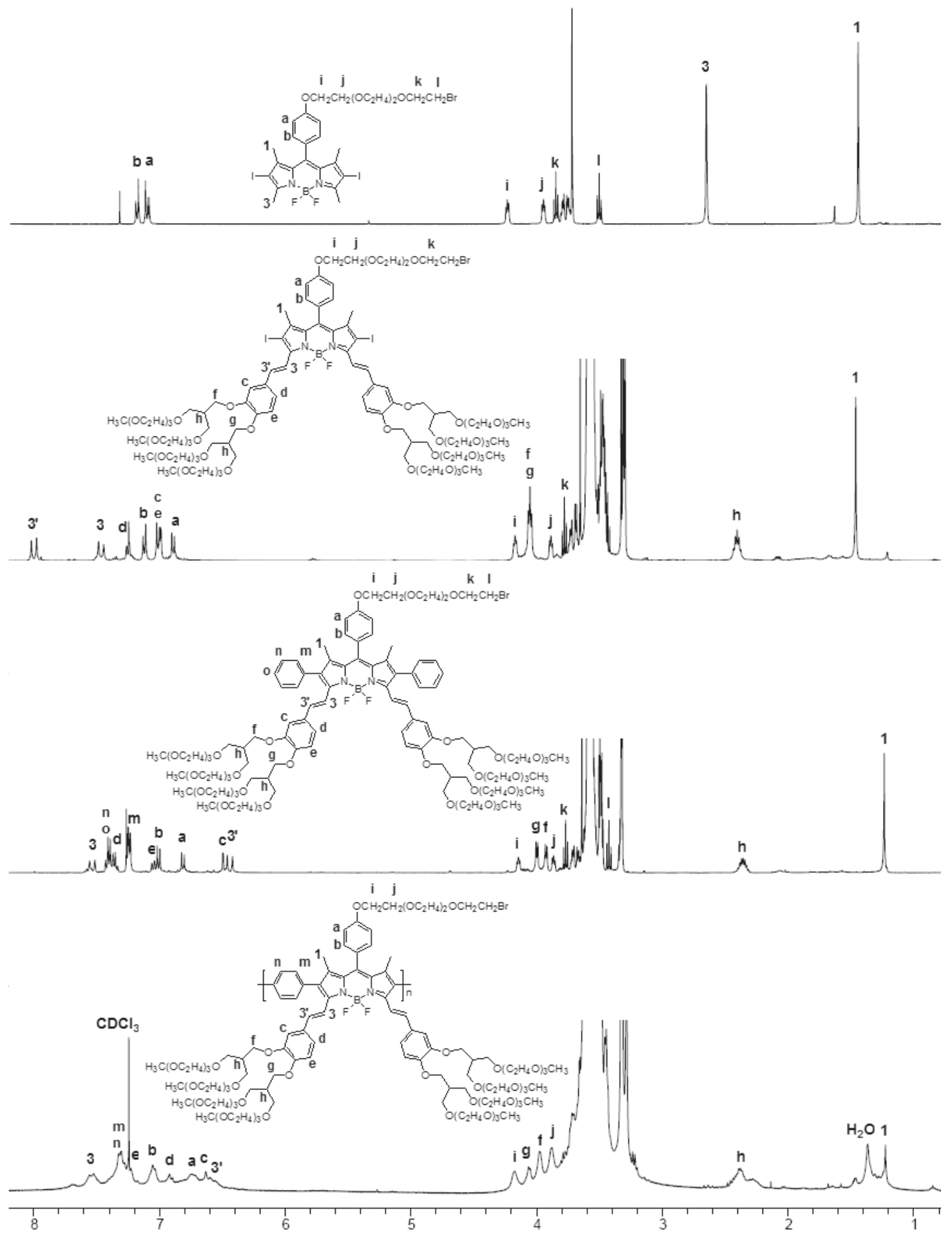

Figure F.1. Comparison of ${ }^{1} \mathrm{H}$ NMR spectra of BODIPY dyes 8.5, 8.7, 8.A and polymer 8-A. 


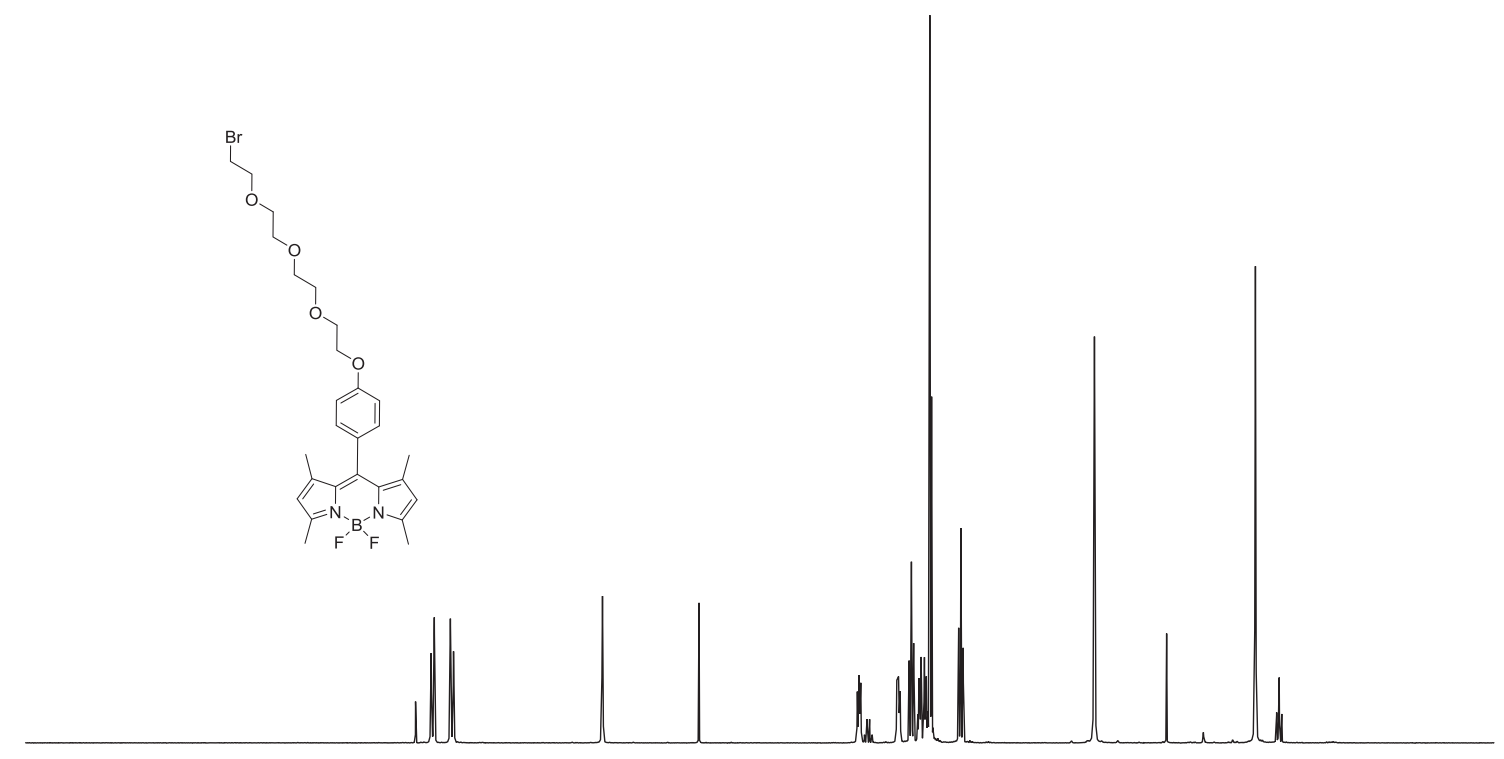

$\begin{array}{llllllllllllllllllll}9.5 & 9.0 & 8.5 & 8.0 & 7.5 & 7.0 & 6.5 & 6.0 & 5.5 & \underset{\mathrm{ppm}}{5.0} & 4.5 & 4.0 & 3.5 & 3.0 & 2.5 & 2.0 & 1.5 & 1.0 & 0.5 & 0.0\end{array}$

Figure F.2. $\quad{ }^{1} \mathrm{H}$ NMR spectrum of BODIPY dye $\mathbf{8 . 4}$ in $\mathrm{CDCl}_{3}$ solution.

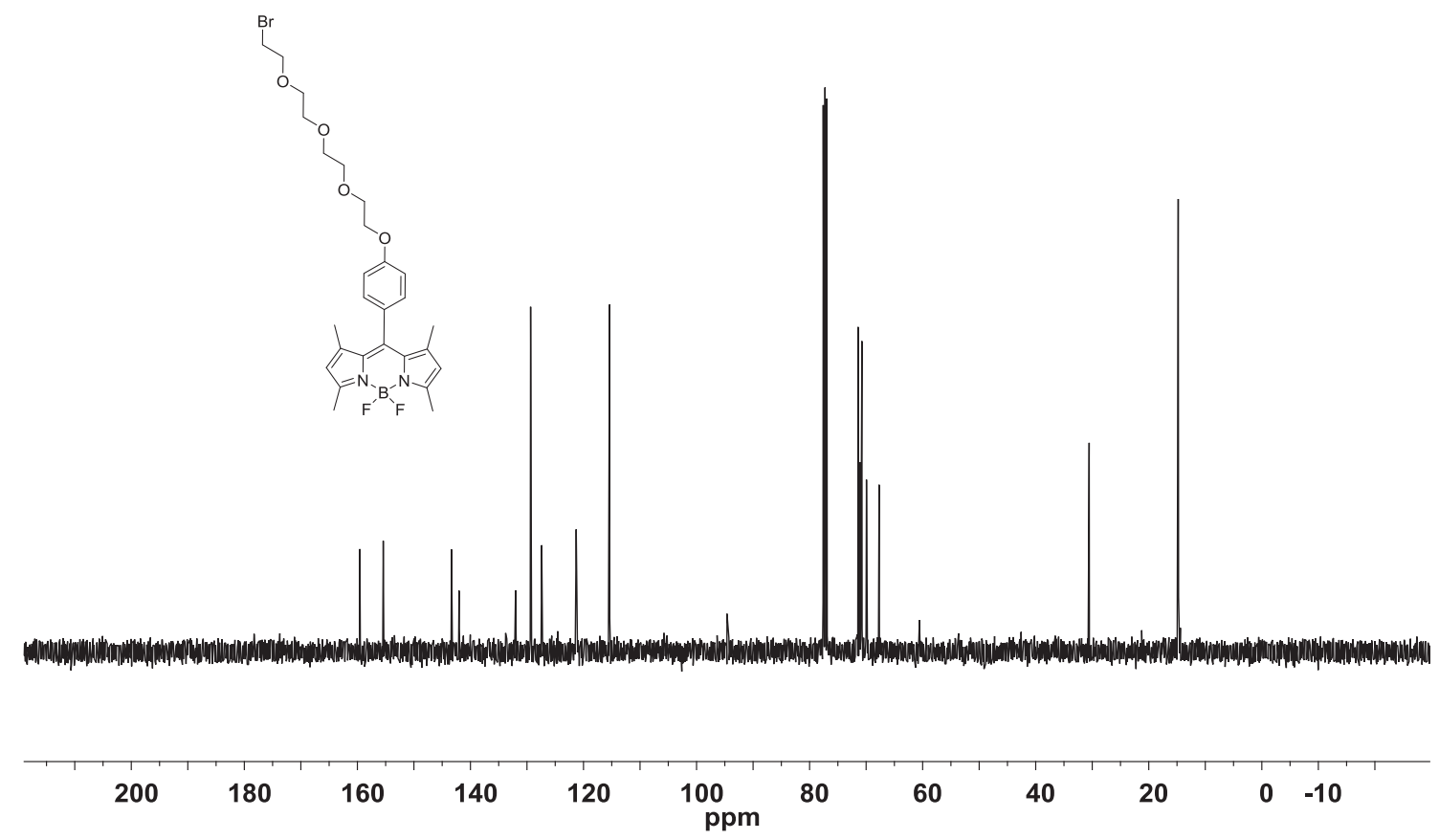

Figure F.3. $\quad{ }^{13} \mathrm{C}$ NMR spectrum of BODIPY dye 8.4 in $\mathrm{CDCl}_{3}$ solution. 


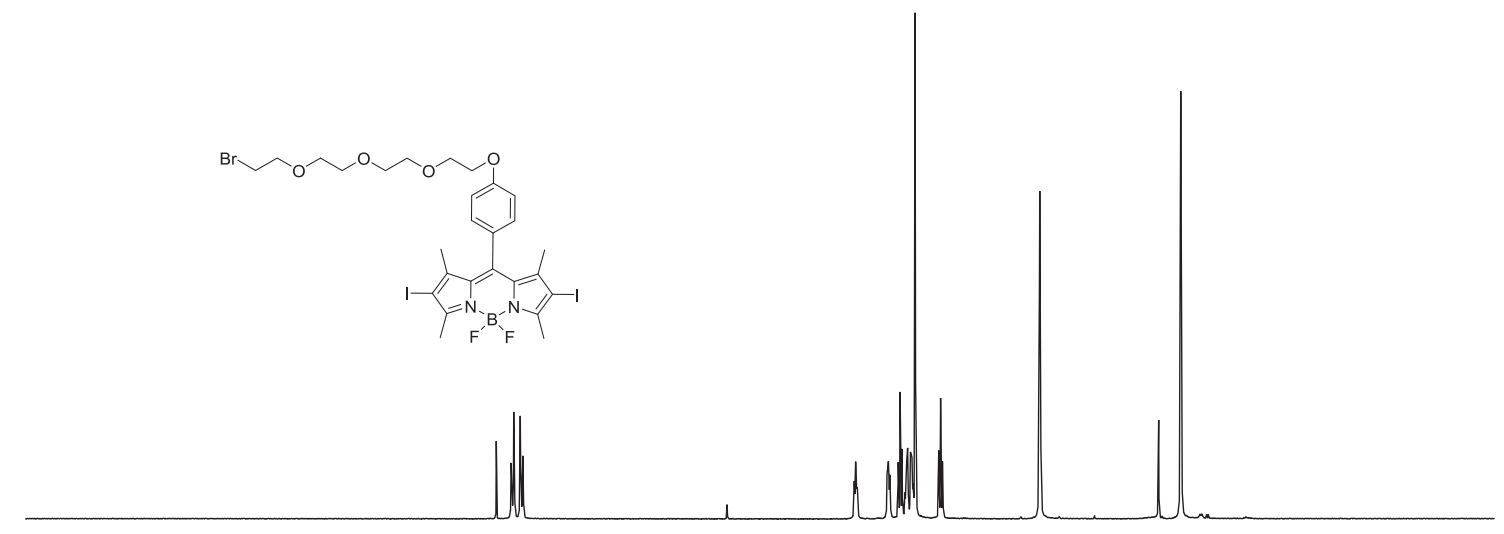

\begin{tabular}{llllllllllllll}
\hline 10.5 & 9.5 & 8.5 & 7.5 & 6.5 & 5.5 & 4.5 & 3.5 & 2.5 & 1.5 & 0.5 & -0.5 & ppm
\end{tabular}

Figure F.4. $\quad{ }^{1} \mathrm{H}$ NMR spectrum of BODIPY dye $\mathbf{8 . 5}$ in $\mathrm{CDCl}_{3}$ solution.

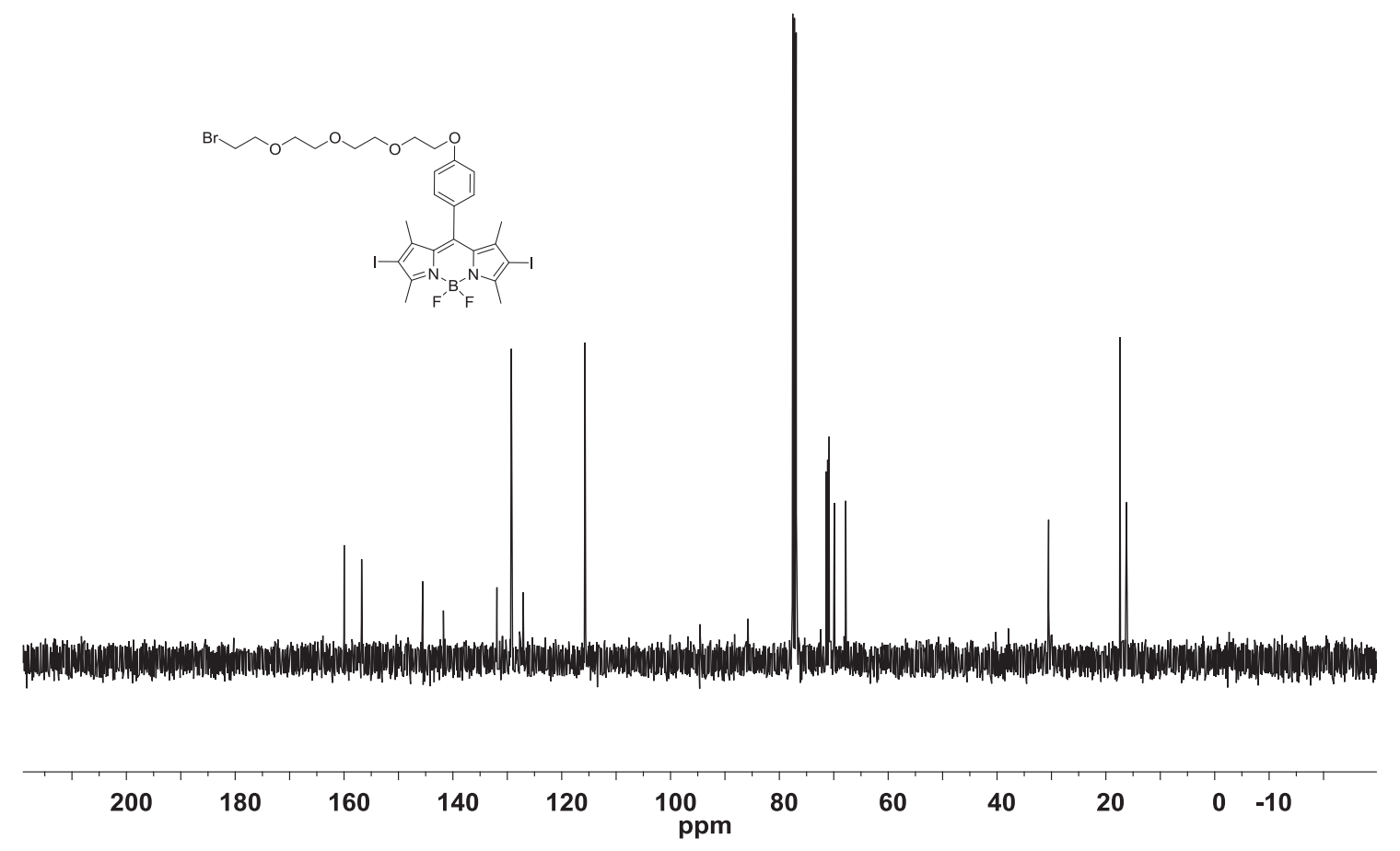

Figure F.5. $\quad{ }^{13} \mathrm{C}$ NMR spectrum of BODIPY dye $\mathbf{8 . 5}$ in $\mathrm{CDCl}_{3}$ solution. 


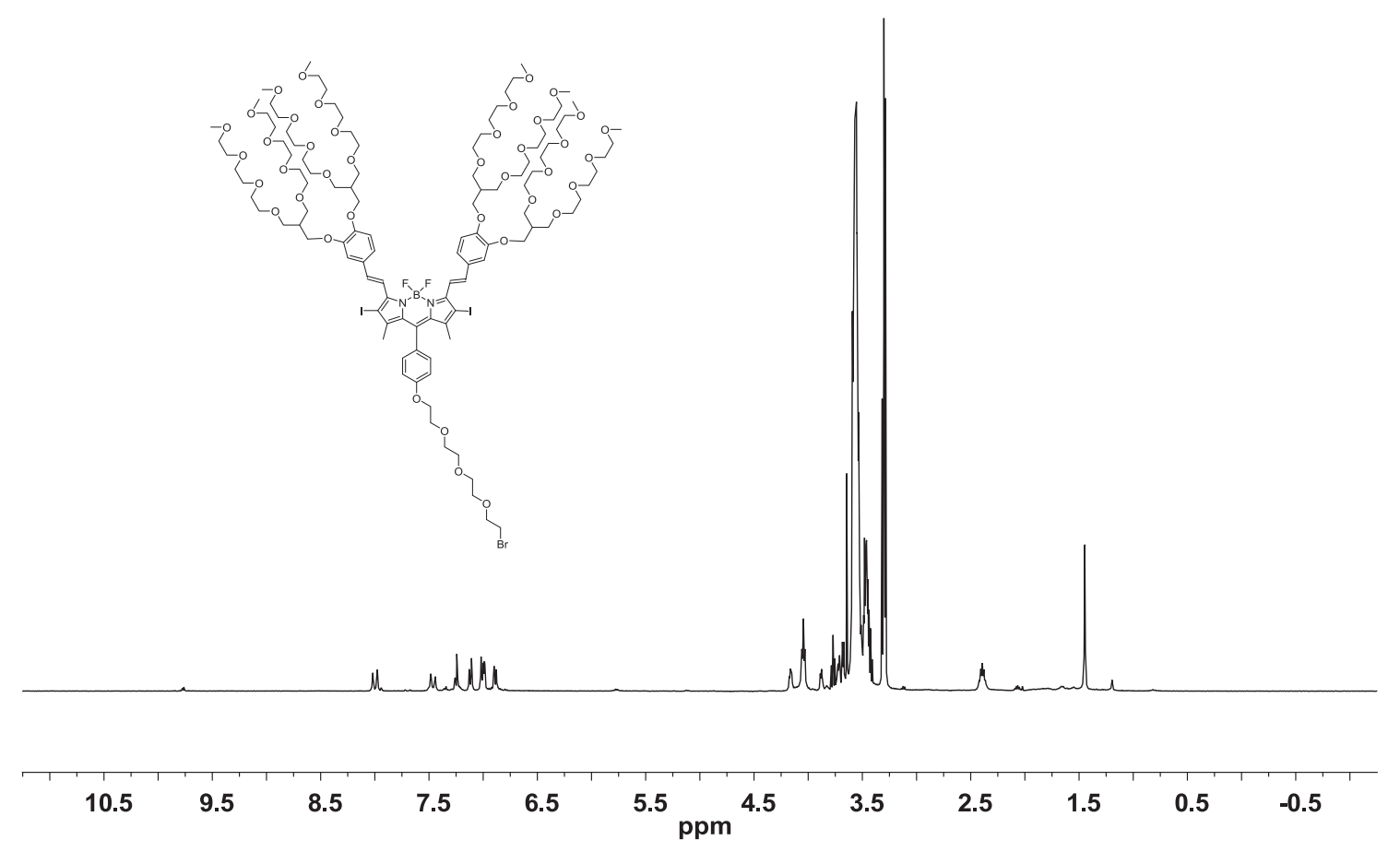

Figure F.6. $\quad{ }^{1} \mathrm{H}$ NMR spectrum of BODIPY dye $\mathbf{8 . 7}$ in $\mathrm{CDCl}_{3}$ solution.

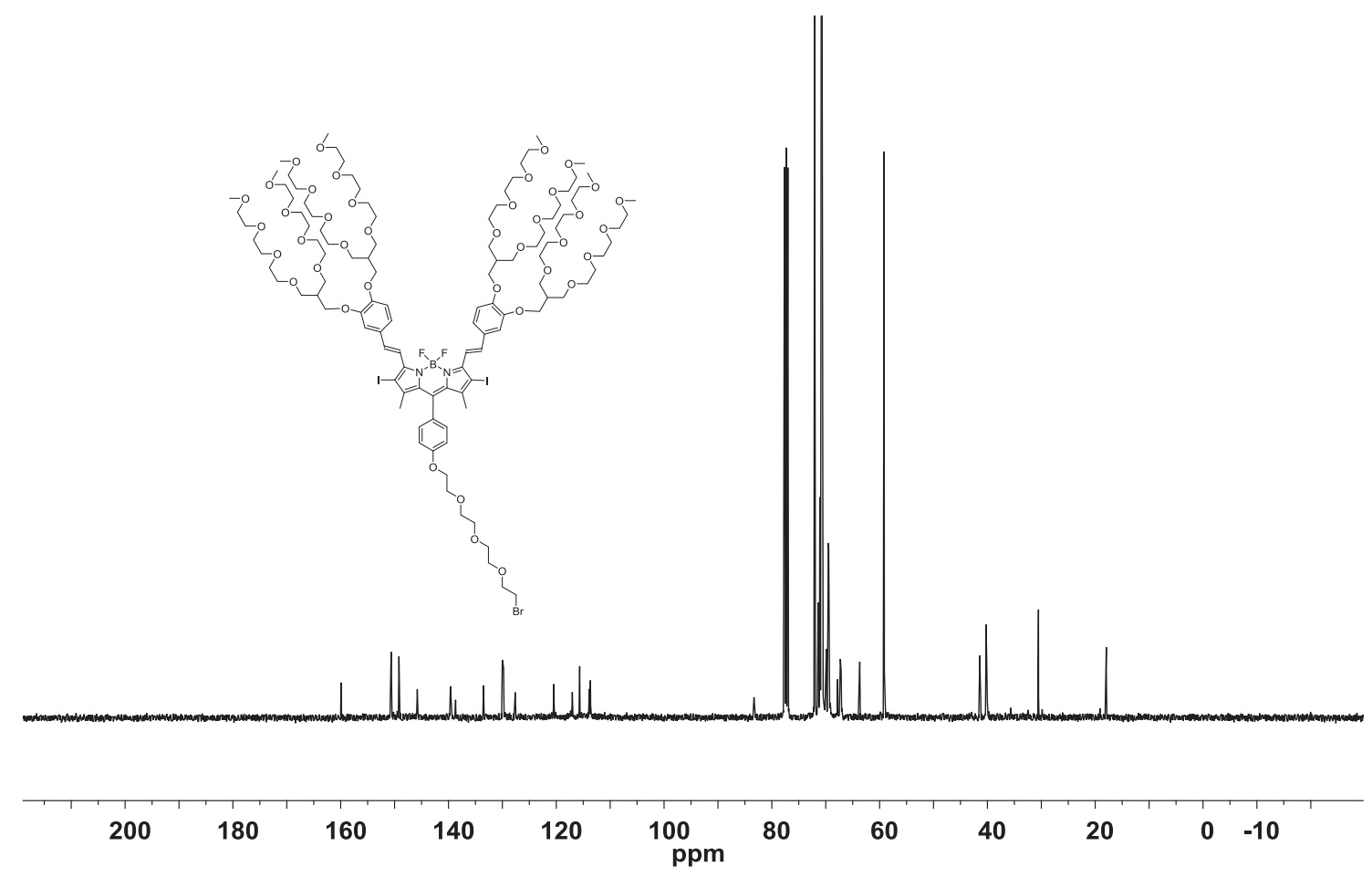

Figure F.7. $\quad{ }^{13} \mathrm{C}$ NMR spectrum of BODIPY dye 8.7 in $\mathrm{CDCl}_{3}$ solution. 


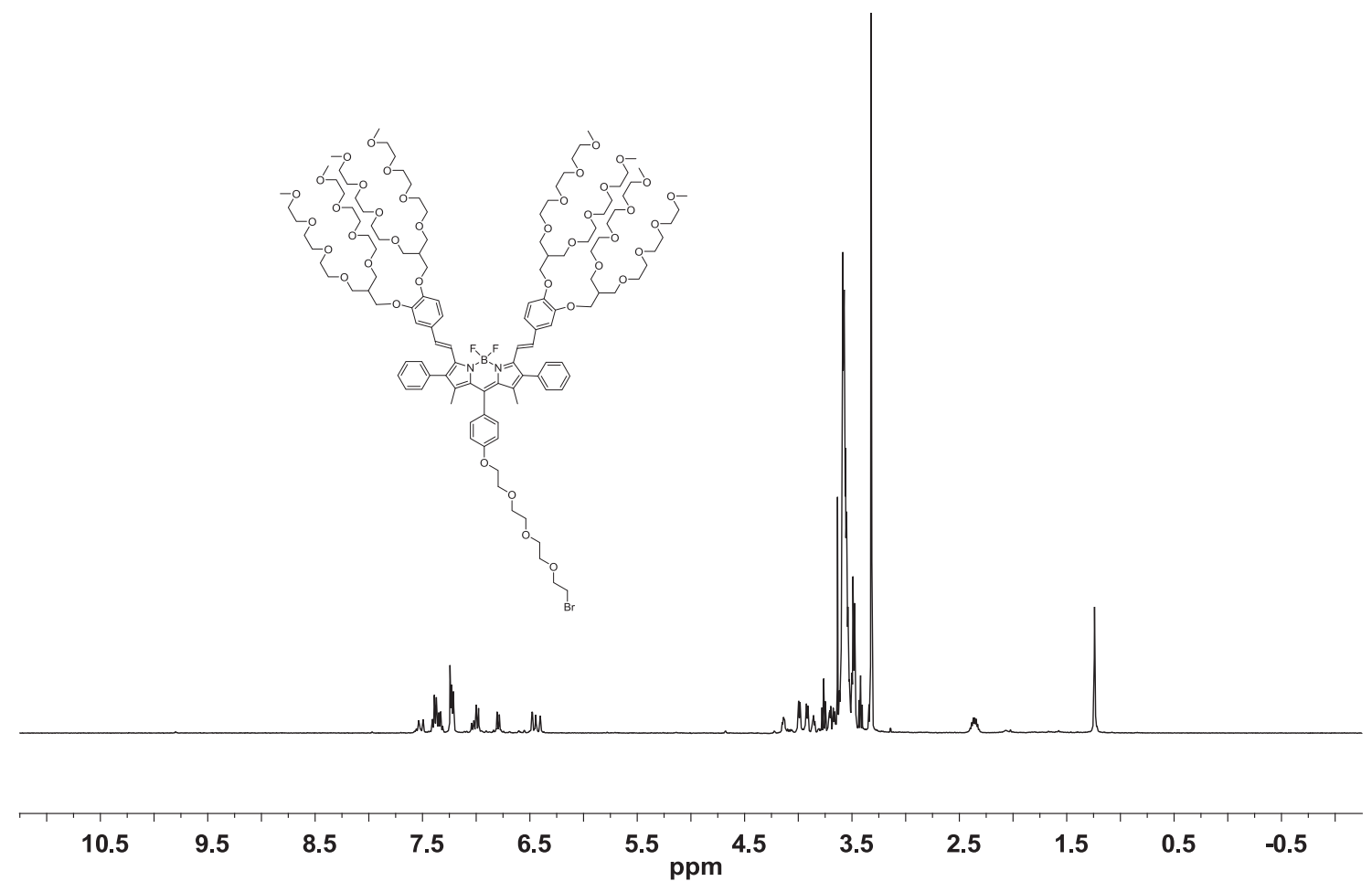

Figure F.8. $\quad{ }^{1} \mathrm{H}$ NMR spectrum of BODIPY dye 8.A in $\mathrm{CDCl}_{3}$ solution.

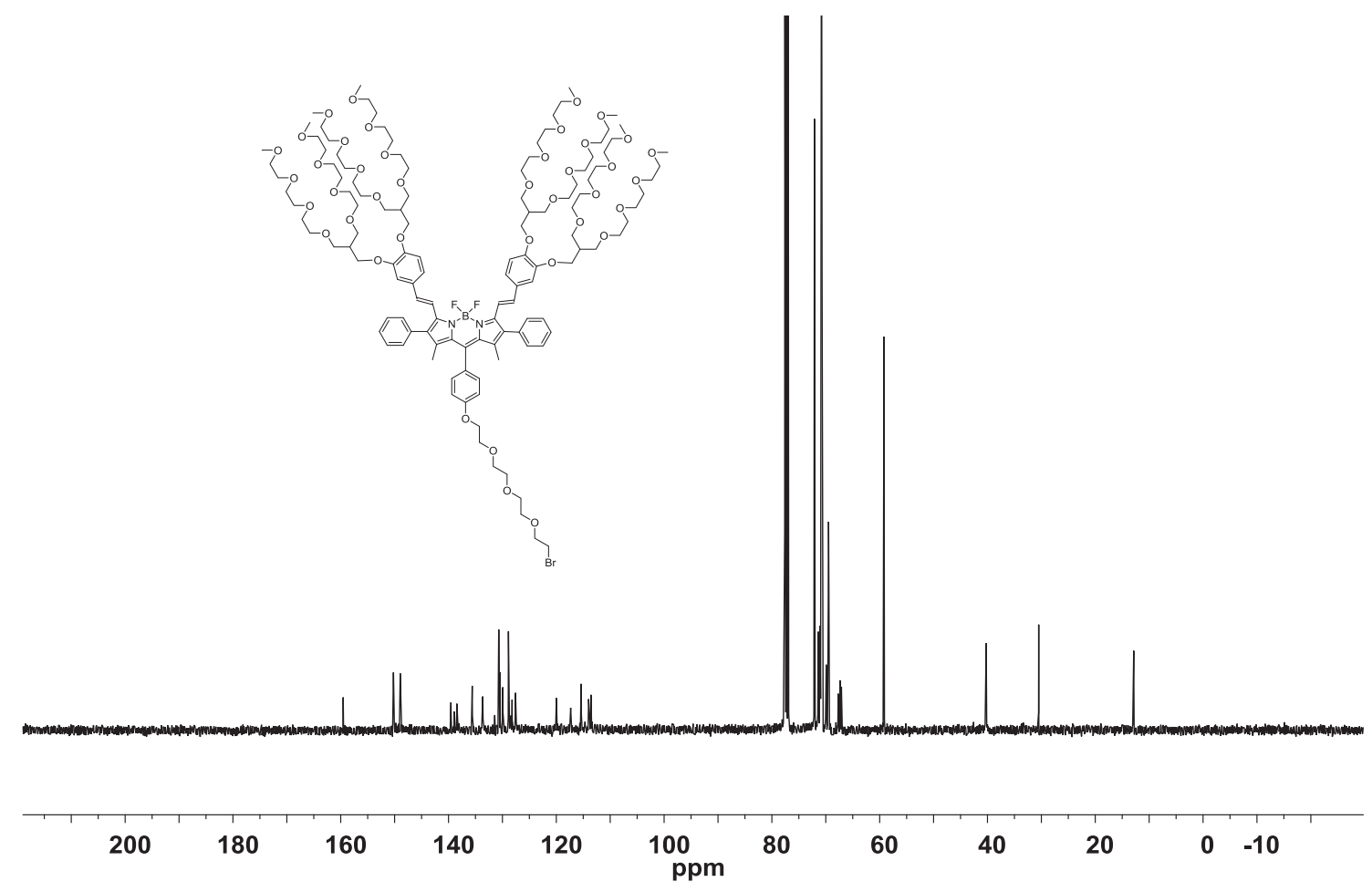

Figure F.9. ${ }^{13} \mathrm{C}$ NMR spectrum of BODIPY dye 8.A in $\mathrm{CDCl}_{3}$ solution. 


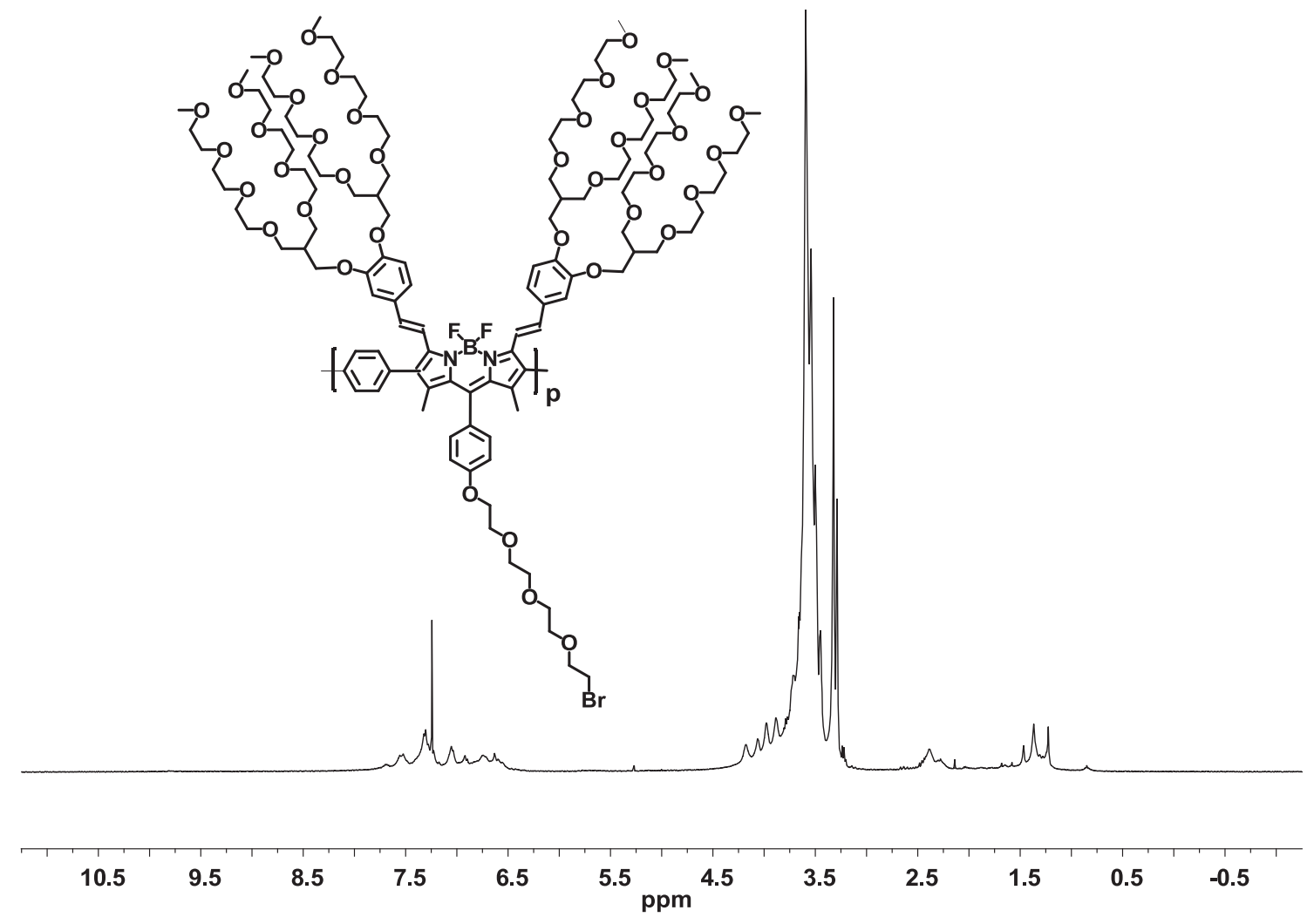

Figure F.10. ${ }^{1} \mathrm{H}$ NMR spectrum of Polymer 8-A in $\mathrm{CDCl}_{3}$ solution. 


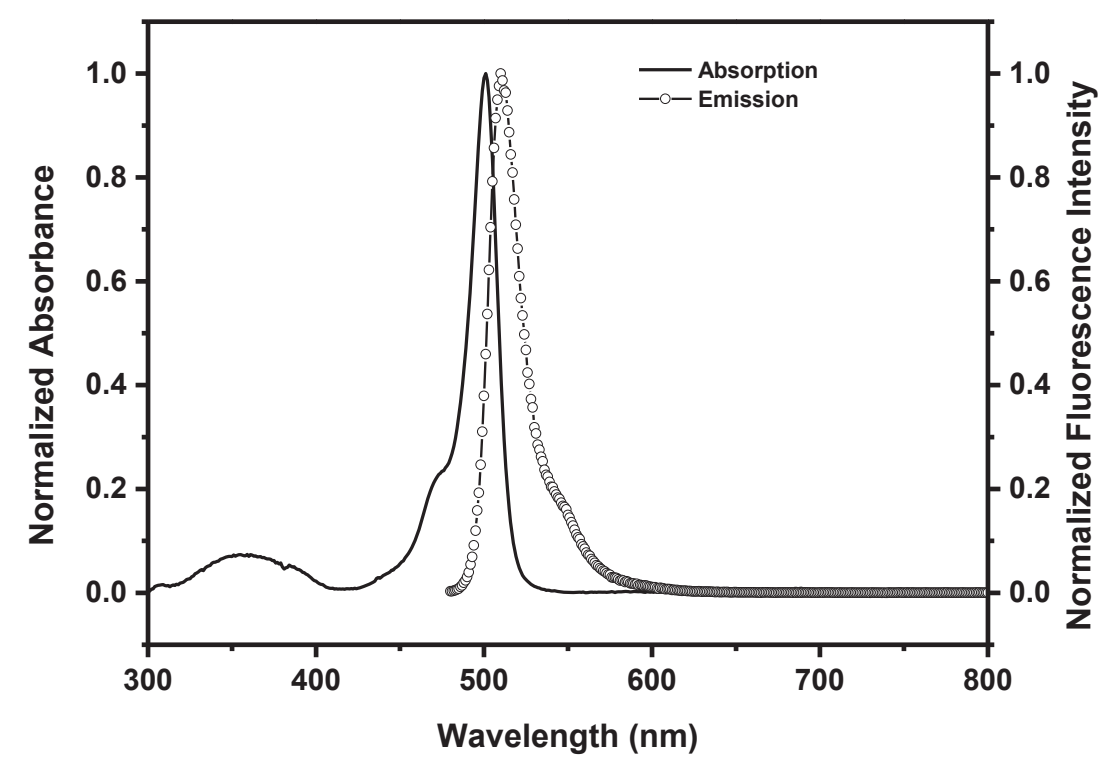

Figure F.11. Absorption and emission spectra of BODIPY dye $\mathbf{8 . 4}$ in methylene chloride solution $\left(\lambda_{\mathrm{ex}}=470 \mathrm{~nm}\right)$.

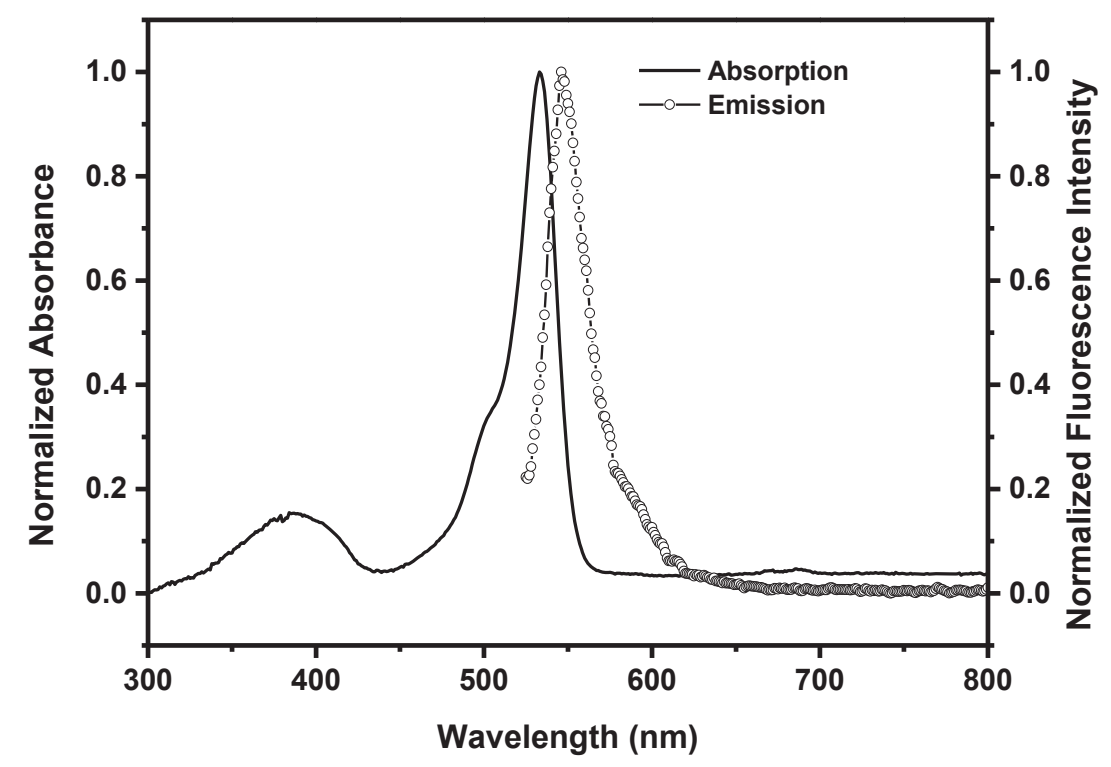

Figure F.12. Absorption and emission spectra of BODIPY dye $\mathbf{8 . 5}$ in methylene chloride solution $\left(\lambda_{\mathrm{ex}}=515 \mathrm{~nm}\right)$. 


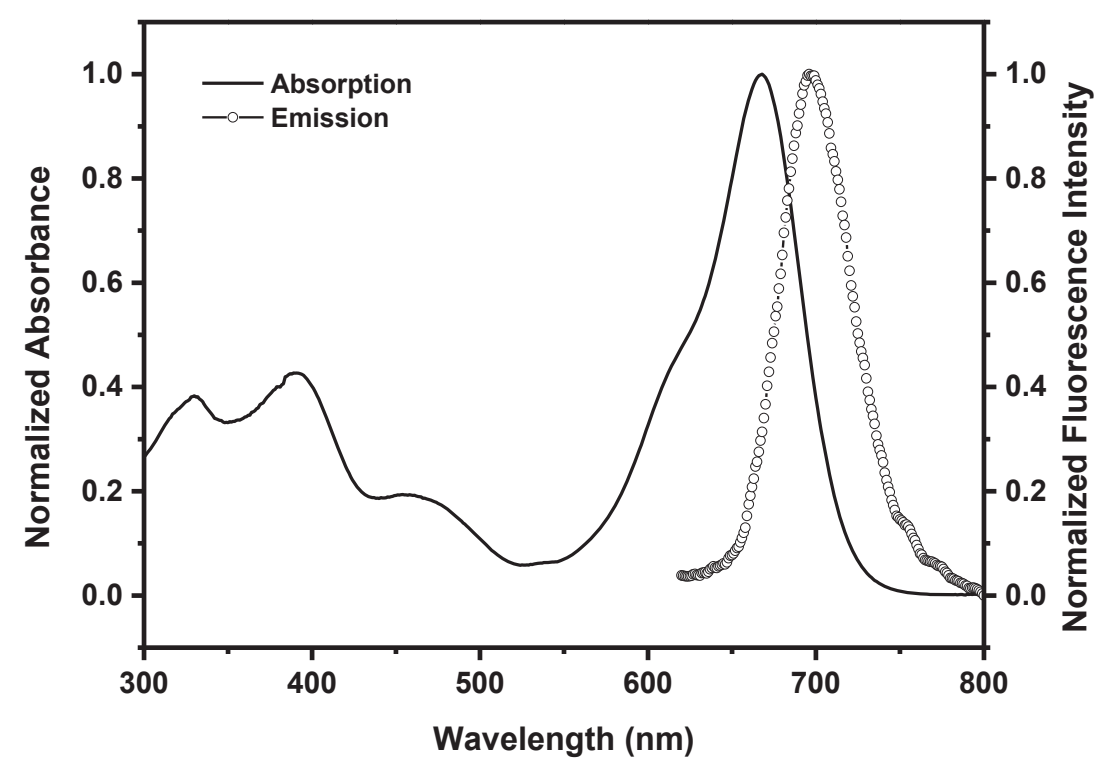

Figure F.13. Absorption and emission spectra of BODIPY dye $\mathbf{8 . 7}$ in methylene chloride solution $\left(\lambda_{\mathrm{ex}}=610 \mathrm{~nm}\right)$.

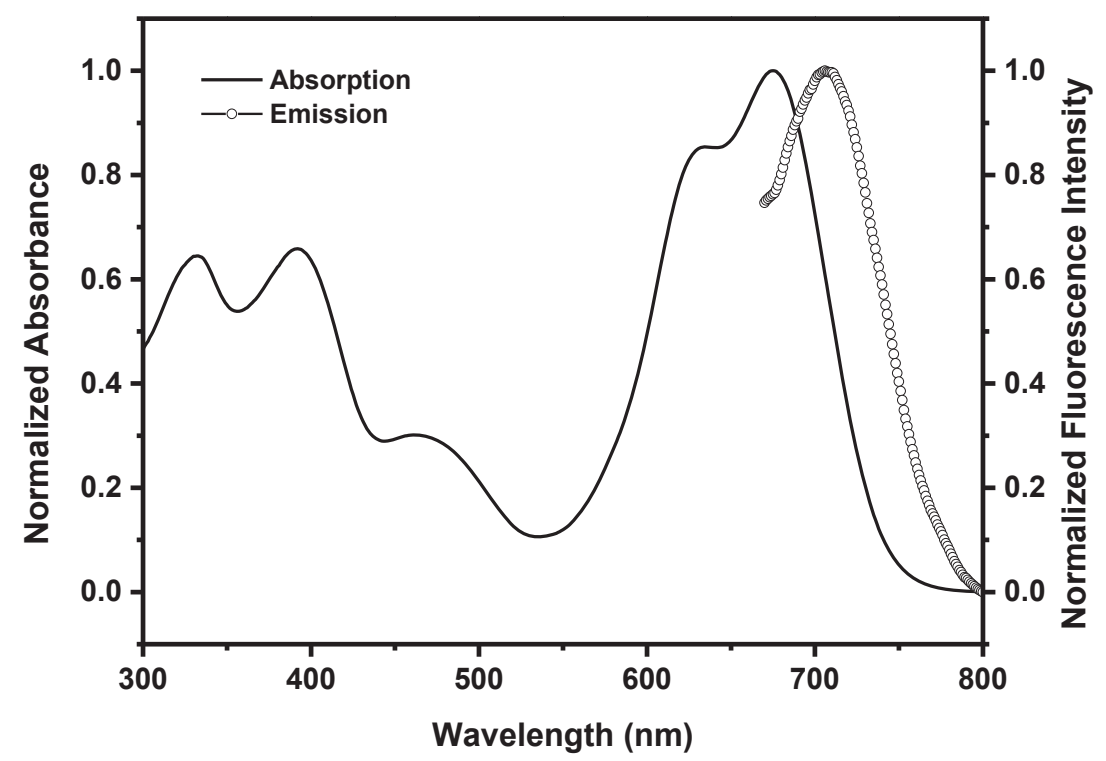

Figure F.14. Absorption and emission spectra of BODIPY dye 8.7 in aqueous solution $\left(\lambda_{\mathrm{ex}}=620 \mathrm{~nm}\right)$. 


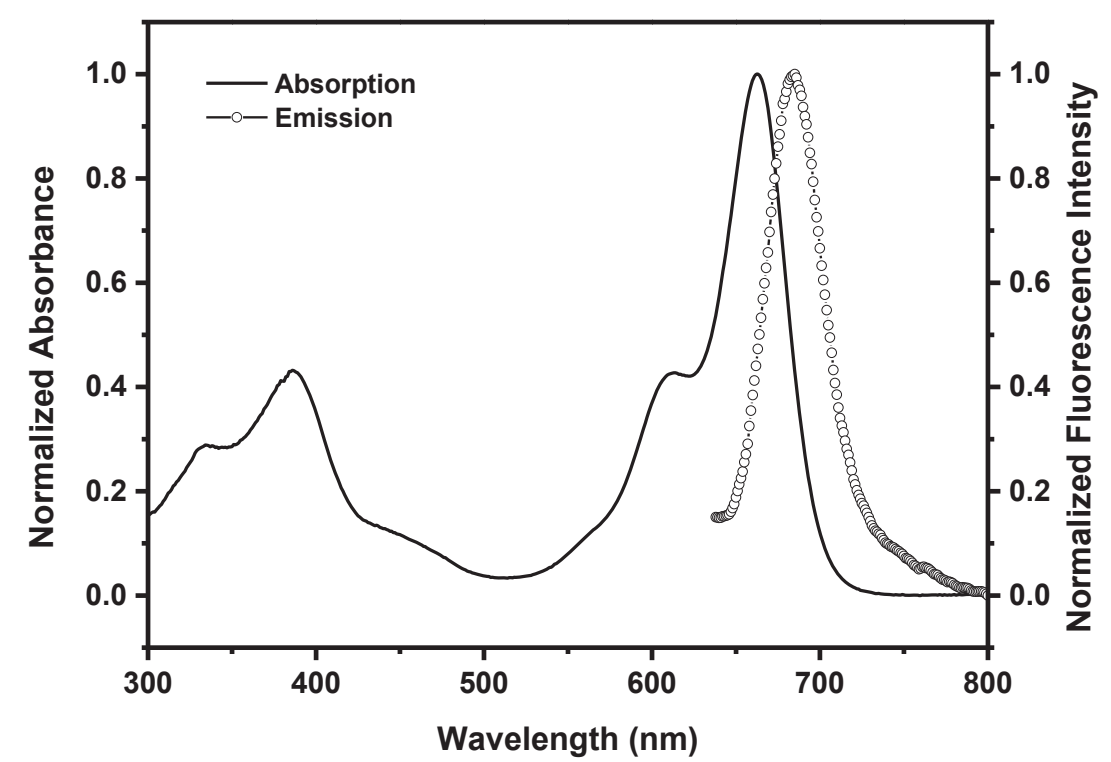

Figure F.15. Absorption and emission spectra of BODIPY dye 8.A in methylene chloride solution $\left(\lambda_{\mathrm{ex}}=610 \mathrm{~nm}\right)$.

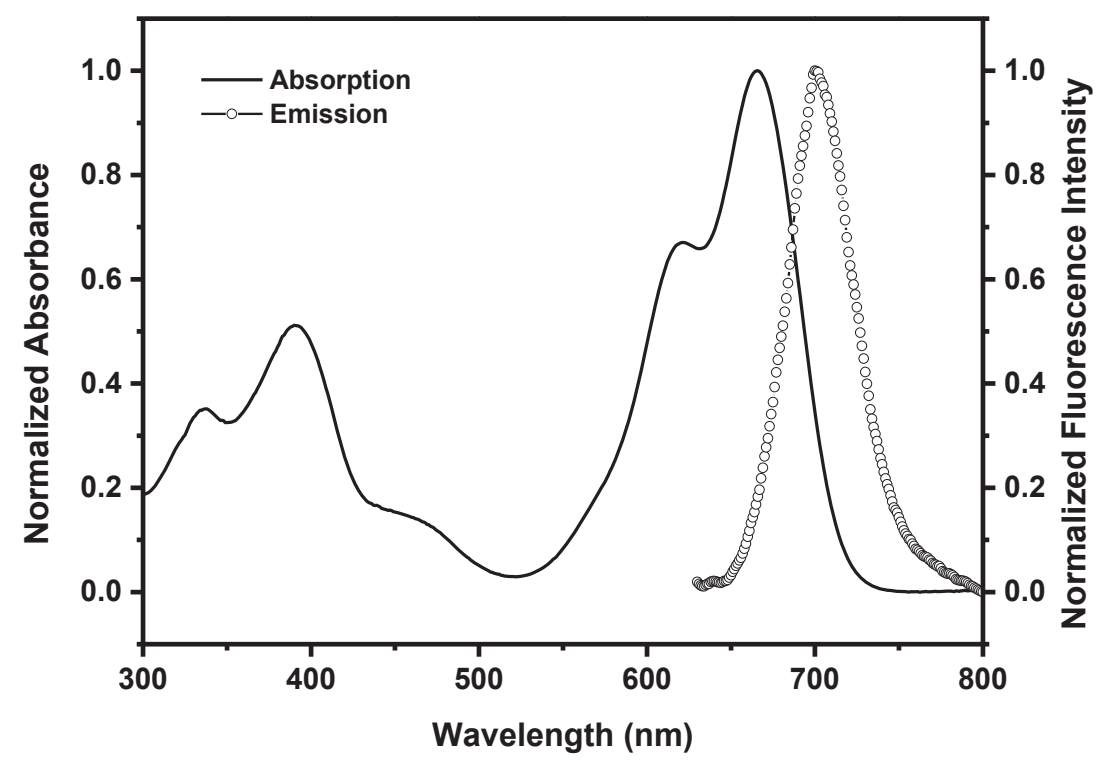

Figure F.16. Absorption and emission spectra of BODIPY dye 8.A in aqueous solution $\left(\lambda_{\mathrm{ex}}=620 \mathrm{~nm}\right)$. 


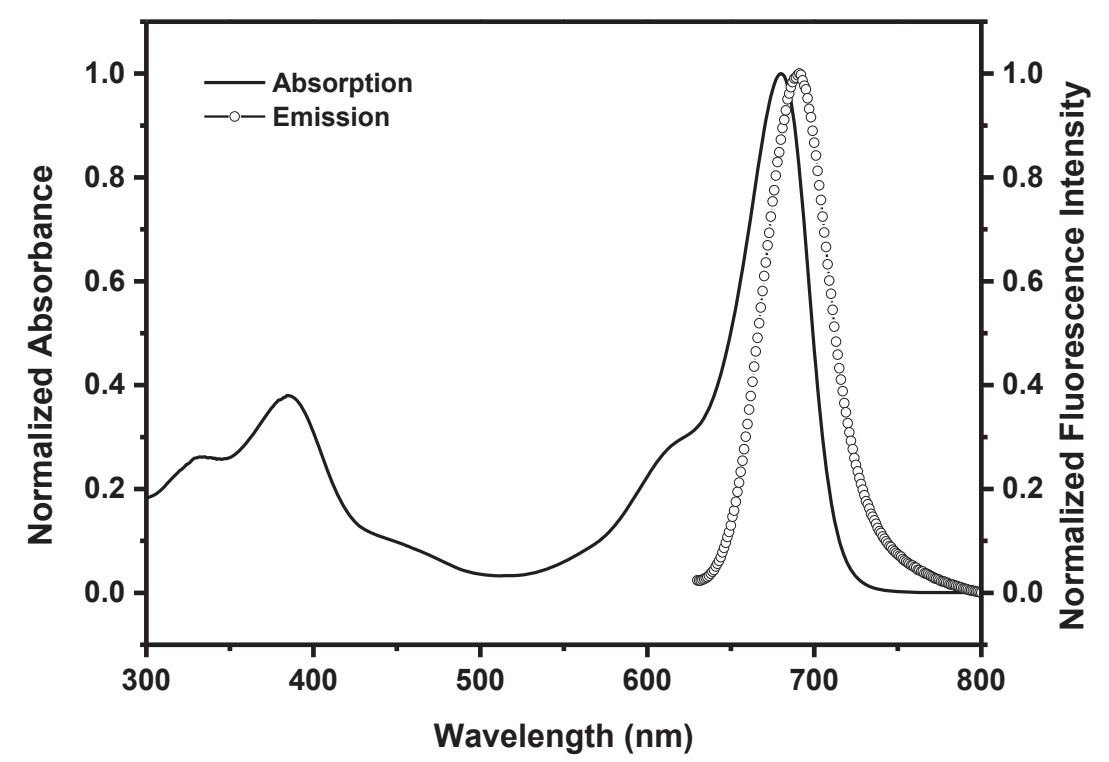

Figure F.17. Absorption and emission spectra of polymer 8-A in methylene chloride solution $\left(\lambda_{\mathrm{ex}}=610 \mathrm{~nm}\right)$.

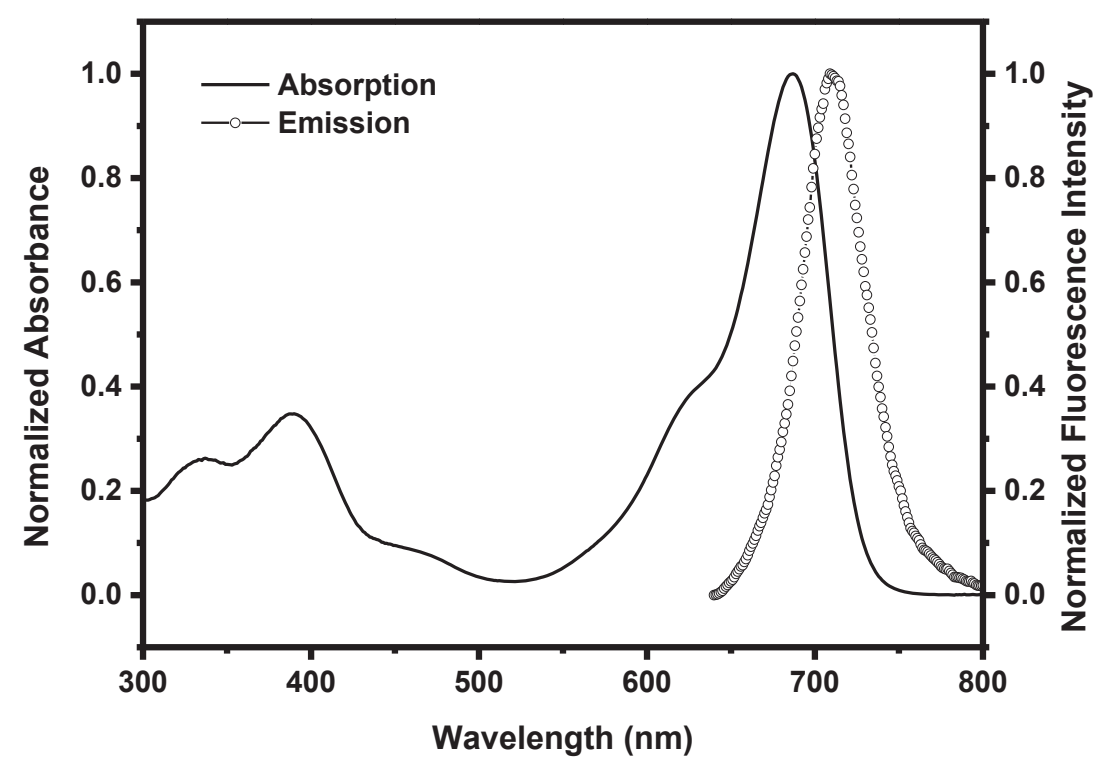

Figure F.18. Absorption and emission spectra of polymer 8-A in aqueous solution $\left(\lambda_{\mathrm{ex}}\right.$ $=620 \mathrm{~nm})$. 


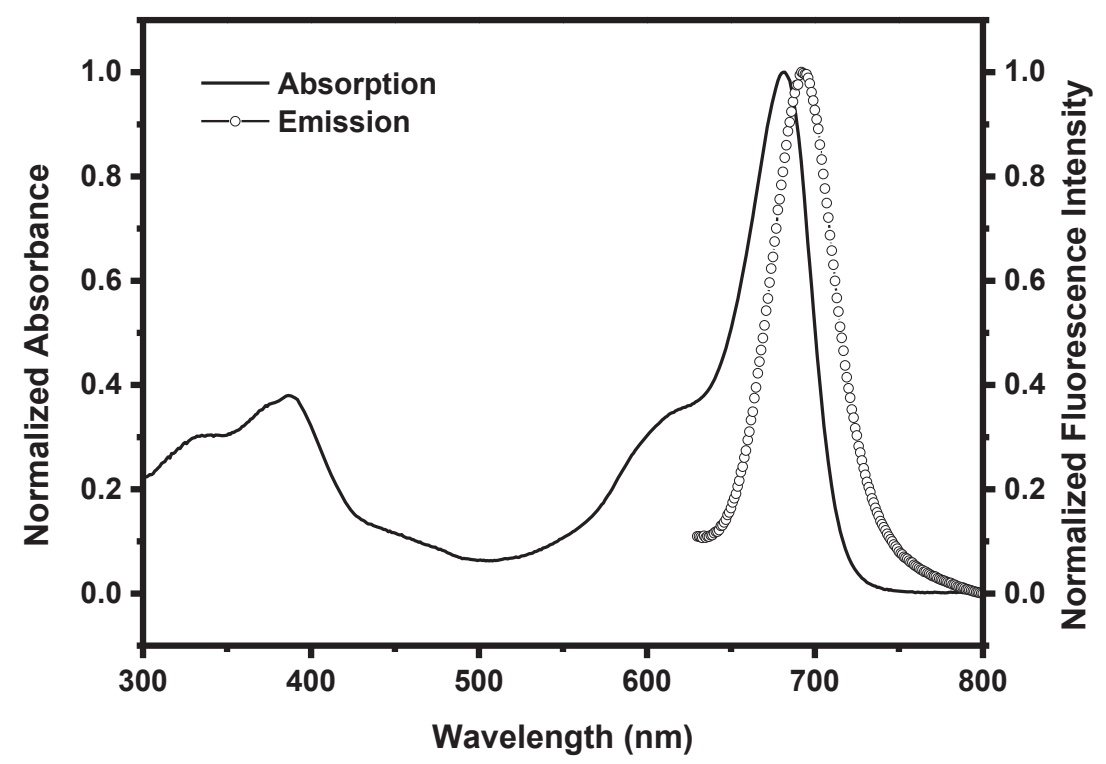

Figure F.19. Absorption and emission spectra of polymer 8-B in methylene chloride solution (excitation wavelength $=610 \mathrm{~nm})$.

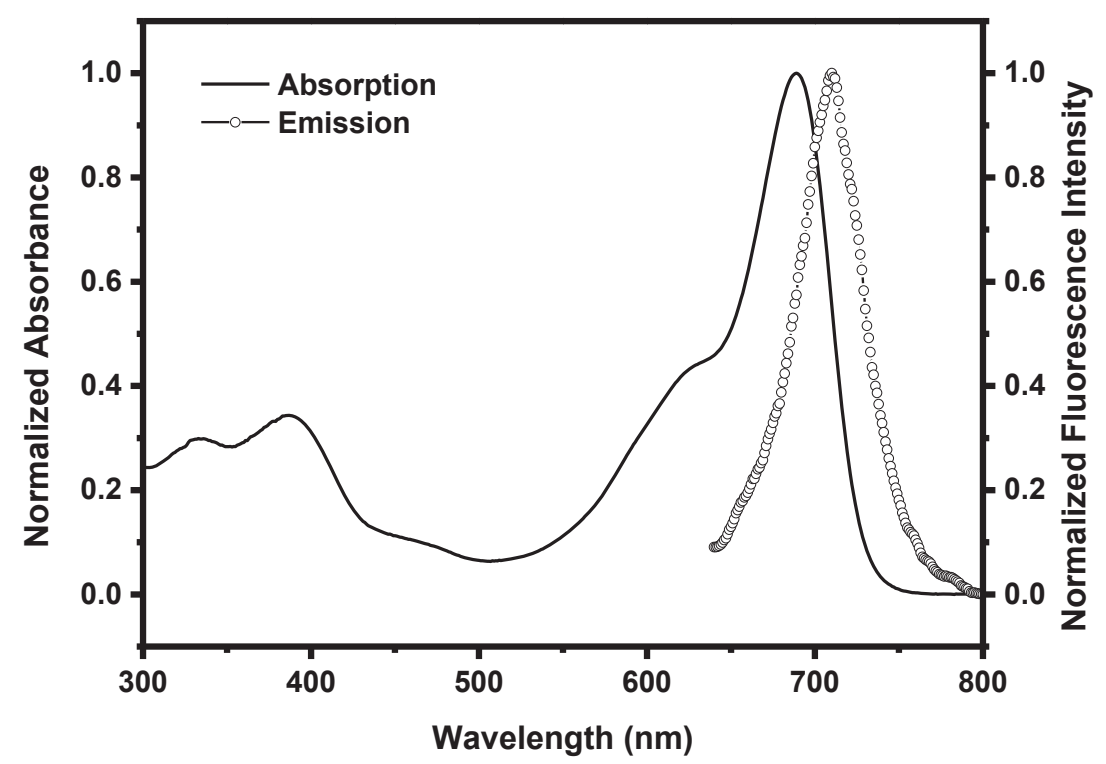

Figure F.20. Absorption and emission spectra of polymer 8-B in aqueous solution $\left(\lambda_{\text {ex }}\right.$ $=630 \mathrm{~nm})$. 
Brightfield

A

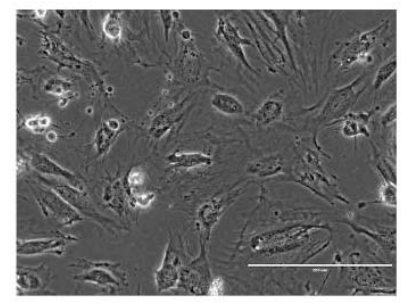

B

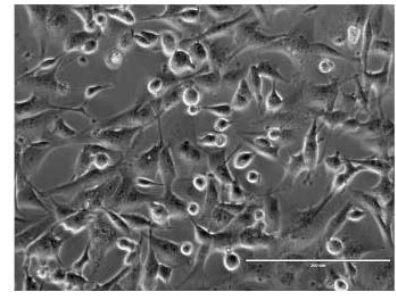

C

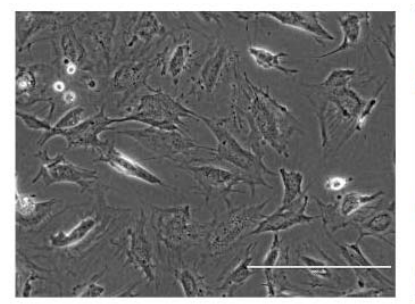

D

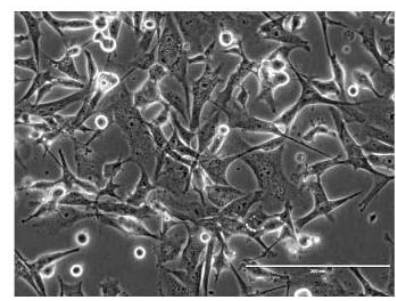

Hoechst
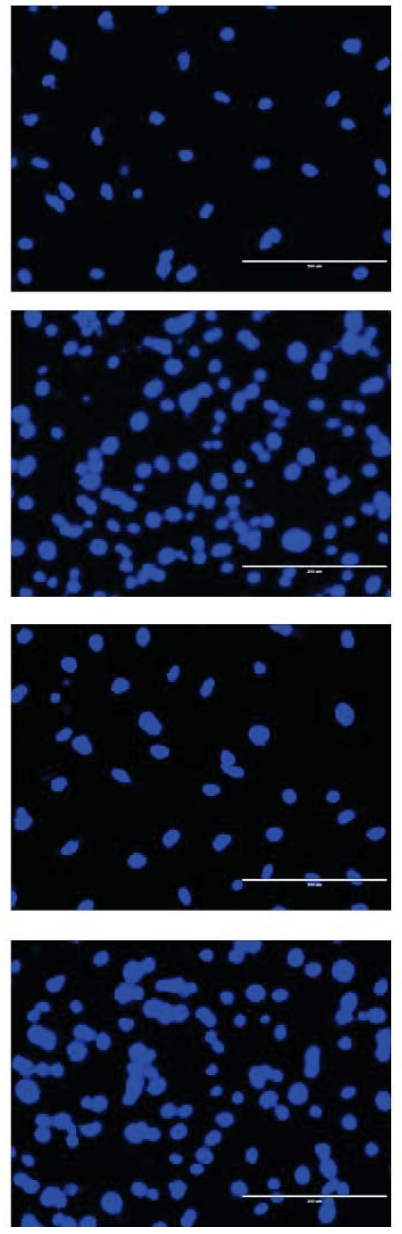

Polymer 8-A or 8-B
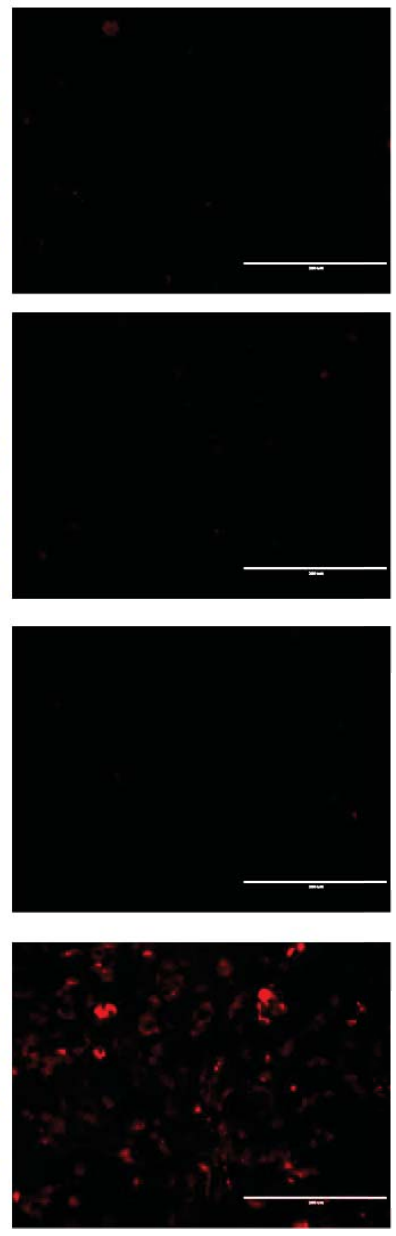

HUVEC

with

Polymer 8-

$$
\text { A }
$$

$(50 \mu \mathrm{g} / \mathrm{mL})$

MDA-MB

$-231$

Polymer 8-

A

$(50 \mu \mathrm{g} / \mathrm{mL})$

HUVEC

with

Polymer 8B

$(50 \mu \mathrm{g} / \mathrm{mL})$

MDA-MB

$-231$

Polymer 8B

$(50 \mu \mathrm{g} / \mathrm{mL})$

Figure F.21. Brightfield and fluorescence images of HUVEC-C cells (A) and (C), and MDA-MB-231 cells (B) and (D) after incubation with $50 \mu \mathrm{g} / \mathrm{ml}$ of polymer 8-A (control BODIPY dye) or polymer 8-B (RGD-bearing polymeric BODIPY dye) for 4 hours and then washing the cells with PBS before imaging.

(The bright-field images were acquired at 20X magnification with transmitted light and fluorescence images were acquired using DAPI (for Hoechst stain) and Cy5 filters (for polymers 8-A or 8-B). Hoechst stain was used for nuclear localization and is shown in the middle panel (blue)) 


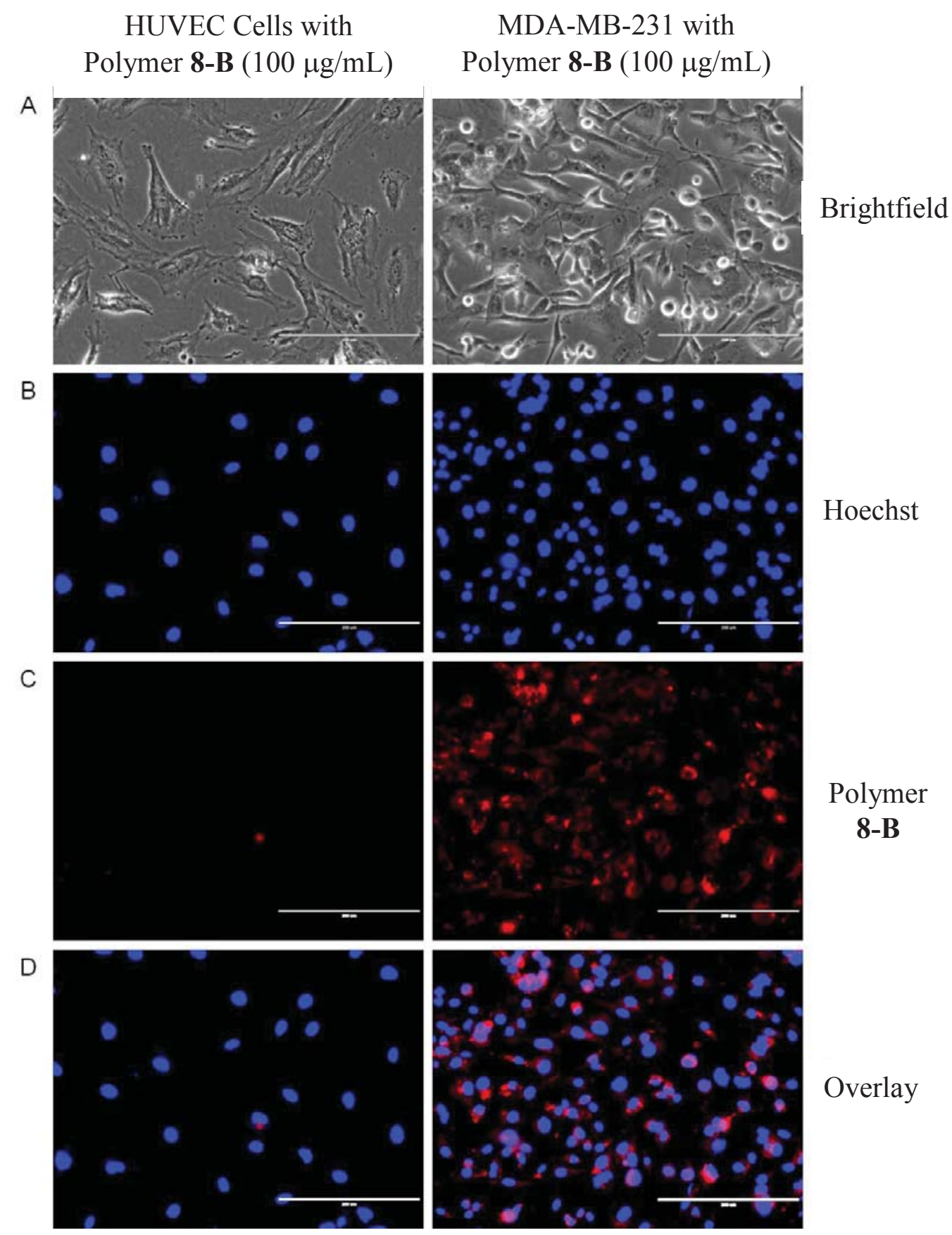

Figure F.22. Brightfield (panel A) and fluorescence images (panels B and C) of HUVEC-C cells and MDA-MB-231 cells after incubation with $100 \mu \mathrm{g} / \mathrm{ml}$ of polymer 8B (RGD-bearing polymeric BODIPY dye) for 4 hours and then washing the cells with PBS before imaging (adapted from Figure 4). 
(The bright-field images were acquired at 20X magnification with transmitted light and fluorescence images were acquired using DAPI (for Hoechst stain; panel C) and Cy5 filters (for polymer 8-B). Hoechst stain was used for nuclear localization and is shown in the middle panel (blue). Fluorescence overlay (Hoechst and Polymer 8-B) shows perinuclear localization of polymer 8-B in MDA-MB-231 cells (Panel D) with little or no incorporation in HUVEC cells)
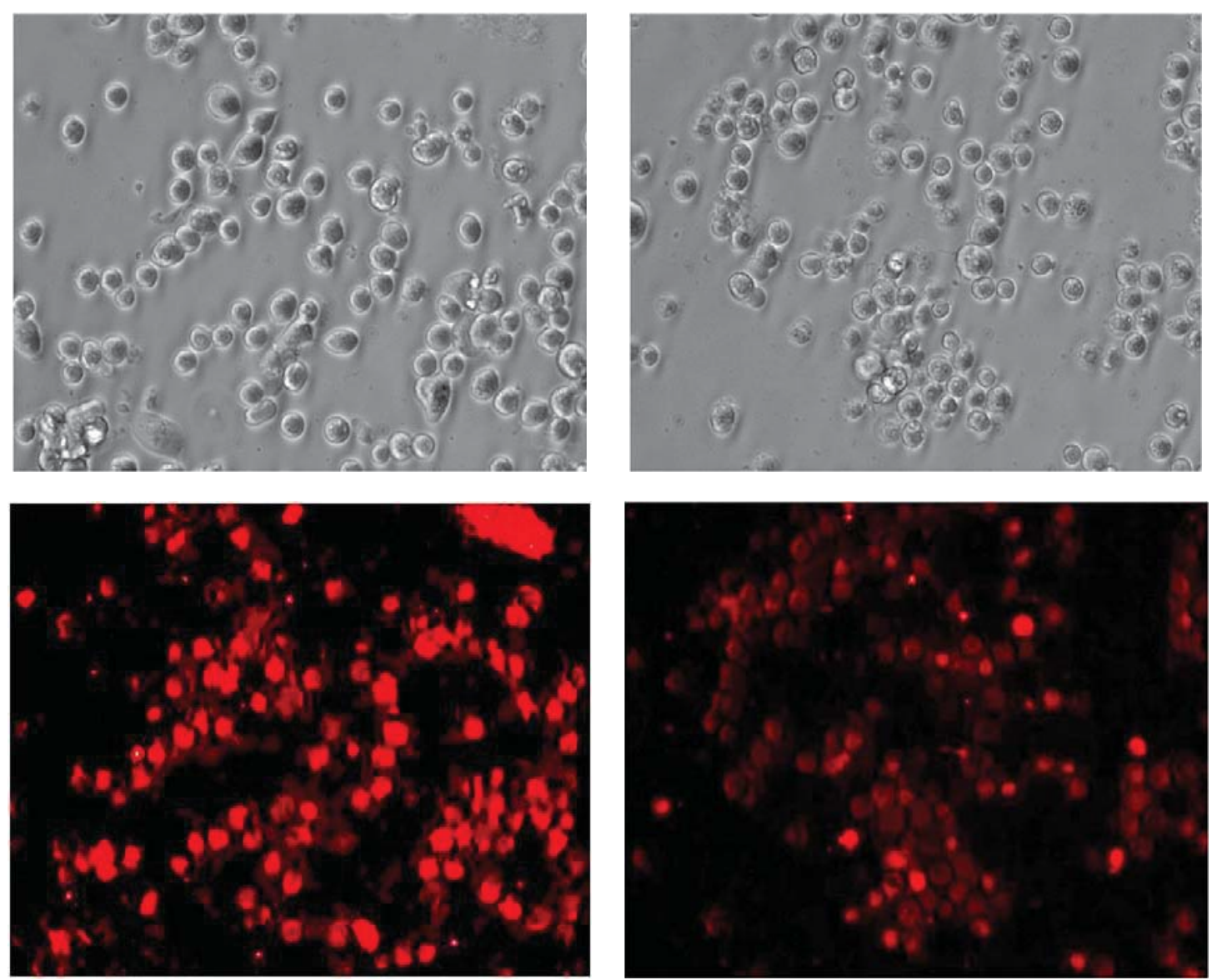

Figure F.23. Images of brightfield (upper panels) and fluorescence (lower panels) of MDA-MB-231 breast cancer cells after 4-hour incubation of $80 \mu \mathrm{g} / \mathrm{mL}$ (left panels) and $40 \mu \mathrm{g} / \mathrm{mL}$ (right panels) polymer 8-B, respectively. 


\title{
Copyright Permissions
}

\author{
This letter is for Chapter 3.
}

\section{Publication contents reuse permission from American Chemical Society (ACS)}

\author{
American Chemical Society's Policy on Theses and Dissertations \\ If your university requires you to obtain permission, you must use the RightsLink permission system. \\ See RightsLink instructions at http://pubs.acs.org/page/copvright/permissions.html.
}

This is regarding request for permission to include your paper(s) or portions of text from your paper(s) in your thesis. Permission is now automatically granted; please pay special attention to the implications paragraph below. The Copyright Subcommittee of the Joint Board/Council Committees on Publications approved the following:

Copyright permission for published and submitted material from theses and dissertations ACS extends blanket permission to students to include in their theses and dissertations their own articles, or portions thereof, that have been published in ACS journals or submitted to ACS journals for publication, provided that the ACS copyright credit line is noted on the appropriate page(s).

Publishing implications of electronic publication of theses and dissertation material Students and their mentors should be aware that posting of theses and dissertation material on the Web prior to submission of material from that thesis or dissertation to an ACS journal may affect publication in that journal. Whether Web posting is considered prior publication may be evaluated on a case-by-case basis by the journal's editor. If an ACS journal editor considers Web posting to be "prior publication", the paper will not be accepted for publication in that journal. If you intend to submit your unpublished paper to ACS for publication, check with the appropriate editor prior to posting your manuscript electronically.

Reuse/Republication of the Entire Work in Theses or Collections: Authors may reuse all or part of the Submitted, Accepted or Published Work in a thesis or dissertation that the author writes and is required to submit to satisfy the criteria of degree-granting institutions. Such reuse is permitted subject to the ACS' "Ethical Guidelines to Publication of Chemical Research" (http://pubs.acs.org/page/policy/ethics/index.html); the author should secure written confirmation (via letter or email) from the respective ACS journal editor(s) to avoid potential conflicts with journal prior publication*/embargo policies. Appropriate citation of the Published Work must be made. If the thesis or dissertation to be published is in electronic format, a direct link to the Published Work must also be included using the ACS Articles on Request author-directed link - see http:/pubs.acs.org/page/policy/articlesonrequest/index.html

* Prior publication policies of ACS journals are posted on the ACS website at http://pubs.acs.org/page/policy/prior/index.html

If vour paper has not vet been published by ACS, please print the following credit line on the first page of your article: "Reproduced (or 'Reproduced in part') with permission from [JOURNAL NAME], in press (or 'submitted for publication'). Unpublished work copyright [CURRENT YEAR] American Chemical Society." Include appropriate information.

If your paper has already been published by ACS and you want to include the text or portions of the text in your thesis/dissertation, please print the ACS copyright credit line on the first page of your article: "Reproduced (or 'Reproduced in part') with permission from [FULL REFERENCE CITATION.] Copyright [YEAR] American Chemical Society." Include appropriate information.

Submission to a Dissertation Distributor: If you plan to submit your thesis to UMI or to another dissertation distributor, you should not include the unpublished ACS paper in your thesis if the thesis will be disseminated electronically, until ACS has published your paper. After publication of the paper by ACS, you may release the entire thesis (not the individual ACS article by itself) for electronic dissemination through the distributor; ACS's copyright credit line should be printed on the first page of the ACS paper. 


\section{This letter is for the Chapter 4, 5, 6 and 7.}

\section{Publication contents permission from Royal Society of Chemistry (RSC)}

$\mathrm{RSC} \mid \begin{aligned} & \text { Advancing the } \\ & \text { Chemical Sciences }\end{aligned}$

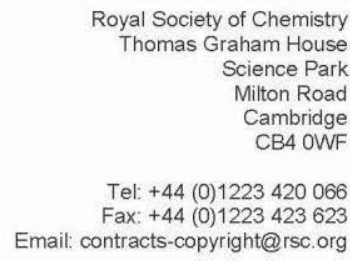

unw.rsc.org

Acknowledgements to be used by RSC authors

Authors of RSC books and journal articles can reproduce material (for example a figure) from the RSC publication in a non-RSC publication, including theses, without formally requesting permission providing that the correct acknowledgement is given to the RSC publication. This permission extends to reproduction of large portions of text or the whole article or book chapter when being reproduced in a thesis.

The acknowledgement to be used depends on the RSC publication in which the material was published and the form of the acknowledgements is as follows:

- For material being reproduced from an article in New Journal of Chemistry the acknowledgement should be in the form:

- [Original citation] - Reproduced by permission of The Royal Society of Chemistry (RSC) on behalf of the Centre National de la Recherche Scientifique (CNRS) and the RSC

- For material being reproduced from an article Photochemical \& Photobiological Sciences the acknowledgement should be in the form:

- [Original citation] - Reproduced by permission of The Royal Society of Chemistry (RSC) on behalf of the European Society for Photobiology, the European Photochemistry Association, and RSC

- For material being reproduced from an article in Physical Chemistry Chemical Physics the acknowledgement should be in the form:

- [Original citation] - Reproduced by permission of the PCCP Owner Societies

- For material reproduced from books and any other journal the acknowledgement should be in the form:

- [Original citation] - Reproduced by permission of The Royal Society of Chemistry

The acknowledgement should also include a hyperlink to the article on the RSC website.

The form of the acknowledgement is also specified in the RSC agreement/licence signed by the corresponding author.

Except in cases of republication in a thesis, this express permission does not cover the reproduction of large portions of text from the RSC publication or reproduction of the whole article or book chapter.

A publisher of a non-RSC publication can use this document as proof that permission is granted to use the material in the non-RSC publication. 
This letter is for the Figure 2.7 and Figure 2.8.

Publication contents reuse permission from Elsevier Inc.

ELSEVIER LICENSE

TERMS AND CONDITIONS

Dec 09, 2014

This is a License Agreement between Jingtuo Zhang ("You") and Elsevier ("Elsevier") provided by Copyright Clearance Center ("CCC"). The license consists of your order details, the terms and conditions provided by Elsevier, and the payment terms and conditions.

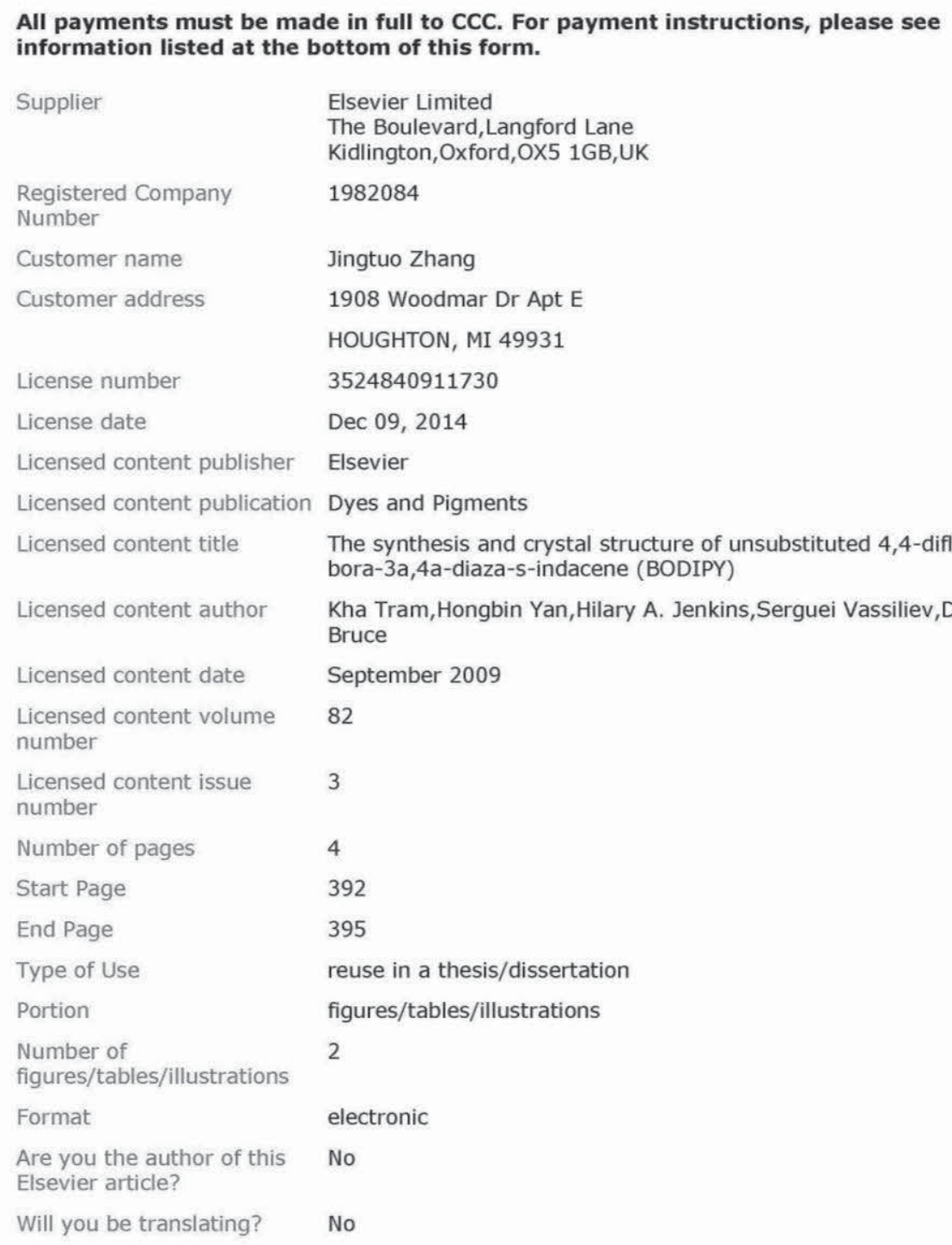


This letter is for Chapter 8 .

Publication contents reuse permission from Elsevier Inc.

ELSEVIER LICENSE

TERMS AND CONDITIONS

Dec 09, 2014

This is a License Agreement between Jingtuo Zhang ("You") and Elsevier ("Elsevier") provided by Copyright Clearance Center ("CCC"). The license consists of your order details, the terms and conditions provided by Elsevier, and the payment terms and conditions.

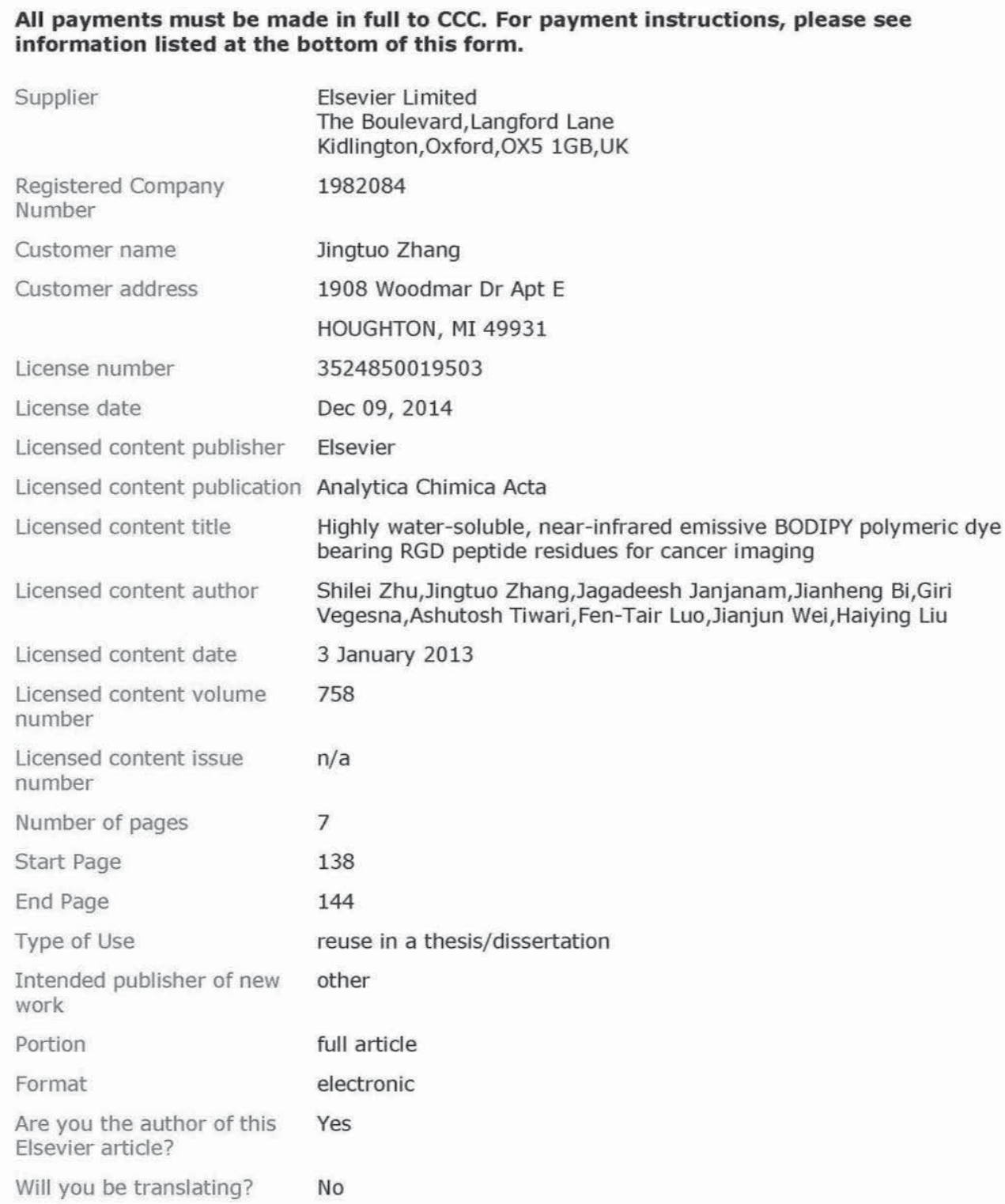

